\title{
Catalytic Enantioselective Pinacol and Meinwald Rearrangements for the Construction of Quaternary Stereocenters
}

\author{
Hua Wu, Qian Wang, and Jieping Zhu* \\ Laboratory of Synthesis and Natural Products, Institute of Chemical Sciences and Engineering, Ecole \\ Polytechnique Fédérale de Lausanne, EPFL-SB-ISIC-LSPN, BCH5304, CH-1015 Lausanne, Switzerland \\ E-mail: jieping.zhu@epfl.ch
}

\section{Table of Contents}

1. General information $\quad$ S2

2. Optimization of the reaction conditions $\quad \mathrm{S} 3$

3. General procedure S6

4. Synthesis and characterization data of compounds $\mathbf{1}$ and $\mathbf{2} \quad$ S7

5. Synthesis and characterization data of compounds 3 and $6 \quad$ S22

6. Synthetic transformations $\quad$ S42

$\begin{array}{ll}\text { 7. Crystallographic data for } \mathbf{1 a} & \text { S53 }\end{array}$

8. Copies of NMR spectra and SFC chromatograms S63

$\begin{array}{ll}\text { 9. References } & \text { S168 }\end{array}$ 


\section{General information}

NMR spectra were recorded on AV2 400 or AV2 500 MHz Bruker spectrometers. Chemical shifts are given in ppm. The spectra are calibrated to the residual ${ }^{1} \mathrm{H}$ and ${ }^{13} \mathrm{C}$ signals of the solvents. Multiplicities are abbreviated as follows: singlet (s), doublet (d), triplet (t), quartet (q), doublet-doublet (dd), quintet (quint), sextet (sext), septet (sept), multiplet (m), and broad (b). Infrared spectra were recorded on a JASCO FT/IR-4100 spectrometer. Mass spectra were determined with a Waters ACQUITY H-class UPLC/MS ACQ-SQD by electron ionization (EI positive and negative) or a Finnigan TSQ7000 by electrospray ionization (ESI+). The accurate masses were measured by the mass spectrometry service of the EPFL by ESI-TOF using a QTOF Ultima from Waters or APPI-FT-ICR using a linear ion trap Fourier transform ion cyclotron resonance mass spectrometer from Thermo Scientific. Optical rotations $\alpha_{D}$ were obtained with a Jasco P-2000 polarimeter (589 nm). Enantiomeric excesses were determined with a Thar SFC Investigator system using chiral stationary phase columns by comparing the samples with the appropriate racemic samples, column and elution details specified in each entry. Melting points were measured using a Stuart SMP30.

Materials and Methods: Unless otherwise stated, starting materials were purchased from commercial sources (Aldrich, Acros, Merck, Fluka and VWR international). More sensitive compounds were stored in a desiccator or in a glove-box if required. Solvents were purchased in HPLC quality, degassed by purging thoroughly with nitrogen and dried over activated molecular sieves of appropriate size. Alternatively, they were purged with argon and passed through alumina columns in a solvent purification system (Innovative Technology). Reactions were monitored by thin layer chromatography (TLC) using Merck TLC silica gel 60 F254. Compounds were visualized by UV-light at $254 \mathrm{~nm}$ and by dipping the plates in an ethanolic vanillin/sulfuric acid solution or an aqueous potassium permanganate solution followed by heating. Flash column chromatography was performed over silica gel (230-400 mesh). The $\mathrm{CDCl}_{3}$ used in the NMR experiments was stored over anhydrous $\mathrm{K}_{2} \mathrm{CO}_{3}$ before use. 


\section{Optimization of the reaction conditions}

Table S1. Optimization of the reaction conditions of pinacol rearrangement. ${ }^{[a]}$

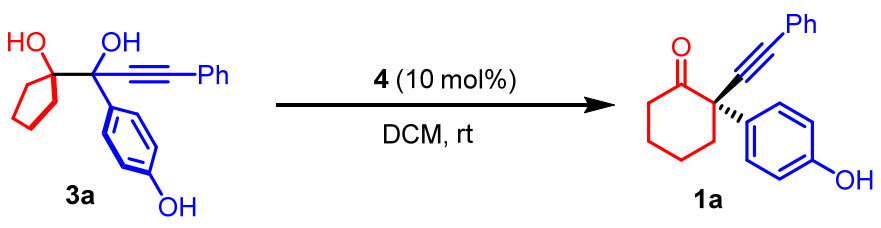

\begin{tabular}{cccccc}
\hline Entry & $\mathbf{4}$ & Solvent & $\mathrm{T}\left({ }^{\circ} \mathrm{C}\right)$ & Yield $(\%)^{[\mathrm{b}]}$ & $E e(\%)^{[\mathrm{c}]}$ \\
\hline 1 & $\mathbf{4 a}$ & DCM & rt & 95 & 45 \\
2 & $\mathbf{4 b}$ & DCM & $\mathrm{rt}$ & 96 & 22 \\
3 & $\mathbf{4 c}$ & DCM & $\mathrm{rt}$ & 89 & 25 \\
4 & $\mathbf{4 d}$ & DCM & $\mathrm{rt}$ & 97 & 0 \\
5 & $\mathbf{4 e}$ & DCM & $\mathrm{rt}$ & 88 & 44 \\
6 & $\mathbf{4 f}$ & DCM & $\mathrm{rt}$ & 94 & 13 \\
7 & $\mathbf{4 g}$ & DCM & $\mathrm{rt}$ & 97 & 44 \\
8 & $\mathbf{4 h}$ & DCM & $\mathrm{rt}$ & 87 & 32 \\
9 & $\mathbf{4 i}$ & DCM & $\mathrm{rt}$ & 93 & 38 \\
10 & $\mathbf{4 a}$ & THF & $\mathrm{rt}$ & $-[\mathrm{e}]$ & - \\
11 & $\mathbf{4 a}$ & EtOAc & $\mathrm{rt}$ & $-[\mathrm{e}]$ & - \\
12 & $\mathbf{4 a}$ & CyH & $\mathrm{rt}$ & 92 & 70 \\
13 & $\mathbf{4 a}$ & Toluene & $\mathrm{rt}$ & 98 & 80 \\
14 & $\mathbf{4 a}$ & PhF & $\mathrm{rt}$ & 96 & 75 \\
$15^{[\mathrm{d}]}$ & $\mathbf{4 a}$ & Toluene & 10 & 97 & 86 \\
$16^{[\mathrm{d}]}$ & $\mathbf{4 a}$ & Toluene & 0 & 99 & 88 \\
$17^{[\mathrm{d}]}$ & $\mathbf{4 a}$ & Toluene & -5 & 99 & $90^{[\mathrm{e}]}$
\end{tabular}

[a] 3a (0.1 mmol), chiral Brønsted acid $4(0.01 \mathrm{mmol})$, solvent $(2.0 \mathrm{~mL}), \mathrm{T}{ }^{\circ} \mathrm{C}, 12 \mathrm{~h}$. [b] Isolated yields. [c] Determined by SFC analysis on a chiral stationary phase. [d] $3 \AA$ molecular sieves $(100 \mathrm{mg}), 3 \mathrm{~d}$. [e] No reaction.<smiles>CNP(=O)(Oc1cnccn1)Oc1c(Br)cc2ccccc2c1-c1cc2ccccc2cc1Br</smiles>

4a $\mathrm{Ar}=2,4,6-\left(\mathrm{Pr}_{3}\right)_{3} \mathrm{C}_{6} \mathrm{H}_{2}$ 4b $\mathrm{Ar}=9$-anthracenyl 4c $\mathrm{Ar}=4-\mathrm{NO}_{2} \mathrm{C}_{6} \mathrm{H}_{4}$ 4d $\mathrm{Ar}=\mathrm{SiPh}_{3}$<smiles>O=P(O)(O)Oc1c(Br)cc2ccccc2c1-c1c(Br)cc2ccccc2c1Br</smiles>

4e $\mathrm{Ar}=9$-phenanthracenyl 4f $\mathrm{Ar}=2,4,6-(c-\mathrm{Pent})_{3} \mathrm{C}_{6} \mathrm{H}_{2}$ 4g $\mathrm{Ar}=2,4,6-\left(\mathrm{Pr}_{3}\right)_{3} \mathrm{C}_{6} \mathrm{H}_{2}$ 4h $\mathrm{Ar}=$ biphenyl<smiles>O=P(O)(N=P(O)(Oc1c(Br)cc2ccccc2c1Br)Oc1c(Br)cc2ccccc2c1-c1cc2ccccc2cc1Br)Oc1c(Br)cc2ccccc2c1Br</smiles>

4i $\mathrm{Ar}=2-\mathrm{iPr}-5-\mathrm{MeC}_{6} \mathrm{H}_{3}$ 
Table S2. Optimization of the reaction conditions of Meinwald rearrangement. ${ }^{\text {[a] }}$

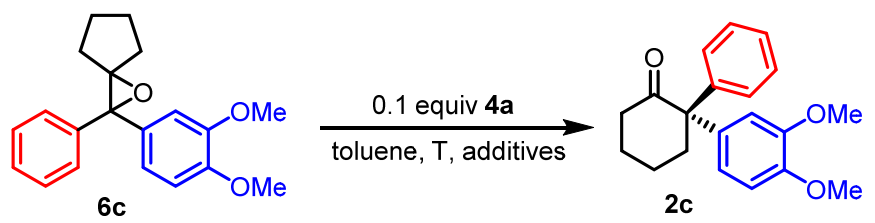

\begin{tabular}{ccccc}
\hline Entry & Additives & $\mathrm{T}\left({ }^{\circ} \mathrm{C}\right)$ & ${\text { Yield }(\%)^{[\mathrm{b}]}}^{\text {Ee }(\%)^{[\mathrm{cc}]}}$ \\
\hline 1 & - & -40 & 95 & 81 \\
2 & $3 \AA \mathrm{MS}$ & -40 & 99 & 81 \\
3 & - & -78 & 97 & 78 \\
4 & $5 \AA \mathrm{MS}$ & -40 & 93 & 82 \\
5 & $4 \AA \mathrm{MS}$ & -20 & 96 & 84 \\
6 & $4 \AA \mathrm{MS}$ & -40 & 97 & 85 \\
7 & $4 \AA \mathrm{MS}$ & -60 & 96 & 88 \\
8 & $4 \AA \mathrm{MS}$ & -78 & 97 & 89
\end{tabular}

[a] 6c $(0.1 \mathrm{mmol})$, chiral Brønsted acid $4 \mathbf{a}(0.01 \mathrm{mmol})$, toluene $(2.0 \mathrm{~mL})$, additives $(100 \mathrm{mg}), \mathrm{T}^{\circ} \mathrm{C}, 12 \mathrm{~h}$. [b] Isolated yields. [c] Determined by HPLC.

Table S3. Details for the adjustments to temperature for different types of substrates. ${ }^{[a]}$

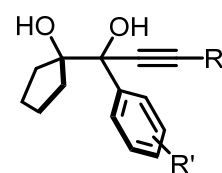

$3 \mathrm{j}, 3 \mathrm{~m}, 3 \mathrm{o}, 3 \mathrm{r}, 3 \mathrm{u}$

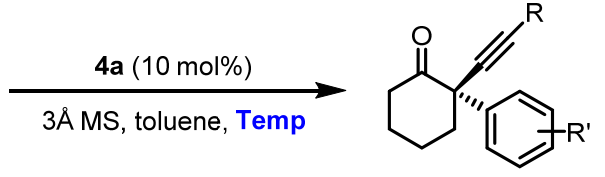

$1 \mathrm{j}, 1 \mathrm{~m}, 1 \mathrm{o}, 1 \mathrm{r}, 1 \mathrm{u}$

\begin{tabular}{cccccc}
\hline Entry & Substrate & Temp $\left({ }^{\circ} \mathrm{C}\right)$ & Time $(\mathrm{d})$ & Yield $(\%)^{[\mathrm{b}]}$ & $E e(\%)^{[\mathrm{c}]}$ \\
\hline 1 & $3 \mathrm{j}$ & -5 & 3 & 95 & 81 \\
2 & $3 \mathrm{j}$ & -10 & 3 & 96 & 84 \\
3 & $3 \mathrm{~m}$ & $\mathrm{RT}$ & 0.5 & 93 & 62 \\
4 & $3 \mathrm{~m}$ & -10 & 1 & 97 & 72 \\
5 & $3 \mathrm{~m}$ & -40 & 2 & 94 & 84 \\
6 & $3 \mathrm{~m}$ & -50 & 2 & 95 & 84 \\
7 & $3 \mathrm{~m}$ & -78 & 2 & trace & [d] \\
8 & $3 \mathrm{~d}$ & -50 & 3 & 81 & 92 \\
9 & 30 & -40 & 4 & 94 & 92 \\
10 & $3 \mathrm{r}$ & -50 & 2 & 44 & 84 \\
11 & $3 \mathrm{r}$ & -40 & 2 & 73 & 83 \\
12 & $3 \mathbf{r}$ & -30 & 3 & 82 & 83 \\
13 & $3 \mathrm{u}$ & -10 & 1 & 85 & 66 \\
14 & $3 \mathrm{u}$ & -50 & 3 & 83 & $81^{[\mathrm{e}]}$ \\
15 & $6 \mathrm{j}$ & -60 & 0.5 & 83 & 57 \\
\hline
\end{tabular}




\begin{tabular}{llllll}
\hline 16 & $6 \mathbf{j}$ & -90 & 2 & 76 & 65
\end{tabular}

[a] $3(0.1 \mathrm{mmol})$, chiral Brønsted acid $4 \mathbf{a}(0.01 \mathrm{mmol})$, toluene $(2.0 \mathrm{~mL}), 3 \AA \mathrm{MS}(100 \mathrm{mg}), \mathrm{T}^{\circ} \mathrm{C} .[\mathrm{b}]$ Isolated yields. [c] Determined by SFC analysis on a chiral stationary phase. [d] Not detected. [e] $15 \mathrm{~mol} \%$ of $\mathbf{4 a}$.

\section{Scheme S1. Comparison of reactivity between 1,2-diols and epoxides.}

(a)<smiles>COc1ccc(C2(c3cc(C)cc(C)c3)OC23CCCC3)cc1OC</smiles>

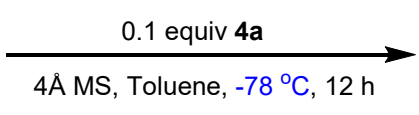<smiles>COc1ccc(C2(c3cc(C)cc(C)c3)CCCCC2=O)cc1OC</smiles>

(b)<smiles>COc1ccc(C(O)(c2cc(C)cc(C)c2)C2(O)CCCC2)cc1OC</smiles>

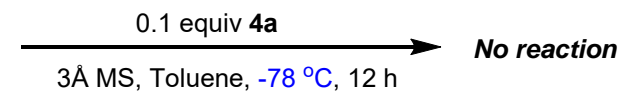

(c)<smiles>COc1ccc(C(O)(c2cc(C)cc(C)c2)C2(O)CCCC2)cc1OC</smiles><smiles>COc1cc(C)cc([C@@]2(c3ccc(OC)c(OC)c3)CCCCC2=O)c1</smiles>
$98 \%$ yield, $77 \%$ ee

(d)<smiles>OC1(C(O)(C#Cc2ccccc2)c2ccccc2)CCCC1</smiles>

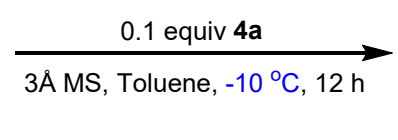

No reaction

(e)<smiles>OC1(C(O)(C#Cc2ccccc2)c2ccccc2)CCCC1</smiles>

(f)

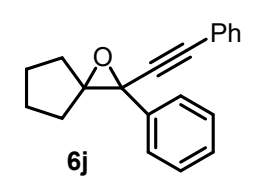

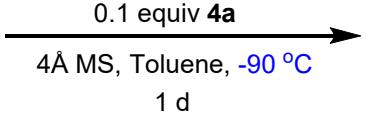

$1 \mathrm{~d}$

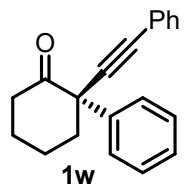

$7 \%$ yield, $34 \%$ ee low conversion of $3 w$

$$
76 \% \text { yield, } 65 \% \text { ee }
$$
full conversion of $\mathbf{6 j}$

Conclusion: Much higher reactivity of tetrasubstituted epoxides than their 1,2-diols form towards the rearrangement has been observed. 


\section{General procedure}

General procedure for the synthesis of racemic 1 and 2

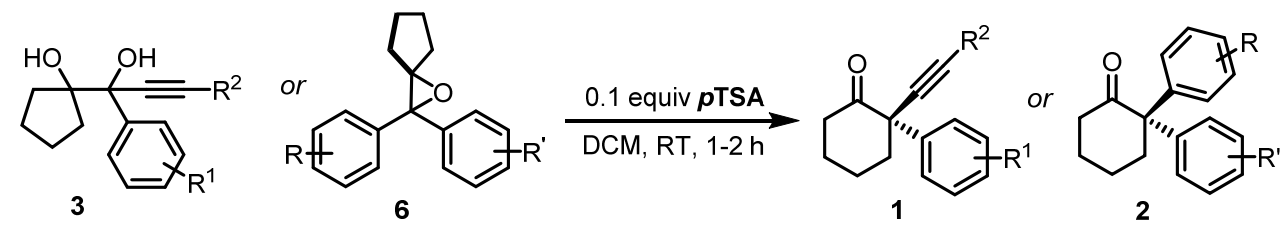

To a solution of 3 or $\mathbf{6}(0.1 \mathrm{mmol}, 1.0$ equiv $)$ in DCM $(1.0 \mathrm{~mL})$ was added pTSA $(0.01 \mathrm{mmol}$, 0.1 equiv). The reaction mixture was stirred at room temperature in 2 hours. After completion of the reaction (monitored by TLC), the solvent was removed under vacuum. The residue was purified by column chromatography on silica gel, eluting with petroleum ether/ethyl acetate to afford the products $\mathbf{1}$ or $\mathbf{2}$ in good to high yields.

General procedure for the catalytic enantioselective pinacol rearrangement

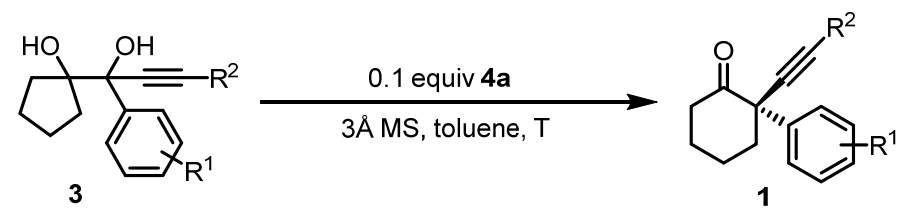

To a dry sealed tube charged with $3 \AA$ molecular sieves $(100 \mathrm{mg})$ was added a solution of $3(0.1$ mmol, 1.0 equiv) in toluene $(1.0 \mathrm{~mL})$. A solution of $4 \mathbf{a}(0.01 \mathrm{mmol}, 0.1$ equiv) in toluene (1.0 $\mathrm{mL}$ ) was added to the above mixture at the indicated temperature. The reaction mixture was stirred at the same temperature for the indicated time. After completion of the reaction (monitored by TLC), $\mathrm{MeOH}(0.5 \mathrm{~mL})$ was added and the solvent was removed under vacuum. The residue was purified by column chromatography on silica gel, eluting with DCM/THF to afford the products 1 .

General procedure for the catalytic enantioselective Meinwald rearrangement

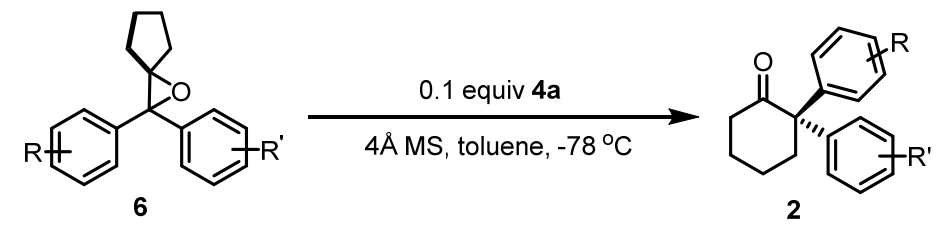

To a dry sealed tube charged with $4 \AA$ molecular sieves $(100 \mathrm{mg})$ was added a solution of $6(0.1$ mmol, 1.0 equiv) in toluene $(1.0 \mathrm{~mL})$. A solution of $\mathbf{4 a}(0.01 \mathrm{mmol}, 0.1$ equiv) in toluene (1.0 $\mathrm{mL}$ ) was added to the above mixture at $-78{ }^{\circ} \mathrm{C}$. The reaction mixture was stirred at the same temperature for the indicated time. After completion of the reaction (monitored by TLC), 
$\mathrm{MeOH}(0.5 \mathrm{~mL})$ was added and the solvent was removed under vacuum. The residue was purified by column chromatography on silica gel, eluting with DCM/THF to afford the products 2 .

\section{Synthesis and characterization data of compounds 1 and 2}

(S)-2-(4-hydroxyphenyl)-2-(phenylethynyl)cyclohexan-1-one (1a)

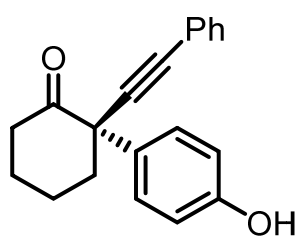

$-5{ }^{\circ} \mathrm{C}, 3$ d. $99 \%$ yield, $90 \%$ ee, colorless crystal from DCM/THF. M.p $=178-180{ }^{\circ} \mathrm{C}$.

${ }^{1}$ H NMR (400 MHz, Chloroform-d) $\delta 7.54-7.42(\mathrm{~m}, 2 \mathrm{H}), 7.38$ - 7.31 (m, 5H), 6.88 - 6.76

(m, 2H), $3.17(\mathrm{td}, J=13.3,6.0 \mathrm{~Hz}, 1 \mathrm{H}), 2.58-2.43(\mathrm{~m}, 1 \mathrm{H}), 2.40-2.24(\mathrm{~m}, 3 \mathrm{H}), 2.20-2.11$ (m, 1H), $1.99-1.74(\mathrm{~m}, 2 \mathrm{H})$.

${ }^{13}$ C NMR (101 MHz, Chloroform- $d$ ) $\delta$ 207.6, 155.0, 131.8, 131.5, 129.2, 128.6, 128.5, 123.0, $115.3,89.7,88.8,56.0,41.7,39.4,27.9,22.8$.

IR $\left(v_{\max }, \mathrm{cm}^{-1}\right) 3422(\mathrm{w}), 2947(\mathrm{~m}), 2365$ (w), 1713 (s), 1513 (s), 1223 (s), $910(\mathrm{~m}), 832(\mathrm{~m})$, 757 (s), 690 (s).

HRMS (ESI/QTOF) m/z: [M + Na $]^{+}$Calcd for $\mathrm{C}_{20} \mathrm{H}_{18} \mathrm{NaO}_{2}{ }^{+}$313.1199; Found 313.1203.

$[\alpha]^{26} \mathrm{D}+177.5$ (c $\left.1.85, \mathrm{THF}\right), 90 \%$ ee.

SFC: OD-H column, $10.0 \% \mathrm{MeOH}$ in supercritical $\mathrm{CO}_{2}$ as eluent, $4 \mathrm{~mL} / \mathrm{min} . \mathrm{tR}=6.5 \mathrm{~min}$ (minor), 7.6 min (major).

(S)-2-(4-hydroxyphenyl)-2-(p-tolylethynyl)cyclohexan-1-one (1b)

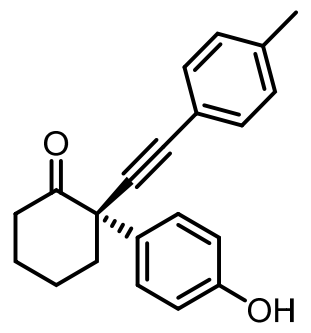

$-5{ }^{\circ} \mathrm{C}, 3$ d. $98 \%$ yield, $94 \%$ ee, colorless oil.

${ }^{1}$ H NMR (400 MHz, Chloroform- $d$ ) $\delta 7.40-7.32(\mathrm{~m}, 4 \mathrm{H}), 7.14(\mathrm{~d}, J=7.4 \mathrm{~Hz}, 2 \mathrm{H}), 6.81$ (d, $J$ $=7.3 \mathrm{~Hz}, 2 \mathrm{H}), 5.09(\mathrm{~s}, 1 \mathrm{H}), 3.18(\mathrm{td}, J=13.3,5.8 \mathrm{~Hz}, 1 \mathrm{H}), 2.54-2.41(\mathrm{~m}, 1 \mathrm{H}), 2.36(\mathrm{~s}, 3 \mathrm{H})$, $2.34-2.23(\mathrm{~m}, 3 \mathrm{H}), 2.21-2.08(\mathrm{~m}, 1 \mathrm{H}), 1.95-1.76(\mathrm{~m}, 2 \mathrm{H})$. 
${ }^{13}$ C NMR (101 MHz, Chloroform- $d$ ) $\delta$ 207.5, 154.9, 138.7, 131.7, 131.7, 129.3, 129.2, 119.9, $115.2,88.9,88.9,56.0,41.8,39.4,27.9,22.9,21.6$.

IR $\left(v_{\max }, \mathrm{cm}^{-1}\right) 2340(\mathrm{~m}), 2160$ (s), 2019 (m), $1729(\mathrm{w}), 1513$ (m), 760 (s), 730 (s), 691 (s), 664 $(\mathrm{s})$.

HRMS (ESI/QTOF) m/z: [M + Na] ${ }^{+}$Calcd for $\mathrm{C}_{21} \mathrm{H}_{20} \mathrm{NaO}_{2}{ }^{+}$327.1356; Found 327.1357.

$[\alpha]^{26} \mathrm{D}+273.7$ ( c $\left.0.52, \mathrm{DCM}\right), 94 \%$ ee.

SFC: OD-H column, $7.0 \% \mathrm{MeOH}$ in supercritical $\mathrm{CO}_{2}$ as eluent, $4 \mathrm{~mL} / \mathrm{min}$. $\mathrm{tR}=12.1 \mathrm{~min}$ (minor), $14.2 \mathrm{~min}$ (major).

(S)-2-([1,1'-biphenyl]-4-ylethynyl)-2-(4-hydroxyphenyl)cyclohexan-1-one (1c)

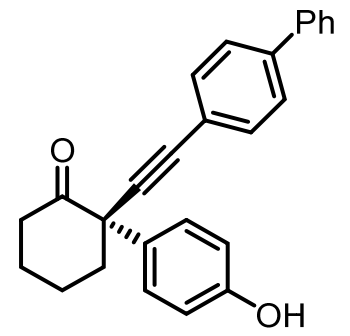

$-5{ }^{\circ} \mathrm{C}, 5$ d. $75 \%$ yield, $91 \%$ ee, white solid. M.p $=150-152{ }^{\circ} \mathrm{C}$.

${ }^{1}$ H NMR (400 MHz, Chloroform-d) $\delta 7.63$ - $7.52(\mathrm{~m}, 6 \mathrm{H}), 7.52$ - 7.42 (m, 2H), 7.42 - 7.31 (m, 3H), $6.87-6.81(\mathrm{~m}, 2 \mathrm{H}), 4.89$ (brs, 1H), $3.24-3.14(\mathrm{~m}, 1 \mathrm{H}), 2.55-2.44(\mathrm{~m}, 1 \mathrm{H}), 2.43-$ $2.24(\mathrm{~m}, 3 \mathrm{H}), 2.24-2.09(\mathrm{~m}, 1 \mathrm{H}), 1.98-1.75(\mathrm{~m}, 2 \mathrm{H})$.

${ }^{13}$ C NMR (101 MHz, Chloroform-d) $\delta$ 207.0, 154.8, 141.2, 140.3, 132.1, 131.6, 129.1, 128.9, $127.7,127.0,121.8,115.1,90.3,88.5,55.9,41.7,39.3,27.7,22.8$.

IR $\left(v_{\max }, \mathrm{cm}^{-1}\right) 3428(\mathrm{w}), 2947(\mathrm{w}), 2359(\mathrm{w}), 1719(\mathrm{~m}), 1517(\mathrm{~m}), 839(\mathrm{~m}), 761(\mathrm{~s}), 700(\mathrm{~m})$, $1224(\mathrm{~m})$.

HRMS (ESI/QTOF) m/z: [M+ H $]^{+}$Calcd for $\mathrm{C}_{26} \mathrm{H}_{23} \mathrm{O}_{2}{ }^{+}$367.1693; Found 367.1696.

$[\alpha]^{26} \mathrm{D}+212.0$ ( с $\left.0.32, \mathrm{DCM}\right), 91 \%$ ee.

SFC: OD-H column, $20.0 \% \mathrm{MeOH}$ in supercritical $\mathrm{CO}_{2}$ as eluent, $4 \mathrm{~mL} / \mathrm{min}$. $\mathrm{tR}=7.2 \mathrm{~min}$ (minor), 8.4 min (major).

(S)-2-((4-fluorophenyl)ethynyl)-2-(4-hydroxyphenyl)cyclohexan-1-one (1d)

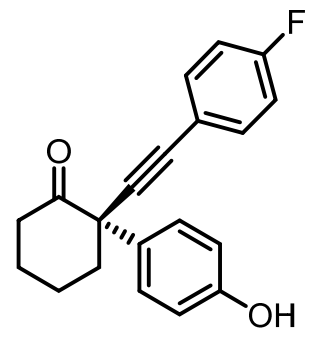


$-5{ }^{\circ} \mathrm{C}, 2$ d. $97 \%$ yield, $92 \%$ ee, colorless oil.

${ }^{1}$ H NMR (400 MHz, Chloroform-d) $\delta 7.52$ - 7.40 (m, 2H), 7.34 - 7.28 (m, 2H), 7.06 - 6.98 $(\mathrm{m}, 2 \mathrm{H}), 6.85-6.73(\mathrm{~m}, 2 \mathrm{H}), 5.39(\mathrm{~s}, 1 \mathrm{H}), 3.20-3.03(\mathrm{~m}, 1 \mathrm{H}), 2.56-2.41(\mathrm{~m}, 1 \mathrm{H}), 2.41-$ $2.20(\mathrm{~m}, 3 \mathrm{H}), 2.19-2.07(\mathrm{~m}, 1 \mathrm{H}), 1.98-1.74(\mathrm{~m}, 2 \mathrm{H})$.

${ }^{13}$ C NMR (101 MHz, Chloroform-d) $\delta 207.7,162.7$ (d, $\left.J=249.6 \mathrm{~Hz}\right), 155.1,133.7$ (d, $J=8.4$ Hz), 131.3, 129.1, 119.0 (d, $J=3.6 \mathrm{~Hz}), 115.8$ (d, $J=22.2 \mathrm{~Hz}), 115.3,89.5$ (d, $J=1.5 \mathrm{~Hz})$, $87.6,55.9,41.5,39.4,27.8,22.8$.

${ }^{19}$ F NMR (377 MHz, Chloroform- $\left.d\right) \delta-110.7$.

IR $\left(v_{\max }, \mathrm{cm}^{-1}\right) 3399(\mathrm{w}), 2936(\mathrm{w}), 2158(\mathrm{w}), 1715(\mathrm{~m}), 1509$ (s), 1226 (s), $836(\mathrm{~s})$.

HRMS (ESI/QTOF) m/z: [M+ H] ${ }^{+}$Calcd for $\mathrm{C}_{20} \mathrm{H}_{18} \mathrm{FO}_{2}{ }^{+}$309.1285; Found 309.1294.

$[\alpha]^{26} \mathrm{D}+205.2$ ( c 1.50, DCM), 92\% ee.

SFC: OD-H column, $6.0 \% \mathrm{MeOH}$ in supercritical $\mathrm{CO}_{2}$ as eluent, $4 \mathrm{~mL} / \mathrm{min}$. $\mathrm{tR}=11.6 \mathrm{~min}$ (minor), 13.4 min (major).

(S)-2-(4-hydroxyphenyl)-2-((4-methoxyphenyl)ethynyl)cyclohexan-1-one (1e)

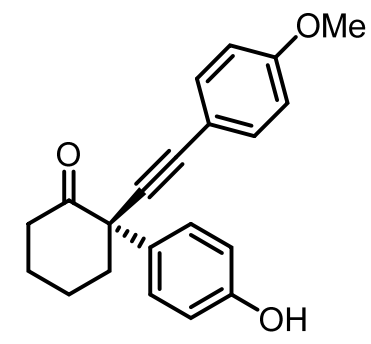

$-5{ }^{\circ} \mathrm{C}, 4$ d. $81 \%$ yield, $87 \%$ ee, colorless oil.

${ }^{1}$ H NMR (400 MHz, Chloroform- $d$ ) $\delta 7.42(\mathrm{~d}, J=8.8 \mathrm{~Hz}, 2 \mathrm{H}), 7.33(\mathrm{~d}, J=8.7 \mathrm{~Hz}, 2 \mathrm{H}), 6.86$ $(\mathrm{d}, J=8.8 \mathrm{~Hz}, 2 \mathrm{H}), 6.81$ (d, $J=8.7 \mathrm{~Hz}, 2 \mathrm{H}), 5.53(\mathrm{~s}, 1 \mathrm{H}), 3.82(\mathrm{~s}, 3 \mathrm{H}), 3.17(\mathrm{td}, J=13.5,6.1$ $\mathrm{Hz}, 1 \mathrm{H}), 2.50-2.42(\mathrm{~m}, 1 \mathrm{H}), 2.35-2.23(\mathrm{~m}, 3 \mathrm{H}), 2.20-2.07(\mathrm{~m}, 1 \mathrm{H}), 1.93-1.76(\mathrm{~m}, 2 \mathrm{H})$. ${ }^{13}$ C NMR (101 MHz, Chloroform-d) $\delta$ 207.9, 159.8, 155.1, 133.2, 131.6, 129.2, 115.3, 115.1, $114.1,88.7,88.2,56.0,55.5,41.8,39.3,27.8,22.8$.

IR $\left(v_{\max }, \mathrm{cm}^{-1}\right) 2936(\mathrm{~m}), 2161(\mathrm{~s}), 2022(\mathrm{~s}), 1716(\mathrm{~m}), 1606$ (m), 1510 (s), 1249 (s), 1178 (s), $838(\mathrm{~s}), 759$ (s).

HRMS (ESI/QTOF) m/z: [M+ H $]^{+}$Calcd for $\mathrm{C}_{21} \mathrm{H}_{21} \mathrm{O}_{3}{ }^{+}$321.1485; Found 321.1486.

$[\alpha]^{26} \mathrm{D}+199.3$ (c 1.1, DCM), 87\% ee.

SFC: OD-H column, $10.0 \% \mathrm{MeOH}$ in supercritical $\mathrm{CO}_{2}$ as eluent, $4 \mathrm{~mL} / \mathrm{min}$. $\mathrm{tR}=8.9 \mathrm{~min}$ (minor), 10.2 min (major).

(S)-2-((3-fluorophenyl)ethynyl)-2-(4-hydroxyphenyl)cyclohexan-1-one (1f) 


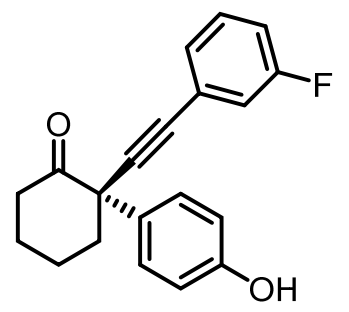

$-5{ }^{\circ} \mathrm{C}, 3$ d. $94 \%$ yield, $91 \%$ ee, white solid. M.p $=180-182{ }^{\circ} \mathrm{C}$.

${ }^{1}$ H NMR (400 MHz, Chloroform-d) $\delta 7.31$ - 7.14 (m, 4H), 7.13 - 7.08 (m, 1H), 7.00 - 6.94 (m, 1H), $6.81-6.70(\mathrm{~m}, 2 \mathrm{H}), 4.94(\mathrm{~s}, 1 \mathrm{H}), 3.11-2.95(\mathrm{~m}, 1 \mathrm{H}), 2.45-2.38(\mathrm{~m}, 1 \mathrm{H}), 2.33-$ $2.01(\mathrm{~m}, 4 \mathrm{H}), 1.93-1.68(\mathrm{~m}, 2 \mathrm{H})$.

${ }^{13}$ C NMR (101 MHz, Chloroform- $d$ ) $\delta 207.0,162.5(\mathrm{~d}, J=246.6 \mathrm{~Hz}), 155.0,131.3,130.1$ (d, $J=8.7 \mathrm{~Hz}), 129.2,127.7(\mathrm{~d}, J=3.1 \mathrm{~Hz}), 124.8(\mathrm{~d}, J=9.6 \mathrm{~Hz}), 118.6(\mathrm{~d}, J=22.8 \mathrm{~Hz}), 115.9$ $(\mathrm{d}, J=21.2 \mathrm{~Hz}), 115.3,90.8,87.4,55.9,41.5,39.4,27.9,22.8$.

${ }^{19}$ F NMR (377 MHz, Chloroform- $d$ ) $\delta-112.85$.

IR $\left(v_{\max }, \mathrm{cm}^{-1}\right) 3412(\mathrm{~m}), 2930(\mathrm{~m}), 2159(\mathrm{~m}), 1716(\mathrm{~m}), 1514(\mathrm{~s}), 1267(\mathrm{~m}), 837(\mathrm{~m}), 748(\mathrm{~s})$, $726(\mathrm{~s})$.

HRMS (ESI/QTOF) m/z: [M+ H] ${ }^{+}$Calcd for $\mathrm{C}_{20} \mathrm{H}_{18} \mathrm{FO}_{2}{ }^{+}$309.1285; Found 309.1290.

$[\alpha]^{26} \mathrm{D}+158.1$ (c 1.0, THF), $91 \%$ ee.

SFC: OD-H column, $7.0 \% \mathrm{MeOH}$ in supercritical $\mathrm{CO}_{2}$ as eluent, $4 \mathrm{~mL} / \mathrm{min}$. $\mathrm{tR}=8.8 \mathrm{~min}$ (minor), 10.4 min (major).

(S)-2-((3-chlorophenyl)ethynyl)-2-(4-hydroxyphenyl)cyclohexan-1-one (1g)

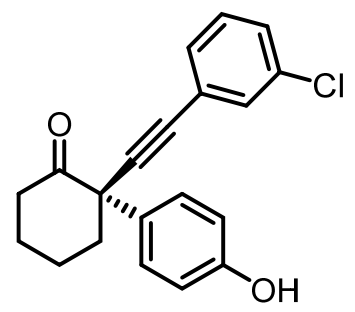

$-5{ }^{\circ} \mathrm{C}, 48$ h. $98 \%$ yield, $88 \%$ ee, white solid. M.p $=164-166^{\circ} \mathrm{C}$.

${ }^{1}$ H NMR (400 MHz, Chloroform-d) $\delta 7.47(\mathrm{~s}, 1 \mathrm{H}), 7.38-7.24(\mathrm{~m}, 5 \mathrm{H}), 6.83(\mathrm{~d}, J=8.4 \mathrm{~Hz}$, 2H), 5.12 (s, 1H), 3.11 (td, $J=13.1,5.9 \mathrm{~Hz}, 1 \mathrm{H}), 2.52-2.45(\mathrm{dt}, J=13.8,4.3 \mathrm{~Hz}, 1 \mathrm{H}), 2.41-$ $2.09(\mathrm{~m}, 4 \mathrm{H}), 1.96-1.75(\mathrm{~m}, 2 \mathrm{H})$.

${ }^{13}$ C NMR (101 MHz, Chloroform-d) $\delta$ 207.0, 155.1, 134.3, 131.7, 131.3, 129.9, 129.7, 129.1, $128.9,124.7,115.3,91.1,87.2,55.9,41.5,39.4,27.8,22.8$.

IR $\left(v_{\max }, \mathrm{cm}^{-1}\right) 3409$ (m), 2941 (m), 1707 (s), 1517 (s), 1178 (s), 790 (s), 756 (s), 691 (s).

HRMS (ESI/QTOF) m/z: [M + Na] ${ }^{+}$Calcd for $\mathrm{C}_{20} \mathrm{H}_{17} \mathrm{ClNaO}_{2}{ }^{+} 347.0809$; Found 347.0811. $[\alpha]^{26} \mathrm{D}+190.7$ (c 1.60, DCM), 88\% ee. 
SFC: OD-H column, $10.0 \% \mathrm{MeOH}$ in supercritical $\mathrm{CO}_{2}$ as eluent, $4 \mathrm{~mL} / \mathrm{min}$. $\mathrm{tR}=7.8 \mathrm{~min}$ (minor), $9.1 \mathrm{~min}$ (major).

(S)-2-(4-hydroxyphenyl)-2-((3-methoxyphenyl)ethynyl)cyclohexan-1-one (1h)

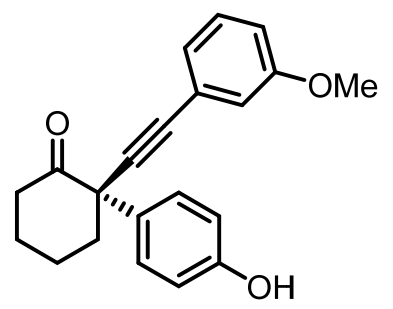

$-5{ }^{\circ} \mathrm{C}, 3$ d. $93 \%$ yield, $92 \%$ ee, colorless oil.

${ }^{1}$ H NMR (400 MHz, Chloroform- $d$ ) $\delta 7.38$ (d, $\left.J=7.8 \mathrm{~Hz}, 2 \mathrm{H}\right), 7.31-7.27$ (m, 1H), 7.12 (d, $J$ $=7.4 \mathrm{~Hz}, 1 \mathrm{H}), 7.04(\mathrm{~s}, 1 \mathrm{H}), 6.93(\mathrm{~d}, J=8.3 \mathrm{~Hz}, 1 \mathrm{H}), 6.86(\mathrm{~d}, J=7.8 \mathrm{~Hz}, 2 \mathrm{H}), 5.12(\mathrm{~s}, 1 \mathrm{H})$, $3.85(\mathrm{~s}, 3 \mathrm{H}), 3.20(\mathrm{td}, J=13.2,5.7 \mathrm{~Hz}, 1 \mathrm{H}), 2.55-2.47(\mathrm{~m}, 1 \mathrm{H}), 2.43-2.28(\mathrm{~m}, 3 \mathrm{H}), 2.25$ $2.14(\mathrm{~m}, 1 \mathrm{H}), 2.00-1.82(\mathrm{~m}, 2 \mathrm{H})$.

${ }^{13}$ C NMR (101 MHz, Chloroform-d) $\delta$ 207.3, 159.5, 155.0, 131.6, 129.6, 129.2, 124.4, 124.0, $116.7,115.2,115.1,89.6,88.6,56.0,55.5,41.7,39.4,27.9,22.9$.

IR $\left(v_{\max }, \mathrm{cm}^{-1}\right) 3396(\mathrm{~m}), 2947(\mathrm{~m}), 2371(\mathrm{~m}), 2162(\mathrm{~s}), 2023(\mathrm{~m}), 1707$ (s), $1600(\mathrm{~s}), 1513$ (s), $1211(\mathrm{~s}), 1176(\mathrm{~s}), 1043$ (m), 791 (s), 720 (s).

HRMS (ESI/QTOF) m/z: [M+ H $]^{+}$Calcd for $\mathrm{C}_{21} \mathrm{H}_{21} \mathrm{O}_{3}{ }^{+}$321.1485; Found 321.1492.

$[\alpha]^{26} \mathrm{D}+246.1$ ( с $\left.0.35, \mathrm{DCM}\right), 92 \%$ ee.

SFC: OD-H column, $6.0 \% \mathrm{MeOH}$ in supercritical $\mathrm{CO}_{2}$ as eluent, $4 \mathrm{~mL} / \mathrm{min}$. $\mathrm{tR}=20.1 \mathrm{~min}$ (minor), $22.9 \mathrm{~min}$ (major).

(S)-2-(4-hydroxyphenyl)-2-(o-tolylethynyl)cyclohexan-1-one (1i)

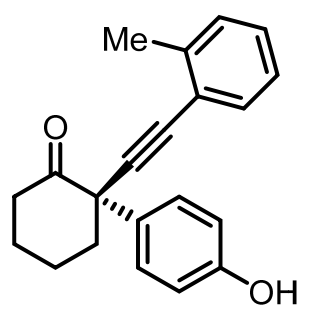

$-5{ }^{\circ} \mathrm{C}, 3$ d. $97 \%$ yield, $90 \%$ ee, colorless oil.

${ }^{1}$ H NMR (400 MHz, Chloroform-d) $\delta 7.46(\mathrm{~d}, J=7.6 \mathrm{~Hz}, 1 \mathrm{H}), 7.36(\mathrm{~d}, J=8.6 \mathrm{~Hz}, 2 \mathrm{H}), 7.25$ $-7.20(\mathrm{~m}, 2 \mathrm{H}), 7.19-7.13(\mathrm{~m}, 1 \mathrm{H}), 6.81(\mathrm{~d}, J=8.7 \mathrm{~Hz}, 2 \mathrm{H}), 5.18(\mathrm{~s}, 1 \mathrm{H}), 3.19$ (td, $J=13.4$, $6.1 \mathrm{~Hz}, 1 \mathrm{H}), 2.53-2.49(\mathrm{~m}, 1 \mathrm{H}), 2.47$ (s, 3H), $2.40-2.29$ (m, 3H), $2.22-2.12(\mathrm{~m}, 1 \mathrm{H}), 1.97$ - 1.77 (m, 2H).

${ }^{13}$ C NMR (101 MHz, Chloroform-d) $\delta$ 207.3, 154.9, 140.2, 132.1, 131.6, 129.5, 129.1, 128.4, $125.6,122.7,115.1,93.5,87.6,56.1,41.7,39.2,27.7,22.7,21.0$. 
IR $\left(v_{\max }, \mathrm{cm}^{-1}\right) 3393(\mathrm{~m}), 2949$ (m), 2360 (m), 1716 (s), 1516 (s), 1444 (s), 1219 (s), 828 (m), $759(\mathrm{~s}), 720(\mathrm{~m})$.

HRMS (ESI/QTOF) m/z: [M + Na $]^{+}$Calcd for $\mathrm{C}_{21} \mathrm{H}_{20} \mathrm{NaO}_{2}{ }^{+}$327.1356; Found 327.1364.

$[\alpha]^{26} \mathrm{D}+197.3$ (c $\left.0.75, \mathrm{DCM}\right), 90 \%$ ee.

SFC: OD-H column, $10.0 \% \mathrm{MeOH}$ in supercritical $\mathrm{CO}_{2}$ as eluent, $4 \mathrm{~mL} / \mathrm{min}$. $\mathrm{tR}=6.7 \mathrm{~min}$ (minor), 7.9 min (major).

(S)-2-(4-hydroxyphenyl)-2-(thiophen-3-ylethynyl)cyclohexan-1-one (1j)

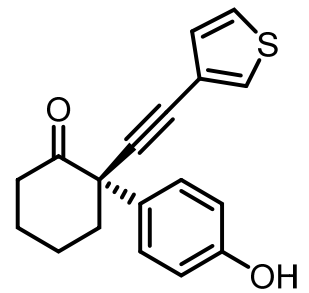

$-10{ }^{\circ} \mathrm{C}, 3$ d. $96 \%$ yield, $84 \%$ ee, colorless oil.

${ }^{1}$ H NMR (400 MHz, Chloroform- $d$ ) $\delta 7.48(\mathrm{dd}, J=2.9,1.2 \mathrm{~Hz}, 1 \mathrm{H}), 7.34-7.26(\mathrm{~m}, 3 \mathrm{H})$,

$7.15(\mathrm{dd}, J=4.9,1.2 \mathrm{~Hz}, 1 \mathrm{H}), 6.87-6.76(\mathrm{~m}, 2 \mathrm{H}), 5.28(\mathrm{~s}, 1 \mathrm{H}), 3.20-3.07$ (m, 1H), 2.53 -

$2.41(\mathrm{~m}, 1 \mathrm{H}), 2.38-2.20(\mathrm{~m}, 3 \mathrm{H}), 2.18-2.08(\mathrm{~m}, 1 \mathrm{H}), 1.96-1.75(\mathrm{~m}, 2 \mathrm{H})$.

${ }^{13}$ C NMR (101 MHz, Chloroform-d) $\delta$ 207.5, 155.1, 131.5, 130.0, 129.2, 128.9, 125.5, 122.0,

$115.3,89.3,83.8,56.0,41.6,39.4,27.8,22.8$.

IR $\left(v_{\max }, \mathrm{cm}^{-1}\right) 3371(\mathrm{~m}), 2947(\mathrm{~m}), 1713$ (s), $1512(\mathrm{~s}), 1222(\mathrm{~m}), 1179(\mathrm{~m}), 789$ (s), 692 (s).

HRMS (ESI/QTOF) m/z: [M+ Na] $]^{+}$Calcd for $\mathrm{C}_{18} \mathrm{H}_{16} \mathrm{NaO}_{2} \mathrm{~S}^{+} 319.0763$; Found 319.0760.

$[\alpha]^{26} \mathrm{D}+221.8$ ( c 1.35, DCM), 84\% ee.

SFC: AD-H column, $5.0 \% \mathrm{MeOH}$ in supercritical $\mathrm{CO}_{2}$ as eluent, $4 \mathrm{~mL} / \mathrm{min}$. $\mathrm{tR}=28.0 \mathrm{~min}$ (major), $33.6 \mathrm{~min}$ (minor).

(S)-2-(3,3-dimethylbut-1-yn-1-yl)-2-(4-hydroxyphenyl)cyclohexan-1-one (1k)

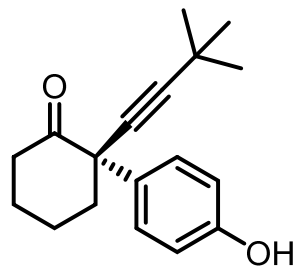

$-5{ }^{\circ} \mathrm{C}, 48$ h. $99 \%$ yield, $94 \%$ ee, white solid. M.p $=197-199{ }^{\circ} \mathrm{C}$.

${ }^{1}$ H NMR (400 MHz, Chloroform-d) $\delta 7.27(\mathrm{~d}, J=8.7 \mathrm{~Hz}, 2 \mathrm{H}), 6.76(\mathrm{~d}, J=8.7 \mathrm{~Hz}, 2 \mathrm{H}), 5.39$ (s, 1H), 3.15 (td, $J=13.6,6.0 \mathrm{~Hz}, 1 \mathrm{H}), 2.44-2.33$ (m, 1H), $2.31-2.18$ (m, 1H), $2.18-2.10$ (m, 3H), $1.88-1.70(\mathrm{~m}, 2 \mathrm{H}), 1.29(\mathrm{~s}, 9 \mathrm{H})$.

${ }^{13}$ C NMR (101 MHz, Chloroform-d) $\delta$ 208.6, 154.7, 132.0, 129.1, 114.9, 98.0, 78.8, 55.3, 42.1, 39.0, 31.0, 27.8, 27.7, 22.7. 
IR $\left(v_{\max }, \mathrm{cm}^{-1}\right) 3403$ (s), 2966 (s), 2363 (m), 1711 (s), 1515 (s), 1439 (m), 1272 (s), 1181 (m), $1124(\mathrm{~m}), 1615$ (m), $826(\mathrm{~m}), 761(\mathrm{~m})$.

HRMS (ESI/QTOF) m/z: [M + Na $]^{+}$Calcd for $\mathrm{C}_{18} \mathrm{H}_{22} \mathrm{NaO}_{2}{ }^{+}$293.1512; Found 293.1508.

$[\alpha]^{26} \mathrm{D}+187.4$ (c 1.35, DCM), 94\% ee.

SFC: OD-H column, $3.0 \% \mathrm{MeOH}$ in supercritical $\mathrm{CO}_{2}$ as eluent, $4 \mathrm{~mL} / \mathrm{min}$. $\mathrm{tR}=10.1 \mathrm{~min}$ (minor), $11.3 \mathrm{~min}$ (major).

(S)-2-(hex-1-yn-1-yl)-2-(4-hydroxyphenyl)cyclohexan-1-one (11)

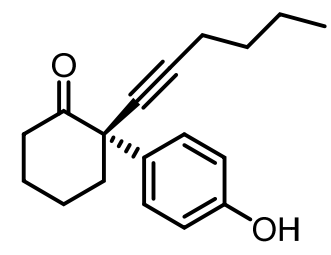

$-5^{\circ} \mathrm{C}, 4$ d. $91 \%$ yield, $94 \%$ ee, colorless oil.

${ }^{1}$ H NMR (400 MHz, Chloroform-d) $\delta 7.25-7.15(\mathrm{~m}, 2 \mathrm{H}), 6.75-6.65(\mathrm{~m}, 2 \mathrm{H}), 5.07$ (s, 1H), $3.04(\mathrm{td}, J=13.4,6.0 \mathrm{~Hz}, 1 \mathrm{H}), 2.39-2.28(\mathrm{~m}, 1 \mathrm{H}), 2.25(\mathrm{t}, J=7.0 \mathrm{~Hz}, 2 \mathrm{H}), 2.20-1.98(\mathrm{~m}$, $4 \mathrm{H}), 1.81-1.61(\mathrm{~m}, 2 \mathrm{H}), 1.54-1.43(\mathrm{~m}, 2 \mathrm{H}), 1.43-1.33(\mathrm{~m}, 2 \mathrm{H}), 0.86(\mathrm{t}, J=7.3 \mathrm{~Hz}, 3 \mathrm{H})$.

${ }^{13}$ C NMR (101 MHz, Chloroform-d) $\delta$ 208.5, 154.9, 132.2, 129.2, 115.1, 89.4, 80.4, 55.5, 41.9, $39.1,31.0,27.8,22.7,22.2,18.8,13.7$.

IR $\left(v_{\max }, \mathrm{cm}^{-1}\right) 3386(\mathrm{~m}), 2936(\mathrm{~m}), 2867(\mathrm{~m}), 1710$ (s), 1515 (s), 1444 (m), 1226 (m), 1181 (m), $824(\mathrm{~m})$.

HRMS (ESI/QTOF) m/z: [M+ H $]^{+}$Calcd for $\mathrm{C}_{18} \mathrm{H}_{23} \mathrm{O}_{2}{ }^{+}$271.1693; Found 271.1699.

$[\alpha]^{26} \mathrm{D}+176.5$ ( с $\left.0.85, \mathrm{DCM}\right), 94 \%$ ee.

SFC: OD-H column, $3.0 \% \mathrm{MeOH}$ in supercritical $\mathrm{CO}_{2}$ as eluent, $4 \mathrm{~mL} / \mathrm{min}$. $\mathrm{tR}=23.3 \mathrm{~min}$ (minor), 26.4 min (major).

(S)-2-(4-methoxyphenyl)-2-(phenylethynyl)cyclohexan-1-one (1 m)

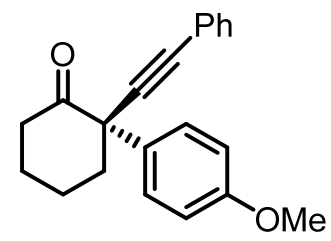

$-50{ }^{\circ} \mathrm{C}, 2 \mathrm{~d} .95 \%$ yield, $84 \%$ ee, colorless oil.

${ }^{1}$ H NMR (400 MHz, Chloroform-d) $\delta 7.45-7.38$ (m, 2H), $7.37-7.29$ (m, 2H), $7.29-7.22$ $(\mathrm{m}, 3 \mathrm{H}), 6.88-6.80(\mathrm{~m}, 2 \mathrm{H}), 3.73(\mathrm{~s}, 3 \mathrm{H}), 3.09(\mathrm{td}, J=13.3,6.0 \mathrm{~Hz}, 1 \mathrm{H}), 2.40(\mathrm{dt}, J=13.8$, $3.9 \mathrm{~Hz}, 1 \mathrm{H}), 2.33-2.15(\mathrm{~m}, 3 \mathrm{H}), 2.11-1.98(\mathrm{~m}, 1 \mathrm{H}), 1.89-1.69$ (m, 2H).

${ }^{13}$ C NMR (101 MHz, Chloroform-d) $\delta$ 206.9, 158.9, 131.8, 131.6, 129.0, 128.5, 128.5, 123.0, $113.7,89.8,88.7,56.0,55.4,41.7,39.4,27.9,22.9$. 
IR $\left(v_{\max }, \mathrm{cm}^{-1}\right) 2941(\mathrm{~m}), 2359(\mathrm{w}), 2359$ (w), 2359 (w), 1720 (s), 1511 (s), $1251(\mathrm{~s}), 1183(\mathrm{~m})$, $1033(\mathrm{~m}), 759(\mathrm{~s})$.

HRMS (ESI/QTOF) m/z: [M + Na $]^{+}$Calcd for $\mathrm{C}_{21} \mathrm{H}_{20} \mathrm{NaO}_{2}{ }^{+}$327.1356; Found 327.1357.

$[\alpha]^{26} \mathrm{D}+203.6$ (c 1.50, DCM), 84\% ee.

SFC: OD-H column, $10.0 \% \mathrm{MeOH}$ in supercritical $\mathrm{CO}_{2}$ as eluent, $4 \mathrm{~mL} / \mathrm{min}$. $\mathrm{tR}=3.6 \mathrm{~min}$ (minor), $4.5 \mathrm{~min}$ (major).

(S)-2-(5-chloropent-1-yn-1-yl)-2-(3,4-dimethoxyphenyl)cyclohexan-1-one (1n)

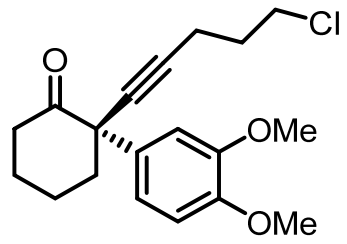

$-50{ }^{\circ} \mathrm{C}, 2$ d. $95 \%$ yield, $91 \%$ ee, colorless oil.

${ }^{1}$ H NMR (400 MHz, Chloroform- $\left.d\right) \delta 6.95(\mathrm{dd}, J=8.4,2.2 \mathrm{~Hz}, 1 \mathrm{H}), 6.93(\mathrm{~d}, J=2.2 \mathrm{~Hz}, 1 \mathrm{H})$, $6.85(\mathrm{~d}, J=8.3 \mathrm{~Hz}, 1 \mathrm{H}), 3.87(\mathrm{~s}, 3 \mathrm{H}), 3.87(\mathrm{~s}, 3 \mathrm{H}), 3.67(\mathrm{t}, J=6.3 \mathrm{~Hz}, 2 \mathrm{H}), 3.02(\mathrm{ddd}, J=13.7$, 12.2, $5.9 \mathrm{~Hz}, 1 \mathrm{H}), 2.53(\mathrm{t}, J=6.8 \mathrm{~Hz}, 2 \mathrm{H}), 2.44-2.36(\mathrm{~m}, 1 \mathrm{H}), 2.31-2.06(\mathrm{~m}, 4 \mathrm{H}), 2.01$ (p, $J=6.6 \mathrm{~Hz}, 2 \mathrm{H}), 1.90-1.73(\mathrm{~m}, 2 \mathrm{H})$.

${ }^{13}$ C NMR (101 MHz, Chloroform-d) $\delta$ 207.3, 148.6, 148.3, 132.4, 119.7, 111.6, 110.8, 86.8, $81.9,56.0,56.0,55.6,43.7,41.5,39.1,31.5,27.7,22.7,16.5$.

IR $\left(v_{\max }, \mathrm{cm}^{-1}\right) 2942(\mathrm{~m}), 1721$ (s), 1516 (s), 1445 (m), 1262 (s), 1150 (s), 1029 (s), 800 (m), 760 (s).

HRMS (ESI/QTOF) m/z: [M + H $]^{+}$Calcd for $\mathrm{C}_{19} \mathrm{H}_{24} \mathrm{ClO}_{3}{ }^{+} 335.1408$; Found 335.1420. $[\alpha]^{26} \mathrm{D}+111.9$ (c 1.60, DCM), 91\% ee.

SFC: OD-H column, $2.0 \% \mathrm{MeOH}$ in supercritical $\mathrm{CO}_{2}$ as eluent, $4 \mathrm{~mL} / \mathrm{min}$. $\mathrm{tR}=14.9 \mathrm{~min}$ (major), $18.0 \mathrm{~min}$ (minor).

(S)-2-(3,4-dimethoxyphenyl)-2-((trimethylsilyl)ethynyl)cyclohexan-1-one (10)

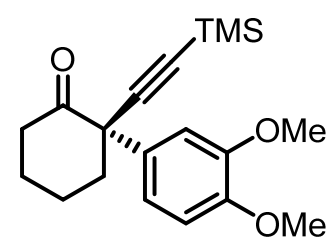

$-40{ }^{\circ} \mathrm{C}, 4$ d. $94 \%$ yield, $92 \%$ ee, white solid. M.p $=104-106{ }^{\circ} \mathrm{C}$.

${ }^{1}$ H NMR (400 MHz, Chloroform-d) $\delta 7.02(\mathrm{~d}, J=2.1 \mathrm{~Hz}, 1 \mathrm{H}), 6.94(\mathrm{dd}, J=8.4,2.2 \mathrm{~Hz}, 1 \mathrm{H})$, $6.86(\mathrm{~d}, J=8.4 \mathrm{~Hz}, 1 \mathrm{H}), 3.87(\mathrm{~s}, 6 \mathrm{H}), 3.14(\mathrm{td}, J=13.5,6.0 \mathrm{~Hz}, 1 \mathrm{H}), 2.44-2.38(\mathrm{~m}, 1 \mathrm{H}), 2.33$ $-2.10(\mathrm{~m}, 4 \mathrm{H}), 1.90-1.71(\mathrm{~m}, 2 \mathrm{H}), 0.22(\mathrm{~s}, 9 \mathrm{H})$. 
${ }^{13}$ C NMR (101 MHz, Chloroform-d) $\delta$ 206.7, 148.5, 148.3, 131.8, 119.9, 111.9, 110.8, 106.1, $93.8,56.9,56.0,55.9,41.7,39.2,27.8,22.8,0.05$.

IR $\left(v_{\max }, \mathrm{cm}^{-1}\right) 2949(\mathrm{w}), 2367$ (w), 2167 (w), 1724 (m), 1517 (s), 1460 (w), $1410(\mathrm{w}), 1260$ (s), $1149(\mathrm{~m}), 1030(\mathrm{~m}), 842(\mathrm{~s}), 761(\mathrm{~m}), 695(\mathrm{w})$.

HRMS (ESI/QTOF) m/z: [M + Na] ${ }^{+}$Calcd for $\mathrm{C}_{19} \mathrm{H}_{26} \mathrm{NaO}_{3} \mathrm{Si}^{+} 353.1543$; Found 353.1543. $[\alpha]^{26} \mathrm{D}+161.6$ ( c $\left.0.60, \mathrm{DCM}\right), 92 \%$ ee.

SFC: OD-H column, $1.0 \% \mathrm{MeOH}$ in supercritical $\mathrm{CO}_{2}$ as eluent, $4 \mathrm{~mL} / \mathrm{min}$. $\mathrm{tR}=8.5 \mathrm{~min}$ (major), $11.1 \mathrm{~min}$ (minor).

(S)-2-(4-(benzyloxy)but-1-yn-1-yl)-2-(3,4-dimethoxyphenyl)cyclohexan-1-one (1p)

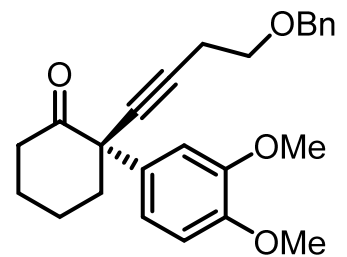

$-50{ }^{\circ} \mathrm{C}, 2$ d. $90 \%$ yield, $87 \%$ ee, colorless oil.

${ }^{1}$ H NMR (400 MHz, Chloroform- $d$ ) $\delta 7.36-7.32(\mathrm{~m}, 4 \mathrm{H}), 7.32-7.27$ (m, 1H), 6.98 (dd, $J=$ 8.4, 2.2 Hz, 1H), 6.94 (d, J=2.2 Hz, 1H), 6.82 (d, J=8.4 Hz, 1H), 4.56 (s, 2H), 3.86 (s, 3H), $3.83(\mathrm{~s}, 3 \mathrm{H}), 3.66(\mathrm{t}, J=6.8 \mathrm{~Hz}, 2 \mathrm{H}), 3.08(\mathrm{ddd}, J=13.6,12.6,6.0 \mathrm{~Hz}, 1 \mathrm{H}), 2.65(\mathrm{t}, J=6.8 \mathrm{~Hz}$, $2 \mathrm{H}), 2.42-2.34(\mathrm{~m}, 1 \mathrm{H}), 2.27-2.15(\mathrm{~m}, 3 \mathrm{H}), 2.12-2.01(\mathrm{~m}, 1 \mathrm{H}), 1.89-1.72(\mathrm{~m}, 2 \mathrm{H})$.

${ }^{13}$ C NMR (101 MHz, Chloroform-d) $\delta$ 207.3, 148.4, 148.1, 138.1, 132.3, 128.4, 127.7, 127.6, $119.8,111.6,110.7,85.8,81.6,73.0,68.6,55.9,55.6,41.5,39.0,27.6,22.6,20.5$.

IR $\left(v_{\max }, \mathrm{cm}^{-1}\right) 2936(\mathrm{~m}), 2867(\mathrm{w}), 1721(\mathrm{~s}), 1516(\mathrm{~s}), 1456(\mathrm{~m}), 1263$ (s), 1149 (s), 1107 (s), 1029 (s), 759 (m), $743(\mathrm{~m})$.

HRMS (ESI/QTOF) m/z: [M + Na $]^{+}$Calcd for $\mathrm{C}_{25} \mathrm{H}_{28} \mathrm{NaO}_{4}{ }^{+} 415.1880$; Found 415.1893.

$[\alpha]^{26} \mathrm{D}+110.3$ (c 1.20, DCM), 87\% ee.

SFC: AD-H column, $4.0 \% \mathrm{MeOH}$ in supercritical $\mathrm{CO}_{2}$ as eluent, $4 \mathrm{~mL} / \mathrm{min}$. $\mathrm{tR}=10.9 \mathrm{~min}$ (major), $13.8 \mathrm{~min}$ (minor).

(S)-2-(3,4-dimethoxyphenyl)-2-(phenylethynyl)cyclohexan-1-one (1q)

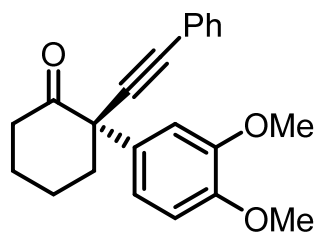

$-50{ }^{\circ} \mathrm{C}, 2$ d. $85 \%$ yield, $90 \%$ ee, colorless oil. 
${ }^{1}$ H NMR (400 MHz, Chloroform-d) $\delta 7.55$ - $7.44(\mathrm{~m}, 2 \mathrm{H}), 7.37$ - 7.31 (m, 3H), 7.09 - 7.00 (m, 2H), 6.89 (d, J = 8.2 Hz, 1H), 3.89 (s, 3H), 3.89 (s, 3H), $3.17(\mathrm{td}, J=13.3,6.0 \mathrm{~Hz}, 1 \mathrm{H})$, $2.48(\mathrm{dt}, J=13.4,3.5 \mathrm{~Hz}, 1 \mathrm{H}), 2.42-2.23(\mathrm{~m}, 3 \mathrm{H}), 2.22-2.09(\mathrm{~m}, 1 \mathrm{H}), 1.98-1.78(\mathrm{~m}, 2 \mathrm{H})$. ${ }^{13}$ C NMR (101 MHz, Chloroform-d) $\delta$ 206.8, 148.6, 148.4, 132.0, 131.7, 128.6, 128.5, 123.0, $120.0,111.7,110.9,89.8,88.7,56.2,56.0,56.0,41.6,39.4,27.8,22.8$.

IR $\left(v_{\max }, \mathrm{cm}^{-1}\right) 2942(\mathrm{~m}), 2157(\mathrm{~m}), 1723(\mathrm{~s}), 1517(\mathrm{~s}), 1449(\mathrm{~m}), 1260$ (s), $1148(\mathrm{~m}), 1027(\mathrm{~s})$, 759 (s), $693(\mathrm{~m})$.

HRMS (ESI/QTOF) m/z: [M + Na $]^{+}$Calcd for $\mathrm{C}_{22} \mathrm{H}_{22} \mathrm{NaO}_{3}{ }^{+} 357.1461$; Found 357.1462. $[\alpha]^{26} \mathrm{D}+203.2$ (c 1.30, DCM), 90\% ee.

SFC: OD-H column, $3.0 \% \mathrm{MeOH}$ in supercritical $\mathrm{CO}_{2}$ as eluent, $4 \mathrm{~mL} / \mathrm{min}$. $\mathrm{tR}=15.1 \mathrm{~min}$ (minor), $16.3 \mathrm{~min}$ (major).

(S)-2-(benzo[d][1,3]dioxol-5-yl)-2-((trimethylsilyl)ethynyl)cyclohexan-1-one (1r)

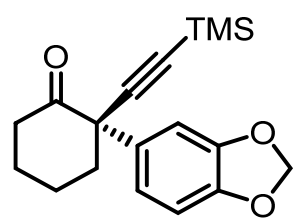

$-30{ }^{\circ} \mathrm{C}, 3 \mathrm{~d} .82 \%$ yield, $83 \%$ ee, white solid. M.p $=110-112{ }^{\circ} \mathrm{C}$.

${ }^{1}$ H NMR (400 MHz, Chloroform-d) $\delta 6.93(\mathrm{dd}, J=8.1,1.9 \mathrm{~Hz}, 1 \mathrm{H}), 6.90(\mathrm{~d}, J=1.9 \mathrm{~Hz}, 1 \mathrm{H})$, $6.79(\mathrm{~d}, J=8.1 \mathrm{~Hz}, 1 \mathrm{H}), 5.96$ (d. $J=1.5 \mathrm{~Hz}, 1 \mathrm{H}), 5.95$ (d, $J=1.5 \mathrm{~Hz}, 1 \mathrm{H}), 3.13$ (td, $J=13.6$, $6.1 \mathrm{~Hz}, 1 \mathrm{H}), 2.46-2.34(\mathrm{~m}, 1 \mathrm{H}), 2.32-2.09(\mathrm{~m}, 4 \mathrm{H}), 1.91-1.69(\mathrm{~m}, 2 \mathrm{H}), 0.22(\mathrm{~s}, 9 \mathrm{H})$. ${ }^{13}$ C NMR (101 MHz, Chloroform-d) $\delta$ 206.7, 147.5, 146.9, 133.1, 121.1, 108.9, 107.9, 105.9, $101.2,94.1,56.9,41.9,39.1,27.7,22.8,0.05$.

IR $\left(v_{\max }, \mathrm{cm}^{-1}\right) 2960(\mathrm{w}), 2166(\mathrm{w}), 1725(\mathrm{~s}), 1505(\mathrm{~m}), 1490(\mathrm{~m}), 1437$ (m), 1242 (s), 1125 (m), $1042(\mathrm{~m}), 936(\mathrm{~m}), 842$ (s), 761 (s).

HRMS (ESI/QTOF) m/z: [M+ H] $]^{+}$Calcd for $\mathrm{C}_{18} \mathrm{H}_{23} \mathrm{O}_{3} \mathrm{Si}^{+}$315.1411; Found 315.1408. $[\alpha]^{26} \mathrm{D}+111.8$ (c $\left.0.80, \mathrm{DCM}\right), 83 \%$ ee.

SFC: OD-H column, $3.0 \% \mathrm{MeOH}$ in supercritical $\mathrm{CO}_{2}$ as eluent, $4 \mathrm{~mL} / \mathrm{min}$. $\mathrm{tR}=2.2 \mathrm{~min}$ (minor), $2.9 \mathrm{~min}$ (major).

(S)-2-(2,3-dihydrobenzofuran-5-yl)-2-(phenylethynyl)cyclohexan-1-one (1s)

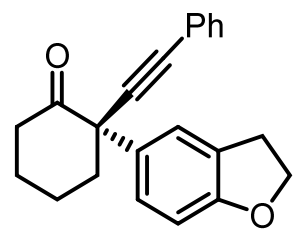

$-50{ }^{\circ} \mathrm{C}, 2$ d. $84 \%$ yield, $82 \%$ ee, colorless oil. 
${ }^{1}$ H NMR (400 MHz, Chloroform- $d$ ) $\delta 7.59-7.48(\mathrm{~m}, 2 \mathrm{H}), 7.43-7.30(\mathrm{~m}, 4 \mathrm{H}), 7.27$ (d, $J=$ $8.3 \mathrm{~Hz}, 1 \mathrm{H}), 6.81$ (d, $J=8.4 \mathrm{~Hz}, 1 \mathrm{H}), 4.60(\mathrm{t}, J=8.7 \mathrm{~Hz}, 2 \mathrm{H}), 3.25$ (t, $J=8.9 \mathrm{~Hz}, 2 \mathrm{H}), 3.18$ $(\mathrm{dt}, J=13.3,5.9 \mathrm{~Hz}, 1 \mathrm{H}), 2.51(\mathrm{~d}, J=14.0 \mathrm{~Hz}, 1 \mathrm{H}), 2.43-2.27(\mathrm{~m}, 3 \mathrm{H}), 2.18(\mathrm{~d}, J=13.5 \mathrm{~Hz}$, $1 \mathrm{H}), 2.00-1.76(\mathrm{~m}, 2 \mathrm{H})$.

${ }^{13}$ C NMR (101 MHz, Chloroform-d) $\delta$ 207.1, 159.5, 131.8, 131.5, 128.5, 128.5, 127.6, 127.0, $124.7,123.1,108.9,90.0,88.7,71.5,56.2,41.9,39.4,30.0,27.8,22.9$.

IR $\left(v_{\max }, \mathrm{cm}^{-1}\right) 2936(\mathrm{~m}), 1721$ (s), 1492 (s), 1239 (s), 1109 (m), 1075 (m), 983 (m), 947 (m), $815(\mathrm{~m}), 759$ (s), $692(\mathrm{~s})$.

HRMS (ESI/QTOF) m/z: [M + Na $]^{+}$Calcd for $\mathrm{C}_{22} \mathrm{H}_{20} \mathrm{NaO}_{2}{ }^{+}$339.1356; Found 339.1362. $[\alpha]^{26} \mathrm{D}+216.5$ (c 1.00, DCM), 82\% ee.

SFC: OD-H column, $2.0 \% \mathrm{MeOH}$ in supercritical $\mathrm{CO}_{2}$ as eluent, $4 \mathrm{~mL} / \mathrm{min}$. $\mathrm{tR}=14.9 \mathrm{~min}$ (minor), 17.2 min (major).

(S)-2-(phenylethynyl)-2-(thiophen-2-yl)cyclohexan-1-one (1t)

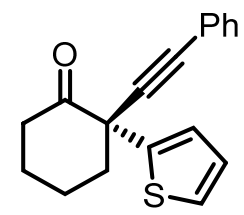

$-50{ }^{\circ} \mathrm{C}, 2$ d. $90 \%$ yield, $83 \%$ ee, pale yellow solid. M.p $=85-87{ }^{\circ} \mathrm{C}$.

${ }^{1}$ H NMR (400 MHz, Chloroform-d) $\delta 7.52-7.46(\mathrm{~m}, 2 \mathrm{H}), 7.39-7.28(\mathrm{~m}, 4 \mathrm{H}), 7.14$ (dd, $J=$ 3.6, $1.2 \mathrm{~Hz}, 1 \mathrm{H}), 7.03$ (dd, $J=5.1,3.6 \mathrm{~Hz}, 1 \mathrm{H}), 3.19$ (td, $J=13.3,6.1 \mathrm{~Hz}, 1 \mathrm{H}), 2.58-2.47$ (m, 2H), $2.39-2.24(\mathrm{~m}, 2 \mathrm{H}), 2.22-2.12(\mathrm{~m}, 1 \mathrm{H}), 1.99-1.89(\mathrm{~m}, 1 \mathrm{H}), 1.88-1.76(\mathrm{~m}, 1 \mathrm{H})$.

${ }^{13}$ C NMR (101 MHz, Chloroform-d) $\delta$ 205.3, 143.8, 131.8, 128.7, 128.5, 126.6, 125.8, 125.1, $122.7,89.2,87.5,53.6,43.2,39.0,27.9,22.9$.

IR $\left(v_{\max }, \mathrm{cm}^{-1}\right) 2942(\mathrm{w}), 1726(\mathrm{~s}), 1490(\mathrm{w}), 1441(\mathrm{w}), 1123(\mathrm{w}), 1071(\mathrm{w}), 758(\mathrm{~s}), 694$ (s).

HRMS (ESI/QTOF) m/z: [M+ H] $]^{+}$Calcd for $\mathrm{C}_{18} \mathrm{H}_{17} \mathrm{OS}^{+} 281.0995$; Found 281.0998.

$[\alpha]^{26} \mathrm{D}+253.2$ (c 1.20, DCM), 83\% ee.

SFC: OD-H column, $3.0 \% \mathrm{MeOH}$ in supercritical $\mathrm{CO}_{2}$ as eluent, $4 \mathrm{~mL} / \mathrm{min}$. $\mathrm{tR}=6.8 \mathrm{~min}$ (minor), $7.9 \min$ (major).

(R)-2-(benzofuran-2-yl)-2-(phenylethynyl)cyclohexan-1-one (1u)

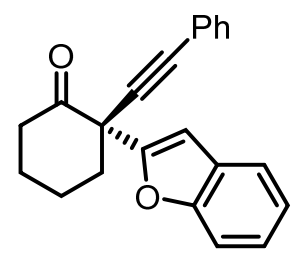

$15 \mathrm{~mol} \%$ of $4 \mathrm{a},-50{ }^{\circ} \mathrm{C}, 3 \mathrm{~d} .83 \%$ yield, $81 \%$ ee, pale yellow oil. 
${ }^{1}$ H NMR (400 MHz, Chloroform-d) $\delta 7.58$ - 7.45 (m, 4H), 7.38 - 7.32 (m, 3H), 7.29 - 7.18 $(\mathrm{m}, 2 \mathrm{H}), 6.88(\mathrm{~d}, J=1.0 \mathrm{~Hz}, 1 \mathrm{H}), 3.15-3.06(\mathrm{~m}, 1 \mathrm{H}), 2.66-2.57(\mathrm{~m}, 1 \mathrm{H}), 2.55-2.41(\mathrm{~m}$, $2 \mathrm{H}), 2.35-2.22(\mathrm{~m}, 1 \mathrm{H}), 2.21-2.10(\mathrm{~m}, 1 \mathrm{H}), 2.03-1.81(\mathrm{~m}, 2 \mathrm{H})$.

${ }^{13}$ C NMR (101 MHz, Chloroform-d) $\delta$ 203.9, 155.8, 155.4, 132.0, 128.8, 128.5, 128.3, 124.1, $122.8,122.6,121.1,111.4,105.3,87.5,86.9,53.0,39.0,38.8,27.5,22.2$.

IR $\left(v_{\max }, \mathrm{cm}^{-1}\right) 2937(\mathrm{w}), 1724(\mathrm{~s}), 1450(\mathrm{~m}), 1252(\mathrm{~m}), 1120(\mathrm{~m}), 1005(\mathrm{~s}), 806(\mathrm{~m}), 752(\mathrm{~m})$, $688(\mathrm{~m})$.

$[\alpha]^{24} \mathrm{D}+220.0($ c $0.82, \mathrm{DCM}), 81 \%$ ee.

HRMS (ESI/QTOF) m/z: [M + H $]^{+}$Calcd for $\mathrm{C}_{22} \mathrm{H}_{19} \mathrm{O}_{2}{ }^{+}$315.1380; Found 315.1382.

SFC: OD-H column, $5.0 \% \mathrm{MeOH}$ in supercritical $\mathrm{CO}_{2}$ as eluent, $4 \mathrm{~mL} / \mathrm{min}$. $\mathrm{tR}=5.9 \mathrm{~min}$ (minor), $7.2 \min$ (major).

(S)-2-phenyl-2-(phenylethynyl)cyclohexan-1-one (1w)

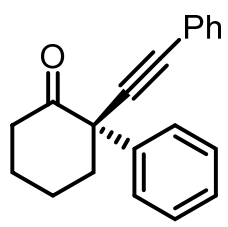

$76 \%$ yield, $65 \%$ ee. White solid, m.p $=77-79^{\circ} \mathrm{C}$.

${ }^{1}$ H NMR (400 MHz, Chloroform-d) $\delta 7.55-7.47$ (m, 4H), $7.45-7.28$ (m, 6H), 3.19 (td, $J=$ 13.4, 6.0 Hz, 1H), $2.59-2.44(\mathrm{~m}, 1 \mathrm{H}), 2.42-2.25(\mathrm{~m}, 3 \mathrm{H}), 2.23-2.12(\mathrm{~m}, 1 \mathrm{H}), 2.01-1.77$ $(\mathrm{m}, 2 \mathrm{H})$.

${ }^{13}$ C NMR (101 MHz, Chloroform-d) $\delta$ 206.4, 139.6, 131.8, 128.6, 128.5, 128.3, 128.0, 127.5, $123.1,89.6,89.0,56.7,41.7,39.4,27.8,22.8$.

IR ( $\left.v_{\max }, \mathrm{cm}^{-1}\right) 2940(\mathrm{w}), 1722(\mathrm{~s}), 1487(\mathrm{~m}), 1442(\mathrm{~m}), 1120$ (w), $1074(\mathrm{~m}), 908$ (s), $756(\mathrm{~s})$, $688(\mathrm{~m})$.

$[\alpha]^{26} \mathrm{D}+230.2$ (c 1.60, DCM), 65\% ee.

HRMS (ESI/QTOF) m/z: [M+ H] ${ }^{+}$Calcd for $\mathrm{C}_{20} \mathrm{H}_{19} \mathrm{O}^{+}$275.1430; Found 275.1431.

SFC: OD-H column, $2.0 \% \mathrm{MeOH}$ in supercritical $\mathrm{CO}_{2}$ as eluent, $4 \mathrm{~mL} / \mathrm{min}$. $\mathrm{tR}=7.8 \mathrm{~min}$ (minor), 9.0 min (major)

(R)-2-(4-hydroxyphenyl)-2-phenylcyclohexan-1-one (2a)

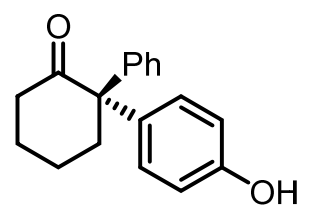

$-78{ }^{\circ} \mathrm{C}, 1$ d. $95 \%$ yield, $84 \%$ ee, white solid. M.p $=136-138{ }^{\circ} \mathrm{C}$. 
${ }^{1}$ H NMR (400 MHz, Chloroform- $d$ ) $\delta 7.38-7.22(\mathrm{~m}, 3 \mathrm{H}), 7.07$ (d, $\left.J=7.7 \mathrm{~Hz}, 2 \mathrm{H}\right), 6.92(\mathrm{~d}, J$ $=8.1 \mathrm{~Hz}, 2 \mathrm{H}), 6.77(\mathrm{~d}, J=8.2 \mathrm{~Hz}, 2 \mathrm{H}), 5.53(\mathrm{~s}, 1 \mathrm{H}), 2.65-2.50(\mathrm{~m}, 4 \mathrm{H}), 2.01-1.92(\mathrm{~m}, 2 \mathrm{H})$, $1.89-1.80(\mathrm{~m}, 2 \mathrm{H})$.

${ }^{13}$ C NMR (101 MHz, Chloroform-d) $\delta$ 212.6, 154.6, 142.8, 134.2, 129.9, 128.6, 128.5, 126.9, $115.4,63.5,40.7,39.5,27.9,22.2$.

IR $\left(v_{\max }, \mathrm{cm}^{-1}\right) 3396(\mathrm{~m}), 2941$ (m), 2359 (m), 1697 (s), 1514 (s), 1448 (m), 1235 (m), 1181 (m), $1118(\mathrm{~m}), 812$ (m), 754 (m), 702 (s).

HRMS (ESI/QTOF) m/z: [M + Na $]^{+}$Calcd for $\mathrm{C}_{18} \mathrm{H}_{18} \mathrm{NaO}_{2}{ }^{+}$289.1199; Found 289.1206.

$[\alpha]^{26} \mathrm{D}-39.5$ (c 1.05, DCM), $84 \%$ ee.

SFC: OD-H column, $5.0 \% \mathrm{MeOH}$ in supercritical $\mathrm{CO}_{2}$ as eluent, $4 \mathrm{~mL} / \mathrm{min}$. $\mathrm{tR}=19.5 \mathrm{~min}$ (minor), $22.6 \mathrm{~min}$ (major).

(S)-2-(4-fluorophenyl)-2-(4-hydroxyphenyl)cyclohexan-1-one (2b)

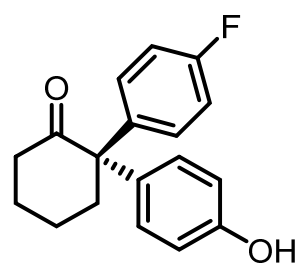

$-78{ }^{\circ} \mathrm{C}, 1$ d. $98 \%$ yield, $72 \%$ ee, colorless oil.

${ }^{1}$ H NMR (400 MHz, Chloroform- $d$ ) $\delta 7.01-6.94(\mathrm{~m}, 4 \mathrm{H}), 6.93(\mathrm{~d}, J=8.6 \mathrm{~Hz}, 2 \mathrm{H}), 6.78$ (d, $J$ $=8.6 \mathrm{~Hz}, 2 \mathrm{H}), 5.48(\mathrm{~s}, 1 \mathrm{H}), 2.67-2.40(\mathrm{~m}, 4 \mathrm{H}), 2.04-1.73(\mathrm{~m}, 4 \mathrm{H})$.

${ }^{13}$ C NMR (101 MHz, Chloroform-d) $\delta 212.4,161.6(\mathrm{~d}, J=246.0 \mathrm{~Hz}), 154.8,139.0(\mathrm{~d}, J=3.1$ Hz), 133.6, 130.3 (d, $J=7.8 \mathrm{~Hz}), 129.8,115.7,115.1$ (d, $J=21.2 \mathrm{~Hz}), 62.9,40.6,39.7,27.9$, 22.2 .

${ }^{19}$ F NMR (377 MHz, Chloroform-d) $\delta$-116.20.

IR $\left(v_{\max }, \mathrm{cm}^{-1}\right) 3398$ (s), 2947 (s), 2357 (s), 1695 (s), 1508 (s), 1225 (s), 825 (s).

$[\alpha]^{26} \mathrm{D}-44.5$ (с $\left.0.5, \mathrm{DCM}\right), 72 \%$ ee.

HRMS (ESI/QTOF) m/z: [M + Na] $]^{+}$Calcd for $\mathrm{C}_{18} \mathrm{H}_{17} \mathrm{FNaO}_{2}{ }^{+}$307.1105; Found 307.1106.

Chiral HPLC: Chiralpak IG, hexane: $i \mathrm{PrOH}=90: 10,1.0 \mathrm{~mL} / \mathrm{min}, 254 \mathrm{~nm}$; $\mathrm{tR}=12.3 \mathrm{~min}$ (major), 13.7 min (minor).

(R)-2-(3,4-dimethoxyphenyl)-2-phenylcyclohexan-1-one (2c)

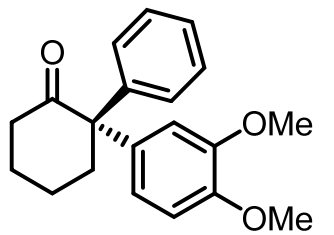

$-78{ }^{\circ} \mathrm{C}, 1$ d. $97 \%$ yield, $89 \%$ ee, colorless oil. 
${ }^{1}$ H NMR (400 MHz, Chloroform-d) $\delta 7.33$ - 7.27 (m, 2H), 7.27 - 7.20 (m, 1H), 7.09 - 6.98 $(\mathrm{m}, 2 \mathrm{H}), 6.83(\mathrm{~d}, J=8.5 \mathrm{~Hz}, 1 \mathrm{H}), 6.64(\mathrm{dd}, J=8.4,2.3 \mathrm{~Hz}, 1 \mathrm{H}), 6.59(\mathrm{~d}, J=2.2 \mathrm{~Hz}, 1 \mathrm{H}), 3.87$ (s, 3H), 3.77 (s, 3H), $2.68-2.46(\mathrm{~m}, 4 \mathrm{H}), 2.06-1.75(\mathrm{~m}, 4 \mathrm{H})$.

${ }^{13}$ C NMR (101 MHz, Chloroform-d) $\delta$ 211.6, 148.9, 148.0, 143.2, 134.3, 128.7, 128.3, 126.9, $120.9,112.1,110.9,63.7,56.0,55.9,40.7,39.6,27.8,22.3$.

IR $\left(v_{\max }, \mathrm{cm}^{-1}\right) 2941$ (s), 2360 (s), 2152 (s), 1707 (s), 1514 (s), 1448 (s), 1255 (s), 1147 (s), 1024 (s), 903 (s), 800 (s), 729 (s), 698 (s).

$[\alpha]^{26} \mathrm{D}-25.7$ (c $\left.0.65, \mathrm{DCM}\right), 89 \%$ ee.

HRMS (APCI/QTOF) m/z: [M + Na] ${ }^{+}$Calcd for $\mathrm{C}_{20} \mathrm{H}_{22} \mathrm{NaO}_{3}{ }^{+} 333.1461$; Found 333.1460.

Chiral HPLC: Chiralpak IG, hexane: $\mathrm{PrOH}=90: 10,1.0 \mathrm{~mL} / \mathrm{min}, 254 \mathrm{~nm}$; $\mathrm{tR}=20.2 \mathrm{~min}$ (major), $21.8 \mathrm{~min}$ (minor).

(R)-2-(3,4-dimethoxyphenyl)-2-(p-tolyl)cyclohexan-1-one (2d)

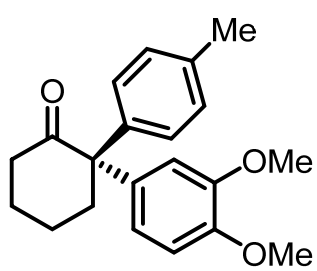

$-78{ }^{\circ} \mathrm{C}, 1 \mathrm{~d} .96 \%$ yield, $91 \%$ ee, colorless oil.

${ }^{1}$ H NMR (400 MHz, Chloroform-d) $\delta 7.12(\mathrm{~d}, J=8.0 \mathrm{~Hz}, 2 \mathrm{H}), 6.97-6.88(\mathrm{~m}, 2 \mathrm{H}), 6.83(\mathrm{~d}, J$ $=8.3 \mathrm{~Hz}, 1 \mathrm{H}), 6.68-6.55(\mathrm{~m}, 2 \mathrm{H}), 3.87(\mathrm{~s}, 3 \mathrm{H}), 3.78(\mathrm{~s}, 3 \mathrm{H}), 2.58-2.48(\mathrm{~m}, 4 \mathrm{H}), 2.33(\mathrm{~s}$, $3 \mathrm{H}), 2.01-1.77(\mathrm{~m}, 4 \mathrm{H})$.

${ }^{13}$ C NMR (101 MHz, Chloroform-d) $\delta$ 211.7, 148.8, 147.9, 140.0, 136.4, 134.4, 129.0, 128.4, $120.7,112.1,110.8,63.3,55.9,55.8,40.6,39.5,27.7,22.2,21.0$.

IR $\left(v_{\max }, \mathrm{cm}^{-1}\right) 2937$ (s), 2154 (s), 1703 (s), 1510 (s), 1450 (s), 1255 (s), 1147 (s), 1026 (s), 806 (s), $729(\mathrm{~s})$.

$[\alpha]^{26} \mathrm{D}-38.0$ (c $\left.0.75, \mathrm{DCM}\right), 91 \%$ ee.

HRMS (APCI/QTOF) m/z: [M + Na $]^{+}$Calcd for $\mathrm{C}_{21} \mathrm{H}_{24} \mathrm{NaO}_{3}{ }^{+}$347.1618; Found 347.1614.

Chiral HPLC: Chiralpak ID, hexane: $\mathrm{PrOH}=80: 20,1.0 \mathrm{~mL} / \mathrm{min}, 254 \mathrm{~nm}$; $\mathrm{tR}=13.1 \mathrm{~min}$ (minor), 13.7 min (major).

(R)-2-(3,4-dimethoxyphenyl)-2-(3,5-dimethylphenyl)cyclohexan-1-one (2e)<smiles>COc1cc(C)cc([C@@]2(c3ccc(OC)c(OC)c3)CCCCC2=O)c1</smiles> 
$-78{ }^{\circ} \mathrm{C}, 1$ d. $99 \%$ yield, $91 \%$ ee, colorless oil.

${ }^{1}$ H NMR (400 MHz, Chloroform- $d$ ) $\delta 6.89(\mathrm{~s}, 1 \mathrm{H}), 6.82(\mathrm{~d}, J=8.1 \mathrm{~Hz}, 1 \mathrm{H}), 6.66(\mathrm{~s}, 2 \mathrm{H}), 6.61$ - $6.57(\mathrm{~m}, 2 \mathrm{H}), 3.87(\mathrm{~s}, 3 \mathrm{H}), 3.78(\mathrm{~s}, 3 \mathrm{H}), 2.63-2.44(\mathrm{~m}, 4 \mathrm{H}), 2.27$ (s, 6H), 1.99 - 1.90 (m, $2 \mathrm{H}), 1.89-1.81(\mathrm{~m}, 2 \mathrm{H})$.

${ }^{13}$ C NMR (101 MHz, Chloroform-d) $\delta$ 211.9, 148.7, 147.9, 142.7, 137.8, 134.8, 128.7, 126.4, $120.9,112.3,110.8,63.6,56.0,55.9,40.8,39.6,27.8,22.3,21.6$.

IR $\left(v_{\max }, \mathrm{cm}^{-1}\right) 2941$ (s), 1707 (s), 1599 (s), 1514 (s), 1460 (s), 1257 (s), 1149 (s), 1025 (s), 731 (s).

$[\alpha]^{26} \mathrm{D}-5.9$ (c $\left.0.50, \mathrm{DCM}\right), 91 \%$ ee.

HRMS (ESI/QTOF) m/z: [M + Na $]^{+}$Calcd for $\mathrm{C}_{22} \mathrm{H}_{26} \mathrm{NaO}_{3}{ }^{+}$361.1774; Found 361.1763.

Chiral HPLC: Chiralpak ID, hexane: $i \mathrm{PrOH}=90: 10,1.0 \mathrm{~mL} / \mathrm{min}, 254 \mathrm{~nm}$; $\mathrm{tR}=14.7 \mathrm{~min}$ (minor), $15.2 \mathrm{~min}$ (major).

\section{(S)-2-(3-chlorophenyl)-2-(3,4-dimethoxyphenyl)cyclohexan-1-one (2f)}

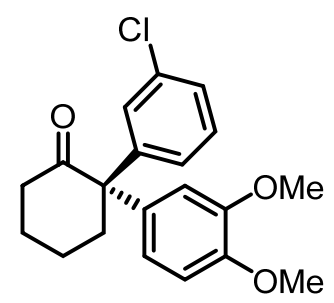

$-78{ }^{\circ} \mathrm{C}, 3$ d. $95 \%$ yield, $87 \%$ ee, colorless oil.

${ }^{1}$ H NMR (400 MHz, Chloroform- $d$ ) $\delta 7.21-7.18(\mathrm{~m}, 2 \mathrm{H}), 6.95(\mathrm{~s}, 1 \mathrm{H}), 6.89-6.83(\mathrm{~m}, 2 \mathrm{H})$, $6.72-6.67(\mathrm{~m}, 1 \mathrm{H}), 6.62-6.58(\mathrm{~m}, 1 \mathrm{H}), 3.89(\mathrm{~s}, 3 \mathrm{H}), 3.79(\mathrm{~s}, 3 \mathrm{H}), 2.69-2.58(\mathrm{~m}, 1 \mathrm{H}), 2.56$ $-2.34(\mathrm{~m}, 3 \mathrm{H}), 2.03-1.73(\mathrm{~m}, 4 \mathrm{H})$.

${ }^{13}$ C NMR (101 MHz, Chloroform-d) $\delta$ 210.9, 149.3, 148.4, 146.1, 134.1, 132.7, 129.3, 128.9, 127.1, 127.0, 120.8, 111.8, 111.2, 63.5, 56.1, 56.0, 40.6, 39.5, 27.6, 22.2.

IR ( $\left.v_{\max }, \mathrm{cm}^{-1}\right) 2949$ (m), 1707 (s), 1515 (s), 1462 (m), 1258 (s), 1150 (s), 1026 (s), 765 (m), $731(\mathrm{~s}), 691(\mathrm{~m})$.

$[\alpha]^{26} \mathrm{D}-38.0$ (c $\left.0.8, \mathrm{DCM}\right), 87 \%$ ee.

HRMS (ESI/QTOF) m/z: [M + Na] ${ }^{+}$Calcd for $\mathrm{C}_{20} \mathrm{H}_{21} \mathrm{ClNaO}_{3}{ }^{+} 367.1071$; Found 367.1077.

Chiral HPLC: Chiralpak ID, hexane: $\mathrm{PrOH}=90: 10,1.0 \mathrm{~mL} / \mathrm{min}, 254 \mathrm{~nm}$; $\mathrm{tR}=14.7 \mathrm{~min}$ (major), $16.2 \mathrm{~min}$ (minor). 


\section{Synthesis and characterization data of compounds 3 and 6}

\section{Synthesis of $\alpha$-bromoketone}

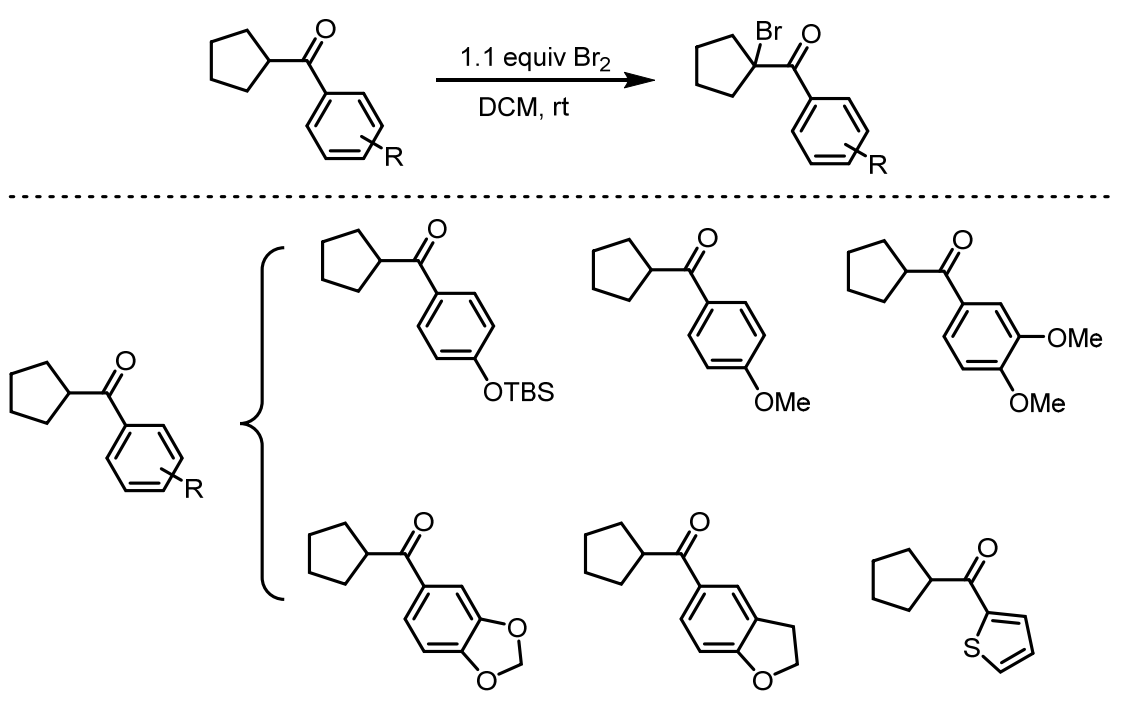

$\mathrm{Br}_{2}$ (1.1 equiv) was added dropwise to a solution of cyclopentyl(aryl)methanone (1.0 equiv) in $\operatorname{DCM}(0.5 \mathrm{M})$ at room temperature. The reaction mixture was stirred at room temperature for $2 \mathrm{~h}$. After completion of the reaction (monitored by TLC), a saturated solution of $\mathrm{Na}_{2} \mathrm{SO}_{3}(\mathrm{aq})$ was added to quench the reaction and the mixture was extracted with DCM. The organic layers were washed with water and brine, dried over $\mathrm{Na}_{2} \mathrm{SO}_{4}$, filtered, and evaporated. The residue was purified by column chromatography on silica gel, eluting with ethyl acetate/petroleum ether to give the corresponding $\alpha$-bromoketone in high yield.

\section{General procedure A}

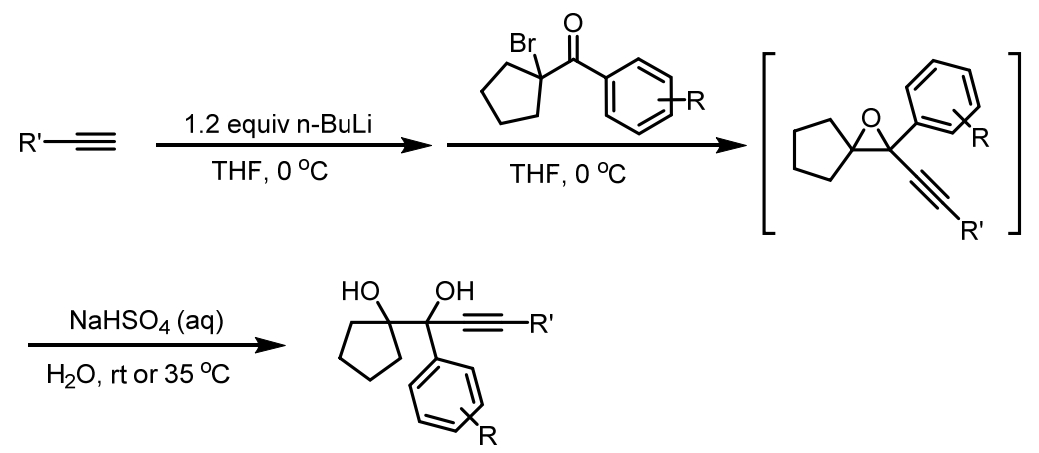

To a solution of the alkyne (1.1 equiv) in THF $(1.0 \mathrm{M})$ was added dropwise n-BuLi $(2.5 \mathrm{M}$ solution in hexane, 1.2 equiv) at $0{ }^{\circ} \mathrm{C}$. The solution was then stirred at the same temperature for $0.5 \mathrm{~h}$. A solution of the $\alpha$-bromoketone (1.0 equiv) in THF $(1.0 \mathrm{M})$ was added dropwise at $0{ }^{\circ} \mathrm{C}$. The resulting solution was allowed to slowly reach room temperature and stirred overnight. The reaction was quenched with water and an unstable epoxide intermediate was observed by TLC. Subsequently, a saturated solution of $\mathrm{NaHSO}_{4}$ (aq) was added and the 
reaction mixture was stirred at room temperature or $35^{\circ} \mathrm{C}$. After completion of the reaction (monitored by TLC, normally in 2 hours), the reaction mixture was extracted with EtOAc. The organic layers were washed with water and brine, dried over $\mathrm{Na}_{2} \mathrm{SO}_{4}$, filtered, and evaporated. The residue was purified by column chromatography on silica gel, eluting with ethyl acetate/petroleum ether to give the 1,2-diol in good yield over two steps.

\section{General procedure B}

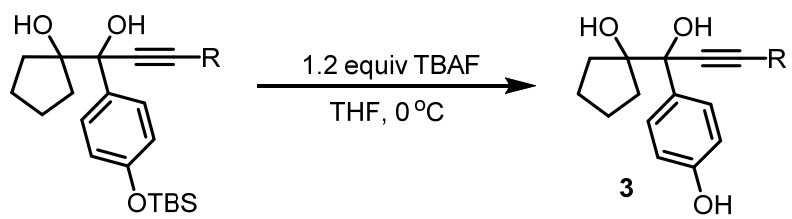

To a solution of the corresponding 1,2-diol (1.0 equiv) in THF (0.5 M) was added a solution of TBAF (1.0 M in THF, 1.2 equiv) at $0{ }^{\circ} \mathrm{C}$. The reaction was stirred at the same temperature for $1 \mathrm{~h}$. After completion of the reaction (monitored by TLC), water was added to quench the reaction and the mixture was extracted with EtOAc. The organic layers were washed with water and brine, dried over $\mathrm{Na}_{2} \mathrm{SO}_{4}$, filtered, and evaporated. The residue was purified by column chromatography on silica gel, eluting with ethyl acetate/petroleum ether to give the desired product 3 in good to high yield.

\section{Synthesis of the tetrasubstituted alkenes (McMurry cross-coupling)}
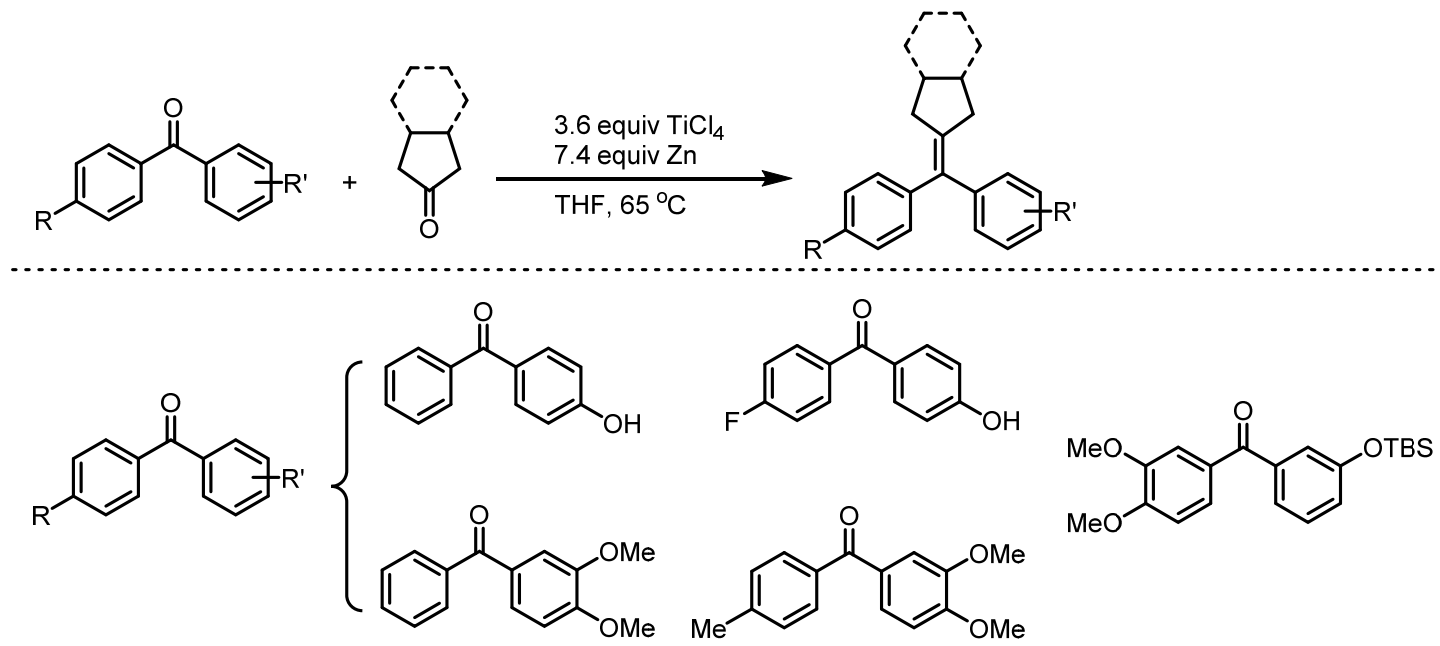

To a stirred suspension of zinc powder ( 7.4 equiv) in anhydrous THF was slowly added, via syringe, $\mathrm{TiCl}_{4}$ (3.6 equiv) at $0{ }^{\circ} \mathrm{C}$ under a nitrogen atmosphere. The stirred reaction mixture was heated at $65^{\circ} \mathrm{C}$ under a nitrogen atmosphere for $2 \mathrm{~h}$. To the refluxing reaction mixture was added a solution of diaryl methanone (1.0 equiv) and cyclopentanone (3.0 equiv) in THF. After completion of the reaction (monitored by TLC), the reaction mixture was cooled to $0{ }^{\circ} \mathrm{C}$ and water was slowly added. The reaction mixture was filtered through a pad of Celite and the 
Celite pad was washed with EtOAc. The filtrate was transferred to a separatory funnel and the organic layer was separated. The aqueous layer was extracted with EtOAc. The organic layers were washed with water and brine, dried over $\mathrm{Na}_{2} \mathrm{SO}_{4}$, filtered, and evaporated. The residue was purified by column chromatography on silica gel, eluting with ethyl acetate/petroleum ether to give the desired alkene in high yield.

\section{General procedure $\mathbf{C}$}

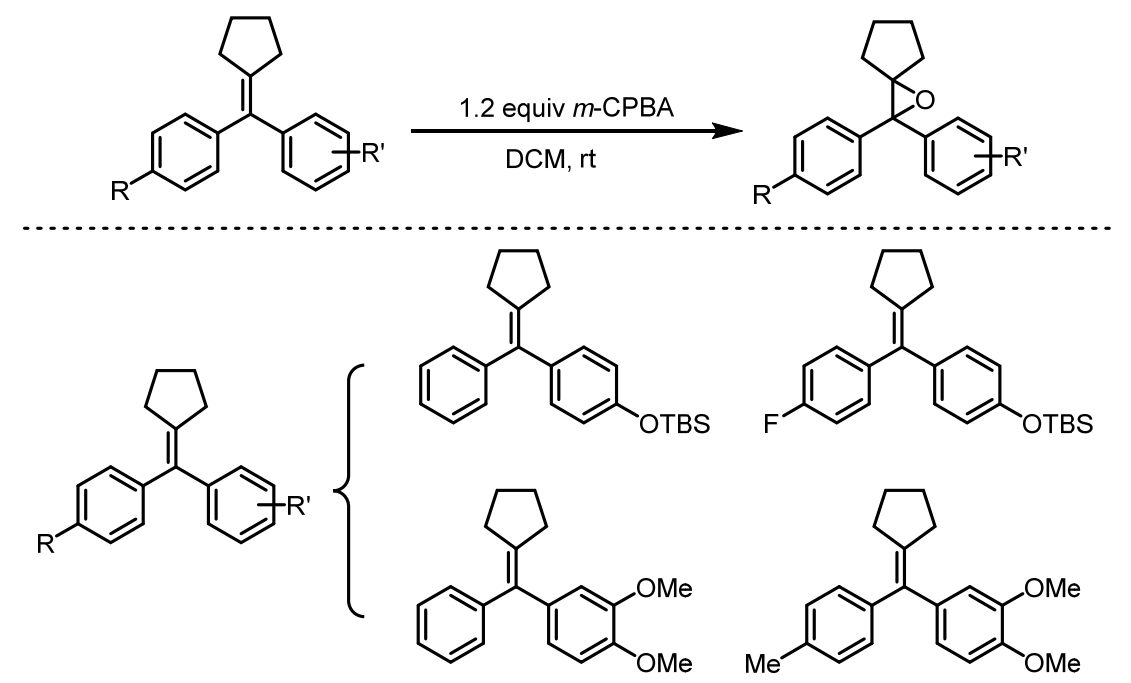

To a solution of the tetrasubstituted alkene in DCM $(0.5 \mathrm{M})$ was added $m$-CPBA (1.2 equiv) at room temperature. The reaction mixture was stirred at room temperature for $3 \mathrm{~h}$. After completion of the reaction (monitored by TLC), a saturated solution of $\mathrm{Na}_{2} \mathrm{CO}_{3}$ (aq) was added to quench the reaction. The resulting mixture was extracted with DCM. The organic layers were washed with water and brine, dried over $\mathrm{Na}_{2} \mathrm{SO}_{4}$, filtered, and evaporated. The residue was purified by column chromatography on silica gel, eluting with ethyl acetate/petroleum ether to give the desired product in good to high yield.

\section{General procedure D}

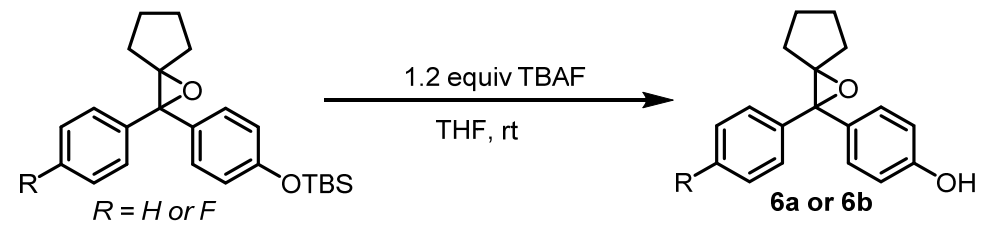

To a solution of the corresponding epoxide (1.0 equiv) in THF $(0.5 \mathrm{M})$ was added a solution of TBAF (1.0 M in THF, 1.2 equiv) at $0{ }^{\circ} \mathrm{C}$. The reaction was stirred at the same temperature for $1 \mathrm{~h}$. After completion of the reaction (monitored by TLC), water was added to quench the reaction and the mixture was extracted with EtOAc. The organic layers were washed with water and brine, dried over $\mathrm{Na}_{2} \mathrm{SO}_{4}$, filtered, and evaporated. The residue was purified by column 
chromatography on silica gel, eluting with ethyl acetate/petroleum ether to give the desired product $\mathbf{5 a}$ or $\mathbf{5 b}$.

4-(1-hydroxy-1-(1-hydroxycyclopentyl)-3-phenylprop-2-yn-1-yl)phenol (3a)

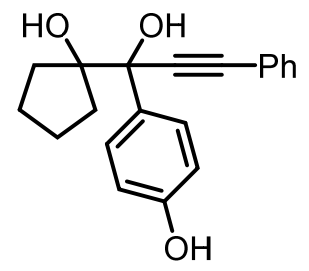

Compound 3a was synthesized according to general procedure A and B starting from (1bromocyclopentyl)(4-((tert-butyldimethylsilyl)oxy)phenyl)methanone (10.0 mmol). Substrate 3a was obtained in 54\% yield over three steps as a white solid. M.p $=150-152{ }^{\circ} \mathrm{C}$.

${ }^{1}$ H NMR (400 MHz, DMSO-d6) $\delta 9.24(\mathrm{~s}, 1 \mathrm{H}), 7.54-7.28(\mathrm{~m}, 7 \mathrm{H}), 6.72-6.67(\mathrm{~m}, 2 \mathrm{H}), 5.91$ (s, 1H), $4.16(\mathrm{~s}, 1 \mathrm{H}), 2.11-1.94(\mathrm{~m}, 2 \mathrm{H}), 1.73-1.59(\mathrm{~m}, 2 \mathrm{H}), 1.57-1.44(\mathrm{~m}, 2 \mathrm{H}), 1.41-$ $1.29(\mathrm{~m}, 2 \mathrm{H})$.

${ }^{13}$ C NMR (101 MHz, DMSO-d6) $\delta$ 156.2, 133.5, 131.3, 128.6, 128.3, 122.9, 113.5, 94.3, 86.3, 83.4, 76.1, 35.8, 35.4, 24.2, 24.1 .

IR $\left(v_{\max }, \mathrm{cm}^{-1}\right) 3361(\mathrm{~m}), 2961$ (m), 2167 (m), 1516 (s), 1441 (s), 1241 (s), 1366 (s), 1015 (s), $834(s)$.

HRMS (ESI/QTOF) m/z: [M + Na $]^{+}$Calcd for $\mathrm{C}_{20} \mathrm{H}_{20} \mathrm{NaO}_{3}{ }^{+} 331.1305$; Found 331.1308.

4-(1-hydroxy-1-(1-hydroxycyclopentyl)-3-(p-tolyl)prop-2-yn-1-yl)phenol (3b)

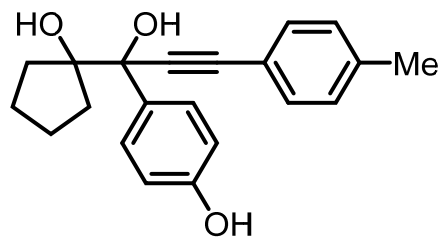

Compound 3b was synthesized according to general procedure A and B starting from (1bromocyclopentyl)(4-((tert-butyldimethylsilyl)oxy)phenyl)methanone (10.0 mmol). Substrate 3b was obtained in 51\% yield over three steps as a white solid. M.p $=165-167{ }^{\circ} \mathrm{C}$.

${ }^{1}$ H NMR (400 MHz, DMSO-d6) $\delta 9.26(\mathrm{~s}, 1 \mathrm{H}), 7.48(\mathrm{~d}, J=8.6 \mathrm{~Hz}, 2 \mathrm{H}), 7.38(\mathrm{~d}, J=8.0 \mathrm{~Hz}$, 2H), $7.23(\mathrm{~d}, J=7.9 \mathrm{~Hz}, 2 \mathrm{H}), 6.72(\mathrm{~d}, J=8.6 \mathrm{~Hz}, 2 \mathrm{H}), 5.90$ (s, 1H), 4.16 (s, 1H), 2.36 (s, 3H), $2.13-1.97(\mathrm{~m}, 2 \mathrm{H}), 1.75-1.63(\mathrm{~m}, 2 \mathrm{H}), 1.61-1.46(\mathrm{~m}, 2 \mathrm{H}), 1.42-1.32(\mathrm{~m}, 2 \mathrm{H})$.

${ }^{13}$ C NMR (101 MHz, DMSO-d6) $\delta$ 156.7, 138.4, 134.1, 131.7, 129.7, 129.1, 120.4, 113.9, 94.1, $86.8,84.0,76.5,36.3,35.9,24.7,24.6,21.5$.

IR $\left(v_{\max }, \mathrm{cm}^{-1}\right) 3390(\mathrm{~m}), 2966(\mathrm{~m}), 2164(\mathrm{~m}), 1513$ (s), $1349(\mathrm{~m}), 1174(\mathrm{~m}), 1008(\mathrm{~m}), 813(\mathrm{~s})$, $761(\mathrm{~m})$. 
HRMS (ESI/QTOF) m/z: [M + Na $]^{+}$Calcd for $\mathrm{C}_{21} \mathrm{H}_{22} \mathrm{NaO}_{3}{ }^{+} 345.1461$; Found 345.1461.

4-(3-([1,1'-biphenyl]-4-yl)-1-hydroxy-1-(1-hydroxycyclopentyl)prop-2-yn-1-yl)phenol (3c)

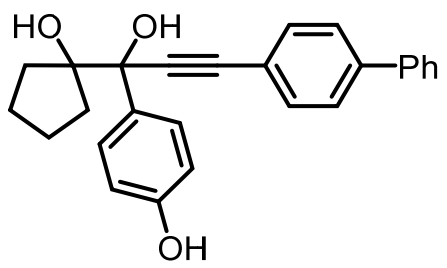

Compound 3c was synthesized according to general procedure A and B starting from (1bromocyclopentyl)(4-((tert-butyldimethylsilyl)oxy)phenyl)methanone (10.0 mmol). Substrate 3c was obtained in $57 \%$ yield over three steps as a white solid. M.p $=220-222^{\circ} \mathrm{C}$.

${ }^{1}$ H NMR (400 MHz, DMSO-d $d_{6} \delta 9.25(\mathrm{~s}, 1 \mathrm{H}), 7.75-7.65(\mathrm{~m}, 4 \mathrm{H}), 7.60-7.33(\mathrm{~m}, 7 \mathrm{H}), 6.70$ $(\mathrm{d}, J=7.5 \mathrm{~Hz}, 2 \mathrm{H}), 5.93(\mathrm{~s}, 1 \mathrm{H}), 4.18(\mathrm{~s}, 1 \mathrm{H}), 2.12-1.96(\mathrm{~m}, 2 \mathrm{H}), 1.72-1.60(\mathrm{~m}, 2 \mathrm{H}), 1.58$ $-1.45(\mathrm{~m}, 2 \mathrm{H}), 1.42-1.28(\mathrm{~m}, 2 \mathrm{H})$.

${ }^{13}$ C NMR (101 MHz, DMSO-d $) \delta 156.7,140.3,139.7,134.0,132.4,129.5,129.1,128.3$, $127.3,127.1,122.4,114.0,95.6,86.8,83.8,76.6,36.3,35.9,24.7,24.6$.

IR ( $\left.v_{\max }, \mathrm{cm}^{-1}\right) 3346$ (m), 2960 (m), 2359 (s), 1612 (m), 1511 (s), 1245 (s), 1061 (s), 846 (s), 764 (s).

HRMS (ESI/QTOF) m/z: [M + Na $]^{+}$Calcd for $\mathrm{C}_{26} \mathrm{H}_{24} \mathrm{NaO}_{3}{ }^{+} 407.1618$; Found 407.1615.

4-(3-(4-fluorophenyl)-1-hydroxy-1-(1-hydroxycyclopentyl)prop-2-yn-1-yl)phenol (3d)

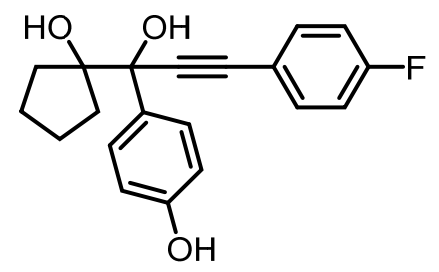

Compound 3d was synthesized according to general procedure A and B starting from (1bromocyclopentyl)(4-((tert-butyldimethylsilyl)oxy)phenyl)methanone $(10.0 \mathrm{mmol})$. Substrate 3d was obtained in $67 \%$ yield over three steps as a white solid. M.p $=136-138^{\circ} \mathrm{C}$.

${ }^{1}$ H NMR (400 MHz, DMSO-d6) $\delta 9.25(\mathrm{~s}, 1 \mathrm{H}), 7.57-7.35(\mathrm{~m}, 4 \mathrm{H}), 7.31-7.15(\mathrm{~m}, 2 \mathrm{H}), 6.73$ $-6.64(\mathrm{~m}, 2 \mathrm{H}), 5.91(\mathrm{~s}, 1 \mathrm{H}), 4.17(\mathrm{~s}, 1 \mathrm{H}), 2.08-1.94(\mathrm{~m}, 2 \mathrm{H}), 1.74-1.25(\mathrm{~m}, 6 \mathrm{H})$.

${ }^{13}$ C NMR (101 MHz, DMSO-d6) $\delta 162.2(\mathrm{~d}, J=246.9 \mathrm{~Hz}), 156.6,134.0$ (d, $\left.J=8.5 \mathrm{~Hz}\right), 133.9$, 129.0, $119.6(\mathrm{~d}, J=3.3 \mathrm{~Hz}), 116.3$ (d, $J=21.9 \mathrm{~Hz}), 114.0,94.2,86.9,82.9$, 76.5, 36.2, 35.8, 24.6, 24.5.

${ }^{19}$ F NMR (377 MHz, DMSO- $\left.d_{6}\right) \delta-111.2$.

IR $\left(v_{\max }, \mathrm{cm}^{-1}\right) 3301(\mathrm{~m}), 2947(\mathrm{~m}), 1599$ (m), $1511(\mathrm{~s}), 1226$ (s), 1055 (m), $1011(\mathrm{~m}), 834$ (s), $2365(w)$. 
HRMS (ESI/QTOF) m/z: [M+ Na] ${ }^{+}$Calcd for $\mathrm{C}_{20} \mathrm{H}_{19} \mathrm{FNaO}_{3}{ }^{+}$349.1210; Found 349.1217.

4-(1-hydroxy-1-(1-hydroxycyclopentyl)-3-(4-methoxyphenyl)prop-2-yn-1-yl)phenol (3e)

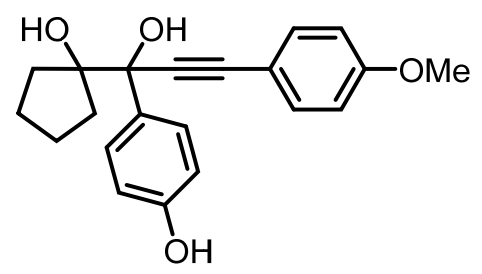

Compound 3e was synthesized according to general procedure A and B starting from (1bromocyclopentyl)(4-((tert-butyldimethylsilyl)oxy)phenyl)methanone (10.0 mmol). Substrate 3e was obtained in $44 \%$ yield over three steps as a white solid. M.p $=178-180{ }^{\circ} \mathrm{C}$.

${ }^{1}$ H NMR (400 MHz, DMSO-d $) \delta 9.22(\mathrm{~s}, 1 \mathrm{H}), 7.44(\mathrm{~d}, J=8.1 \mathrm{~Hz}, 2 \mathrm{H}), 7.38(\mathrm{~d}, J=8.1 \mathrm{~Hz}$, 2H), $6.94(\mathrm{~d}, J=8.3 \mathrm{~Hz}, 2 \mathrm{H}), 6.68$ (d, $J=8.2 \mathrm{~Hz}, 2 \mathrm{H}), 5.83(\mathrm{~s}, 1 \mathrm{H}), 4.10$ (s, 1H), 3.77 (s, 3H), $2.11-1.92(\mathrm{~m}, 2 \mathrm{H}), 1.74-1.59(\mathrm{~m}, 2 \mathrm{H}), 1.57-1.43(\mathrm{~m}, 2 \mathrm{H}), 1.41-1.27(\mathrm{~m}, 2 \mathrm{H})$.

${ }^{13}$ C NMR $\left(101 \mathrm{MHz}, \mathrm{DMSO}-d_{6}\right) \delta 159.1,156.2,133.7,132.8,128.6,114.9,114.2,113.5,92.7$, $86.4,83.4,76.1,55.2,35.8,35.4,24.2,24.2$.

IR $\left(v_{\max }, \mathrm{cm}^{-1}\right) 3301(\mathrm{~m}), 2966(\mathrm{~m}), 2352(\mathrm{~m}), 1612(\mathrm{~m}), 1511(\mathrm{~s}), 1251$ (s), $834(\mathrm{~m}), 760(\mathrm{~s})$.

HRMS (ESI/QTOF) m/z: [M + Na] ${ }^{+}$Calcd for $\mathrm{C}_{21} \mathrm{H}_{22} \mathrm{NaO}_{4}{ }^{+}$361.1410; Found 361.1414.

4-(3-(3-fluorophenyl)-1-hydroxy-1-(1-hydroxycyclopentyl)prop-2-yn-1-yl)phenol (3f)

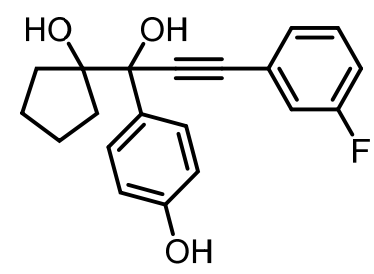

Compound 3f was synthesized according to general procedure A and B starting from (1bromocyclopentyl)(4-((tert-butyldimethylsilyl)oxy)phenyl)methanone (10.0 mmol). Substrate 3f was obtained in $37 \%$ yield over three steps as a white solid. M.p $=155-157^{\circ} \mathrm{C}$.

${ }^{1}$ H NMR (400 MHz, DMSO-d6) $\delta 9.24(\mathrm{~s}, 1 \mathrm{H}), 7.55$ - $7.10(\mathrm{~m}, 6 \mathrm{H}), 6.74-6.62(\mathrm{~m}, 2 \mathrm{H}), 5.95$ (s, 1H), 4.19 (s, 1H), $2.11-1.89(\mathrm{~m}, 2 \mathrm{H}), 1.72-1.21(\mathrm{~m}, 6 \mathrm{H})$.

${ }^{13}$ C NMR (101 MHz, DMSO-d6) $\delta 162.3(\mathrm{~d}, J=244.3 \mathrm{~Hz}), 156.7,133.7,131.2(\mathrm{~d}, J=8.7 \mathrm{~Hz})$, $129.1,128.1(\mathrm{~d}, J=2.8 \mathrm{~Hz}), 125.3(\mathrm{~d}, J=9.0 \mathrm{~Hz}), 118.3$ (d, $J=22.7 \mathrm{~Hz}), 116.1$ (d, $J=20.6$ Hz), 114.0, 96.0, 86.8, 82.6, 76.5, 36.3, 35.6, 24.6, 24.5.

${ }^{19}$ F NMR (377 MHz, DMSO-d6) $\delta-112.79$.

IR $\left(v_{\max }, \mathrm{cm}^{-1}\right) 3380(\mathrm{~m}), 2967(\mathrm{~m}), 1610(\mathrm{~s}), 1582(\mathrm{~s}), 1514(\mathrm{~s}), 1446$ (s), 1238 (s), 1175 (s), 1055 (s), 1012 (s), 833 (s), 731 (s), 679 (s). 
HRMS (ESI/QTOF) m/z: [M+ Na] ${ }^{+}$Calcd for $\mathrm{C}_{20} \mathrm{H}_{19} \mathrm{FNaO}_{3}{ }^{+}$349.1210; Found 349.1209.

4-(3-(3-chlorophenyl)-1-hydroxy-1-(1-hydroxycyclopentyl)prop-2-yn-1-yl)phenol (3g)

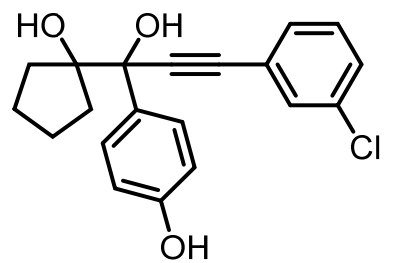

Compound 3g was synthesized according to general procedure A and B starting from (1bromocyclopentyl)(4-((tert-butyldimethylsilyl)oxy)phenyl)methanone (10.0 mmol). Substrate 3g was obtained in $18 \%$ yield over three steps as a white solid. M.p $=170-172{ }^{\circ} \mathrm{C}$.

${ }^{1}$ H NMR (400 MHz, DMSO-d $) \delta 9.25(\mathrm{~s}, 1 \mathrm{H}), 7.64-7.30(\mathrm{~m}, 6 \mathrm{H}), 6.70(\mathrm{~d}, J=8.3 \mathrm{~Hz}, 2 \mathrm{H})$, $5.96(\mathrm{~s}, 1 \mathrm{H}), 4.22(\mathrm{~s}, 1 \mathrm{H}), 2.07-1.95(\mathrm{~m}, 2 \mathrm{H}), 1.75-1.59(\mathrm{~m}, 2 \mathrm{H}), 1.57-1.45(\mathrm{~m}, 2 \mathrm{H}), 1.43$ $-1.26(\mathrm{~m}, 2 \mathrm{H})$.

${ }^{13}$ C NMR (101 MHz, DMSO-d6) $\delta$ 156.8, 133.7, 133.6, 131.3, 131.0, 130.4, 129.1, 128.9, $125.3,114.0,96.4,86.8,82.4,76.5,36.2,35.6,24.59,24.55$.

IR $\left(v_{\max }, \mathrm{cm}^{-1}\right) 3368$ (s), 2974 (s), 2359 (m), 2162 (m), 1596 (s), 1511 (s), 1447 (s), 1369 (s), 1242 (s), 1174 (s), 1012 (s), 783 (s), 685 (s), 812 (m).

HRMS (ESI/QTOF) m/z: [M + Na] ${ }^{+}$Calcd for $\mathrm{C}_{20} \mathrm{H}_{19} \mathrm{ClNaO}_{3}{ }^{+}$365.0915; Found 365.0920.

4-(1-hydroxy-1-(1-hydroxycyclopentyl)-3-(3-methoxyphenyl)prop-2-yn-1-yl)phenol (3h)

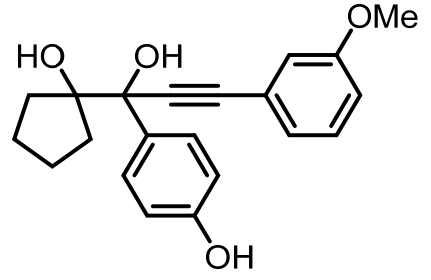

Compound 3h was synthesized according to general procedure A and B starting from (1bromocyclopentyl)(4-((tert-butyldimethylsilyl)oxy)phenyl)methanone (10.0 mmol). Substrate 3h was obtained in $15 \%$ yield over three steps as a pale yellow solid. M.p $=100-102{ }^{\circ} \mathrm{C}$.

${ }^{1}$ H NMR (400 MHz, DMSO-d6) $\delta 9.27(\mathrm{~s}, 1 \mathrm{H}), 7.48(\mathrm{~d}, J=8.3 \mathrm{~Hz}, 2 \mathrm{H}), 7.34(\mathrm{t}, J=7.9 \mathrm{~Hz}$, 1H), $7.07(\mathrm{~d}, J=7.6 \mathrm{~Hz}, 1 \mathrm{H}), 7.04-6.95(\mathrm{~m}, 2 \mathrm{H}), 6.72(\mathrm{~d}, J=8.3 \mathrm{~Hz}, 2 \mathrm{H}), 5.93(\mathrm{~s}, 1 \mathrm{H}), 4.19$ $(\mathrm{s}, 1 \mathrm{H}), 3.81(\mathrm{~s}, 3 \mathrm{H}), 2.13-1.98(\mathrm{~m}, 2 \mathrm{H}), 1.76-1.63(\mathrm{~m}, 2 \mathrm{H}), 1.62-1.45(\mathrm{~m}, 2 \mathrm{H}), 1.44-$ $1.32(\mathrm{~m}, 2 \mathrm{H})$.

${ }^{13}$ C NMR (101 MHz, DMSO-d6) $\delta$ 159.6, 156.7, 134.0, 130.2, 129.1, 124.4, 124.2, 116.6, $115.1,114.0,94.7,86.8,83.8,76.5,55.7,36.3,35.8,24.6,24.6$.

IR ( $\left.v_{\max }, \mathrm{cm}^{-1}\right) 3403$ (m), 2953 (m), 2161 (s), 1592 (s), 1513 (s), 1207 (s), 1176 (s), 1011 (s), 866 (s), 832 (s), 759 (s), 732 (s). 
HRMS (ESI/QTOF) m/z: [M + Na] ${ }^{+}$Calcd for $\mathrm{C}_{21} \mathrm{H}_{22} \mathrm{NaO}_{4}{ }^{+}$361.1410; Found 361.1408.

4-(1-hydroxy-1-(1-hydroxycyclopentyl)-3-(o-tolyl)prop-2-yn-1-yl)phenol (3i)

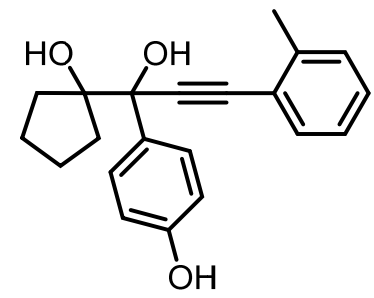

Compound 3i was synthesized according to general procedure A and B starting from (1bromocyclopentyl)(4-((tert-butyldimethylsilyl)oxy)phenyl)methanone (10.0 mmol). Substrate 3i was obtained in $14 \%$ yield over three steps as a pale yellow solid. M.p $=116-118{ }^{\circ} \mathrm{C}$.

${ }^{1}$ H NMR (400 MHz, DMSO-d6) $\delta 9.23(\mathrm{~s}, 1 \mathrm{H}), 7.47$ (d, $\left.J=8.1 \mathrm{~Hz}, 2 \mathrm{H}\right), 7.39$ (d, $J=7.5 \mathrm{~Hz}$, 1H), $7.31-7.14(\mathrm{~m}, 3 \mathrm{H}), 6.69$ (d, $J=8.2 \mathrm{~Hz}, 2 \mathrm{H}), 5.90(\mathrm{~s}, 1 \mathrm{H}), 4.13(\mathrm{~s}, 1 \mathrm{H}), 2.43(\mathrm{~s}, 3 \mathrm{H}), 2.14$ - $1.96(\mathrm{~m}, 2 \mathrm{H}), 1.73-1.59(\mathrm{~m}, 2 \mathrm{H}), 1.57-1.44(\mathrm{~m}, 2 \mathrm{H}), 1.41-1.28(\mathrm{~m}, 2 \mathrm{H})$.

${ }^{13}$ C NMR (101 MHz, DMSO-d6) $\delta$ 156.7, 140.0, 134.1, 132.1, 130.0, 129.1, 128.7, 126.3, $123.2,114.0,98.7,86.8,82.7,76.8,36.4,36.0,24.7,24.6,20.9$.

IR $\left(v_{\max }, \mathrm{cm}^{-1}\right) 3384$ (s), 2953 (m), 2365 (s), 2161 (s), 1511 (s), 1447 (s), 1367 (s), 1237 (s), $1010(\mathrm{~s}), 814(\mathrm{~s}), 757$ (s).

HRMS (ESI/QTOF) m/z: [M + Na $]^{+}$Calcd for $\mathrm{C}_{21} \mathrm{H}_{22} \mathrm{NaO}_{3}{ }^{+} 345.1461$; Found 345.1457.

4-(1-hydroxy-1-(1-hydroxycyclopentyl)-3-(thiophen-3-yl)prop-2-yn-1-yl)phenol (3j)

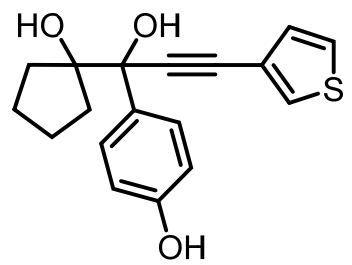

Compound 3j was synthesized according to general procedure A and B starting from (1bromocyclopentyl)(4-((tert-butyldimethylsilyl)oxy)phenyl)methanone (10.0 mmol). Substrate 3j was obtained in $10 \%$ yield over three steps as a white solid. M.p $=148-150{ }^{\circ} \mathrm{C}$.

${ }^{1}$ H NMR (400 MHz, DMSO-d6) $\delta 9.23(\mathrm{~s}, 1 \mathrm{H}), 7.75-7.73(\mathrm{~m}, 1 \mathrm{H}), 7.60(\mathrm{dd}, J=5.0,3.0 \mathrm{~Hz}$, 1H), $7.44(\mathrm{~d}, J=8.4 \mathrm{~Hz}, 2 \mathrm{H}), 7.17(\mathrm{~d}, J=4.9 \mathrm{~Hz}, 1 \mathrm{H}), 6.68$ (d, $J=8.4 \mathrm{~Hz}, 2 \mathrm{H}), 5.88(\mathrm{~s}, 1 \mathrm{H})$, $4.13(\mathrm{~s}, 1 \mathrm{H}), 2.07-1.93(\mathrm{~m}, 2 \mathrm{H}), 1.74-1.57(\mathrm{~m}, 2 \mathrm{H}), 1.55-1.45(\mathrm{~m}, 2 \mathrm{H}), 1.37-1.28(\mathrm{~m}$, $2 \mathrm{H})$.

${ }^{13}$ C NMR (101 MHz, DMSO- $\left.d_{6}\right) \delta 156.7,134.0,130.2,129.5,129.1,127.1,122.2,113.9,94.0$, $86.8,79.3,76.6,36.3,35.8,24.6,24.6$. 
IR $\left(v_{\max }, \mathrm{cm}^{-1}\right) 3352(\mathrm{~s}), 2960(\mathrm{~m}), 2359$ (s), $2156(\mathrm{~m}), 1612$ (m), 1511 (s), $1442(\mathrm{~m}), 1364$ (m), $1236(\mathrm{~s}), 1012(\mathrm{~s}), 785(\mathrm{~s})$.

HRMS (ESI/QTOF) m/z: [M + Na $]^{+}$Calcd for $\mathrm{C}_{18} \mathrm{H}_{18} \mathrm{NaO}_{3} \mathrm{~S}^{+} 337.0869$; Found 337.0873.

4-(1-hydroxy-1-(1-hydroxycyclopentyl)-4,4-dimethylpent-2-yn-1-yl)phenol (3k)

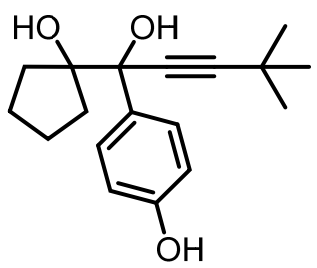

Compound 3k was synthesized according to general procedure A and B starting from (1bromocyclopentyl)(4-((tert-butyldimethylsilyl)oxy)phenyl)methanone (10.0 mmol). Substrate 3k was obtained in $27 \%$ yield over three steps as a white solid. M.p $=118-120{ }^{\circ} \mathrm{C}$.

${ }^{1}$ H NMR (400 MHz, DMSO-d6) $\delta 9.17(\mathrm{~s}, 1 \mathrm{H}), 7.37$ (d, $\left.J=8.7 \mathrm{~Hz}, 2 \mathrm{H}\right), 6.64(\mathrm{~d}, J=8.7 \mathrm{~Hz}$, 2H), $5.47(\mathrm{~s}, 1 \mathrm{H}), 3.77(\mathrm{~s}, 1 \mathrm{H}), 2.13-2.00(\mathrm{~m}, 1 \mathrm{H}), 1.92-1.78(\mathrm{~m}, 1 \mathrm{H}), 1.71-1.54(\mathrm{~m}, 2 \mathrm{H})$, $1.54-1.43(\mathrm{~m}, 2 \mathrm{H}), 1.41-1.34(\mathrm{~m}, 1 \mathrm{H}), 1.23(\mathrm{~s}, 9 \mathrm{H}), 1.15-1.06(\mathrm{~m}, 1 \mathrm{H})$.

${ }^{13}$ C NMR (101 MHz, DMSO-d $) \delta$ 156.5, 134.5, 129.1, 113.7, 92.7, 86.9, 83.3, 76.2, 36.4, $36.3,31.3,27.5,24.9,24.8$.

IR $\left(v_{\max }, \mathrm{cm}^{-1}\right) 3282(\mathrm{~s}), 2966(\mathrm{~s}), 2360(\mathrm{~m}), 2158(\mathrm{~s}), 1510(\mathrm{~m}), 1363(\mathrm{~m}), 1243(\mathrm{~m}), 1008(\mathrm{~m})$, 758 (s).

HRMS (ESI/QTOF) m/z: [M + Na $]^{+}$Calcd for $\mathrm{C}_{18} \mathrm{H}_{24 \mathrm{NaO}_{3}}{ }^{+} 311.1618$; Found 311.1620.

4-(1-hydroxy-1-(1-hydroxycyclopentyl)hept-2-yn-1-yl)phenol (3I)

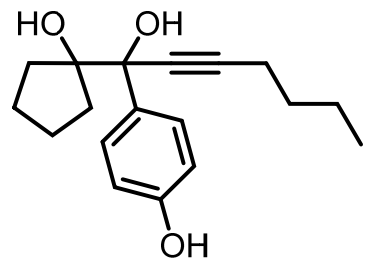

Compound 3l was synthesized according to general procedure A and B starting from (1bromocyclopentyl)(4-((tert-butyldimethylsilyl)oxy)phenyl)methanone (10.0 mmol). Substrate 31 was obtained in $28 \%$ yield over three steps as a white solid. M.p $=145-147^{\circ} \mathrm{C}$.

${ }^{1}$ H NMR (400 MHz, DMSO-d $) \delta 9.16(\mathrm{~s}, 1 \mathrm{H}), 7.37$ (d, $\left.J=8.1 \mathrm{~Hz}, 2 \mathrm{H}\right), 6.64(\mathrm{~d}, J=8.2 \mathrm{~Hz}$, 2H), $5.54(\mathrm{~s}, 1 \mathrm{H}), 3.86(\mathrm{~s}, 1 \mathrm{H}), 2.25(\mathrm{t}, J=6.4 \mathrm{~Hz}, 2 \mathrm{H}), 2.08-1.97(\mathrm{~m}, 1 \mathrm{H}), 1.92-1.82(\mathrm{~m}$, $1 \mathrm{H}), 1.68-1.56(\mathrm{~m}, 2 \mathrm{H}), 1.53-1.28(\mathrm{~m}, 7 \mathrm{H}), 1.23-1.12(\mathrm{~m}, 1 \mathrm{H}), 0.90(\mathrm{t}, J=6.9 \mathrm{~Hz}, 3 \mathrm{H})$. ${ }^{13}$ C NMR (101 MHz, DMSO-d6) $\delta 156.5,134.6,129.1,113.7,86.8,85.0,84.3,76.3,36.2$, $36.1,30.9,24.7,24.6,21.8,18.3,13.9$. 
IR $\left(v_{\max }, \mathrm{cm}^{-1}\right) 3361$ (s), 2955 (m), 1597 (m), $1514(\mathrm{~s}), 1453$ (m), 1375 (s), 1243 (s), 1002 (s), $806(\mathrm{~s})$.

HRMS (ESI/QTOF) m/z: [M + Na] ${ }^{+}$Calcd for $\mathrm{C}_{18} \mathrm{H}_{24} \mathrm{NaO}_{3}{ }^{+}$311.1618; Found 311.1619.

1-(1-hydroxy-1-(4-methoxyphenyl)-3-phenylprop-2-yn-1-yl)cyclopentan-1-ol (3m)

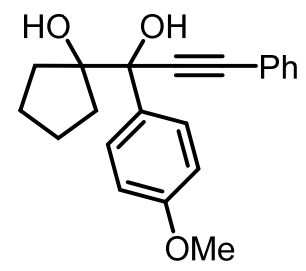

Compound 3m was synthesized according to general procedure A starting from (1bromocyclopentyl)(4-methoxyphenyl)methanone (10.0 mmol). Substrate $3 \mathbf{m}$ was obtained in $47 \%$ yield over two steps as a yellow oil.

${ }^{1}$ H NMR (400 MHz, DMSO-d $) \delta 7.60$ - $7.55(\mathrm{~m}, 2 \mathrm{H}), 7.51$ - $7.31(\mathrm{~m}, 5 \mathrm{H}), 6.90$ - 6.84 (m, 2H), $6.00(\mathrm{~s}, 1 \mathrm{H}), 4.23(\mathrm{~s}, 1 \mathrm{H}), 3.75(\mathrm{~s}, 3 \mathrm{H}), 2.11-1.97(\mathrm{~m}, 2 \mathrm{H}), 1.72-1.61(\mathrm{~m}, 2 \mathrm{H}), 1.58-$ $1.46(\mathrm{~m}, 2 \mathrm{H}), 1.40-1.28(\mathrm{~m}, 2 \mathrm{H})$.

${ }^{13}$ C NMR (101 MHz, DMSO-d $) \delta 158.7,135.8,131.8,129.1,129.1,128.8,123.3,112.6,94.6$, $86.8,84.0,76.4,55.5,36.4,35.8,24.7,24.6$.

IR $\left(v_{\max }, \mathrm{cm}^{-1}\right) 3460(\mathrm{~m}), 2953(\mathrm{~m}), 2359$ (s), 1612 (m), 1511 (s), 1251 (s), 1175 (s), 1036 (s), $758(\mathrm{~s})$.

HRMS (ESI/QTOF) m/z: [M + Na $]^{+}$Calcd for $\mathrm{C}_{21} \mathrm{H}_{22} \mathrm{NaO}_{3}{ }^{+} 345.1461$; Found 345.1458.

1-(6-chloro-1-(3,4-dimethoxyphenyl)-1-hydroxyhex-2-yn-1-yl)cyclopentan-1-ol (3n)

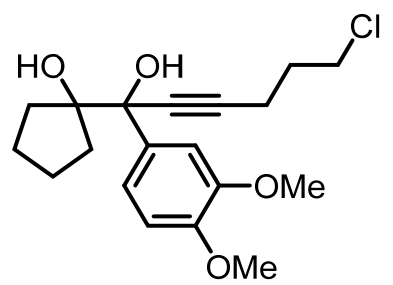

Compound 3n was synthesized according to general procedure A starting from (1bromocyclopentyl)(3,4-dimethoxyphenyl)methanone (10.0 mmol). Substrate 3n was obtained in $51 \%$ yield over two steps as a pale yellow oil.

${ }^{1}$ H NMR (400 MHz, DMSO-d $) \delta 7.23(\mathrm{~d}, J=2.0 \mathrm{~Hz}, 1 \mathrm{H}), 7.17-7.12(\mathrm{~m}, 1 \mathrm{H}), 6.89$ (d, $J=$ $8.4 \mathrm{~Hz}, 1 \mathrm{H}), 5.78(\mathrm{~s}, 1 \mathrm{H}), 4.06(\mathrm{~s}, 1 \mathrm{H}), 3.82(\mathrm{t}, J=6.5 \mathrm{~Hz}, 2 \mathrm{H}), 3.77(\mathrm{~s}, 3 \mathrm{H}), 3.76(\mathrm{~s}, 3 \mathrm{H}), 2.46$ $(\mathrm{t}, J=6.8 \mathrm{~Hz}, 2 \mathrm{H}), 2.08-1.90(\mathrm{~m}, 4 \mathrm{H}), 1.73-1.60(\mathrm{~m}, 2 \mathrm{H}), 1.58-1.45(\mathrm{~m}, 2 \mathrm{H}), 1.36-1.26$ $(\mathrm{m}, 2 \mathrm{H})$.

${ }^{13}$ C NMR (101 MHz, DMSO-d6) $\delta 148.1,147.5,136.8,120.2,112.6,110.6,86.7,85.7,82.7$, $76.2,55.9,55.9,44.7,36.3,35.8,31.7,24.6,24.6,16.2$. 
IR $\left(v_{\max }, \mathrm{cm}^{-1}\right) 3480(\mathrm{w}), 2949(\mathrm{~m}), 2160$ (m), 1513 (s), 1456 (m), 1260 (s), 1135 (s), 1027 (s), $797(\mathrm{~m}), 763(\mathrm{~m})$.

HRMS (ESI/QTOF) m/z: [M + Na $]^{+}$Calcd for $\mathrm{C}_{19} \mathrm{H}_{25} \mathrm{ClNaO}_{4}{ }^{+}$375.1334; Found 375.1334.

1-(1-(3,4-dimethoxyphenyl)-1-hydroxy-3-(trimethylsilyl)prop-2-yn-1-yl)cyclopentan-1-ol (30)

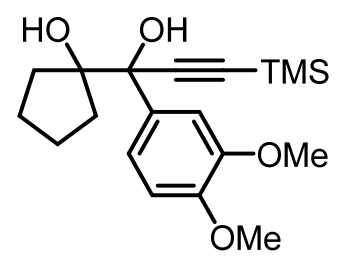

Compound 3o was synthesized according to general procedure A starting from (1bromocyclopentyl)(3,4-dimethoxyphenyl)methanone (10.0 mmol). Substrate 30 was obtained in $60 \%$ yield over two steps as a pale yellow solid. M.p $=95-97{ }^{\circ} \mathrm{C}$.

${ }^{1}$ H NMR (400 MHz, DMSO-d $) \delta 7.24(\mathrm{~d}, J=2.1 \mathrm{~Hz}, 1 \mathrm{H}), 7.15$ (dd, $\left.J=8.4,2.1 \mathrm{~Hz}, 1 \mathrm{H}\right), 6.91$ $(\mathrm{d}, J=8.5 \mathrm{~Hz}, 1 \mathrm{H}), 5.92(\mathrm{~s}, 1 \mathrm{H}), 4.09(\mathrm{~s}, 1 \mathrm{H}), 3.78(\mathrm{~s}, 3 \mathrm{H}), 3.76(\mathrm{~s}, 3 \mathrm{H}), 2.16-2.05(\mathrm{~m}, 1 \mathrm{H})$, $1.99-1.90(\mathrm{~m}, 1 \mathrm{H}), 1.73-1.61(\mathrm{~m}, 2 \mathrm{H}), 1.60-1.46(\mathrm{~m}, 2 \mathrm{H}), 1.45-1.36(\mathrm{~m}, 1 \mathrm{H}), 1.29-1.20$ $(\mathrm{m}, 1 \mathrm{H}), 0.22(\mathrm{~s}, 9 \mathrm{H})$.

${ }^{13}$ C NMR (101 MHz, DMSO-d6) $\delta$ 148.2, 147.5, 135.9, 120.2, 112.7, 111.1, 110.7, 88.4, 86.6, $76.7,56.0,55.8,36.3,36.2,24.8,24.7,0.4$.

IR $\left(v_{\max }, \mathrm{cm}^{-1}\right) 3487(\mathrm{w}), 2955$ (w), 1514 (m), 1459 (w), 1408 (w), 1259 (s), 1145 (m), 1026 (m), $843(\mathrm{~s}), 762(\mathrm{~m})$.

HRMS (ESI/QTOF) m/z: [M + Na] ${ }^{+}$Calcd for $\mathrm{C}_{19} \mathrm{H}_{28} \mathrm{NaO}_{4} \mathrm{Si}^{+}$371.1649; Found 371.1644.

1-(5-(benzyloxy)-1-(3,4-dimethoxyphenyl)-1-hydroxypent-2-yn-1-yl)cyclopentan-1-ol (3p)

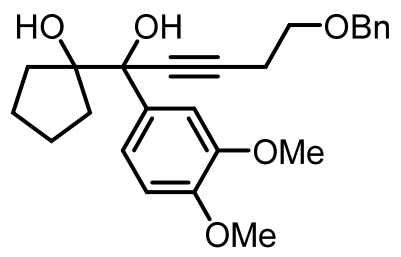

Compound 3p was synthesized according to general procedure A starting from (1bromocyclopentyl)(3,4-dimethoxyphenyl)methanone (10.0 mmol). Substrate 3p was obtained in $37 \%$ yield over two steps as a pale yellow oil.

${ }^{1}$ H NMR (400 MHz, DMSO-d6) $\delta 7.40-7.36(\mathrm{~m}, 4 \mathrm{H}), 7.36-7.29(\mathrm{~m}, 1 \mathrm{H}), 7.25$ (d, $J=2.1$ Hz, 1H), $7.17(\mathrm{dd}, J=8.4,2.1 \mathrm{~Hz}, 1 \mathrm{H}), 6.84(\mathrm{~d}, J=8.4 \mathrm{~Hz}, 1 \mathrm{H}), 5.75(\mathrm{~s}, 1 \mathrm{H}), 4.57$ (s, 2H), $4.01(\mathrm{~s}, 1 \mathrm{H}), 3.76(\mathrm{~s}, 3 \mathrm{H}), 3.72(\mathrm{~s}, 3 \mathrm{H}), 3.62$ (t, $J=6.7 \mathrm{~Hz}, 2 \mathrm{H}), 2.60(\mathrm{t}, J=6.7 \mathrm{~Hz}, 2 \mathrm{H}), 2.12$ 
$-2.03(\mathrm{~m}, 1 \mathrm{H}), 2.01-1.91(\mathrm{~m}, 1 \mathrm{H}), 1.72-1.59(\mathrm{~m}, 2 \mathrm{H}), 1.57-1.45(\mathrm{~m}, 2 \mathrm{H}), 1.42-1.33(\mathrm{~m}$, $1 \mathrm{H}), 1.29-1.20(\mathrm{~m}, 1 \mathrm{H})$.

${ }^{13}$ C NMR (101 MHz, DMSO-d $) \delta$ 148.1, 147.5, 138.8, 136.7, 128.7, 127.9, 127.8, 120.4, 112.8, 110.6, 86.8, 86.8, 85.4, 82.0, 76.4, 72.3, 68.7, 56.0, 55.9, 36.2, 36.0, 24.6, 24.6, 20.1.

IR $\left(v_{\max }, \mathrm{cm}^{-1}\right) 3480(\mathrm{w}), 2936(\mathrm{w}), 2867(\mathrm{w}), 2160(\mathrm{w}), 1513$ (s), 1457 (m), 1263 (s), 1135 (s), 1027 (s), $802(\mathrm{~m}), 742(\mathrm{~m}), 698(\mathrm{~m})$.

HRMS (ESI/QTOF) m/z: [M + Na $]^{+}$Calcd for $\mathrm{C}_{25} \mathrm{H}_{30} \mathrm{NaO}_{5}{ }^{+}$433.1985; Found 433.1986.

1-(1-(3,4-dimethoxyphenyl)-1-hydroxy-3-phenylprop-2-yn-1-yl)cyclopentan-1-ol (3q)

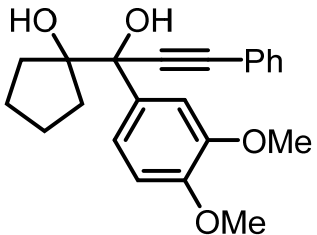

Compound 3q was synthesized according to general procedure A starting from (1bromocyclopentyl)(3,4-dimethoxyphenyl)methanone (10.0 mmol). Substrate $\mathbf{3 q}$ was obtained in $43 \%$ yield over two steps as a pale yellow oil.

${ }^{1}$ H NMR (400 MHz, DMSO-d6) $\delta 7.53-7.39(\mathrm{~m}, 5 \mathrm{H}), 7.33-7.30(\mathrm{~m}, 1 \mathrm{H}), 7.26-7.21(\mathrm{~m}$, $1 \mathrm{H}), 6.94(\mathrm{~d}, J=8.5 \mathrm{~Hz}, 1 \mathrm{H}), 6.07(\mathrm{~s}, 1 \mathrm{H}), 4.27(\mathrm{~s}, 1 \mathrm{H}), 3.79(\mathrm{~s}, 6 \mathrm{H}), 2.16-2.02(\mathrm{~m}, 2 \mathrm{H}), 1.76$ $-1.66(\mathrm{~m}, 2 \mathrm{H}), 1.64-1.50(\mathrm{~m}, 2 \mathrm{H}), 1.45-1.34(\mathrm{~m}, 2 \mathrm{H})$.

${ }^{13}$ C NMR (101 MHz, DMSO-d6) $\delta$ 148.3, 147.6, 136.3, 131.8, 129.1, 128.8, 123.3, 120.3, $112.6,110.8,94.6,86.8,84.0,76.6,56.0,55.9,36.4,35.9,24.7,24.6$.

IR $\left(v_{\max }, \mathrm{cm}^{-1}\right) 3455$ (w), 2949 (w), 1512 (s), 1442 (m), 1263 (s), 1141 (s), 1029 (s), 758 (s), $691(\mathrm{~m})$.

HRMS (ESI/QTOF) m/z: [M + Na $]^{+}$Calcd for $\mathrm{C}_{22} \mathrm{H}_{24} \mathrm{NaO}_{4}{ }^{+}$375.1567; Found 375.1571.

1-(1-(benzo[d][1,3]dioxol-5-yl)-1-hydroxy-3-(trimethylsilyl)prop-2-yn-1-yl)cyclopentan1-ol (3r)

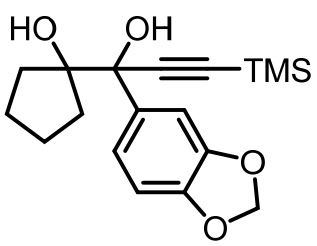

Compound 3r was synthesized according to general procedure A starting from benzo[d][1,3]dioxol-5-yl(1-bromocyclopentyl)methanone $(10.0 \mathrm{mmol})$. Substrate $3 \mathbf{r}$ was obtained in $12 \%$ yield over two steps as a pale yellow oil. 
${ }^{1}$ H NMR (400 MHz, DMSO-d $d_{6} \delta 7.11-7.05(\mathrm{~m}, 2 \mathrm{H}), 6.84-6.78(\mathrm{~m}, 1 \mathrm{H}), 5.99-5.97(\mathrm{~m}$, 2H), $5.94(\mathrm{~s}, 1 \mathrm{H}), 4.13(\mathrm{~s}, 1 \mathrm{H}), 2.09-1.99(\mathrm{~m}, 1 \mathrm{H}), 1.93-1.84(\mathrm{~m}, 1 \mathrm{H}), 1.69-1.58(\mathrm{~m}, 2 \mathrm{H})$, $1.53-1.45(\mathrm{~m}, 2 \mathrm{H}), 1.37-1.30(\mathrm{~m}, 1 \mathrm{H}), 1.22-1.16(\mathrm{~m}, 1 \mathrm{H}), 0.18(\mathrm{~s}, 9 \mathrm{H})$.

${ }^{13}$ C NMR $\left(101 \mathrm{MHz}, \mathrm{DMSO}-d_{6}\right) \delta 145.5,145.4,136.5,120.2,110.0,108.1,106.0,100.2,87.6$, $85.6,75.8,35.5,35.2,23.9,23.8,0.5$.

IR $\left(v_{\max }, \mathrm{cm}^{-1}\right) 3467(\mathrm{w}), 2960(\mathrm{w}), 2366(\mathrm{w}), 1487$ (m), 1434 (m), 1242 (s), 1039 (m), 843 (s), $802(\mathrm{~m}), 762(\mathrm{~m})$.

HRMS (ESI/QTOF) m/z: [M + Na] ${ }^{+}$Calcd for $\mathrm{C}_{18} \mathrm{H}_{24} \mathrm{NaO}_{4} \mathrm{Si}^{+}$355.1336; Found 355.1334.

1-(1-(2,3-dihydrobenzofuran-5-yl)-1-hydroxy-3-phenylprop-2-yn-1-yl)cyclopentan-1-ol (3s)

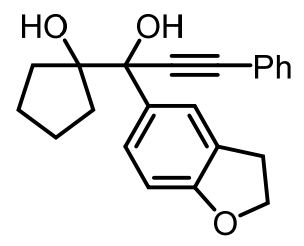

Compound 3s was synthesized according to general procedure A starting from (1bromocyclopentyl)(2,3-dihydrobenzofuran-5-yl)methanone $(10.0 \mathrm{mmol})$. Substrate $3 \mathrm{~s}$ was obtained in $43 \%$ yield over two steps as a pale yellow solid. M.p $=50-52{ }^{\circ} \mathrm{C}$.

${ }^{1}$ H NMR (400 MHz, DMSO-d6) $\delta 7.54(\mathrm{~s}, 1 \mathrm{H}), 7.52$ - 7.47 (m, 2H), 7.47 - $7.38(\mathrm{~m}, 4 \mathrm{H}), 6.72$ (d, $J=8.4 \mathrm{~Hz}, 1 \mathrm{H}), 6.01(\mathrm{~s}, 1 \mathrm{H}), 4.55(\mathrm{t}, J=8.7 \mathrm{~Hz}, 2 \mathrm{H}), 4.24(\mathrm{~s}, 1 \mathrm{H}), 3.20(\mathrm{t}, J=8.8 \mathrm{~Hz}, 2 \mathrm{H})$, $2.14-1.98(\mathrm{~m}, 2 \mathrm{H}), 1.77-1.67(\mathrm{~m}, 2 \mathrm{H}), 1.62-1.50(\mathrm{~m}, 2 \mathrm{H}), 1.45-1.32(\mathrm{~m}, 2 \mathrm{H})$.

${ }^{13}$ C NMR (101 MHz, DMSO-d6) $\delta$ 159.1, 135.8, 131.8, 129.1, 128.8, 127.5, 125.9, 124.9, $123.3,107.4,94.8,86.8,83.9,76.6,71.4,36.4,35.8,29.7,24.7,24.6$.

IR $\left(v_{\max }, \mathrm{cm}^{-1}\right) 2974(\mathrm{~m}), 2342(\mathrm{~m}), 1537$ (s), $1384(\mathrm{~m}), 1179$ (s), 1012 (s), 757 (s).

HRMS (nanochip-ESI/LTQ-Orbitrap) m/z: $[\mathrm{M}+\mathrm{Na}]^{+}$Calcd for $\mathrm{C}_{22} \mathrm{H}_{22} \mathrm{NaO}_{3}{ }^{+} 357.1461$; Found 357.1455.

\section{1-(1-hydroxy-3-phenyl-1-(thiophen-2-yl)prop-2-yn-1-yl)cyclopentan-1-ol (3t)}

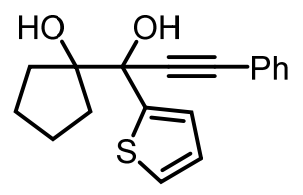

Compound 3t was synthesized according to general procedure A starting from (1bromocyclopentyl)(3,4-dimethoxyphenyl)methanone (10.0 mmol). Substrate $\mathbf{3 t}$ was obtained in $47 \%$ yield over two steps as a brown oil. 
${ }^{1}$ H NMR (400 MHz, DMSO-d6) $\delta 7.54-7.47(\mathrm{~m}, 2 \mathrm{H}), 7.46-7.40(\mathrm{~m}, 4 \mathrm{H}), 7.25(\mathrm{dd}, J=3.6$, $1.3 \mathrm{~Hz}, 1 \mathrm{H}), 7.00(\mathrm{dd}, J=5.1,3.5 \mathrm{~Hz}, 1 \mathrm{H}), 6.53(\mathrm{~s}, 1 \mathrm{H}), 4.57(\mathrm{~s}, 1 \mathrm{H}), 2.22-2.13(\mathrm{~m}, 1 \mathrm{H}), 2.12$ $-2.01(\mathrm{~m}, 1 \mathrm{H}), 1.80-1.68(\mathrm{~m}, 2 \mathrm{H}), 1.66-1.42(\mathrm{~m}, 4 \mathrm{H})$.

${ }^{13}$ C NMR (101 MHz, DMSO- $\left.d_{6}\right) \delta 148.4,131.8,129.1,129.0,126.0,125.9,125.6,122.9,93.5$, $86.6,84.0,75.2,36.7,36.2,25.2,25.0$.

IR $\left(v_{\max }, \mathrm{cm}^{-1}\right) 3415(\mathrm{~m}), 2953$ (m), 2352 (w), 1490 (m), 1443 (m), 1357 (m), 1234 (m), 1009 (s), 755 (s), 694 (s).

HRMS (ESI/QTOF) m/z: [M + Na] $]^{+}$Calcd for $\mathrm{C}_{18} \mathrm{H}_{18} \mathrm{NaO}_{2} \mathrm{~S}^{+} 321.0920$; Found 321.0921 .

1-(1-(benzofuran-2-yl)-1-hydroxy-3-phenylprop-2-yn-1-yl)cyclopentan-1-ol (3u)

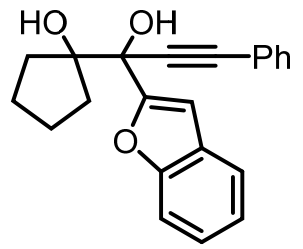

Compound 3u was synthesized according to general procedure A starting from benzofuran-2yl(1-bromocyclopentyl)methanone $(10.0 \mathrm{mmol})$. Substrate $3 \mathbf{u}$ was obtained in $78 \%$ yield over two steps as a pale yellow oil.

${ }^{1}$ H NMR (400 MHz, Methylene Chloride- $\left.d_{2}\right) \delta 7.60$ - $7.56(\mathrm{~m}, 1 \mathrm{H}), 7.54-7.45(\mathrm{~m}, 3 \mathrm{H}), 7.40$ $-7.32(\mathrm{~m}, 3 \mathrm{H}), 7.32-7.21(\mathrm{~m}, 2 \mathrm{H}), 6.98(\mathrm{~d}, J=0.9 \mathrm{~Hz}, 1 \mathrm{H}), 3.74(\mathrm{~s}, 1 \mathrm{H}), 2.45-2.36(\mathrm{~m}, 1 \mathrm{H})$, $2.36(\mathrm{~s}, 1 \mathrm{H}), 2.22-2.06(\mathrm{~m}, 1 \mathrm{H}), 1.90-1.74(\mathrm{~m}, 3 \mathrm{H}), 1.73-1.49(\mathrm{~m}, 3 \mathrm{H})$.

${ }^{13}$ C NMR (101 MHz, Methylene Chloride- $\left.d_{2}\right) \delta 157.0,155.0,131.8,128.9,128.4,127.8,124.4$, 123.0, 122.0, 121.2, 111.3, 105.5, 88.0, 87.8, 86.0, 74.6, 36.7, 36.5, 24.8, 24.8.

IR $\left(v_{\max }, \mathrm{cm}^{-1}\right) 3398(\mathrm{w}), 2956(\mathrm{~s}), 1450(\mathrm{w}), 1249$ (m), 1058 (m), 1008 (m), 750 (s), $688(\mathrm{~m})$.

HRMS (ESI/QTOF) m/z: [M + Na] ${ }^{+}$Calcd for $\mathrm{C}_{22} \mathrm{H}_{20} \mathrm{NaO}_{3}{ }^{+}$355.1305; Found 355.1310.

1-((3,4-dimethoxyphenyl)(3,5-dimethylphenyl)(hydroxy)methyl)cyclopentan-1-ol (3v)

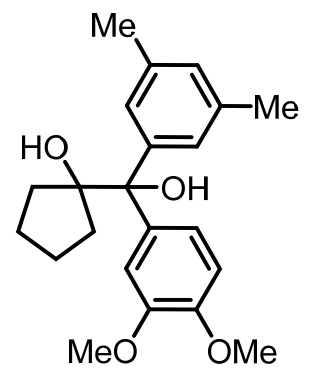

Compound $\mathbf{3 v}$ was synthesized from hydrolysis of compound $\mathbf{6 e}$. 
${ }^{1}$ H NMR (400 MHz, Methylene Chloride- $\left.d_{2}\right) \delta 7.27$ (d, $\left.J=2.0 \mathrm{~Hz}, 1 \mathrm{H}\right), 7.21(\mathrm{~s}, 2 \mathrm{H}), 7.10$ $7.06(\mathrm{~m}, 1 \mathrm{H}), 6.88(\mathrm{~s}, 1 \mathrm{H}), 6.76(\mathrm{~d}, J=8.5 \mathrm{~Hz}, 1 \mathrm{H}), 3.79(\mathrm{~s}, 3 \mathrm{H}), 3.77(\mathrm{~s}, 3 \mathrm{H}), 2.85(\mathrm{~s}, 1 \mathrm{H})$, $2.27(\mathrm{~s}, 6 \mathrm{H}), 2.18-2.04(\mathrm{~m}, 2 \mathrm{H}), 1.87$ (brs, 1H), $1.80-1.68(\mathrm{~m}, 2 \mathrm{H}), 1.65-1.46(\mathrm{~m}, 4 \mathrm{H})$.

${ }^{13}$ C NMR (101 MHz, Methylene Chloride- $d_{2}$ ) $\delta$ 148.9, 148.7, 145.9, 138.9, 137.6, 129.0, 126.2, $121.1,112.9,111.1,88.5,82.1,56.4,56.3,38.0,38.0,25.5,25.4,21.9$.

IR $\left(v_{\max }, \mathrm{cm}^{-1}\right) 2948(\mathrm{w}), 2152(\mathrm{~s}), 1797(\mathrm{w}), 1510(\mathrm{~m}), 1442(\mathrm{~m}), 1257(\mathrm{~m}), 1143$ (s), 1025 (m), $719(\mathrm{~m})$.

HRMS (ESI/QTOF) m/z: [M + Na $]^{+}$Calcd for $\mathrm{C}_{22} \mathrm{H}_{28} \mathrm{NaO}_{4}{ }^{+}$379.1880; Found 379.1891.

1-(1-hydroxy-1,3-diphenylprop-2-yn-1-yl)cyclopentan-1-ol (3w)

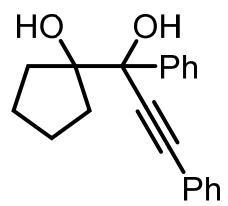

Compound $\mathbf{3 w}$ was synthesized from hydrolysis of compound $\mathbf{6 j}$.

${ }^{1}$ H NMR (400 MHz, DMSO-d $\left.)\right) \delta 7.72-7.69(\mathrm{~m}, 2 \mathrm{H}), 7.53$ - $7.48(\mathrm{~m}, 2 \mathrm{H}), 7.45-7.41(\mathrm{~m}$, 3H), $7.39-7.25$ (m, 3H), 6.14 (s, 1H), 4.34 (s, 1H), $2.17-2.04(\mathrm{~m}, 2 \mathrm{H}), 1.76-1.64(\mathrm{~m}, 2 \mathrm{H})$, $1.64-1.50(\mathrm{~m}, 2 \mathrm{H}), 1.45-1.31(\mathrm{~m}, 2 \mathrm{H})$.

${ }^{13}$ C NMR (101 MHz, DMSO-d6) $\delta 143.8,131.8,129.1,128.9,128.1,127.2,127.2,123.2,94.4$, $86.7,84.1,76.7,36.4,35.8,24.6,24.6$.

IR ( $\left.v_{\max }, \mathrm{cm}^{-1}\right) 3419(\mathrm{w}), 2949(\mathrm{w}), 1486$ (m), $1444(\mathrm{~m}), 1358(\mathrm{w}), 1198(\mathrm{w}), 1009$ (m), 752 (s), $692(\mathrm{~m})$.

\section{4-(2-phenyl-1-oxaspiro[2.4]heptan-2-yl)phenol (6a)}

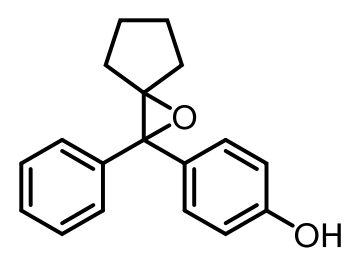

Compound 6a was synthesized according to general procedure $\mathrm{C}$ and $\mathrm{D}$ starting from tertbutyl(4-(cyclopentylidene(phenyl)methyl)phenoxy)dimethylsilane $(8.5 \mathrm{mmol})$. Substrate $6 \mathrm{a}$ was obtained in $60 \%$ yield over two steps as a colorless oil.

${ }^{1}$ H NMR (400 MHz, DMSO-d6) $\delta 9.42(\mathrm{~s}, 1 \mathrm{H}), 7.45-7.41(\mathrm{~m}, 2 \mathrm{H}), 7.36(\mathrm{t}, J=7.5 \mathrm{~Hz}, 2 \mathrm{H})$, $7.30-7.23(\mathrm{~m}, 3 \mathrm{H}), 6.78-6.73(\mathrm{~m}, 2 \mathrm{H}), 1.82-1.51(\mathrm{~m}, 6 \mathrm{H}), 1.42-1.22(\mathrm{~m}, 2 \mathrm{H})$.

${ }^{13}$ C NMR (101 MHz, DMSO-d6) $\delta$ 156.9, 141.3, 131.0, 128.5, 128.4, 127.4, 127.1, 115.3, 76.4, $68.8,31.6,31.6,25.1,25.1$. 
IR $\left(v_{\max }, \mathrm{cm}^{-1}\right) 3380(\mathrm{w}), 2967(\mathrm{w}), 1616(\mathrm{~m}), 1514(\mathrm{~s}), 1450$ (m), 1266 (m), 1223 (s), 1170 (s), 830 (s), 754 (s), 699 (s).

HRMS (APPI/LTQ-Orbitrap) m/z: [M] ${ }^{+}$Calcd for $\mathrm{C}_{18} \mathrm{H}_{18} \mathrm{O}_{2}{ }^{+}$266.1301; Found 266.1289.

4-(2-(4-fluorophenyl)-1-oxaspiro[2.4]heptan-2-yl)phenol (6b)

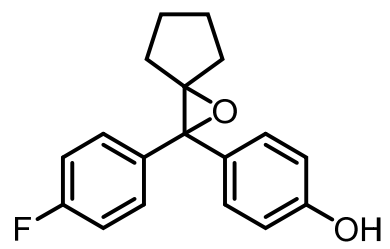

Compound 6b was synthesized according to general procedure $\mathrm{C}$ and $\mathrm{D}$ starting from tertbutyl(4-(cyclopentylidene(4-fluorophenyl)methyl)phenoxy)dimethylsilane $\quad(10.0 \mathrm{mmol})$. Substrate $\mathbf{6 b}$ was obtained in $78 \%$ yield over two steps as a colorless oil.

${ }^{1}$ H NMR (400 MHz, DMSO-d $) \delta 9.41(\mathrm{~s}, 1 \mathrm{H}), 7.48-7.37(\mathrm{~m}, 2 \mathrm{H}), 7.28-7.09$ (m, 4H), 6.79 $-6.66(\mathrm{~m}, 2 \mathrm{H}), 1.77-1.51(\mathrm{~m}, 6 \mathrm{H}), 1.39-1.21(\mathrm{~m}, 2 \mathrm{H})$.

${ }^{13}$ C NMR (101 MHz, DMSO-d6) $\delta 161.5(\mathrm{~d}, J=243.1 \mathrm{~Hz}), 156.9,137.6(\mathrm{~d}, J=3.0 \mathrm{~Hz}), 130.8$, $129.1(\mathrm{~d}, J=8.2 \mathrm{~Hz}), 128.4,115.3(\mathrm{~d}, J=21.3 \mathrm{~Hz}), 115.2,76.6,68.3,31.6,31.5,25.1,25.1$.

${ }^{19}$ F NMR (377 MHz, DMSO-d6) $\delta-115.51$.

IR $\left(v_{\max }, \mathrm{cm}^{-1}\right) 3404$ (s), 2956 (s), 2154 (s), 1604 (s), 1508 (s), 1219 (s), 831 (s).

HRMS (ESI/QTOF) m/z: [M + Na $]^{+}$Calcd for $\mathrm{C}_{18} \mathrm{H}_{17} \mathrm{FNaO}_{2}{ }^{+}$307.1105; Found 307.1102.

\section{2-(3,4-dimethoxyphenyl)-2-phenyl-1-oxaspiro[2.4]heptane (6c)}

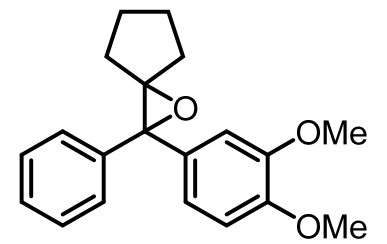

Compound 6c was synthesized according to general procedure $\mathrm{C}$ starting from 4(cyclopentylidene(phenyl)methyl)-1,2-dimethoxybenzene $(10.0 \mathrm{mmol})$. Substrate $\mathbf{6 c}$ was obtained in $80 \%$ yield as a pale yellow oil.

${ }^{1}$ H NMR (400 MHz, Methylene Chloride-d $\left.d_{2}\right) \delta 7.46$ - 7.38 (m, 2H), 7.36 - 7.27 (m, 2H), 7.27 - $7.19(\mathrm{~m}, 1 \mathrm{H}), 6.97-6.92(\mathrm{~m}, 2 \mathrm{H}), 6.83-6.79(\mathrm{~m}, 1 \mathrm{H}), 3.80(\mathrm{~s}, 3 \mathrm{H}), 3.79(\mathrm{~s}, 3 \mathrm{H}), 1.85$ $1.55(\mathrm{~m}, 6 \mathrm{H}), 1.50-1.35(\mathrm{~m}, 2 \mathrm{H})$.

${ }^{13}$ C NMR (101 MHz, Methylene Chloride- $\left.d_{2}\right) \delta 148.9,148.3,140.7,133.0,128.0,127.1,126.9$, $119.4,111.0,110.5,76.9,68.8,55.8,55.8,31.7,31.6,25.1$.

IR $\left(v_{\max }, \mathrm{cm}^{-1}\right) 2943(\mathrm{w}), 2355$ (w), 2156 (m), 1514 (s), 1444 (m), 1246 (m), 1138 (s), 1022 (s), $756(\mathrm{~s}), 702(\mathrm{~s})$.

HRMS (APCI/QTOF) m/z: [M + H $]^{+}$Calcd for $\mathrm{C}_{20} \mathrm{H}_{23} \mathrm{O}_{3}{ }^{+} 311.1647$; Found 311.1642. 
2-(3,4-dimethoxyphenyl)-2-(p-tolyl)-1-oxaspiro[2.4]heptane (6d)

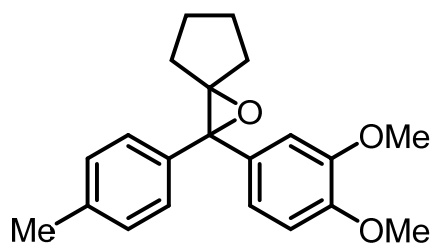

Compound 6d was synthesized according to general procedure $\mathrm{C}$ starting from 4(cyclopentylidene(p-tolyl)methyl)-1,2-dimethoxybenzene $(10.0 \mathrm{mmol})$. Substrate $6 \mathbf{d}$ was obtained in $77 \%$ yield as a pale yellow oil.

${ }^{1}$ H NMR (400 MHz, Methylene Chloride- $\left.d_{2}\right) \delta 7.29(\mathrm{~d}, J=8.1 \mathrm{~Hz}, 2 \mathrm{H}), 7.13(\mathrm{~d}, J=7.9 \mathrm{~Hz}$, 2H), $6.99-6.88(\mathrm{~m}, 2 \mathrm{H}), 6.81(\mathrm{~d}, J=8.8 \mathrm{~Hz}, 1 \mathrm{H}), 3.80(\mathrm{~s}, 3 \mathrm{H}), 3.79(\mathrm{~s}, 3 \mathrm{H}), 2.31(\mathrm{~s}, 3 \mathrm{H}), 1.82$ $-1.59(\mathrm{~m}, 6 \mathrm{H}), 1.50-1.36(\mathrm{~m}, 2 \mathrm{H})$.

${ }^{13}$ C NMR (101 MHz, Methylene Chloride- $\left.d_{2}\right) \delta 148.9,148.3,137.7,136.8,133.3,128.7,126.8$, $119.4,111.0,110.4,76.8,68.7,55.8,55.8,31.7,31.6,25.1,25.1,20.8$.

IR $\left(v_{\max }, \mathrm{cm}^{-1}\right) 2954(\mathrm{w}), 2358(\mathrm{w}), 2156(\mathrm{~m}), 1510(\mathrm{~s}), 1458(\mathrm{~m}), 1250(\mathrm{~m}), 1134(\mathrm{~s}), 1028(\mathrm{~s})$, 808 (s).

HRMS (APCI/QTOF) m/z: [M + Na] ${ }^{+}$Calcd for $\mathrm{C}_{21} \mathrm{H}_{24} \mathrm{NaO}_{3}{ }^{+} 347.1618$; Found 347.1611.

\section{2-(3,4-dimethoxyphenyl)-2-(3,5-dimethylphenyl)-1-oxaspiro[2.4]heptane (6e)}

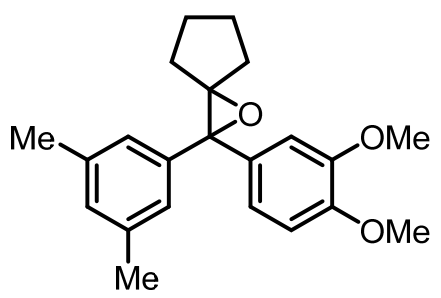

Compound 6e was synthesized according to general procedure $\mathrm{C}$ starting from 4(cyclopentylidene(3,5-dimethylphenyl)methyl)-1,2-dimethoxybenzene (10.0 mmol). Substrate 6e was obtained in $83 \%$ yield as a pale yellow oil.

${ }^{1}$ H NMR (400 MHz, Methylene Chloride-d $\left.d_{2}\right) \delta 7.02(\mathrm{~s}, 2 \mathrm{H}), 6.96-6.91(\mathrm{~m}, 2 \mathrm{H}), 6.88(\mathrm{~s}, 1 \mathrm{H})$, $6.80(\mathrm{~d}, J=8.4 \mathrm{~Hz}, 1 \mathrm{H}), 3.80(\mathrm{~s}, 3 \mathrm{H}), 3.78(\mathrm{~s}, 3 \mathrm{H}), 2.28$ (s, 6H), $1.87-1.68(\mathrm{~m}, 4 \mathrm{H}), 1.70-$ $1.54(\mathrm{~m}, 2 \mathrm{H}), 1.49-1.34(\mathrm{~m}, 2 \mathrm{H})$.

${ }^{13}$ C NMR (101 MHz, Methylene Chloride- $\left.d_{2}\right) \delta$ 149.3, 148.7, 140.9, 138.0, 133.8, 129.1, 125.0, $119.8,111.4,110.8,77.1,69.2,56.2,32.1,32.0,25.5,21.5$.

IR $\left(v_{\max }, \mathrm{cm}^{-1}\right) 2947(\mathrm{w}), 2357(\mathrm{w}), 1604(\mathrm{~m}), 1514(\mathrm{~s}), 1455$ (m), 1257 (m), $1138(\mathrm{~s}), 1026(\mathrm{~s})$, 806 (s), 756 (s).

HRMS (ESI/QTOF) m/z: [M + H $]^{+}$Calcd for $\mathrm{C}_{22} \mathrm{H}_{27} \mathrm{O}_{3}{ }^{+}$339.1955; Found 339.1952.

2-(3-chlorophenyl)-2-(3,4-dimethoxyphenyl)-1-oxaspiro[2.4]heptane (6f) 


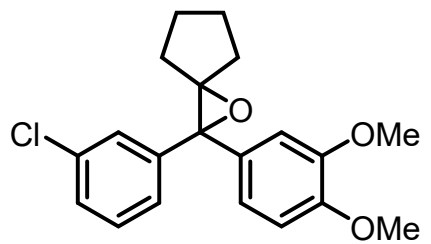

Compound 6f was synthesized according to general procedure $C$ starting from 4-((3chlorophenyl)(cyclopentylidene)methyl)-1,2-dimethoxybenzene $(10.0 \mathrm{mmol})$. Substrate $6 \mathbf{f}$ was obtained in $80 \%$ yield as a pale yellow oil.

${ }^{1}$ H NMR (400 MHz, Methylene Chloride- $\left.d_{2}\right) \delta 7.41$ (s, 1H), 7.32 (d, J= $\left.7.3 \mathrm{~Hz}, 1 \mathrm{H}\right), 7.29$ $7.19(\mathrm{~m}, 2 \mathrm{H}), 6.97-6.89(\mathrm{~m}, 2 \mathrm{H}), 6.82(\mathrm{~d}, J=8.1 \mathrm{~Hz}, 1 \mathrm{H}), 3.79(\mathrm{~s}, 6 \mathrm{H}), 1.83-1.72(\mathrm{~m}, 3 \mathrm{H})$, $1.70-1.58(\mathrm{~m}, 3 \mathrm{H}), 1.48-1.35(\mathrm{~m}, 2 \mathrm{H})$.

${ }^{13}$ C NMR (101 MHz, Methylene Chloride-d 2 ) $\delta$ 149.0, 148.6, 142.9, 133.9, 132.2, 129.4, 127.2, $127.0,125.2,119.5,111.1,110.5,77.1,68.3,55.8,55.8,31.8,31.4,25.1$.

IR $\left(v_{\max }, \mathrm{cm}^{-1}\right) 2955$ (w), 1514 (s), 1246 (s), 1229 (s), 1137 (s), 1029 (s), 783 (s), 760 (s), 740 (s), 696 (s).

HRMS (ESI/QTOF) m/z: [M + Na] ${ }^{+}$Calcd for $\mathrm{C}_{20} \mathrm{H}_{21} \mathrm{ClNaO}_{3}{ }^{+}$367.1071; Found 367.1073.

2-phenyl-2-(phenylethynyl)-1-oxaspiro[2.4]heptane (6j)

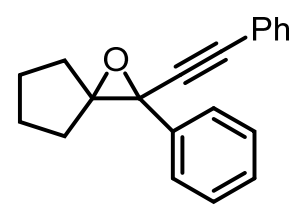

Compound 6j was prepared according to the reported method and the physical and spectroscopic data were in accordance with those reported in the literature. ${ }^{[1]}$

${ }^{1}$ H NMR (400 MHz, Methylene Chloride- $\left.d_{2}\right) \delta 7.57$ - $7.42(\mathrm{~m}, 4 \mathrm{H}), 7.42-7.23(\mathrm{~m}, 6 \mathrm{H}), 2.44$ $-2.28(\mathrm{~m}, 1 \mathrm{H}), 2.01-1.65(\mathrm{~m}, 4 \mathrm{H}), 1.64-1.45(\mathrm{~m}, 2 \mathrm{H}), 1.45-1.31(\mathrm{~m}, 1 \mathrm{H})$.

${ }^{13}$ C NMR (101 MHz, Methylene Chloride- $\left.d_{2}\right) \delta$ 138.0, 132.4, 129.3, 128.9, 128.6, 128.3, 127.0, $122.9,88.4,85.6,79.1,60.7,33.2,30.1,26.0,25.8$.

IR $\left(v_{\max }, \mathrm{cm}^{-1}\right) 2956(\mathrm{w}), 2868(\mathrm{w}), 1490(\mathrm{~m}), 1444(\mathrm{~m}), 1315(\mathrm{w}), 912(\mathrm{~m}), 858(\mathrm{~s}), 752(\mathrm{~s})$, $692(\mathrm{~m})$.

HRMS (APPI/LTQ-Orbitrap) m/z: [M + H] $]^{+}$Calcd for $\mathrm{C}_{20} \mathrm{H}_{19} \mathrm{O}^{+}$275.1430; Found 275.1425. Synthesis of 9 


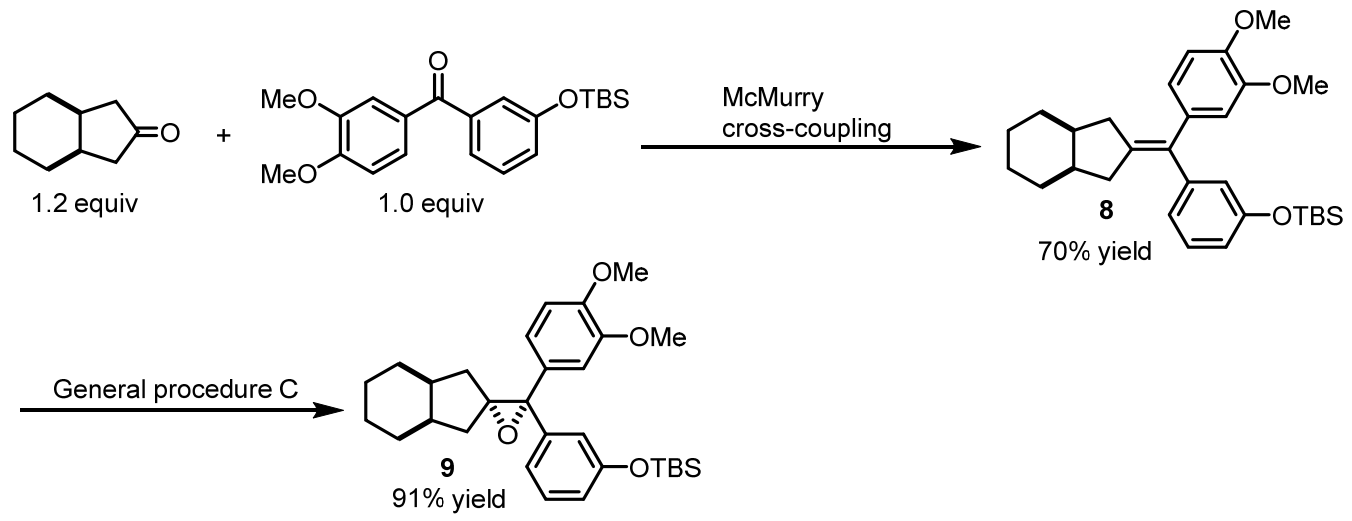

To a stirred suspension of zinc powder ( $4.81 \mathrm{~g}, 74 \mathrm{mmol}, 7.4$ equiv) in anhydrous THF (35 mL) was slowly added, via syringe, $\mathrm{TiCl}_{4}\left(4.0 \mathrm{~mL}, 36 \mathrm{mmol}, 3.6\right.$ equiv) at $0{ }^{\circ} \mathrm{C}$ under a nitrogen atmosphere. The stirred reaction mixture was heated at $65^{\circ} \mathrm{C}$ under a nitrogen atmosphere for $2 \mathrm{~h}$. To the refluxing reaction mixture was added a solution of diaryl methanone $(3.73 \mathrm{~g}, 10$ mmol, 1.0 equiv) and cis-hexahydro- $1 H$-inden-2(3H)-one ${ }^{[2]}(1.66 \mathrm{~g}, 12 \mathrm{mmol}, 1.2$ equiv) in THF (10 mL). After completion of the reaction (monitored by TLC, in 2 hours), the reaction mixture was cooled to $0{ }^{\circ} \mathrm{C}$ and water was slowly added. The reaction mixture was filtered through a pad of Celite and the Celite pad was washed with EtOAc. The filtrate was transferred to a separatory funnel and the organic layer was separated. The aqueous layer was extracted with EtOAc. The organic layers were washed with water and brine, dried over $\mathrm{Na}_{2} \mathrm{SO}_{4}$, filtered, and evaporated. The residue was purified by column chromatography on silica gel, eluting with ethyl acetate/petroleum ether to give the desired alkene $\mathbf{8}$ in $70 \%$ yield ( $3.35 \mathrm{~g})$ as a pale yellow oil.

Compound 9 was synthesized according to general procedure $\mathrm{C}$ starting from 8 (5.0 mmol). Substrate 9 was obtained in $91 \%$ yield (2.25 g, pale yellow oil) as a mixture of two diastereomers $\left(\mathrm{dr}=2 / 1\right.$ from $\left.{ }^{1} \mathrm{H} \mathrm{NMR}\right)$.

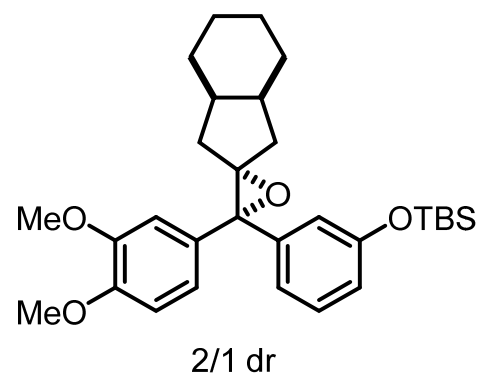

${ }^{1}$ H NMR (400 MHz, Methylene Chloride- $\left.d_{2}\right) \delta 7.20$ - $7.13(\mathrm{~m}, 1 \mathrm{H}), 7.04-6.87$ (m, 4H), 6.84 $-6.79(\mathrm{~m}, 1 \mathrm{H}), 6.74-6.69(\mathrm{~m}, 1 \mathrm{H}), 3.92-3.69(\mathrm{~m}, 6 \mathrm{H}), 2.29-1.98(\mathrm{~m}, 2 \mathrm{H}), 1.78-1.59(\mathrm{~m}$, $2 \mathrm{H}), 1.55-1.23(\mathrm{~m}, 10 \mathrm{H}), 0.96(\mathrm{~m}, 9 \mathrm{H}), 0.17(\mathrm{~m}, 6 \mathrm{H})$. 
${ }^{13}$ C NMR (101 MHz, Methylene Chloride- $\left.d_{2}\right) \delta 156.1,156.0,149.5,149.5,148.9,142.8,142.8$, 133.5, 133.5, 129.7, 129.6, 120.7, 120.5, 120.1, 120.0, 119.3, 119.3, 119.2, 111.6, 111.6, 111.2, $111.1,76.5,76.4,69.4,68.0,56.4,56.3,38.8,38.7,38.4,38.4,37.0,36.8,36.3,36.2,28.2,28.2$, $27.9,27.8,26.0,23.53,23.50,23.4,18.7,-4.07,-4.10,-4.13$.

IR $\left(v_{\max }, \mathrm{cm}^{-1}\right) 2925(\mathrm{w}), 2850(\mathrm{w}), 2154(\mathrm{w}), 1583(\mathrm{~m}), 1514(\mathrm{~m}), 1455(\mathrm{~m}), 1257(\mathrm{~m}), 1026$ (m), $836(\mathrm{~s}), 779(\mathrm{~m})$.

HRMS (ESI/QTOF) m/z: [M + Na] ${ }^{+}$Calcd for $\mathrm{C}_{30} \mathrm{H}_{42} \mathrm{NaO}_{4} \mathrm{Si}^{+}$517.2745; Found 517.2759.

(3S,4aR,8aS)-3-(3-((tert-butyldimethylsilyl)oxy)phenyl)-3-(3,4-

dimethoxyphenyl)octahydronaphthalen-2(1H)-one (10)

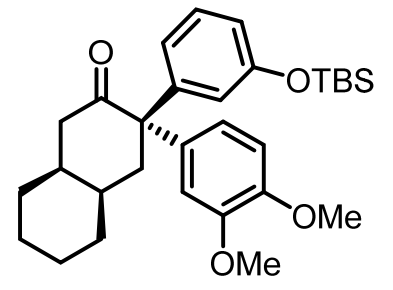

Compound $9(\mathrm{dr}=2 / 1)$ was used as a starting material. This desymmetrizative Meinwald rearrangement was performed on standard reaction conditions: $10 \mathrm{~mol} \% \mathbf{4 a}, 4 \AA \mathrm{MS}$ (100 mg), $-78^{\circ} \mathrm{C}, 2 \mathrm{~d}$. The desired product 10 was isolated in $99 \%$ yield $(\mathrm{dr}=2 / 1)$ and $87 \%$ ee $(44 \%$ ee $)$ as a colorless oil. The ee of $\mathbf{1 0}$ was determined by deprotection to its corresponding phenol. ${ }^{1}$ H NMR (400 MHz, Chloroform-d) $\delta 7.28$ - 7.05 (m, 1H), 6.97 - $6.62(\mathrm{~m}, 4 \mathrm{H}), 6.55-6.20$ $(\mathrm{m}, 2 \mathrm{H}), 3.91,3.82,3.79,3.70$ (four s, 6H), $2.83-2.64(\mathrm{~m}, 2 \mathrm{H}), 2.55-2.43(\mathrm{~m}, 1 \mathrm{H}), 2.43-$ $2.33(\mathrm{~m}, 1 \mathrm{H}), 2.28-2.18(\mathrm{~m}, 1 \mathrm{H}), 2.12-1.96(\mathrm{~m}, 1 \mathrm{H}), 1.84-1.75(\mathrm{~m}, 1 \mathrm{H}), 1.72-1.40(\mathrm{~m}$, $6 \mathrm{H}), 1.36-1.19$ (m, 1H), 0.96, 0.90 (two s, 9H), 0.17, 0.08, 0.07 (three s, 6H).

${ }^{13}$ C NMR (101 MHz, Chloroform- $d$ ) $\delta$ 211.5, 211.4, 156.4, 155.1, 149.5, 148.4, 148.1, 147.6, $147.4,141.8,138.0,132.2,130.0,128.5,121.7,121.3,121.1,120.9,120.4,120.1,119.2,118.0$, 113.0, 111.6, 111.3, 110.5, 62.8, 56.1, 55.9, 55.9, 46.6, 46.4, 39.5, 39.4, 39.2, 39.0, 31.6, 31.6, $30.6,27.6,27.6,26.4,25.8,20.5,20.4,18.4,18.3,-4.2,-4.3$.

IR $\left(v_{\max }, \mathrm{cm}^{-1}\right) 2929(\mathrm{w}), 2152(\mathrm{w}), 1707(\mathrm{~s}), 1583(\mathrm{~m}), 1513(\mathrm{~m}), 1461$ (m), 1255 (m), 1147 (w), 1029 (m), 951 (s), 839 (s), 782 (m).

HRMS (ESI/QTOF) m/z: [M+ Na] ${ }^{+}$Calcd for $\mathrm{C}_{30} \mathrm{H}_{42} \mathrm{NaO}_{4} \mathrm{Si}^{+}$517.2745; Found 517.2745.

SFC: IA column, $5.0 \% \mathrm{MeOH}$ in supercritical $\mathrm{CO}_{2}$ as eluent, $4 \mathrm{~mL} / \mathrm{min}$. $\mathrm{tR}=25.6 \mathrm{~min}$ (minor), $30.4 \min$ (major); $\mathrm{tR}=22.7 \mathrm{~min}$ (major), $29.0 \mathrm{~min}$ (minor). 


\section{Synthetic transformations}

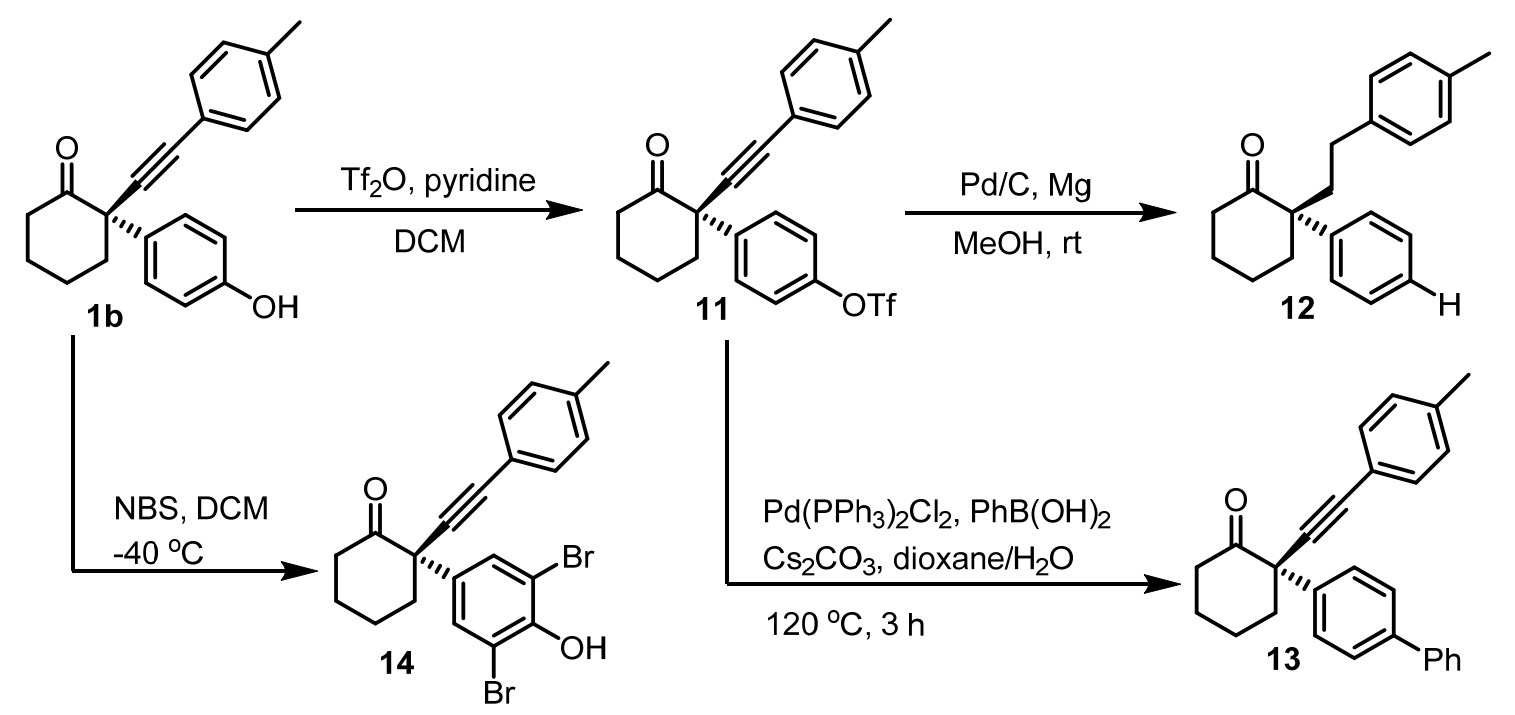

\section{Synthesis of 11}

To a solution of $\mathbf{1 b}(60.8 \mathrm{mg}, 0.2 \mathrm{mmol}, 1.0$ equiv) and pyridine (47.4 mg, $0.6 \mathrm{mmol}, 3.0$ equiv) in anhydrous DCM $(2 \mathrm{~mL})$ was added $\mathrm{Tf}_{2} \mathrm{O}\left(67.7 \mathrm{mg}, 0.24 \mathrm{mmol}, 1.2\right.$ equiv) at $0{ }^{\circ} \mathrm{C}$. The reaction mixture was stirred at room temperature for $2 \mathrm{~h}$. After completion of the reaction (monitored by TLC), a saturated solution of $\mathrm{NaHCO}_{3}$ (aq) was added to quench the reaction and the mixture was extracted with DCM $(5 \mathrm{~mL}$ x 3). The organic layers were washed with water and brine, dried over $\mathrm{Na}_{2} \mathrm{SO}_{4}$, filtered, and evaporated. The residue was purified by column chromatography on silica gel, eluting with ethyl acetate/petroleum ether to give the compound 11 in $98 \%$ yield $(85.5 \mathrm{mg})$ as a white solid.

\section{Synthesis of 12}

Compound 12 was synthesized according to the previously reported procedure. ${ }^{[3]}$ Methanol $(1.0 \mathrm{~mL})$ was added to the mixture of 11 (43.6 mg, $0.1 \mathrm{mmol}, 1.0$ equiv), magnesium powder (12 mg, $0.5 \mathrm{mmol}, 5.0$ equiv) and $\mathrm{Pd} / \mathrm{C}(20 \mathrm{wt} \%$ of substrate $11,8.7 \mathrm{mg})$. The reaction was stirred at room temperature under nitrogen atmosphere. After completion of the reaction (monitored by TLC), the reaction mixture was filtered through Celite and the filtrate was partitioned between EtOAc $(5 \mathrm{~mL})$ and water $(5 \mathrm{~mL})$. The aqueous layer was extracted with EtOAc ( $5 \mathrm{~mL}$ x 3). The organic layers were washed with water and brine, dried over $\mathrm{Na}_{2} \mathrm{SO}_{4}$, filtered, and evaporated. The residue was purified by column chromatography on silica gel, eluting with ethyl acetate/petroleum ether to give the compound 12 in $84 \%$ yield $(24.5 \mathrm{mg})$ as a colorless oil. Note: The triple bond was also reduced under the reported conditions.

\section{Synthesis of 13}


$\mathrm{Pd}\left(\mathrm{PPh}_{3}\right)_{2} \mathrm{Cl}_{2}$ (3.5 mg, $0.005 \mathrm{mmol}, 0.05$ equiv), $\mathrm{Cs}_{2} \mathrm{CO}_{3}(65.2 \mathrm{mg}, 0.2 \mathrm{mmol}, 2.0$ equiv) and $\mathrm{PhB}(\mathrm{OH})_{2}$ (24.4 mg, $0.2 \mathrm{mmol}, 2.0$ equiv) were added to a solution of 11 (43.6 mg, $0.1 \mathrm{mmol}$, 1.0 equiv) in 1,4-dioxane $/ \mathrm{H}_{2} \mathrm{O}(4 / 1,2.0 \mathrm{~mL})$. The reaction mixture was stirred under reflux for $3 \mathrm{~h}$. After completion of the reaction (monitored by TLC), the reaction was quenched with saturated aqueous $\mathrm{NH}_{4} \mathrm{Cl}(5 \mathrm{~mL})$, the resulting mixture was extracted with EtOAc $(5 \mathrm{~mL} \times 3)$. The combined organic layers were washed with water and brine, dried over $\mathrm{Na}_{2} \mathrm{SO}_{4}$, filtered, and evaporated. The residue was purified by column chromatography on silica gel, eluting with ethyl acetate/petroleum ether to give the compound $\mathbf{1 3}$ in $85 \%$ yield (31 mg) as a colorless oil.

\section{Synthesis of $14^{[4]}$}

To a solution of $\mathbf{1 b}(30.4 \mathrm{mg}, 0.1 \mathrm{mmol}, 1.0$ equiv) in DCM (2.0 mL) was added dropwise a solution of NBS (39.2 mg, $0.22 \mathrm{mmol}, 2.2$ equiv) in DCM $(2.0 \mathrm{~mL})$ at $-40{ }^{\circ} \mathrm{C}$. The reaction mixture was stirred at the same temperature for $1 \mathrm{~h}$. After completion of the reaction (monitored by TLC), the reaction was quenched with water $(5 \mathrm{~mL})$, the resulting mixture was extracted with DCM (5 mL x 3). The combined organic layers were washed with water and brine, dried over $\mathrm{Na}_{2} \mathrm{SO}_{4}$, filtered, and evaporated. The residue was purified by column chromatography on silica gel, eluting with ethyl acetate/petroleum ether to give the compound $\mathbf{1 4}$ in $81 \%$ yield (37.4 $\mathrm{mg})$ as a pale yellow oil.

\section{(S)-4-(2-oxo-1-(p-tolylethynyl)cyclohexyl)phenyl trifluoromethanesulfonate (11)}

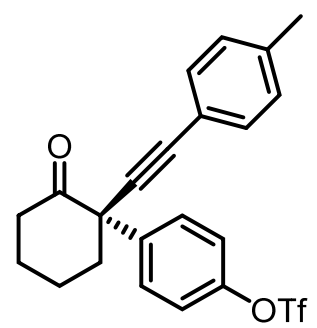

$98 \%$ yield, white solid. M.p $=94-96{ }^{\circ} \mathrm{C}$.

${ }^{1}$ H NMR (400 MHz, Chloroform-d) $\delta 7.66$ - $7.54(\mathrm{~m}, 2 \mathrm{H}), 7.47-7.35$ (m, 2H), $7.34-7.26$ (m, 2H), $7.18(\mathrm{~d}, J=7.9 \mathrm{~Hz}, 2 \mathrm{H}), 3.25(\mathrm{td}, J=13.7,6.1 \mathrm{~Hz}, 1 \mathrm{H}), 2.53-2.46(\mathrm{~m}, 1 \mathrm{H}), 2.38(\mathrm{~s}$, $3 \mathrm{H}), 2.37-2.32(\mathrm{~m}, 2 \mathrm{H}), 2.30-2.16(\mathrm{~m}, 2 \mathrm{H}), 1.98-1.80(\mathrm{~m}, 2 \mathrm{H})$.

${ }^{19}$ F NMR (377 MHz, Chloroform- $d$ ) $\delta-72.86$.

${ }^{13}$ C NMR (101 MHz, Chloroform-d) $\delta$ 205.9, 148.8, 140.3, 139.1, 131.7, 130.2, 129.3, 120.9, 119.4, 118.9 (q, $J=320.9 \mathrm{~Hz}), 90.0,87.6,56.4,42.2,39.2,27.7,22.8,21.6$.

IR ( $\left.v_{\max }, \mathrm{cm}^{-1}\right) 2933$ (s), 1716 (s), 1500 (s), 1412 (s), 1209 (s), 1132 (s), 879 (s), 816 (s). $[\alpha]^{18} \mathrm{D}+202.5$ ( c $\left.0.72, \mathrm{DCM}\right)$.

HRMS (ESI/QTOF) m/z: [M + H] ${ }^{+}$Calcd for $\mathrm{C}_{22} \mathrm{H}_{20} \mathrm{~F}_{3} \mathrm{O}_{4} \mathrm{~S}^{+}$437.1029; Found 437.1026.

(R)-2-(4-methylphenethyl)-2-phenylcyclohexan-1-one (12) 


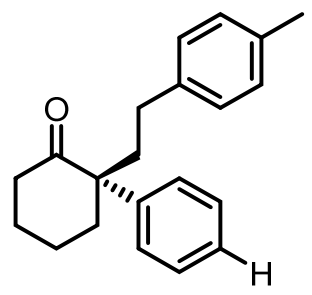

$84 \%$ yield, colorless oil.

${ }^{1}$ H NMR (400 MHz, Chloroform- $\left.d\right) \delta 7.38$ (t, $\left.J=7.6 \mathrm{~Hz}, 2 \mathrm{H}\right), 7.29-7.24$ (m, 1H), 7.21 (d, $J$ $=7.5 \mathrm{~Hz}, 2 \mathrm{H}), 7.02(\mathrm{~d}, J=7.8 \mathrm{~Hz}, 2 \mathrm{H}), 6.95(\mathrm{~d}, J=7.9 \mathrm{~Hz}, 2 \mathrm{H}), 2.83-2.76(\mathrm{~m}, 1 \mathrm{H}), 2.44-$ $2.28(\mathrm{~m}, 3 \mathrm{H}), 2.27(\mathrm{~s}, 3 \mathrm{H}), 2.19(\mathrm{td}, J=12.9,4.5 \mathrm{~Hz}, 1 \mathrm{H}), 2.08(\mathrm{td}, J=13.2,4.5 \mathrm{~Hz}, 1 \mathrm{H}), 2.01$ $-1.94(\mathrm{~m}, 1 \mathrm{H}), 1.89$ (td, $J=13.2,5.2 \mathrm{~Hz}, 1 \mathrm{H}), 1.82-1.66(\mathrm{~m}, 4 \mathrm{H})$.

${ }^{13}$ C NMR (101 MHz, Chloroform- $d$ ) $\delta$ 213.5, 140.9, 139.7, 135.1, 129.0, 129.0, 128.3, 127.1, $126.8,57.6,42.6,40.4,35.4,30.0,28.5,21.9,21.1$.

IR $\left(v_{\max }, \mathrm{cm}^{-1}\right) 2933(\mathrm{~s}), 1703$ (s), 1514 (s), 1437 (s), 802 (s), 752 (s), 700 (s).

$[\alpha]^{18} \mathrm{D}-117.3$ (c $\left.0.42, \mathrm{DCM}\right)$.

HRMS (APPI/LTQ-Orbitrap) m/z: $[\mathrm{M}+\mathrm{Na}]^{+}$Calcd for $\mathrm{C}_{21} \mathrm{H}_{24} \mathrm{NaO}^{+}$315.1719; Found 315.1724 .

(S)-2-([1,1'-biphenyl]-4-yl)-2-(p-tolylethynyl)cyclohexan-1-one (13)

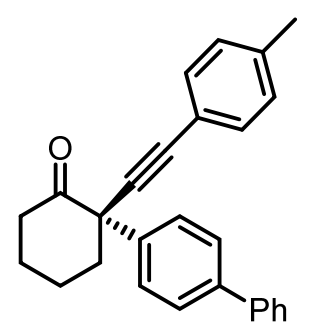

$85 \%$ yield, colorless oil.

${ }^{1}$ H NMR (400 MHz, Chloroform-d) $\delta 7.66$ - 7.55 (m, 6H), 7.49 - 7.39 (m, 4H), 7.39 - 7.33 (m, 1H), 7.17 (d, $J=7.9 \mathrm{~Hz}, 2 \mathrm{H}), 3.24(\mathrm{td}, J=13.5,6.1 \mathrm{~Hz}, 1 \mathrm{H}), 2.59-2.47$ (m, 1H), $2.44-$ $2.32(\mathrm{~m}, 3 \mathrm{H}), 2.39(\mathrm{~s}, 3 \mathrm{H}), 2.28-2.15(\mathrm{~m}, 1 \mathrm{H}), 2.01-1.81(\mathrm{~m}, 2 \mathrm{H})$.

${ }^{13}$ C NMR (101 MHz, Chloroform-d) $\delta$ 206.7, 141.0, 140.3, 138.8, 138.8, 131.7, 129.3, 128.8, $128.5,127.3,127.3,127.0,119.9,89.2,88.7,56.5,41.8,39.4,27.8,22.9,21.6$.

IR $\left(v_{\max }, \mathrm{cm}^{-1}\right) 2933$ (s), 2351 (s), 2021 (s), $1716(\mathrm{~s}), 1475$ (s), 1120 (s), 816 (s), 752 (s), 688 (s).

$[\alpha]^{18} \mathrm{D}+236.5$ ( c $\left.1.00, \mathrm{DCM}\right)$.

HRMS (APCI/QTOF) m/z: [M + H] ${ }^{+}$Calcd for $\mathrm{C}_{27} \mathrm{H}_{25} \mathrm{O}^{+}$365.1908; Found 365.1900.

(S)-2-(3,5-dibromo-4-hydroxyphenyl)-2-(p-tolylethynyl)cyclohexan-1-one (14) 


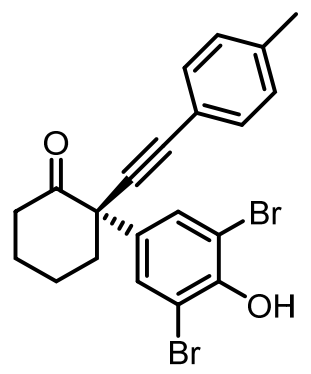

${ }^{1}$ H NMR (400 MHz, Chloroform-d) $\delta 7.57$ (s, 2H), 7.47 - $7.36(\mathrm{~m}, 2 \mathrm{H}), 7.22-7.13(\mathrm{~m}, 2 \mathrm{H})$, $5.87(\mathrm{~s}, 1 \mathrm{H}), 3.19(\mathrm{td}, J=13.7,6.2 \mathrm{~Hz}, 1 \mathrm{H}), 2.52-2.44(\mathrm{~m}, 1 \mathrm{H}), 2.38(\mathrm{~s}, 3 \mathrm{H}), 2.36-2.27(\mathrm{~m}$, $2 \mathrm{H}), 2.24-2.14(\mathrm{~m}, 2 \mathrm{H}), 1.98-1.75(\mathrm{~m}, 2 \mathrm{H})$.

${ }^{13}$ C NMR (101 MHz, Chloroform-d) $\delta$ 205.9, 148.7, 139.2, 134.4, 131.8, 131.7, 129.3, 119.4, $109.5,90.1,87.4,55.7,42.0,39.2,27.7,22.8,21.7$.

IR $\left(v_{\max }, \mathrm{cm}^{-1}\right) 3467$ (s), 2933 (s), 2351 (s), 1716 (s), 1475 (s), 1271 (s), 1157 (s), 1107 (s), 904 (s), $816(\mathrm{~s}), 727(\mathrm{~s}), 675(\mathrm{~s})$.

$[\alpha]^{18} \mathrm{D}+115.1$ ( c $\left.0.80, \mathrm{DCM}\right)$.

HRMS (ESI/QTOF) m/z: $[\mathrm{M}+\mathrm{H}]^{+}$Calcd for $\mathrm{C}_{21} \mathrm{H}_{19}{ }^{79} \mathrm{Br}_{2} \mathrm{O}_{2}{ }^{+}$460.9752; Found 460.9742; Calcd for $\mathrm{C}_{21} \mathrm{H}_{19}{ }^{79} \mathrm{Br}^{81} \mathrm{BrO}_{2}{ }^{+}$462.9733; Found 462.9732; Calcd for $\mathrm{C}_{21} \mathrm{H}_{19}{ }^{81} \mathrm{Br}_{2} \mathrm{O}_{2}{ }^{+}$464.9716; Found 464.9718.
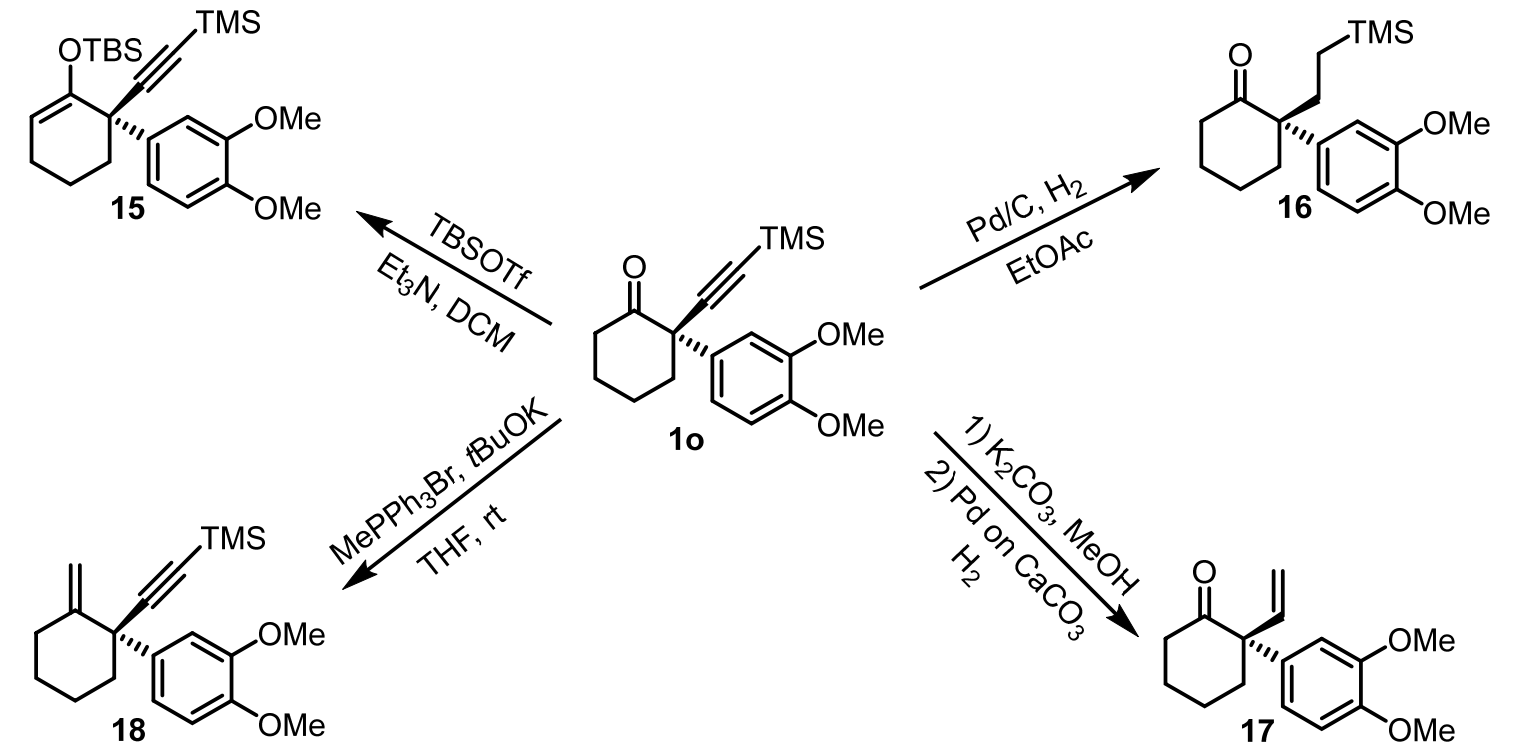

\section{Synthesis of 15}

To a solution of 10 (66.0 mg, $0.2 \mathrm{mmol}, 1.0$ equiv) and $\mathrm{Et}_{3} \mathrm{~N}$ (30.3 mg, $0.3 \mathrm{mmol}, 1.5$ equiv) in anhydrous DCM (2 mL) was added TBSOTf $\left(63.4 \mathrm{mg}, 0.24 \mathrm{mmol}, 1.2\right.$ equiv) at $0{ }^{\circ} \mathrm{C}$. The reaction mixture was stirred at room temperature for $3 \mathrm{~h}$. After completion of the reaction (monitored by TLC), a saturated solution of $\mathrm{NaHCO}_{3}$ (aq) was added to quench the reaction 
and the mixture was extracted with DCM $(5 \mathrm{~mL}$ x 3$)$. The organic layers were washed with water and brine, dried over $\mathrm{Na}_{2} \mathrm{SO}_{4}$, filtered, and evaporated. The residue was purified by column chromatography on silica gel, eluting with ethyl acetate/petroleum ether to give the compound $\mathbf{1 5}$ in $93 \%$ yield $(82.7 \mathrm{mg})$ as a colorless oil.

\section{Synthesis of 16}

To a solution of $10(66.0 \mathrm{mg}, 0.2 \mathrm{mmol}, 1.0$ equiv $)$ in EtOAc was added palladium on activated charcoal $(10 \% \mathrm{Pd}, 6.6 \mathrm{mg})$. The reaction mixture was stirred at room temperature under an $\mathrm{H}_{2}$ atmosphere. After completion of the reaction (monitored by TLC), the reaction mixture was filtered through Celite and concentrated. The residue was purified by column chromatography on silica gel, eluting with ethyl acetate/petroleum ether to give the compound $\mathbf{1 6}$ in $91 \%$ yield $(60.9 \mathrm{mg})$ as a colorless oil.

\section{Synthesis of 17}

To a solution of 10 (66 mg, $0.2 \mathrm{mmol}, 1.0$ equiv) in $\mathrm{MeOH}(2 \mathrm{~mL})$ was added $\mathrm{K}_{2} \mathrm{CO}_{3}(55.2 \mathrm{mg}$, $0.4 \mathrm{mmol}, 2.0$ equiv). The reaction was stirred at room temperature and monitored by TLC. After completion of the reaction, the reaction mixture was filtered through Celite and concentrated. The residue was purified by a short column chromatography on silica gel, eluting with ethyl acetate/petroleum ether to give the terminal alkyne intermediate in quantitative yield. (Note: Prolonging the reaction time after completion will result in the formation of side product.)

To a solution of the obtained terminal alkyne $(51.6 \mathrm{mg}, 0.2 \mathrm{mmol})$ in EtOAc was added palladium on $\mathrm{CaCO}_{3}(5 \%$, lead poisoned, $10 \mathrm{mg})$. The reaction mixture was stirred at room temperature. After completion of the reaction (monitored by TLC), the reaction mixture was filtered through Celite and concentrated. The residue was purified by column chromatography on silica gel, eluting with ethyl acetate/petroleum ether to give the compound $\mathbf{1 7}$ in $86 \%$ yield $(44.7 \mathrm{mg})$ as a colorless oil. (Note: Prolonging the reaction time after completion will result in the formation of over-reduction product.)

\section{Synthesis of 18}

To a stirred suspension of methyltriphenylphosphonium bromide (143 mg, $0.4 \mathrm{mmol}, 2.0$ equiv) in THF ( $2 \mathrm{~mL}$ ) was added potassium tert-butoxide ( $44.8 \mathrm{mg}, 0.4 \mathrm{mmol}, 2.0$ equiv) at $0{ }^{\circ} \mathrm{C}$. The reaction mixture was stirred at room temperature for $1 \mathrm{~h}$, and then cooled to $0{ }^{\circ} \mathrm{C}$. A solution of $10(66.0 \mathrm{mg}, 0.2 \mathrm{mmol}, 1.0$ equiv) in THF ( $1 \mathrm{~mL})$ was added and the reaction mixture was stirred at room temperature for $2 \mathrm{~h}$. After completion of the reaction (monitored by TLC), water $(5.0 \mathrm{~mL})$ was added to quench the reaction and the mixture was extracted with EtOAc $(5 \mathrm{~mL}$ x 3). The organic layers were washed with water and brine, dried over $\mathrm{Na}_{2} \mathrm{SO}_{4}$, filtered, and 
evaporated. The residue was purified by column chromatography on silica gel, eluting with ethyl acetate/petroleum ether to give the compound $\mathbf{1 8}$ in $88 \%$ yield $(57.8 \mathrm{mg}$ ) as a white solid. (S)-tert-butyl((3',4'-dimethoxy-1-((trimethylsilyl)ethynyl)-1,4,5,6-tetrahydro-[1,1'biphenyl]-2-yl)oxy)dimethylsilane (15)

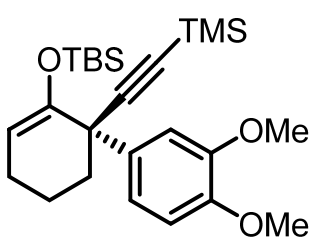

93\% yield, colorless oil.

${ }^{1}$ H NMR (400 MHz, Chloroform- $d$ ) $\delta 7.12(\mathrm{~d}, J=2.1 \mathrm{~Hz}, 1 \mathrm{H}), 7.03(\mathrm{dd}, J=8.4,2.1 \mathrm{~Hz}, 1 \mathrm{H})$, $6.79(\mathrm{~d}, J=8.4 \mathrm{~Hz}, 1 \mathrm{H}), 4.95(\mathrm{t}, J=4.0 \mathrm{~Hz}, 1 \mathrm{H}), 3.87(\mathrm{~s}, 3 \mathrm{H}), 3.86(\mathrm{~s}, 3 \mathrm{H}), 2.20-2.03$ (m, $3 \mathrm{H}), 1.92-1.73(\mathrm{~m}, 2 \mathrm{H}), 1.63-1.55(\mathrm{~m}, 1 \mathrm{H}), 0.69(\mathrm{~s}, 9 \mathrm{H}), 0.19(\mathrm{~s}, 9 \mathrm{H}), 0.12(\mathrm{~s}, 3 \mathrm{H}), 0.06(\mathrm{~s}$, $3 \mathrm{H})$.

${ }^{13}$ C NMR (101 MHz, Chloroform-d) $\delta$ 150.3, 148.2, 147.4, 136.9, 119.4, 111.2, 110.8, 110.6, $104.5,87.0,56.0,55.9,47.4,42.2,25.6,24.4,19.9,18.1,0.3,-4.3,-4.9$.

IR ( $\left.v_{\max }, \mathrm{cm}^{-1}\right) 2359$ (s), 2160 (s), 2023 (s), 1800 (s), 1250 (s), 1083 (s), 762 (s), 735 (s).

HRMS (ESI/QTOF) m/z: [M + Na] ${ }^{+}$Calcd for $\mathrm{C}_{25} \mathrm{H}_{40} \mathrm{NaO}_{3} \mathrm{Si}_{2}{ }^{+}$467.2408; Found 467.2419. $[\alpha]^{26} \mathrm{D}-28.4$ (c $\left.1.55, \mathrm{DCM}\right)$.

(R)-2-(3,4-dimethoxyphenyl)-2-(2-(trimethylsilyl)ethyl)cyclohexan-1-one (16)

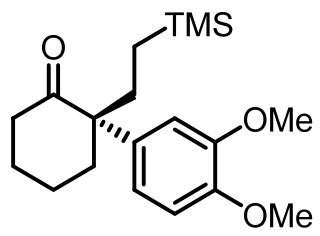

91\% yield, colorless oil.

${ }^{1}$ H NMR (400 MHz, Chloroform-d) $\delta 6.83(\mathrm{~d}, J=8.4 \mathrm{~Hz}, 1 \mathrm{H}), 6.72(\mathrm{~d}, J=8.3 \mathrm{~Hz}, 1 \mathrm{H}), 6.58$ $(\mathrm{s}, 1 \mathrm{H}), 3.86(\mathrm{~s}, 3 \mathrm{H}), 3.83(\mathrm{~s}, 3 \mathrm{H}), 2.67-2.59(\mathrm{~m}, 1 \mathrm{H}), 2.37-2.17(\mathrm{~m}, 2 \mathrm{H}), 1.98-1.87(\mathrm{~m}$, $1 \mathrm{H}), 1.85-1.53(\mathrm{~m}, 6 \mathrm{H}), 0.21(\mathrm{td}, J=14.1,4.2 \mathrm{~Hz}, 1 \mathrm{H}),-0.02--0.11(\mathrm{~m}, 1 \mathrm{H}),-0.12(\mathrm{~s}, 9 \mathrm{H})$. ${ }^{13}$ C NMR (101 MHz, Chloroform-d) $\delta$ 214.2, 149.2, 147.7, 133.3, 119.5, 111.2, 110.5, 57.7, $56.1,55.9,40.4,34.5,34.3,28.2,21.9,9.6,-1.8$.

IR $\left(v_{\max }, \mathrm{cm}^{-1}\right) 2949(\mathrm{~m}), 1704(\mathrm{~s}), 1514(\mathrm{~s}), 1462(\mathrm{~m}), 1251(\mathrm{~s}), 1150(\mathrm{~m}), 1031(\mathrm{~m}), 862(\mathrm{~s})$, 837 (s).

HRMS (ESI/QTOF) m/z: [M + Na ${ }^{+}$Calcd for $\mathrm{C}_{19} \mathrm{H}_{30} \mathrm{NaO}_{3} \mathrm{Si}^{+}$357.1856; Found 357.1856. $[\alpha]^{26} \mathrm{D}-154.9$ (c 1.87, DCM).

(R)-2-(3,4-dimethoxyphenyl)-2-vinylcyclohexan-1-one (17) 


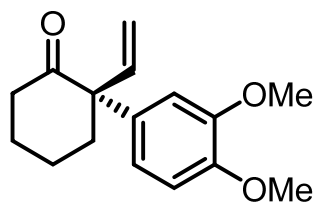

$86 \%$ yield, colorless oil.

${ }^{1}$ H NMR (400 MHz, Chloroform- $d$ ) $\delta 6.84$ (d, $\left.J=8.4 \mathrm{~Hz}, 1 \mathrm{H}\right), 6.76-6.71$ (m, 1H), 6.60 (brs, 1H), 6.21 (dd, $J=17.5,10.6 \mathrm{~Hz}, 1 \mathrm{H}), 5.07$ (d, $J=10.6 \mathrm{~Hz}, 1 \mathrm{H}), 4.55$ (d, $J=17.5 \mathrm{~Hz}, 1 \mathrm{H}), 3.85$ (s, 3H), $3.81(\mathrm{~s}, 3 \mathrm{H}), 2.59-2.50(\mathrm{~m}, 1 \mathrm{H}), 2.46-2.30(\mathrm{~m}, 2 \mathrm{H}), 1.99-1.87(\mathrm{~m}, 2 \mathrm{H}), 1.87-$ $1.65(\mathrm{~m}, 3 \mathrm{H})$.

${ }^{13}$ C NMR (101 MHz, Chloroform-d) $\delta$ 212.3, 149.3, 148.0, 143.3, 131.9, 119.8, 114.8, 111.4, $111.0,60.6,56.0,55.9,40.0,36.4,27.9,21.7$.

IR $\left(v_{\max }, \mathrm{cm}^{-1}\right) 2936(\mathrm{~m}), 1704(\mathrm{~s}), 1513(\mathrm{~s}), 1455$ (m), 1259 (s), 1151 (s), 1028 (s), 926 (m), $813(\mathrm{~m}), 768(\mathrm{~m})$.

$[\alpha]^{26} \mathrm{D}-178.5$ (c 1.75, DCM).

HRMS (ESI/QTOF) m/z: [M + Na $]^{+}$Calcd for $\mathrm{C}_{16} \mathrm{H}_{20} \mathrm{NaO}_{3}{ }^{+}$283.1305; Found 283.1304.

(S)-((1-(3,4-dimethoxyphenyl)-2-methylenecyclohexyl)ethynyl)trimethylsilane (18)

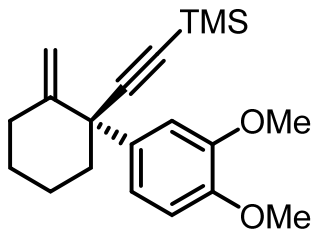

$88 \%$ yield, white solid. M.p $=56-58{ }^{\circ} \mathrm{C}$.

${ }^{1}$ H NMR (400 MHz, Chloroform- $d$ ) $\delta 7.12(\mathrm{~d}, J=2.2 \mathrm{~Hz}, 1 \mathrm{H}), 7.04$ (dd, $\left.J=8.4,2.2 \mathrm{~Hz}, 1 \mathrm{H}\right)$, $6.84(\mathrm{~d}, J=8.4 \mathrm{~Hz}, 1 \mathrm{H}), 4.82(\mathrm{brs}, 1 \mathrm{H}), 4.50$ (d, $J=1.7 \mathrm{~Hz}, 1 \mathrm{H}), 3.88$ (s, 6H), 2.57 (ddd, $J=$ 14.0, 10.1, 4.1 Hz, 1H), $2.33-2.15(\mathrm{~m}, 2 \mathrm{H}), 1.98-1.88(\mathrm{~m}, 2 \mathrm{H}), 1.86-1.62(\mathrm{~m}, 2 \mathrm{H}), 1.60-$ $1.43(\mathrm{~m}, 1 \mathrm{H}), 0.18(\mathrm{~s}, 9 \mathrm{H})$.

${ }^{13}$ C NMR (101 MHz, Chloroform-d) $\delta$ 151.9, 148.5, 147.8, 135.2, 119.7, 111.8, 110.7, 110.6, $110.6,89.5,56.0,55.9,48.3,39.8,33.5,28.2,23.3,0.3$.

IR $\left(v_{\max }, \mathrm{cm}^{-1}\right) 2933$ (s), 1514 (s), 1246 (s), 1146 (s), 1018 (s), 841 (s), 752 (s).

$[\alpha]^{18} \mathrm{D}+25.2(c 1.83, \mathrm{DCM})$.

HRMS (APCI/QTOF) m/z: [M + H] $]^{+}$Calcd for $\mathrm{C}_{20} \mathrm{H}_{29} \mathrm{O}_{2} \mathrm{Si}^{+}$329.1931; Found 329.1936.

(S)-2-(1-benzyl-1H-1,2,3-triazol-4-yl)-2-(3,4-dimethoxyphenyl)cyclohexan-1-one (19)<smiles>COc1ccc([C@]2(c3cn(Cc4ccccc4)nn3)CCCCC2=O)cc1</smiles> 
To a solution of copper iodine ( $3.8 \mathrm{mg}, 0.02 \mathrm{mmol}, 0.1$ equiv), 2,2'-bipyridine ( $3.1 \mathrm{mg}, 0.02$ mmol, 0.1 equiv) in DCM $(1.0 \mathrm{~mL})$ was added a solution of terminal alkyne $(51.6 \mathrm{mg}, 0.2$ mmol, 1.0 equiv) and (azidomethyl)benzene (32.0 mg, $0.24 \mathrm{mmol}, 1.2$ equiv) in DCM (1.0 $\mathrm{mL}$ ). The reaction mixture was stirred in a sealed tube at $40{ }^{\circ} \mathrm{C}$ overnight. After completion of the reaction (monitored by TLC), the reaction mixture was filtered through Celite and concentrated. The residue was purified by column chromatography on silica gel, eluting with ethyl acetate/petroleum ether (3/1 to 2/1) to give the desired compound 19 in $99 \%$ yield (77.7 $\mathrm{mg}$ ) as a light yellow oil.

${ }^{1}$ H NMR (400 MHz, Chloroform-d) $\delta 7.29$ - 7.17 (m, 3H), 7.13 - 7.09 (m, 2H), 7.05 (s, 1H), $6.75(\mathrm{~d}, J=8.4 \mathrm{~Hz}, 1 \mathrm{H}), 6.70(\mathrm{dd}, J=8.5,2.1 \mathrm{~Hz}, 1 \mathrm{H}), 6.61(\mathrm{~d}, J=2.1 \mathrm{~Hz}, 1 \mathrm{H}), 5.36(\mathrm{~d}, J=$ $15.0 \mathrm{~Hz}, 1 \mathrm{H}), 5.32(\mathrm{~d}, J=15.0 \mathrm{~Hz}, 1 \mathrm{H}), 3.77(\mathrm{~s}, 3 \mathrm{H}), 3.69(\mathrm{~s}, 3 \mathrm{H}), 2.96-2.82(\mathrm{~m}, 1 \mathrm{H}), 2.62-$ $2.53(\mathrm{~m}, 1 \mathrm{H}), 2.48-2.34(\mathrm{~m}, 2 \mathrm{H}), 1.99-1.66(\mathrm{~m}, 4 \mathrm{H})$.

${ }^{13}$ C NMR (101 MHz, Chloroform-d) $\delta$ 209.9, 151.5, 149.2, 148.1, 134.9, 132.7, 129.0, 128.5, $127.9,122.7,119.5,111.4,110.5,57.4,55.9,55.8,54.0,40.1,36.8,27.4,21.5$.

IR $\left(v_{\max }, \mathrm{cm}^{-1}\right) 2937(\mathrm{w}), 1706$ (s), $1514(\mathrm{w}), 1454(\mathrm{~m}), 1255$ (m), 1147 (m), 1025 (s), 908 (m), $719(\mathrm{~m})$.

$[\alpha]^{24} \mathrm{D}-81.5$ (c 1.60, DCM), 90\% ee.

HRMS (ESI/QTOF) m/z: [M+ H $]^{+}$Calcd for $\mathrm{C}_{23} \mathrm{H}_{26} \mathrm{~N}_{3} \mathrm{O}_{3}{ }^{+}$392.1969; Found 392.1977.

Gram scale reaction and total synthesis of $(+)$-Mesembrane (20)
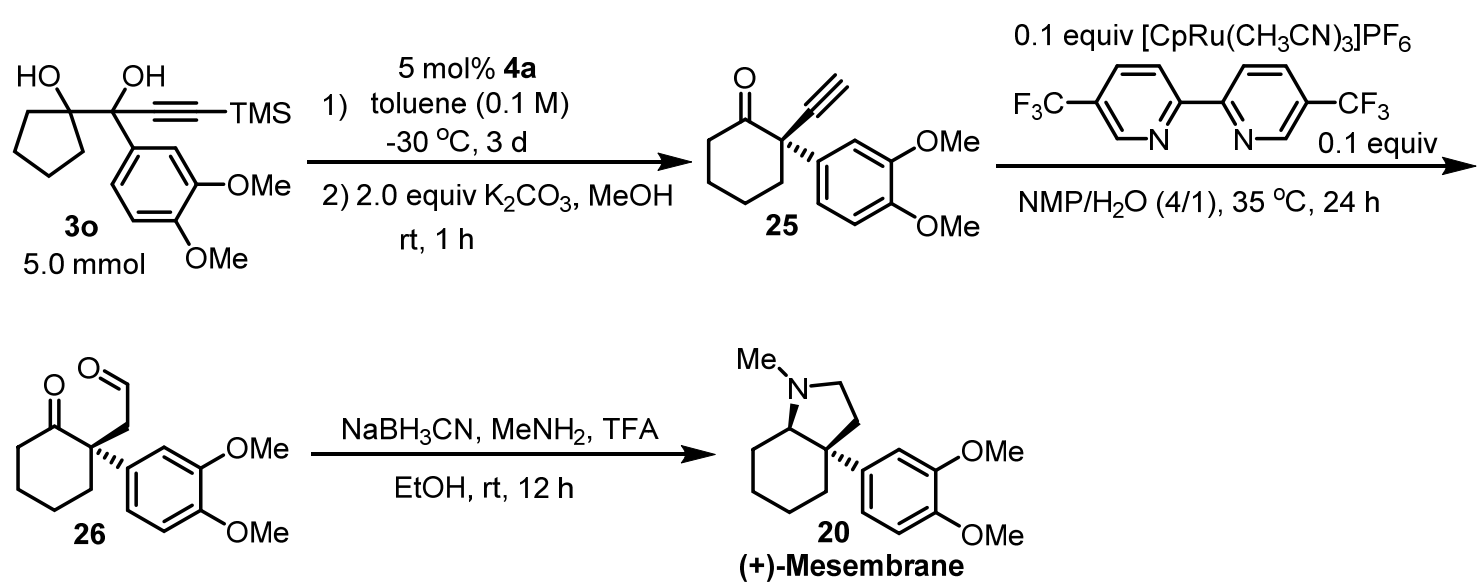

\section{Synthesis of 25}

To a dry flask charged with $3 \AA$ molecular sieves $(5.0 \mathrm{~g})$ was added a solution of 3o (1.74 g, $5.0 \mathrm{mmol}, 1.0$ equiv) in toluene $(40 \mathrm{~mL})$. The mixture was stirred at room temperature for 5 min. A solution of $\mathbf{4 a}(221 \mathrm{mg}, 0.25 \mathrm{mmol}, 0.05$ equiv) in toluene $(10 \mathrm{~mL})$ was added to the above mixture at $-30{ }^{\circ} \mathrm{C}$. The reaction mixture was stirred at the same temperature for 3 days. 
After completion of the reaction (monitored by TLC), $\mathrm{MeOH}(5 \mathrm{~mL})$ was added to quench the reaction. The mixture was filtered through Celite and the filtrate was concentrated under vacuum to afford the crude product 10 (90\% ee), which was directly used for the next step without further purification.

To a solution of the crude product 10 in $\mathrm{MeOH}(20 \mathrm{~mL})$ was added $\mathrm{K}_{2} \mathrm{CO}_{3}(1.38 \mathrm{~g}, 10 \mathrm{mmol}$, 2.0 equiv). The reaction was stirred at room temperature and monitored by TLC. After completion of the reaction, the reaction mixture was filtered through Celite and concentrated. The residue was purified by column chromatography on silica gel, eluting with ethyl acetate/petroleum ether to give the terminal alkyne intermediate 25 in $92 \%$ yield $(1.19 \mathrm{~g})$ over two steps as a white solid. (Note: Prolonging the reaction time after completion will result in the formation of the side product.)

\section{Synthesis of 26}

Compound 26 was synthesized according to the previously reported procedure. ${ }^{[5]}$ To a solution of 5,5'-bis(trifluoromethyl)-2,2'-bipyridine $(11.7 \mathrm{mg}, \quad 0.04 \mathrm{mmol}, \quad 0.1$ equiv) and $\left[\mathrm{CpRu}\left(\mathrm{CH}_{3} \mathrm{CN}\right)_{3}\right] \mathrm{PF}_{6}(17.4 \mathrm{mg}, 0.04 \mathrm{mmol}, 0.1$ equiv $)$ in a mixture of water $/ N$-methyl-2pyrrolidinone (1/4 v/v, $1.0 \mathrm{~mL}$, deoxygenated before using) was added a solution of 25 (110.4 $\mathrm{mg}, 0.4 \mathrm{mmol}, 1.0$ equiv) in $\mathrm{H}_{2} \mathrm{O} / \mathrm{NMP}(1 / 4 \mathrm{v} / \mathrm{v}, 1.0 \mathrm{~mL}$, deoxygenated before using) under Ar. The reaction mixture was stirred at $35^{\circ} \mathrm{C}$ for $24 \mathrm{~h}$. After completion of the reaction (monitored by TLC), the resulting mixture was extracted with EtOAc $(5 \mathrm{~mL}$ x 3$)$. The combined organic layers were washed with water and brine, dried over $\mathrm{Na}_{2} \mathrm{SO}_{4}$, filtered, and evaporated. The residue was purified by column chromatography on silica gel, eluting with ethyl acetate/petroleum ether to give the compound $\mathbf{2 6}$ in $82 \%$ yield $(90.5 \mathrm{mg})$ as a colorless oil.

\section{Synthesis of (+)-Mesembrane (20)}

Compound 20 was synthesized according to the previously reported procedure. ${ }^{[6]}$ An ovendried round-bottom flask was charged with 26 (27.6 mg, $0.1 \mathrm{mmol}, 1.0$ equiv) in EtOH ( $2 \mathrm{~mL})$, followed by addition of $\mathrm{MeNH}_{2}\left(2.0 \mathrm{M}\right.$ in THF, $0.5 \mathrm{~mL}, 1.0 \mathrm{mmol}, 10.0$ equiv), $\mathrm{NaBH}_{3} \mathrm{CN}$ (25.2 mg, 0.4 mmol, 4.0 equiv) and $\mathrm{CF}_{3} \mathrm{COOH}$ (1.1 mg, $0.01 \mathrm{mmol}, 0.1$ equiv), respectively. The reaction mixture was stirred at room temperature for $12 \mathrm{~h}$. After completion of the reaction, the reaction mixture was then basified with a solution of $\mathrm{NaOH}(2 \mathrm{~N}$ in water, $5 \mathrm{~mL})$ and extracted with $\mathrm{CH}_{2} \mathrm{Cl}_{2}(5 \mathrm{~mL} \times 3)$. The combined organic layers were dried over anhydrous

$\mathrm{K}_{2} \mathrm{CO}_{3}$ and concentrated under vacuum. The residue was purified by column chromatography on silica gel, eluting with $\mathrm{CH}_{2} \mathrm{Cl}_{2} /$ methanol to give the compound $\mathbf{2 0}$ in $76 \%$ yield $(21 \mathrm{mg})$ as a pale yellow oil. 
(R)-2-(3,4-dimethoxyphenyl)-2-ethynylcyclohexan-1-one (25)

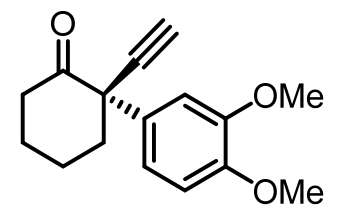

93\% yield, white solid. M.p $=95-97^{\circ} \mathrm{C}$.

${ }^{1}$ H NMR (400 MHz, Chloroform-d) $\delta 7.02-6.90$ (m, 2H), 6.85 (d, J = 8.4 Hz, 1H), 3.86 (s, $3 \mathrm{H}), 3.86$ (s, 3H), 3.01 (ddd, $J=13.7,11.5,5.8 \mathrm{~Hz}, 1 \mathrm{H}), 2.67$ (s, 1H), $2.45-2.12$ (m, 4H), $2.11-2.02(\mathrm{~m}, 1 \mathrm{H}), 1.91-1.75(\mathrm{~m}, 2 \mathrm{H})$.

${ }^{13}$ C NMR (101 MHz, Chloroform-d) $\delta$ 206.5, 148.7, 148.4, 131.2, 119.6, 111.4, 110.9, 84.5, 76.2, 55.9, 55.9, 55.3, 40.8, 38.9, 27.6, 22.3.

IR $\left(v_{\max }, \mathrm{cm}^{-1}\right) 3270$ (w), 2941 (m), 2352 (w), 1724 (s), 1518 (s), 1460 (m), 1261 (s), 1147 (s), $1026(\mathrm{~m})$.

$[\alpha]^{26} \mathrm{D}+89.1$ ( с $\left.0.30, \mathrm{DCM}\right)$.

HRMS (ESI/QTOF) m/z: [M+ H $]^{+}$Calcd for $\mathrm{C}_{16} \mathrm{H}_{19} \mathrm{O}_{3}{ }^{+}$259.1329; Found 259.1337.

(S)-2-(1-(3,4-dimethoxyphenyl)-2-oxocyclohexyl)acetaldehyde (26)

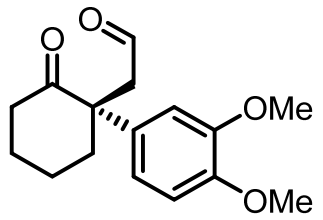

$0.4 \mathrm{mmol}$ scale. $91 \mathrm{mg}, 82 \%$ yield, colorless oil.

${ }^{1}$ H NMR (400 MHz, Chloroform- $d$ ) $\delta 9.55(\mathrm{t}, J=2.5 \mathrm{~Hz}, 1 \mathrm{H}), 6.85(\mathrm{~d}, J=8.4 \mathrm{~Hz}, 1 \mathrm{H}), 6.77$ $(\mathrm{dd}, J=8.4,2.3 \mathrm{~Hz}, 1 \mathrm{H}), 6.65(\mathrm{~d}, J=2.3 \mathrm{~Hz}, 1 \mathrm{H}), 3.86$ (s, 3H), 3.84 (s, 3H), $2.77-2.58$ (m, $3 \mathrm{H}), 2.51-2.27(\mathrm{~m}, 2 \mathrm{H}), 2.04-1.89(\mathrm{~m}, 2 \mathrm{H}), 1.81-1.67$ (m, 3H).

${ }^{13}$ C NMR (101 MHz, Chloroform-d) $\delta$ 212.0, 201.7, 149.6, 148.3, 131.8, 118.9, 111.6, 109.7, $56.0,55.9,55.7,53.4,39.5,35.9,27.7,21.4$.

IR $\left(v_{\max }, \mathrm{cm}^{-1}\right) 2937$ (s), 1701 (s), 1514 (s), 1456 (s), 1255 (s), 1149 (s), 1024 (s), 809 (s), 764 (s).

$[\alpha]^{26} \mathrm{D}-196.3$ (c 1.3, DCM).

HRMS (ESI/QTOF) m/z: [M + Na $]^{+}$Calcd for $\mathrm{C}_{16} \mathrm{H}_{20} \mathrm{NaO}_{4}{ }^{+}$299.1254; Found 299.1251.

(+)-Mesembrane (20)

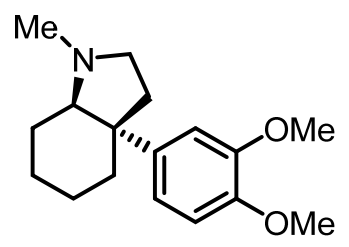


$76 \%$ yield, colorless oil.

${ }^{1}$ H NMR (400 MHz, Chloroform- $d$ ) $\delta 6.91(\mathrm{dd}, J=8.3,2.2 \mathrm{~Hz}, 1 \mathrm{H}), 6.88(\mathrm{~d}, J=2.2 \mathrm{~Hz}, 1 \mathrm{H})$, $6.81(\mathrm{~d}, J=8.3 \mathrm{~Hz}, 1 \mathrm{H}), 3.88(\mathrm{~s}, 3 \mathrm{H}), 3.86(\mathrm{~s}, 3 \mathrm{H}), 3.28(\mathrm{~m}, 1 \mathrm{H}), 2.62(\mathrm{~m}, 1 \mathrm{H}), 2.34(\mathrm{~s}, 3 \mathrm{H})$, $2.33-2.28(\mathrm{~m}, 1 \mathrm{H}), 1.98-1.76(\mathrm{~m}, 5 \mathrm{H}), 1.66-1.56(\mathrm{~m}, 2 \mathrm{H}), 1.53-1.44(\mathrm{~m}, 1 \mathrm{H}), 1.41-1.34$ $(\mathrm{m}, 1 \mathrm{H}), 1.23-1.10(\mathrm{~m}, 1 \mathrm{H})$.

${ }^{13}$ C NMR (101 MHz, Chloroform- $\left.d\right) \delta$ 148.7, 147.0, 140.2, 118.9, 110.81, 110.78, 68.9, 56.1, $56.0,54.4,47.7,41.1,40.8,36.0,23.8,23.0,20.5$.

IR $\left(v_{\max }, \mathrm{cm}^{-1}\right) 2931$ (s), 1518 (s), 1454 (s), 1255 (s), 1147 (s), 1026 (s), 804 (s), 762 (s), 715 (s).

HRMS (APCI/QTOF) m/z: [M + H] ${ }^{+}$Calcd for $\mathrm{C}_{17} \mathrm{H}_{26} \mathrm{NO}_{2}{ }^{+} 276.1958$; Found 276.1960.

$[\alpha]^{26} \mathrm{D}+14.8\left(c\right.$ 0.25, MeOH); lit. $[\alpha]^{20} \mathrm{D}+15.9\left(c\right.$ 0.25, MeOH). ${ }^{[7]}$ 


\section{Crystallographic data for 1a (CCDC 1898915)}
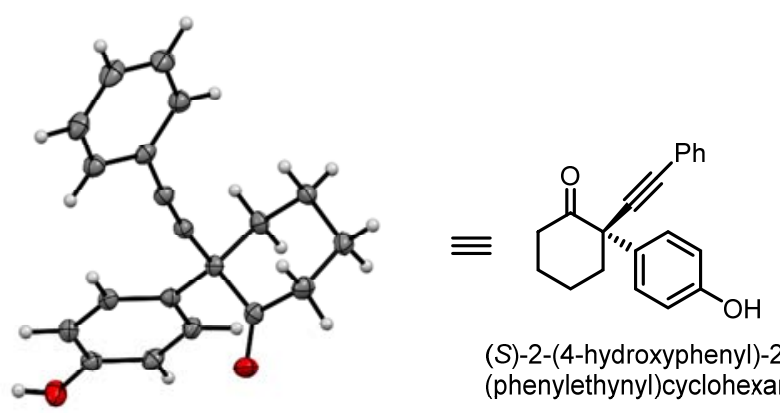

(S)-2-(4-hydroxyphenyl)-2(phenylethynyl)cyclohexan-1-one

Table 1. Crystal data and structure refinement for hwu-six-1.

Identification code

Empirical formula

Formula weight

Temperature

Wavelength

Crystal system

Space group

Unit cell dimensions

Volume

$\mathrm{Z}$

Density (calculated)

Absorption coefficient

$\mathrm{F}(000)$

Crystal size

$\Theta$ range for data collection

Index ranges

Reflections collected

Independent reflections

Completeness to $\theta=67.684^{\circ}$

Absorption correction

Max. and min. transmission

Refinement method

Data / restraints / parameters

Goodness-of-fit on $\mathrm{F}^{2}$

Final $R$ indices $[\mathrm{I}>2 \sigma(\mathrm{I})]$

$\mathrm{R}$ indices (all data)

Absolute structure parameter

Extinction coefficient

Largest diff. peak and hole hwu-six-1

$\mathrm{C}_{20} \mathrm{H}_{18} \mathrm{O}_{2}$

290.34

140.01(10) K

$1.54184 \AA$

Orthorhombic

$P 22_{1} 2_{1}$

$\begin{array}{ll}\mathrm{a}=8.32811(6) \AA & \alpha=90^{\circ} . \\ \mathrm{b}=10.96364(7) \AA & \beta=90^{\circ} . \\ \mathrm{c}=16.54433(9) \AA & \gamma=90^{\circ} .\end{array}$

1510.604(17) $\AA^{3}$

4

$1.277 \mathrm{Mg} / \mathrm{m}^{3}$

$0.640 \mathrm{~mm}^{-1}$

616

$0.591 \times 0.27 \times 0.134 \mathrm{~mm}^{3}$

4.839 to $76.075^{\circ}$.

$-10 \leq \mathrm{h} \leq 9,-13 \leq \mathrm{k} \leq 13,-20 \leq 1 \leq 20$

25302

$3125\left[R_{\text {int }}=0.0236\right]$

$100.0 \%$

Gaussian

1.000 and 0.452

Full-matrix least-squares on $\mathrm{F}^{2}$

3125 / 0 / 202

1.060

$R_{1}=0.0250, w R_{2}=0.0663$

$R_{1}=0.0252, w R_{2}=0.0666$

$0.04(4)$

$0.0050(5)$

0.239 and -0.124 e. $\AA^{-3}$ 
Table 2. Atomic coordinates $\left(\mathrm{x} 10^{4}\right)$ and equivalent isotropic displacement parameters $\left(\AA^{2} \times 10^{3}\right)$ for hwu-six-1. U(eq) is defined as one third of the trace of the orthogonalized $\mathrm{U}^{\mathrm{ij}}$ tensor.

\begin{tabular}{|c|c|c|c|c|}
\hline & $\mathrm{x}$ & $\mathrm{y}$ & $\mathrm{z}$ & $\mathrm{U}(\mathrm{eq})$ \\
\hline $\mathrm{O}(2)$ & $7169(1)$ & $2946(1)$ & $6935(1)$ & $28(1)$ \\
\hline $\mathrm{O}(1)$ & $4487(1)$ & $3718(1)$ & 3661(1) & $31(1)$ \\
\hline$C(14)$ & $2313(2)$ & $2460(1)$ & $7869(1)$ & $22(1)$ \\
\hline$C(4)$ & $3478(2)$ & 2948(1) & $5765(1)$ & $21(1)$ \\
\hline$C(13)$ & $3282(2)$ & $3025(1)$ & 7487(1) & $21(1)$ \\
\hline $\mathrm{C}(12)$ & $6175(2)$ & 3323(1) & 7404(1) & $21(1)$ \\
\hline$C(6)$ & 4433(2) & $3779(1)$ & 6184(1) & $19(1)$ \\
\hline $\mathrm{C}(5)$ & $5374(2)$ & $4586(1)$ & $5732(1)$ & $23(1)$ \\
\hline $\mathrm{C}(1)$ & $4418(2)$ & $3713(1)$ & $4487(1)$ & $23(1)$ \\
\hline$C(3)$ & $3462(2)$ & 2908(1) & 4925(1) & $24(1)$ \\
\hline$C(7)$ & $4514(2)$ & 3799(1) & $7105(1)$ & $19(1)$ \\
\hline$C(20)$ & $649(2)$ & $2395(1)$ & 9089(1) & $27(1)$ \\
\hline$C(10)$ & $6162(2)$ & 4694(1) & $8618(1)$ & $27(1)$ \\
\hline $\mathrm{C}(8)$ & 4308(2) & $5118(1)$ & $7456(1)$ & $23(1)$ \\
\hline $\mathrm{C}(9)$ & $4513(2)$ & $5158(1)$ & 8373(1) & $25(1)$ \\
\hline$C(15)$ & $1189(2)$ & 1831(1) & $8380(1)$ & $22(1)$ \\
\hline$C(2)$ & $5356(2)$ & $4558(1)$ & 4895(1) & $24(1)$ \\
\hline $\mathrm{C}(11)$ & $6470(2)$ & $3396(1)$ & $8300(1)$ & $27(1)$ \\
\hline$C(19)$ & $-423(2)$ & $1799(2)$ & 9595(1) & $32(1)$ \\
\hline $\mathrm{C}(18)$ & $-946(2)$ & $630(2)$ & 9406(1) & $33(1)$ \\
\hline$C(16)$ & $647(2)$ & $659(1)$ & 8193(1) & $26(1)$ \\
\hline$C(17)$ & $-402(2)$ & $61(2)$ & $8710(1)$ & $31(1)$ \\
\hline
\end{tabular}


Table 3. Bond lengths $[\AA]$ and angles $\left[^{\circ}\right]$ for hwu-six-1.

\begin{tabular}{|c|c|}
\hline $\mathrm{O}(2)-\mathrm{C}(12)$ & $1.2076(17)$ \\
\hline $\mathrm{O}(1)-\mathrm{H}(1)$ & $0.86(2)$ \\
\hline $\mathrm{O}(1)-\mathrm{C}(1)$ & $1.3668(16)$ \\
\hline$C(14)-C(13)$ & $1.198(2)$ \\
\hline $\mathrm{C}(14)-\mathrm{C}(15)$ & $1.4370(19)$ \\
\hline $\mathrm{C}(4)-\mathrm{H}(4)$ & 0.9500 \\
\hline$C(4)-C(6)$ & $1.3943(18)$ \\
\hline$C(4)-C(3)$ & $1.3909(18)$ \\
\hline$C(13)-C(7)$ & $1.4737(19)$ \\
\hline$C(12)-C(7)$ & $1.5593(19)$ \\
\hline$C(12)-C(11)$ & $1.5054(19)$ \\
\hline$C(6)-C(5)$ & $1.3982(19)$ \\
\hline$C(6)-C(7)$ & $1.5257(16)$ \\
\hline $\mathrm{C}(5)-\mathrm{H}(5)$ & 0.9500 \\
\hline$C(5)-C(2)$ & $1.3841(19)$ \\
\hline $\mathrm{C}(1)-\mathrm{C}(3)$ & $1.3926(19)$ \\
\hline$C(1)-C(2)$ & $1.388(2)$ \\
\hline $\mathrm{C}(3)-\mathrm{H}(3)$ & 0.9500 \\
\hline$C(7)-C(8)$ & $1.5676(18)$ \\
\hline $\mathrm{C}(20)-\mathrm{H}(20)$ & 0.9500 \\
\hline$C(20)-C(15)$ & $1.400(2)$ \\
\hline$C(20)-C(19)$ & $1.387(2)$ \\
\hline $\mathrm{C}(10)-\mathrm{H}(10 \mathrm{~A})$ & 0.9900 \\
\hline $\mathrm{C}(10)-\mathrm{H}(10 \mathrm{~B})$ & 0.9900 \\
\hline$C(10)-C(9)$ & $1.520(2)$ \\
\hline $\mathrm{C}(10)-\mathrm{C}(11)$ & $1.538(2)$ \\
\hline $\mathrm{C}(8)-\mathrm{H}(8 \mathrm{~A})$ & 0.9900 \\
\hline $\mathrm{C}(8)-\mathrm{H}(8 \mathrm{~B})$ & 0.9900 \\
\hline$C(8)-C(9)$ & $1.5286(18)$ \\
\hline $\mathrm{C}(9)-\mathrm{H}(9 \mathrm{~A})$ & 0.9900 \\
\hline $\mathrm{C}(9)-\mathrm{H}(9 \mathrm{~B})$ & 0.9900 \\
\hline $\mathrm{C}(15)-\mathrm{C}(16)$ & $1.397(2)$ \\
\hline $\mathrm{C}(2)-\mathrm{H}(2)$ & 0.9500 \\
\hline $\mathrm{C}(11)-\mathrm{H}(11 \mathrm{~A})$ & 0.9900 \\
\hline $\mathrm{C}(11)-\mathrm{H}(11 \mathrm{~B})$ & 0.9900 \\
\hline C(19)-H(19) & 0.9500 \\
\hline
\end{tabular}




\begin{tabular}{|c|c|}
\hline $\mathrm{C}(19)-\mathrm{C}(18)$ & $1.390(2)$ \\
\hline $\mathrm{C}(18)-\mathrm{H}(18)$ & 0.9500 \\
\hline $\mathrm{C}(18)-\mathrm{C}(17)$ & $1.385(2)$ \\
\hline $\mathrm{C}(16)-\mathrm{H}(16)$ & 0.9500 \\
\hline $\mathrm{C}(16)-\mathrm{C}(17)$ & $1.387(2)$ \\
\hline $\mathrm{C}(17)-\mathrm{H}(17)$ & 0.9500 \\
\hline $\mathrm{C}(1)-\mathrm{O}(1)-\mathrm{H}(1)$ & 109.5 \\
\hline$C(13)-C(14)-C(15)$ & $175.72(14)$ \\
\hline $\mathrm{C}(6)-\mathrm{C}(4)-\mathrm{H}(4)$ & 119.2 \\
\hline $\mathrm{C}(3)-\mathrm{C}(4)-\mathrm{H}(4)$ & 119.2 \\
\hline$C(3)-C(4)-C(6)$ & $121.52(13)$ \\
\hline$C(14)-C(13)-C(7)$ & $173.27(14)$ \\
\hline $\mathrm{O}(2)-\mathrm{C}(12)-\mathrm{C}(7)$ & $121.22(12)$ \\
\hline $\mathrm{O}(2)-\mathrm{C}(12)-\mathrm{C}(11)$ & $122.67(13)$ \\
\hline$C(11)-C(12)-C(7)$ & $116.08(11)$ \\
\hline$C(4)-C(6)-C(5)$ & $117.86(12)$ \\
\hline$C(4)-C(6)-C(7)$ & $122.05(12)$ \\
\hline$C(5)-C(6)-C(7)$ & $120.06(12)$ \\
\hline $\mathrm{C}(6)-\mathrm{C}(5)-\mathrm{H}(5)$ & 119.5 \\
\hline $\mathrm{C}(2)-\mathrm{C}(5)-\mathrm{C}(6)$ & 121.01(13) \\
\hline $\mathrm{C}(2)-\mathrm{C}(5)-\mathrm{H}(5)$ & 119.5 \\
\hline $\mathrm{O}(1)-\mathrm{C}(1)-\mathrm{C}(3)$ & $123.11(13)$ \\
\hline $\mathrm{O}(1)-\mathrm{C}(1)-\mathrm{C}(2)$ & $117.41(12)$ \\
\hline $\mathrm{C}(2)-\mathrm{C}(1)-\mathrm{C}(3)$ & $119.48(12)$ \\
\hline $\mathrm{C}(4)-\mathrm{C}(3)-\mathrm{C}(1)$ & $119.63(13)$ \\
\hline $\mathrm{C}(4)-\mathrm{C}(3)-\mathrm{H}(3)$ & 120.2 \\
\hline $\mathrm{C}(1)-\mathrm{C}(3)-\mathrm{H}(3)$ & 120.2 \\
\hline$C(13)-C(7)-C(12)$ & $106.81(10)$ \\
\hline$C(13)-C(7)-C(6)$ & $112.92(11)$ \\
\hline$C(13)-C(7)-C(8)$ & $107.26(11)$ \\
\hline $\mathrm{C}(12)-\mathrm{C}(7)-\mathrm{C}(8)$ & $106.74(11)$ \\
\hline $\mathrm{C}(6)-\mathrm{C}(7)-\mathrm{C}(12)$ & $110.57(11)$ \\
\hline $\mathrm{C}(6)-\mathrm{C}(7)-\mathrm{C}(8)$ & $112.21(10)$ \\
\hline $\mathrm{C}(15)-\mathrm{C}(20)-\mathrm{H}(20)$ & 119.8 \\
\hline $\mathrm{C}(19)-\mathrm{C}(20)-\mathrm{H}(20)$ & 119.8 \\
\hline $\mathrm{C}(19)-\mathrm{C}(20)-\mathrm{C}(15)$ & $120.30(14)$ \\
\hline $\mathrm{H}(10 \mathrm{~A})-\mathrm{C}(10)-\mathrm{H}(10 \mathrm{~B})$ & 107.9 \\
\hline
\end{tabular}




\begin{tabular}{|c|c|}
\hline $\mathrm{C}(9)-\mathrm{C}(10)-\mathrm{H}(10 \mathrm{~A})$ & 109.3 \\
\hline $\mathrm{C}(9)-\mathrm{C}(10)-\mathrm{H}(10 \mathrm{~B})$ & 109.3 \\
\hline$C(9)-C(10)-C(11)$ & $111.70(12)$ \\
\hline $\mathrm{C}(11)-\mathrm{C}(10)-\mathrm{H}(10 \mathrm{~A})$ & 109.3 \\
\hline $\mathrm{C}(11)-\mathrm{C}(10)-\mathrm{H}(10 \mathrm{~B})$ & 109.3 \\
\hline $\mathrm{C}(7)-\mathrm{C}(8)-\mathrm{H}(8 \mathrm{~A})$ & 109.1 \\
\hline $\mathrm{C}(7)-\mathrm{C}(8)-\mathrm{H}(8 \mathrm{~B})$ & 109.1 \\
\hline $\mathrm{H}(8 \mathrm{~A})-\mathrm{C}(8)-\mathrm{H}(8 \mathrm{~B})$ & 107.8 \\
\hline $\mathrm{C}(9)-\mathrm{C}(8)-\mathrm{C}(7)$ & $112.46(11)$ \\
\hline $\mathrm{C}(9)-\mathrm{C}(8)-\mathrm{H}(8 \mathrm{~A})$ & 109.1 \\
\hline $\mathrm{C}(9)-\mathrm{C}(8)-\mathrm{H}(8 \mathrm{~B})$ & 109.1 \\
\hline $\mathrm{C}(10)-\mathrm{C}(9)-\mathrm{C}(8)$ & $110.84(12)$ \\
\hline $\mathrm{C}(10)-\mathrm{C}(9)-\mathrm{H}(9 \mathrm{~A})$ & 109.5 \\
\hline $\mathrm{C}(10)-\mathrm{C}(9)-\mathrm{H}(9 \mathrm{~B})$ & 109.5 \\
\hline $\mathrm{C}(8)-\mathrm{C}(9)-\mathrm{H}(9 \mathrm{~A})$ & 109.5 \\
\hline $\mathrm{C}(8)-\mathrm{C}(9)-\mathrm{H}(9 \mathrm{~B})$ & 109.5 \\
\hline $\mathrm{H}(9 \mathrm{~A})-\mathrm{C}(9)-\mathrm{H}(9 \mathrm{~B})$ & 108.1 \\
\hline$C(20)-C(15)-C(14)$ & $119.36(13)$ \\
\hline$C(16)-C(15)-C(14)$ & $121.46(13)$ \\
\hline$C(16)-C(15)-C(20)$ & $119.17(13)$ \\
\hline $\mathrm{C}(5)-\mathrm{C}(2)-\mathrm{C}(1)$ & $120.48(13)$ \\
\hline $\mathrm{C}(5)-\mathrm{C}(2)-\mathrm{H}(2)$ & 119.8 \\
\hline $\mathrm{C}(1)-\mathrm{C}(2)-\mathrm{H}(2)$ & 119.8 \\
\hline$C(12)-C(11)-C(10)$ & $110.99(12)$ \\
\hline $\mathrm{C}(12)-\mathrm{C}(11)-\mathrm{H}(11 \mathrm{~A})$ & 109.4 \\
\hline $\mathrm{C}(12)-\mathrm{C}(11)-\mathrm{H}(11 \mathrm{~B})$ & 109.4 \\
\hline $\mathrm{C}(10)-\mathrm{C}(11)-\mathrm{H}(11 \mathrm{~A})$ & 109.4 \\
\hline $\mathrm{C}(10)-\mathrm{C}(11)-\mathrm{H}(11 \mathrm{~B})$ & 109.4 \\
\hline $\mathrm{H}(11 \mathrm{~A})-\mathrm{C}(11)-\mathrm{H}(11 \mathrm{~B})$ & 108.0 \\
\hline $\mathrm{C}(20)-\mathrm{C}(19)-\mathrm{H}(19)$ & 120.0 \\
\hline C(20)-C(19)-C(18) & $120.03(14)$ \\
\hline $\mathrm{C}(18)-\mathrm{C}(19)-\mathrm{H}(19)$ & 120.0 \\
\hline $\mathrm{C}(19)-\mathrm{C}(18)-\mathrm{H}(18)$ & 120.0 \\
\hline $\mathrm{C}(17)-\mathrm{C}(18)-\mathrm{C}(19)$ & $119.97(14)$ \\
\hline $\mathrm{C}(17)-\mathrm{C}(18)-\mathrm{H}(18)$ & 120.0 \\
\hline $\mathrm{C}(15)-\mathrm{C}(16)-\mathrm{H}(16)$ & 119.9 \\
\hline$C(17)-C(16)-C(15)$ & $120.15(14)$ \\
\hline $\mathrm{C}(17)-\mathrm{C}(16)-\mathrm{H}(16)$ & 119.9 \\
\hline
\end{tabular}




$\begin{array}{ll}\mathrm{C}(18)-\mathrm{C}(17)-\mathrm{C}(16) & 120.36(15) \\ \mathrm{C}(18)-\mathrm{C}(17)-\mathrm{H}(17) & 119.8 \\ \mathrm{C}(16)-\mathrm{C}(17)-\mathrm{H}(17) & 119.8\end{array}$

Symmetry transformations used to generate equivalent atoms: 
Table 4. Anisotropic displacement parameters $\left(\AA^{2} \times 10^{3}\right)$ for hwu-six-1. The anisotropic displacement factor exponent takes the form: $-2 \pi^{2}\left[h^{2} a^{* 2} U^{11}+\ldots+2 h k a^{*} b^{*} U^{12}\right]$

\begin{tabular}{|c|c|c|c|c|c|c|}
\hline & $\mathrm{U}^{11}$ & $U^{22}$ & $\mathrm{U}^{33}$ & $\mathrm{U}^{23}$ & $\mathrm{U}^{13}$ & $\mathrm{U}^{12}$ \\
\hline $\mathrm{O}(2)$ & $25(1)$ & $32(1)$ & $28(1)$ & $-1(1)$ & $3(1)$ & $4(1)$ \\
\hline $\mathrm{O}(1)$ & $31(1)$ & $44(1)$ & $18(1)$ & $1(1)$ & 1(1) & $-8(1)$ \\
\hline$C(14)$ & 21(1) & $22(1)$ & $22(1)$ & $0(1)$ & $0(1)$ & 1(1) \\
\hline$C(4)$ & 21(1) & $18(1)$ & $22(1)$ & $1(1)$ & 1(1) & $-1(1)$ \\
\hline $\mathrm{C}(13)$ & $22(1)$ & $20(1)$ & $19(1)$ & $-1(1)$ & $-2(1)$ & 1(1) \\
\hline $\mathrm{C}(12)$ & $22(1)$ & $18(1)$ & $24(1)$ & $1(1)$ & $0(1)$ & $-2(1)$ \\
\hline$C(6)$ & $19(1)$ & $18(1)$ & $20(1)$ & $1(1)$ & $0(1)$ & $2(1)$ \\
\hline $\mathrm{C}(5)$ & 21(1) & $24(1)$ & $24(1)$ & $1(1)$ & $-1(1)$ & $-4(1)$ \\
\hline $\mathrm{C}(1)$ & $21(1)$ & $28(1)$ & $19(1)$ & $1(1)$ & 1(1) & $3(1)$ \\
\hline $\mathrm{C}(3)$ & $24(1)$ & $23(1)$ & $24(1)$ & $-1(1)$ & $-2(1)$ & $-3(1)$ \\
\hline$C(7)$ & $20(1)$ & $18(1)$ & $20(1)$ & $1(1)$ & $-1(1)$ & $-1(1)$ \\
\hline $\mathrm{C}(20)$ & $25(1)$ & $31(1)$ & $25(1)$ & $-1(1)$ & $-1(1)$ & $-2(1)$ \\
\hline $\mathrm{C}(10)$ & $26(1)$ & $35(1)$ & $21(1)$ & $-3(1)$ & $-2(1)$ & $-4(1)$ \\
\hline $\mathrm{C}(8)$ & $27(1)$ & $19(1)$ & $23(1)$ & $-1(1)$ & $-2(1)$ & $-1(1)$ \\
\hline $\mathrm{C}(9)$ & $27(1)$ & $24(1)$ & $23(1)$ & $-4(1)$ & $1(1)$ & $-2(1)$ \\
\hline $\mathrm{C}(15)$ & $18(1)$ & $26(1)$ & $22(1)$ & $6(1)$ & $-2(1)$ & $1(1)$ \\
\hline $\mathrm{C}(2)$ & 21(1) & $26(1)$ & $25(1)$ & $4(1)$ & $2(1)$ & $-3(1)$ \\
\hline $\mathrm{C}(11)$ & $24(1)$ & $35(1)$ & $23(1)$ & $2(1)$ & $-4(1)$ & $4(1)$ \\
\hline$C(19)$ & $30(1)$ & $47(1)$ & $20(1)$ & $0(1)$ & $3(1)$ & $-3(1)$ \\
\hline $\mathrm{C}(18)$ & $26(1)$ & $47(1)$ & $25(1)$ & 11(1) & 1(1) & $-10(1)$ \\
\hline$C(16)$ & $24(1)$ & $28(1)$ & $24(1)$ & $1(1)$ & 1(1) & $-2(1)$ \\
\hline $\mathrm{C}(17)$ & $27(1)$ & $33(1)$ & $32(1)$ & $5(1)$ & $-2(1)$ & $-9(1)$ \\
\hline
\end{tabular}


Table 5. Hydrogen coordinates $\left(\mathrm{x} 10^{4}\right)$ and isotropic displacement parameters $\left(\AA^{2} \mathrm{x} 10^{3}\right)$ for hwu-six-1.

\begin{tabular}{|c|c|c|c|c|}
\hline & $\mathrm{x}$ & $\mathrm{y}$ & $\mathrm{z}$ & $\mathrm{U}(\mathrm{eq})$ \\
\hline $\mathrm{H}(1)$ & $3830(30)$ & $3180(20)$ & $3472(5)$ & 47 \\
\hline $\mathrm{H}(4)$ & 2824 & 2396 & 6061 & 25 \\
\hline $\mathrm{H}(5)$ & 6035 & 5162 & 6003 & 28 \\
\hline $\mathrm{H}(3)$ & 2801 & 2334 & 4651 & 28 \\
\hline $\mathrm{H}(20)$ & 1019 & 3189 & 9224 & 32 \\
\hline $\mathrm{H}(10 \mathrm{~A})$ & 6248 & 4695 & 9215 & 33 \\
\hline $\mathrm{H}(10 \mathrm{~B})$ & 6995 & 5250 & 8402 & 33 \\
\hline $\mathrm{H}(8 \mathrm{~A})$ & 5111 & 5664 & 7203 & 27 \\
\hline $\mathrm{H}(8 \mathrm{~B})$ & 3227 & 5427 & 7314 & 27 \\
\hline $\mathrm{H}(9 \mathrm{~A})$ & 4377 & 6007 & 8566 & 30 \\
\hline $\mathrm{H}(9 \mathrm{~B})$ & 3675 & 4649 & 8631 & 30 \\
\hline $\mathrm{H}(2)$ & 5991 & 5122 & 4599 & 29 \\
\hline $\mathrm{H}(11 \mathrm{~A})$ & 7594 & 3160 & 8417 & 33 \\
\hline $\mathrm{H}(11 \mathrm{~B})$ & 5753 & 2815 & 8583 & 33 \\
\hline $\mathrm{H}(19)$ & -800 & 2191 & 10070 & 39 \\
\hline $\mathrm{H}(18)$ & -1677 & 221 & 9753 & 39 \\
\hline $\mathrm{H}(16)$ & 996 & 270 & 7711 & 31 \\
\hline $\mathrm{H}(17)$ & -748 & -744 & 8587 & 37 \\
\hline
\end{tabular}


Table 6. Torsion angles $\left[{ }^{\circ}\right]$ for hwu-six-1.

\begin{tabular}{|c|c|}
\hline $\mathrm{O}(2)-\mathrm{C}(12)-\mathrm{C}(7)-\mathrm{C}(13)$ & $119.71(14)$ \\
\hline $\mathrm{O}(2)-\mathrm{C}(12)-\mathrm{C}(7)-\mathrm{C}(6)$ & $-3.50(18)$ \\
\hline $\mathrm{O}(2)-\mathrm{C}(12)-\mathrm{C}(7)-\mathrm{C}(8)$ & $-125.81(14)$ \\
\hline $\mathrm{O}(2)-\mathrm{C}(12)-\mathrm{C}(11)-\mathrm{C}(10)$ & $125.54(15)$ \\
\hline $\mathrm{O}(1)-\mathrm{C}(1)-\mathrm{C}(3)-\mathrm{C}(4)$ & $178.95(13)$ \\
\hline $\mathrm{O}(1)-\mathrm{C}(1)-\mathrm{C}(2)-\mathrm{C}(5)$ & $-178.50(13)$ \\
\hline$C(14)-C(15)-C(16)-C(17)$ & $178.10(14)$ \\
\hline$C(4)-C(6)-C(5)-C(2)$ & $0.0(2)$ \\
\hline$C(4)-C(6)-C(7)-C(13)$ & $-10.56(18)$ \\
\hline$C(4)-C(6)-C(7)-C(12)$ & $109.03(14)$ \\
\hline$C(4)-C(6)-C(7)-C(8)$ & $-131.93(13)$ \\
\hline$C(13)-C(7)-C(8)-C(9)$ & $59.75(14)$ \\
\hline$C(12)-C(7)-C(8)-C(9)$ & $-54.43(14)$ \\
\hline$C(6)-C(4)-C(3)-C(1)$ & $0.1(2)$ \\
\hline$C(6)-C(5)-C(2)-C(1)$ & $-1.0(2)$ \\
\hline$C(6)-C(7)-C(8)-C(9)$ & $-175.70(11)$ \\
\hline$C(5)-C(6)-C(7)-C(13)$ & $171.50(13)$ \\
\hline$C(5)-C(6)-C(7)-C(12)$ & $-68.91(16)$ \\
\hline$C(5)-C(6)-C(7)-C(8)$ & $50.14(17)$ \\
\hline$C(3)-C(4)-C(6)-C(5)$ & $0.5(2)$ \\
\hline$C(3)-C(4)-C(6)-C(7)$ & $-177.50(13)$ \\
\hline$C(3)-C(1)-C(2)-C(5)$ & $1.5(2)$ \\
\hline$C(7)-C(12)-C(11)-C(10)$ & $-52.86(16)$ \\
\hline$C(7)-C(6)-C(5)-C(2)$ & $178.00(13)$ \\
\hline$C(7)-C(8)-C(9)-C(10)$ & $58.96(15)$ \\
\hline$C(20)-C(15)-C(16)-C(17)$ & $-0.6(2)$ \\
\hline $\mathrm{C}(20)-\mathrm{C}(19)-\mathrm{C}(18)-\mathrm{C}(17)$ & $-0.3(2)$ \\
\hline $\mathrm{C}(9)-\mathrm{C}(10)-\mathrm{C}(11)-\mathrm{C}(12)$ & $52.81(16)$ \\
\hline$C(15)-C(20)-C(19)-C(18)$ & $1.1(2)$ \\
\hline$C(15)-C(16)-C(17)-C(18)$ & $1.4(2)$ \\
\hline$C(2)-C(1)-C(3)-C(4)$ & $-1.0(2)$ \\
\hline $\mathrm{C}(11)-\mathrm{C}(12)-\mathrm{C}(7)-\mathrm{C}(13)$ & $-61.87(15)$ \\
\hline$C(11)-C(12)-C(7)-C(6)$ & 174.92(11) \\
\hline $\mathrm{C}(11)-\mathrm{C}(12)-\mathrm{C}(7)-\mathrm{C}(8)$ & $52.62(14)$ \\
\hline $\mathrm{C}(11)-\mathrm{C}(10)-\mathrm{C}(9)-\mathrm{C}(8)$ & $-56.54(15)$ \\
\hline$C(19)-C(20)-C(15)-C(14)$ & $-179.37(14)$ \\
\hline
\end{tabular}


$\mathrm{C}(19)-\mathrm{C}(20)-\mathrm{C}(15)-\mathrm{C}(16)$

Symmetry transformations used to generate equivalent atoms. 
8. Copies of NMR spectra and SFC chromatograms

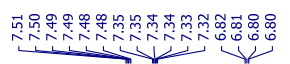

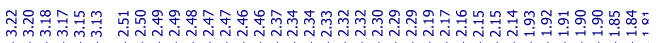

$1 \mathrm{a}$
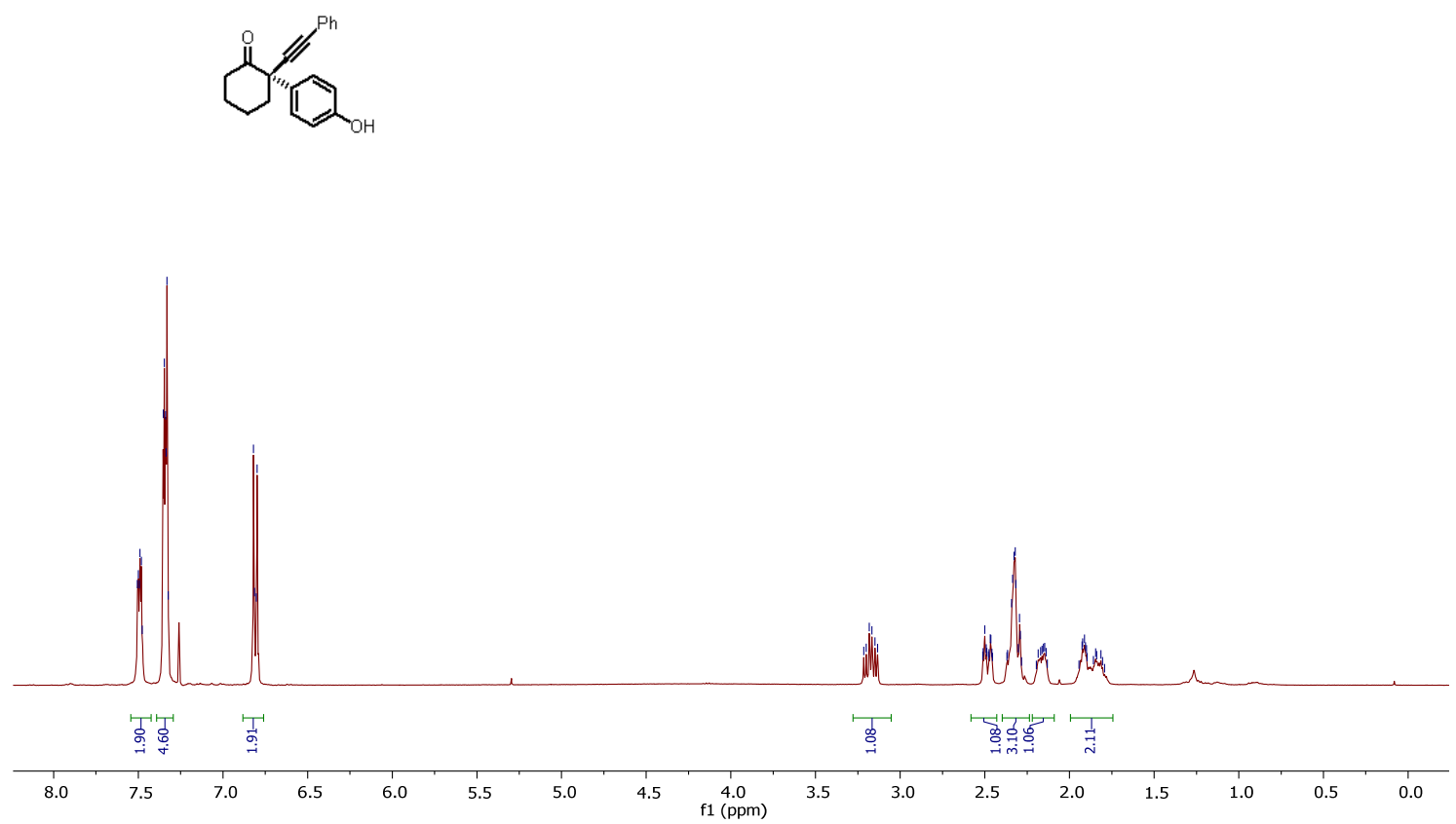

in

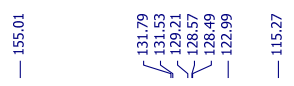

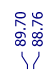

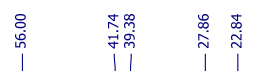
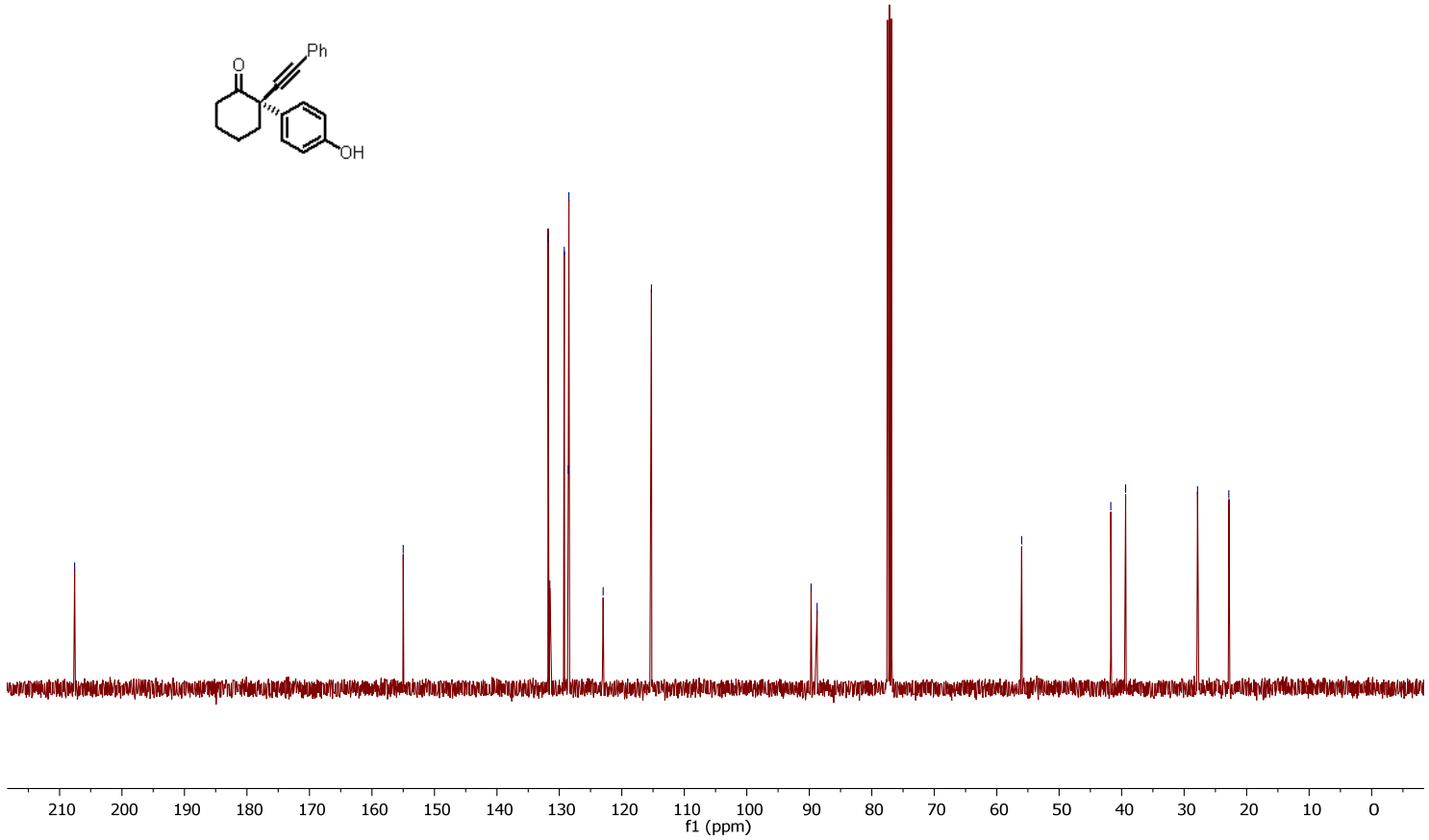

S63 


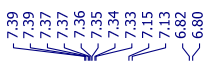

$1 \mathrm{~b}$

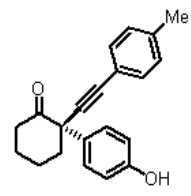

:

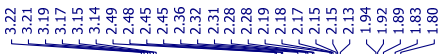

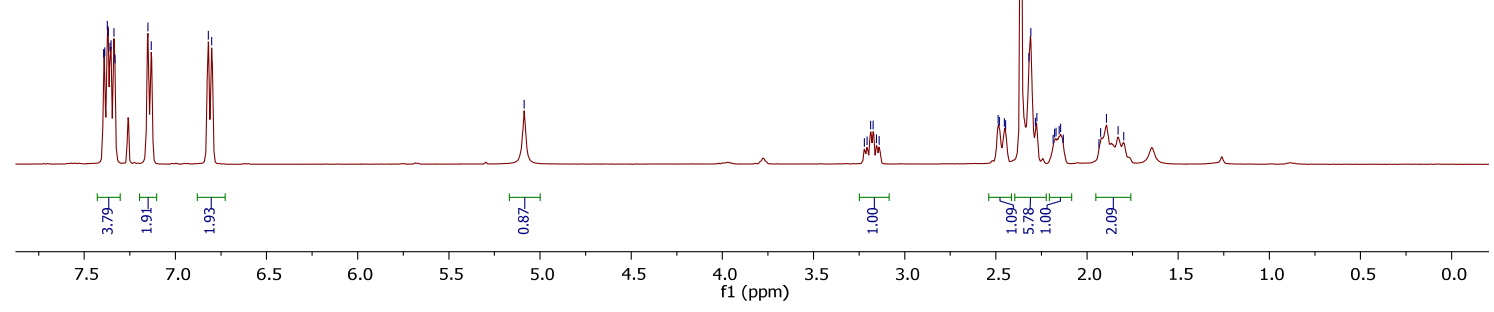

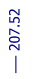

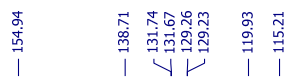

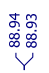

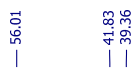

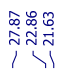
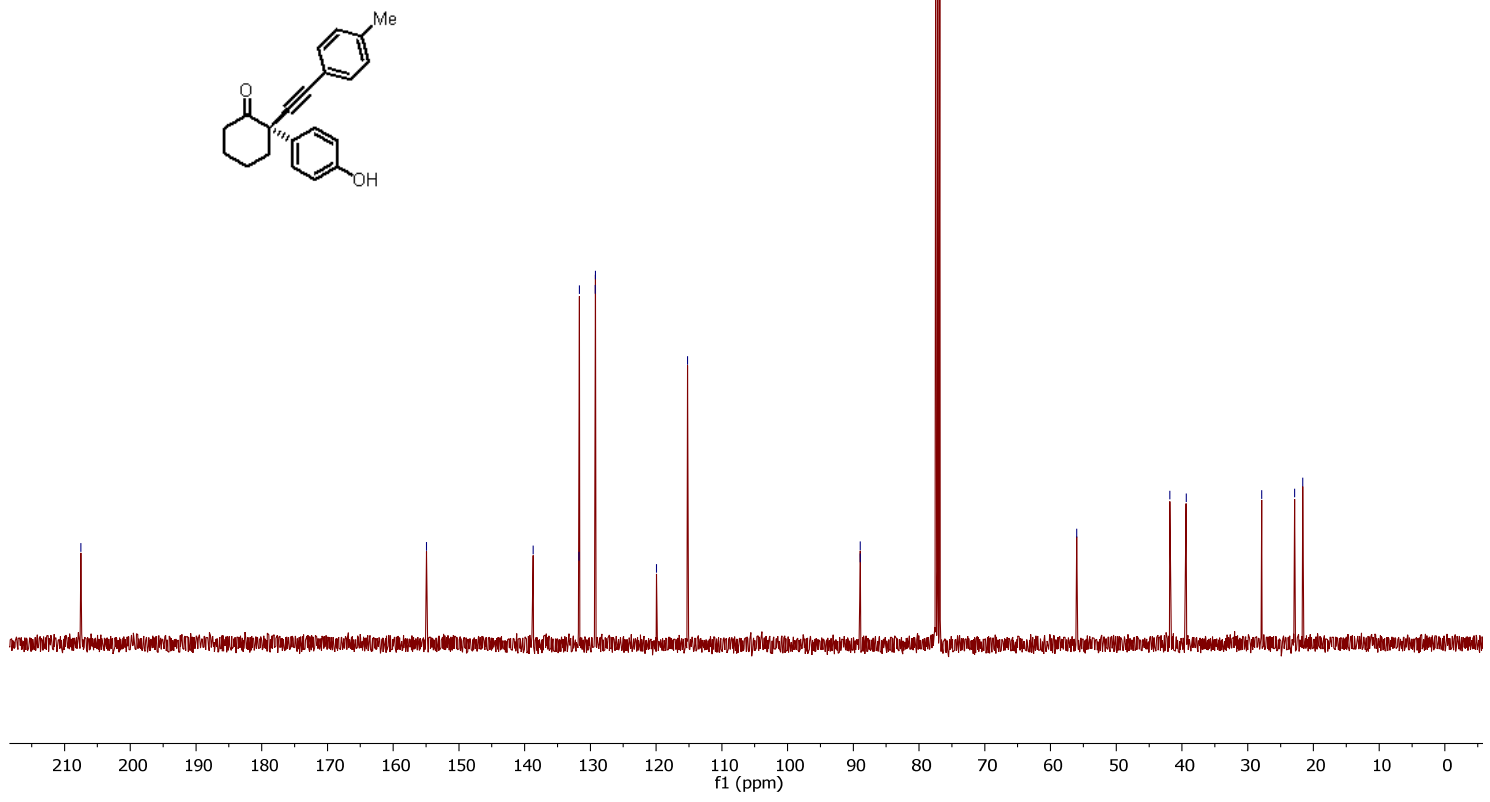

S64 


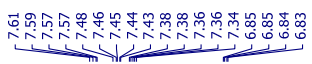

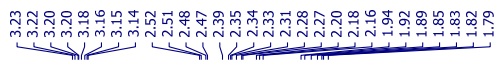

$1 \mathrm{c}$
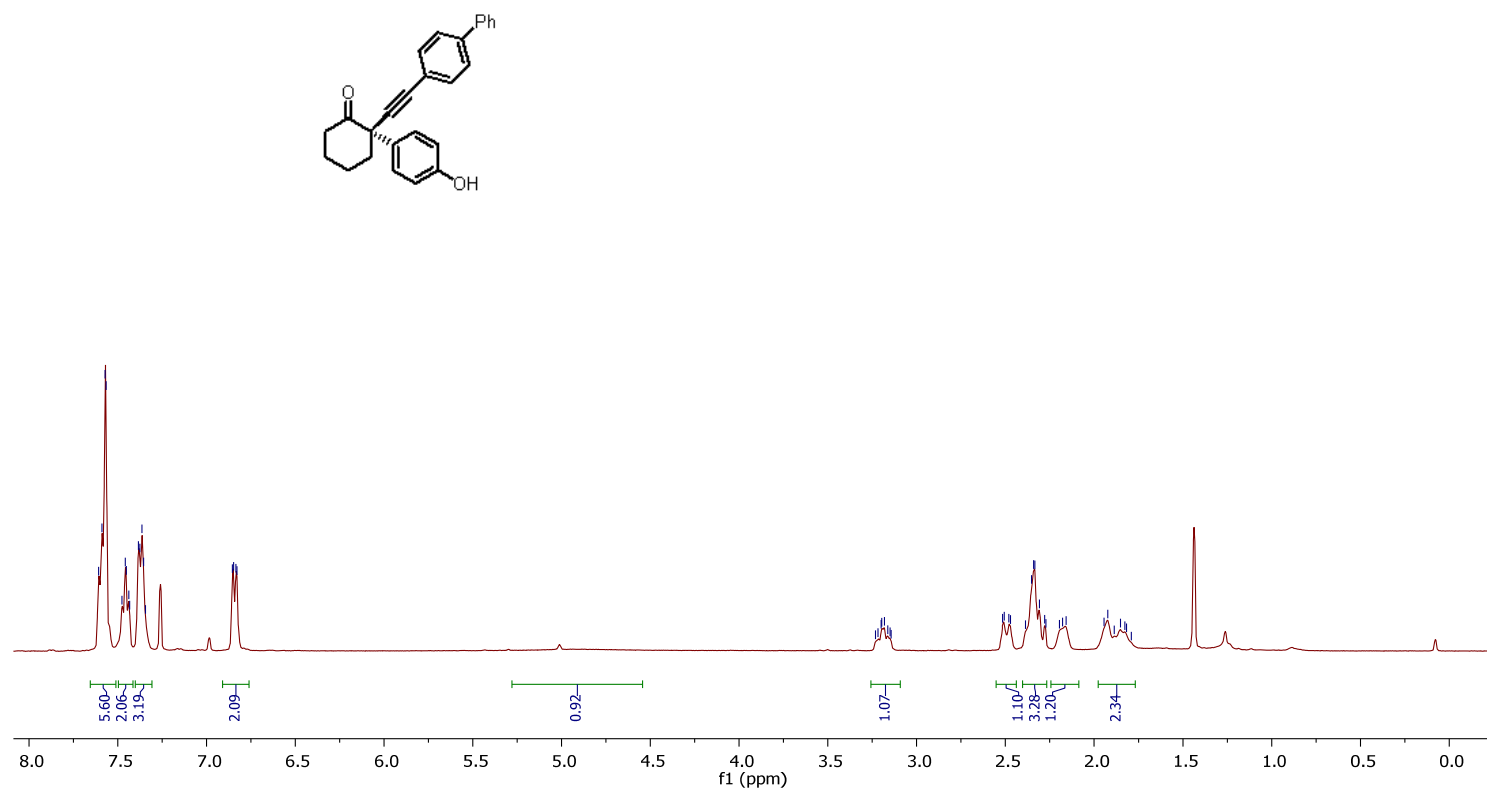

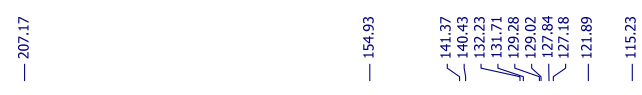

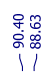

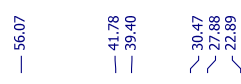
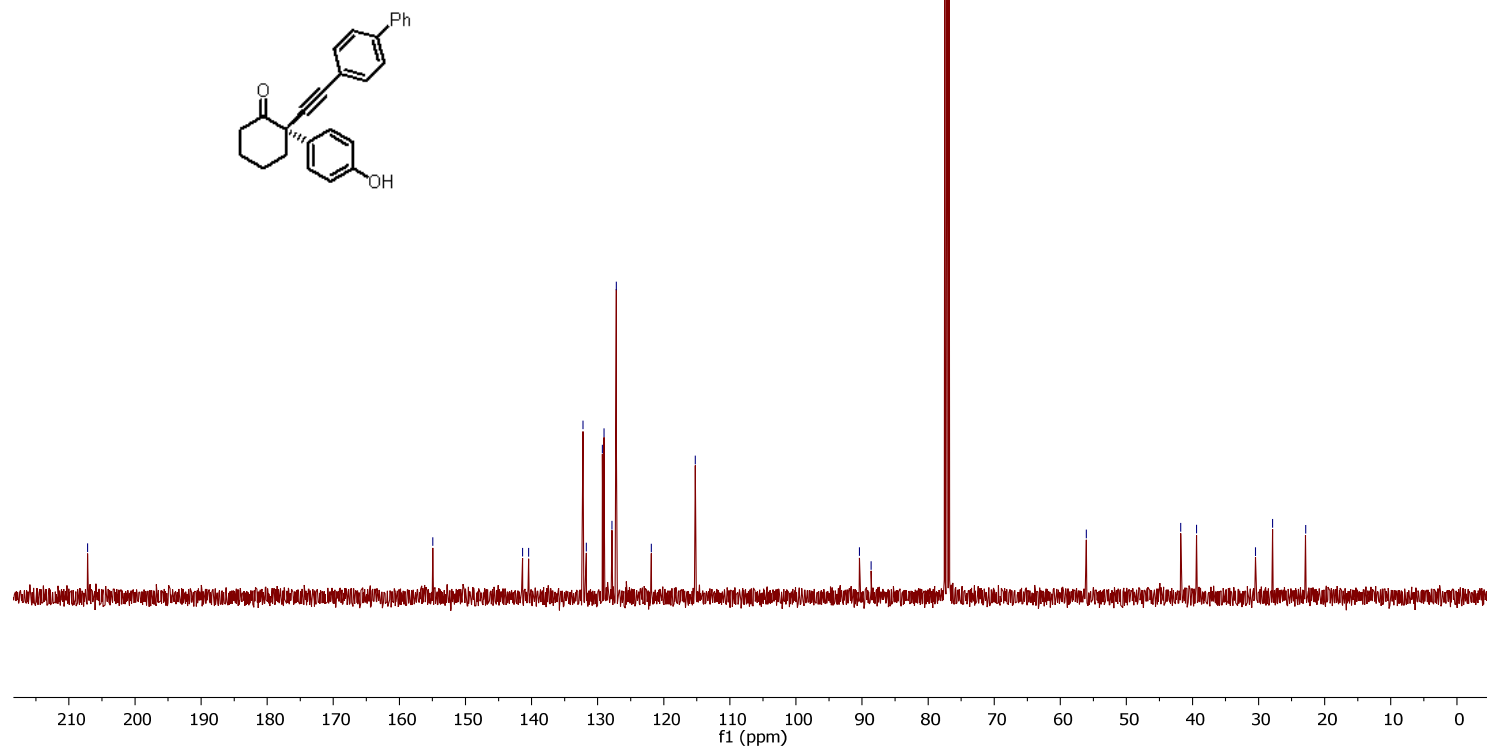

S65 


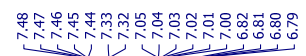

1d
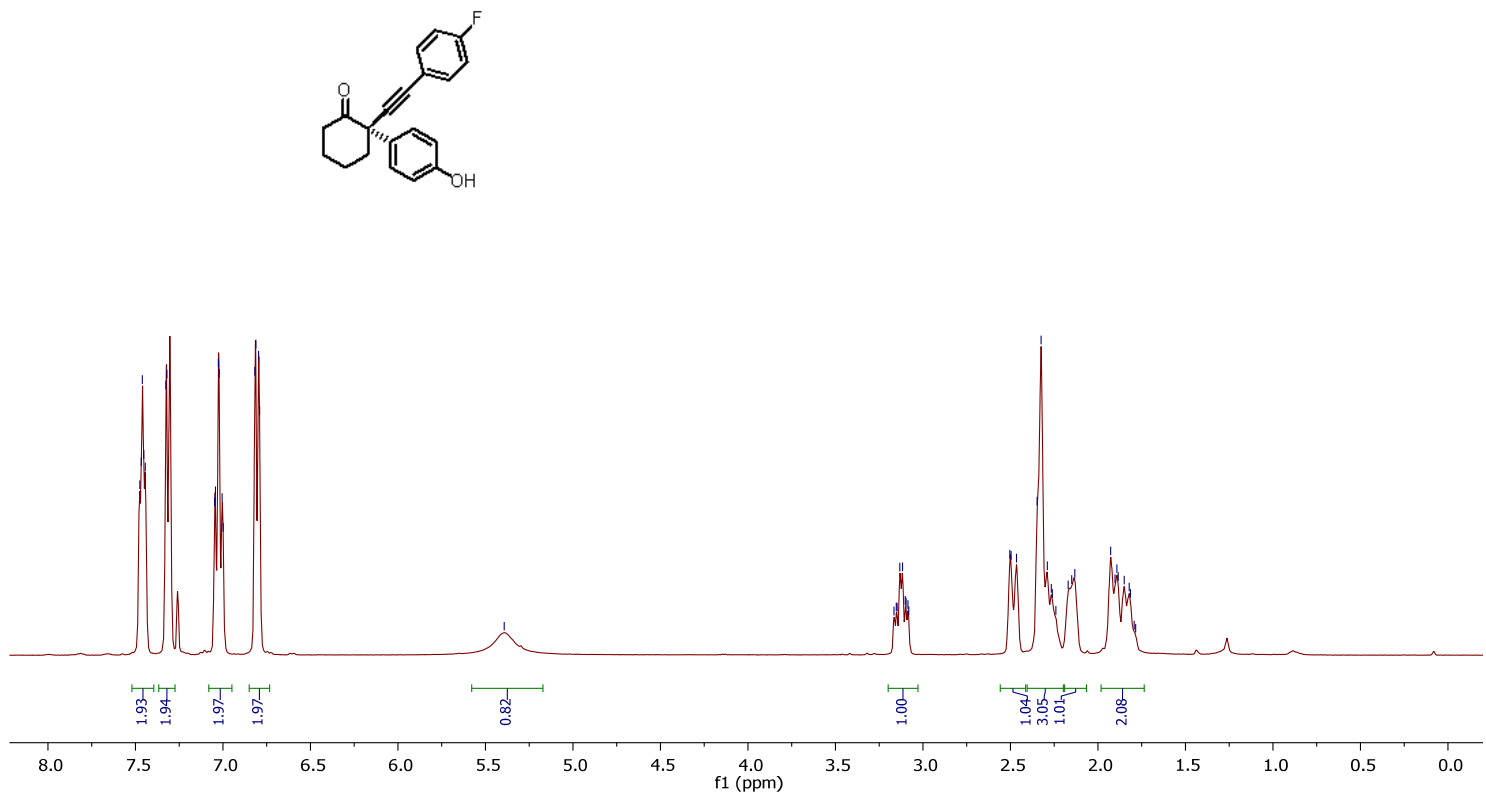

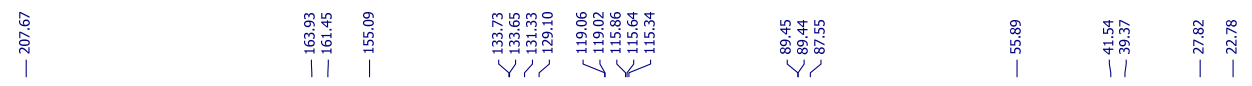

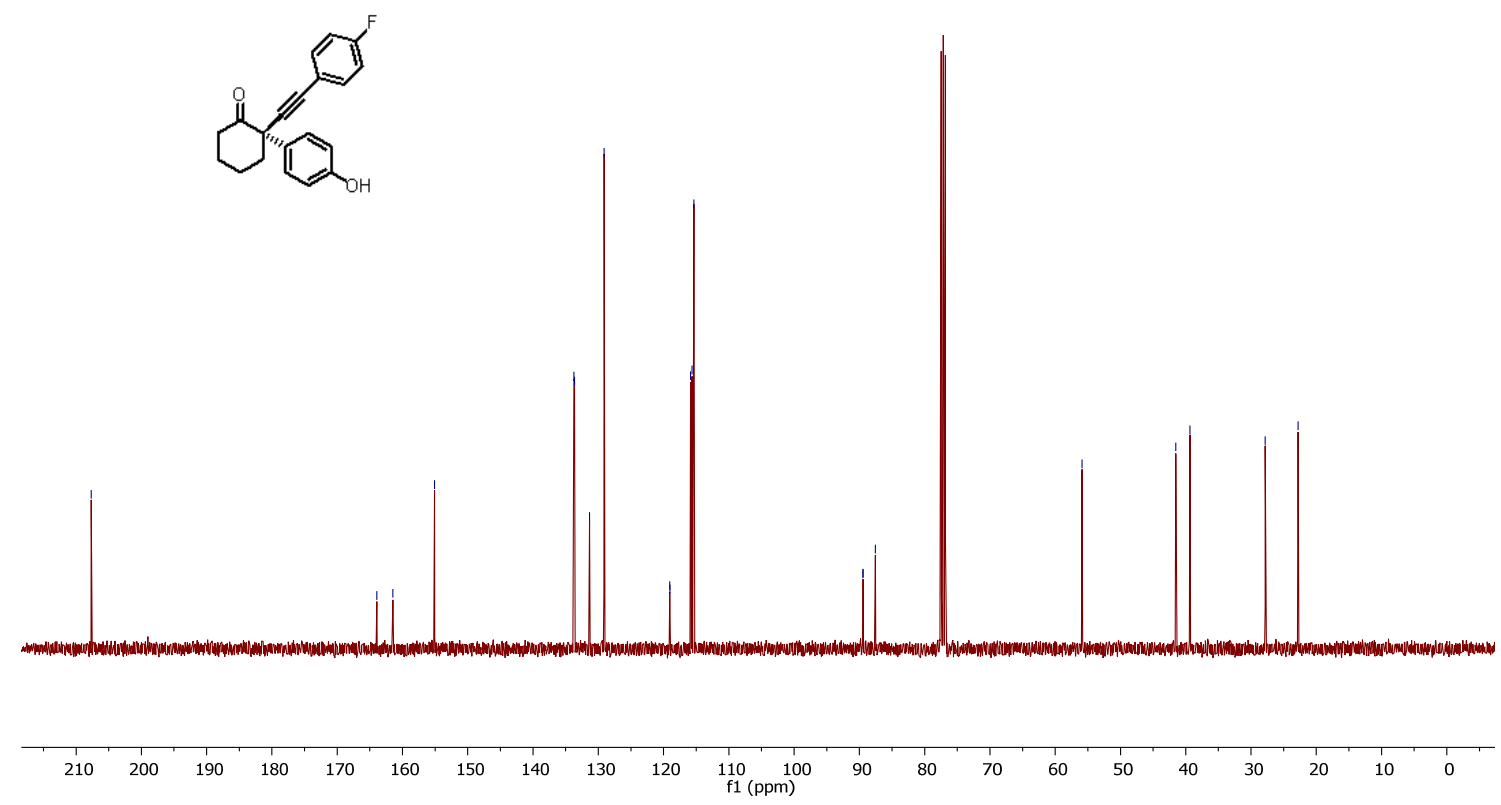



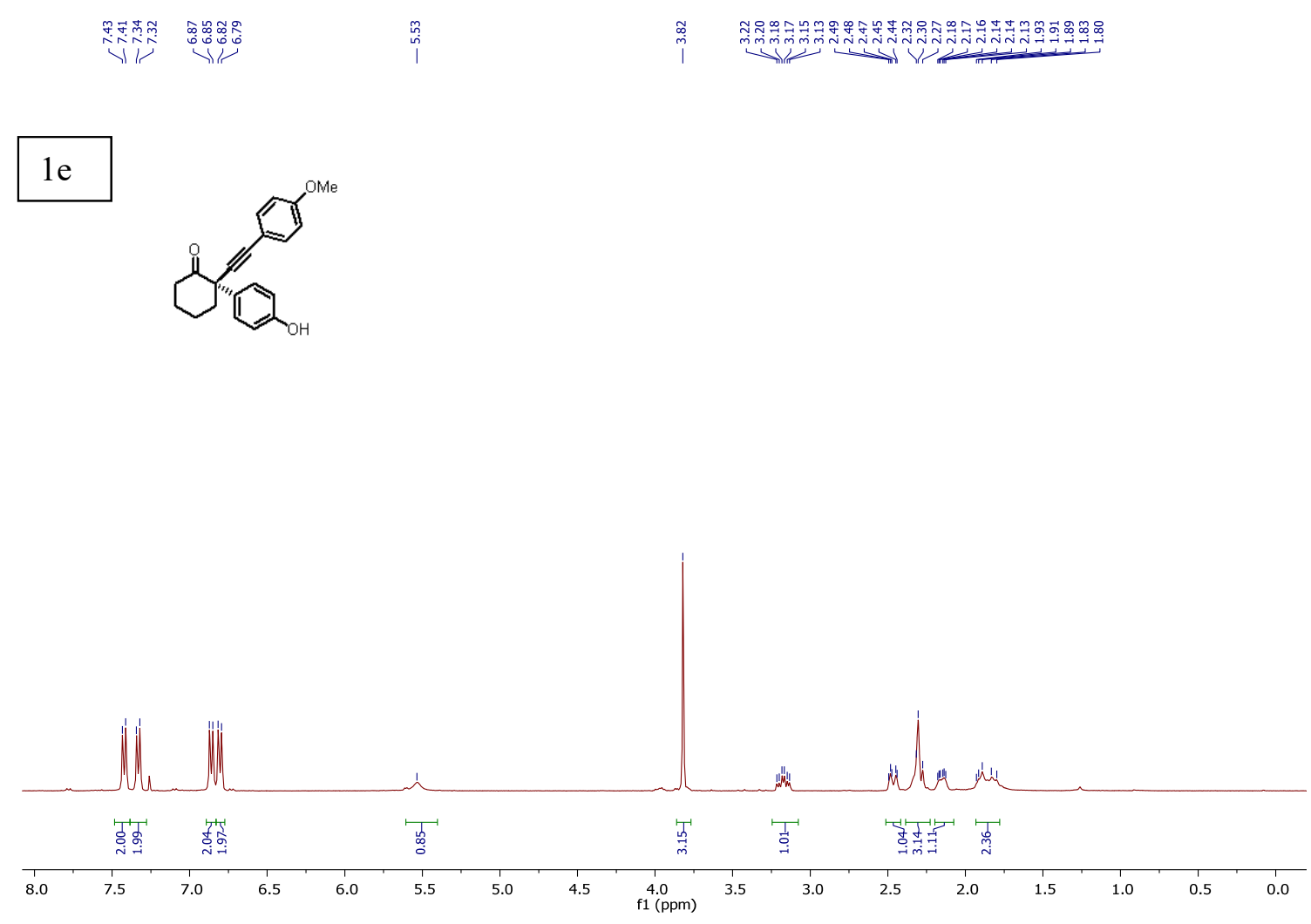

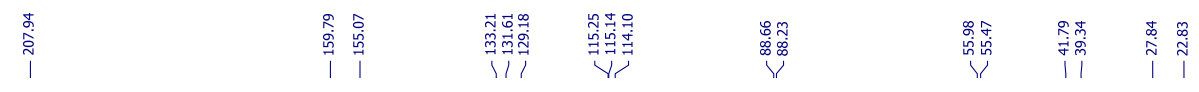

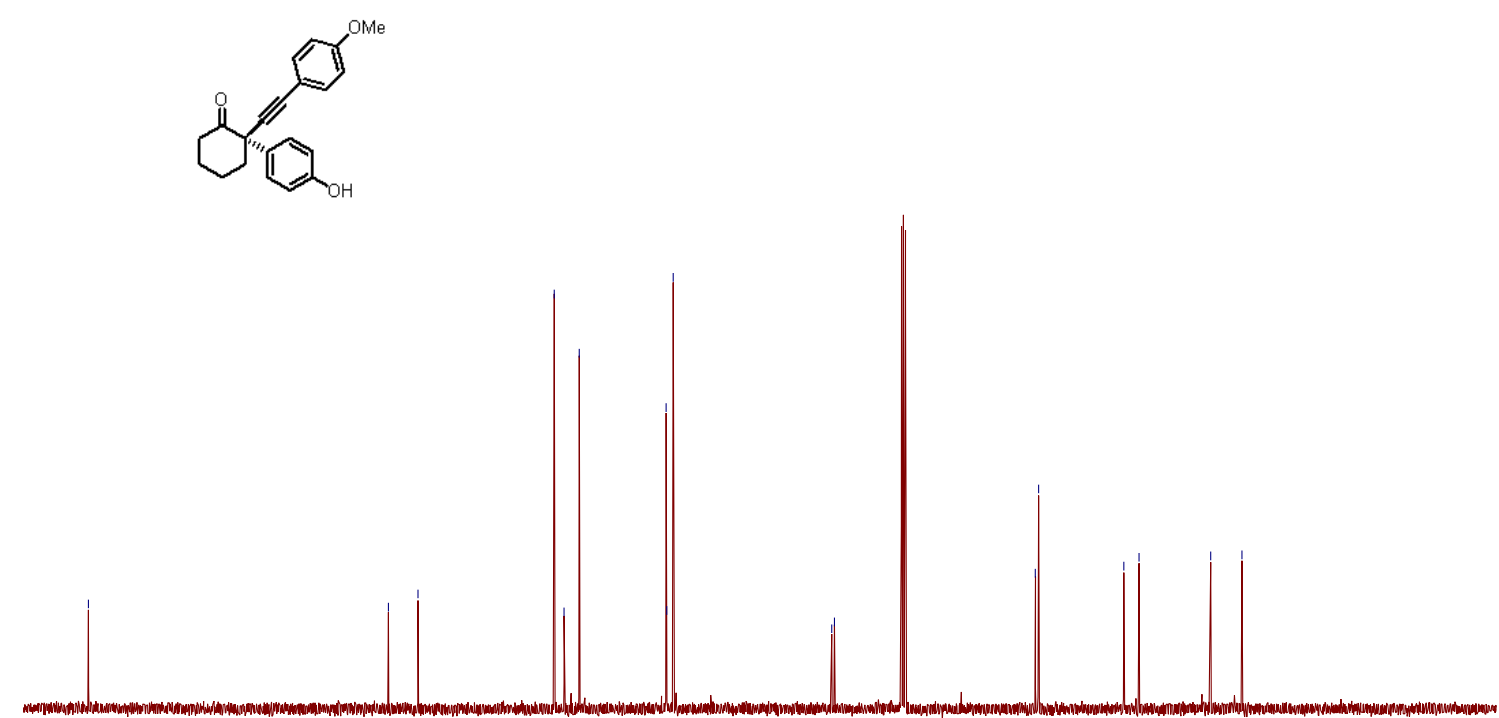

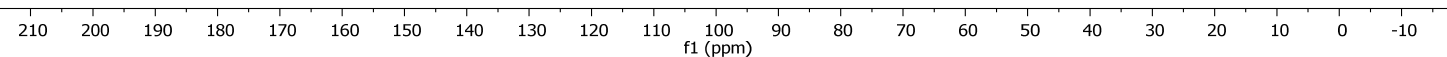




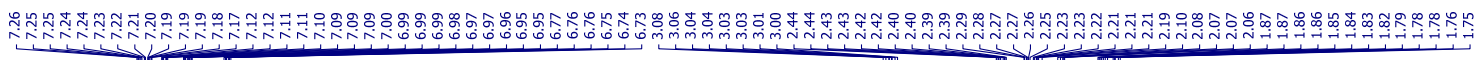

1f
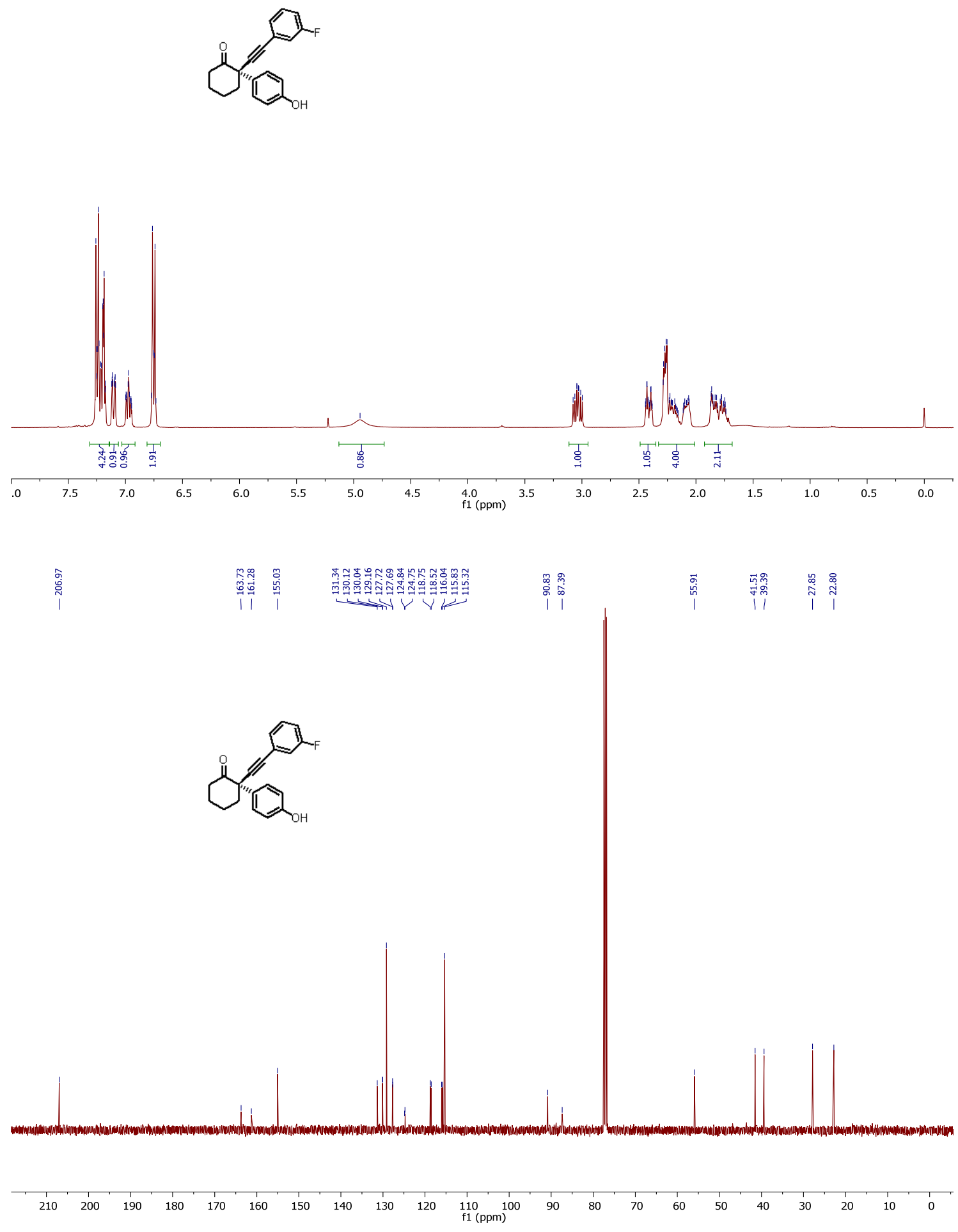

S68 


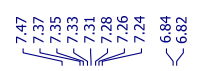

$\stackrel{\approx}{\tilde{*}}$

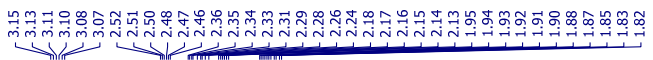

$1 \mathrm{~g}$
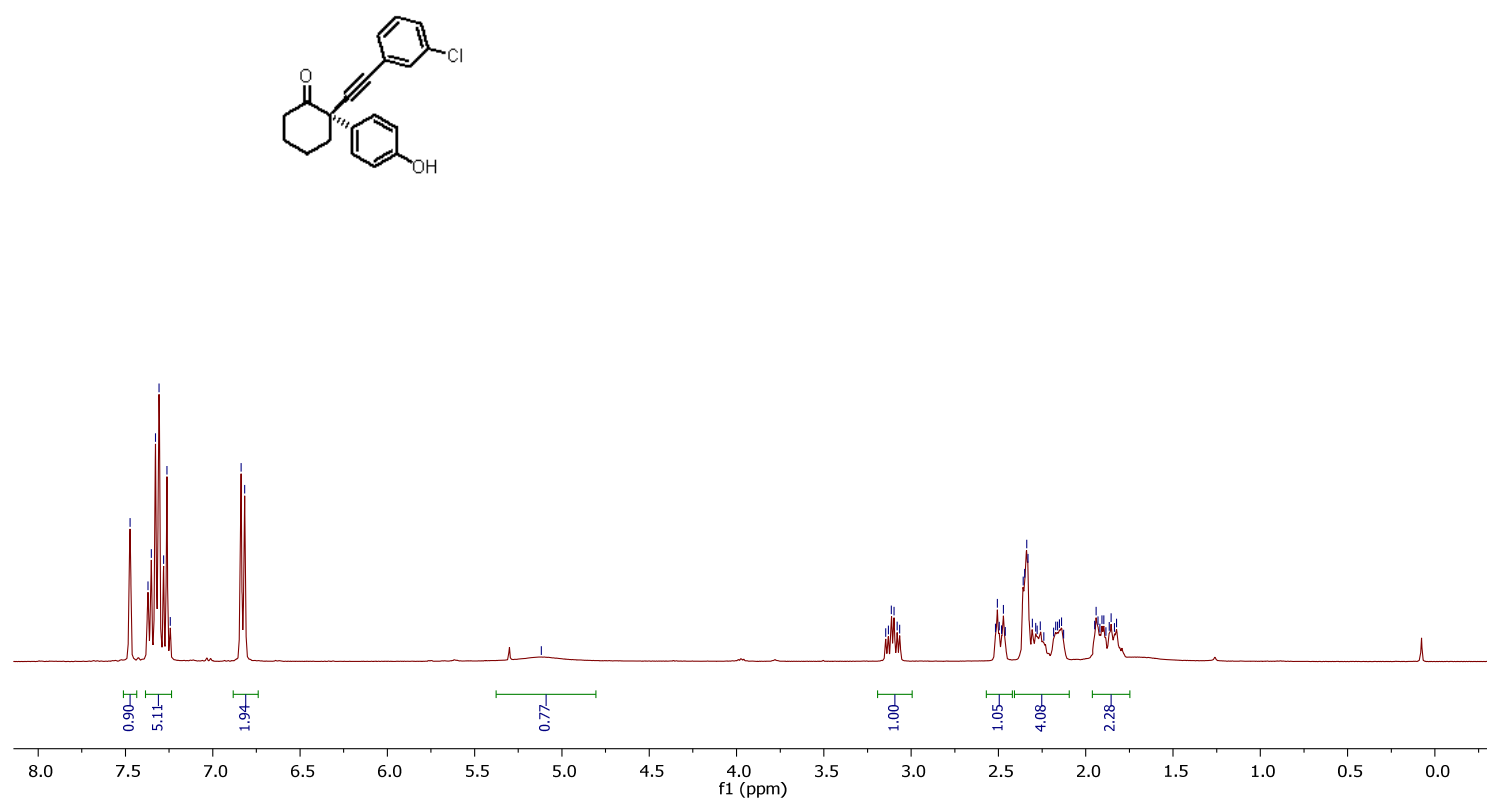

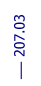

|

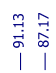

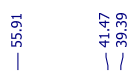

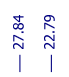
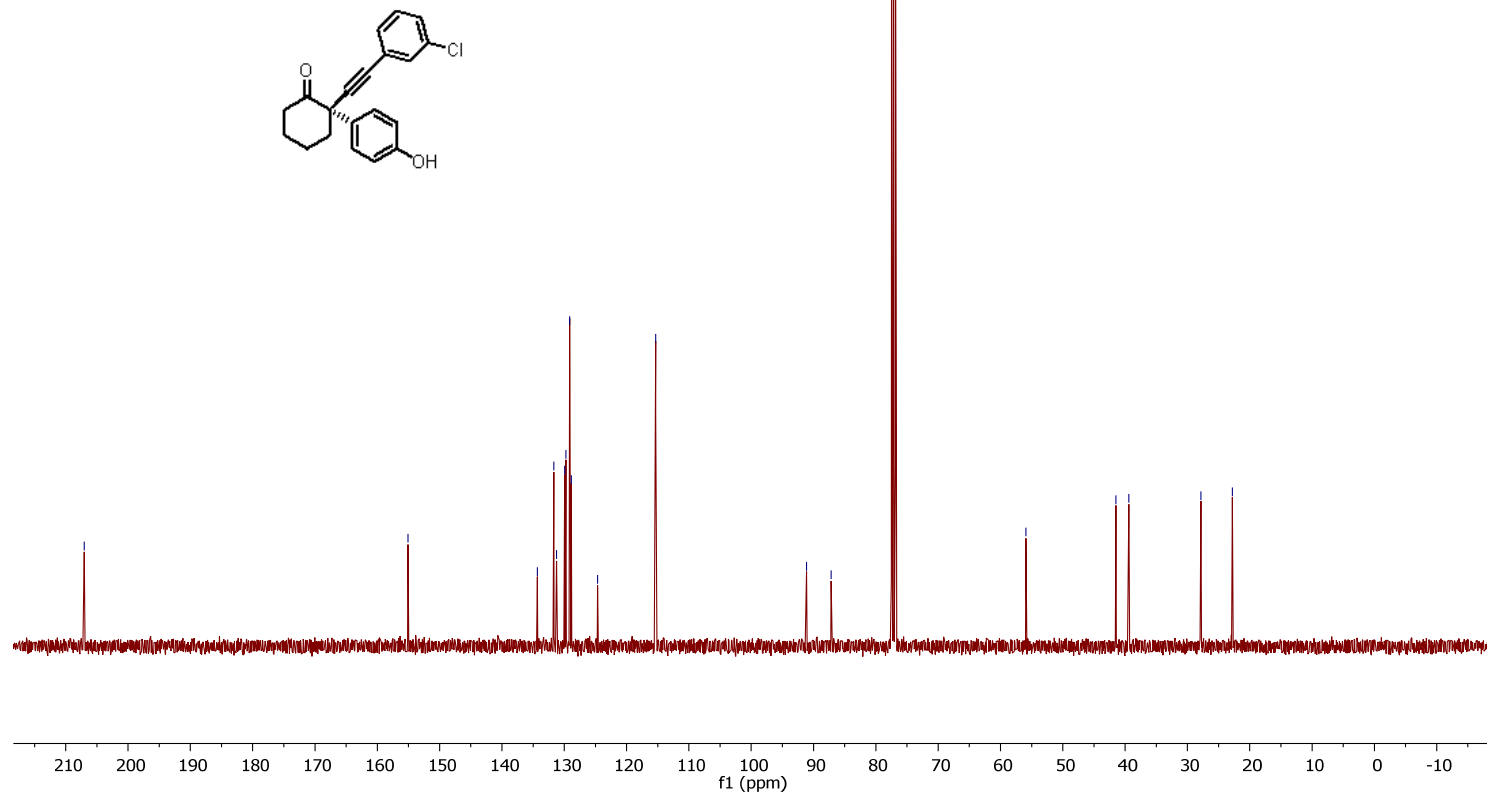

S69 

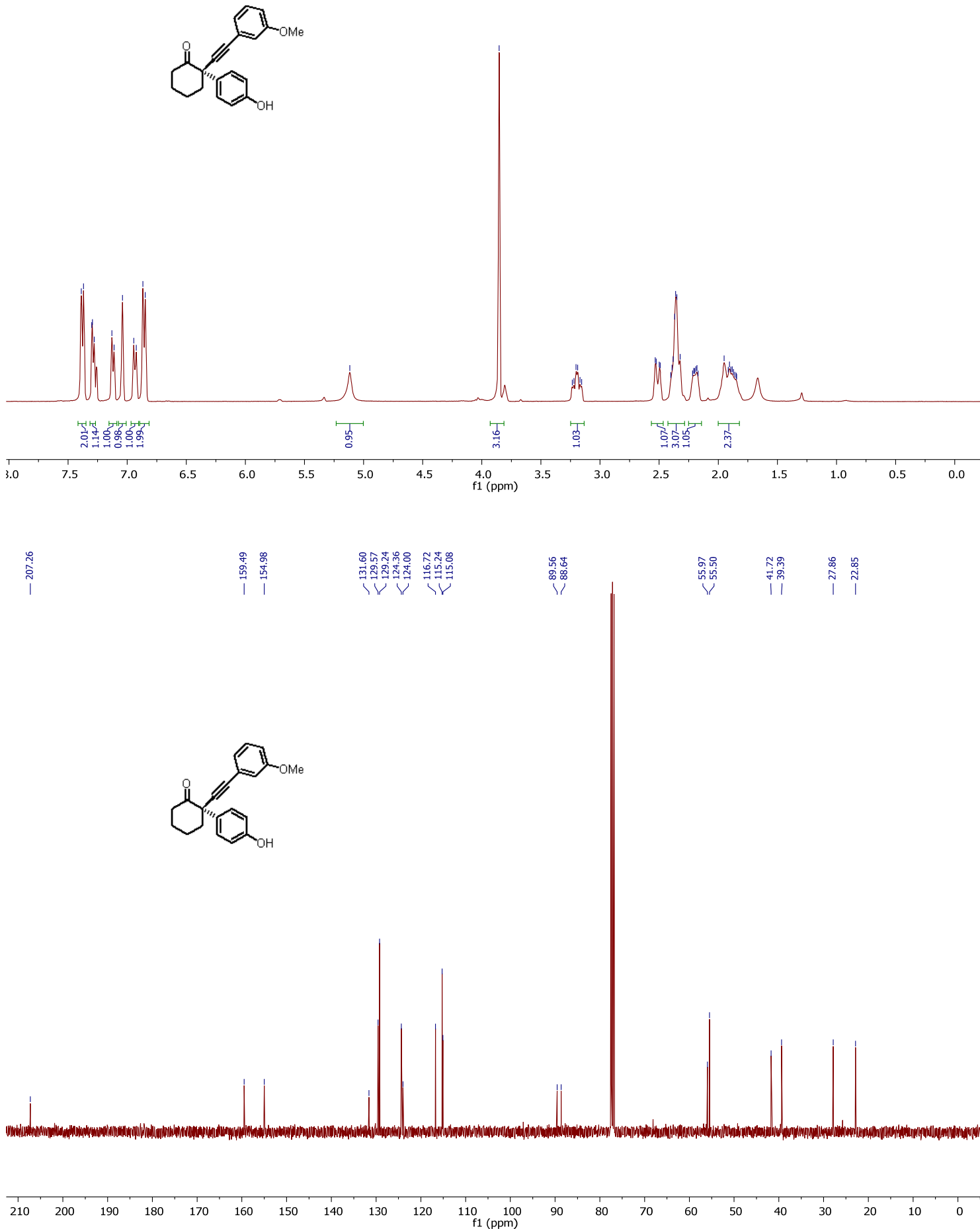

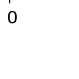




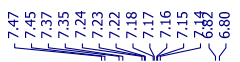

$1 \mathrm{i}$
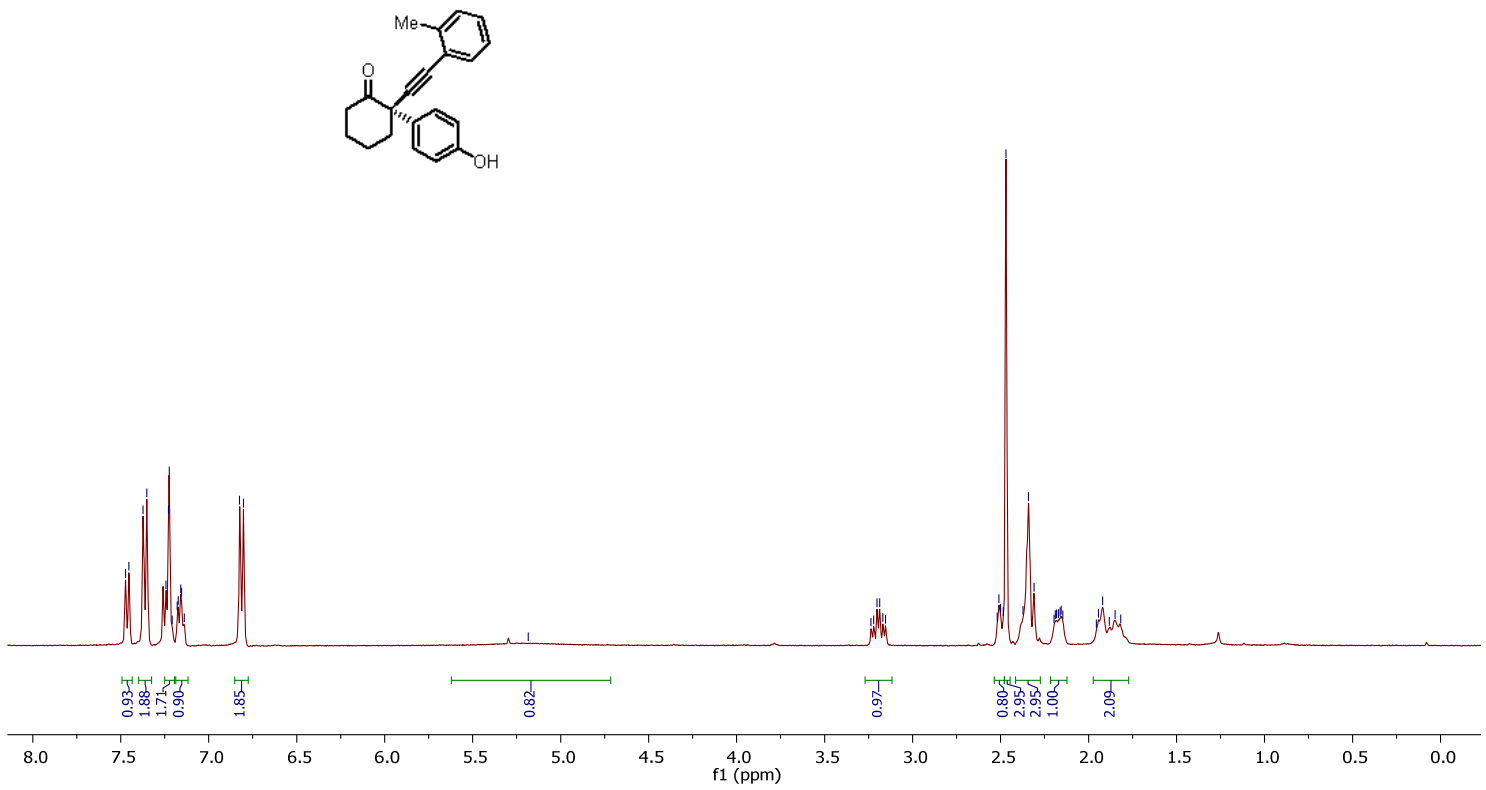

$\stackrel{\widetilde{N}}{\stackrel{\sim}{\sim}}$ $\stackrel{\infty}{\substack{\omega \\ i}}$

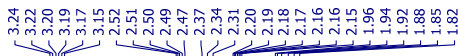

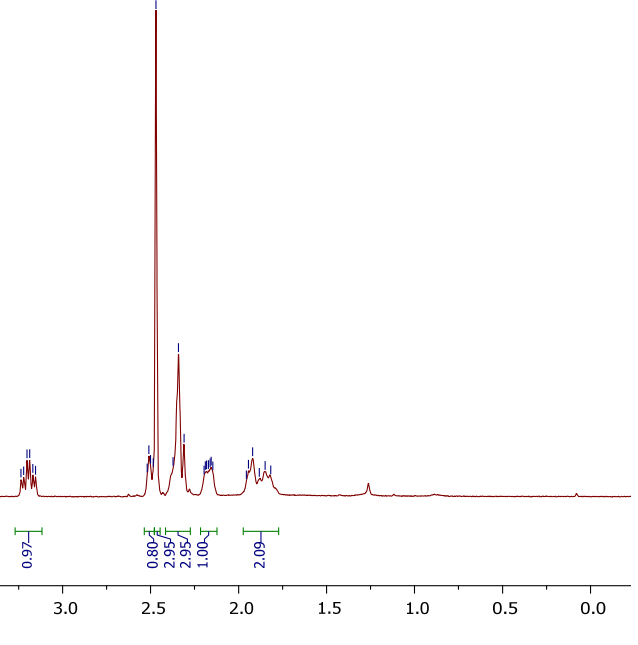

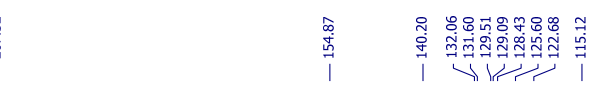

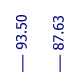

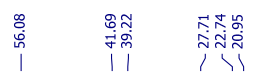

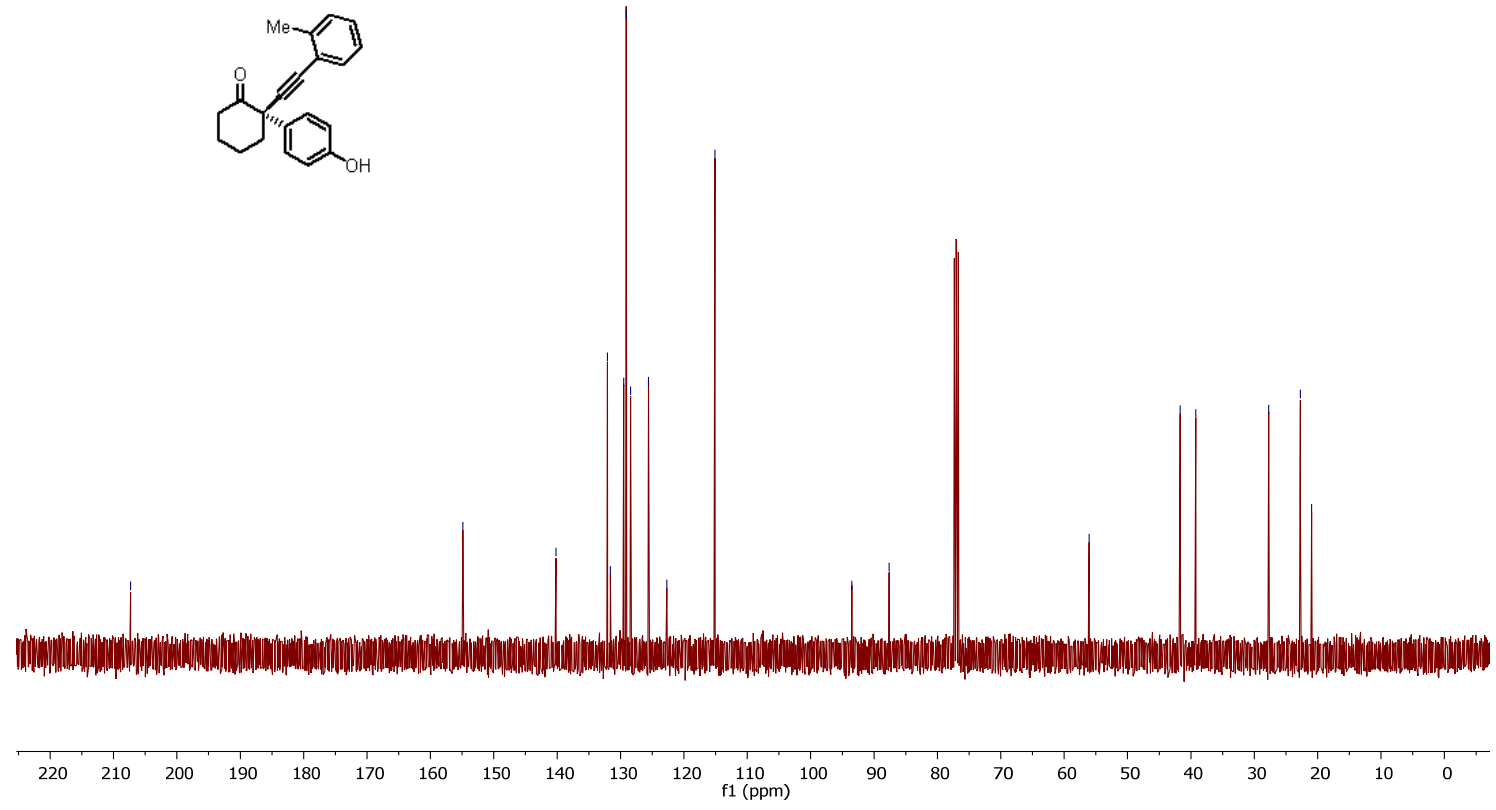

S71 
$1 \mathrm{j}$

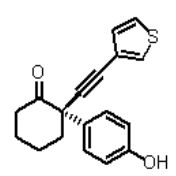

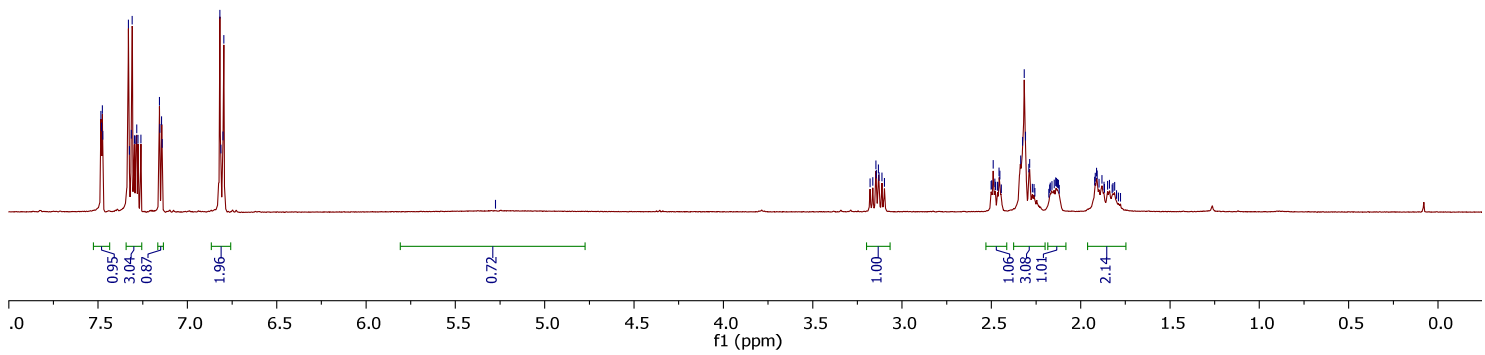

量

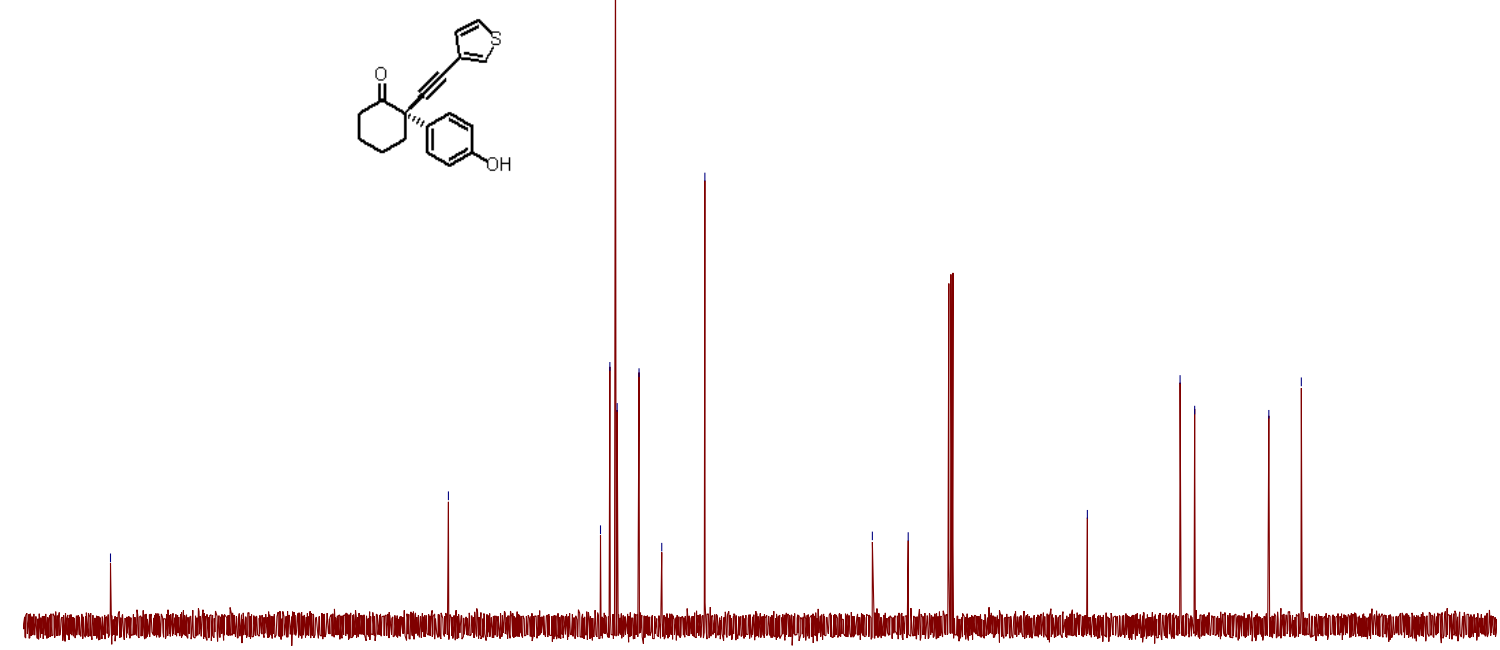

$\begin{array}{lllllllllllllllllllllllll}120 & 210 & 200 & 190 & 180 & 170 & 160 & 150 & 140 & 130 & 120 & \begin{array}{l}110 \\ \mathrm{f} 1(\mathrm{ppm})\end{array} & 90 & 80 & 70 & 60 & 50 & 40 & 30 & 20 & 10 & 0\end{array}$ 


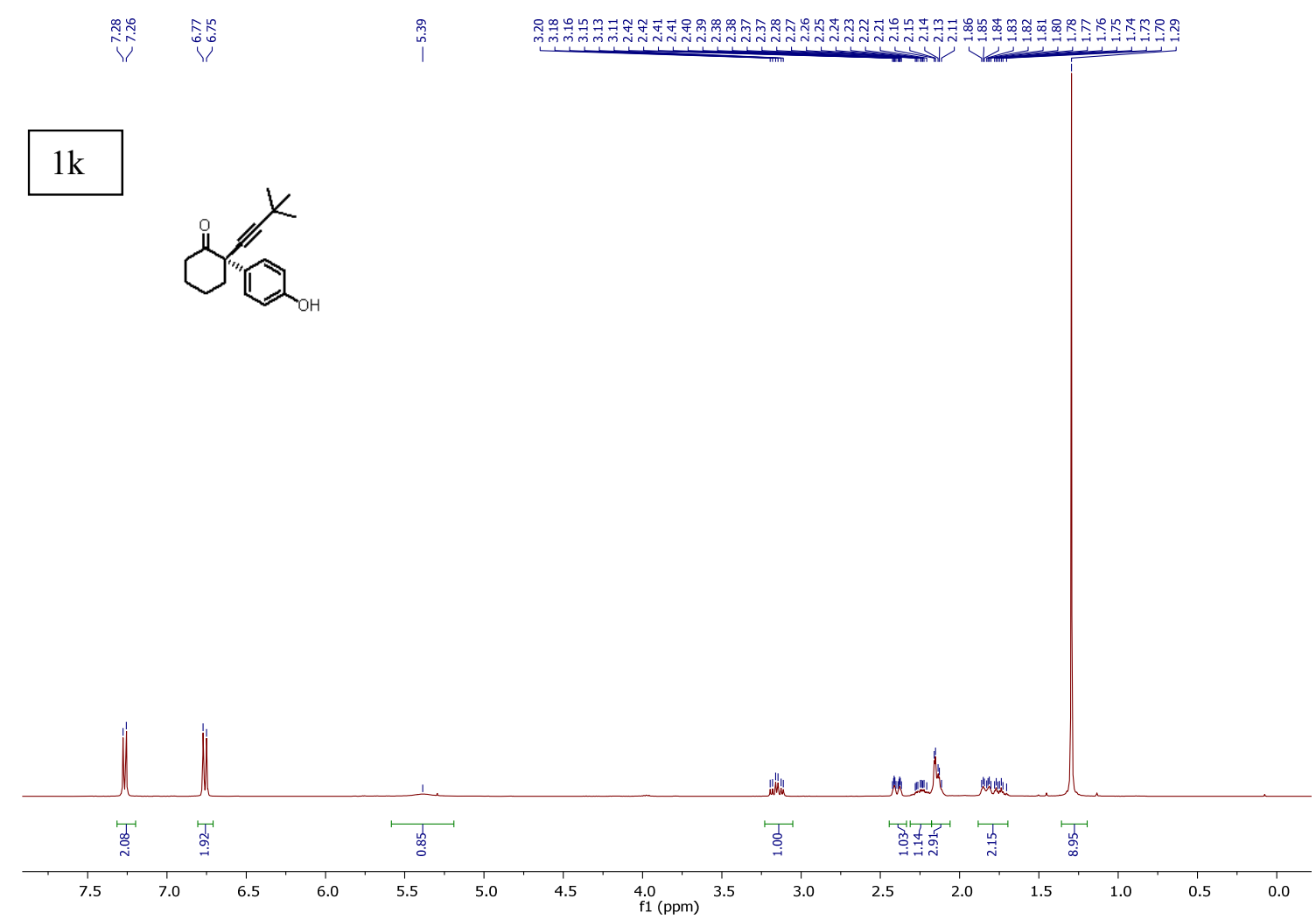

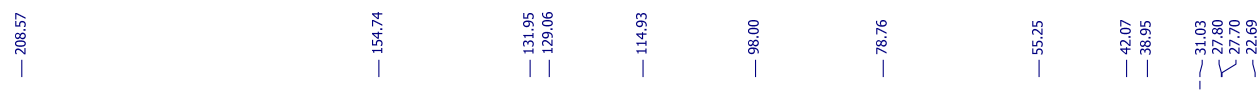
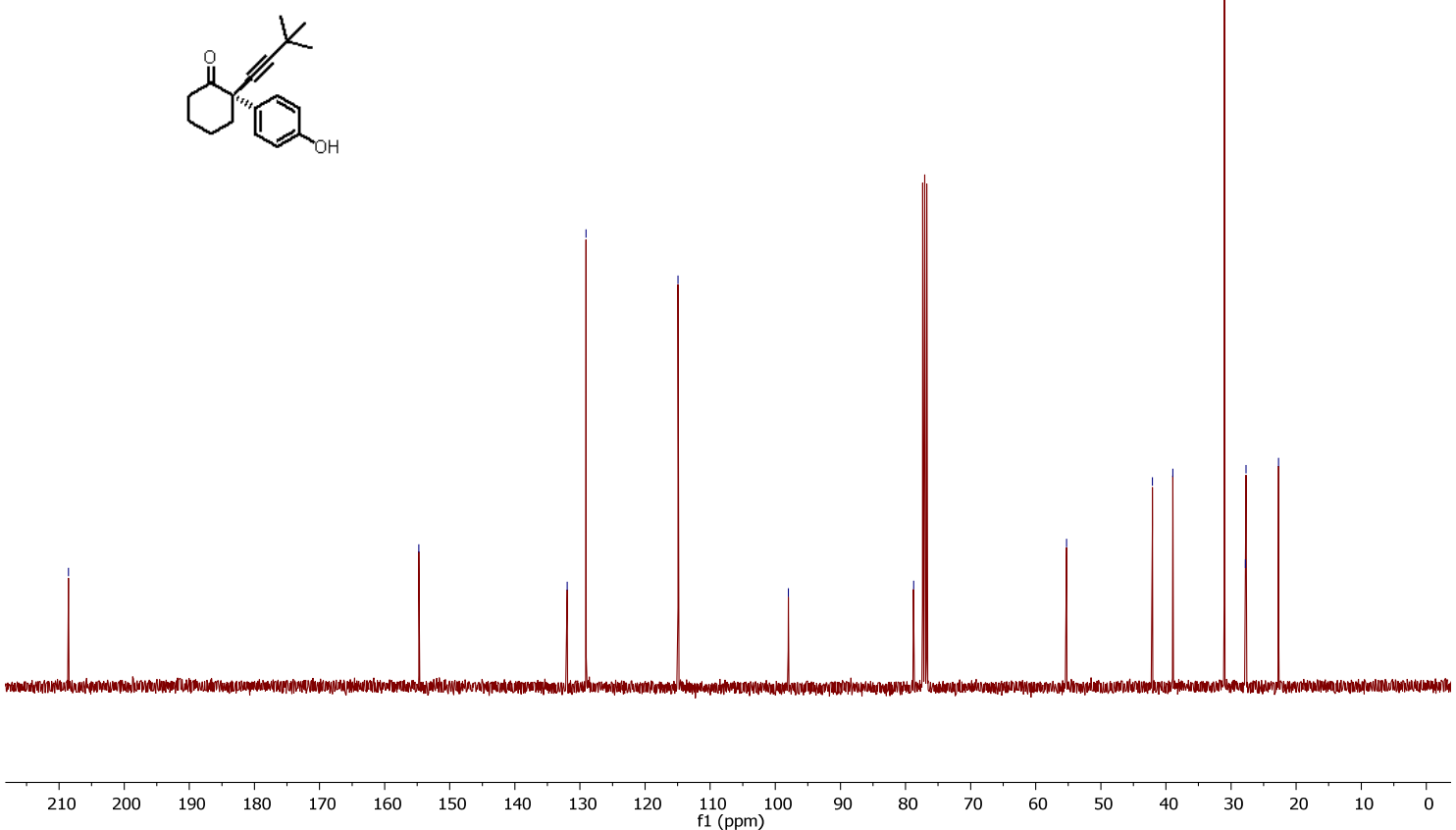


\section{1}<smiles>CCCCC1CCCCC1=O</smiles>
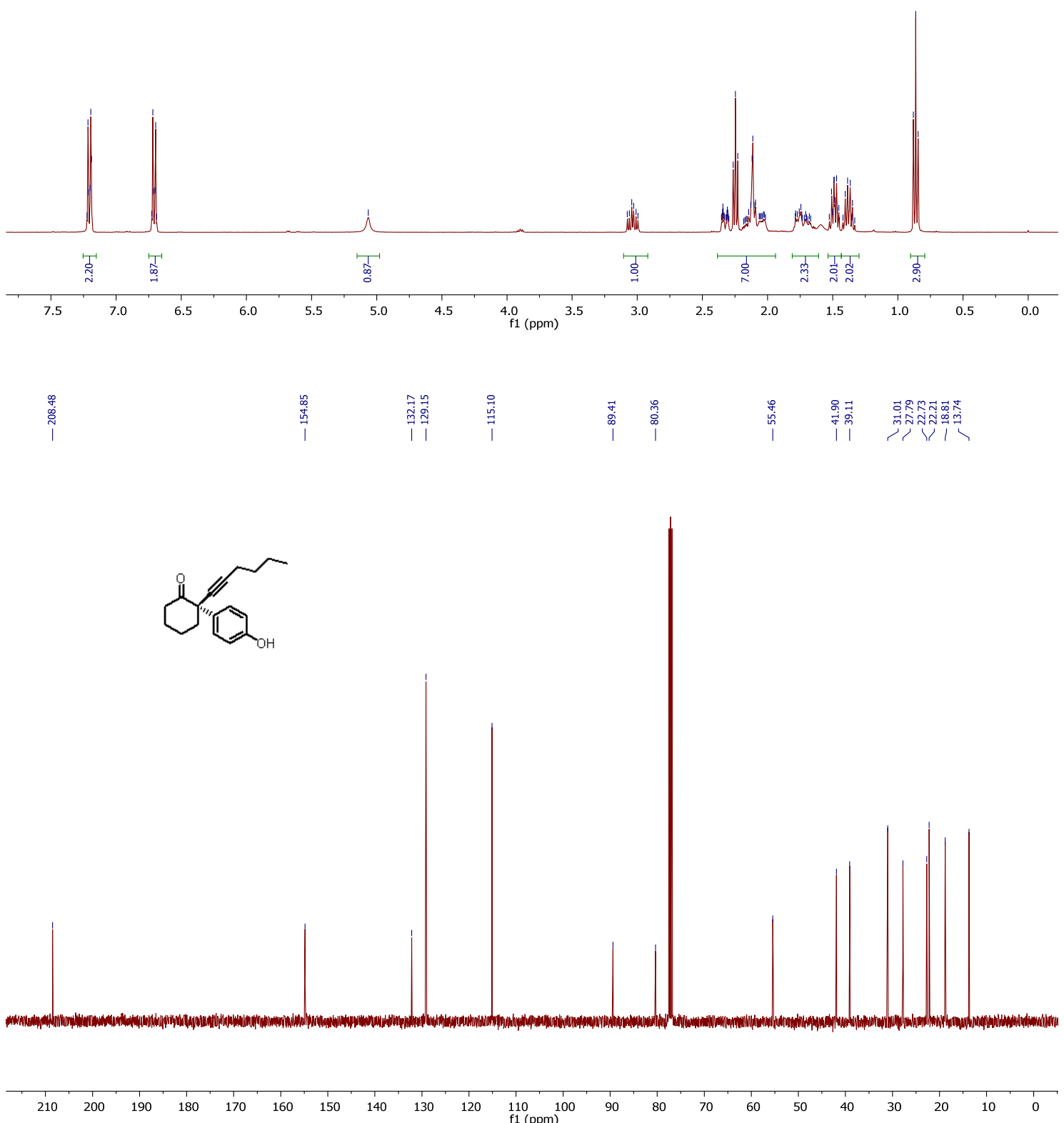


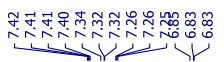

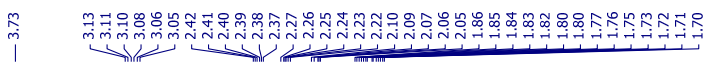

$1 \mathrm{~m}$
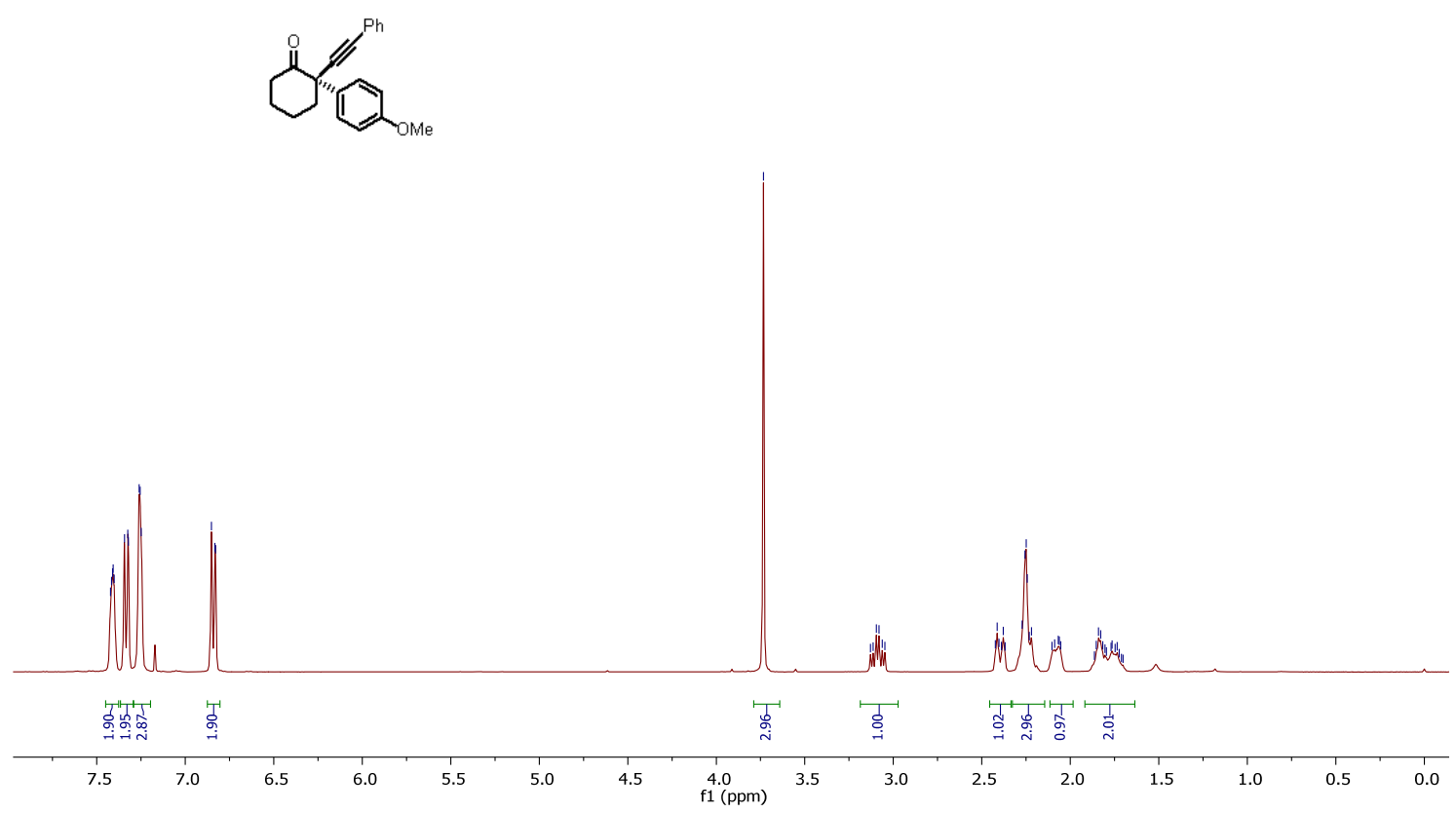

$\underset{\substack{\infty \\ \stackrel{0}{0} \\ \text { I }}}{ }$

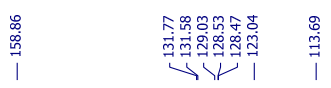

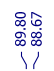

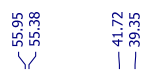

$\stackrel{\infty}{\stackrel{\infty}{\sim}} \stackrel{\stackrel{\infty}{\sim}}{1}$

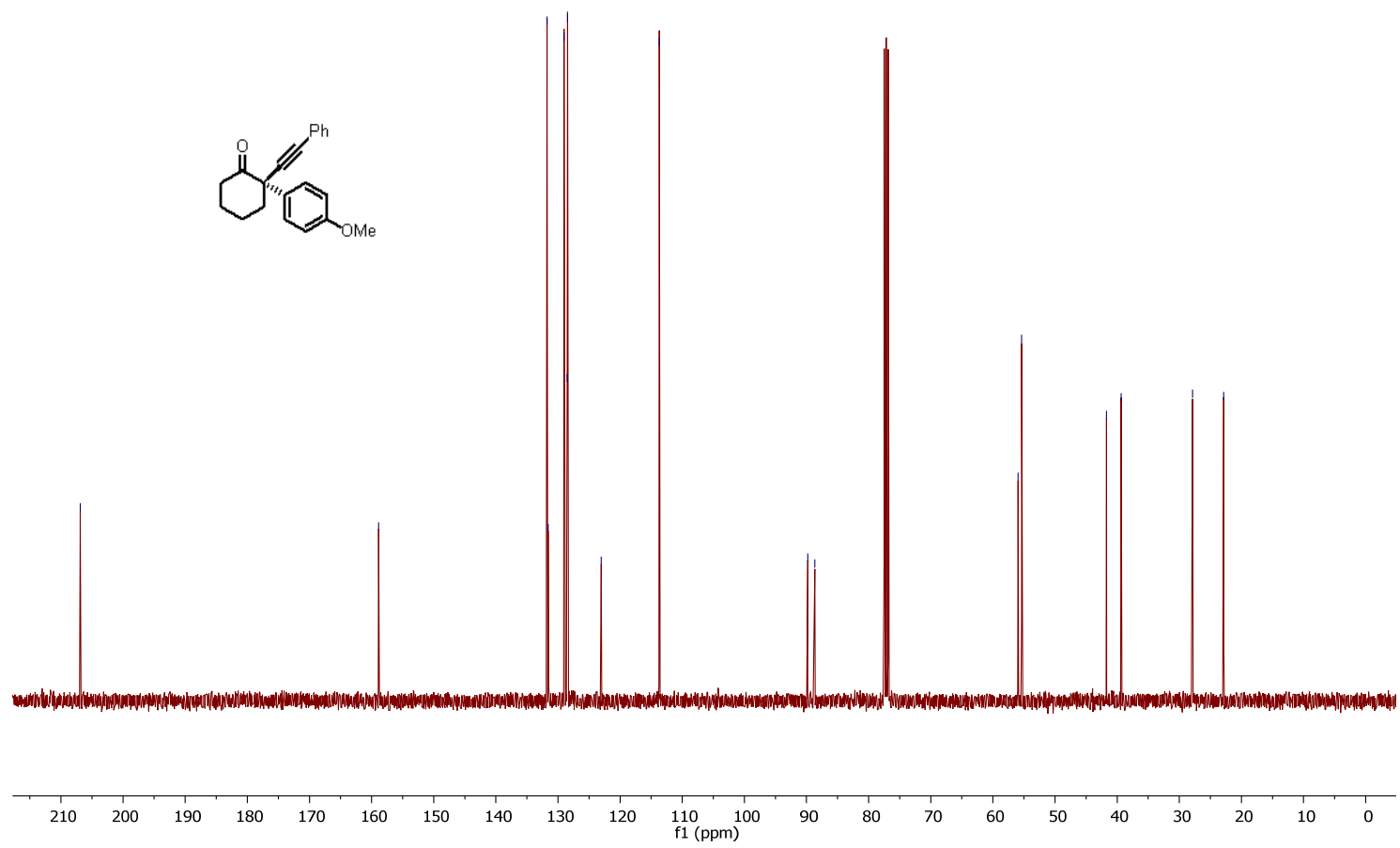

S75 


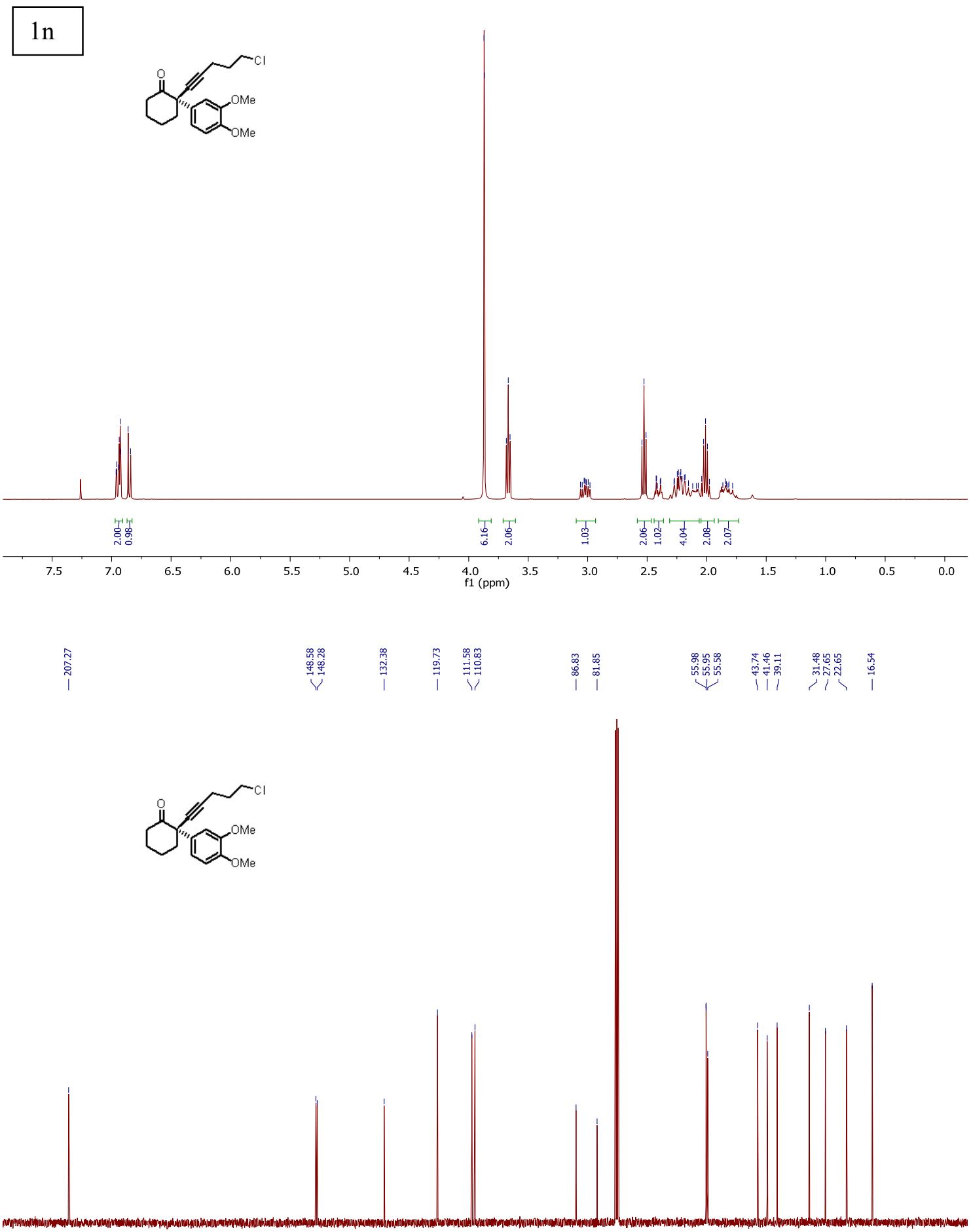

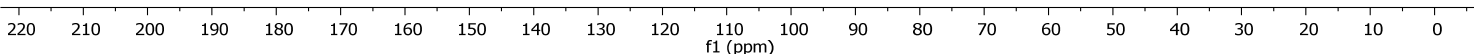


我

10
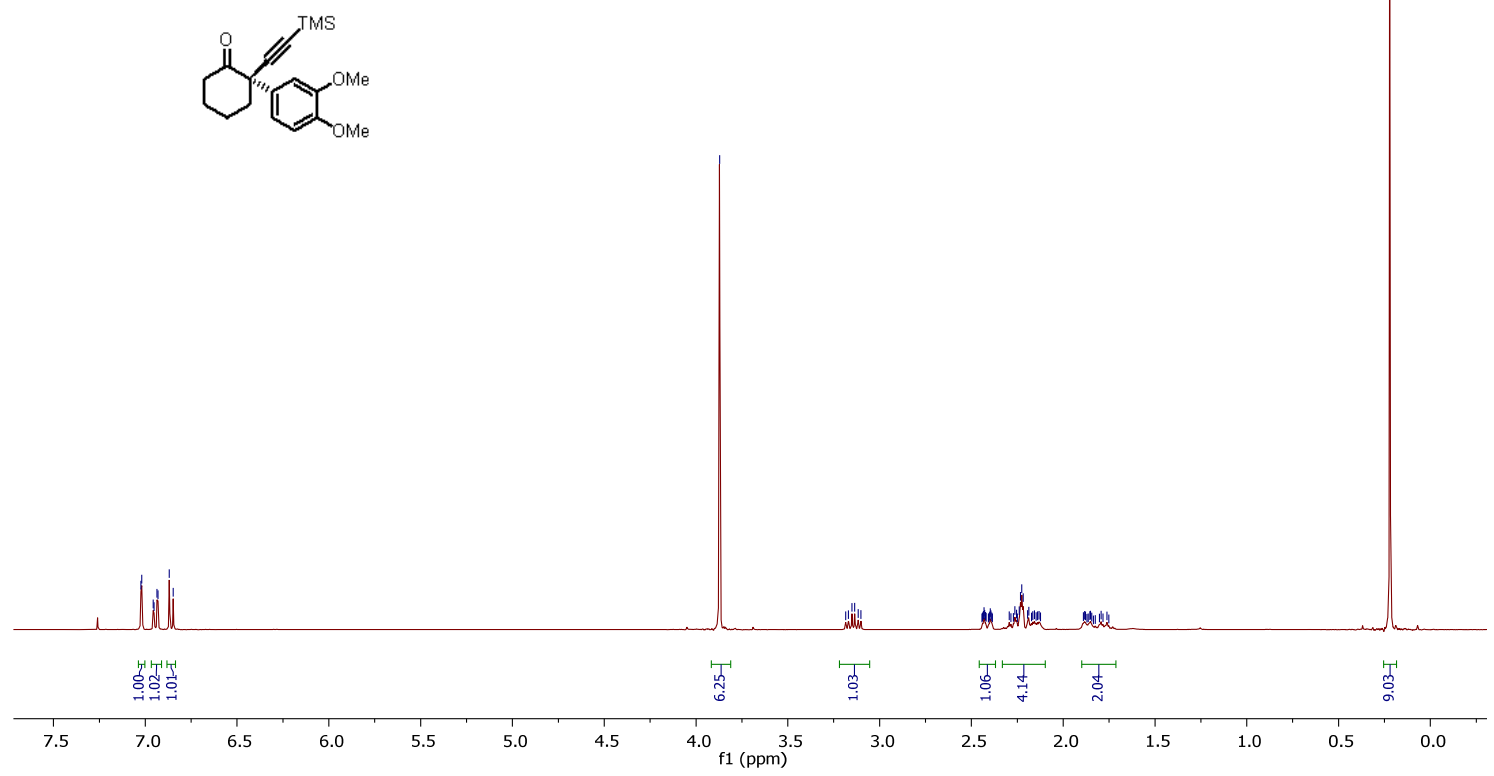

\begin{tabular}{l}
0 \\
0 \\
0 \\
\hline
\end{tabular}

ป

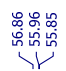

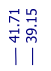

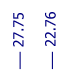

i̊․․
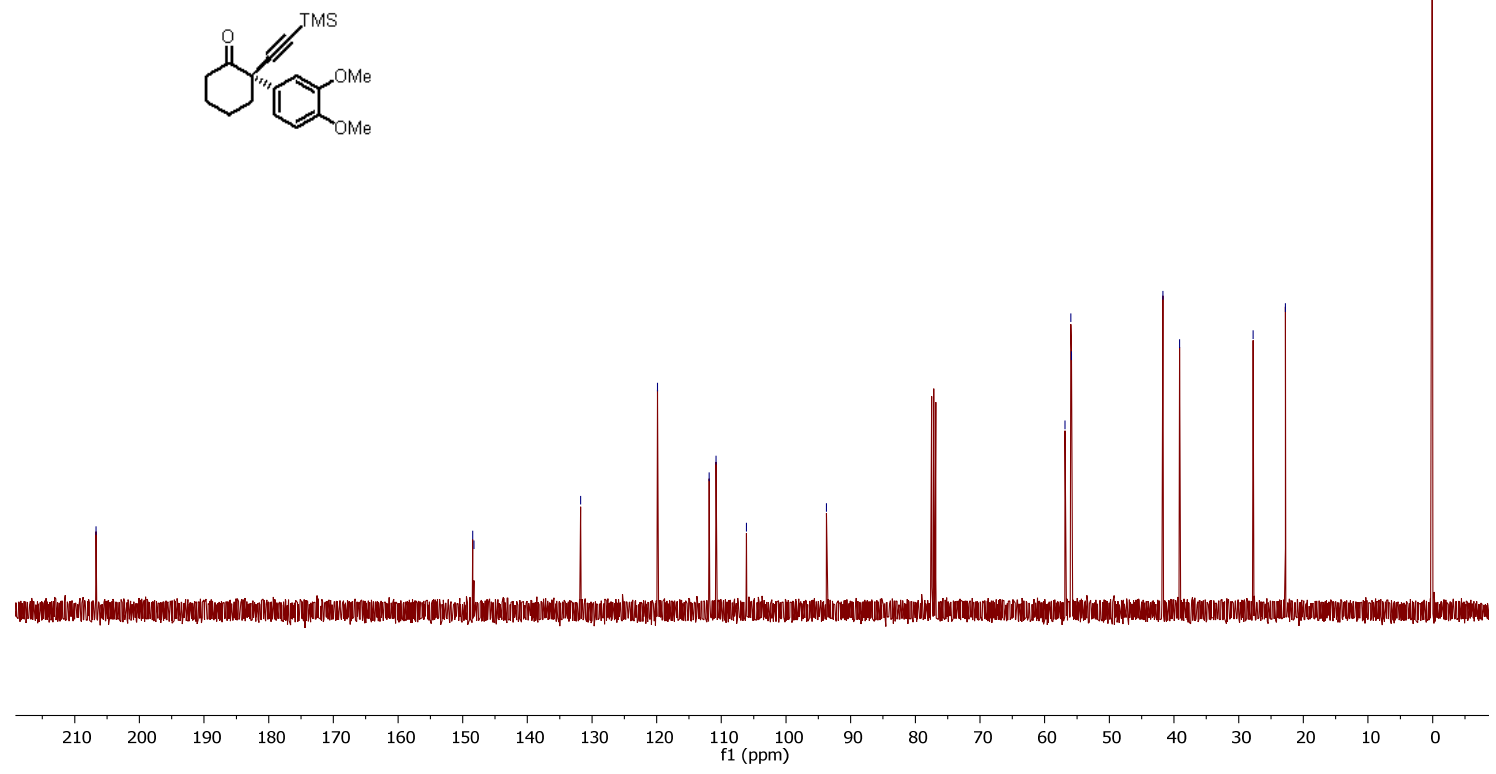

S77 


\section{$1 \mathrm{p}$}
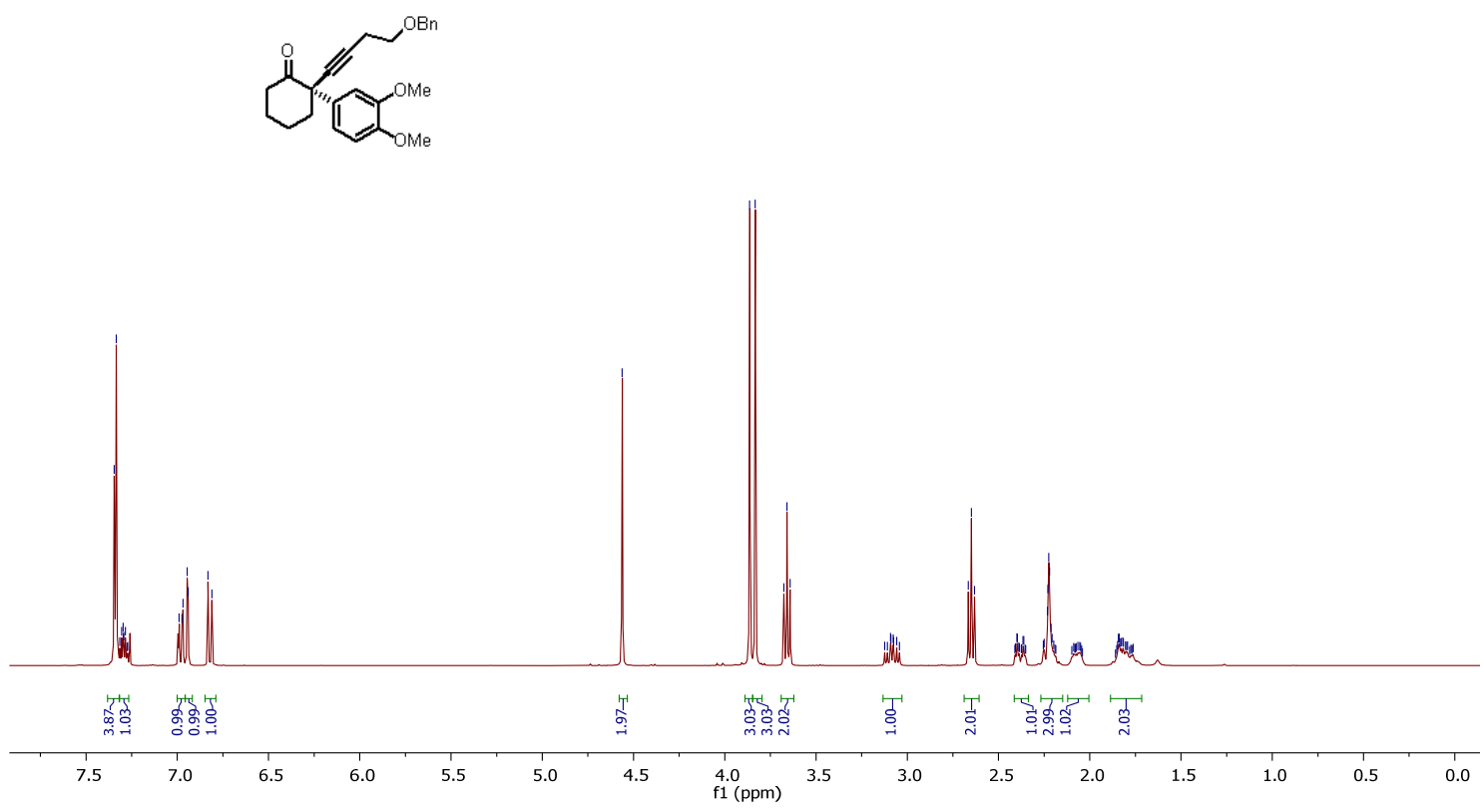

1

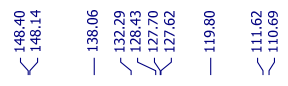

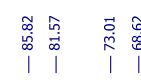

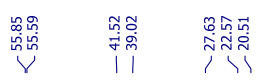
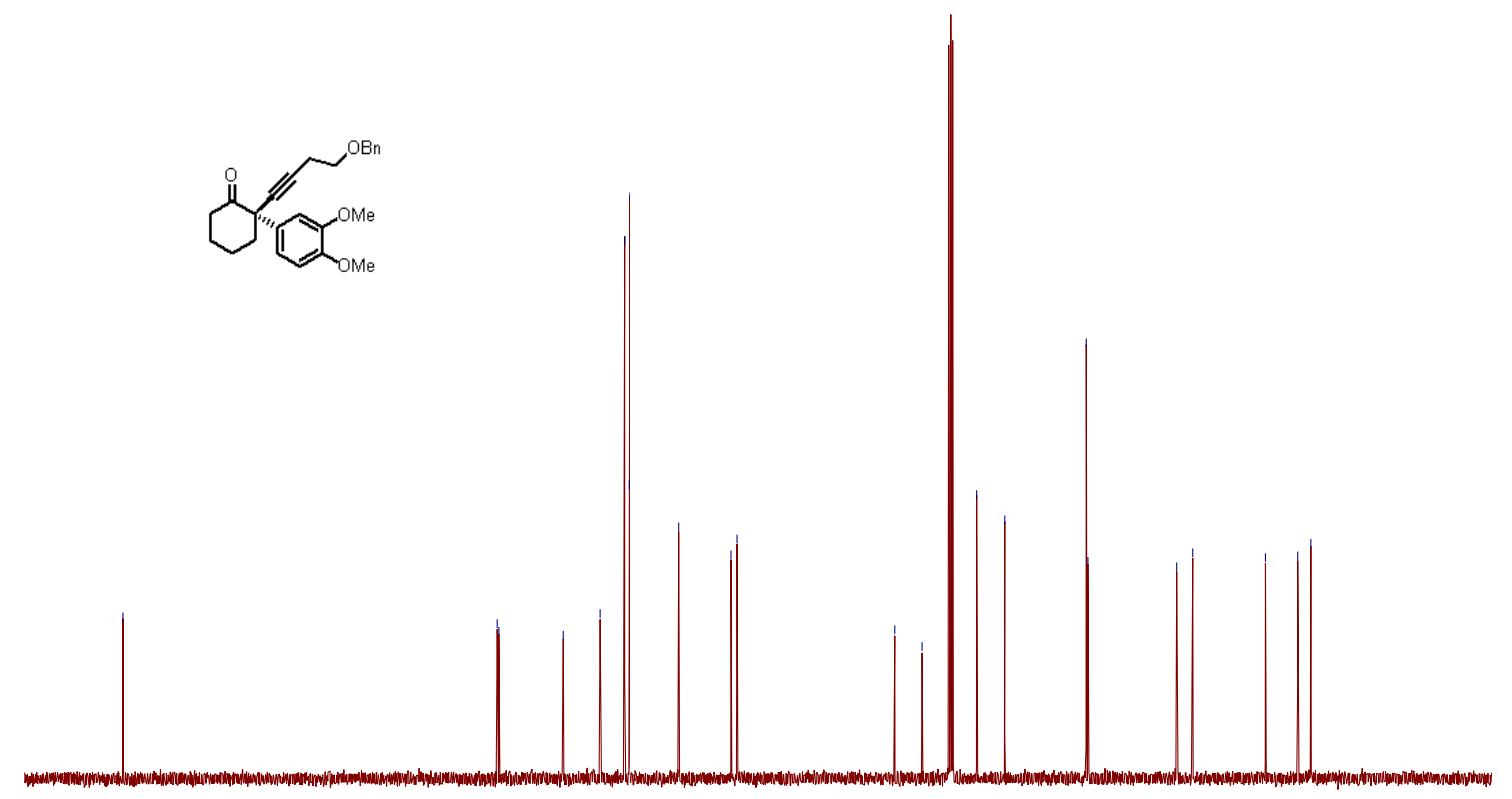

$220 \quad 210 \quad 200$ 
$1 \mathrm{q}$
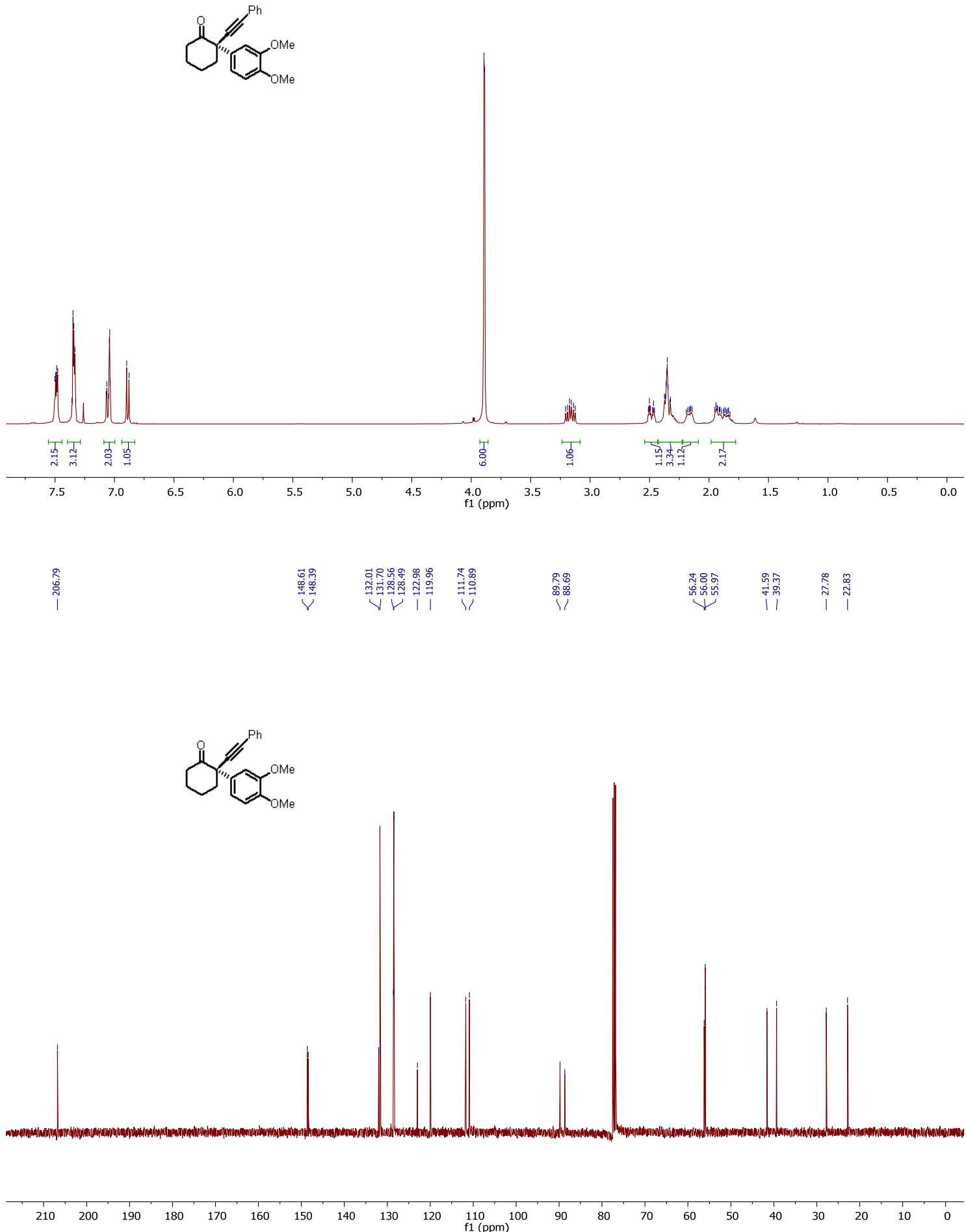


\section{$1 \mathrm{r}$}
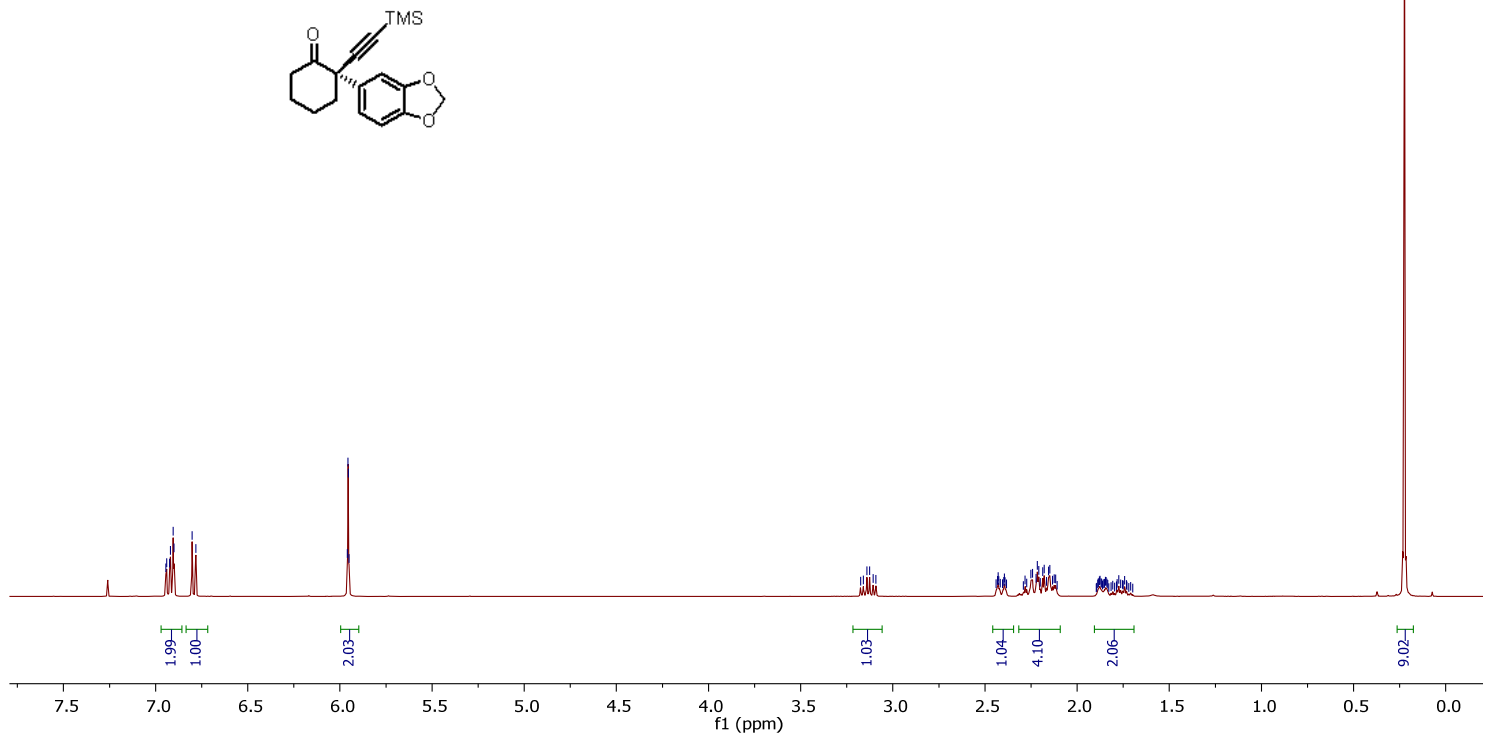

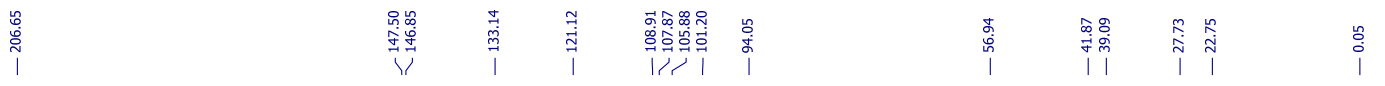

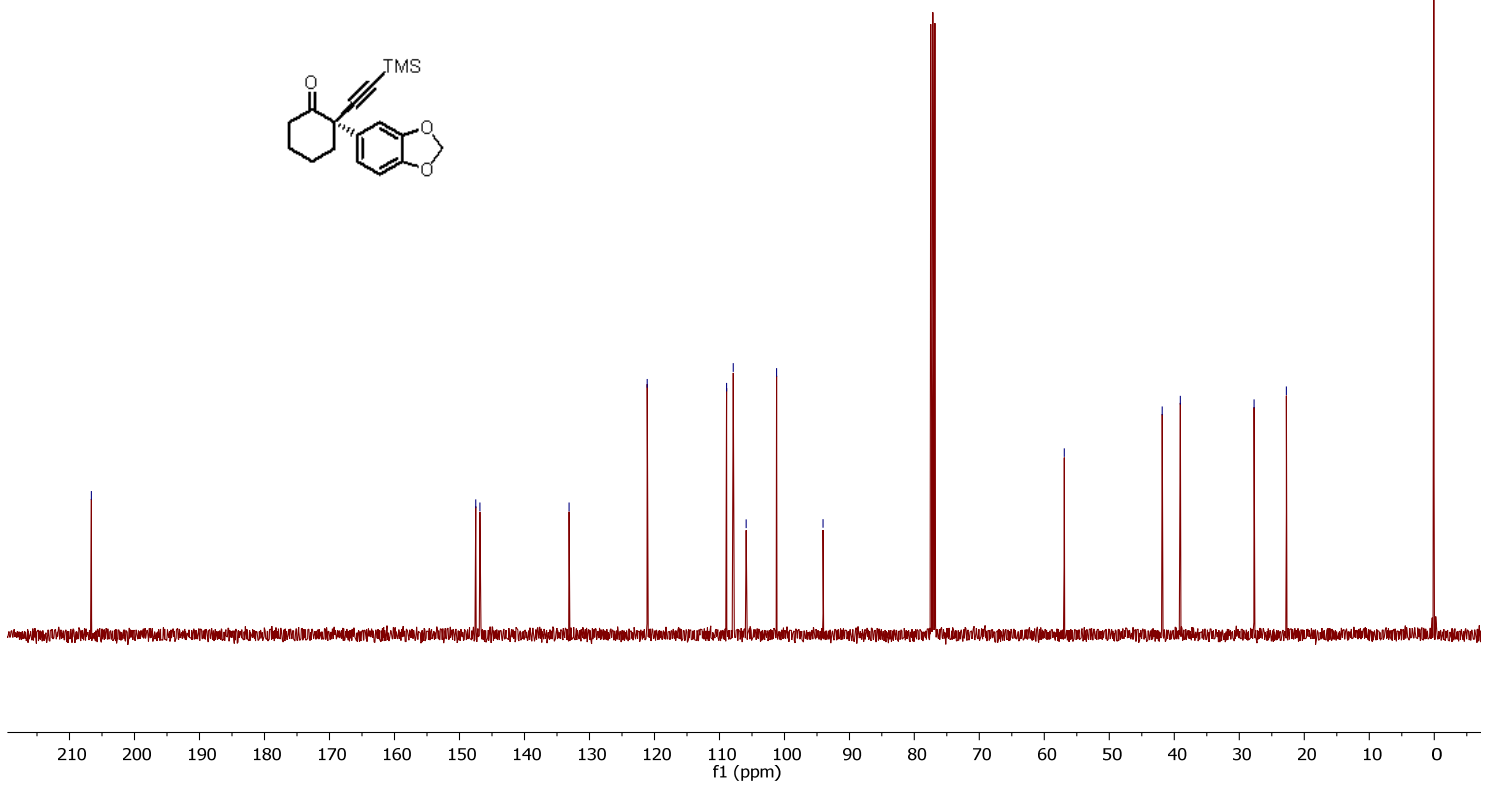




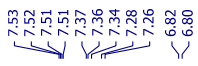

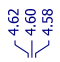

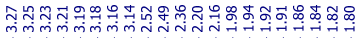

$1 \mathrm{~s}$<smiles>O=C1CCCCC1(C=Cc1ccccc1)Cc1ccccc1</smiles>

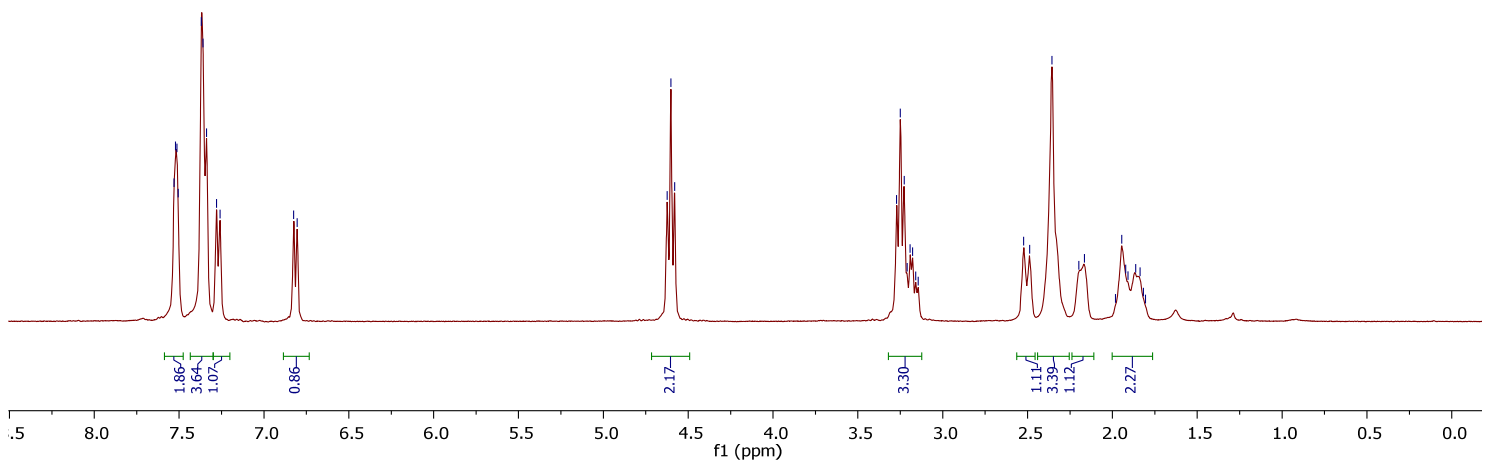

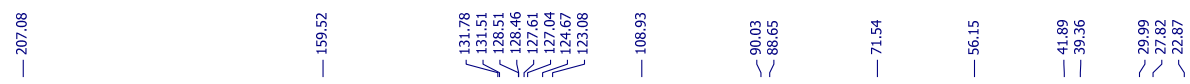

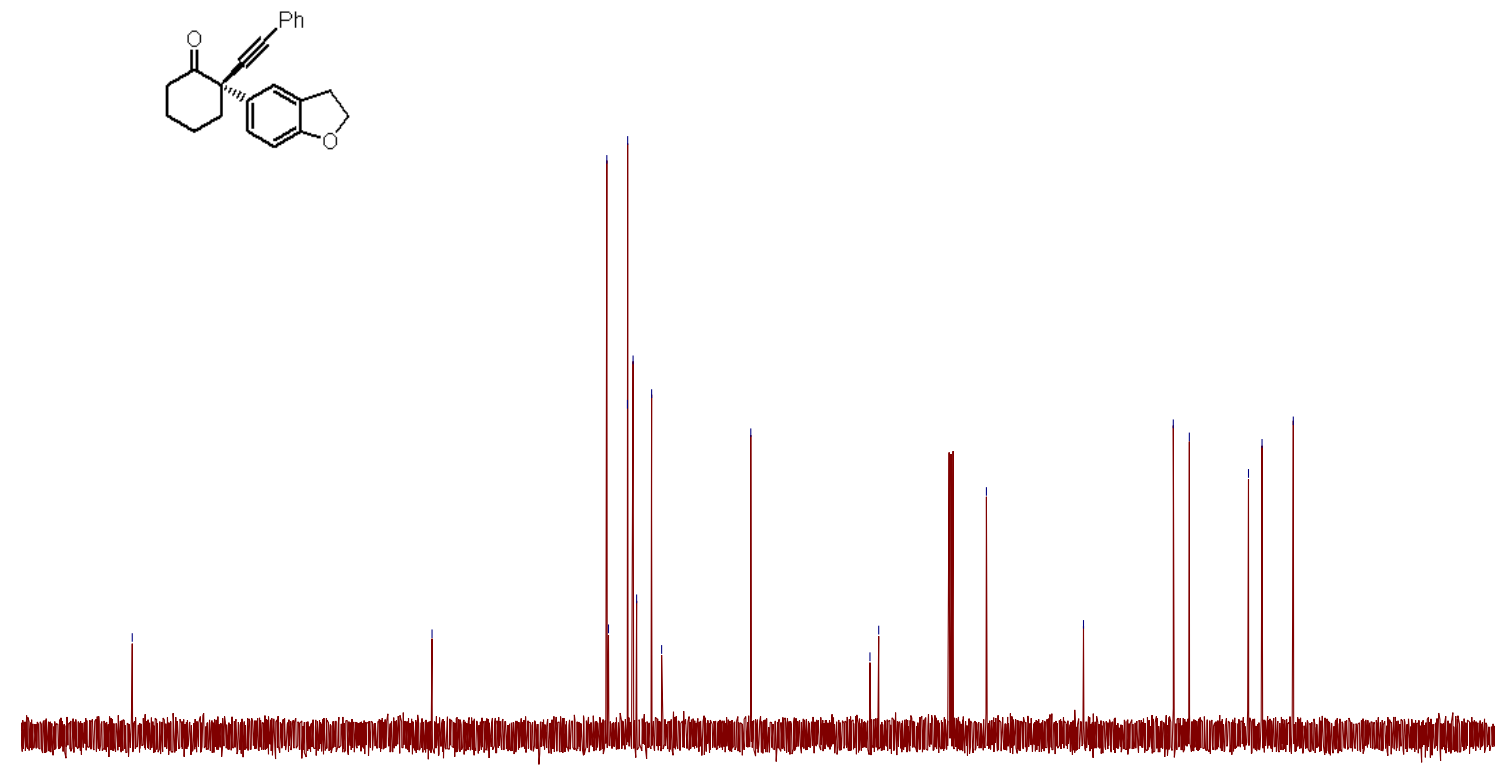

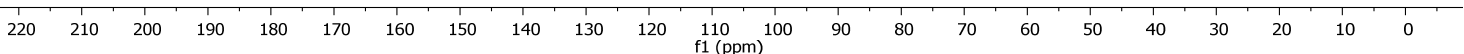




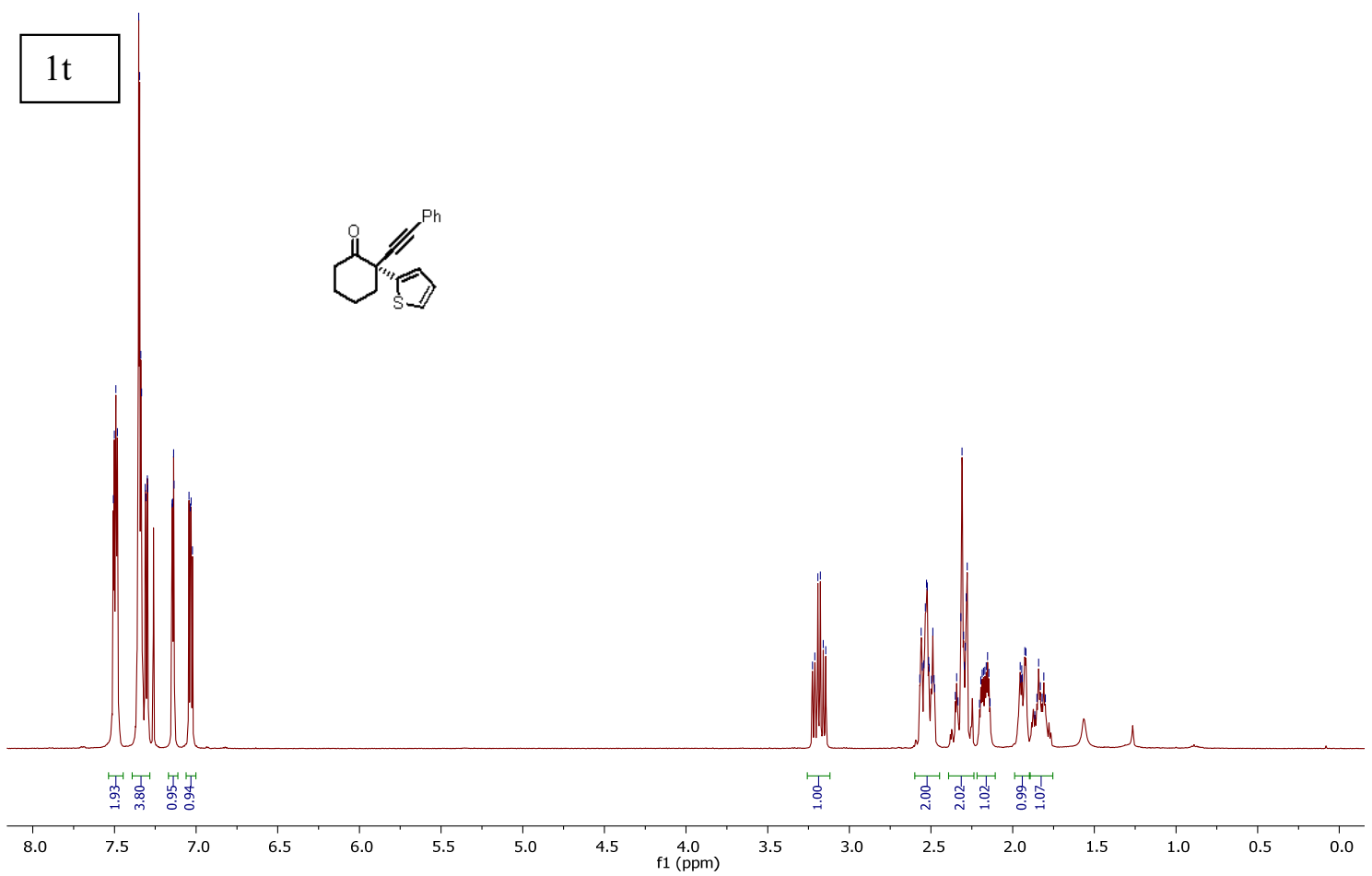

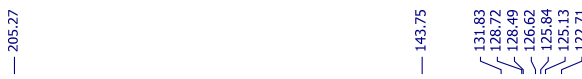
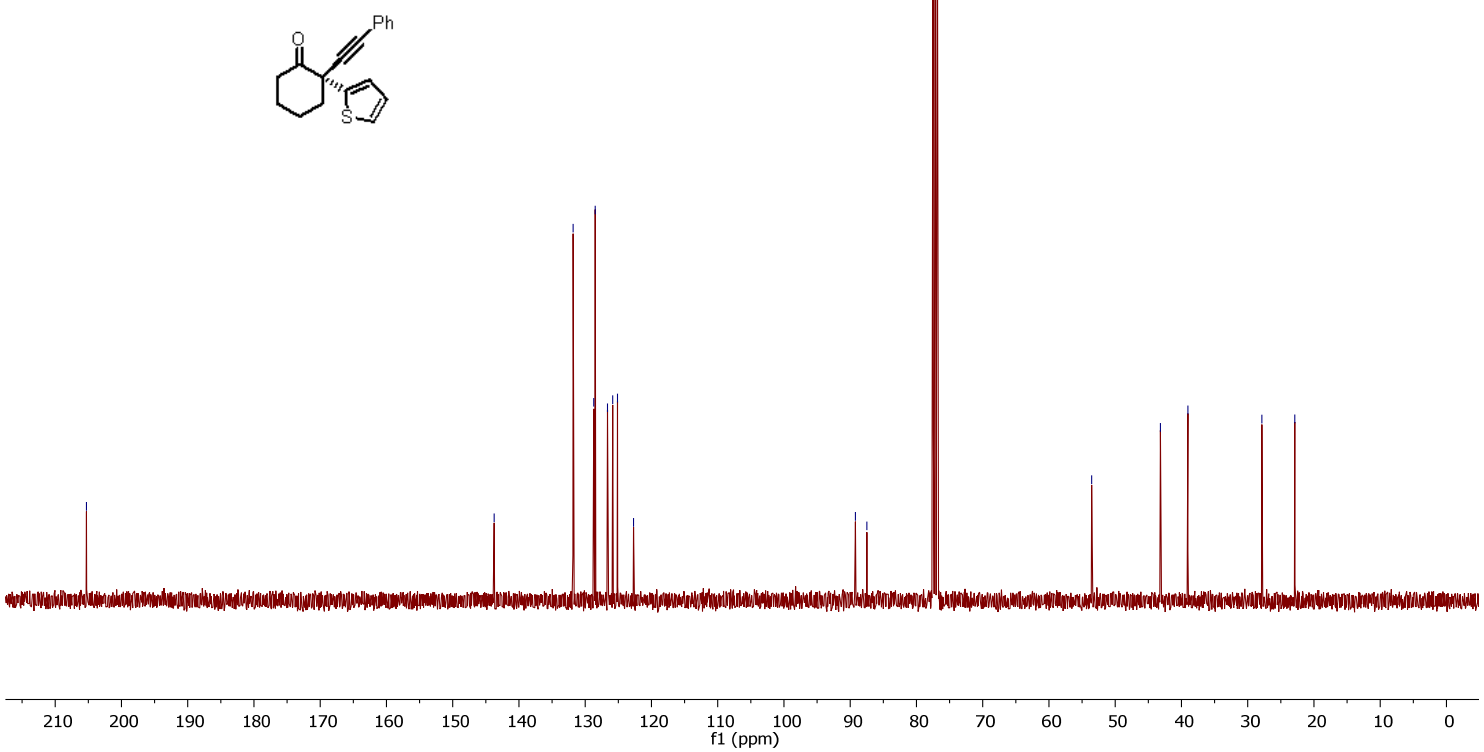


\section{$1 \mathrm{u}$}<smiles>O=C(O)C1C2C=CC(C2)C1C(=O)c1ccccc1</smiles>

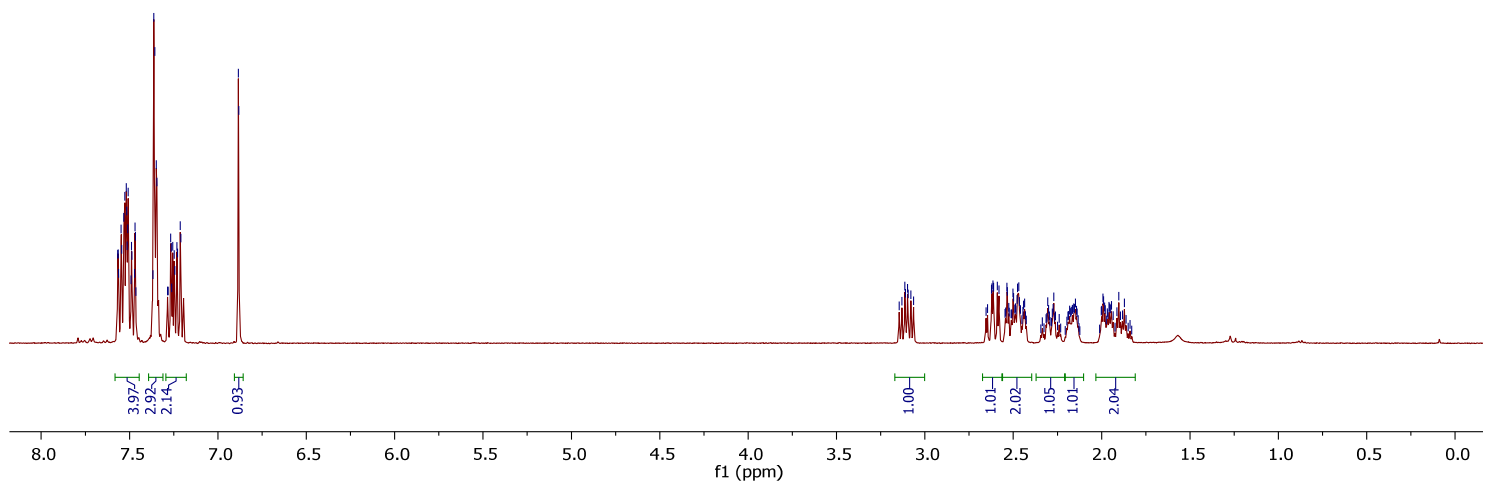

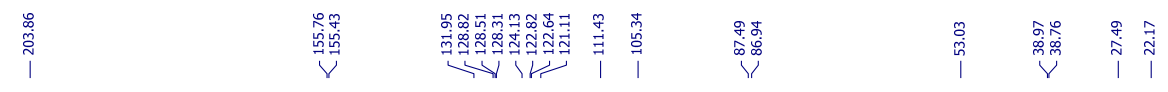

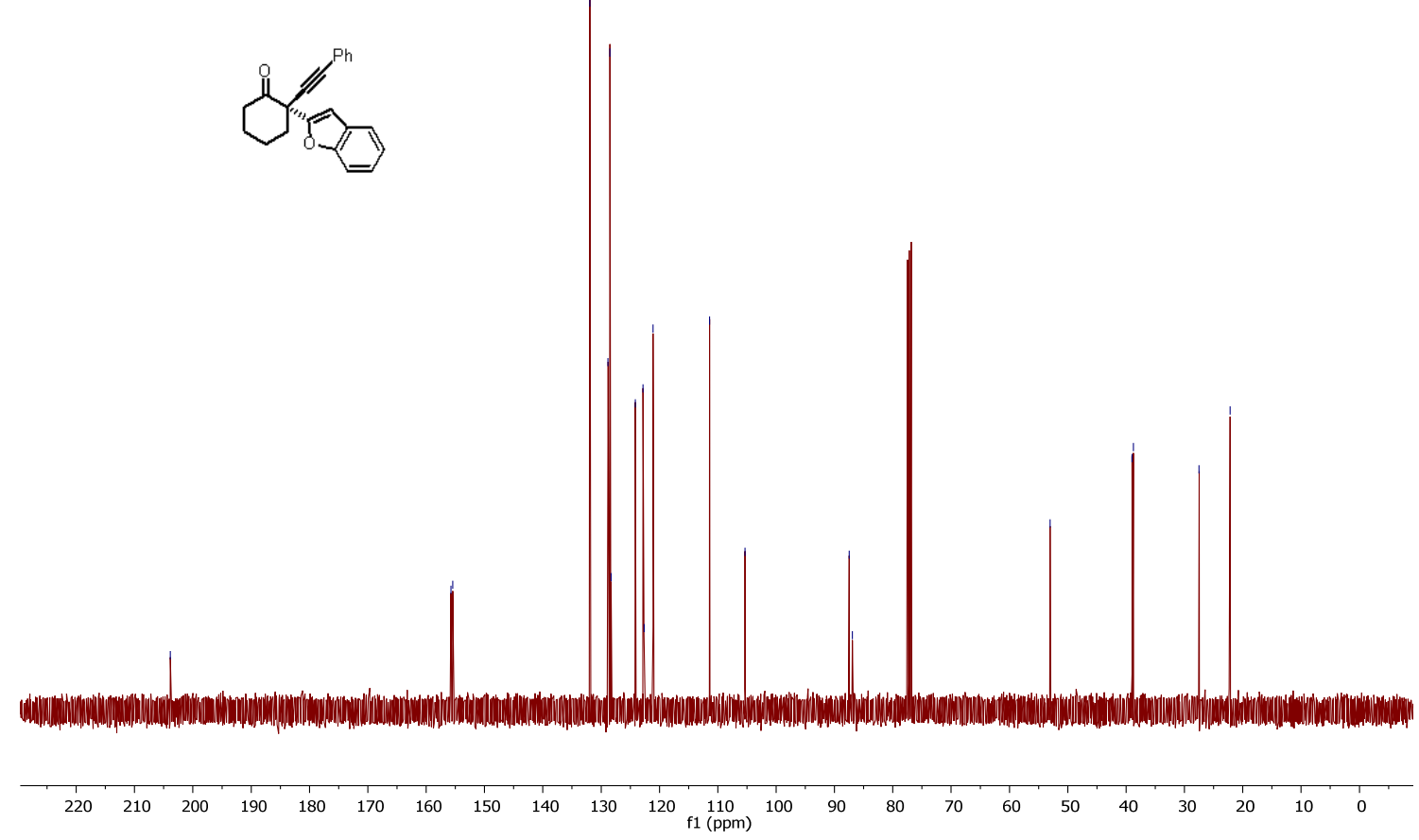




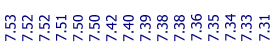

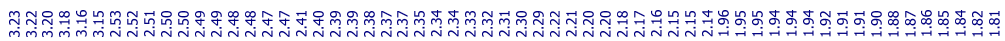

1w
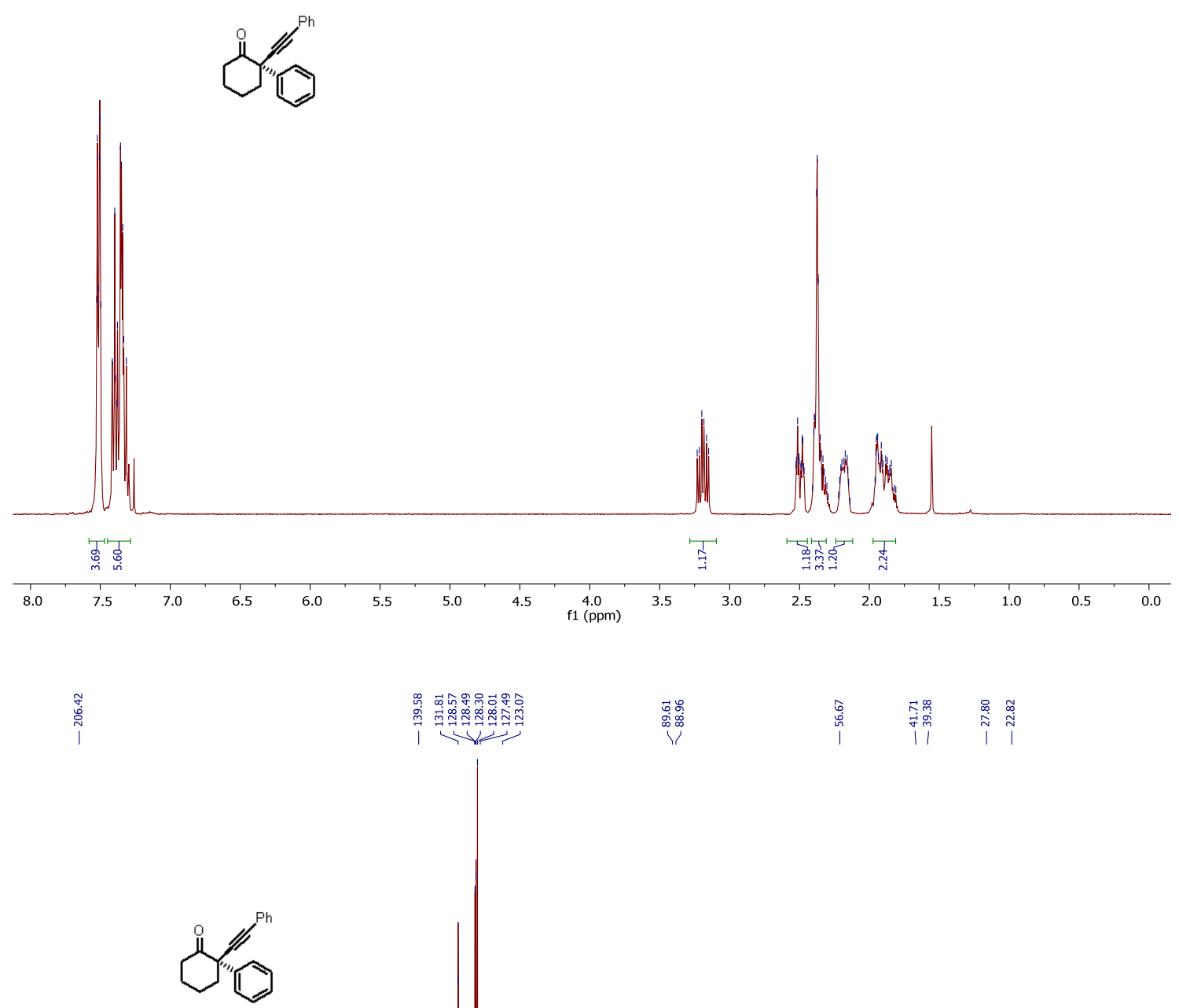

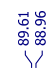

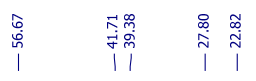
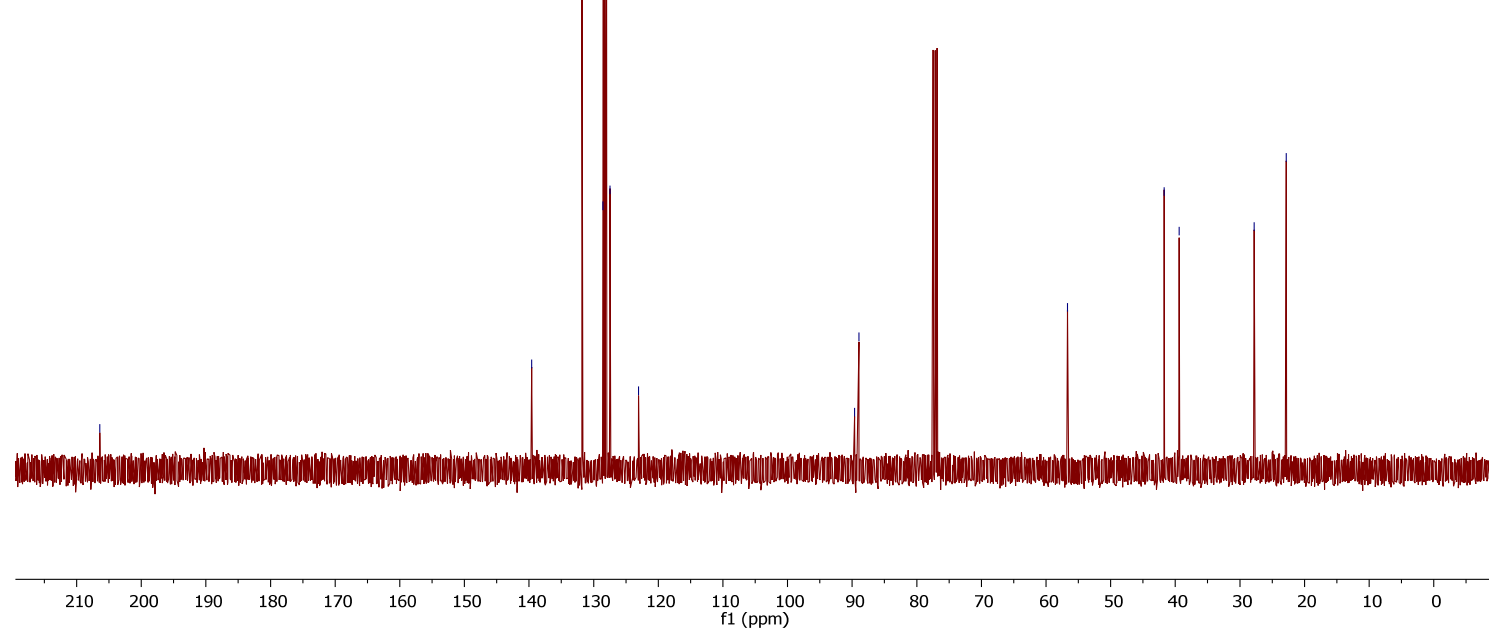

S84 


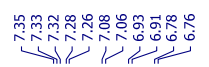

$\stackrel{\mathscr{R}}{0}$

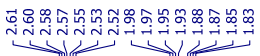

$2 \mathrm{a}$
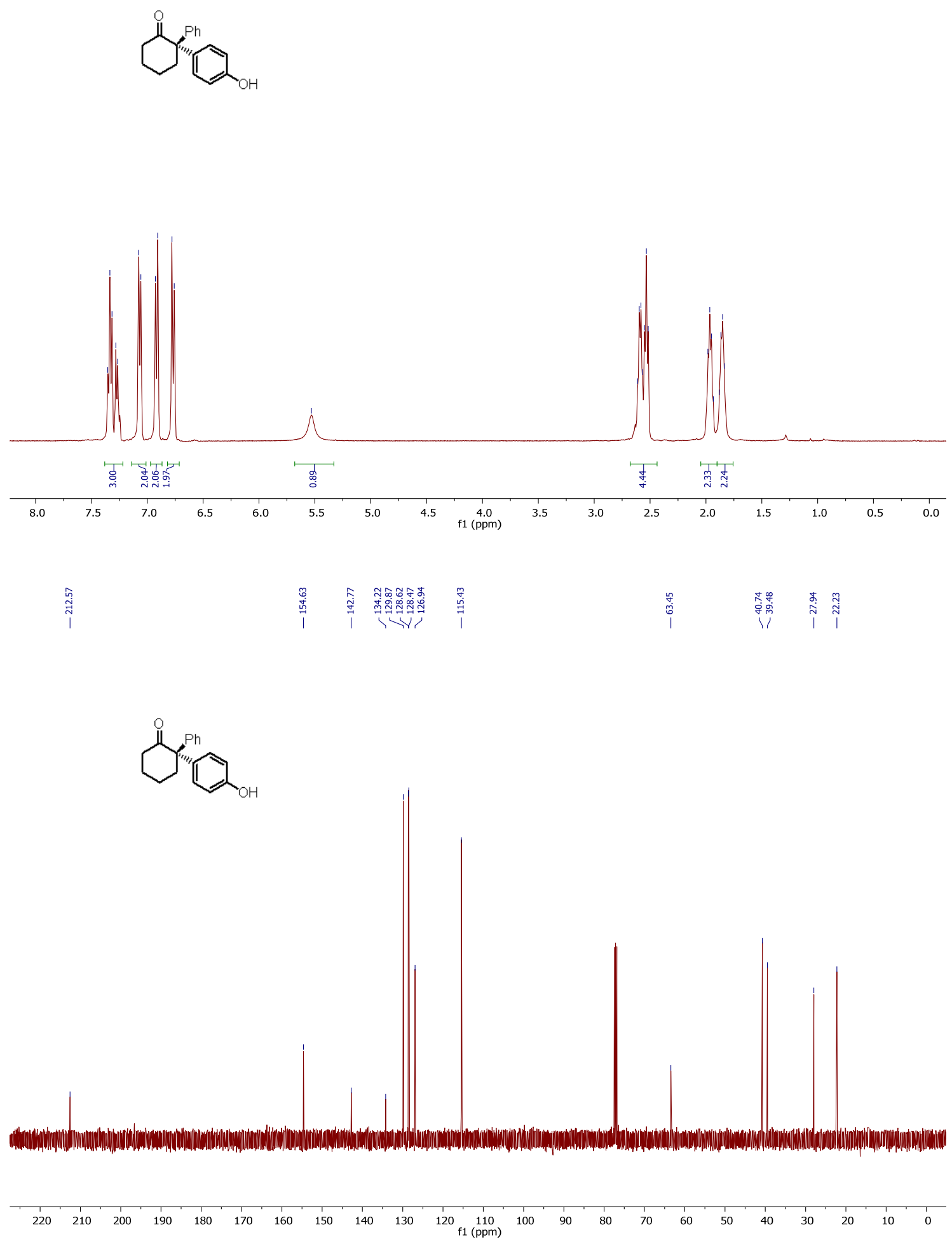

S85 

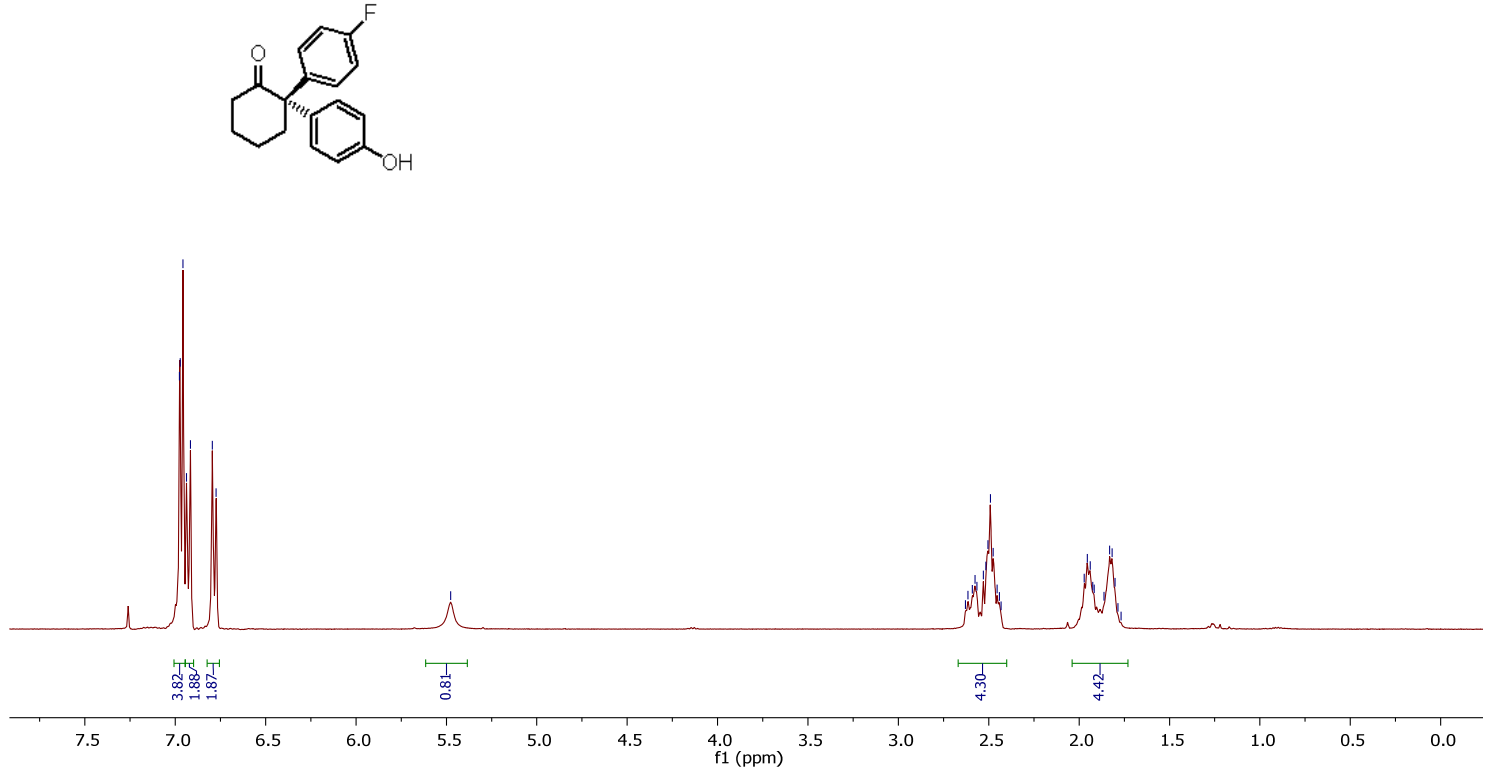

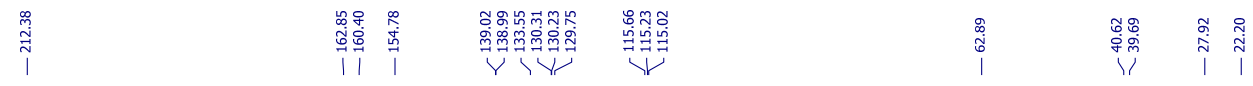

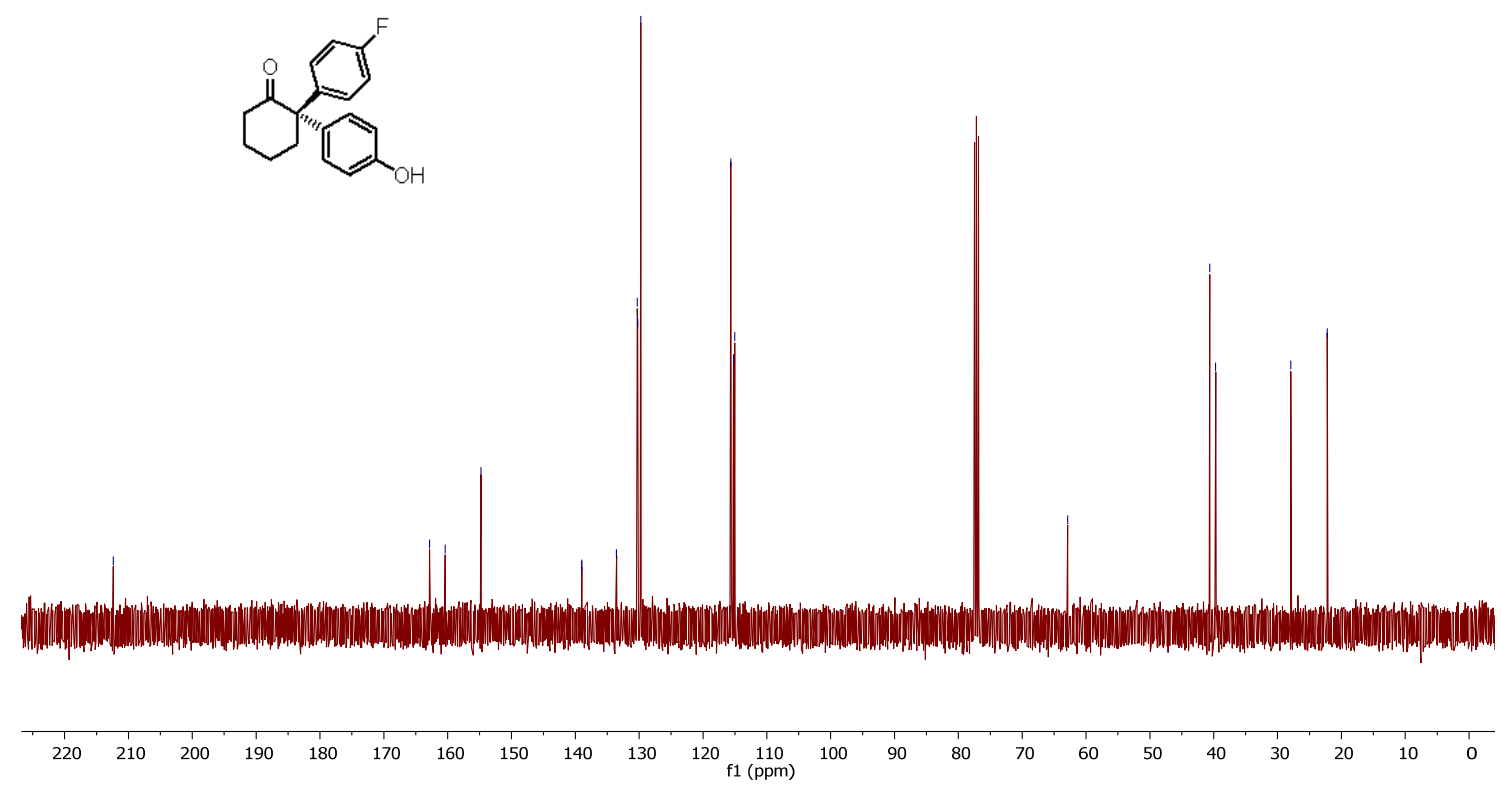


2c
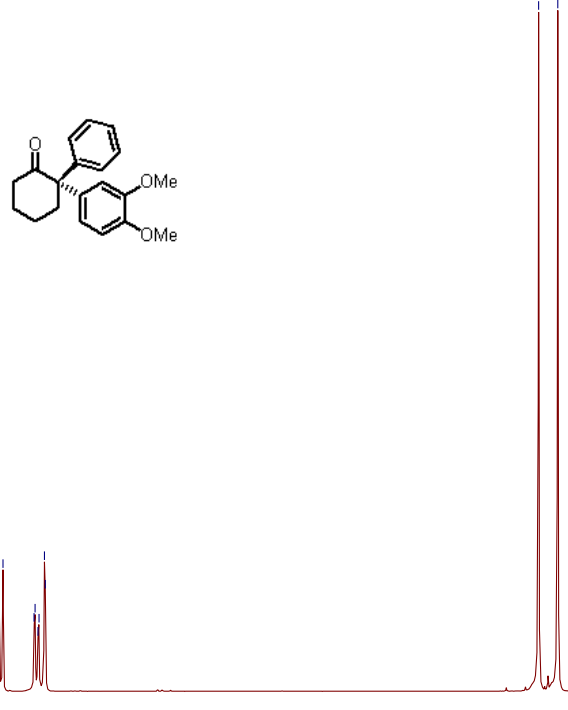

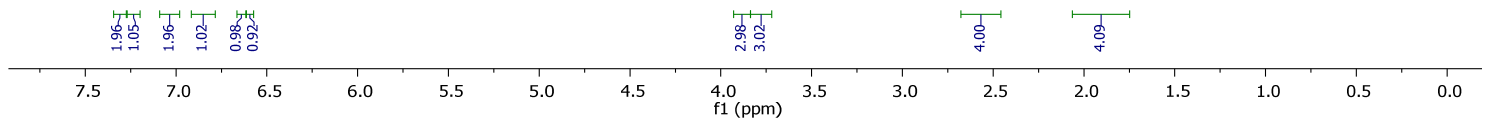

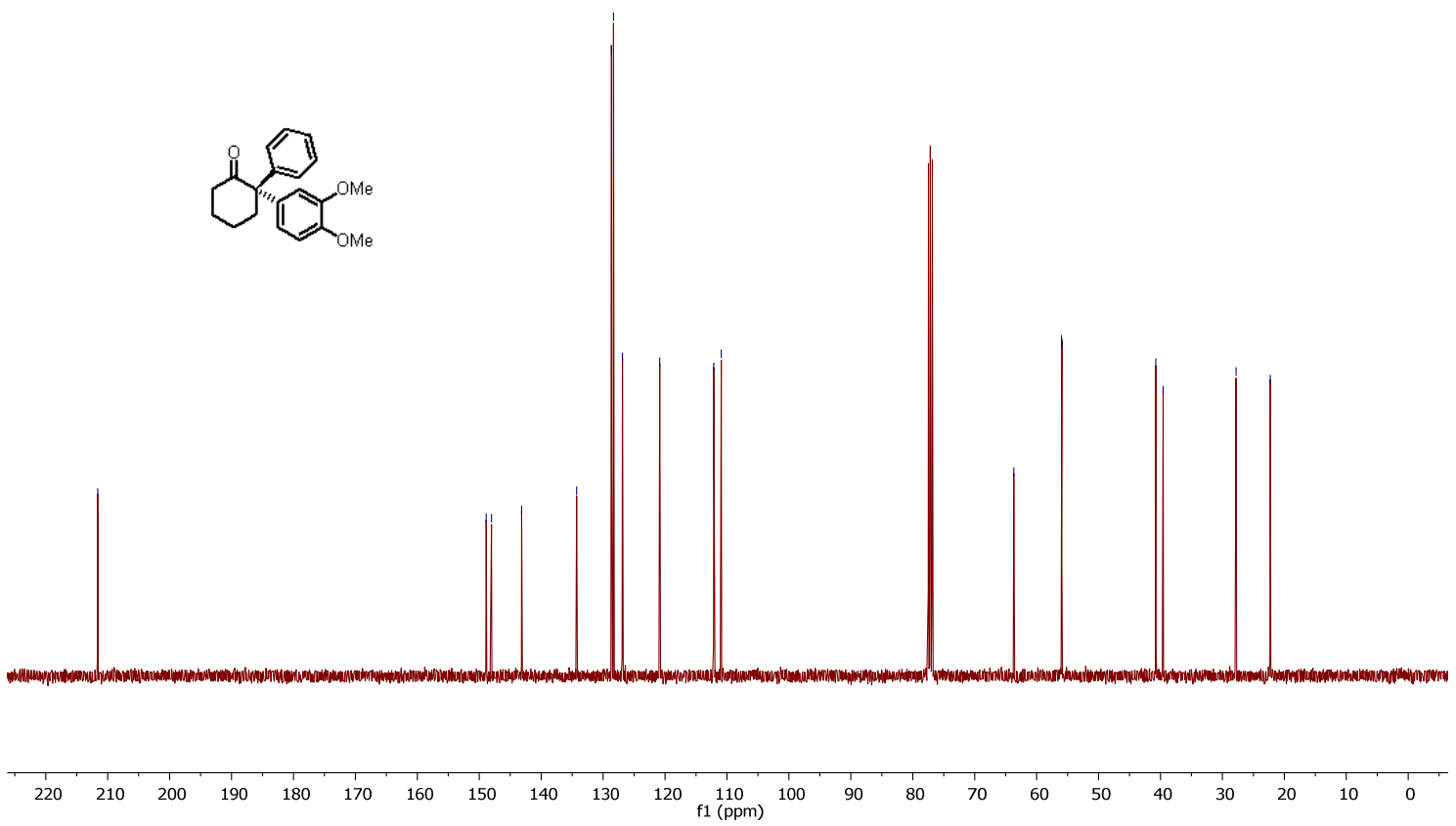




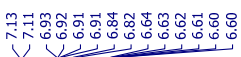

$2 \mathrm{~d}$

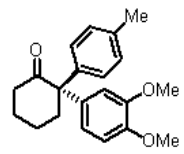

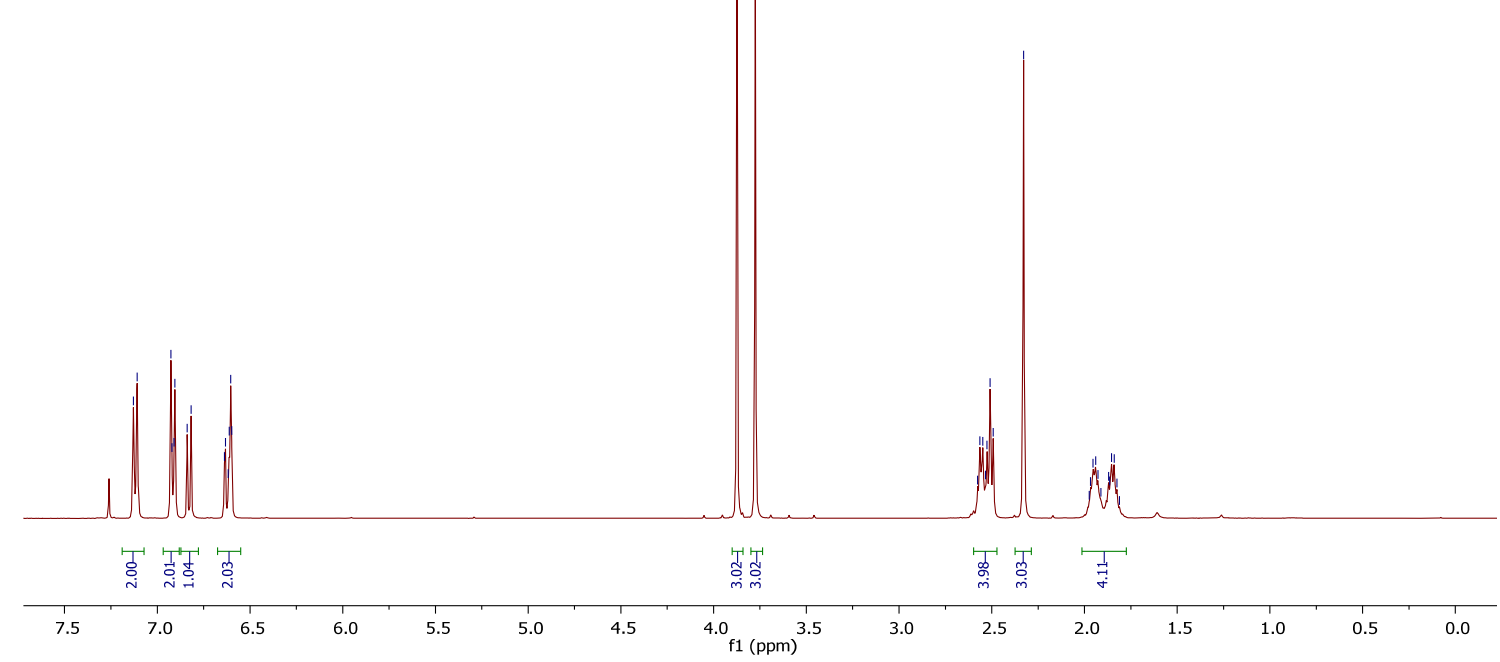

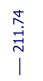

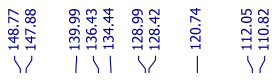

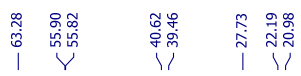
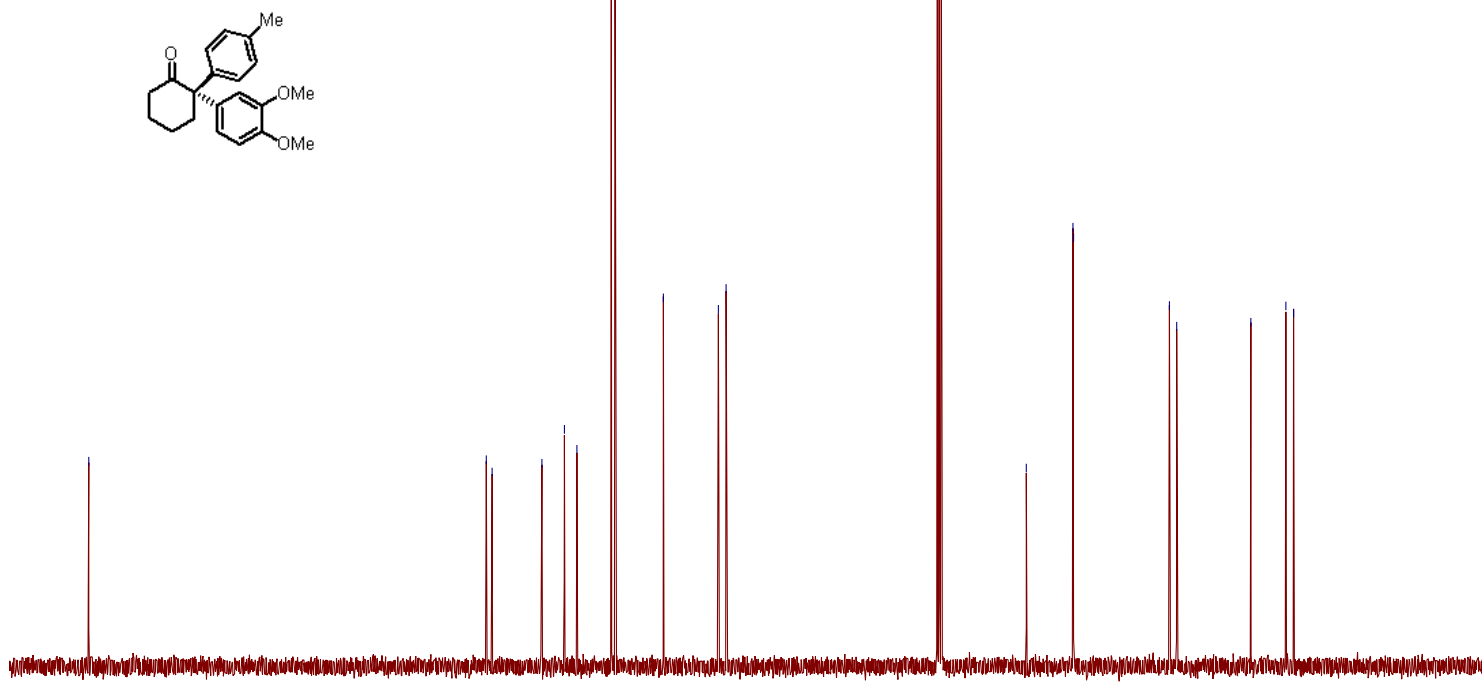

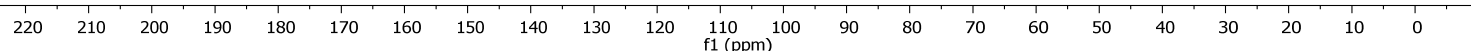




$2 \mathrm{e}$
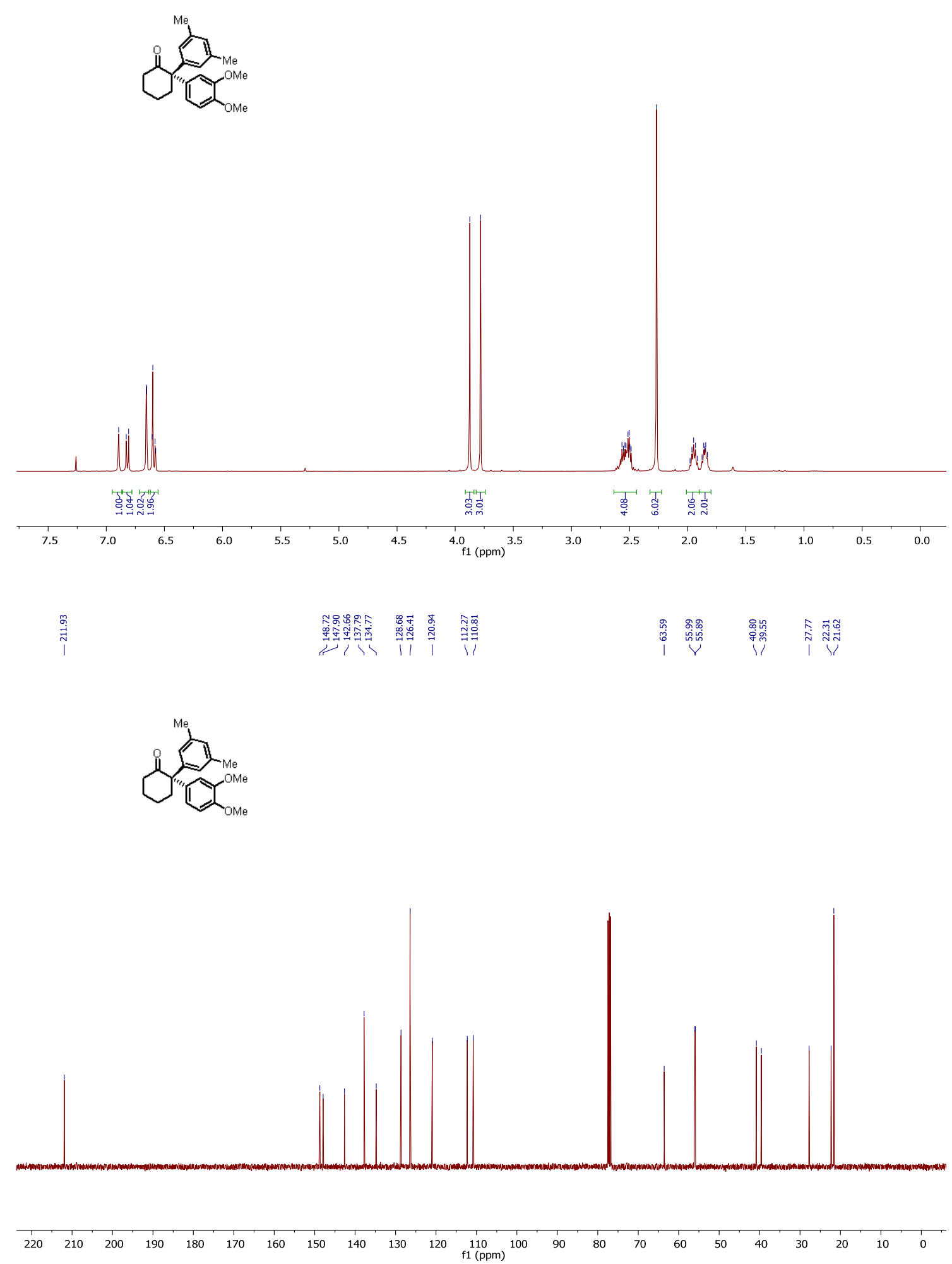


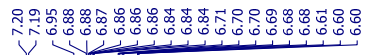

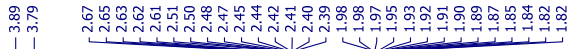

$2 f$<smiles>Cc1ccc(C2(c3ccc(Cl)cc3)CCCCC2=O)c(C)c1</smiles>

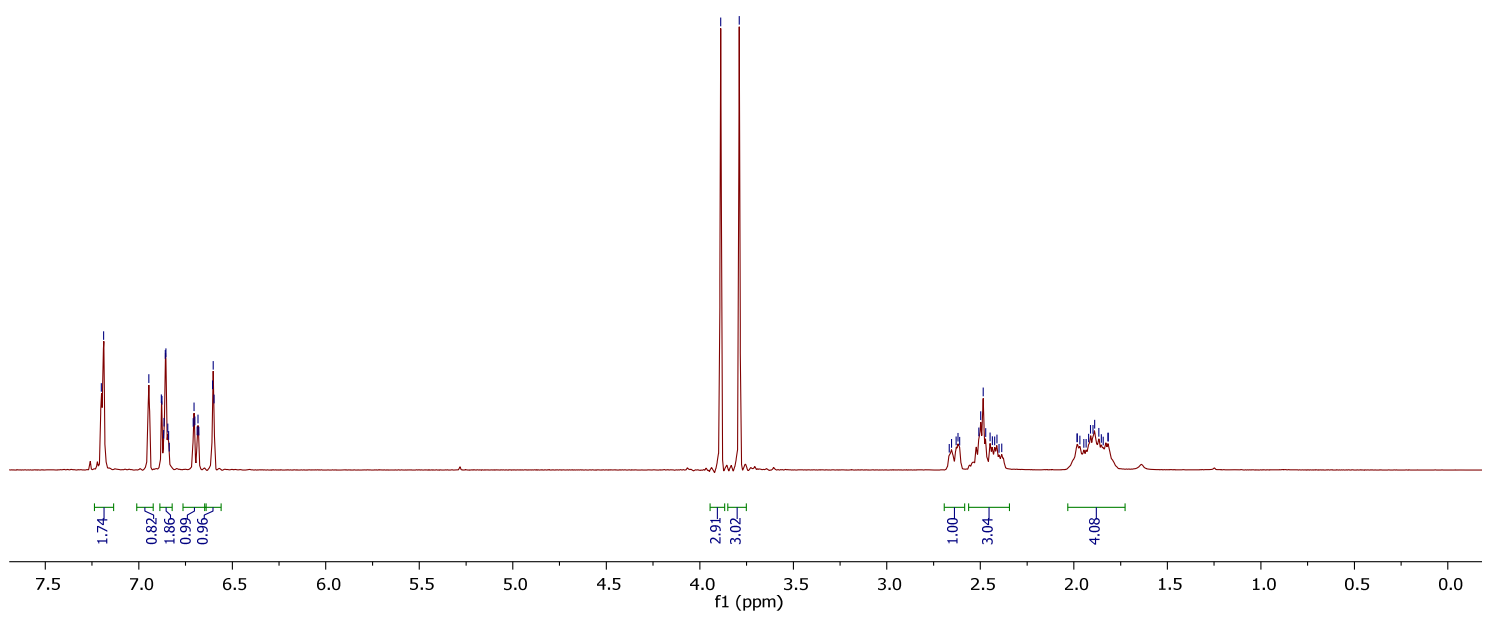

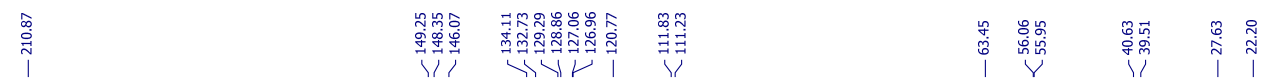
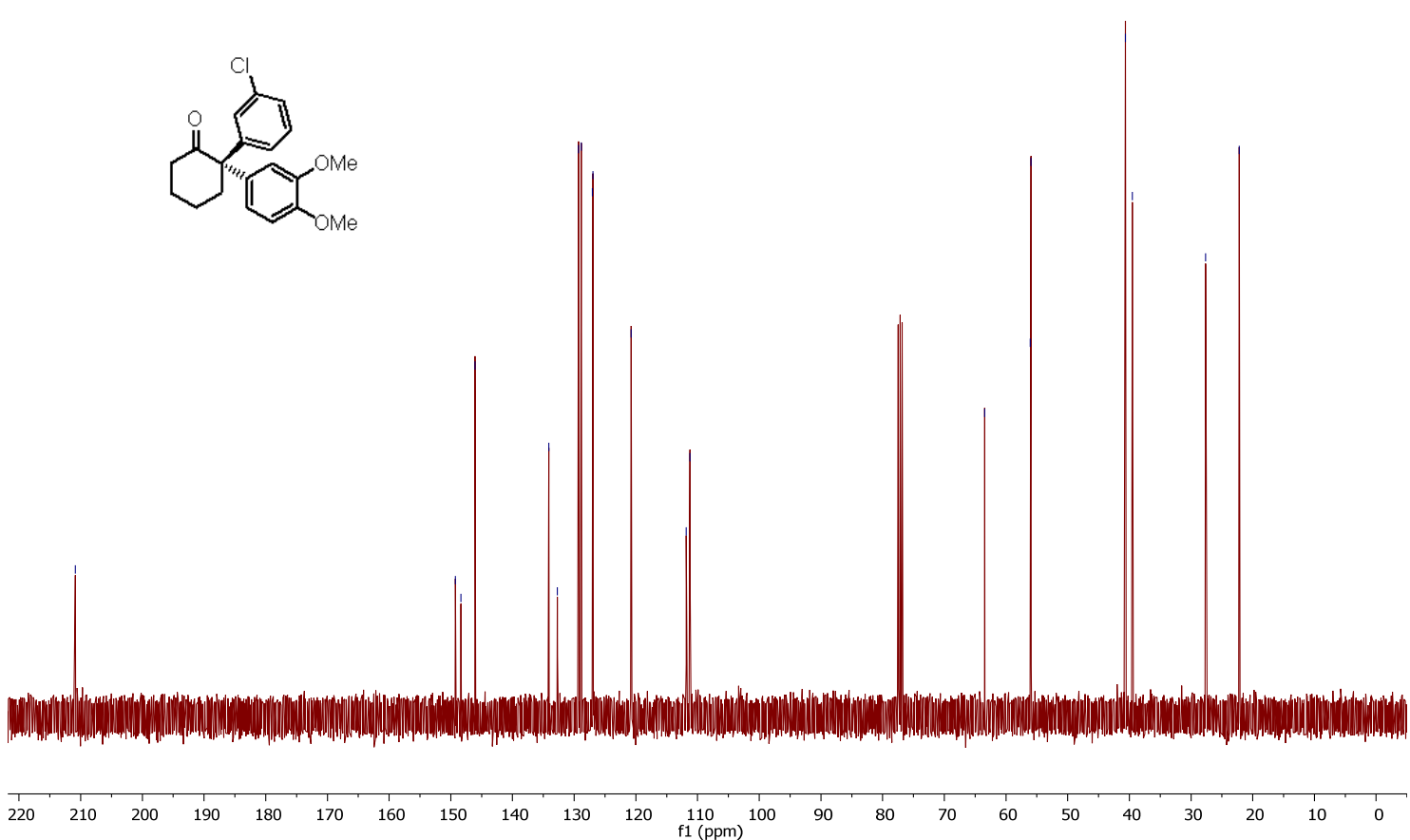


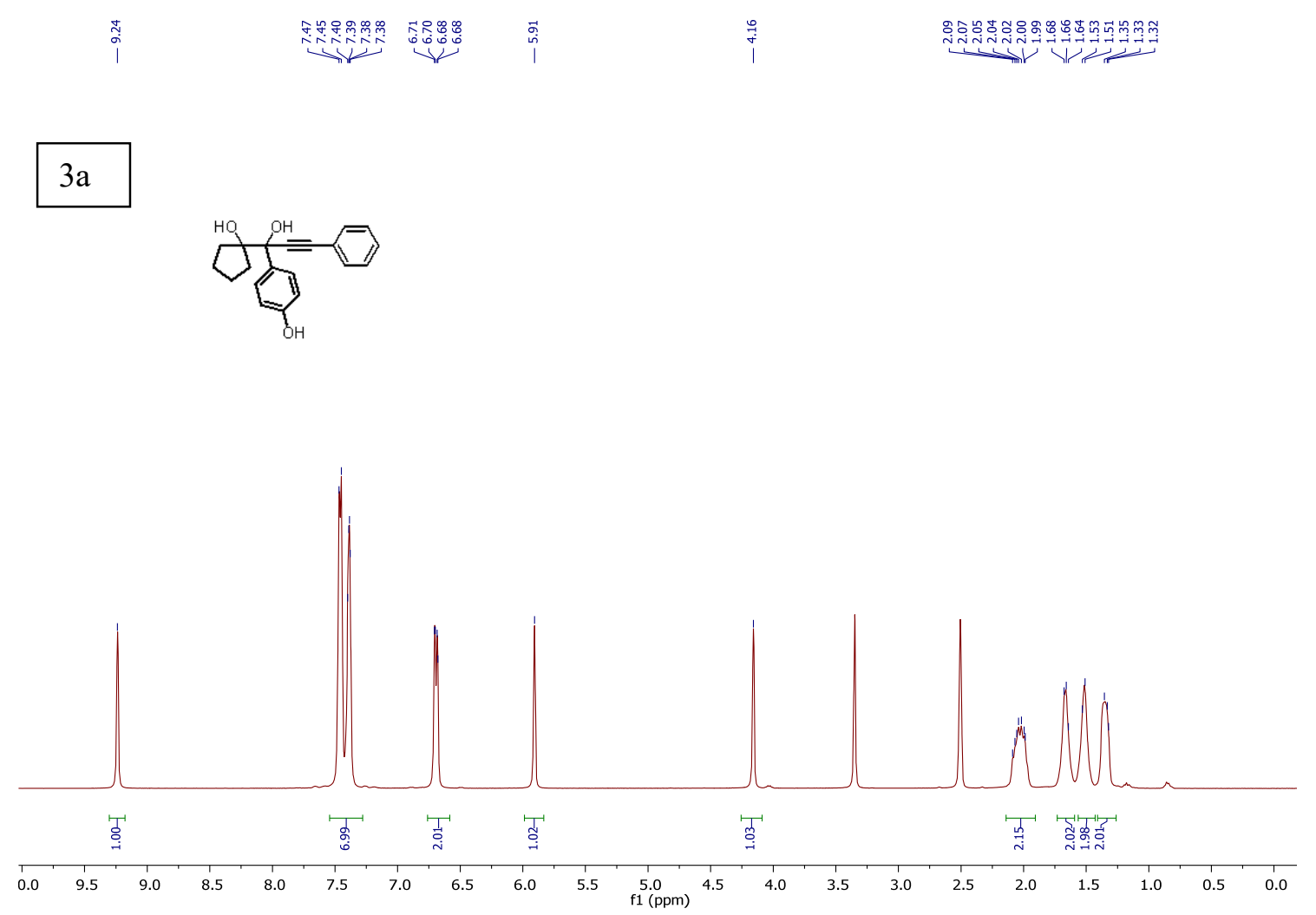

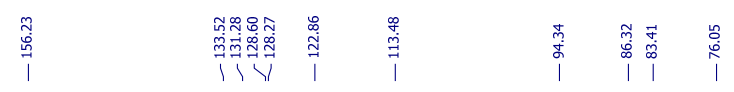
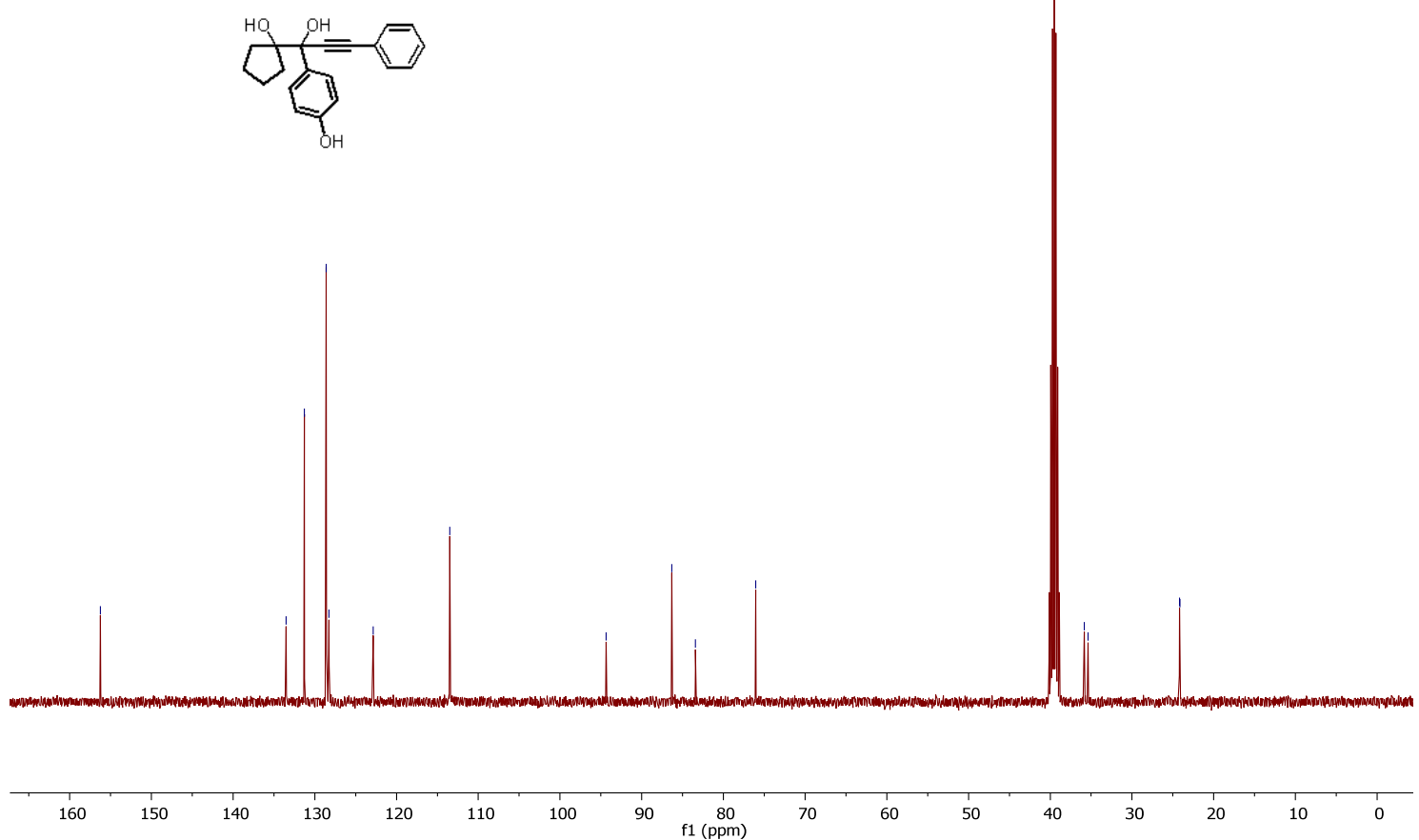

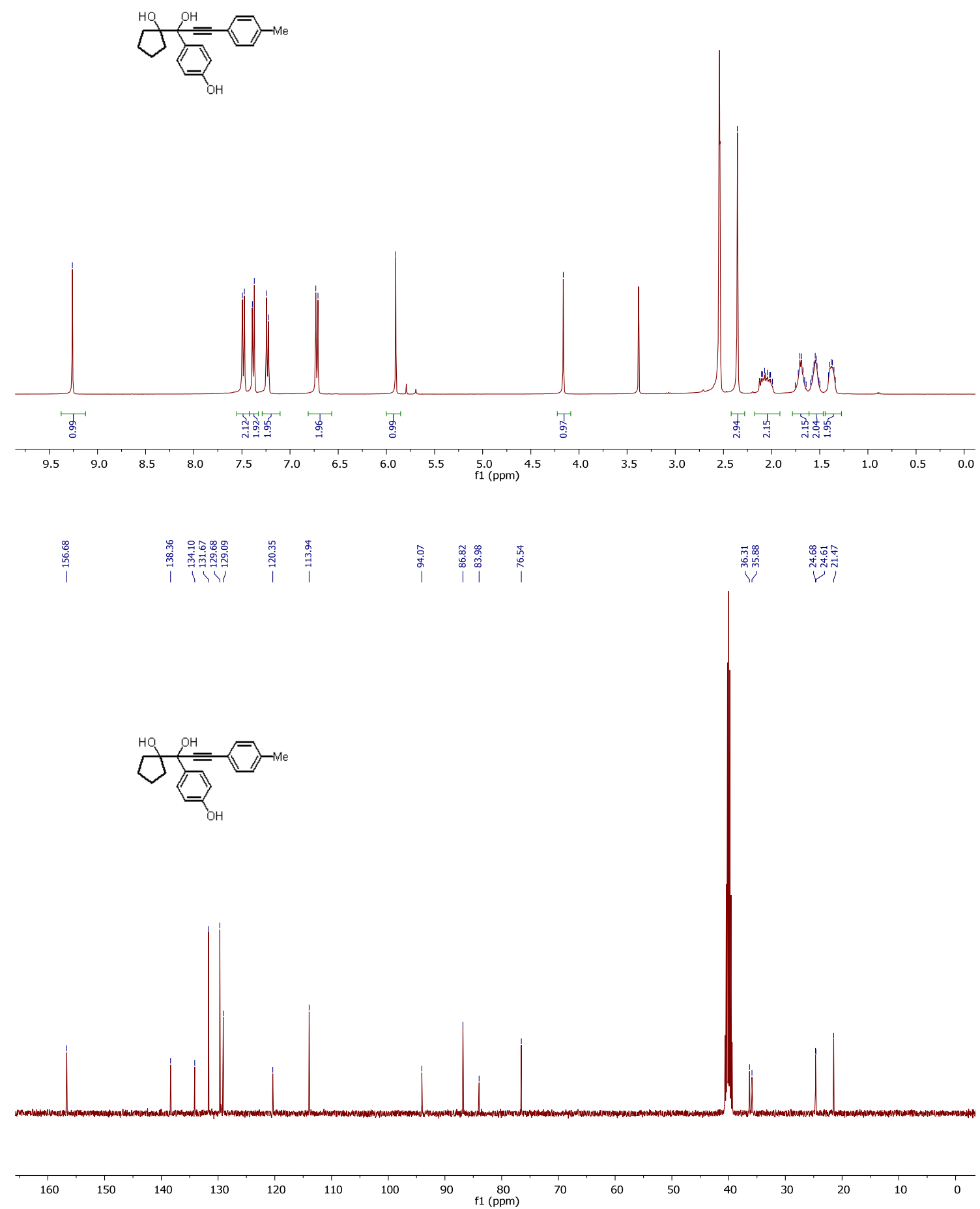


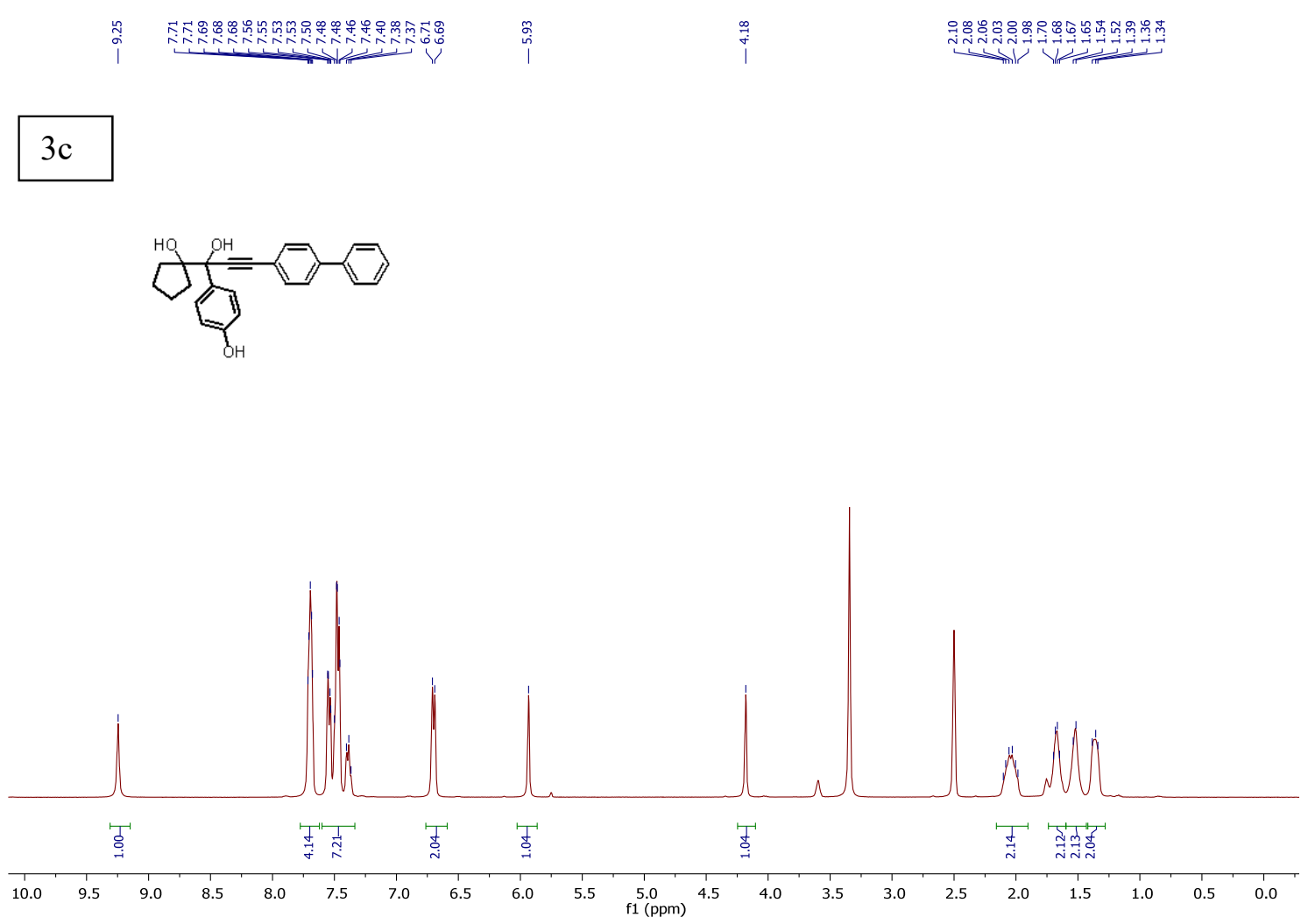

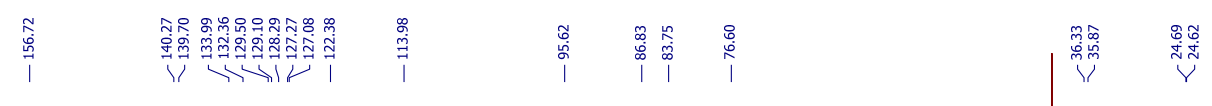
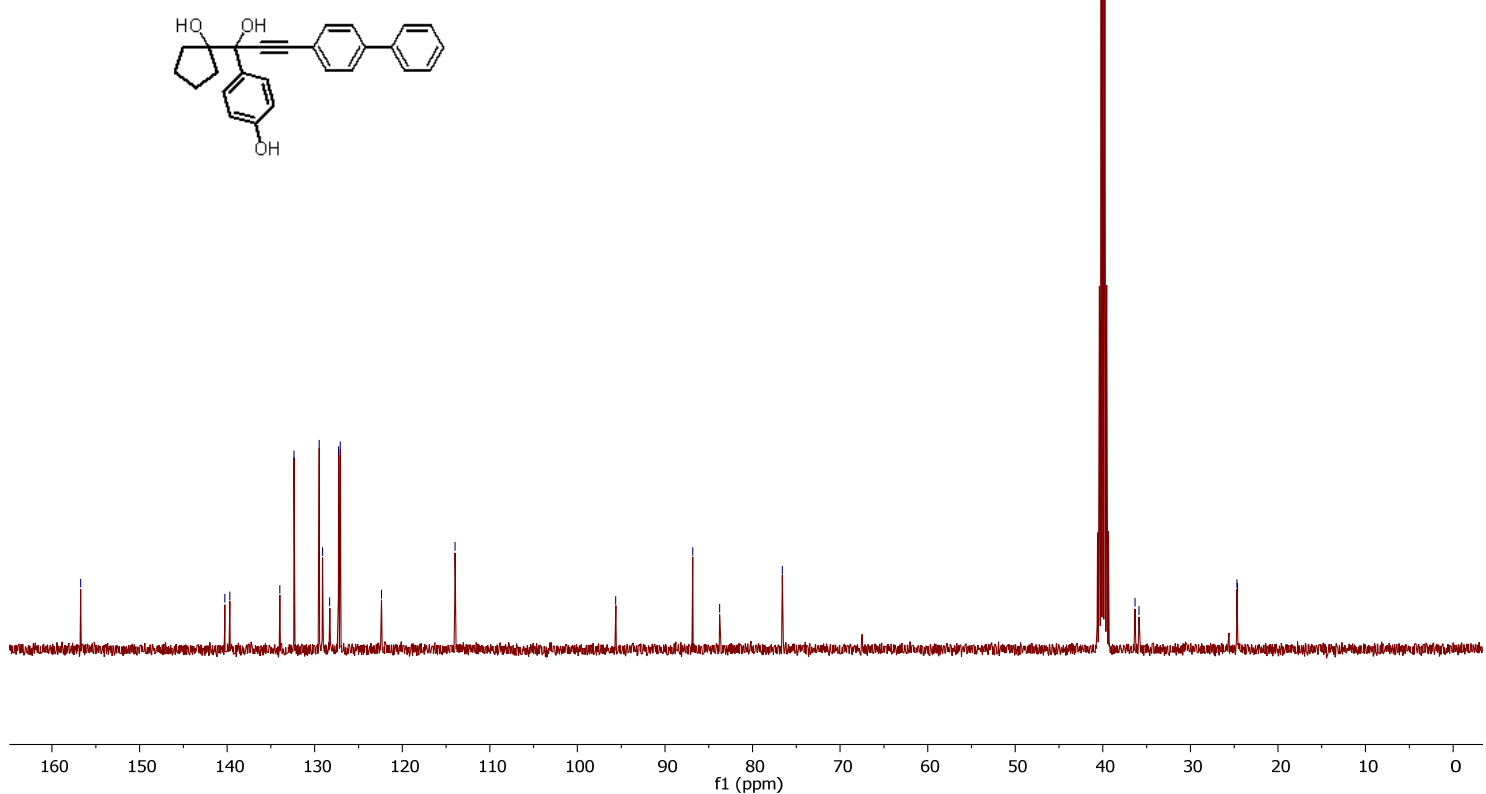


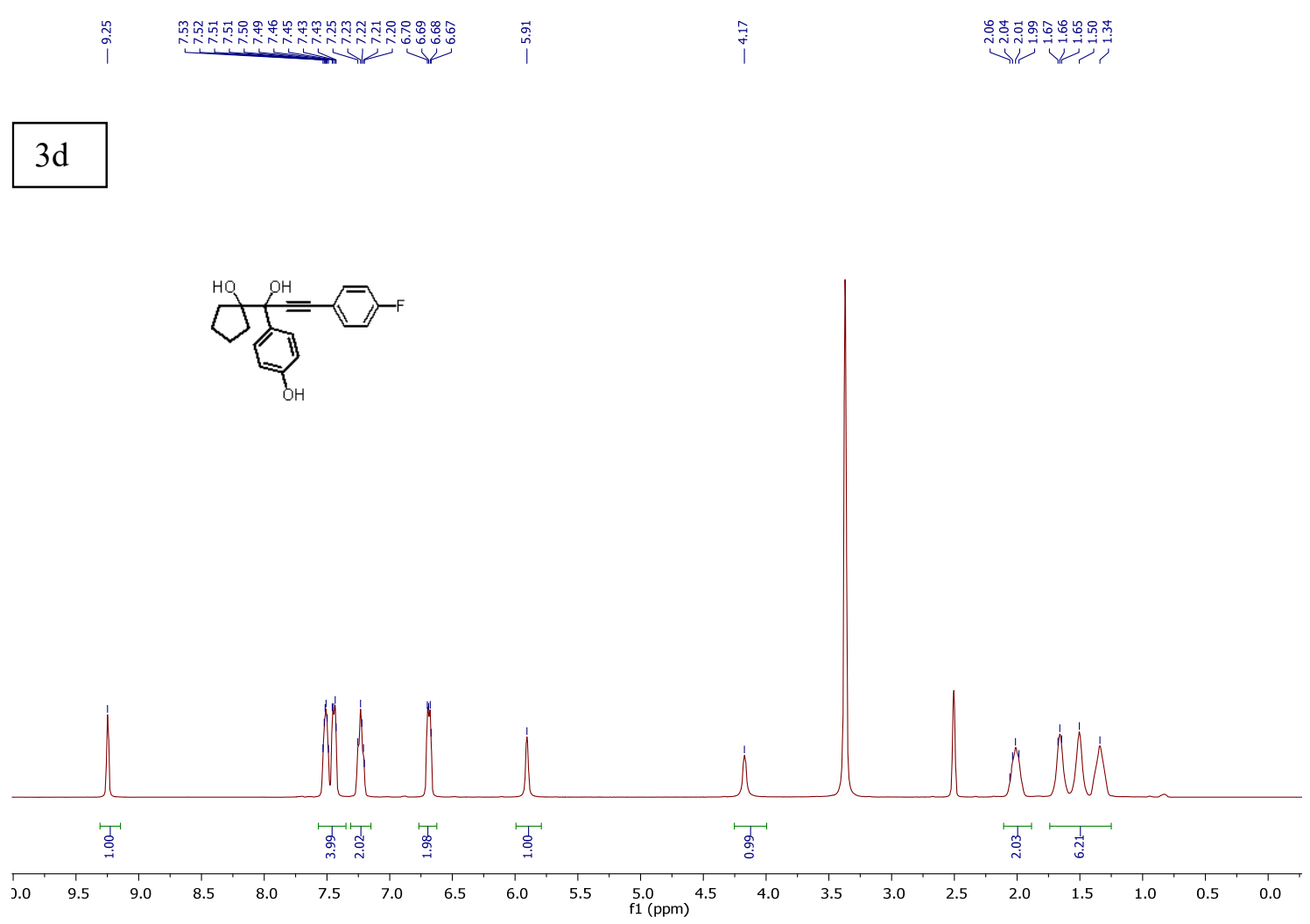

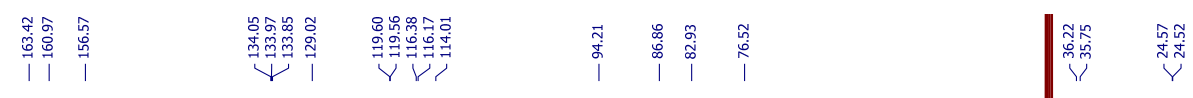<smiles>Oc1ccc(-c2ccc(F)cc2)cc1</smiles>
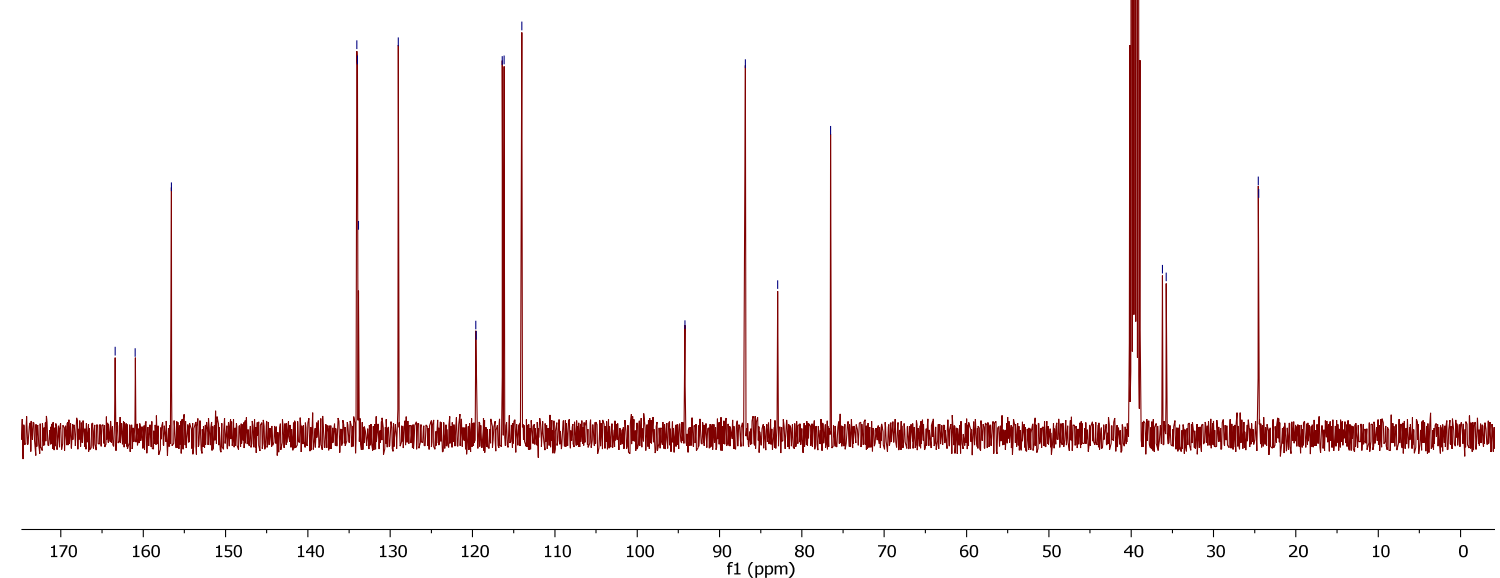

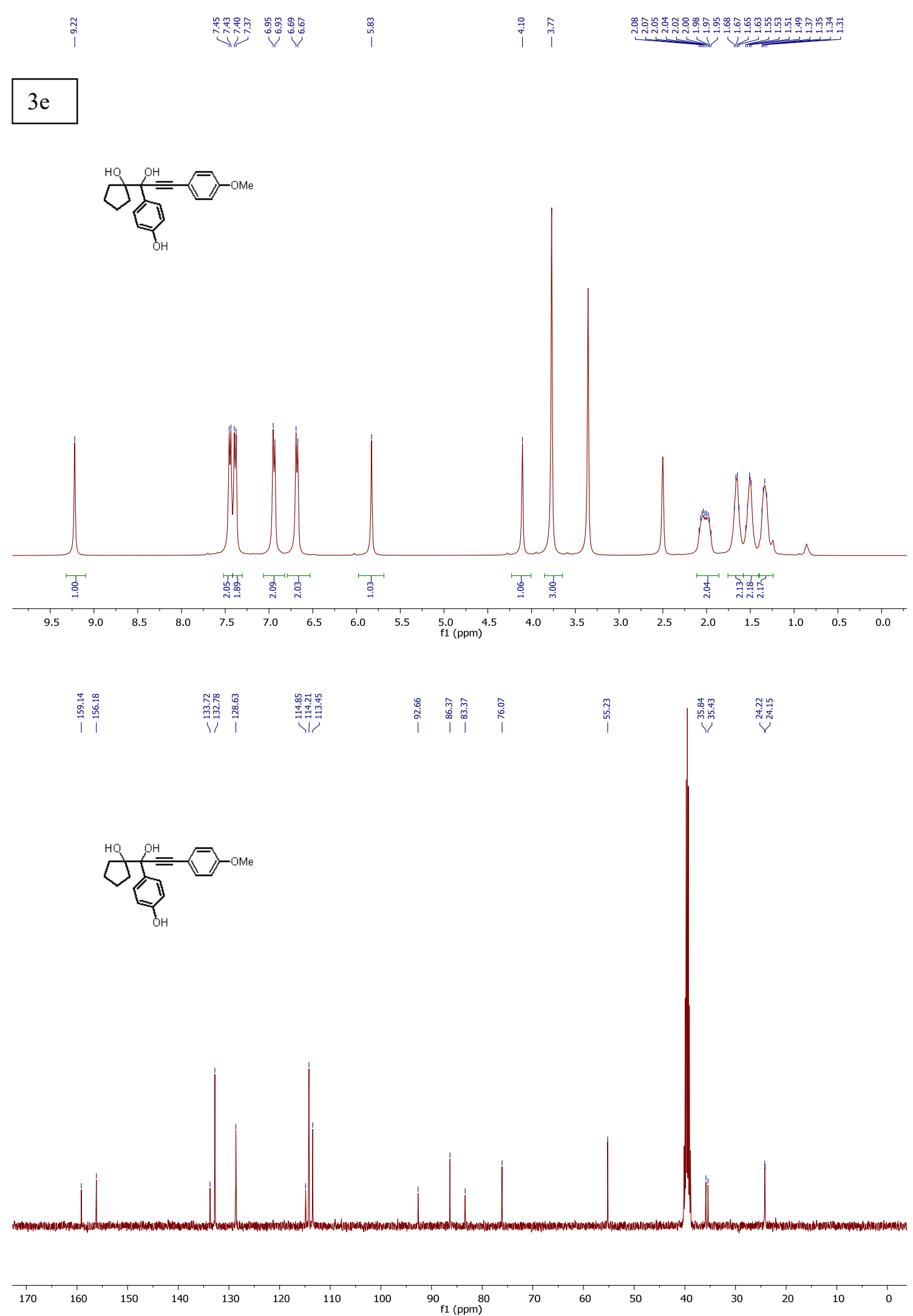

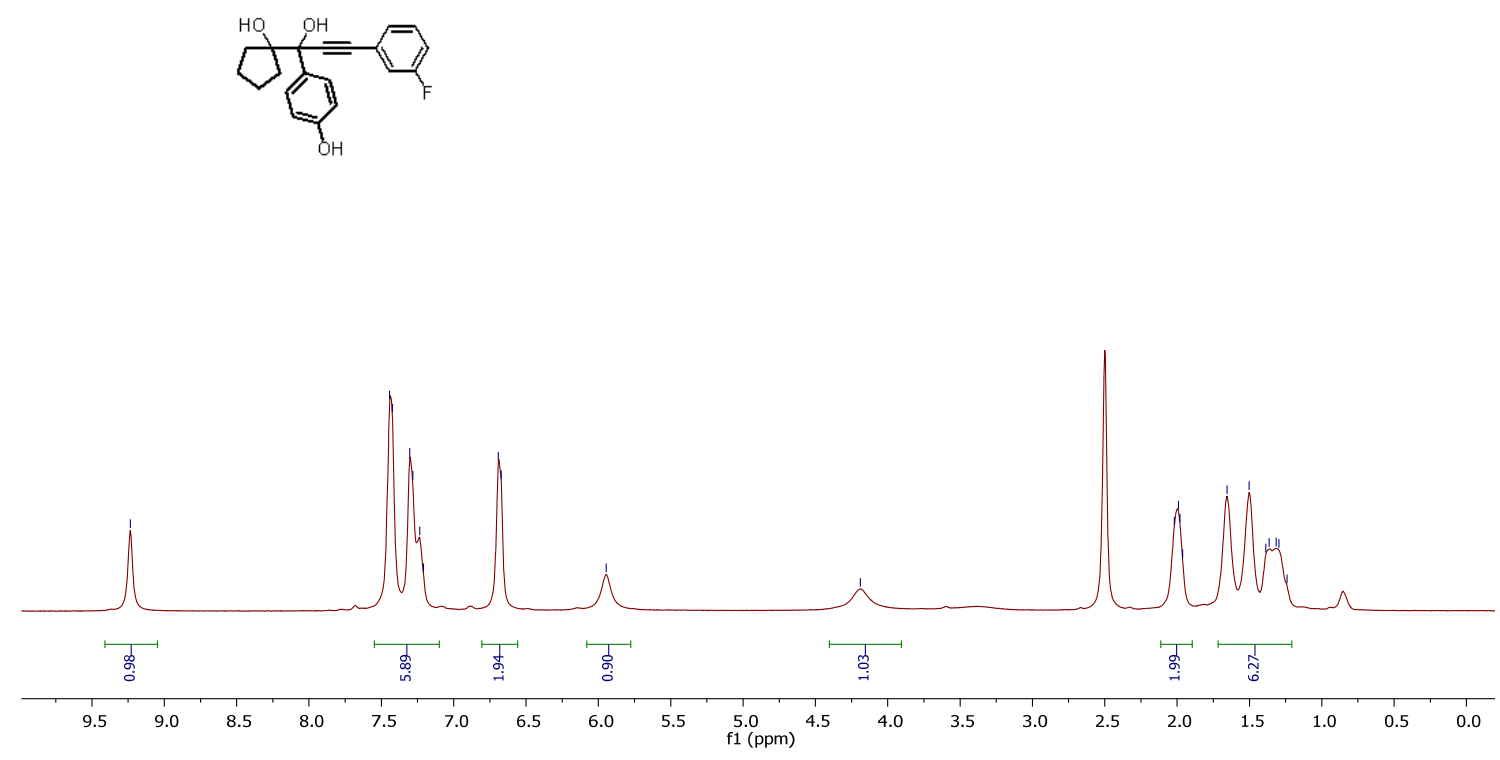

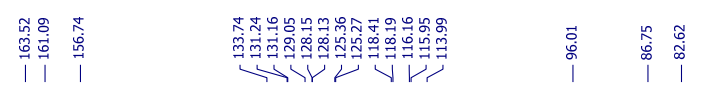<smiles>Oc1ccc(C(O)(C#Cc2cccc(F)c2)c2ccccc2)cc1</smiles>

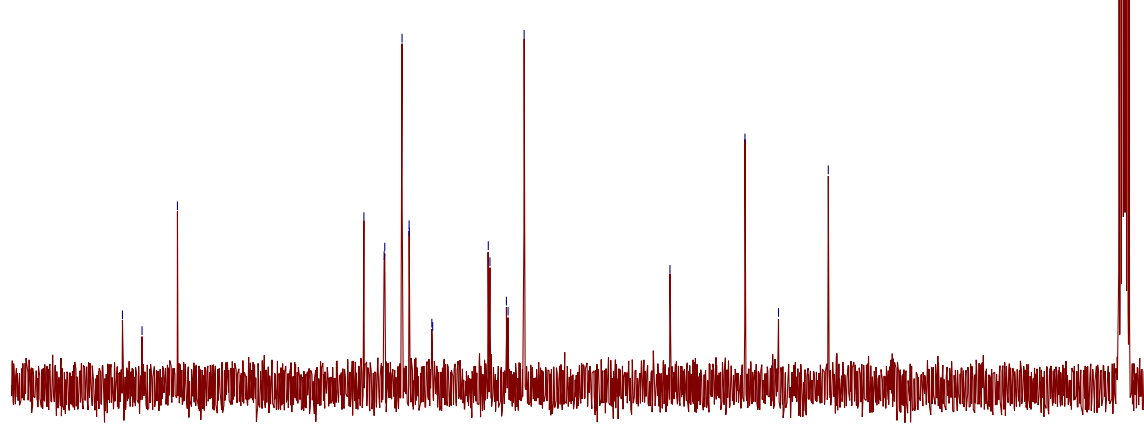

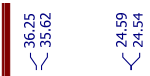

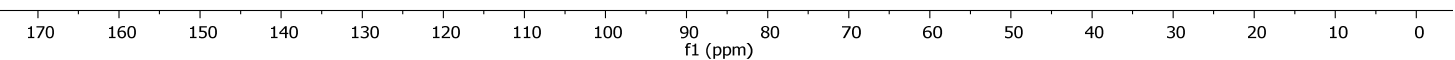



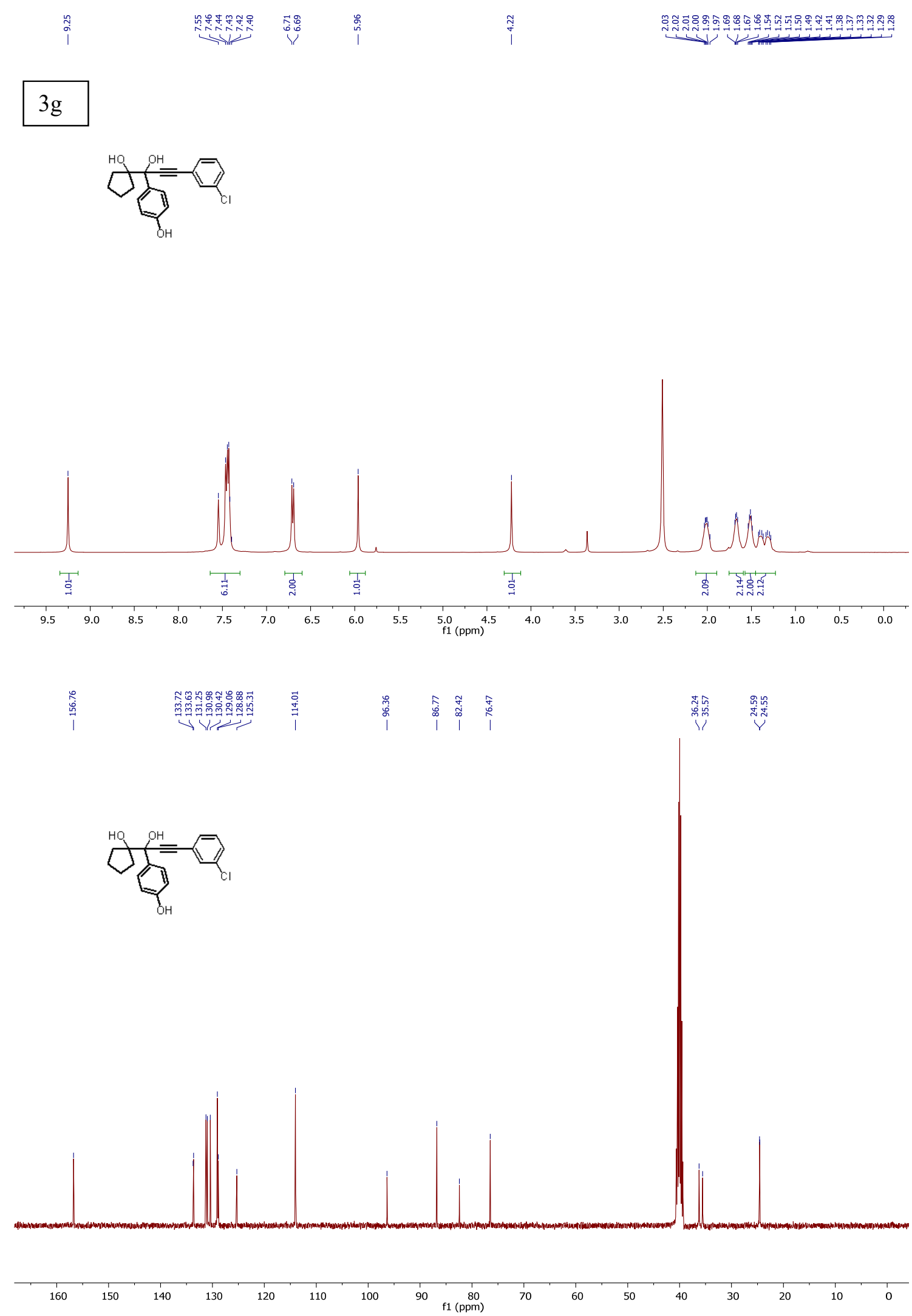

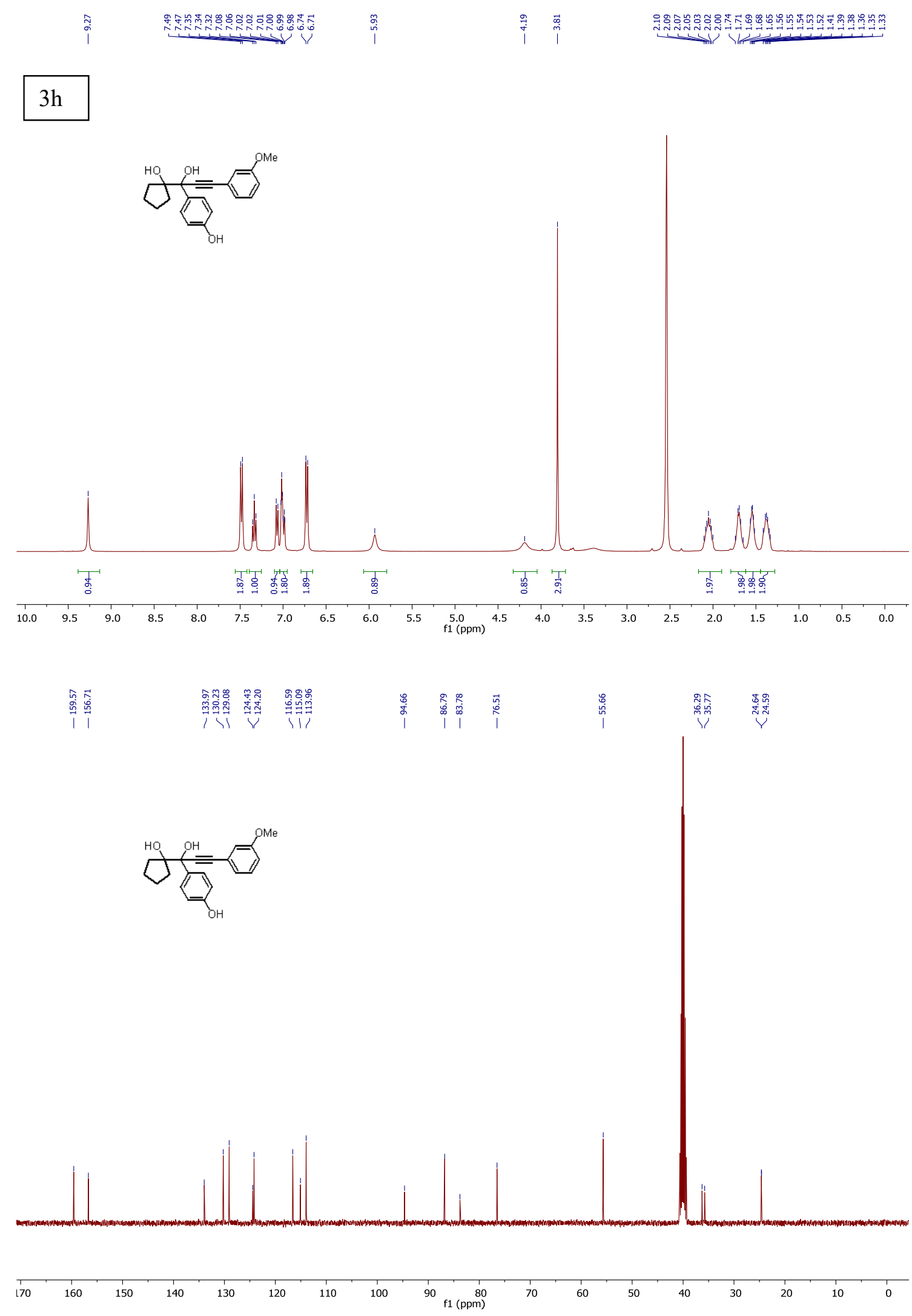

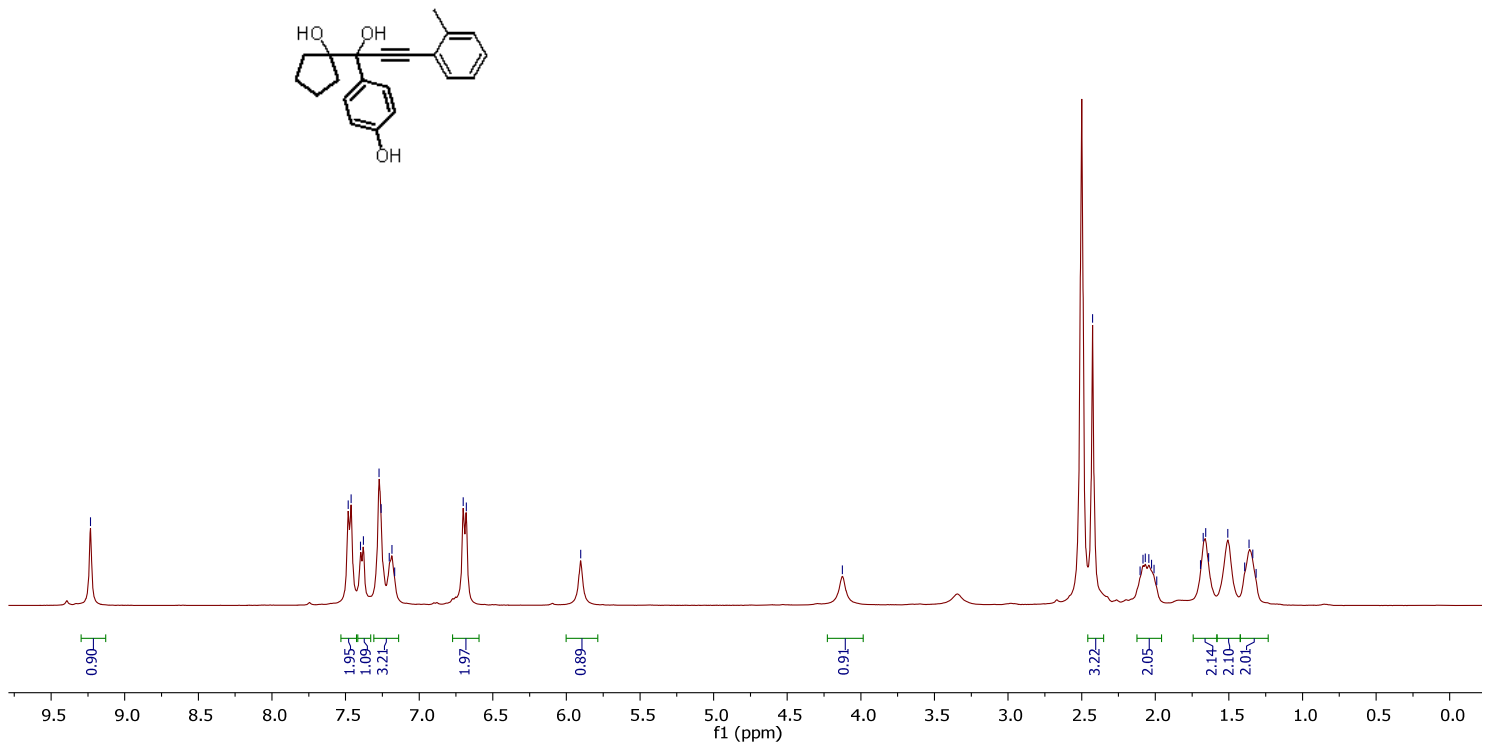

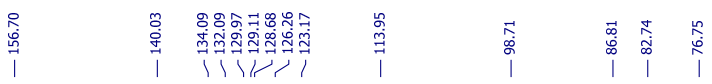
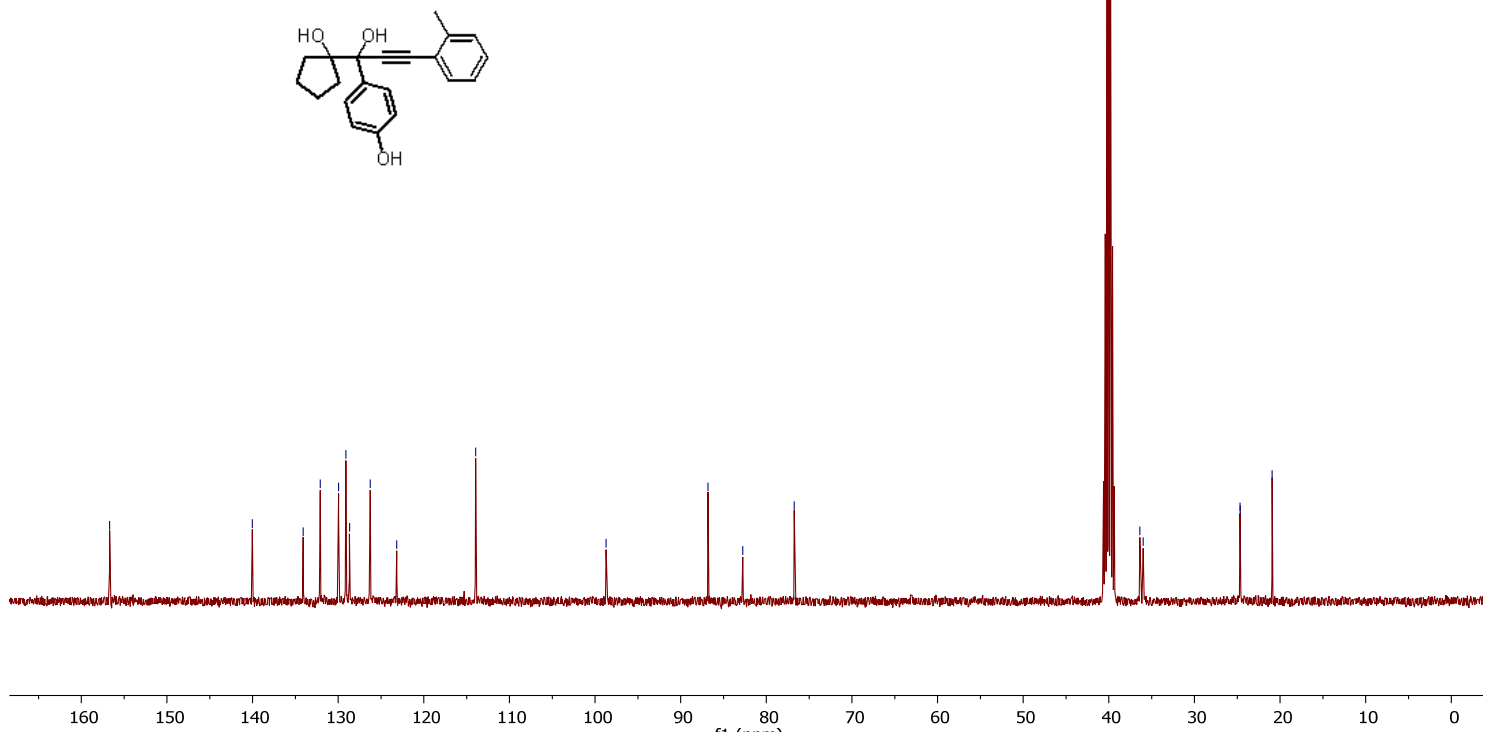

f1 (ppm) 


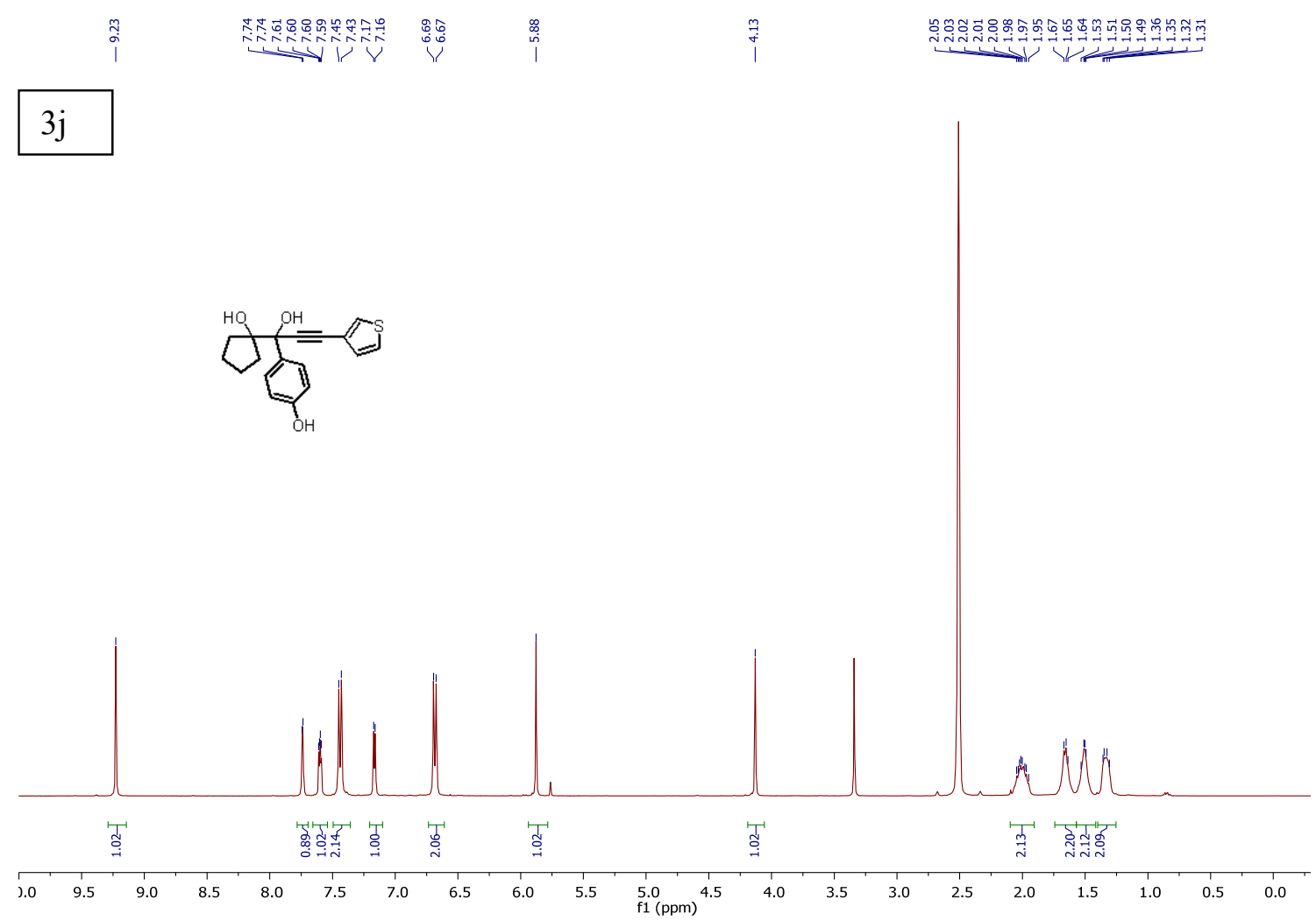

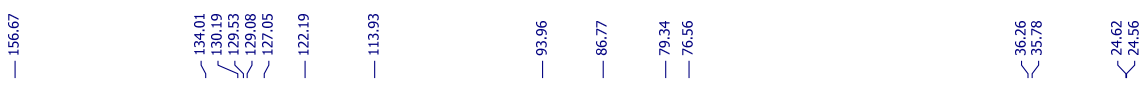
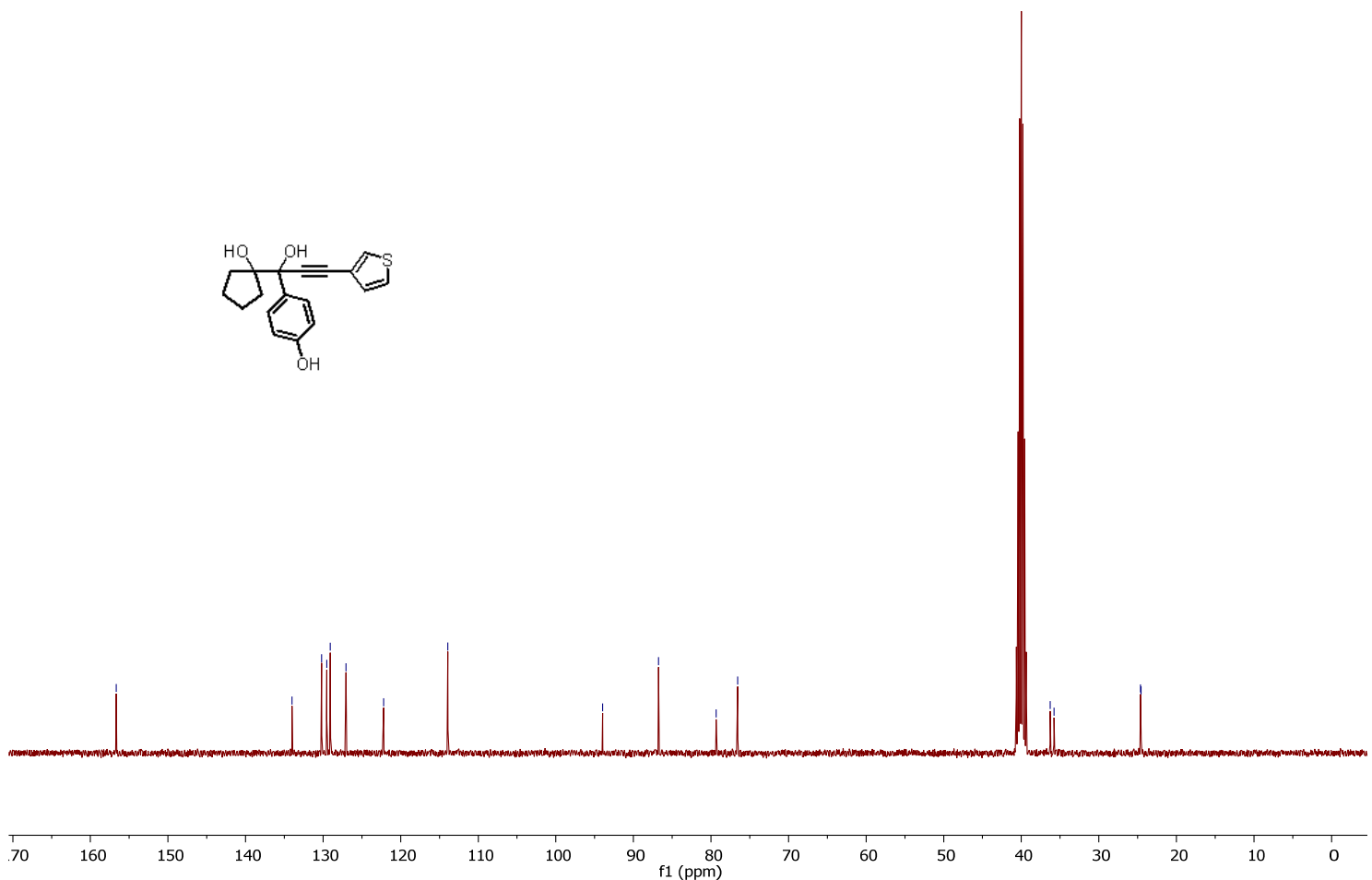


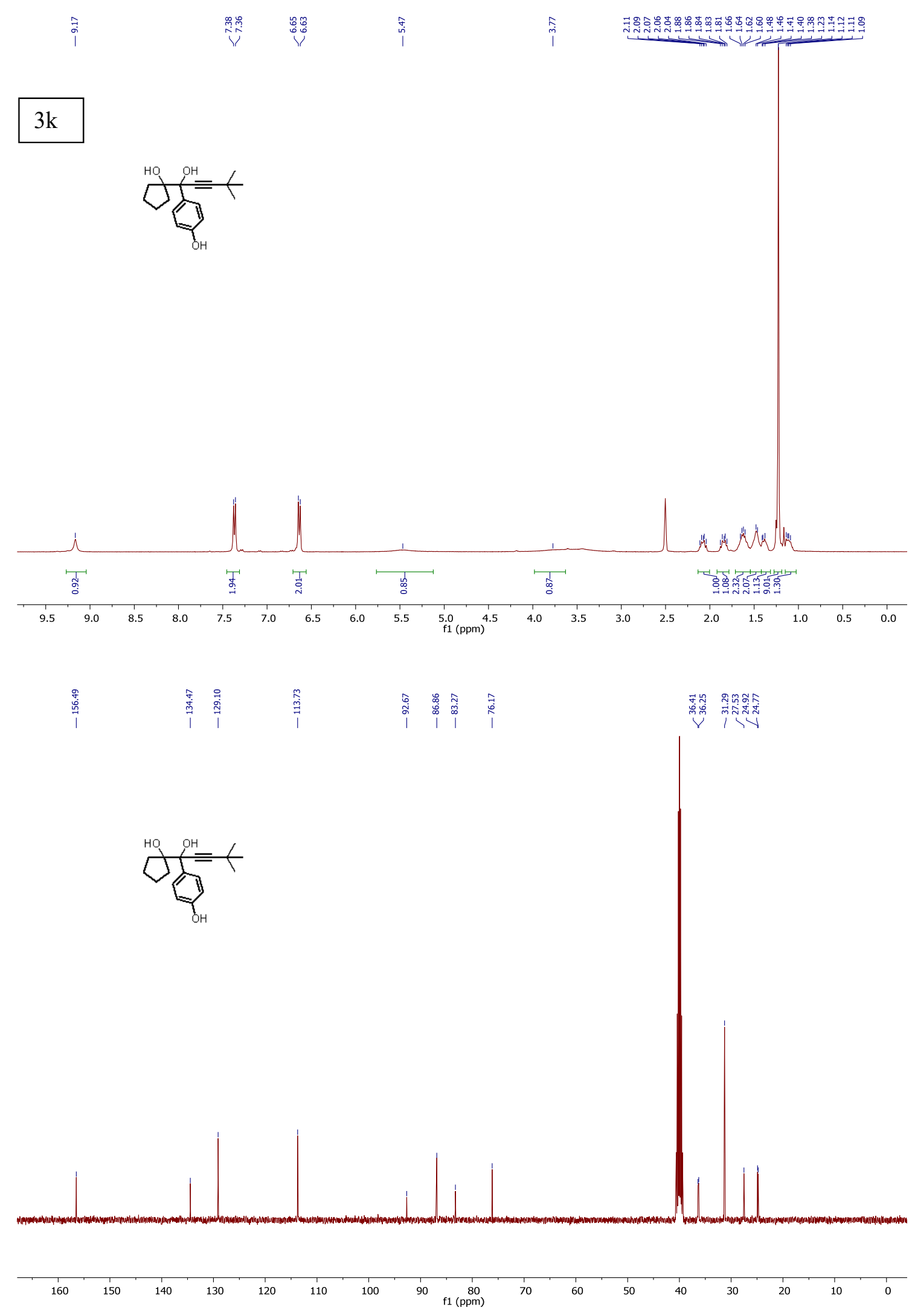



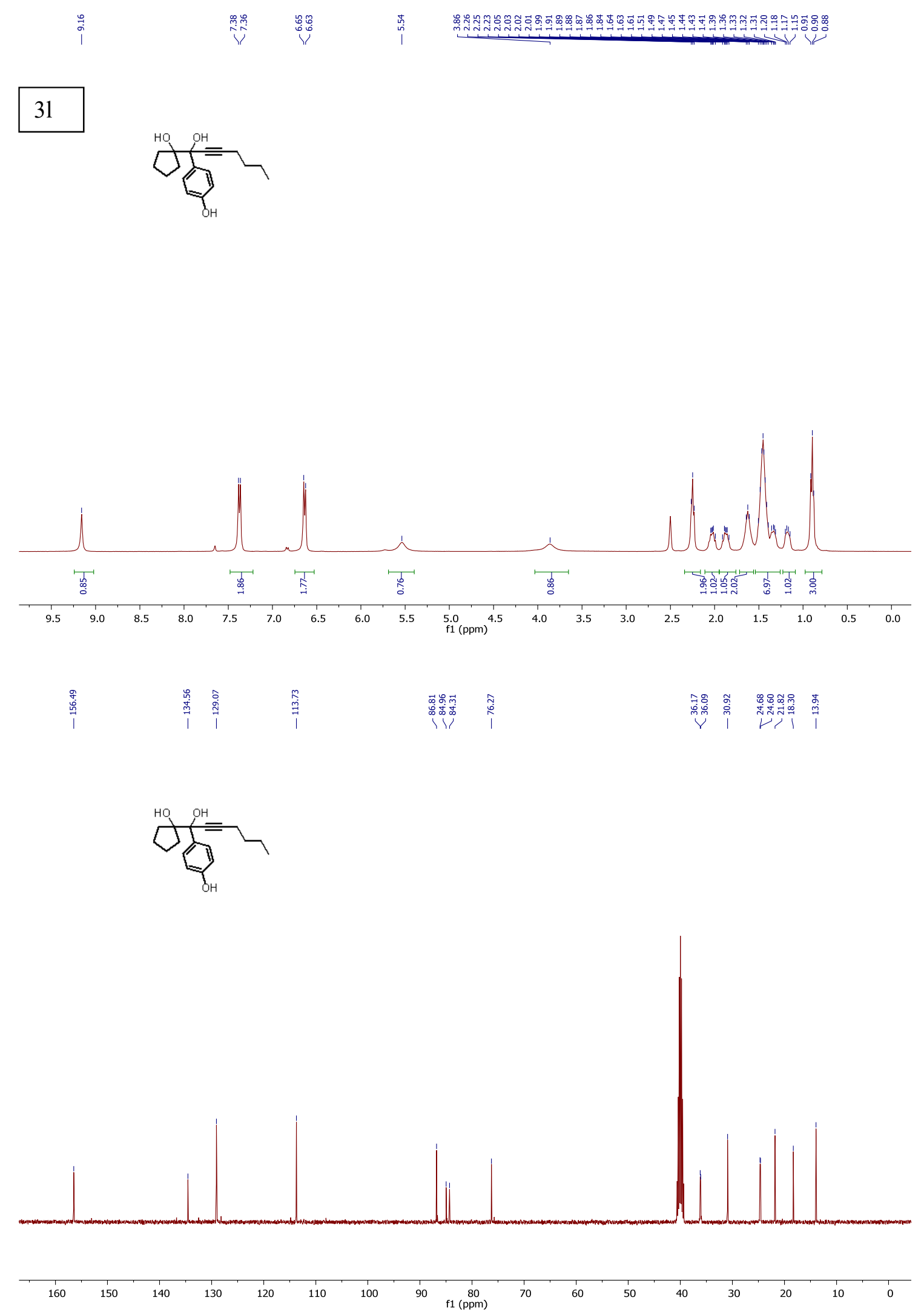


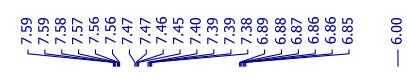

\section{$3 \mathrm{~m}$}

$$
{ }^{\mathrm{HQ}}
$$
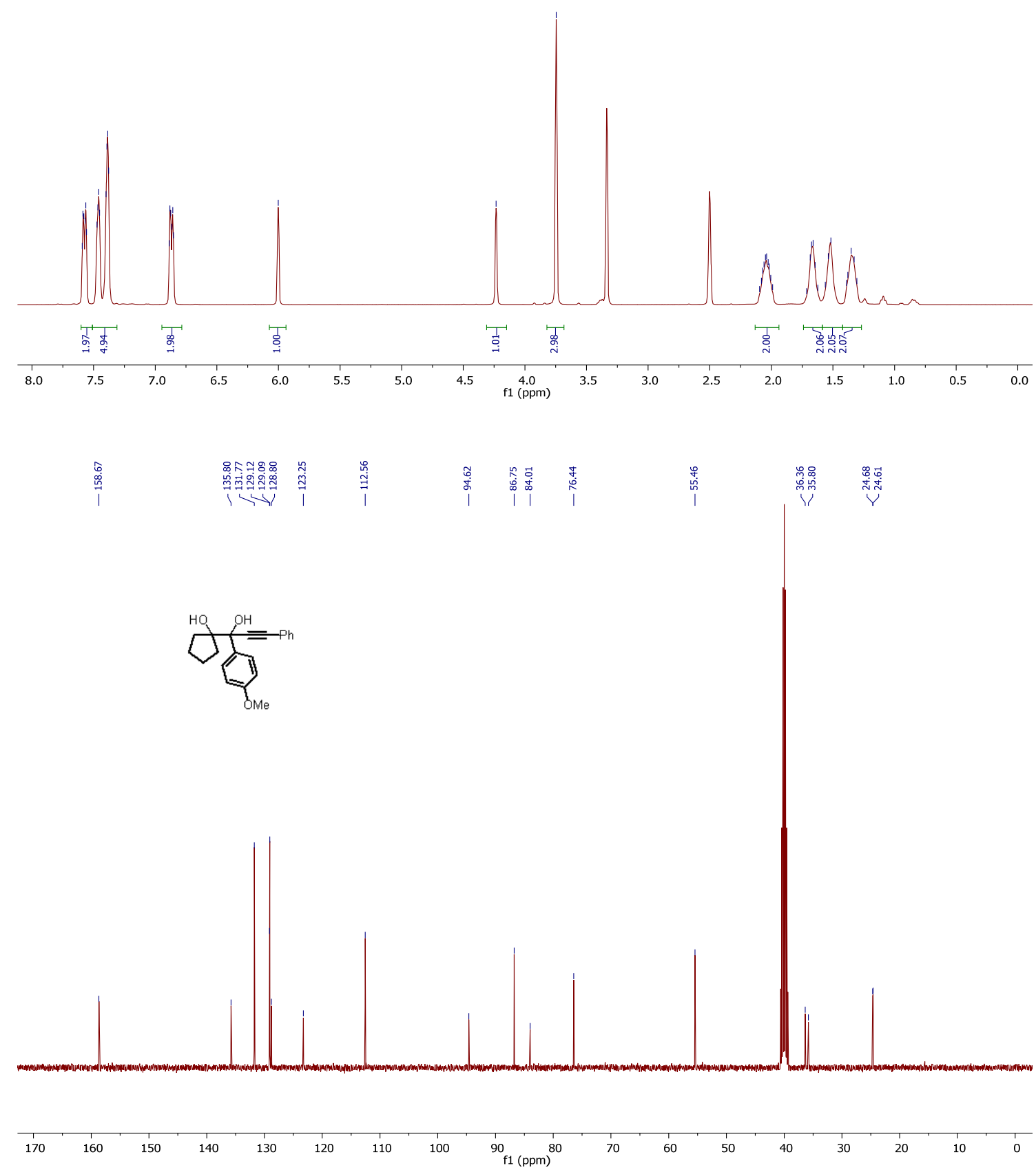


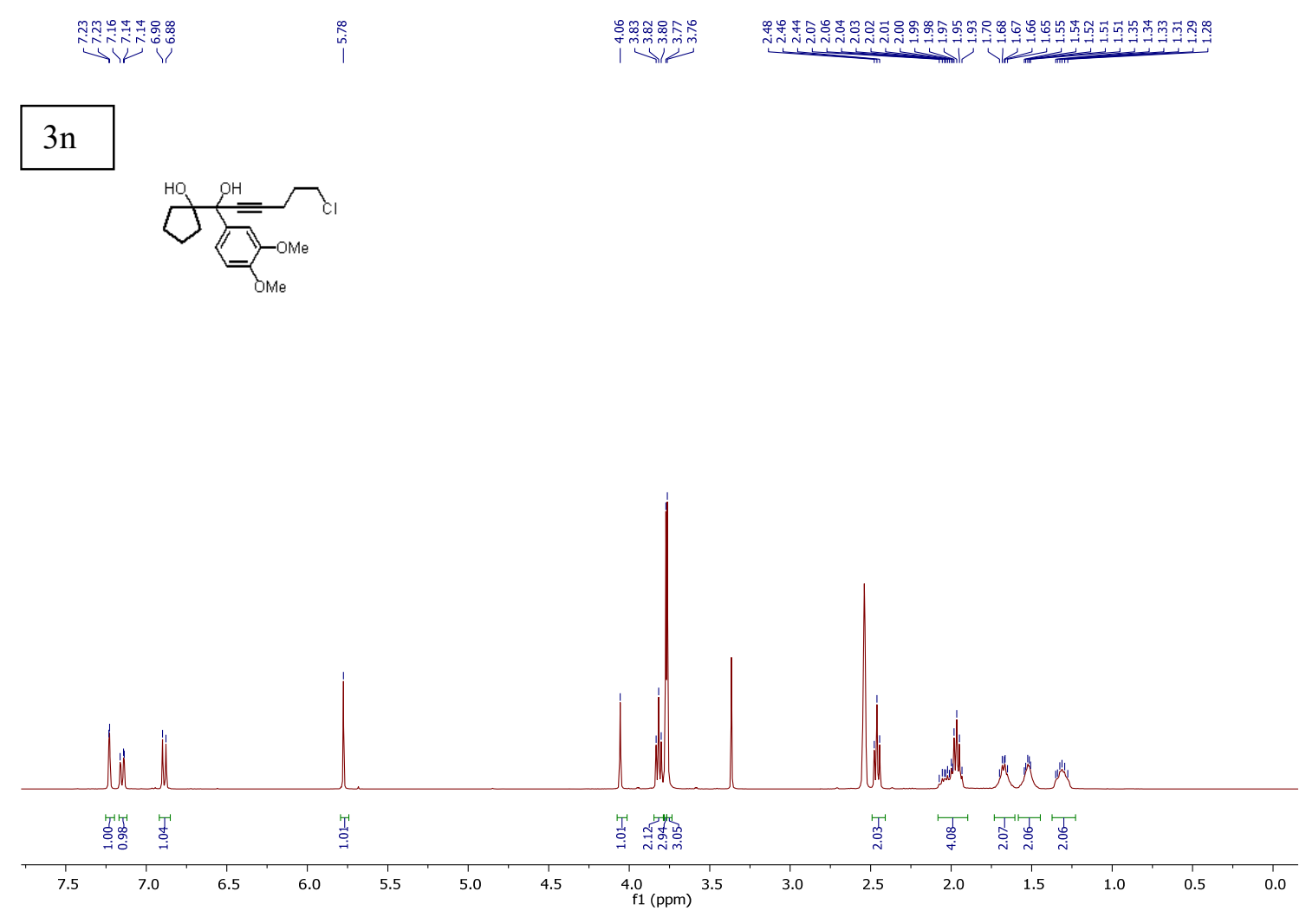

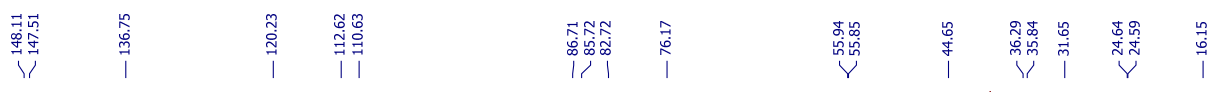
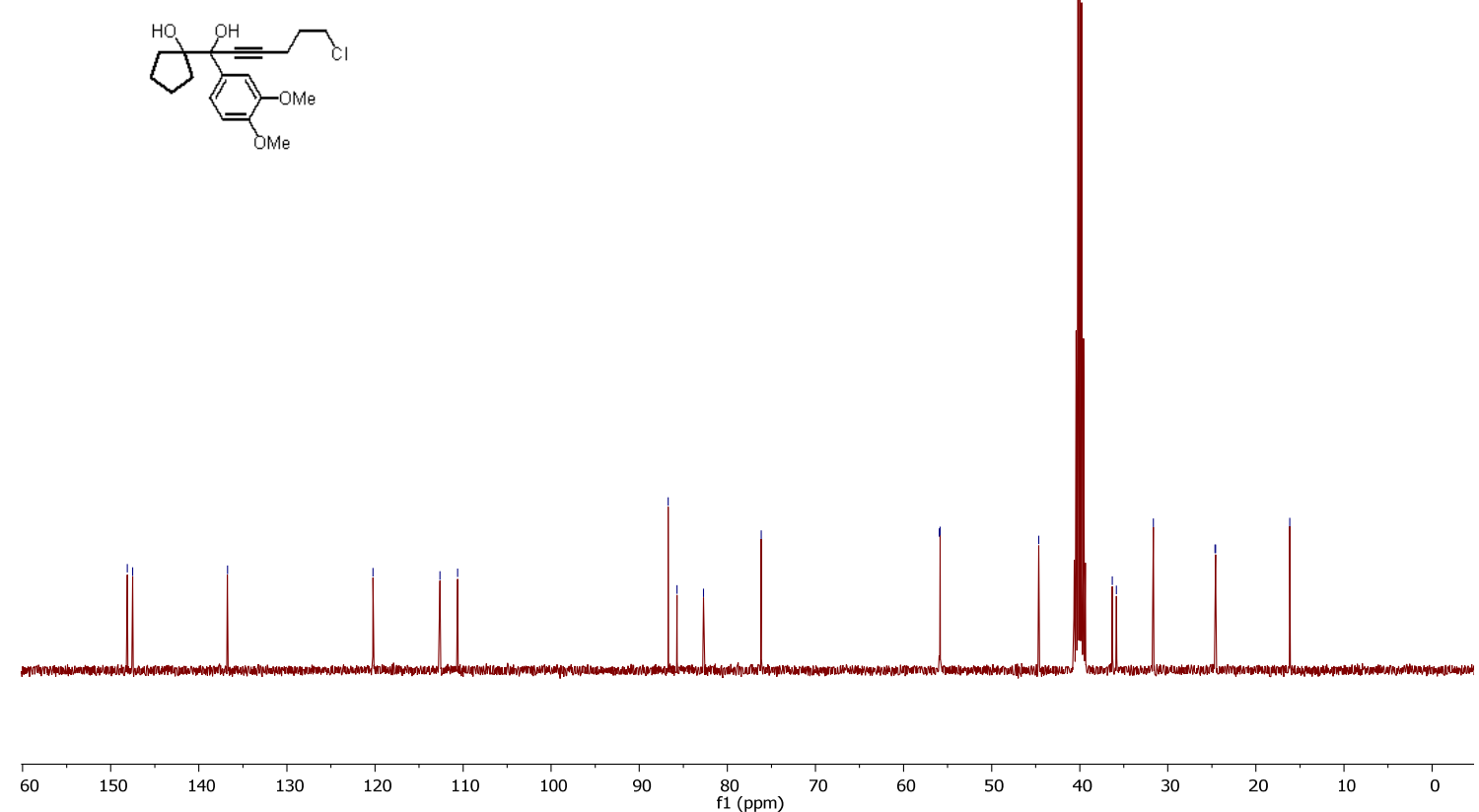


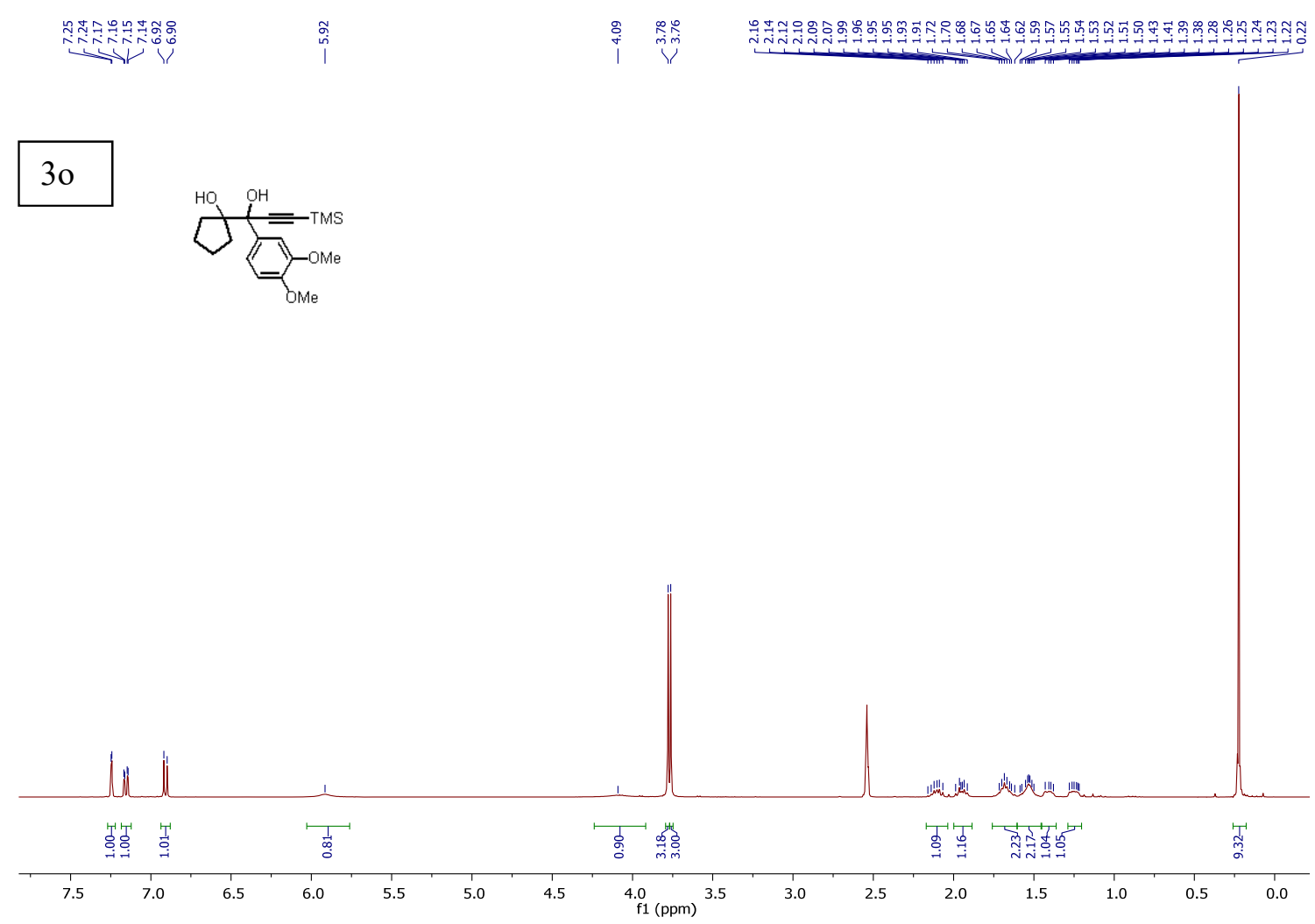

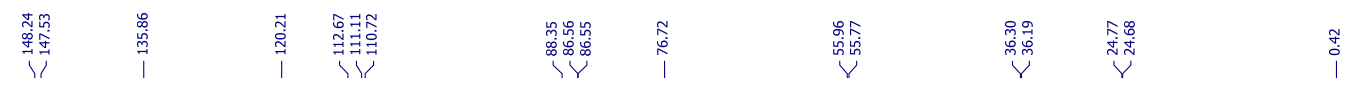

$0 \longdiv { Z _ { \text { -оме } } }$

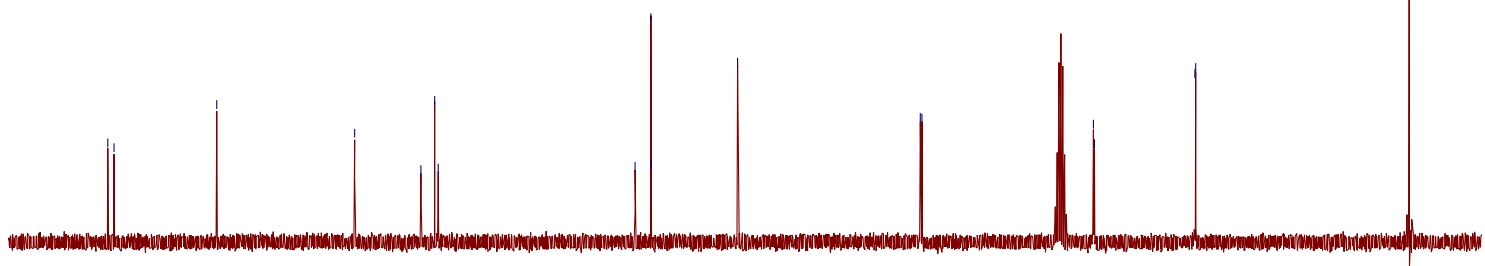

$\begin{array}{llllllllllllll}1 & 1 & 140 & 130 & 120 & 110 & 100 & 90 & 80 & 1 & 1 & 1 & 1 & 1 \\ \mathrm{f} 1(\mathrm{ppm}) & 70 & 60 & 50 & 40 & 30 & 20 & 10 & 0\end{array}$ 

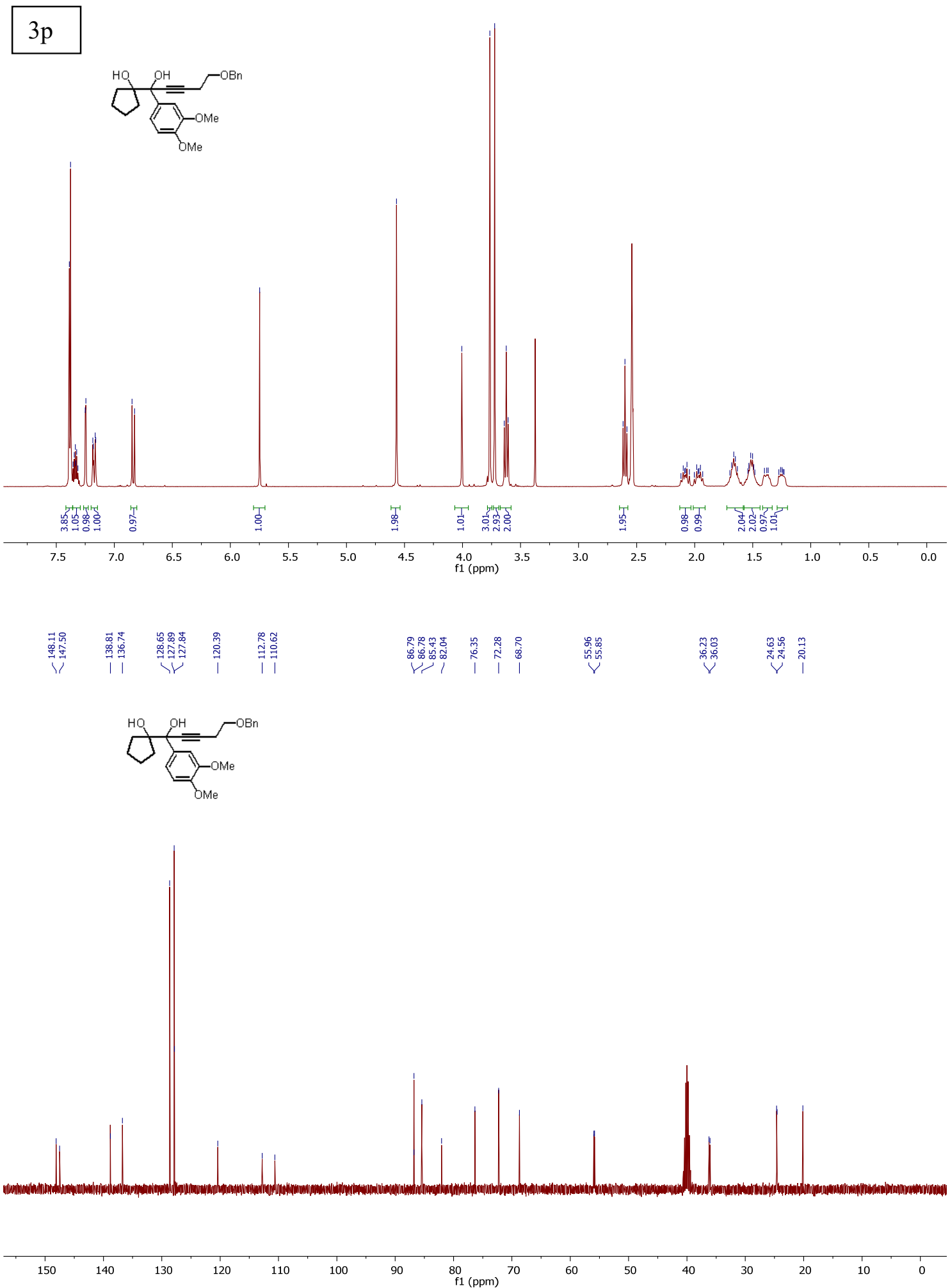


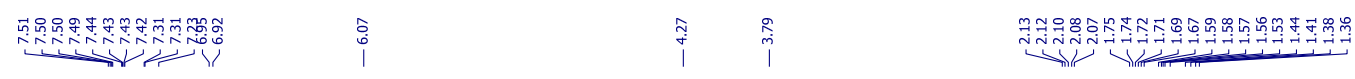

$3 q$
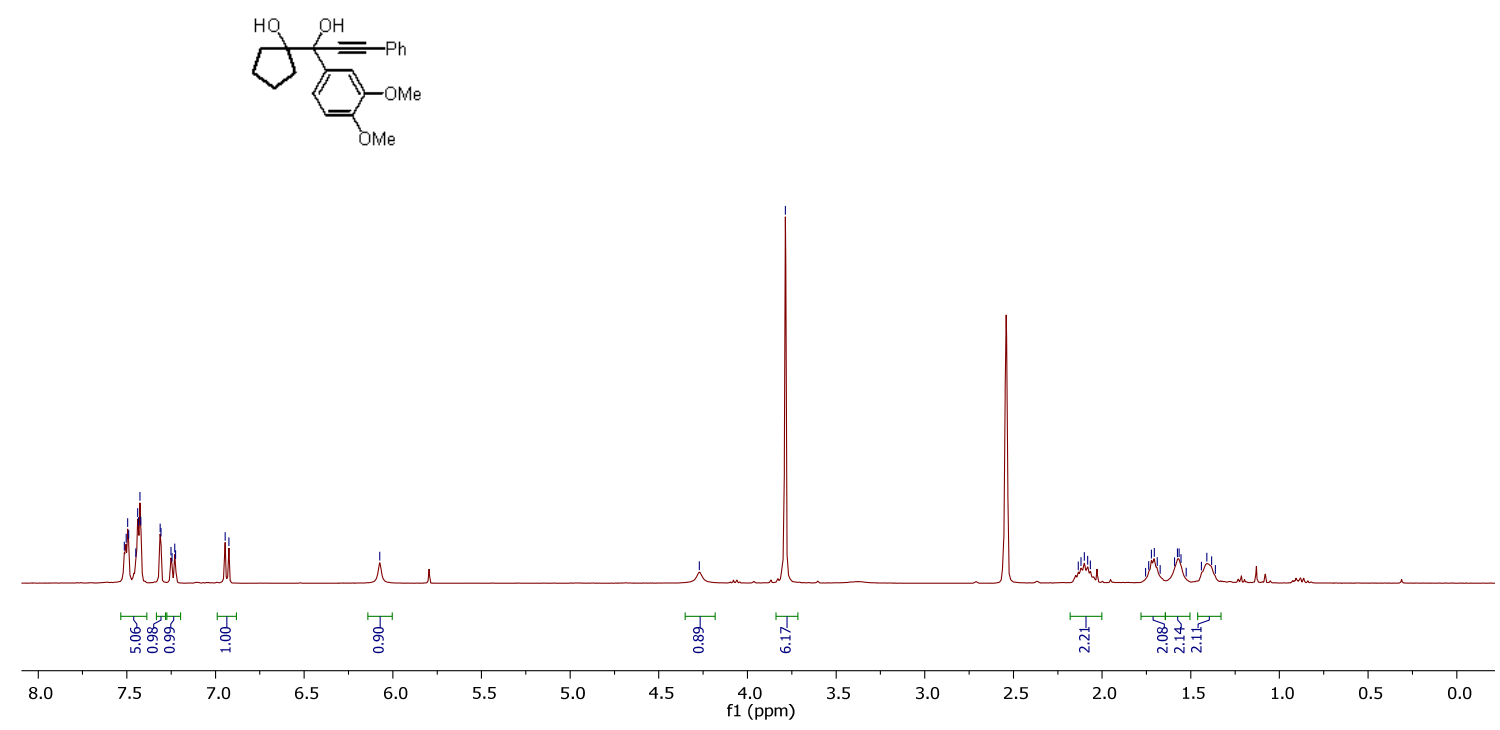

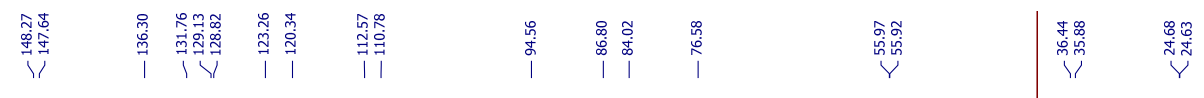
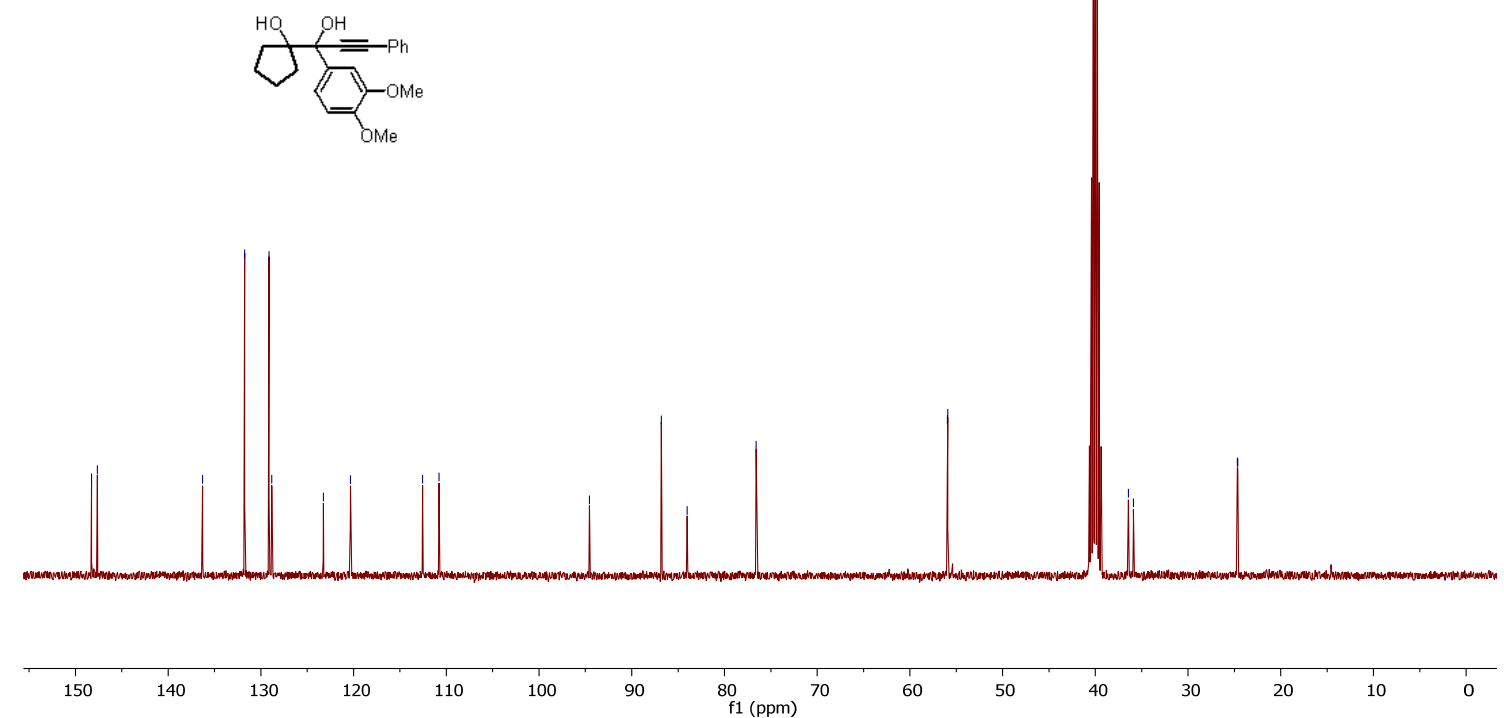


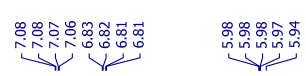

$3 r$
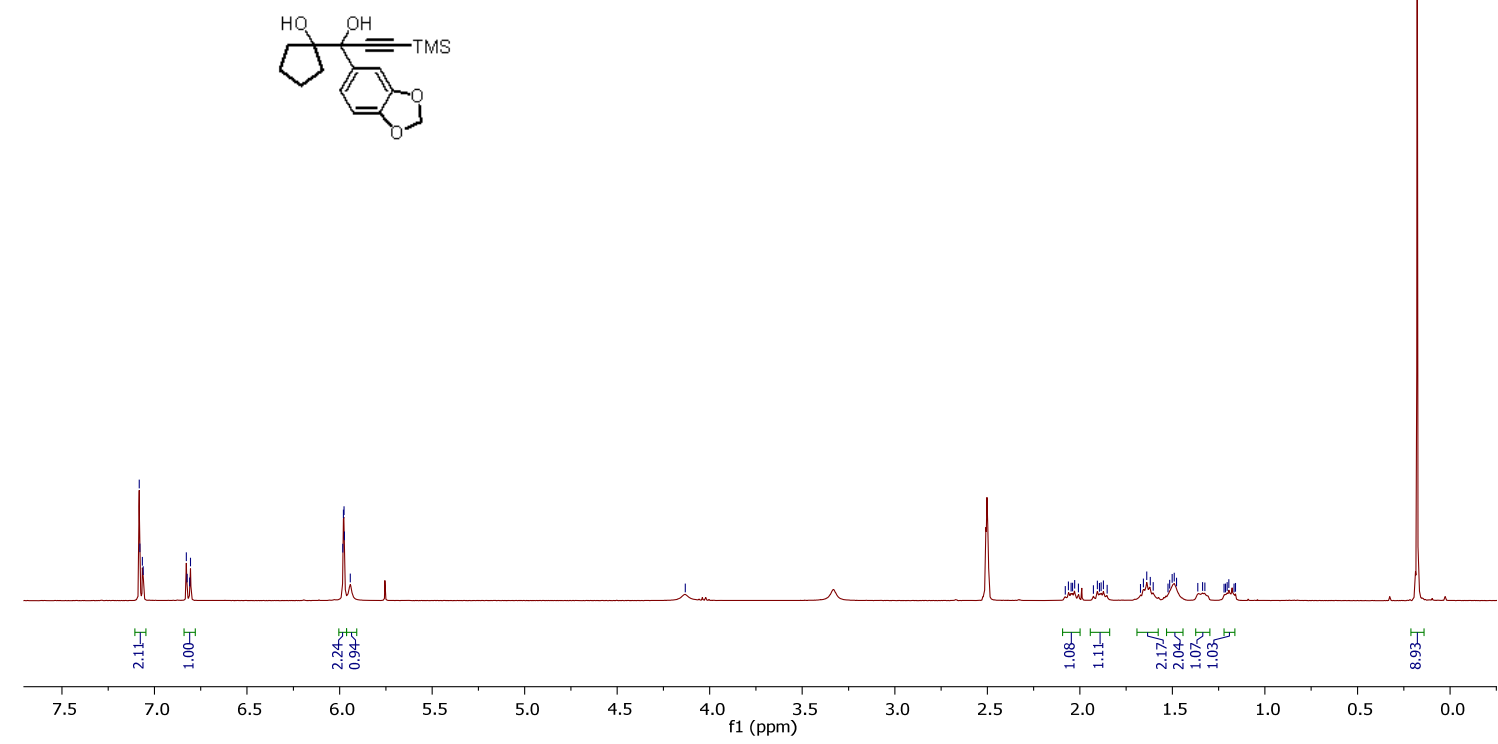

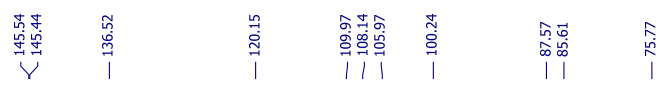
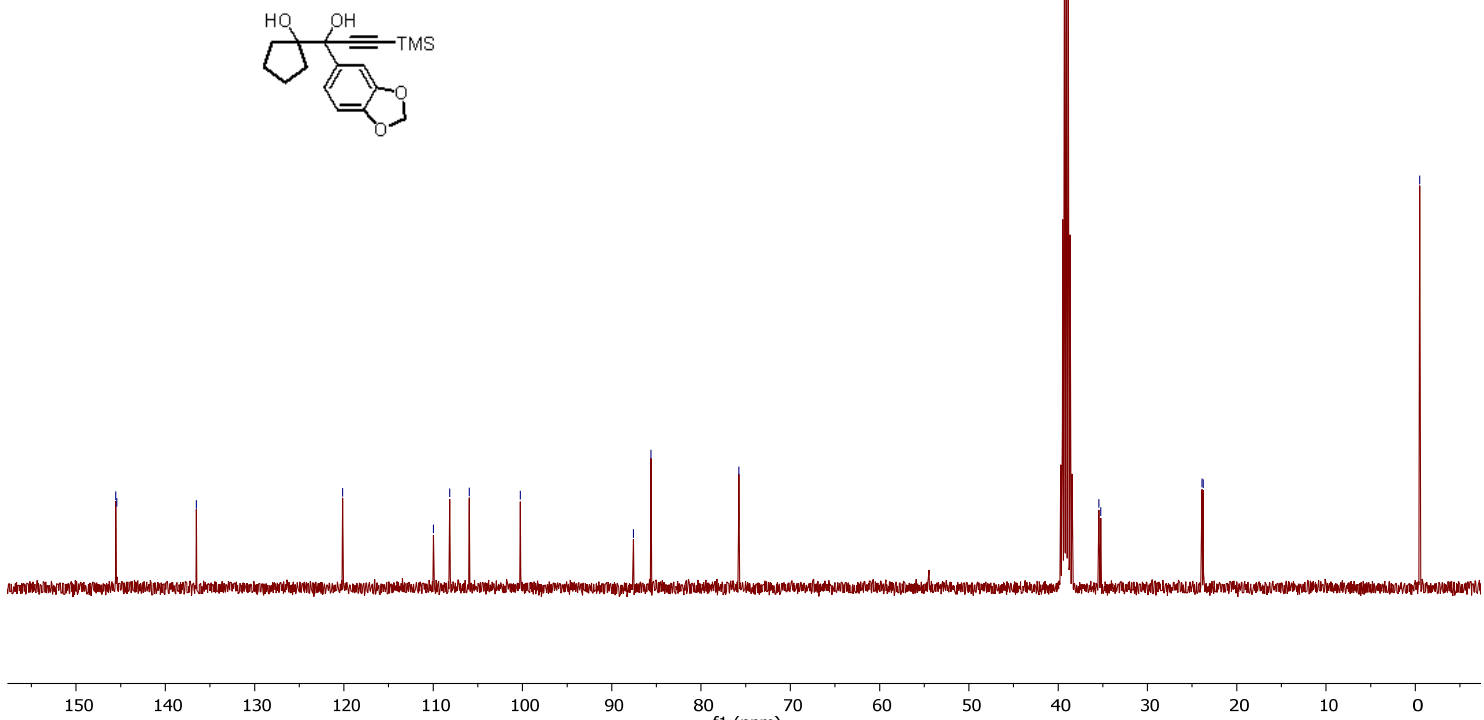

100

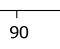

${ }^{81} 1(\mathrm{ppm}){ }^{70}$
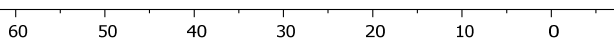


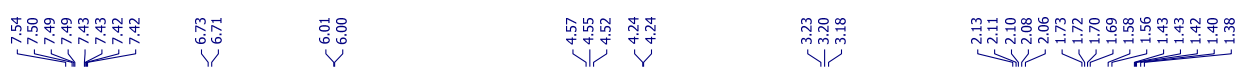

$3 s$

$0 \sum^{\mathrm{HO}}$

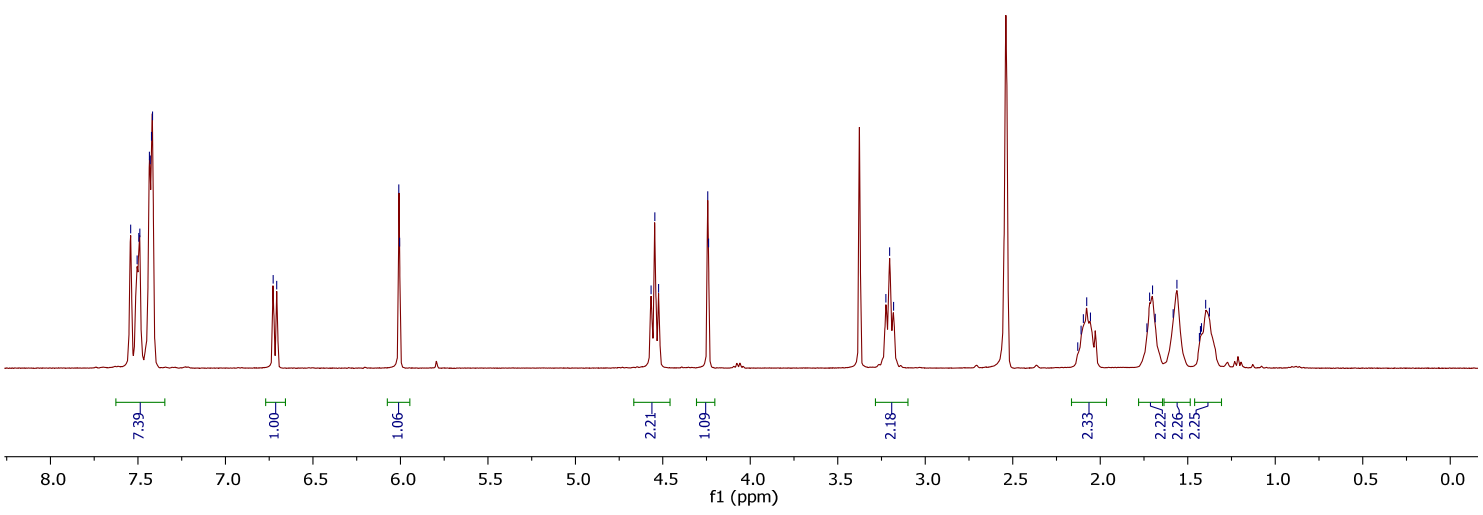

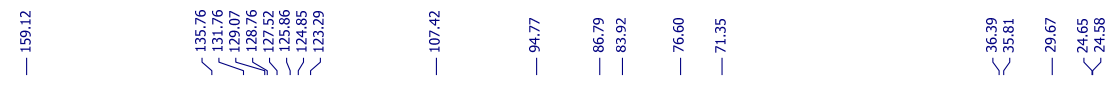

$\Delta \sum^{\mathrm{HO}}$

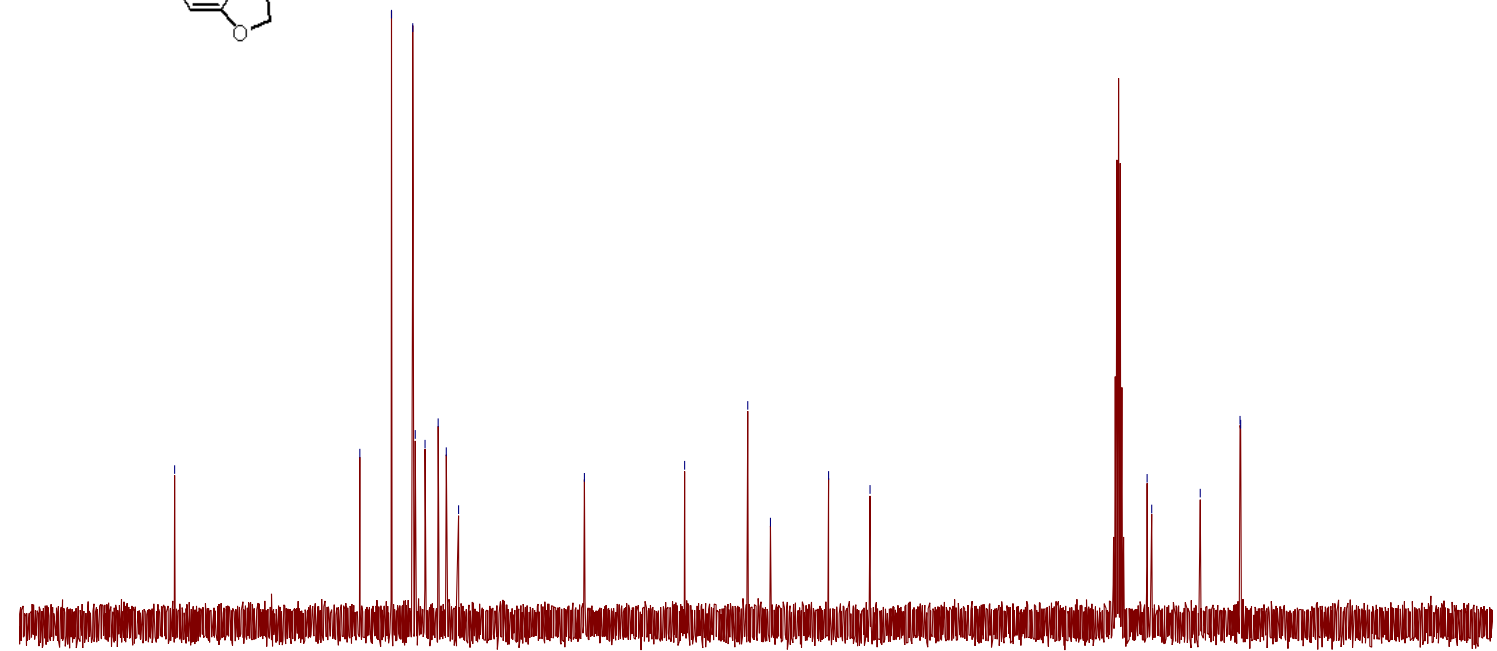

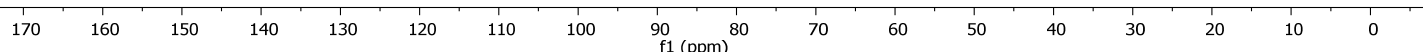




\section{$3 t$}

$\stackrel{\mathrm{HO}}{=}$
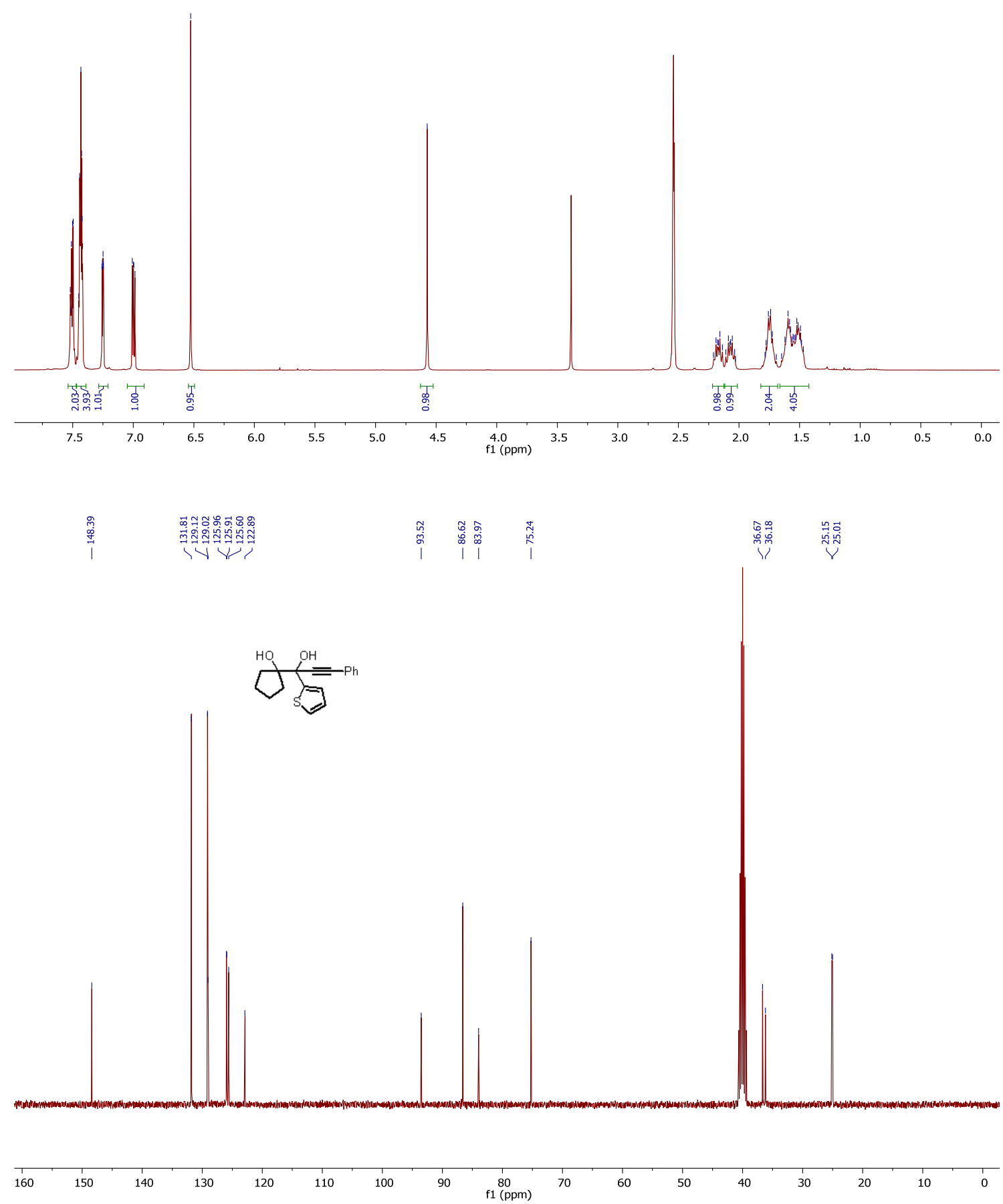


\section{$3 \mathrm{u}$}<smiles>O=S(=O)(c1ccccc1)c1ccccc1</smiles>

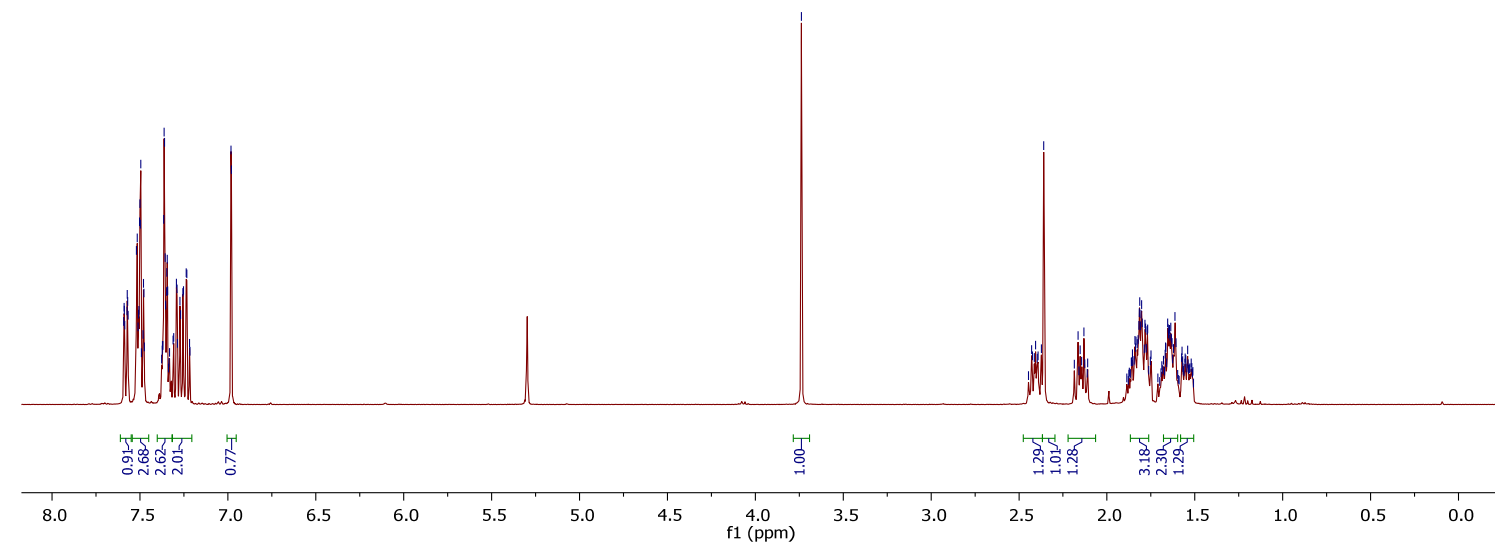

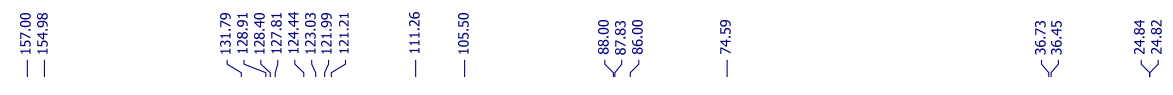
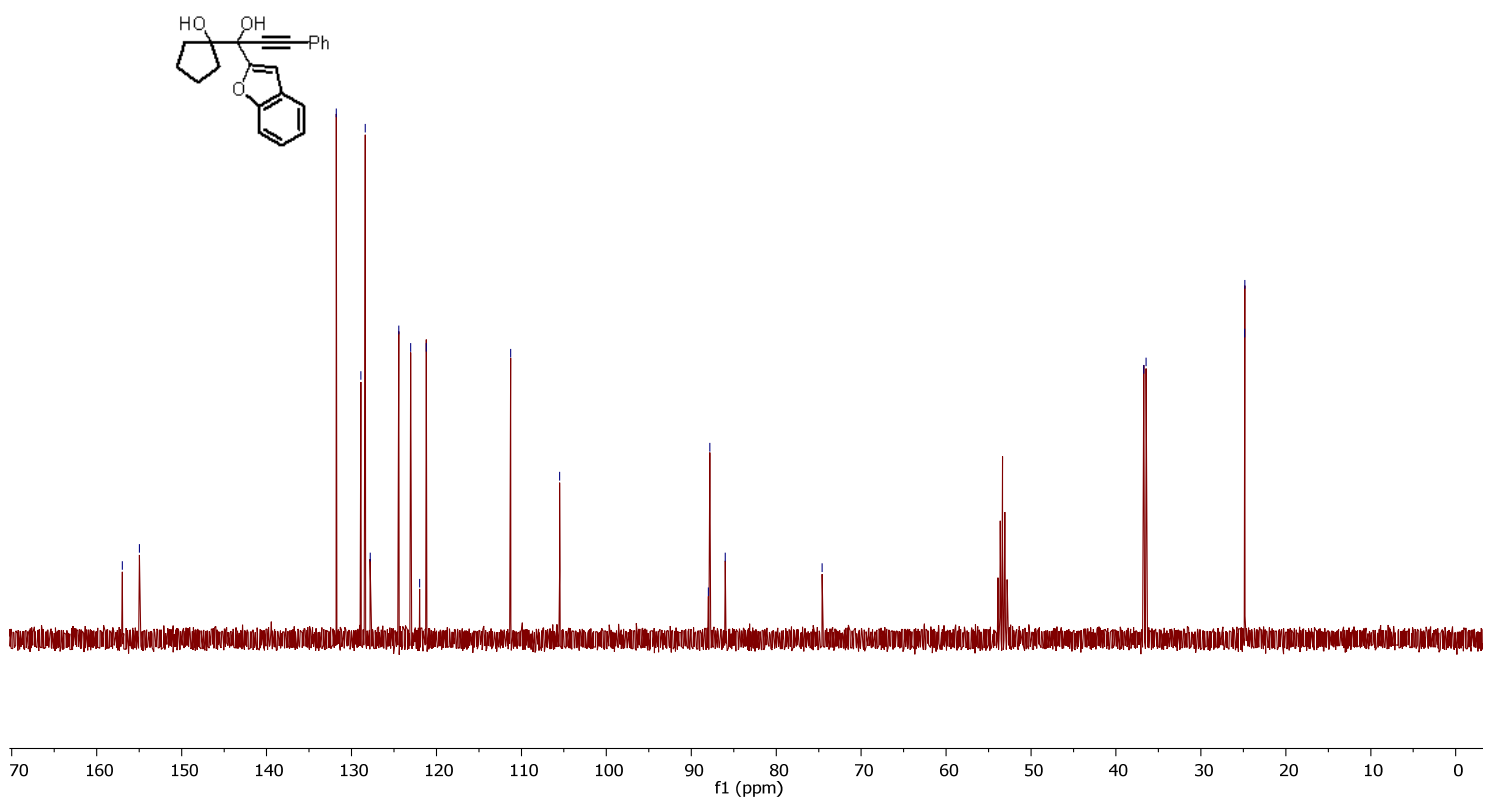
$3 \mathrm{v}$
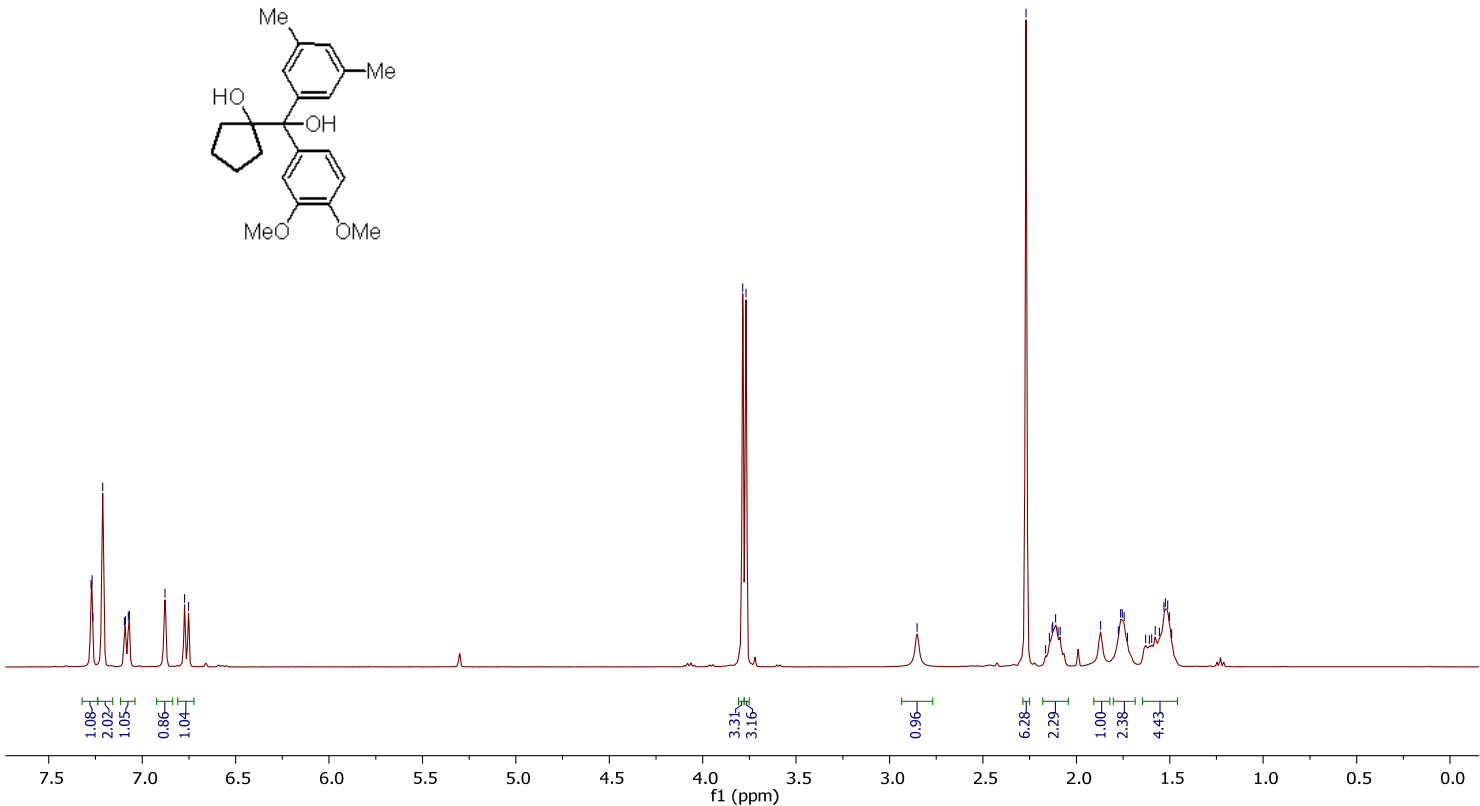

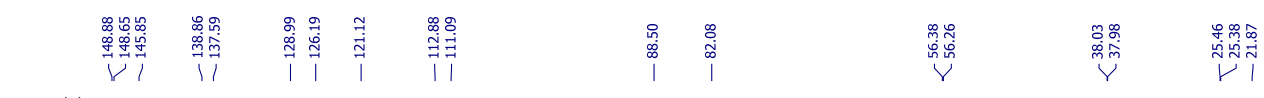<smiles>COc1ccc(C(c2cccc(O)c2)(c2cccc(O)c2)c2cccc([N+](=O)[O-])c2)cc1</smiles>

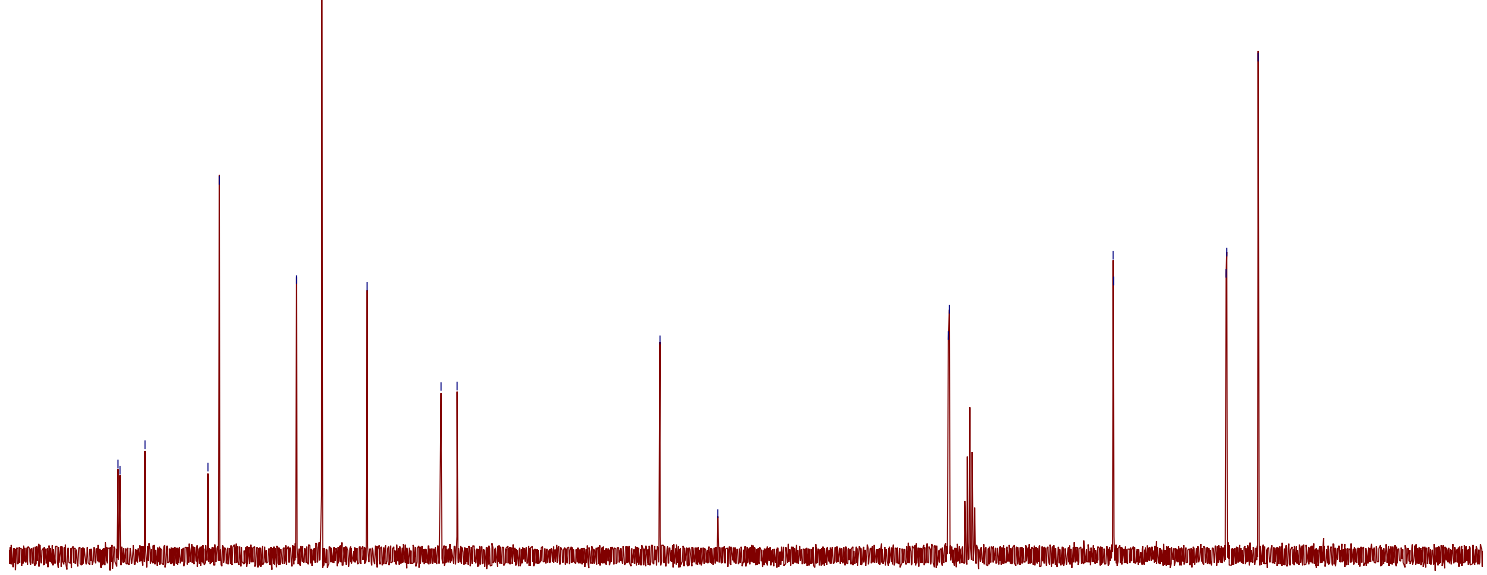

T'160
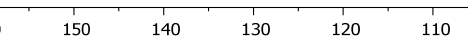

100

90

f1 1 (ppm)

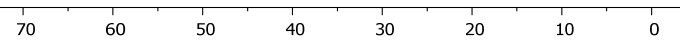




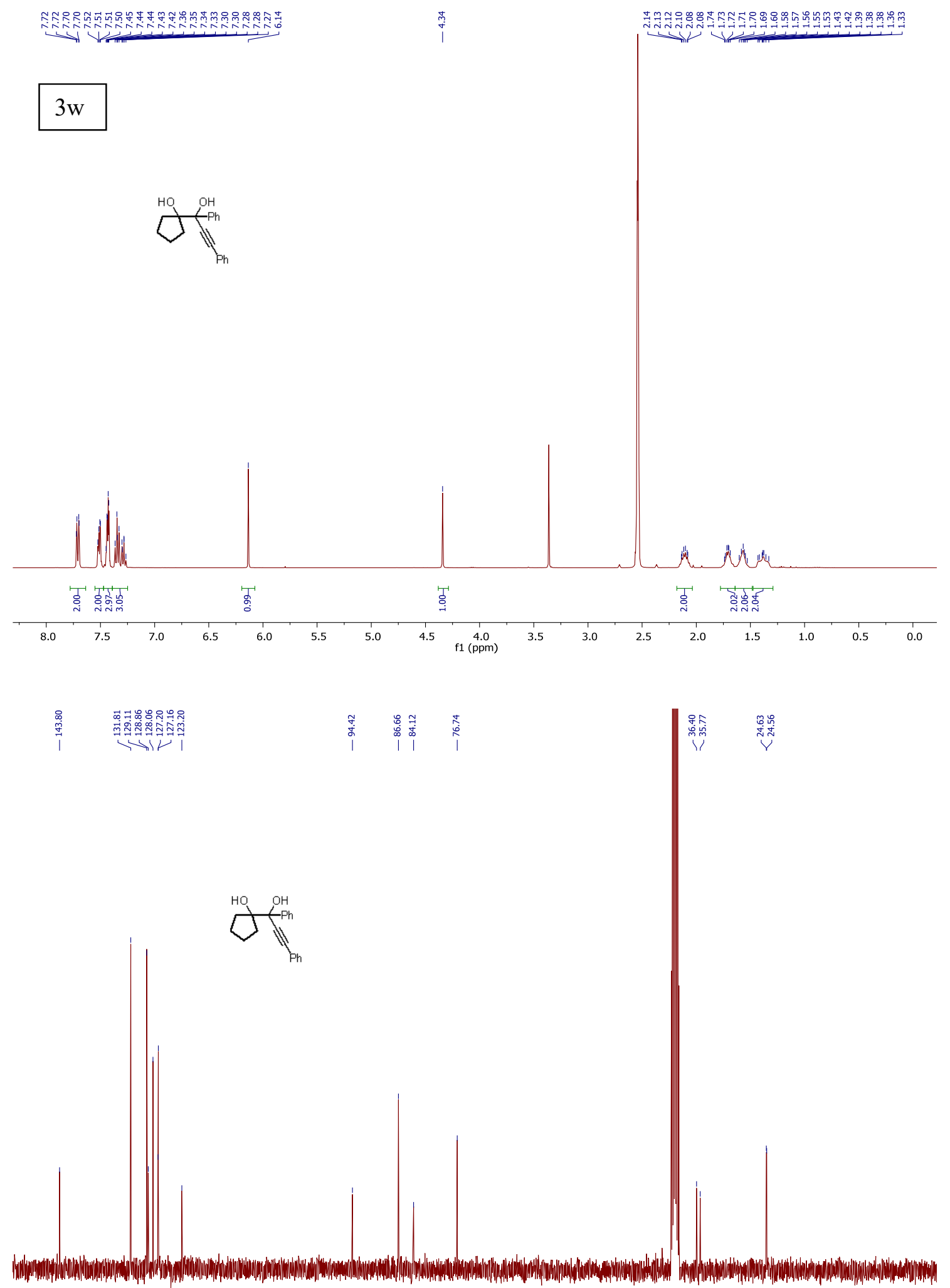

$\begin{array}{lllllllllllllllllllllllllllllllllllllllllllllllllll}150 & 145 & 140 & 135 & 130 & 125 & 120 & 115 & 110 & 105 & 100 & 95 & 90 & 85 & 80 & 75 & 70 & 65 & 60 & 55 & 50 & 45 & 40 & 35 & 30 & 25 & 20 & 15 & 10 & 5 & 0\end{array}$ 

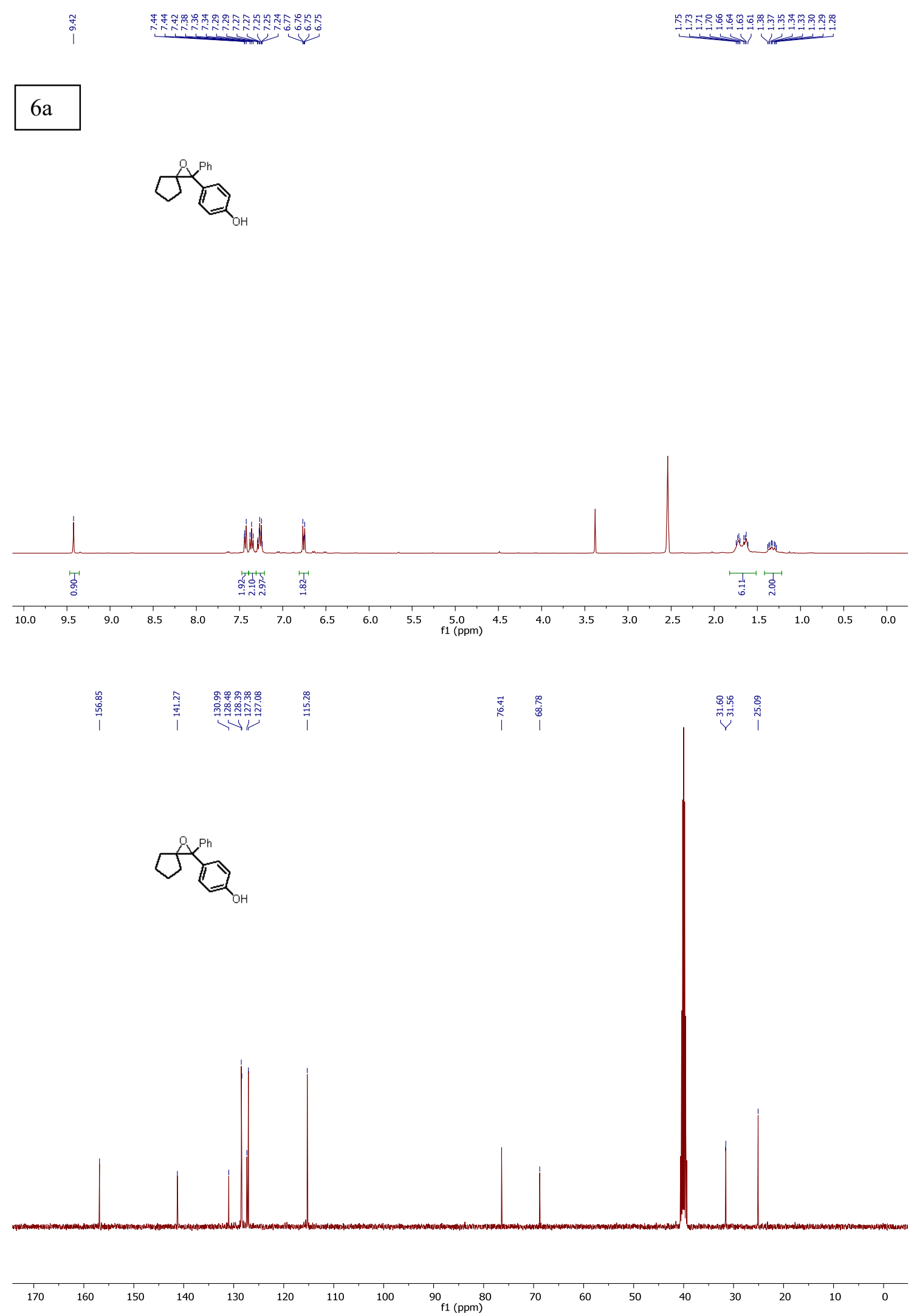
去

$6 b$<smiles>Oc1ccc(C(O)(c2ccc(F)cc2)C2CCCC2)cc1</smiles>

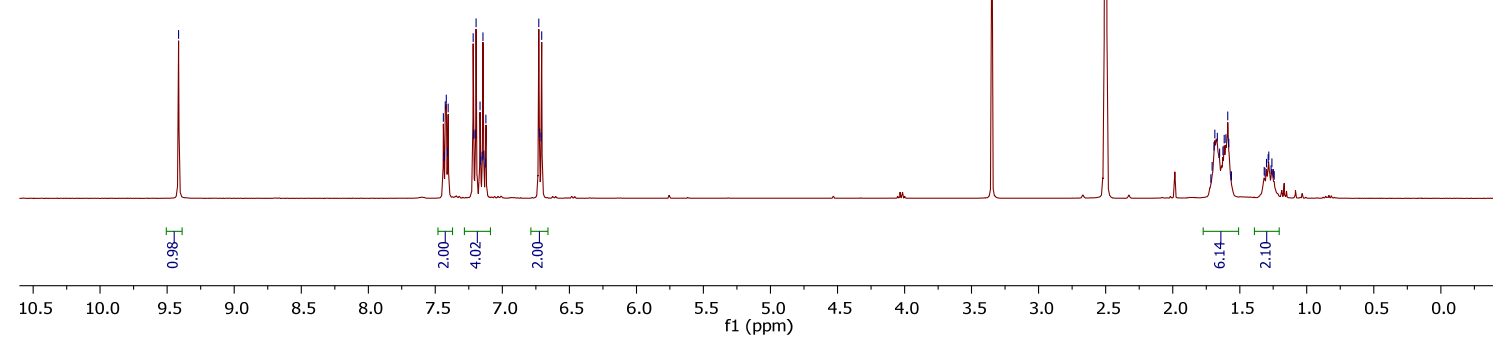<smiles>Oc1ccc(C(O)(c2ccc(F)cc2)C2(c3ccc(F)cc3)CCCC2)cc1</smiles>

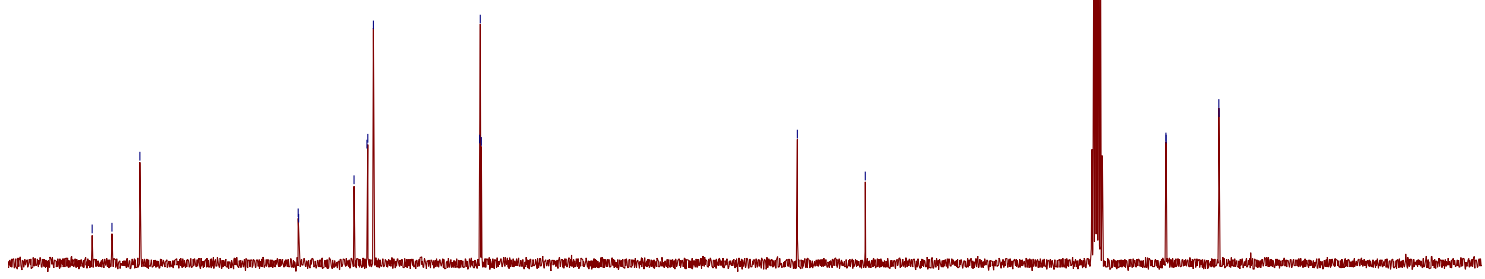




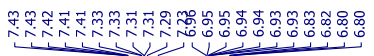

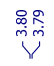

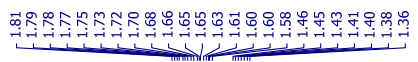

$6 c$
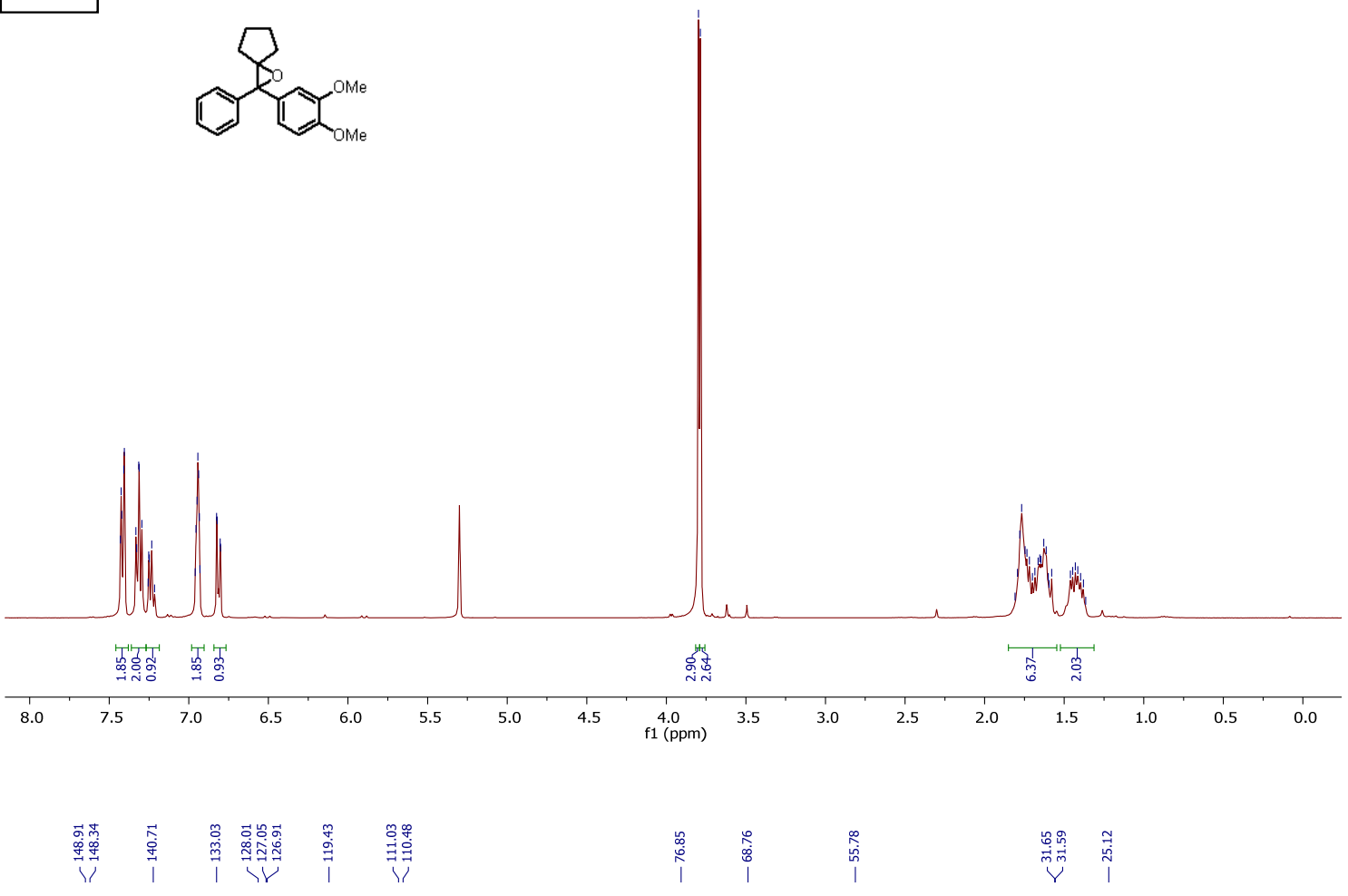

ฟึ)

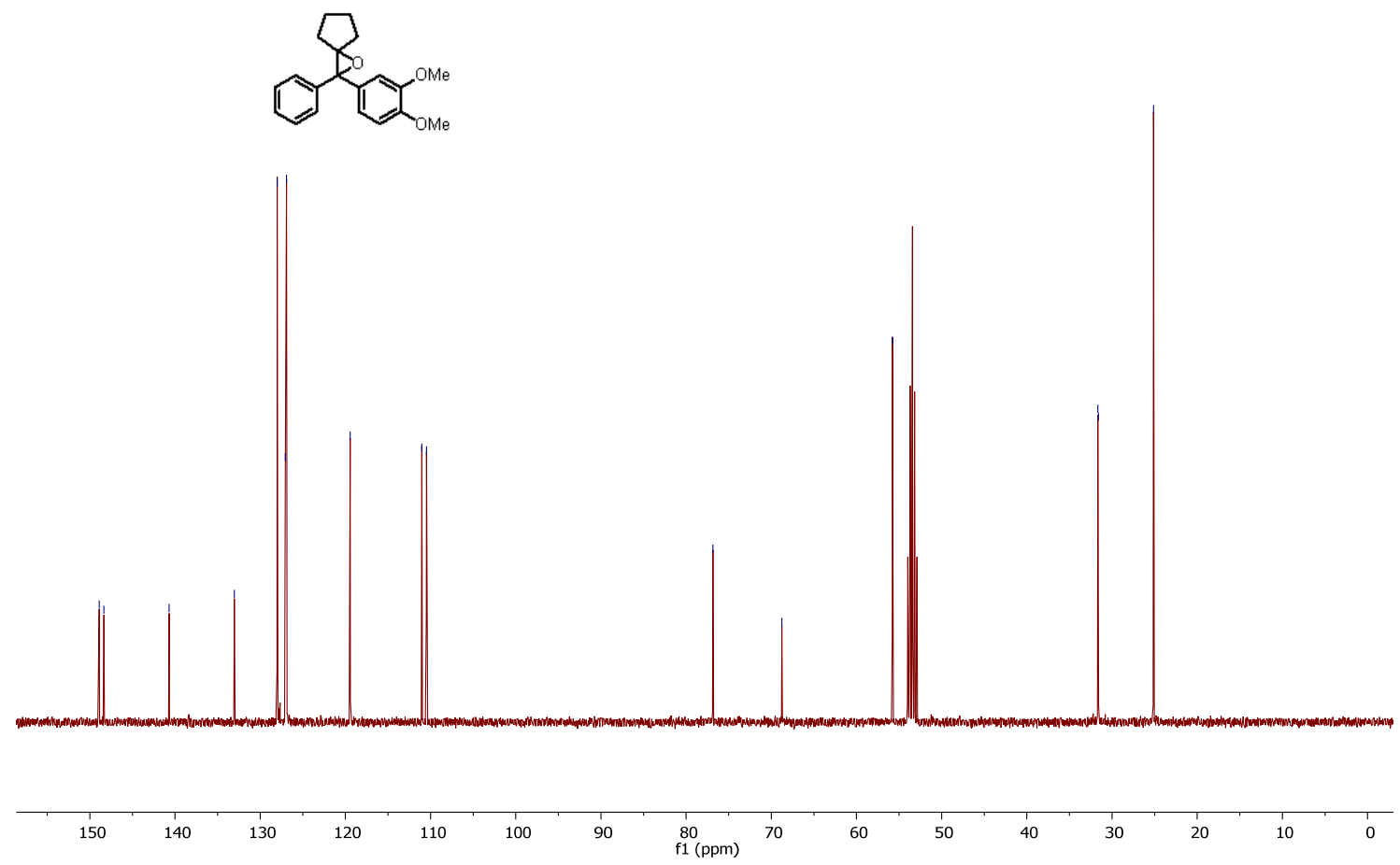

S116 


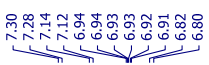

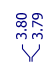

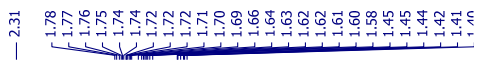

$6 d$
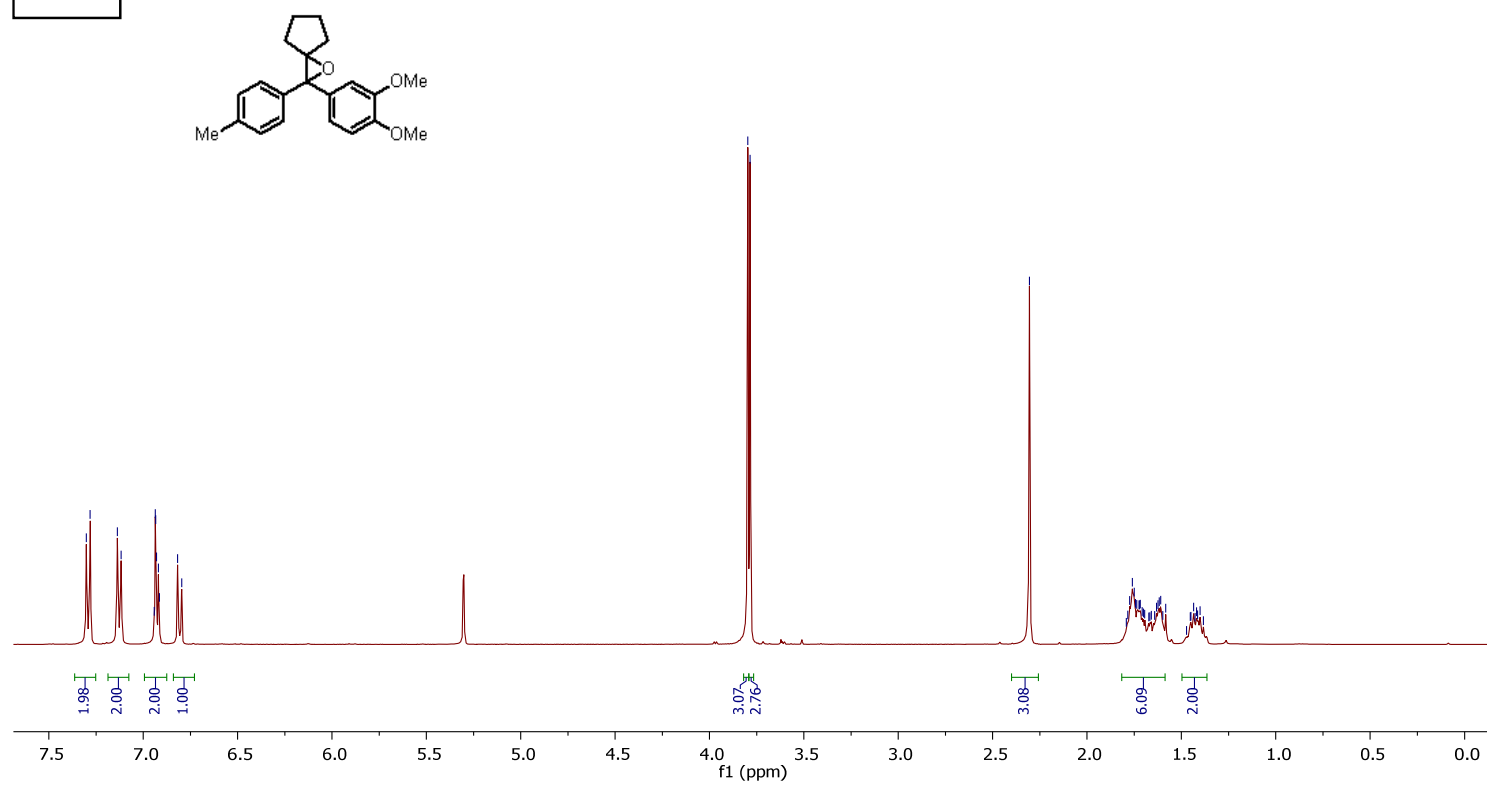

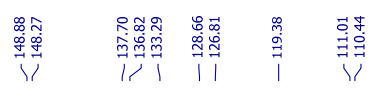

年䉕

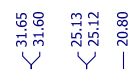

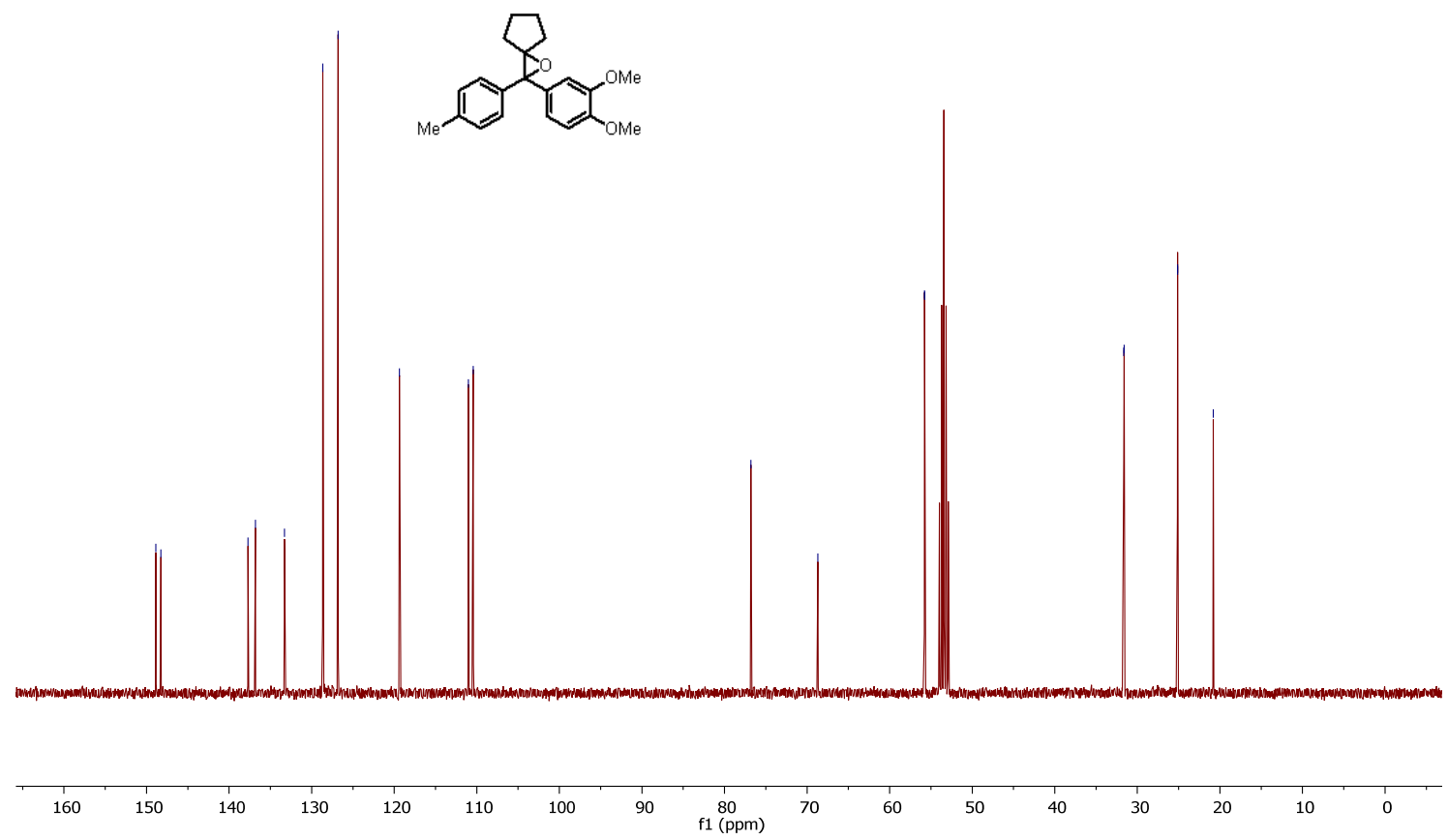

S117 
$6 e$

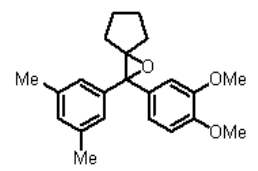

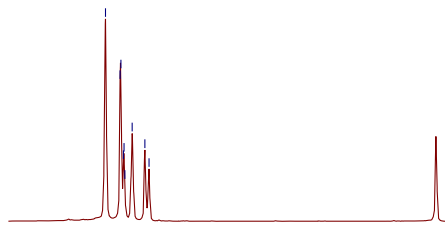

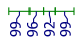

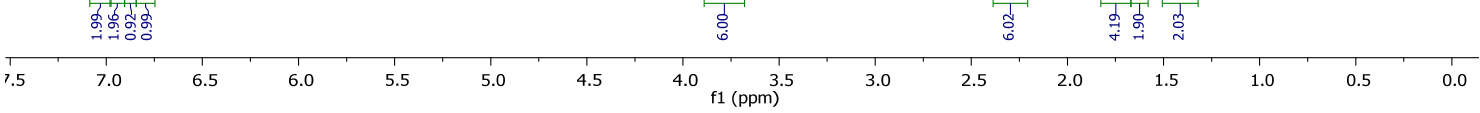

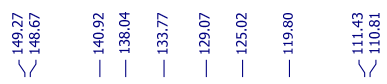

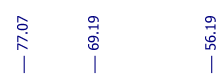

ฟin
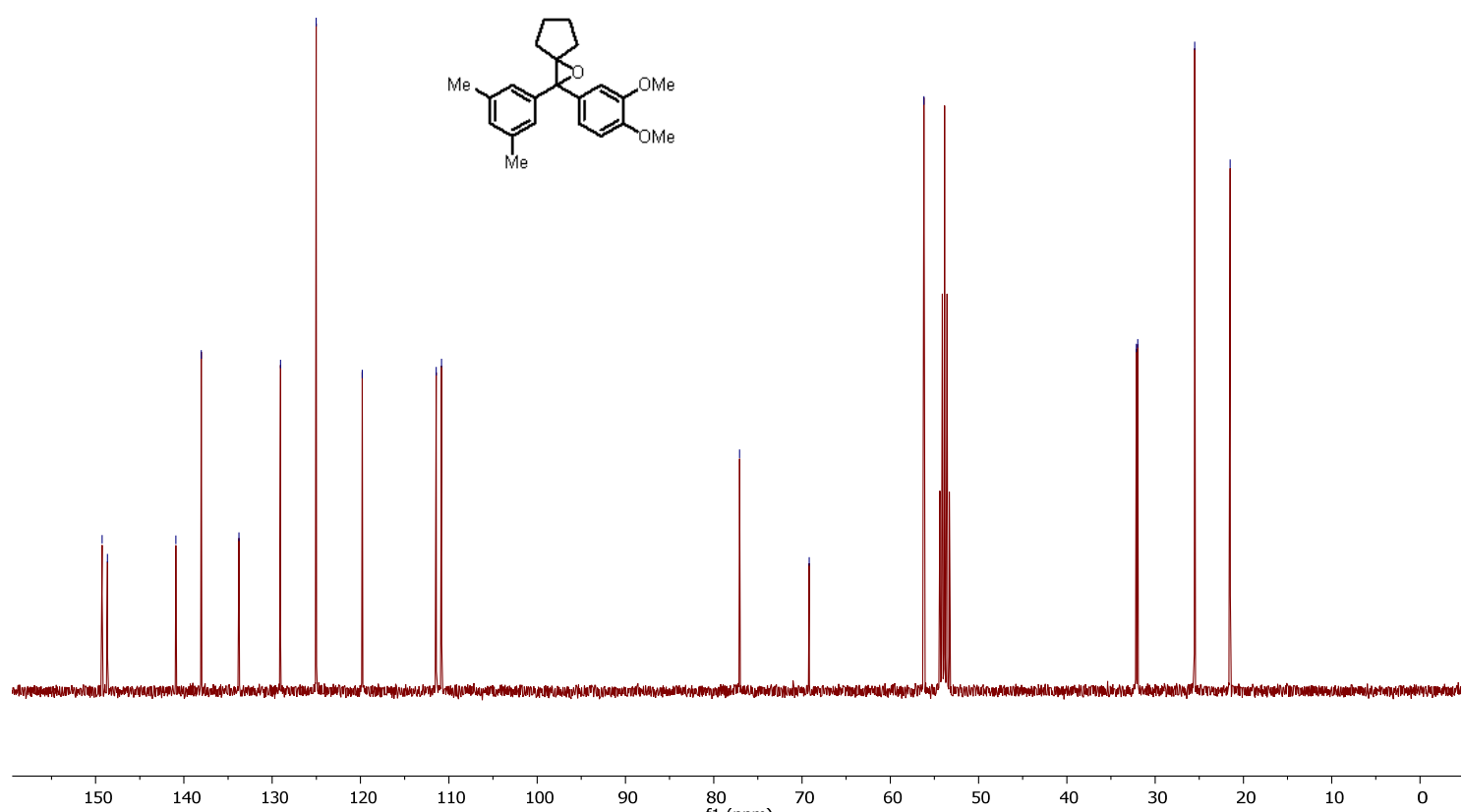

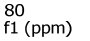
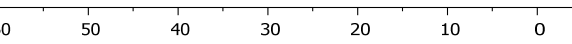
$6 f$<smiles>COc1cc(C2(c3cccc(Cl)c3)CCCC2)ccc1O</smiles>
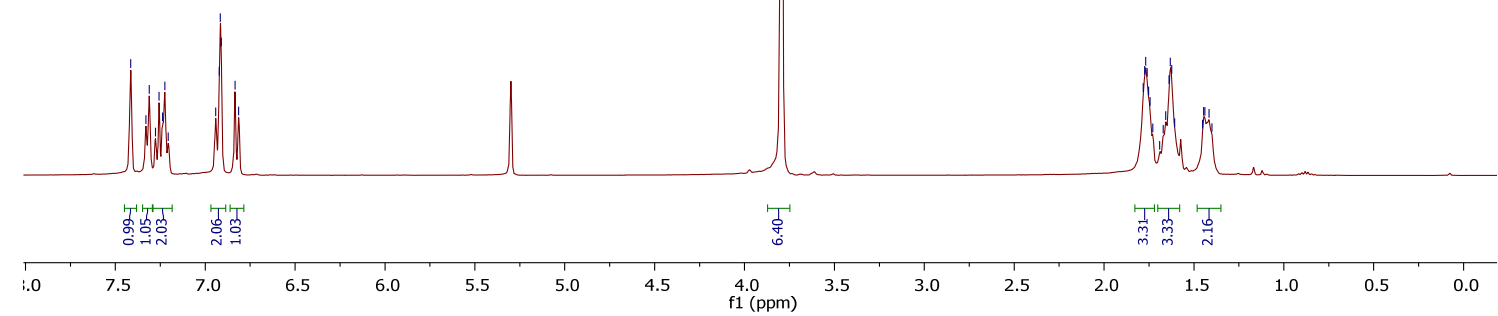

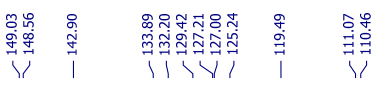

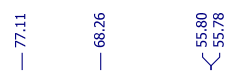

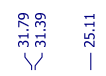<smiles>COc1ccc(C2(c3cccc(Cl)c3)OC23CCCC3)cc1OC</smiles>

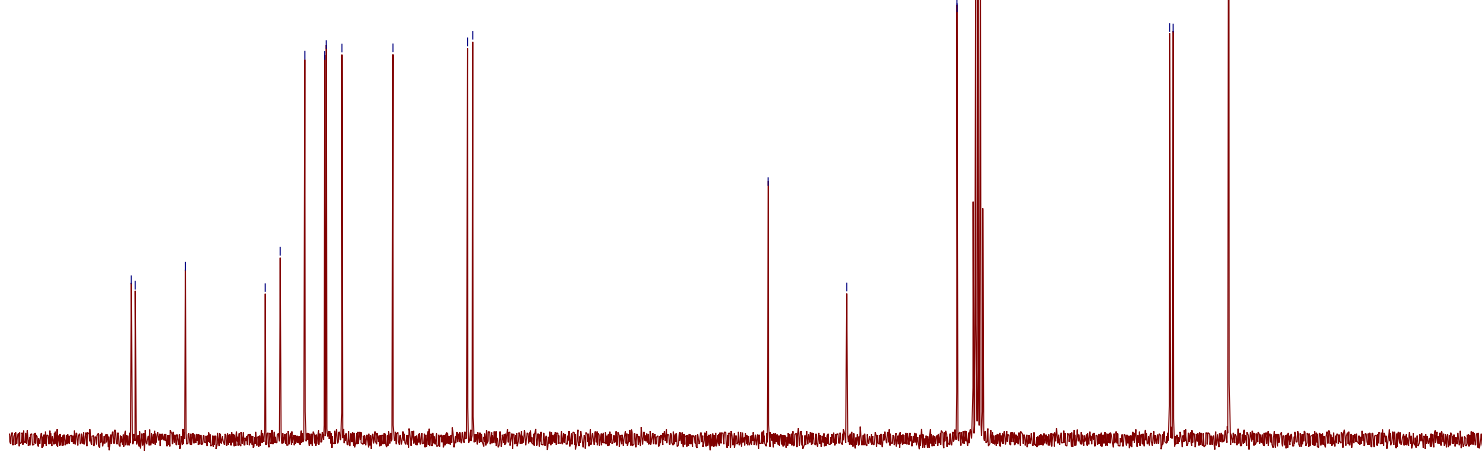

160

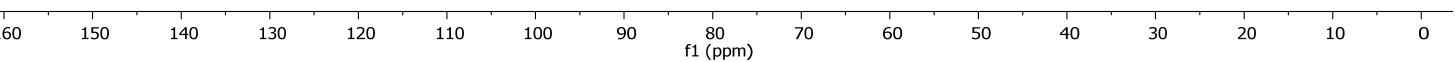


$6 \mathrm{j}$
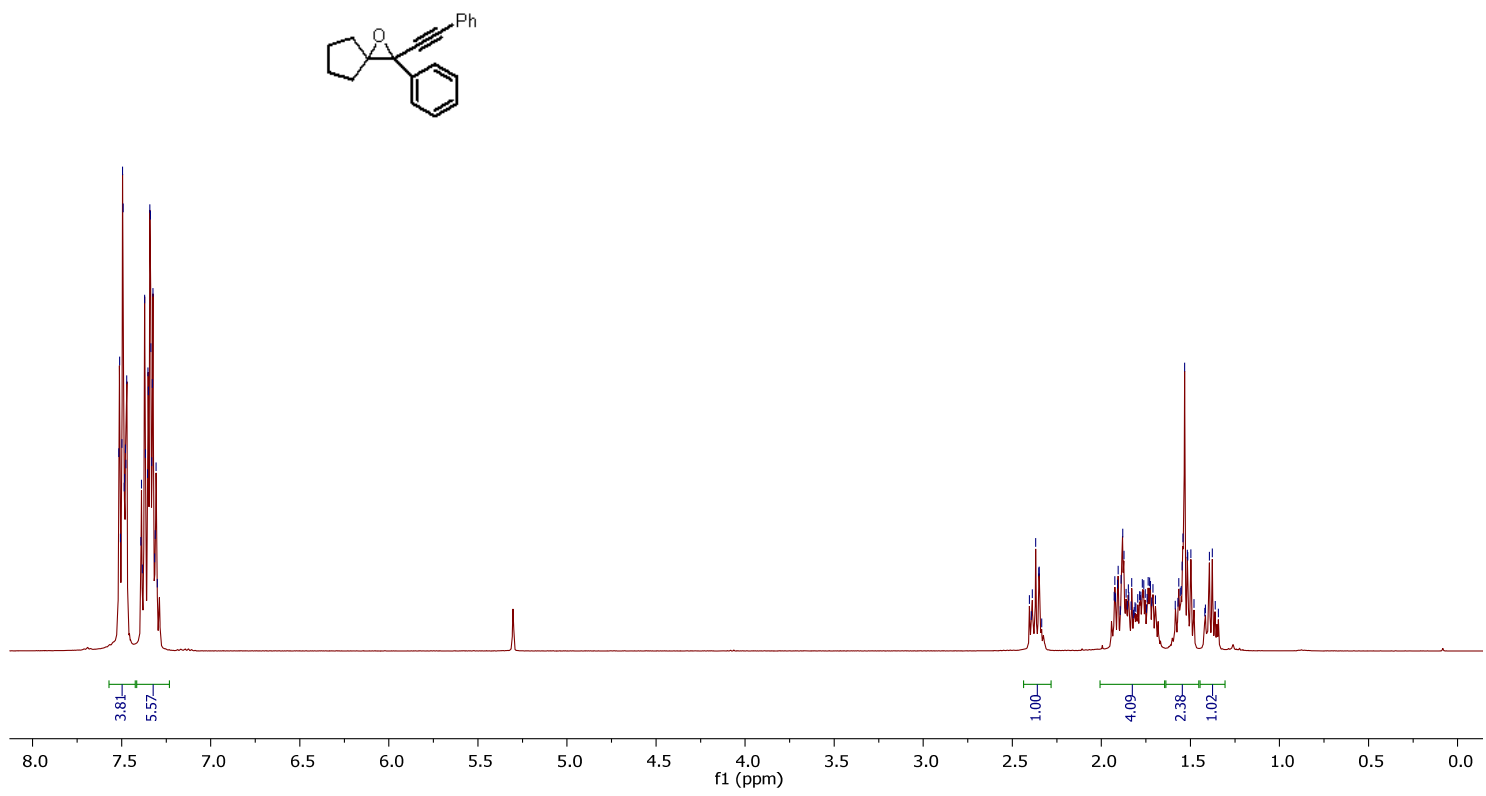

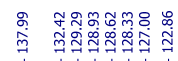

|

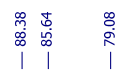

\begin{tabular}{l}
0 \\
0 \\
0 \\
\hline
\end{tabular}

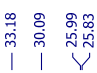

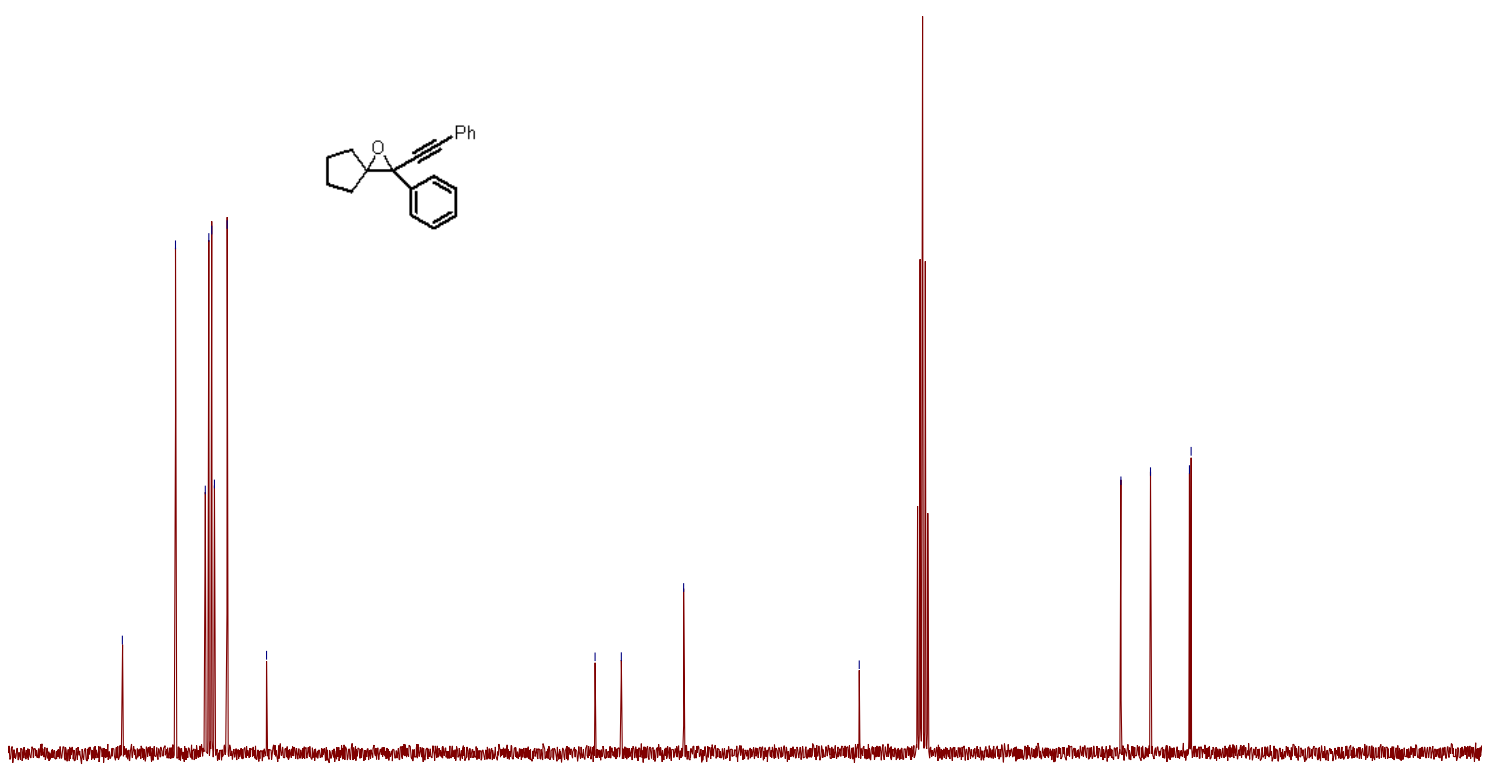

$\begin{array}{llllllllllllllllllllllllllllllllllllll}145 & 140 & 135 & 130 & 125 & 120 & 115 & 110 & 105 & 100 & 95 & 90 & 85 & 80 & 75 & 70 & 65 & 60 & 55 & 50 & 45 & 40 & 35 & 30 & 25 & 20 & 15 & 10 & 5 & 0\end{array}$ 
9

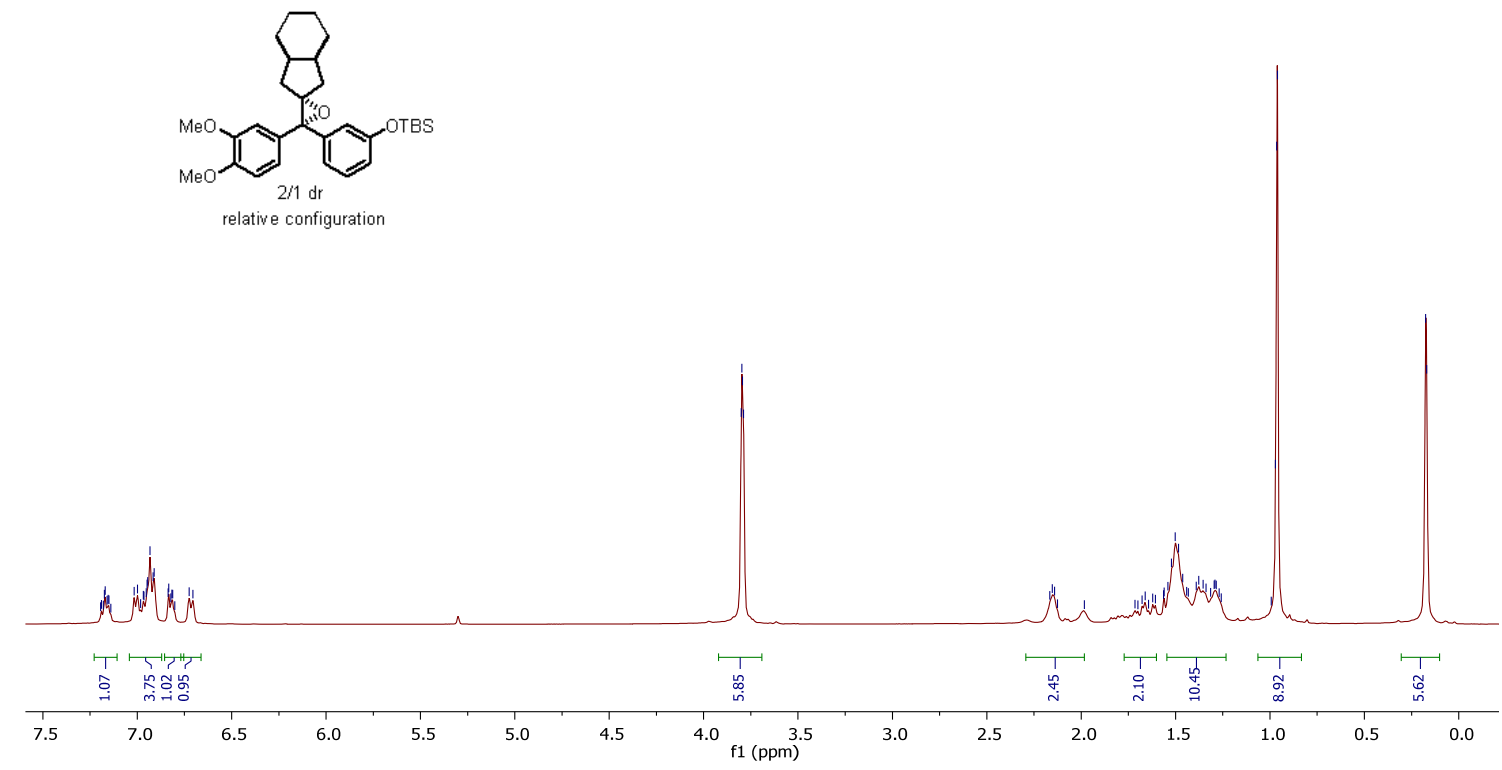

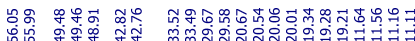

V V V V V

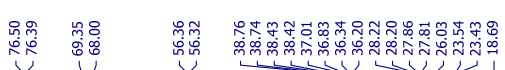

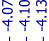
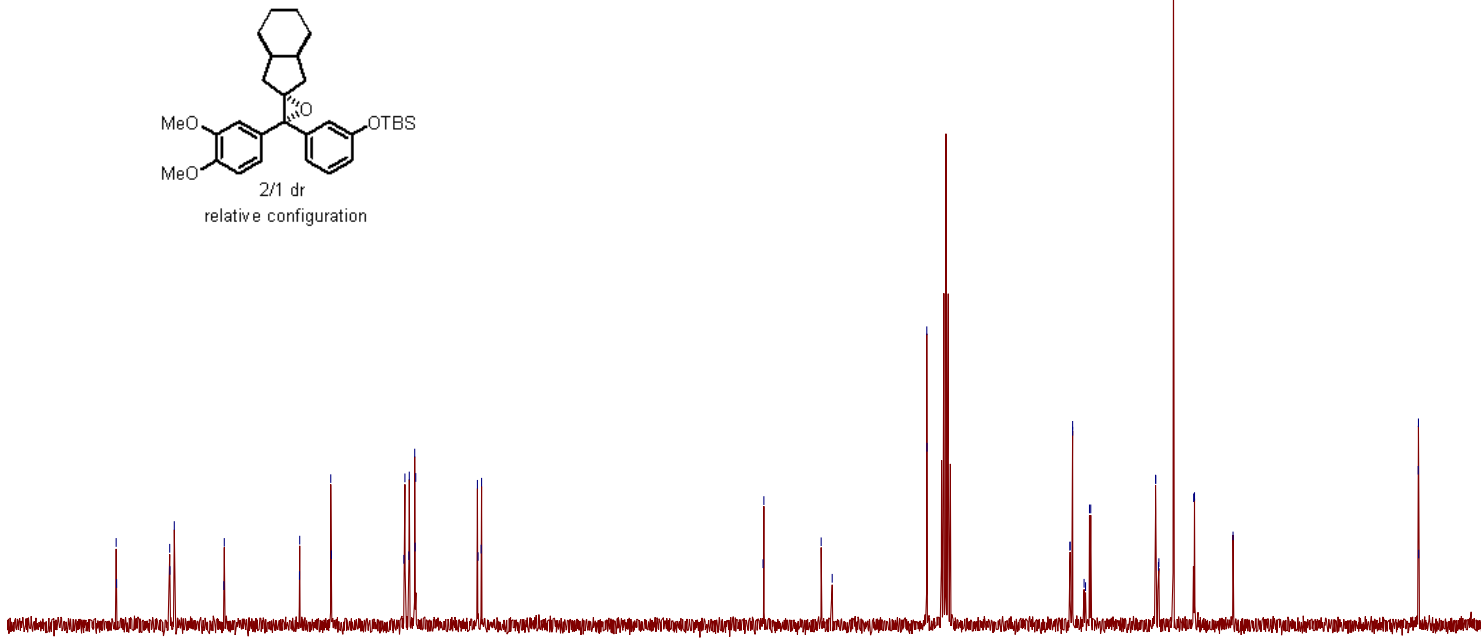

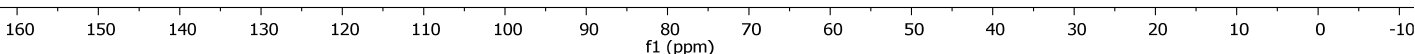




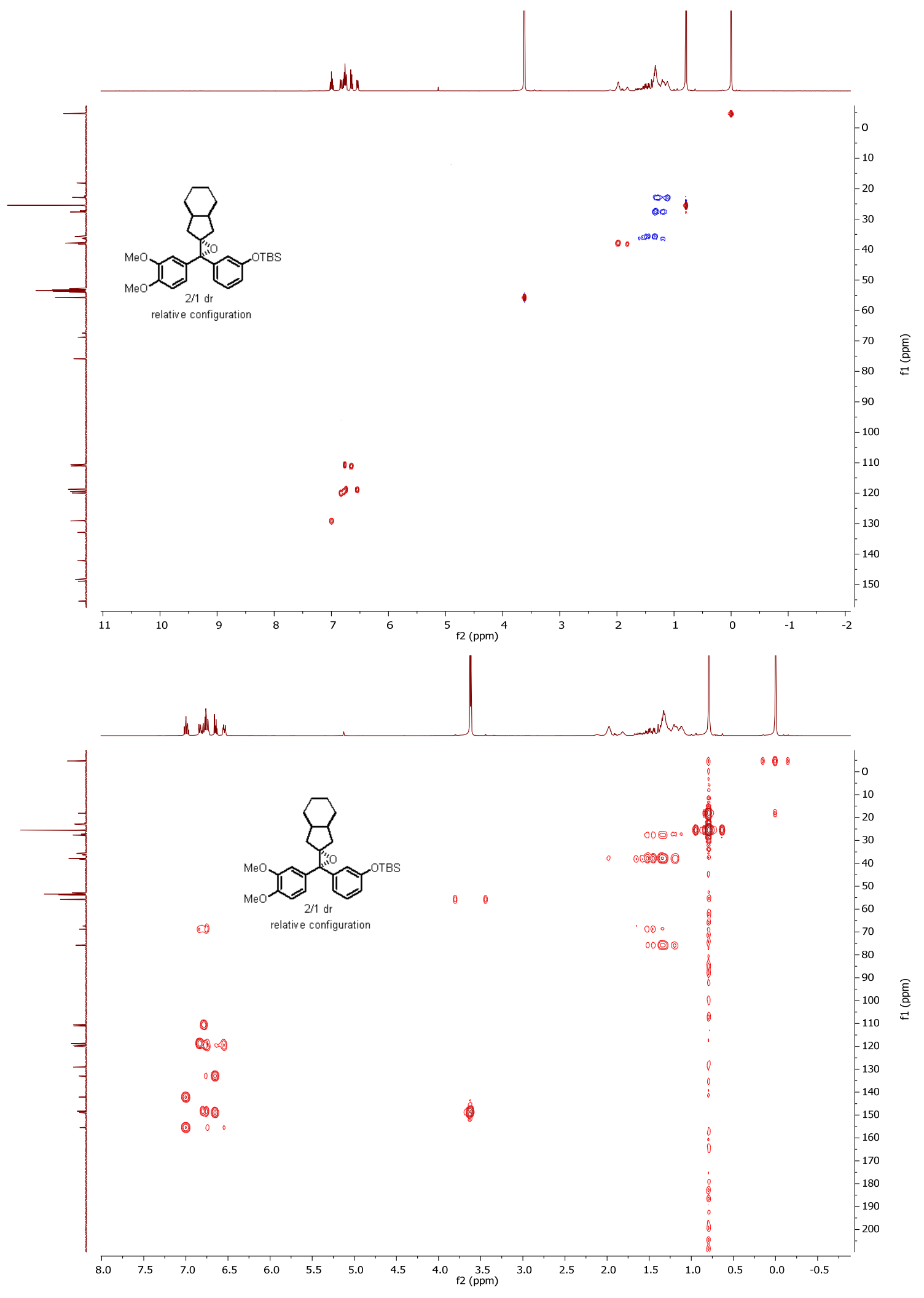




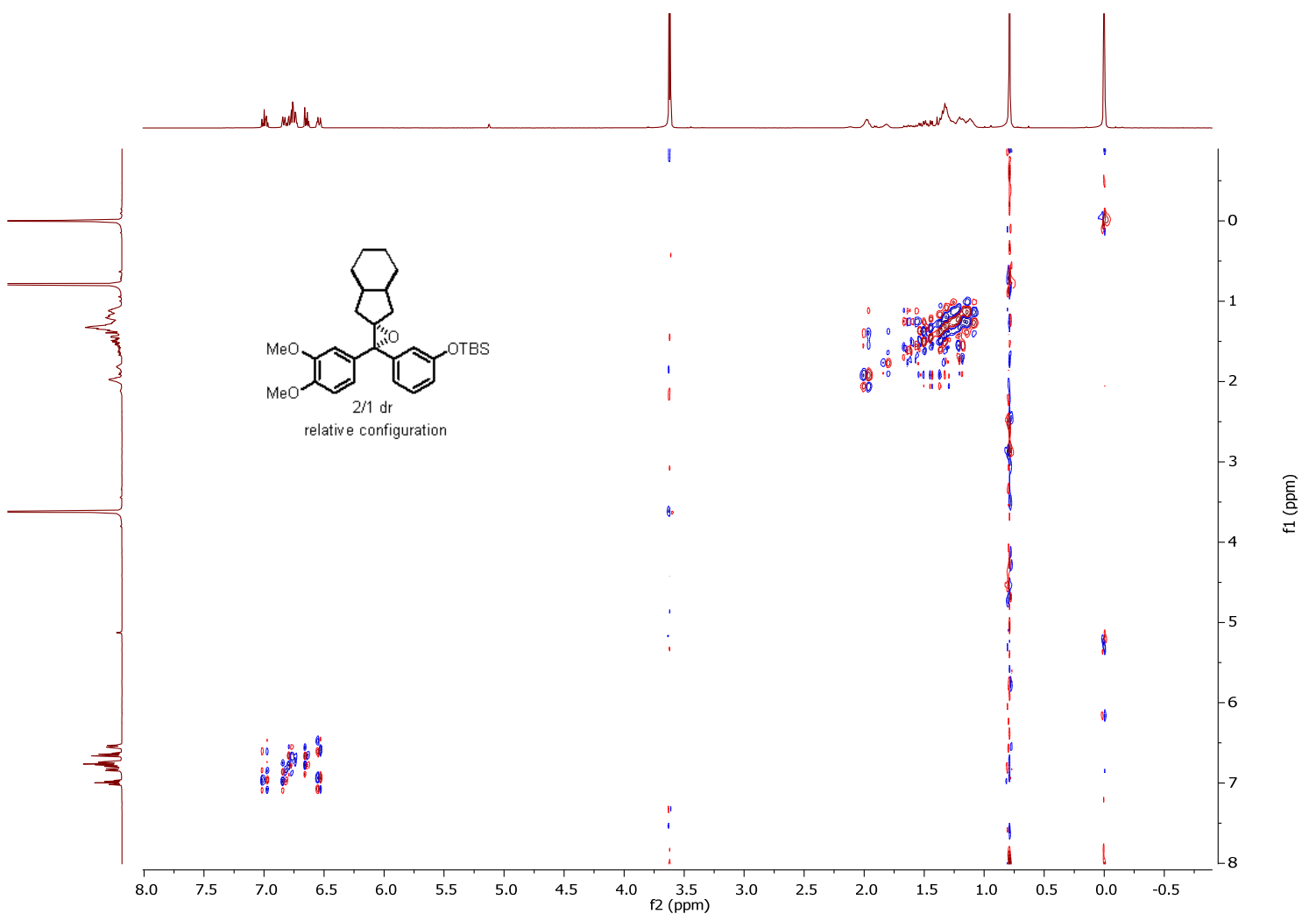




\section{0}

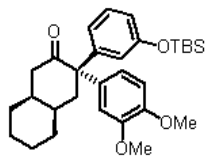

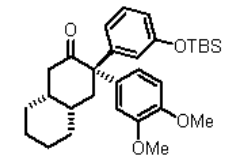

2

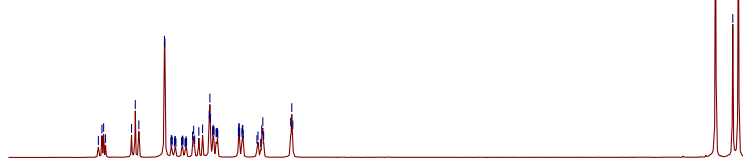

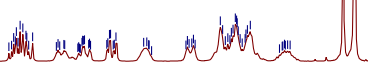

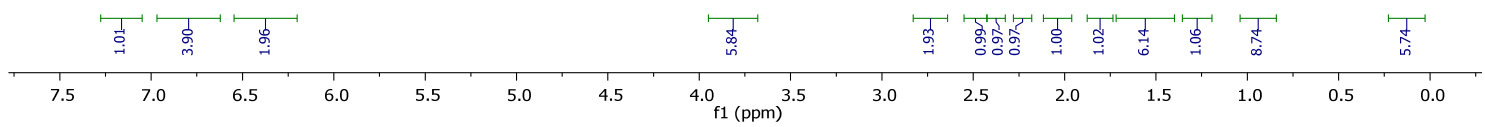

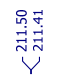
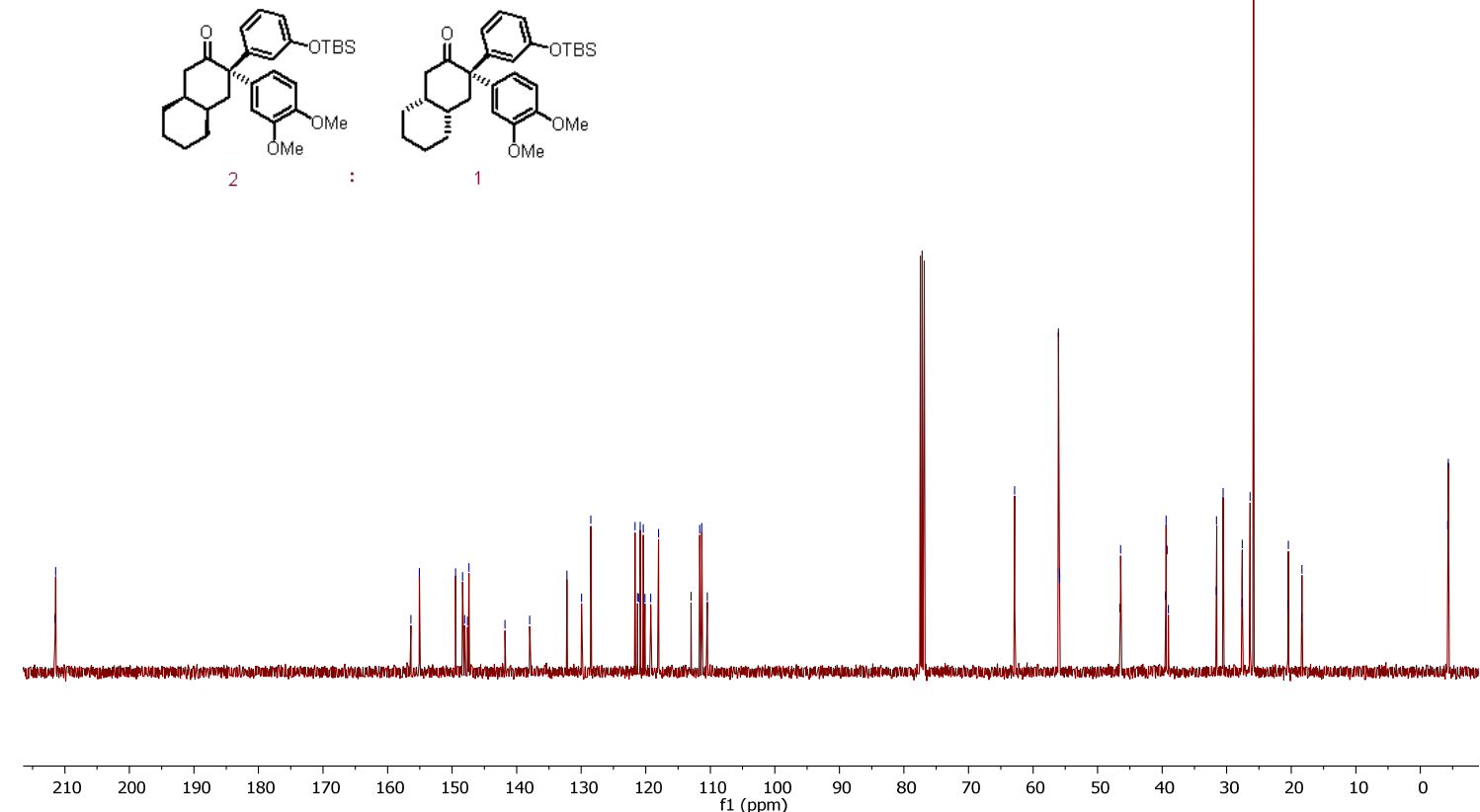


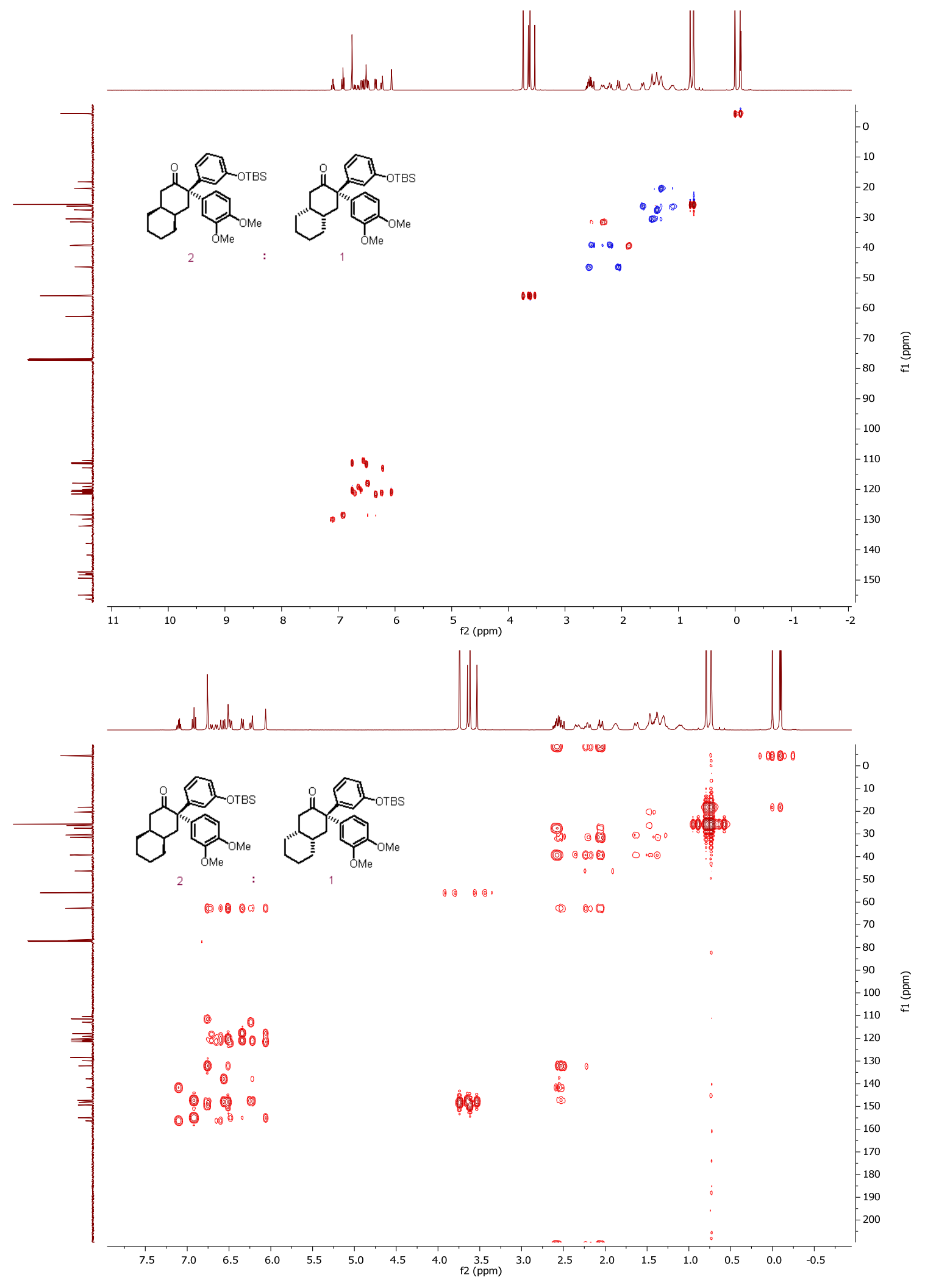




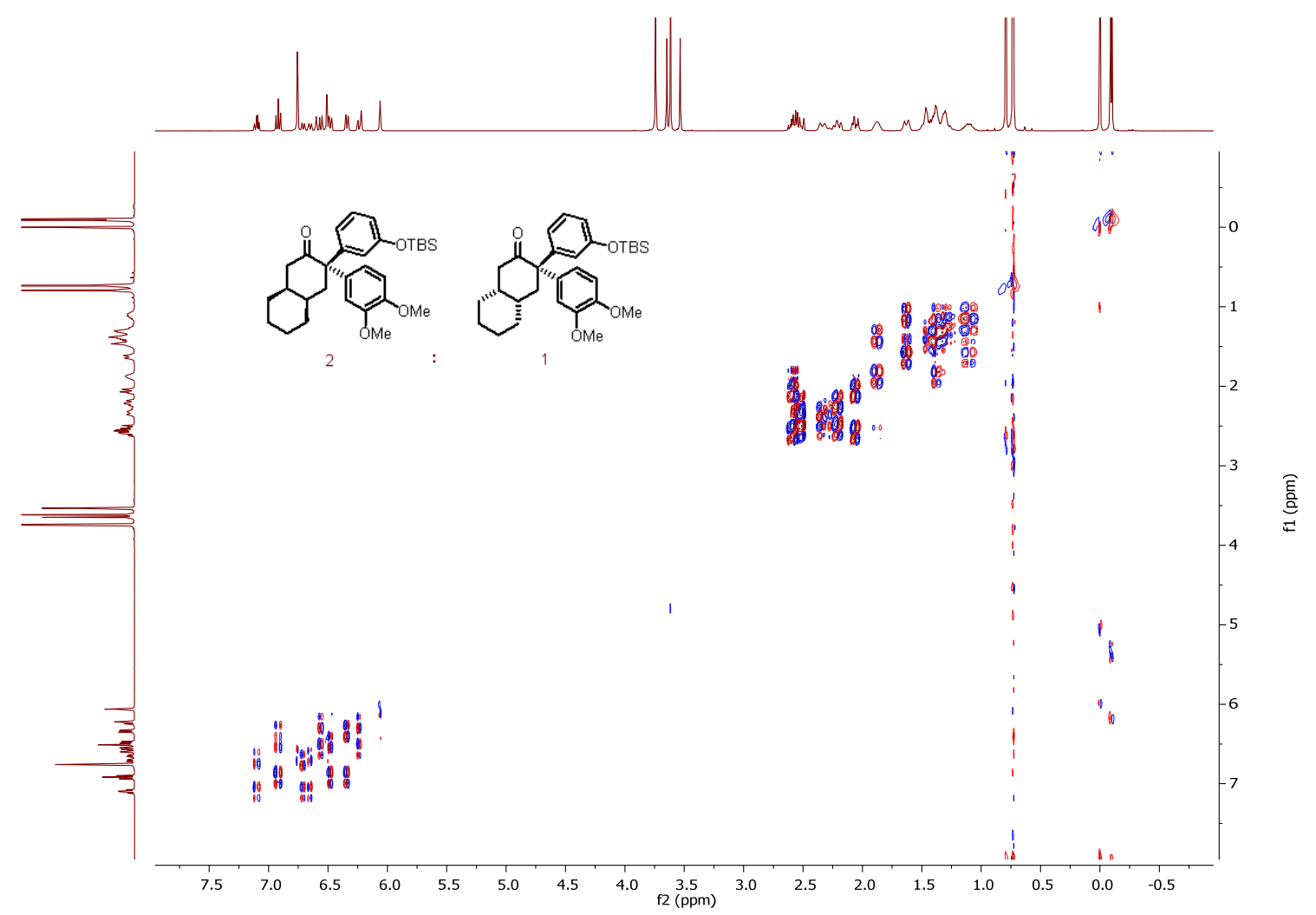


11<smiles>Cc1ccc(C=C2CCCC2=O)cc1</smiles>
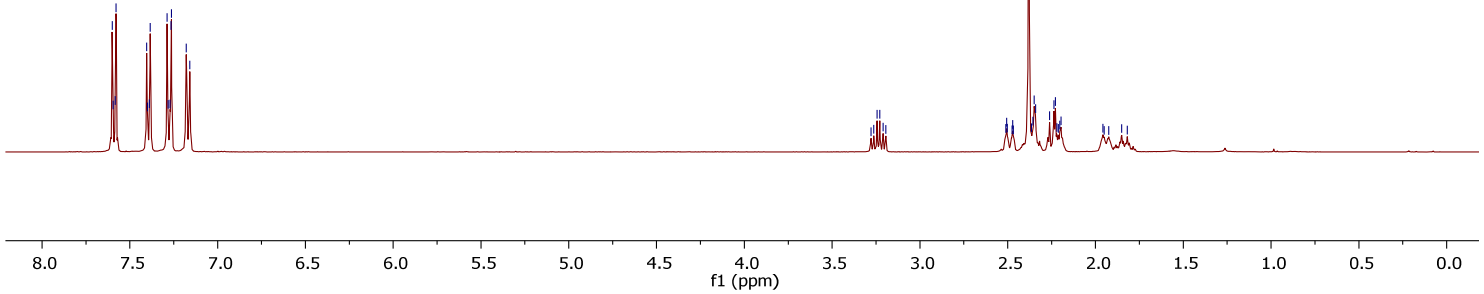

\ْ

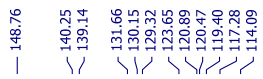

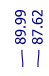

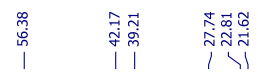

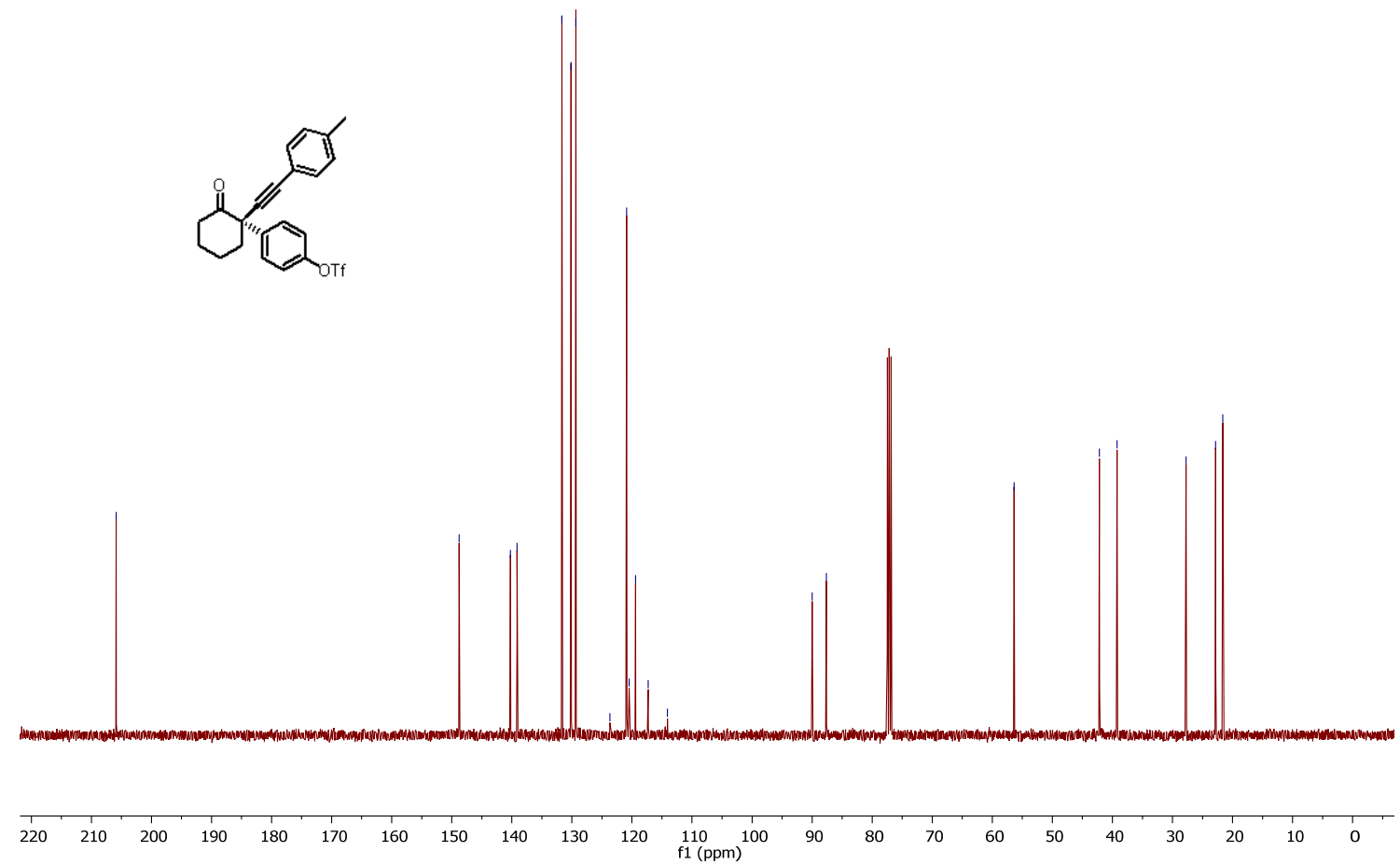


12

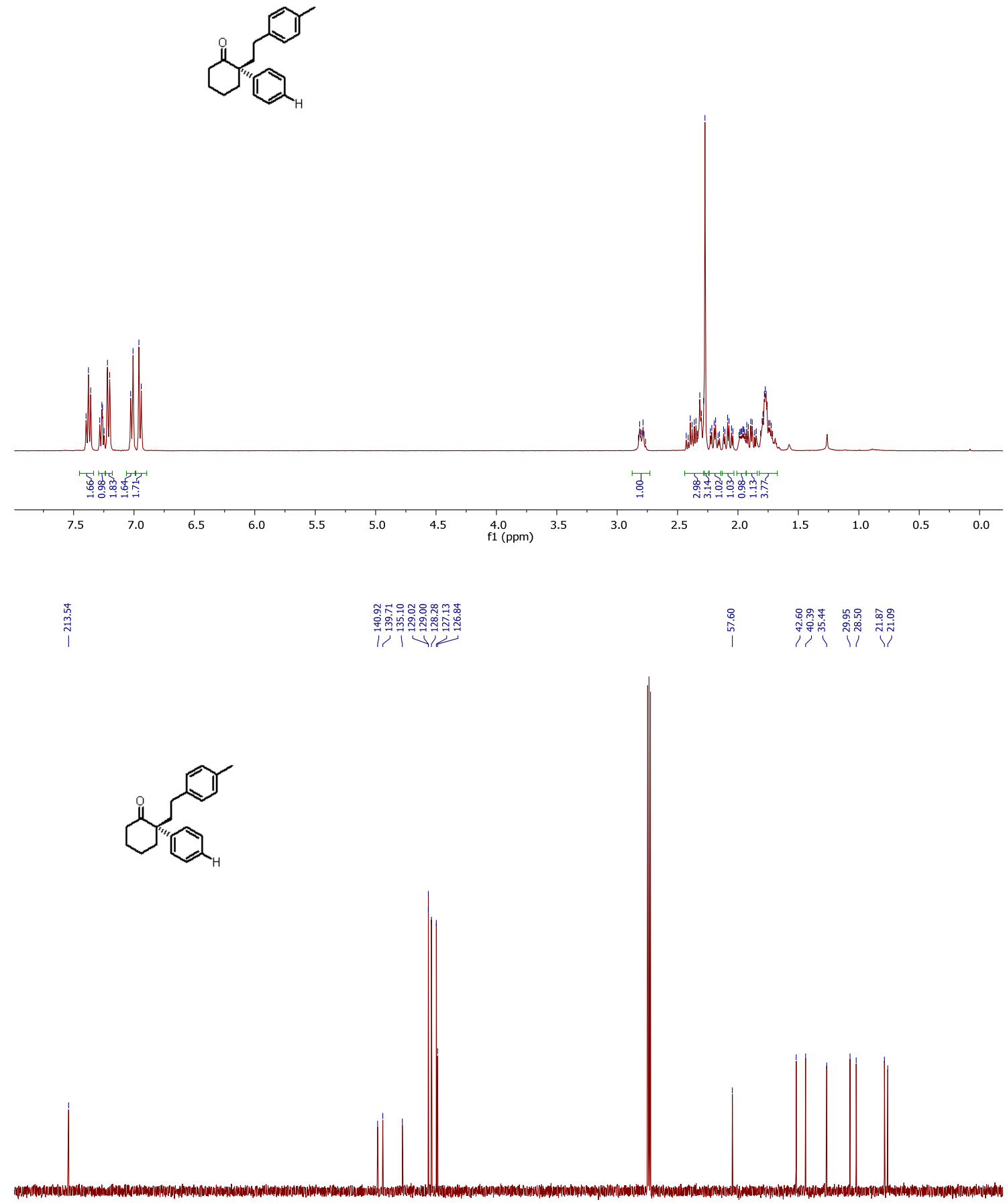

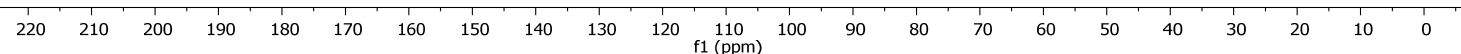




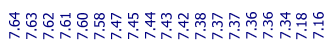

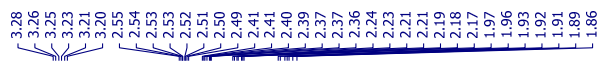

13
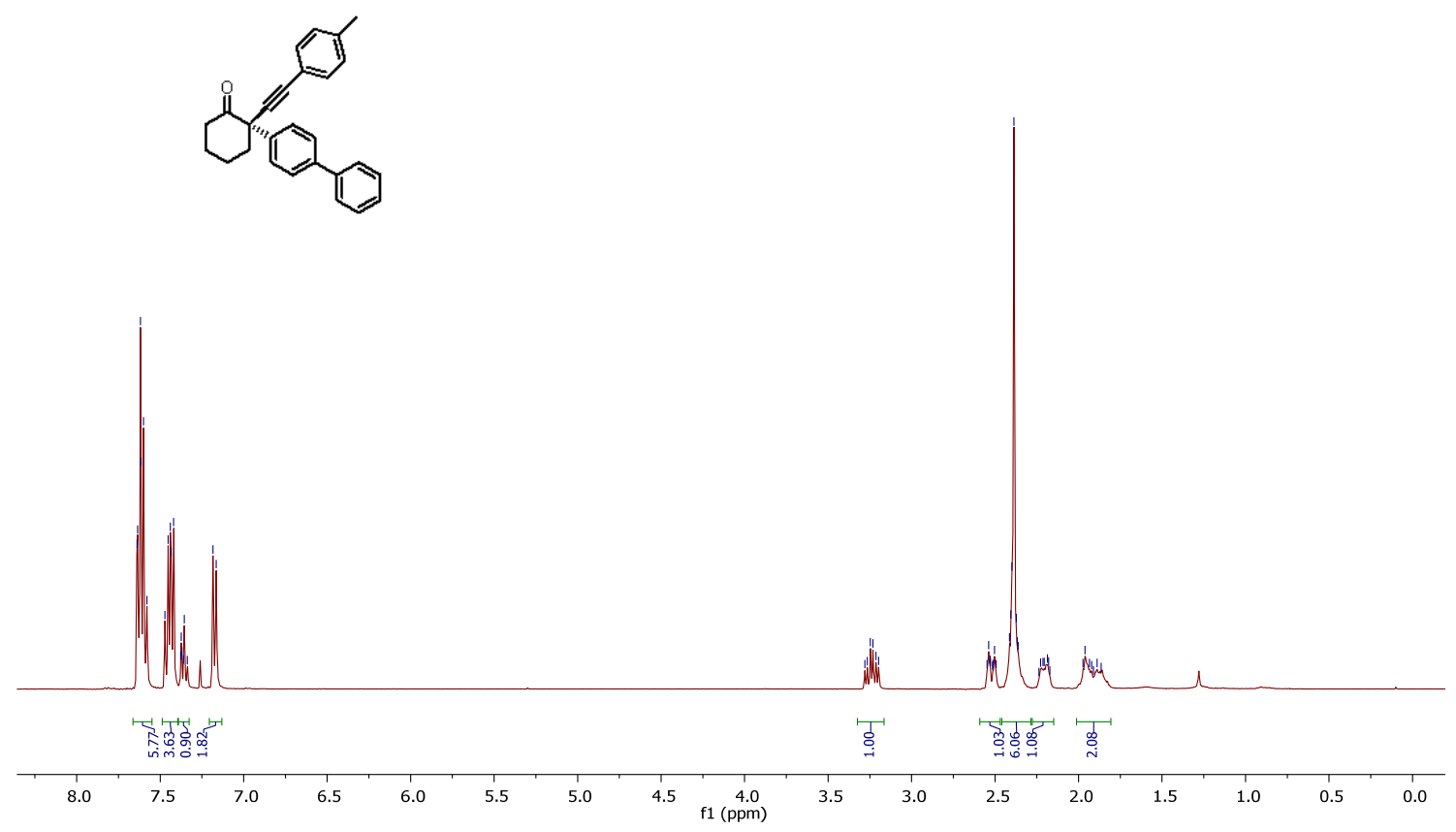

i̊n.

$\underbrace{-10}$

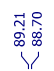

।
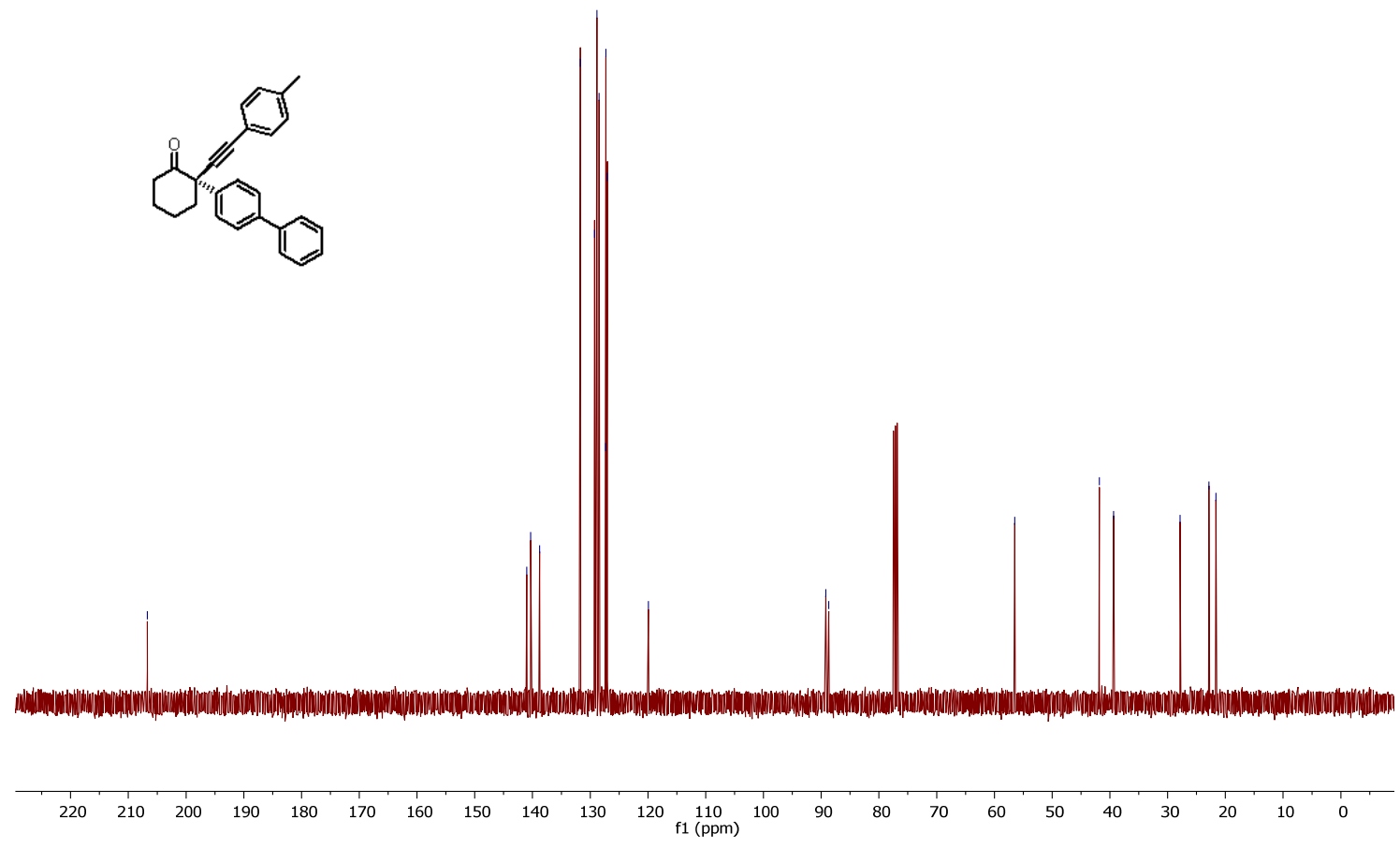

S129 


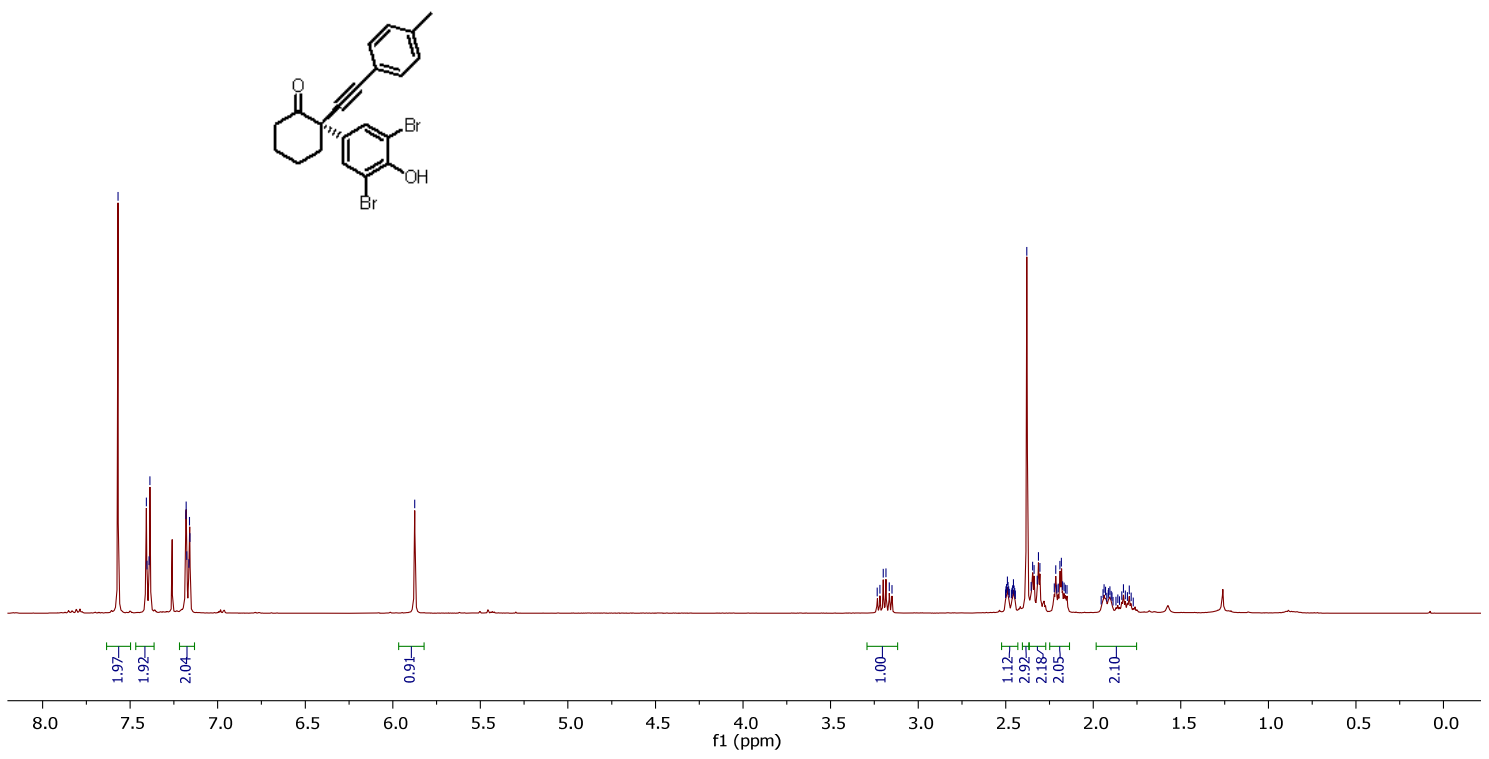

ஸे

| |
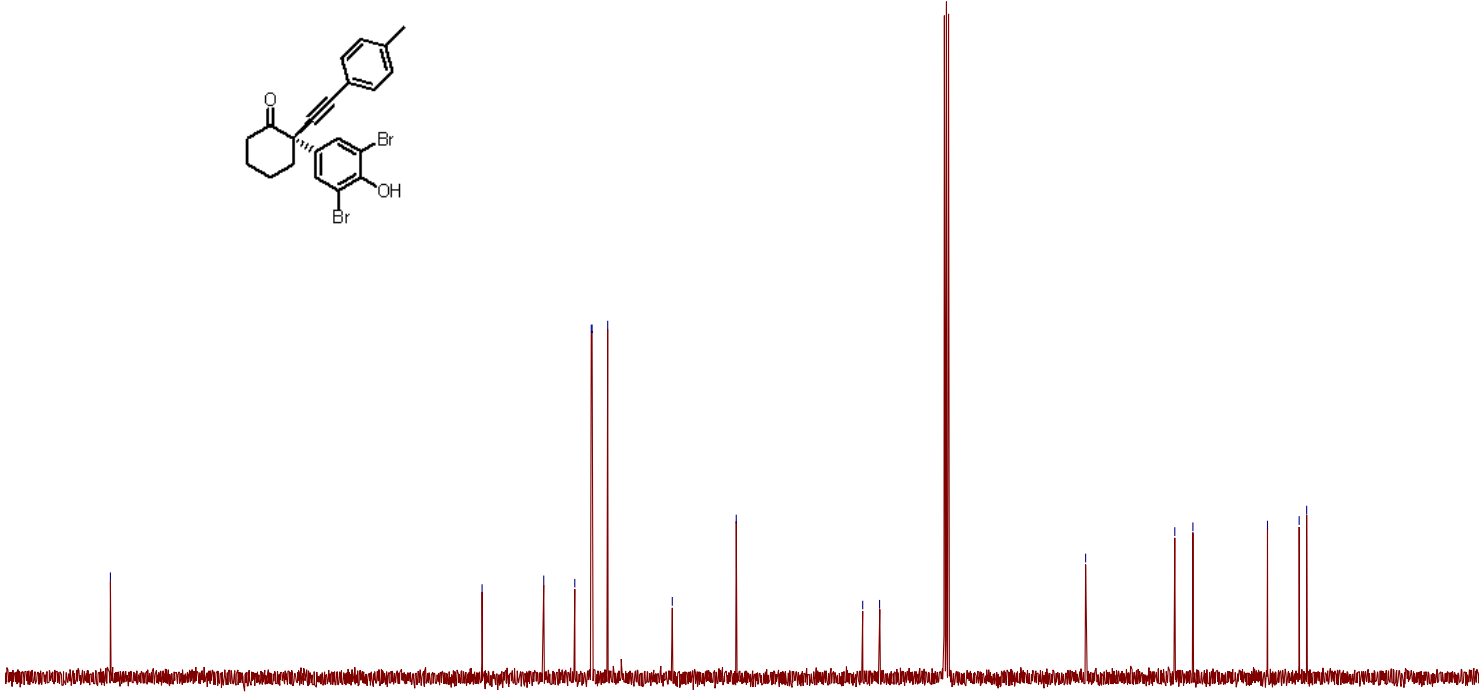
15<smiles>CC#CC1(c2ccc(C)c(C)c2)CCC=C1Cl</smiles>

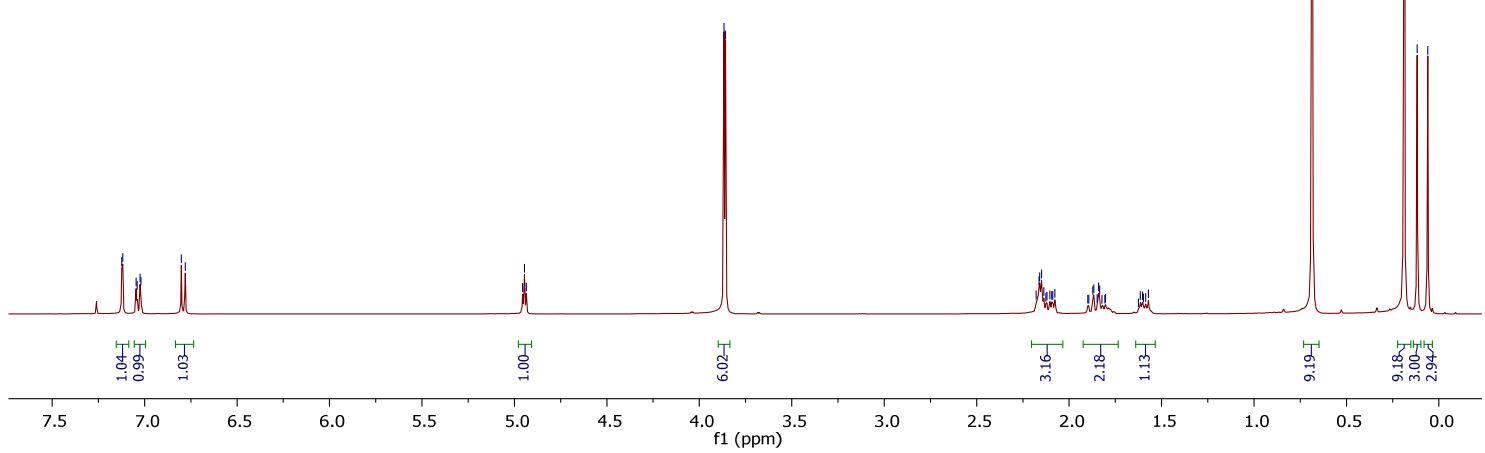

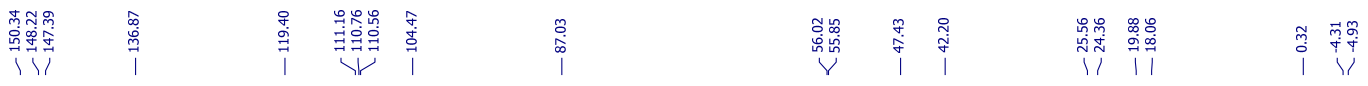<smiles>CCCCCC#CC1(c2ccc(C)c([N+](=O)[O-])c2)CCCC=C1Cl</smiles>
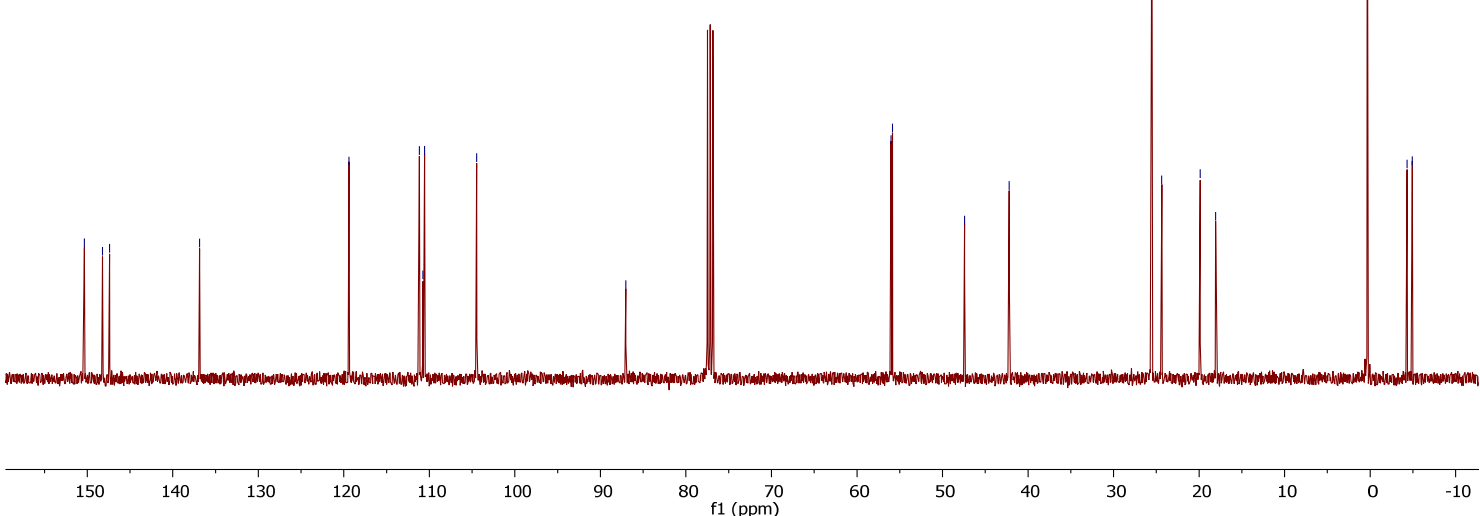

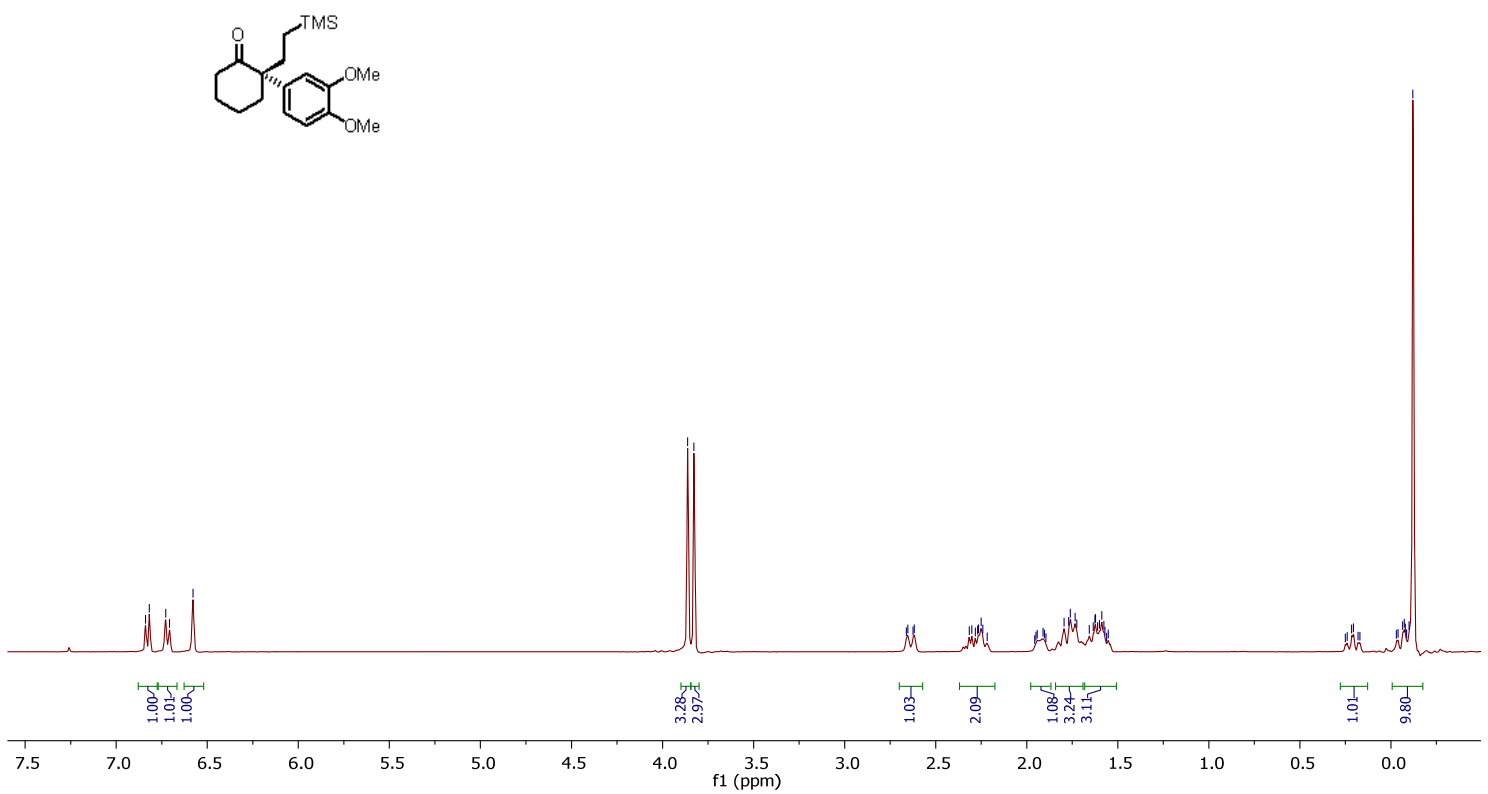

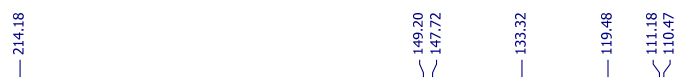
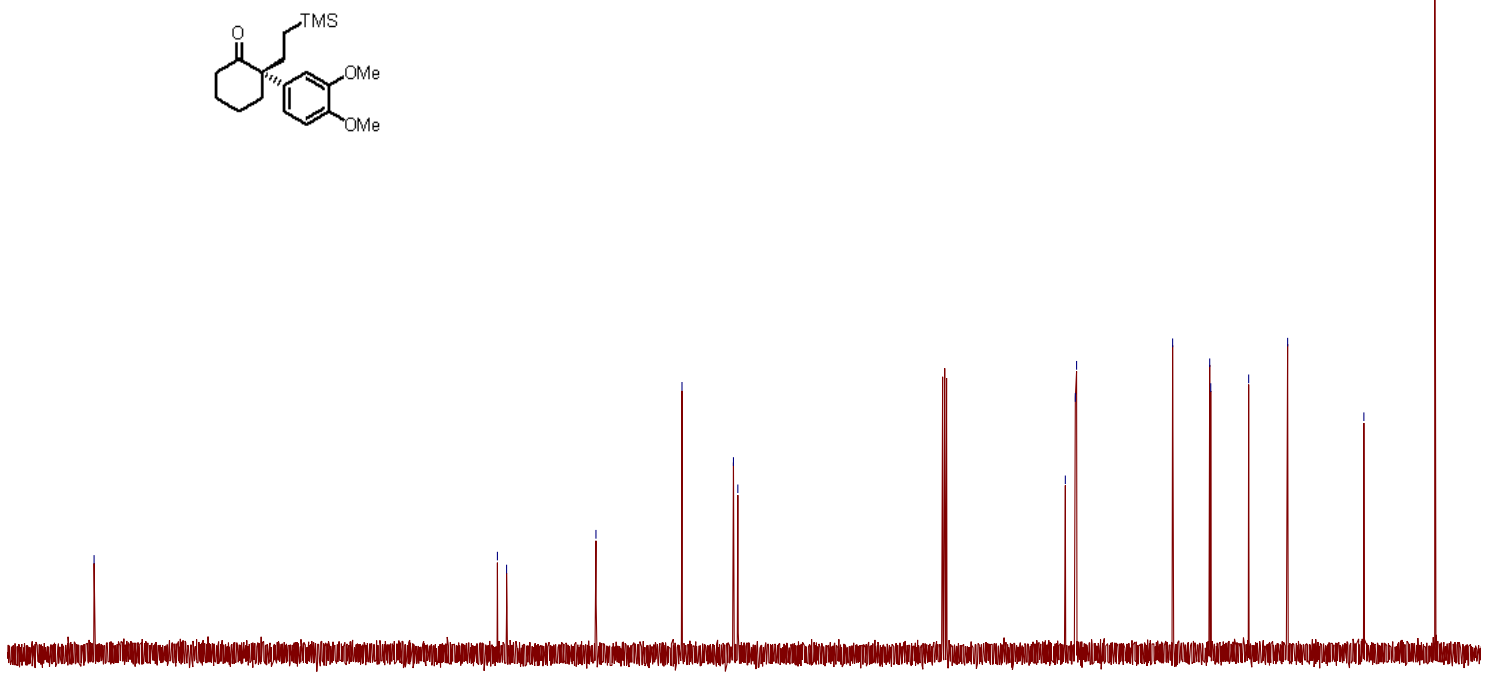

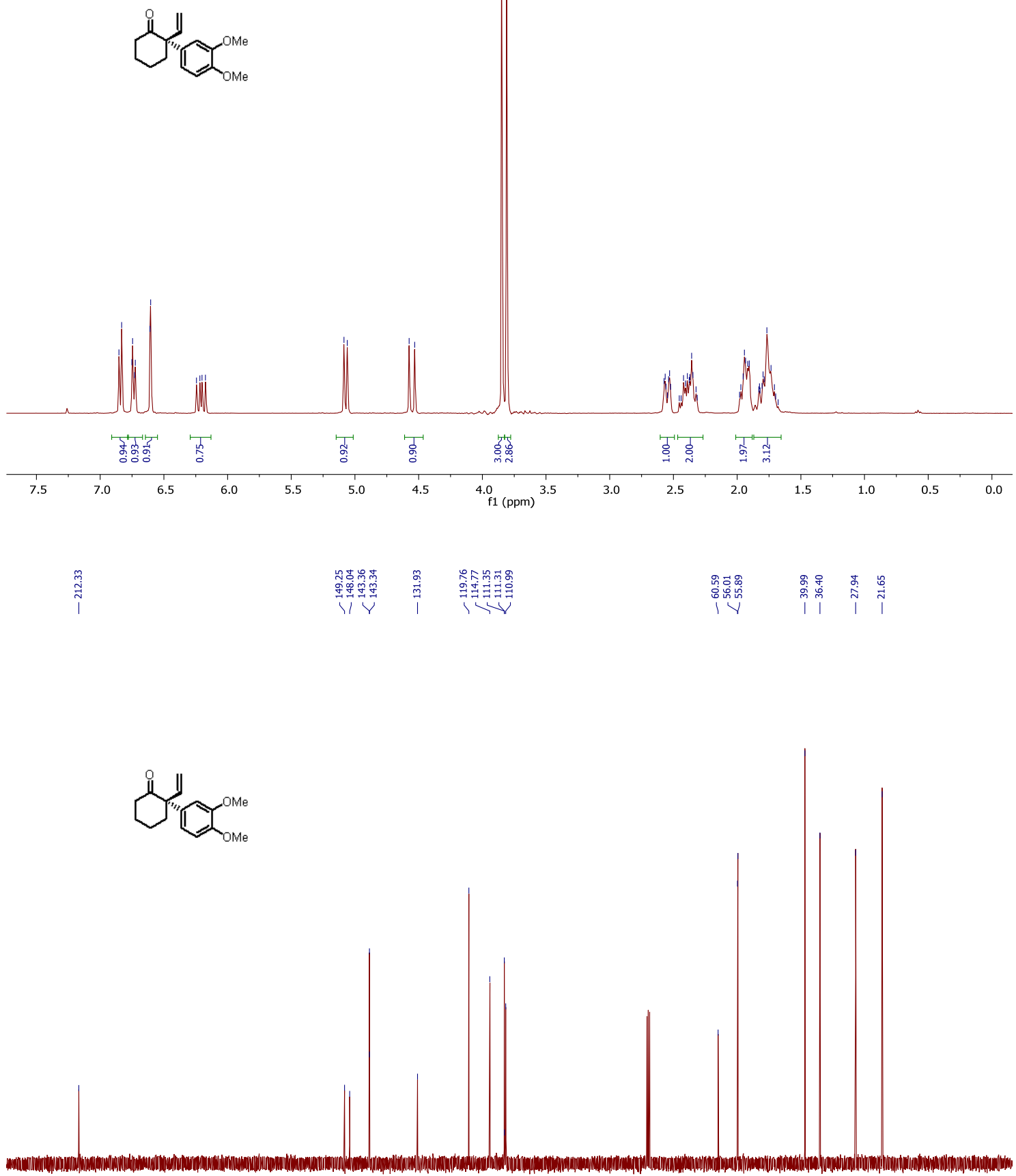

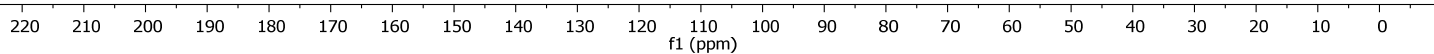



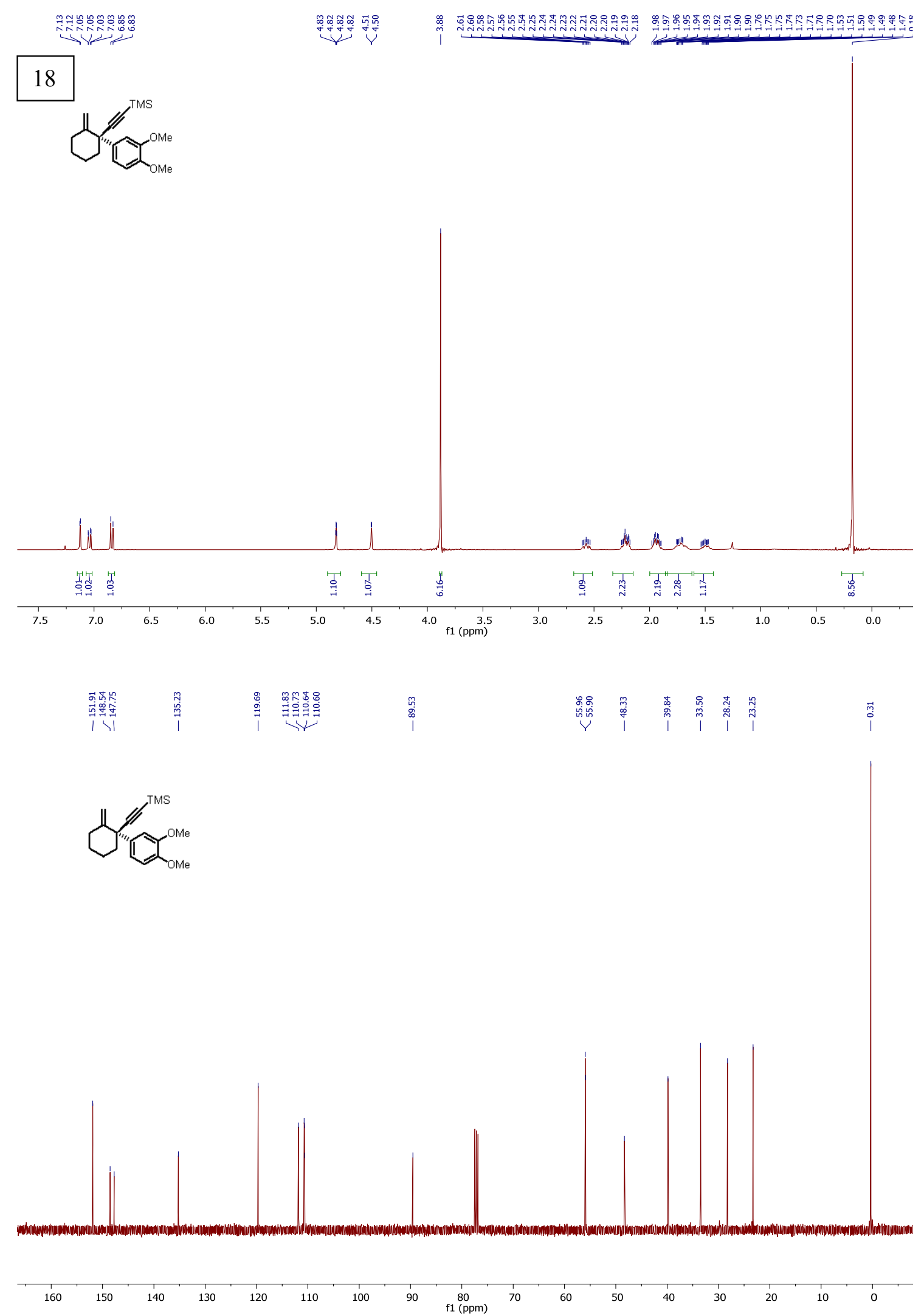
19
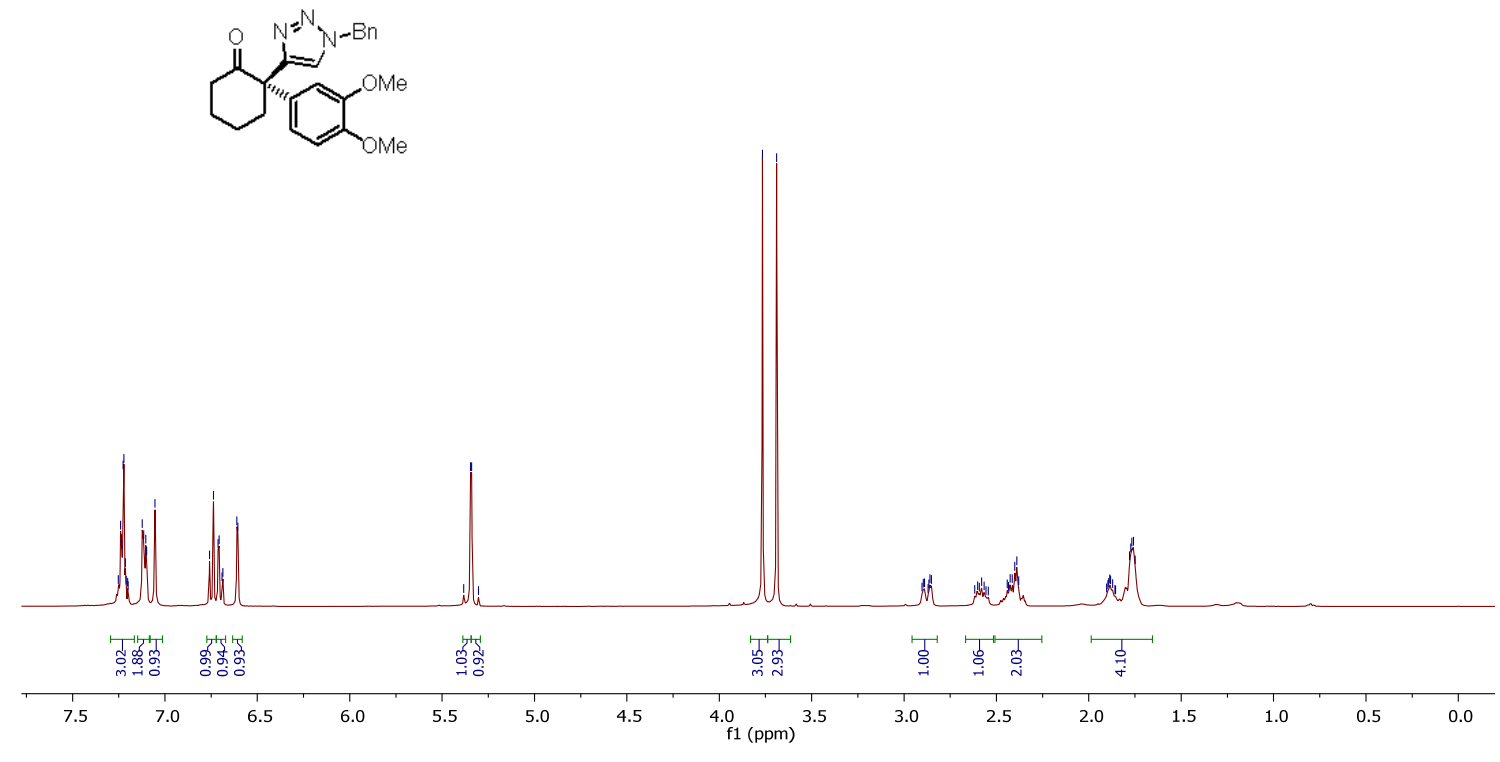

œ

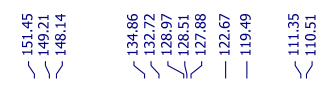

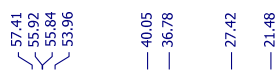
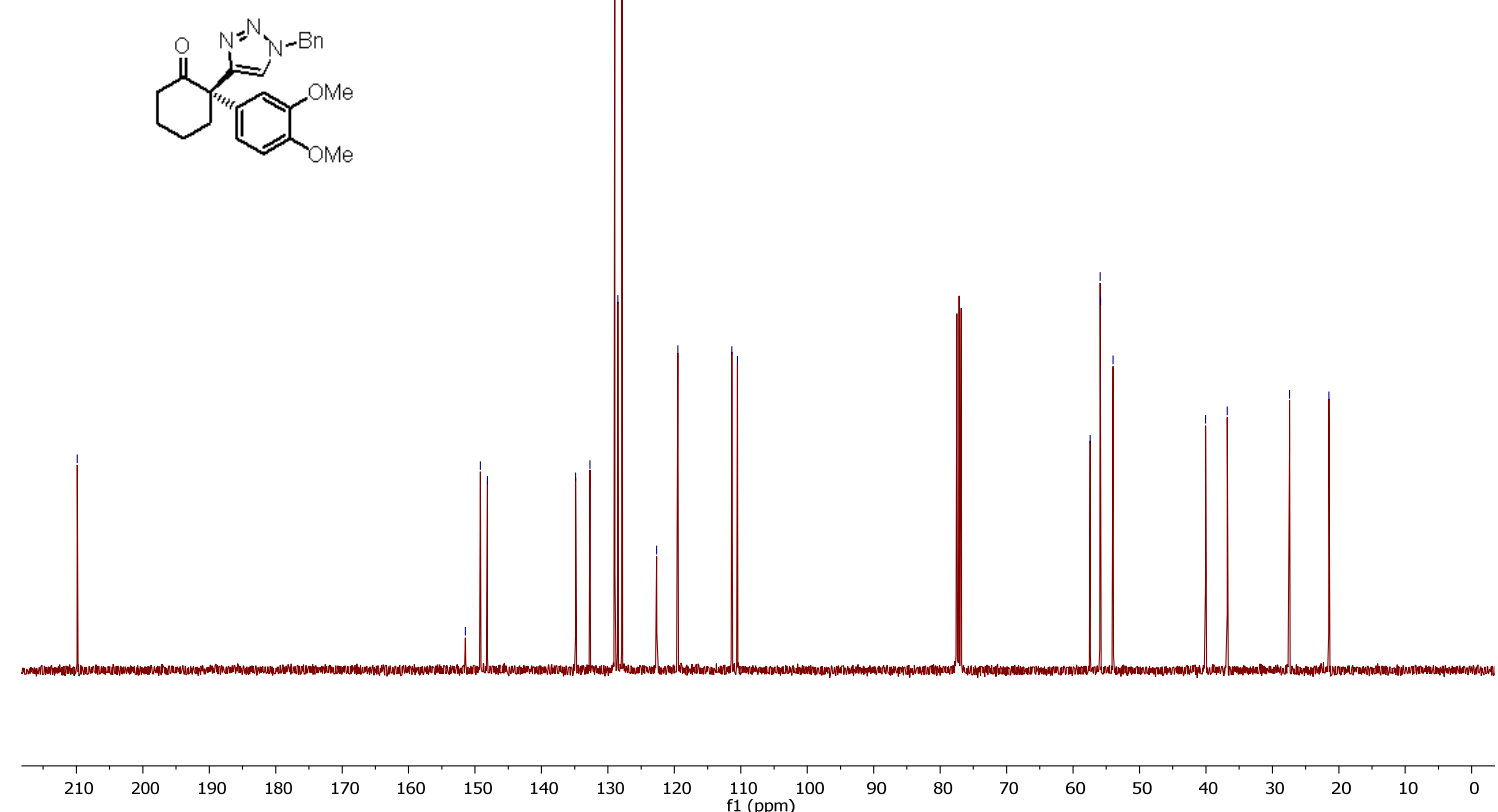
25

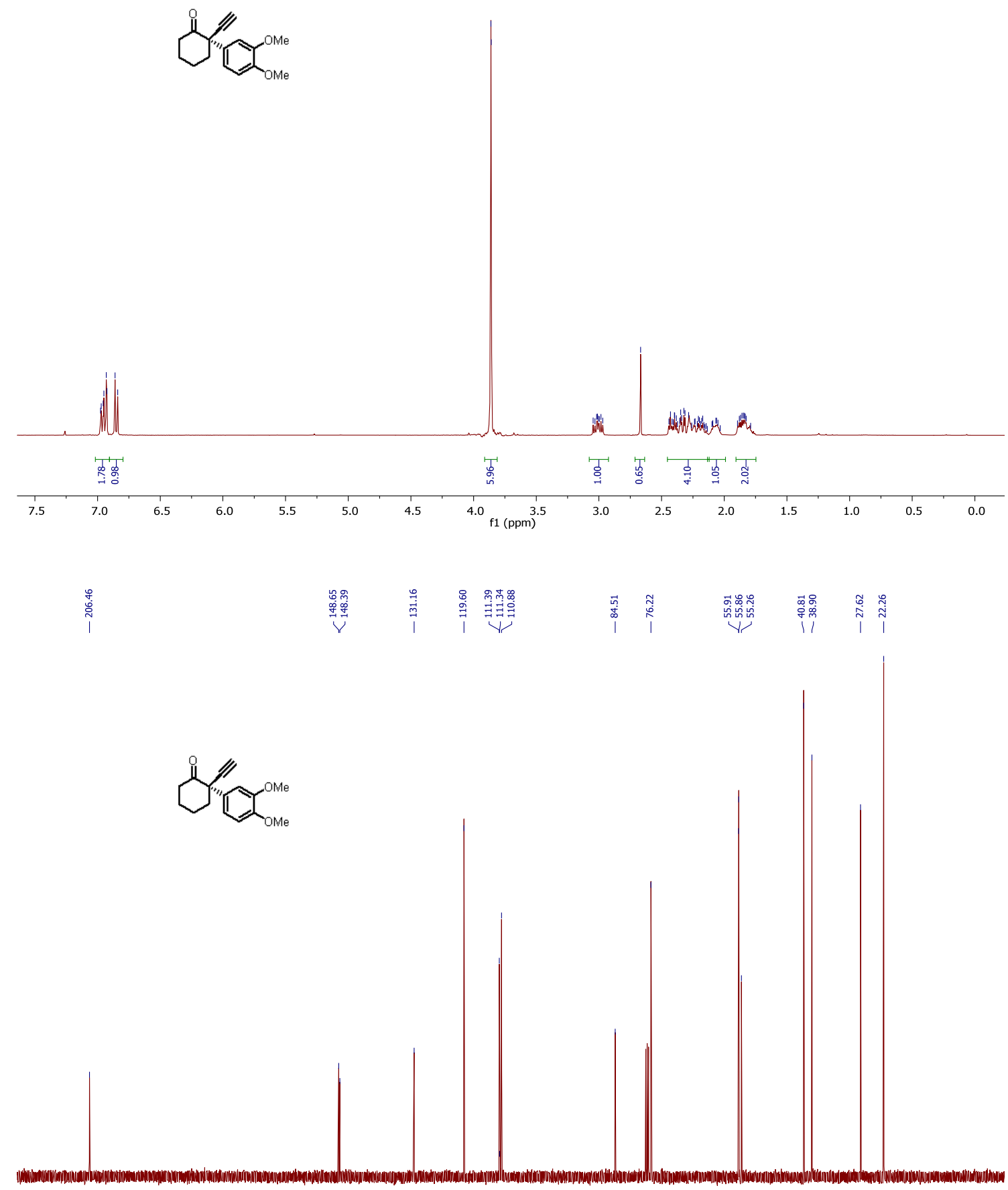

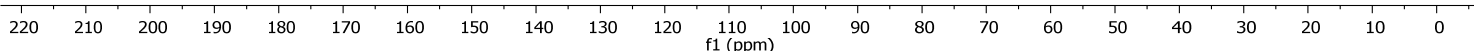



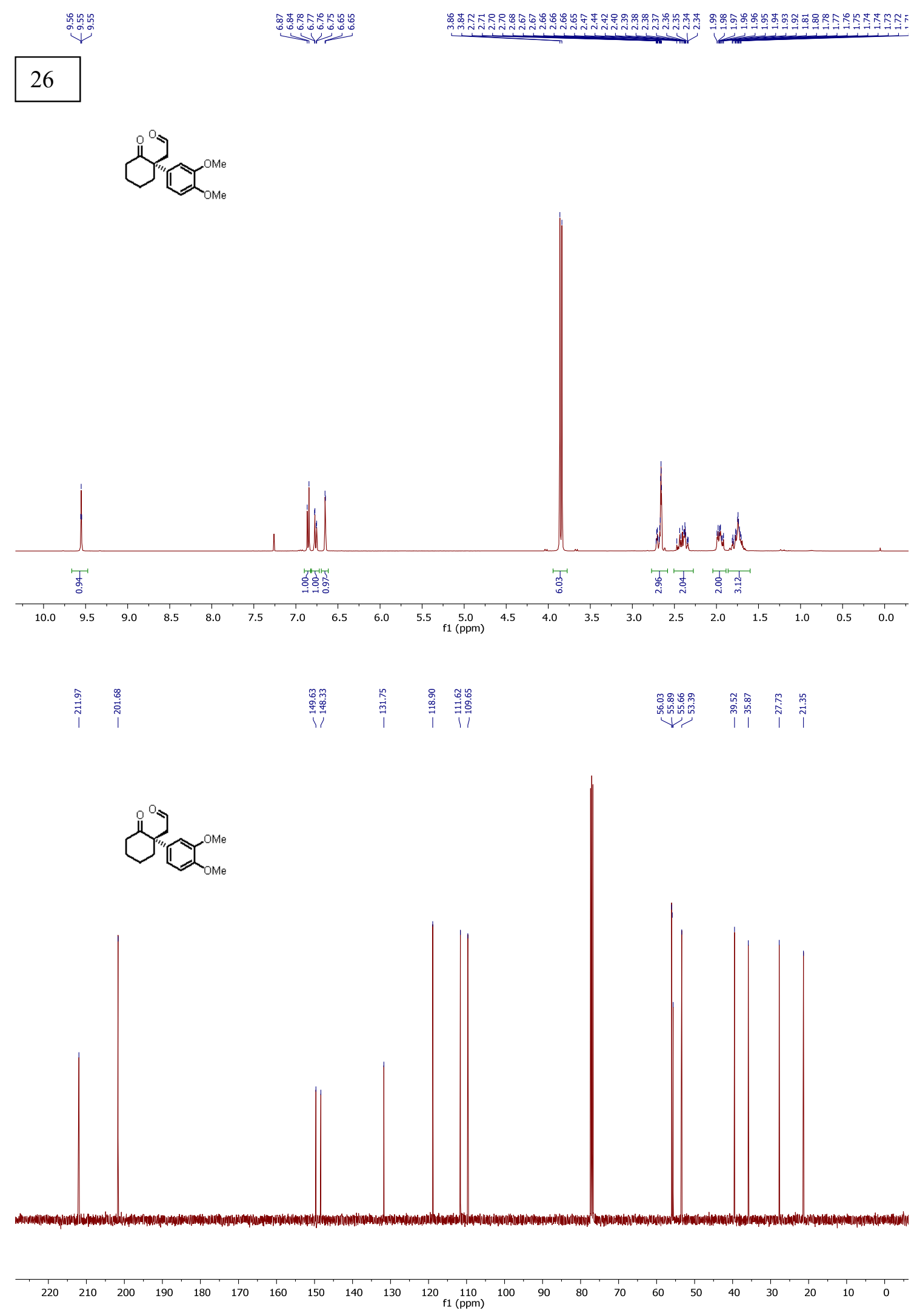

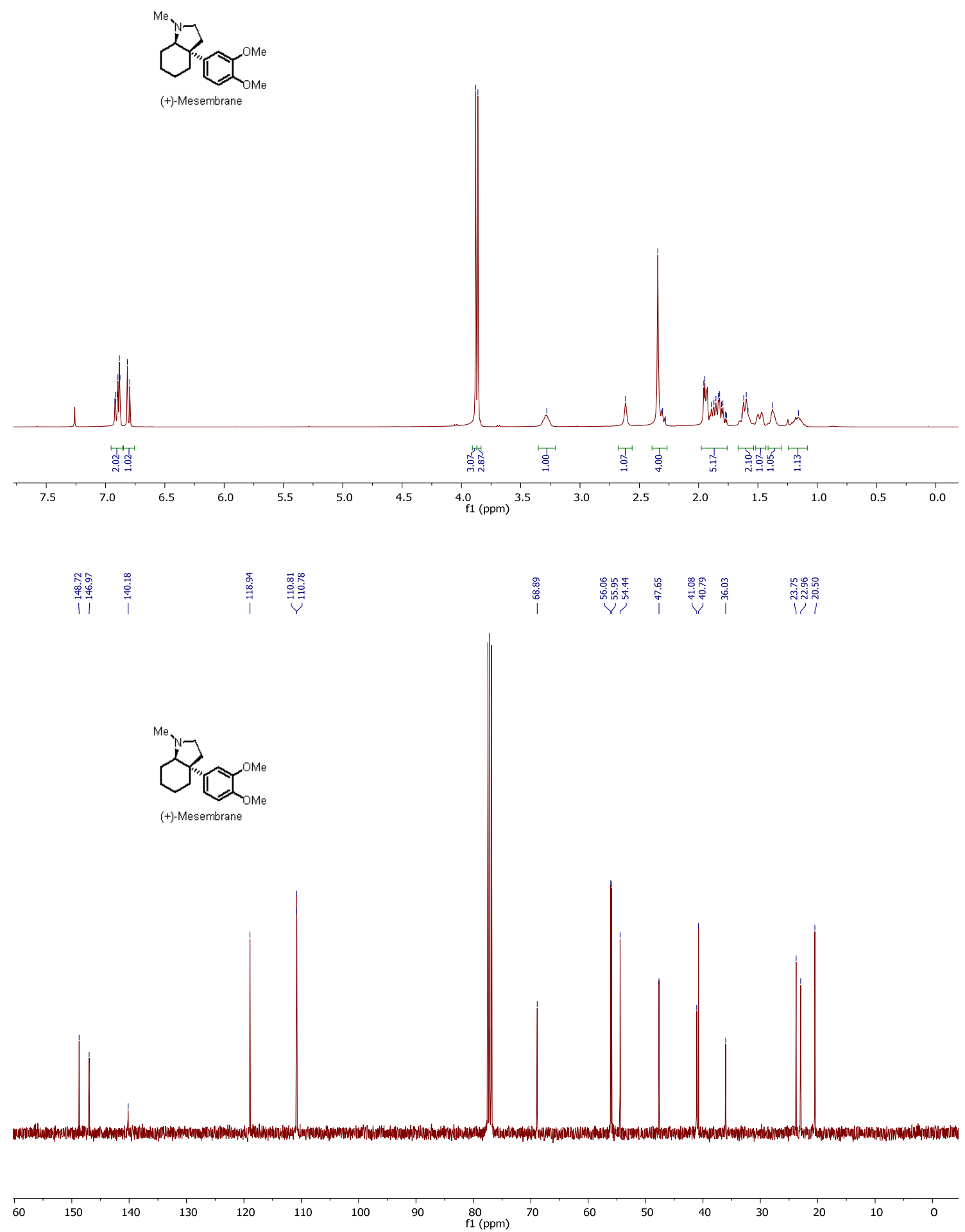


\begin{tabular}{|l|l|l|l|l|}
\hline Co-Solvent \% & Total Flow & Column & Co-Solvent & Back Pressure \\
\hline 10 & 4 & OD-H & MeOH & 150 \\
\hline
\end{tabular}

\begin{tabular}{|l|l|l|l|}
\hline Peak \# & Ret. Time & Area & Area \% \\
\hline 1 & $6.49 \mathrm{~min}$ & 2360.8373 & 49.5416 \\
\hline 2 & $7.52 \mathrm{~min}$ & 2404.5263 & 50.4584 \\
\hline
\end{tabular}

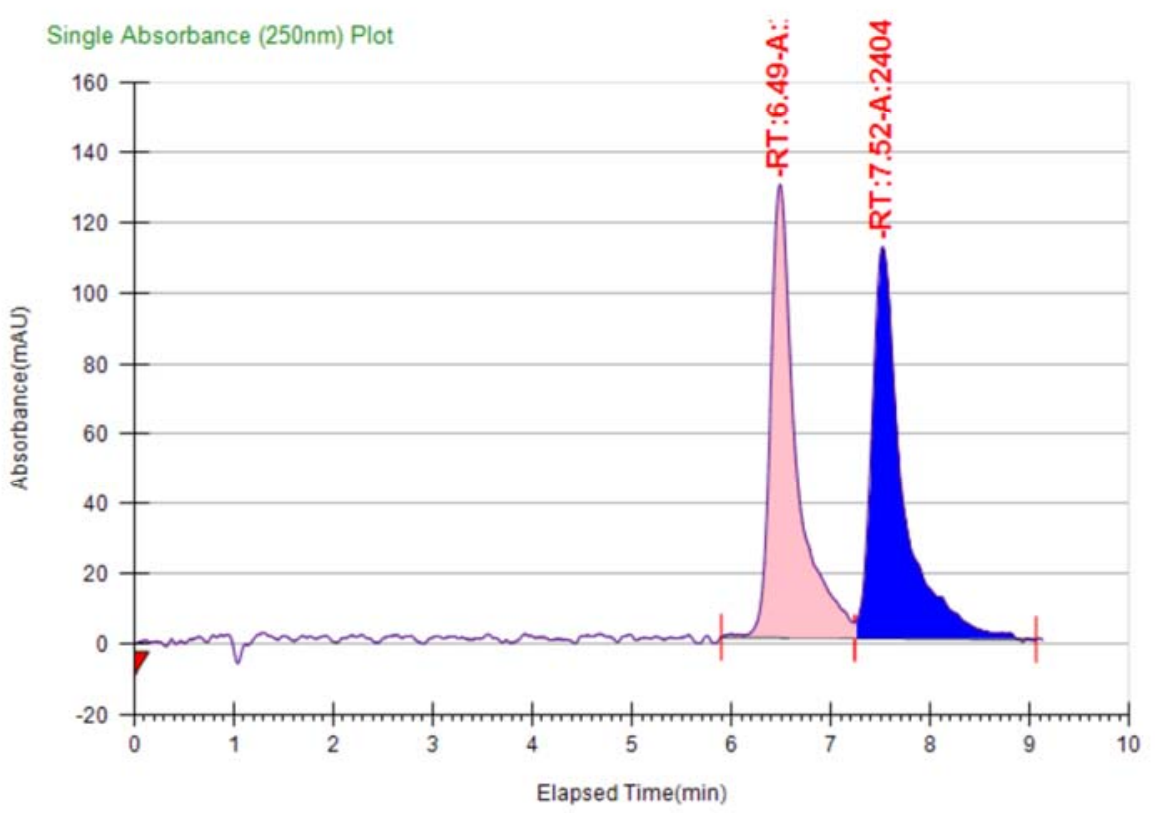

\begin{tabular}{|l|l|l|l|l|}
\hline Co-Solvent \% & Total Flow & Column & Co-Solvent & Back Pressure \\
\hline 10 & 4 & OD-H & MeOH & 150 \\
\hline
\end{tabular}

\begin{tabular}{|l|l|l|l|}
\hline Peak \# & Ret. Time & Area & Area \% \\
\hline 1 & $6.54 \mathrm{~min}$ & 535.9348 & 5.1377 \\
\hline 2 & $7.56 \mathrm{~min}$ & 9895.5626 & 94.8623 \\
\hline
\end{tabular}

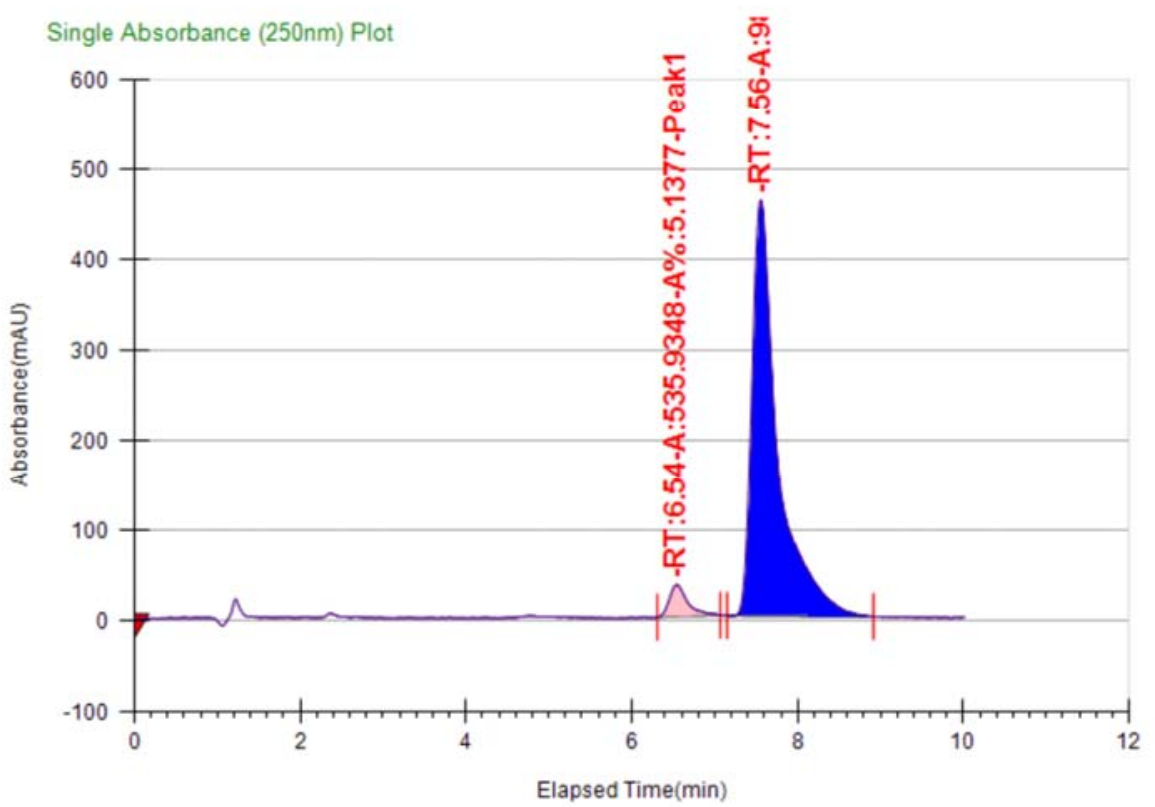




\begin{tabular}{|l|l|l|l|l|}
\hline Co-Solvent \% & Total Flow & Column & Co-Solvent & Back Pressure \\
\hline 7.0018 & 3.999 & OD-H & MeOH & 150 \\
\hline
\end{tabular}

\begin{tabular}{|l|l|l|l|}
\hline Peak \# & Ret. Time & Area & Area \% \\
\hline 1 & $12.09 \mathrm{~min}$ & 1859.6607 & 48.9312 \\
\hline 2 & $14.28 \mathrm{~min}$ & 1940.9001 & 51.0688 \\
\hline
\end{tabular}

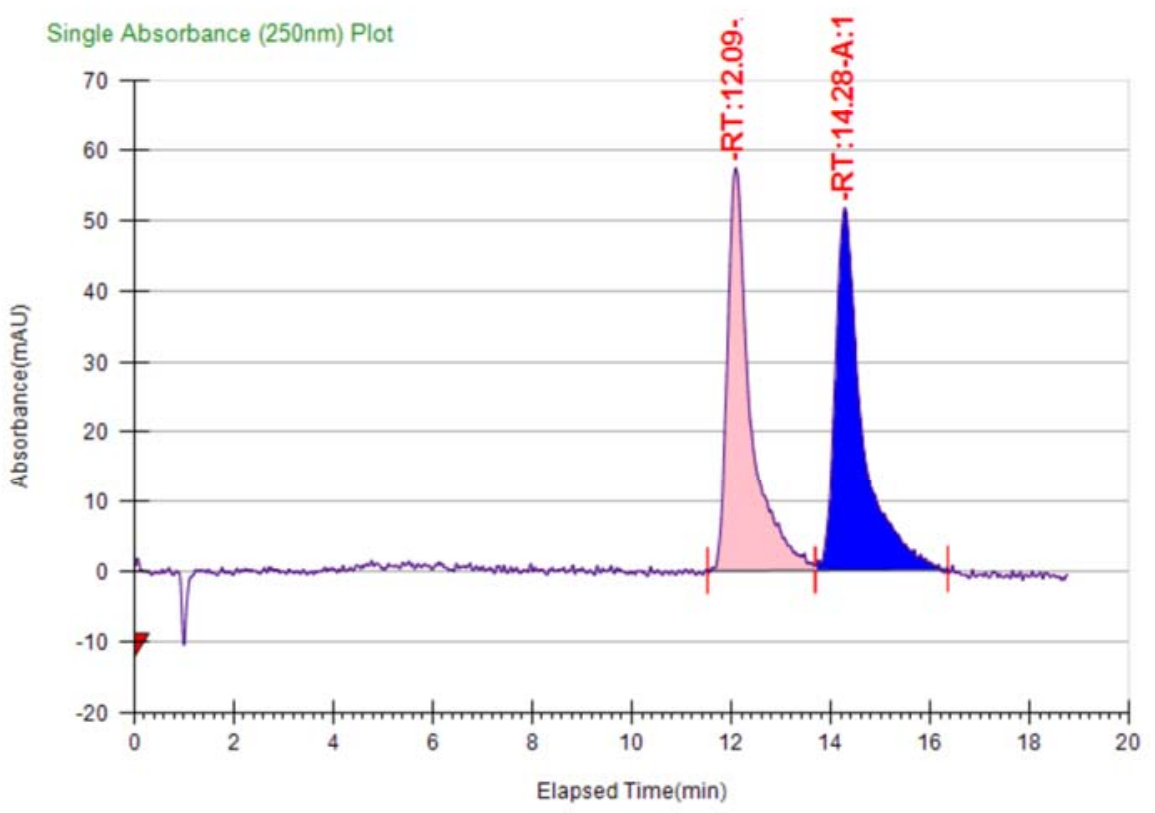

\begin{tabular}{|l|l|l|l|l|}
\hline Co-Solvent \% & Total Flow & Column & Co-Solvent & Back Pressure \\
\hline 7.0018 & 3.999 & OD-H & MeOH & 150 \\
\hline
\end{tabular}

\begin{tabular}{|l|l|l|l|}
\hline Peak \# & Ret. Time & Area & Area \% \\
\hline 1 & $12.08 \mathrm{~min}$ & 488.5595 & 3.1821 \\
\hline 2 & $14.21 \mathrm{~min}$ & 14864.7399 & 96.8179 \\
\hline
\end{tabular}

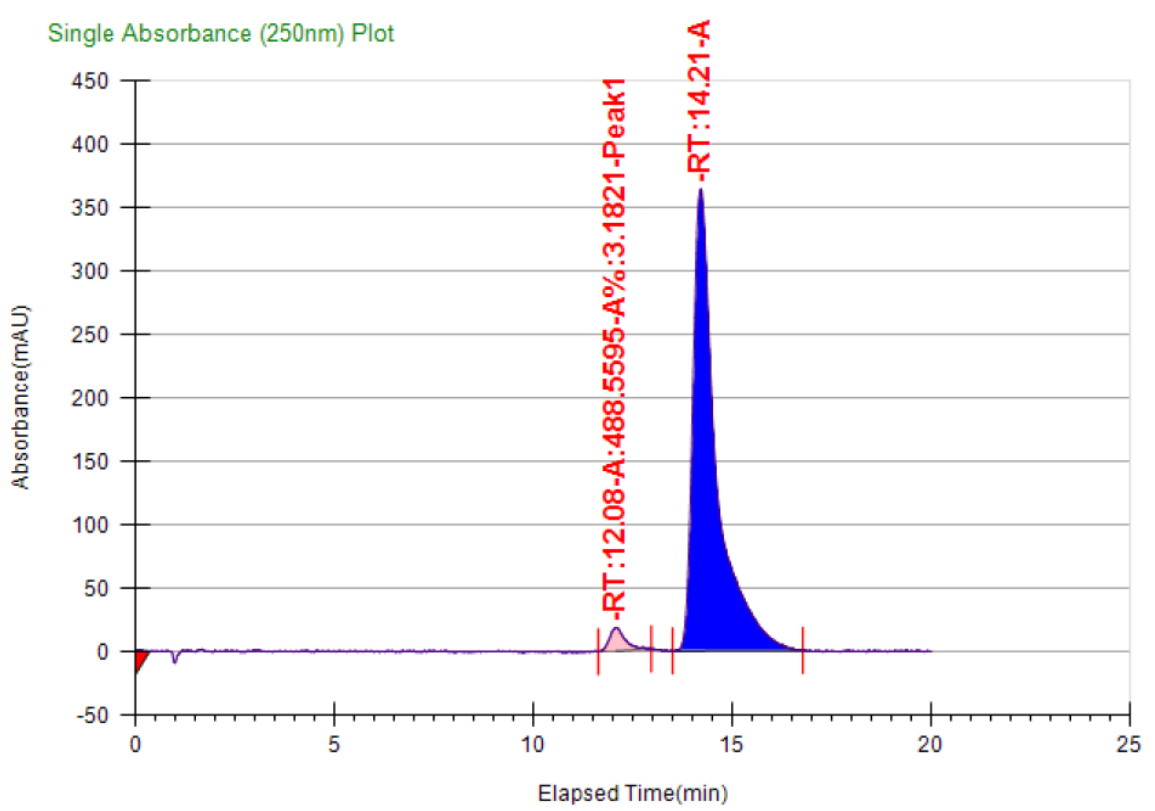




\begin{tabular}{|l|l|l|l|l|}
\hline Co-Solvent \% & Total Flow & Column & Co-Solvent & Back Pressure \\
\hline 20 & 4 & OD-H & MeOH & 150 \\
\hline
\end{tabular}

\begin{tabular}{|l|l|l|l|}
\hline Peak \# & Ret. Time & Area & Area \% \\
\hline 1 & $7.15 \mathrm{~min}$ & 3008.1185 & 49.6583 \\
\hline 2 & $8.43 \mathrm{~min}$ & 3049.5114 & 50.3417 \\
\hline
\end{tabular}

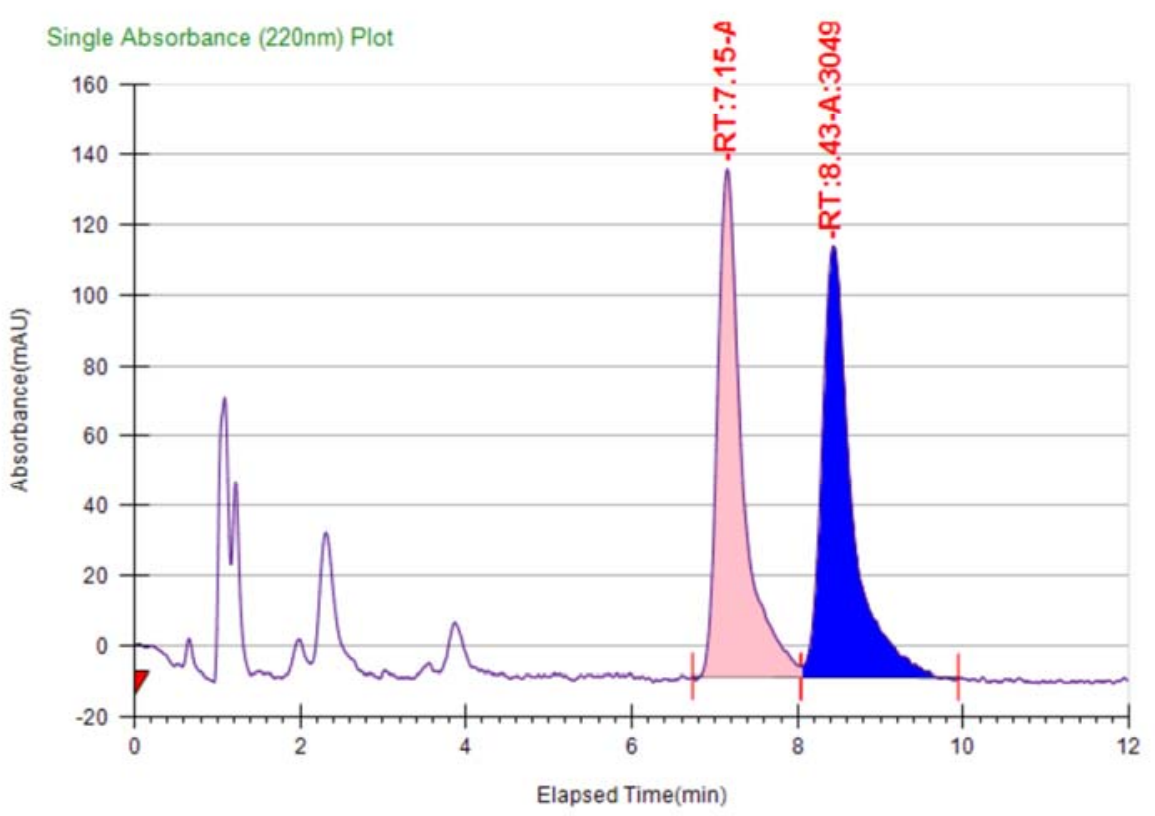

\begin{tabular}{|c|c|c|c|c|}
\hline Co-Solvent $\%$ & Total Flow & Column & Co-Solvent & Back Pressure \\
\hline 20 & 4 & $\mathrm{OD}-\mathrm{H}$ & $\mathrm{MeOH}$ & 150 \\
\hline Peak \# & Ret. Time & Area & Area $\%$ & \\
\hline 1 & $7.17 \mathrm{~min}$ & 390.7795 & 4.5804 & \\
\hline 2 & $8.44 \mathrm{~min}$ & 8140.7727 & 95.4196 & \\
\hline
\end{tabular}

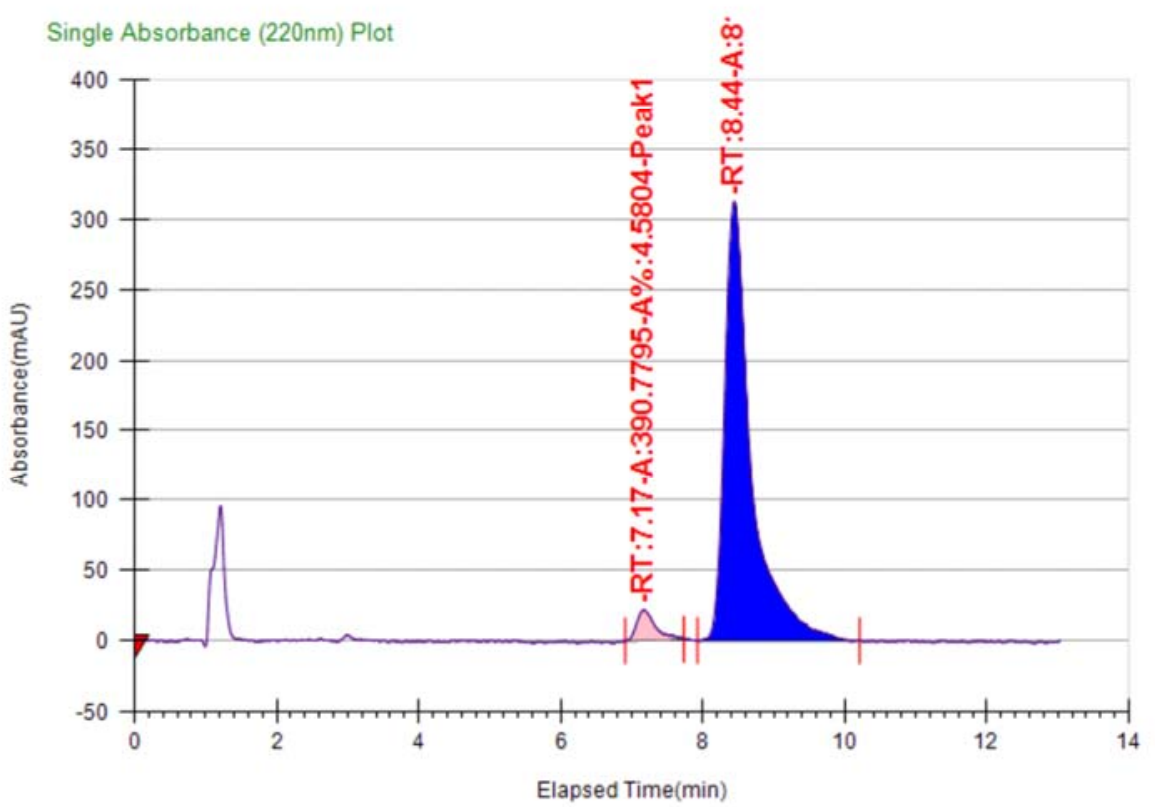




\begin{tabular}{|l|l|l|l|l|}
\hline Co-Solvent \% & Total Flow & Column & Co-Solvent & Back Pressure \\
\hline 6 & 4 & OD-H & MeOH & 150 \\
\hline
\end{tabular}

\begin{tabular}{|l|l|l|l|}
\hline Peak \# & Ret. Time & Area & Area \% \\
\hline 1 & $11.65 \mathrm{~min}$ & 14304.8009 & 49.2294 \\
\hline 2 & $13.52 \mathrm{~min}$ & 14752.6174 & 50.7706 \\
\hline
\end{tabular}

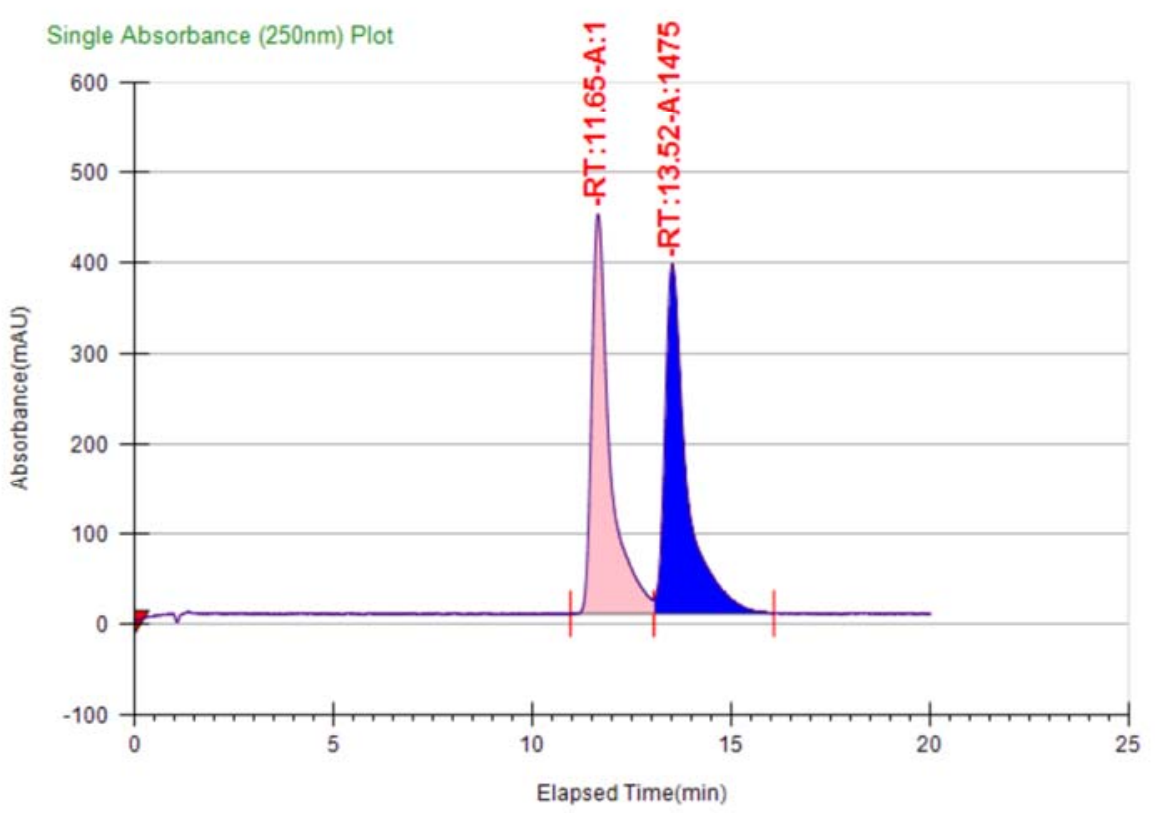

\begin{tabular}{|l|l|l|l|l|}
\hline Co-Solvent \% & Total Flow & Column & Co-Solvent & Back Pressure \\
\hline 6 & 4 & OD-H & MeOH & 150 \\
\hline
\end{tabular}

\begin{tabular}{|l|l|l|l|}
\hline Peak \# & Ret. Time & Area & Area \% \\
\hline 1 & $11.57 \mathrm{~min}$ & 1065.8582 & 3.9909 \\
\hline 2 & $13.39 \mathrm{~min}$ & 25641.4756 & 96.0091 \\
\hline
\end{tabular}

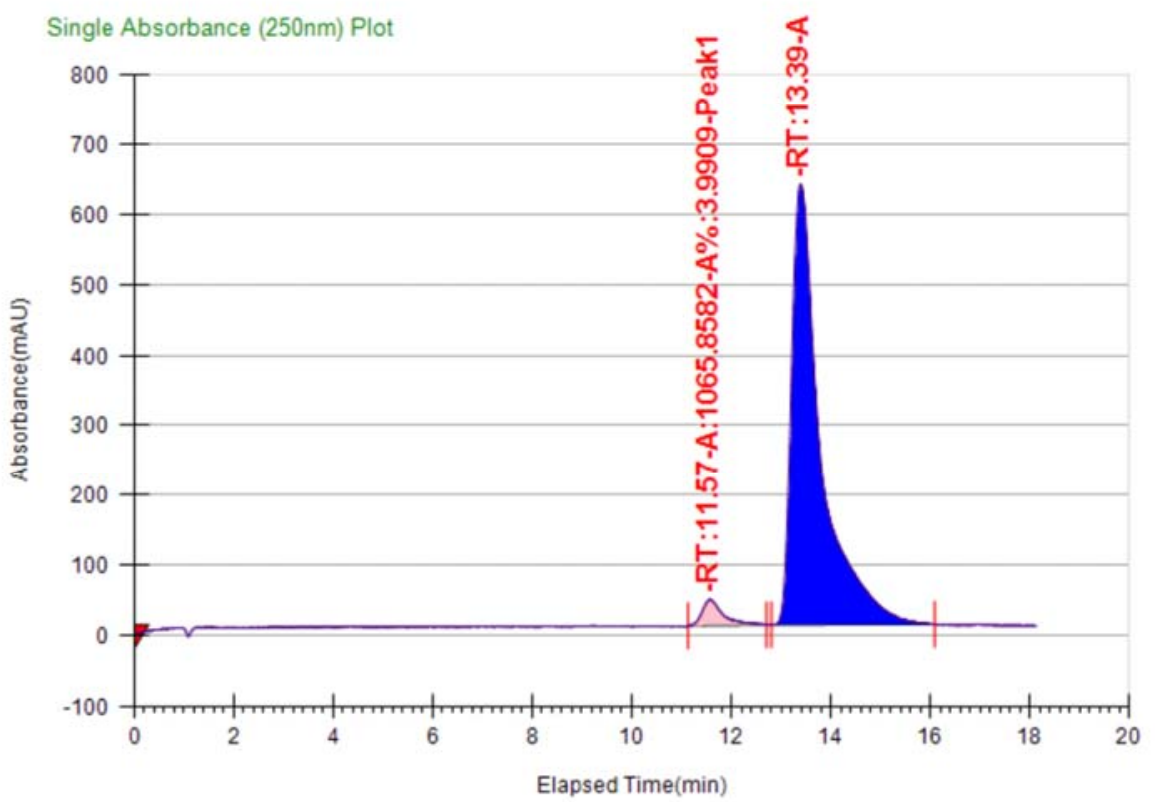




\begin{tabular}{|l|l|l|l|l|}
\hline Co-Solvent \% & Total Flow & Column & Co-Solvent & Back Pressure \\
\hline 10 & 4 & OD-H & MeOH & 150 \\
\hline
\end{tabular}

\begin{tabular}{|l|l|l|l|}
\hline Peak \# & Ret. Time & Area & Area \% \\
\hline 1 & $9.04 \mathrm{~min}$ & 4064.7143 & 49.2496 \\
\hline 2 & $10.39 \mathrm{~min}$ & 4188.5744 & 50.7504 \\
\hline
\end{tabular}

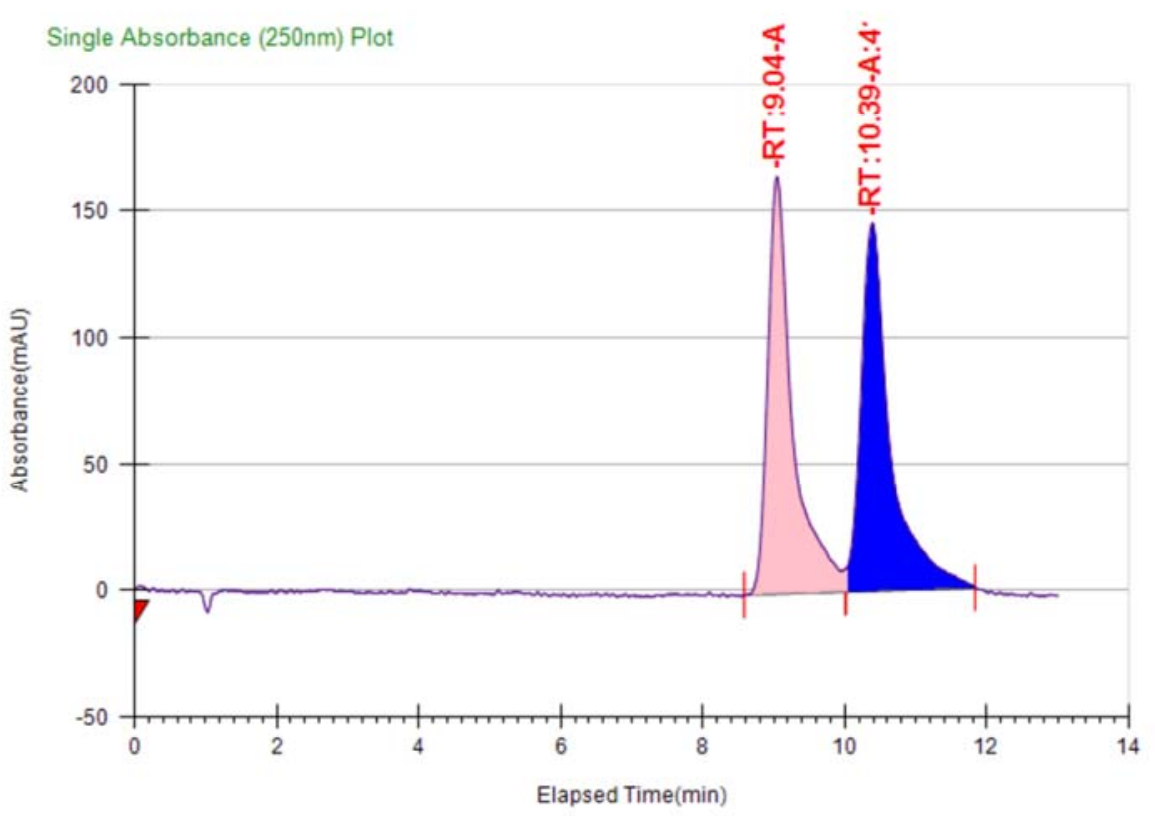

\begin{tabular}{|l|l|l|l|l|}
\hline Co-Solvent \% & Total Flow & Column & Co-Solvent & Back Pressure \\
\hline 10 & 4 & OD-H & MeOH & 150 \\
\hline
\end{tabular}

\begin{tabular}{|l|l|l|l|}
\hline Peak \# & Ret. Time & Area & Area \% \\
\hline 1 & $8.92 \mathrm{~min}$ & 183.2983 & 6.5892 \\
\hline 2 & $10.23 \mathrm{~min}$ & 2598.5203 & 93.4108 \\
\hline
\end{tabular}

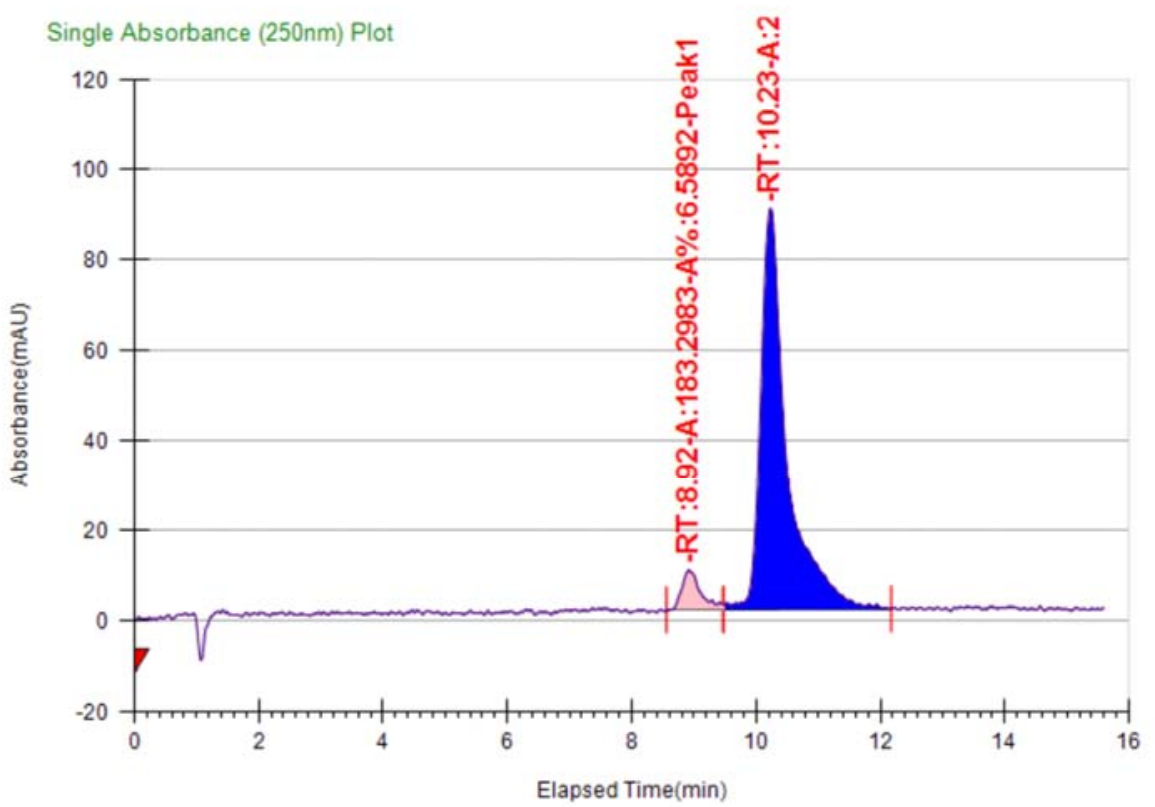




\begin{tabular}{|l|l|l|l|l|}
\hline Co-Solvent \% & Total Flow & Column & Co-Solvent & Back Pressure \\
\hline 7.0018 & 3.999 & OD-H & $\mathrm{MeOH}$ & 150 \\
\hline
\end{tabular}

\begin{tabular}{|l|l|l|l|}
\hline Peak \# & Ret. Time & Area & Area \% \\
\hline 1 & $8.57 \mathrm{~min}$ & 16360.8517 & 49.2669 \\
\hline 2 & $10.06 \mathrm{~min}$ & 16847.7426 & 50.7331 \\
\hline
\end{tabular}

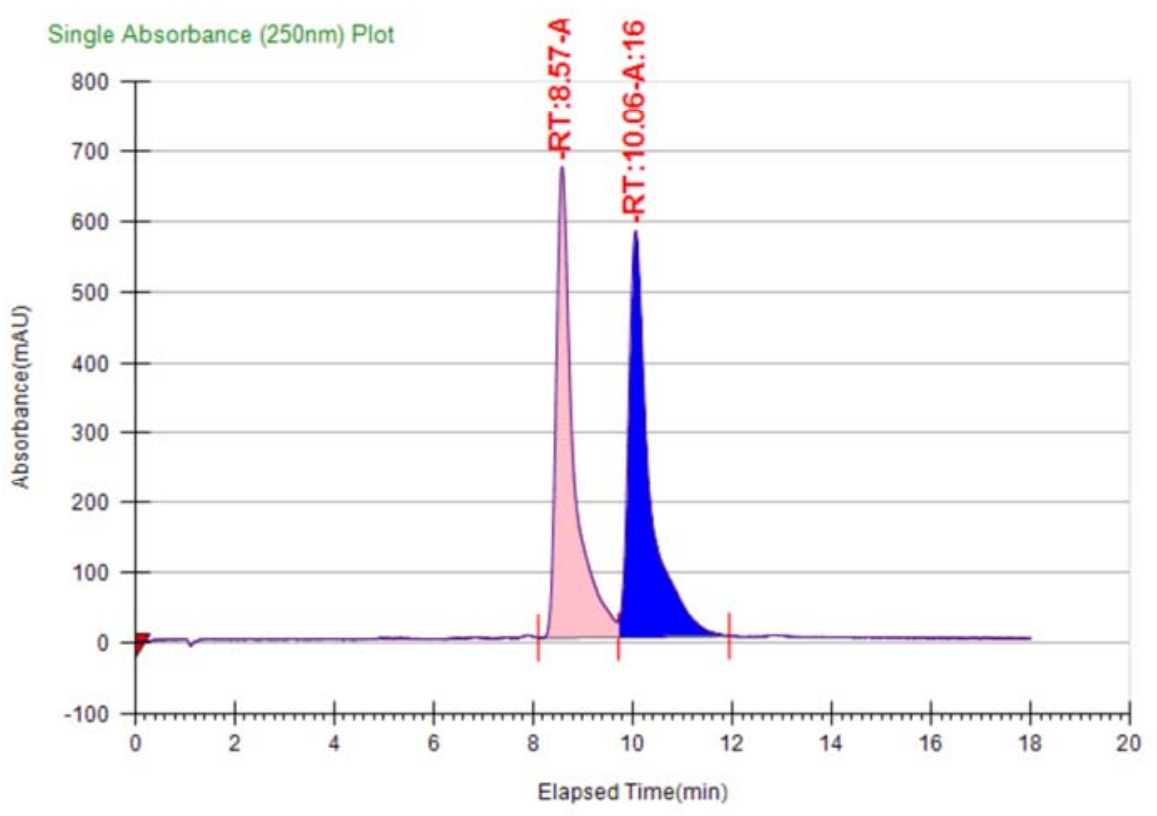

\begin{tabular}{|c|c|c|c|c|}
\hline Co-Solvent \% & Total Flow & Column & Co-Solvent & Back Pressure \\
\hline 7.0018 & 3.999 & $\mathrm{OD}-\mathrm{H}$ & $\mathrm{MeOH}$ & 150 \\
\hline Peak \# & Ret. Time & Area & Area \% & \\
\hline 1 & $8.84 \mathrm{~min}$ & 720.0077 & 4.5929 & \\
\hline 2 & $10.37 \mathrm{~min}$ & 14956.4059 & 95.4071 & \\
\hline
\end{tabular}

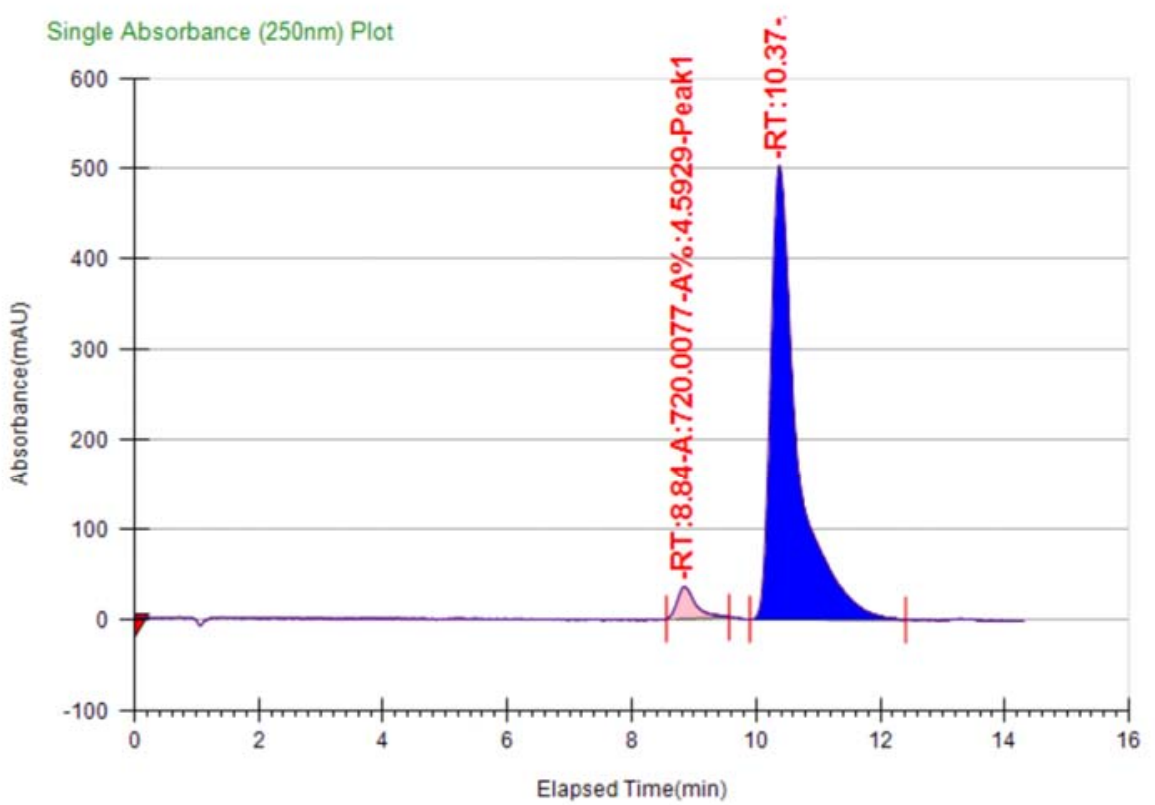




\begin{tabular}{|l|l|l|l|l|}
\hline Co-Solvent \% & Total Flow & Column & Co-Solvent & Back Pressure \\
\hline 10 & 4 & OD-H & MeOH & 150 \\
\hline
\end{tabular}

\begin{tabular}{|l|l|l|l|}
\hline Peak \# & Ret. Time & Area & Area \% \\
\hline 1 & $7.83 \mathrm{~min}$ & 3036.7807 & 49.5219 \\
\hline 2 & $9.17 \mathrm{~min}$ & 3095.4146 & 50.4781 \\
\hline
\end{tabular}

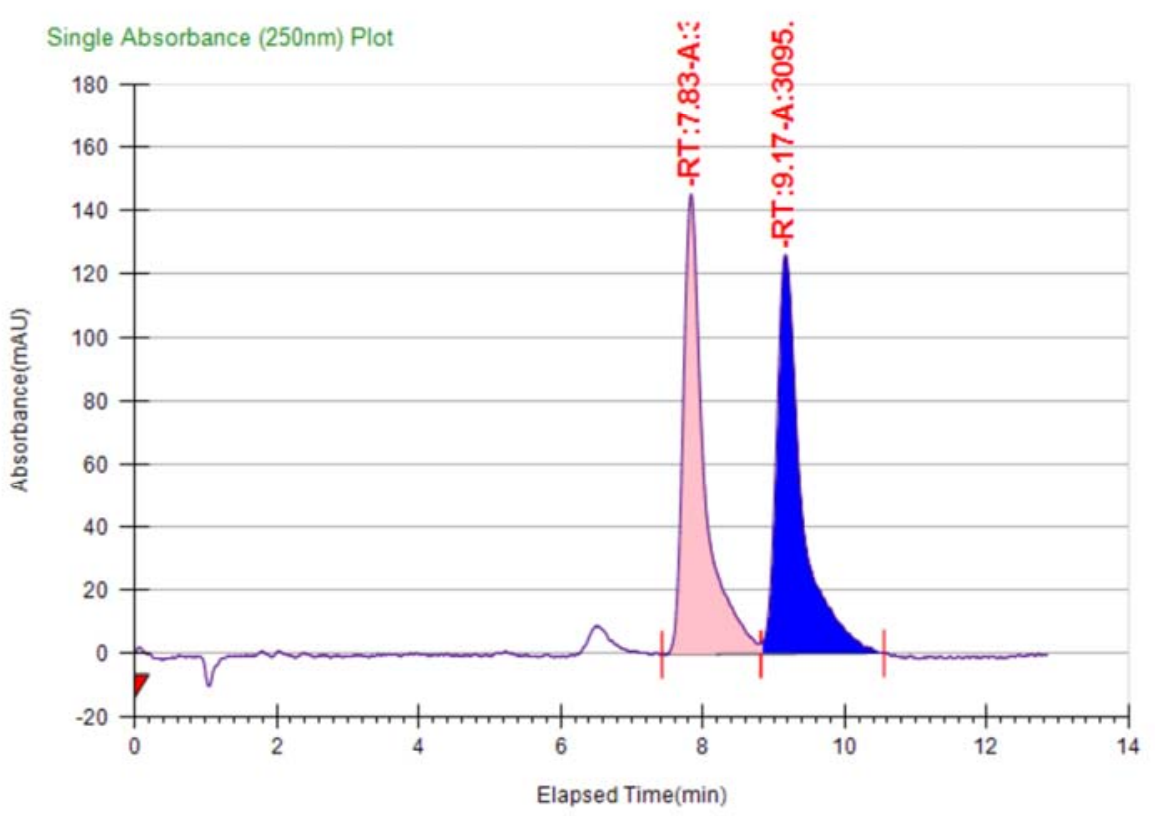

\begin{tabular}{|l|l|l|l|l|}
\hline Co-Solvent \% & Total Flow & Column & Co-Solvent & Back Pressure \\
\hline 10 & 4 & OD-H & MeOH & 150 \\
\hline
\end{tabular}

\begin{tabular}{|l|l|l|l|}
\hline Peak \# & Ret. Time & Area & Area \% \\
\hline 1 & $7.83 \mathrm{~min}$ & 1779.3485 & 5.8517 \\
\hline 2 & $9.12 \mathrm{~min}$ & 28627.8077 & 94.1483 \\
\hline
\end{tabular}

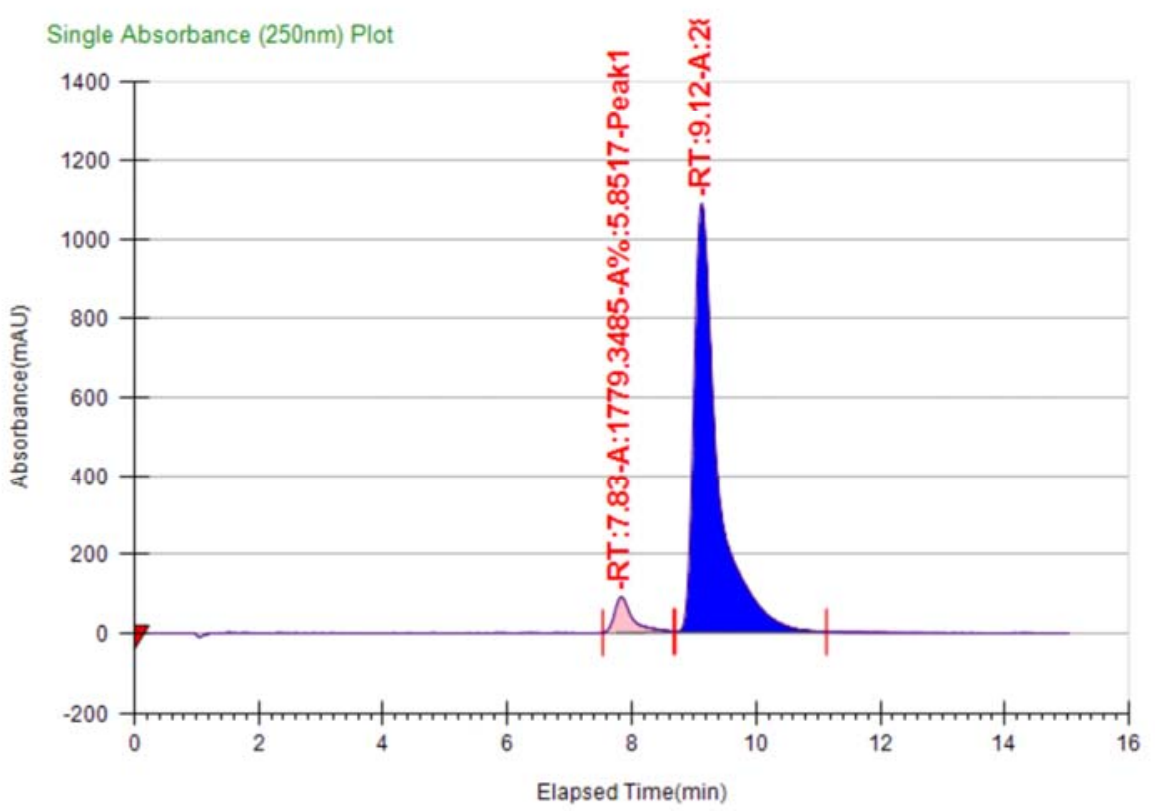




\begin{tabular}{|l|l|l|l|}
\hline Peak \# & Ret. Time & Area & Area \% \\
\hline 1 & $20.38 \mathrm{~min}$ & 4224.6107 & 49.2327 \\
\hline 2 & $23.22 \mathrm{~min}$ & 4356.3011 & 50.7673 \\
\hline
\end{tabular}

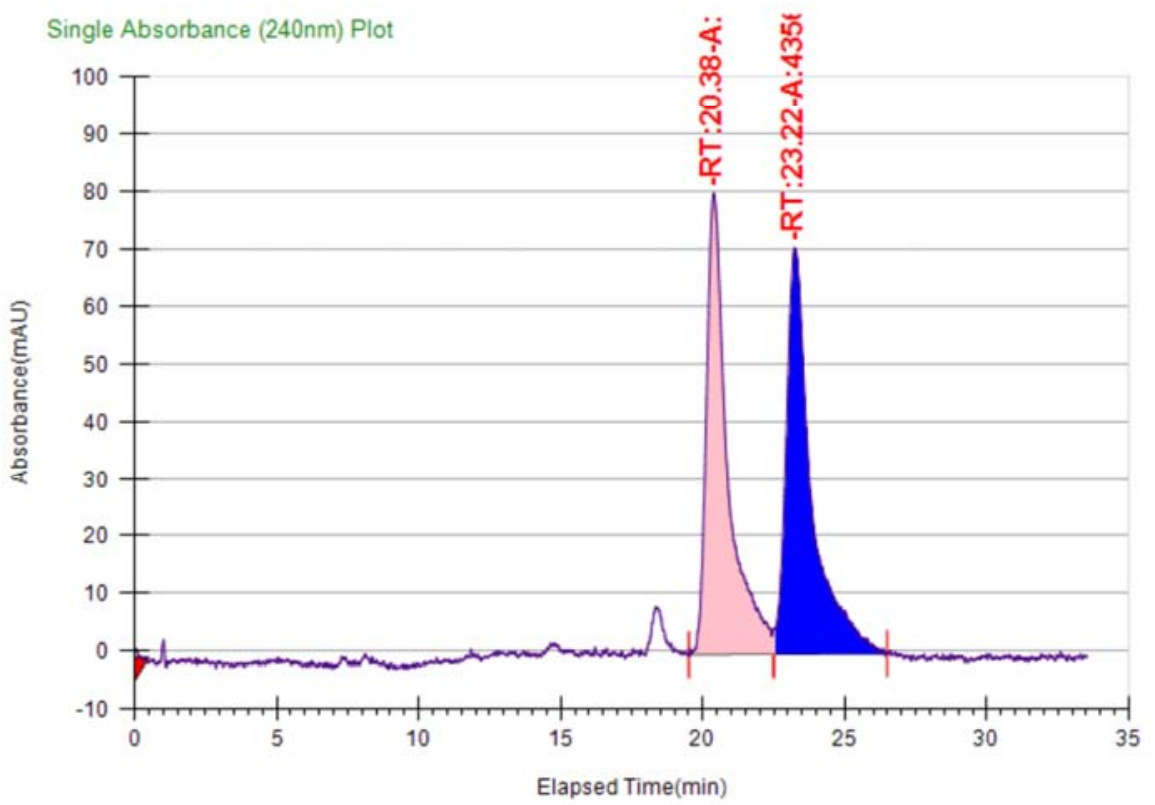

\begin{tabular}{|l|l|l|l|l|}
\hline Co-Solvent \% & Total Flow & Column & Co-Solvent & Back Pressure \\
\hline 6 & 4 & OD-H & MeOH & 150 \\
\hline
\end{tabular}

\begin{tabular}{|l|l|l|l|}
\hline Peak \# & Ret. Time & Area & Area \% \\
\hline 1 & $20.12 \mathrm{~min}$ & 417.5175 & 4.1936 \\
\hline 2 & $22.88 \mathrm{~min}$ & 9538.4774 & 95.8064 \\
\hline
\end{tabular}

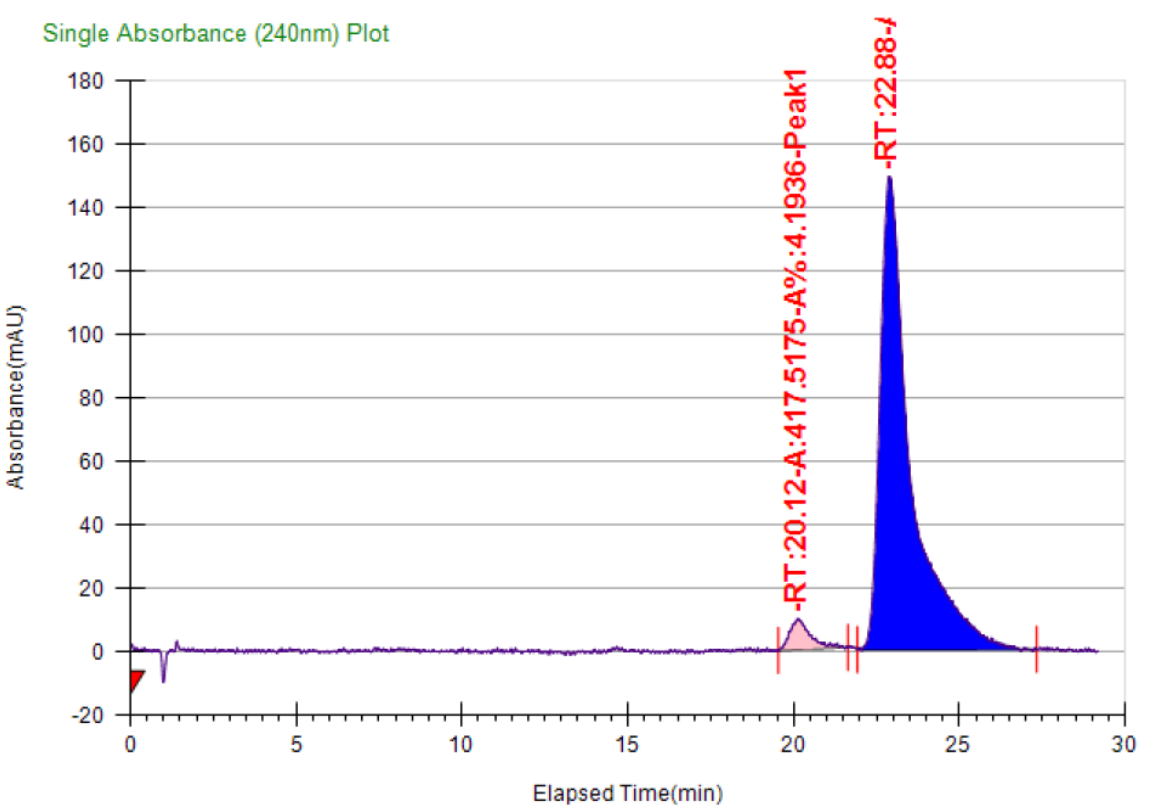




\begin{tabular}{|l|l|l|l|l|}
\hline Co-Solvent \% & Total Flow & Column & Co-Solvent & Back Pressure \\
\hline 10 & 4 & OD-H & MeOH & 150 \\
\hline
\end{tabular}

\begin{tabular}{|l|l|l|l|}
\hline Peak \# & Ret. Time & Area & Area \% \\
\hline 1 & $6.56 \mathrm{~min}$ & 6494.4929 & 49.5417 \\
\hline 2 & $7.76 \mathrm{~min}$ & 6614.6632 & 50.4583 \\
\hline
\end{tabular}

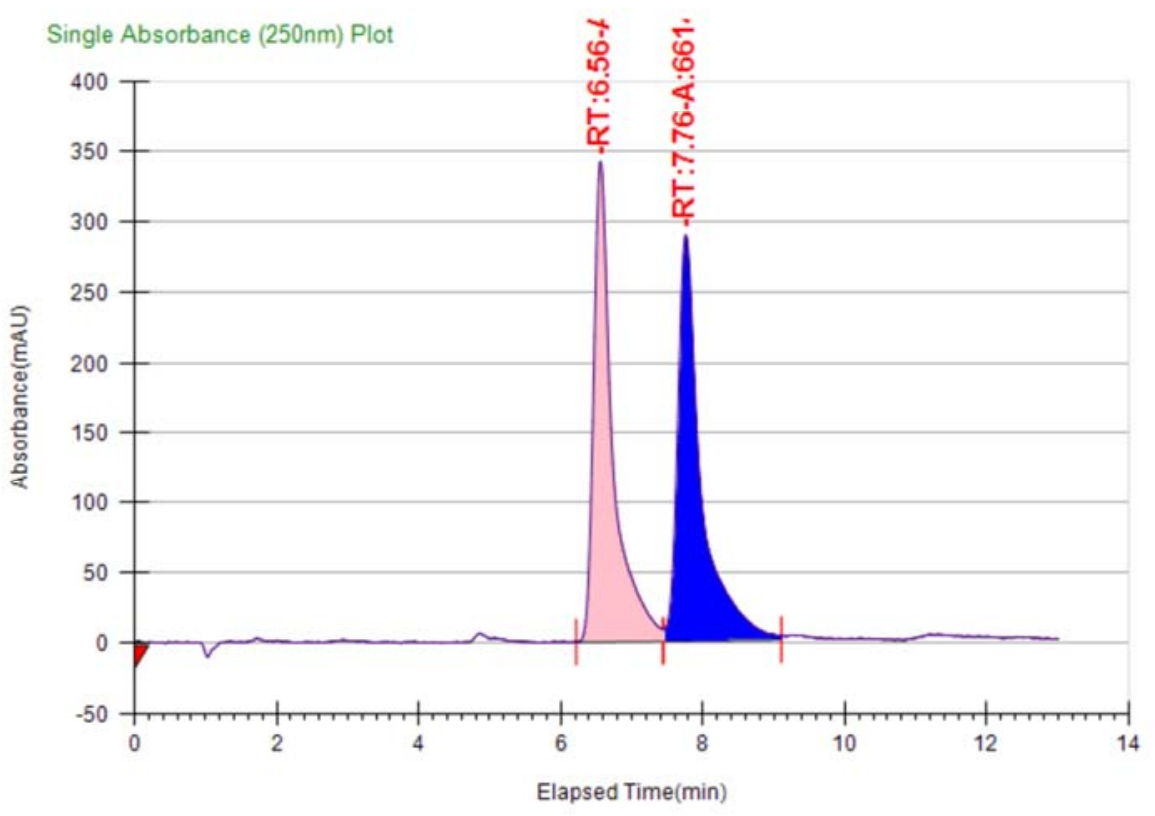

\begin{tabular}{|l|l|l|l|l|}
\hline Co-Solvent \% & Total Flow & Column & Co-Solvent & Back Pressure \\
\hline 10 & 4 & OD-H & MeOH & 150 \\
\hline
\end{tabular}

\begin{tabular}{|l|l|l|l|}
\hline Peak \# & Ret. Time & Area & Area \% \\
\hline 1 & $6.66 \mathrm{~min}$ & 694.0775 & 5.0832 \\
\hline 2 & $7.87 \mathrm{~min}$ & 12960.3632 & 94.9168 \\
\hline
\end{tabular}

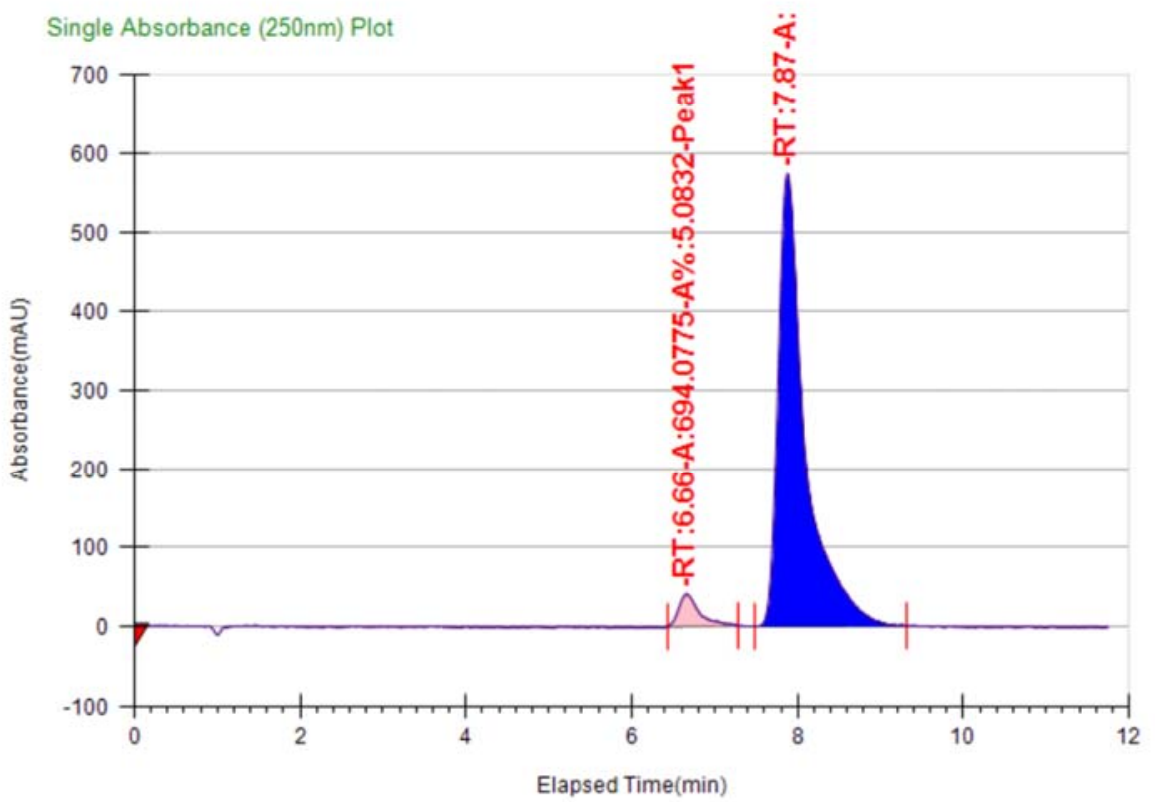




\begin{tabular}{|l|l|l|l|l|}
\hline Co-Solvent \% & Total Flow & Column & Co-Solvent & Back Pressure \\
\hline 5 & 4 & AD-H & MeOH & 150 \\
\hline
\end{tabular}

\begin{tabular}{|l|l|l|l|}
\hline Peak \# & Ret. Time & Area & Area \% \\
\hline 1 & $24.27 \mathrm{~min}$ & 5717.4356 & 48.8614 \\
\hline 2 & $28.1 \mathrm{~min}$ & 5983.8993 & 51.1386 \\
\hline
\end{tabular}

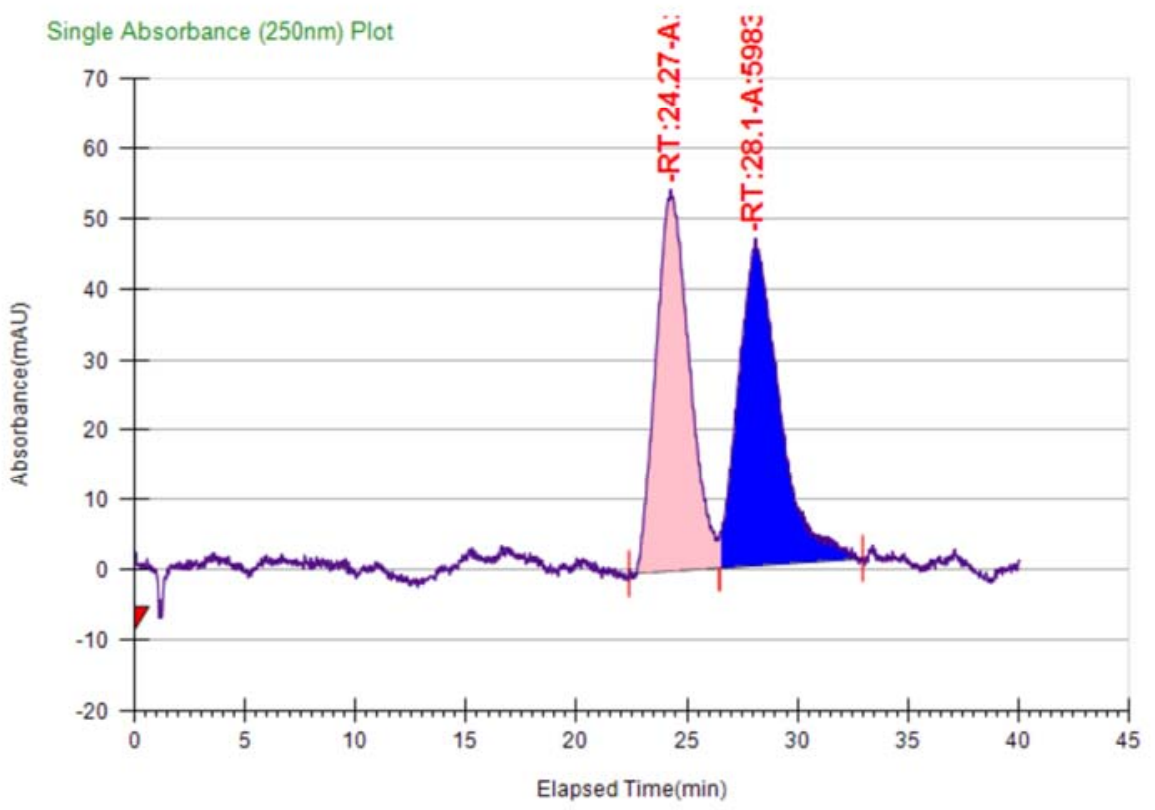

\begin{tabular}{|l|l|l|l|l|}
\hline Co-Solvent \% & Total Flow & Column & Co-Solvent & Back Pressure \\
\hline 5 & 4 & AD-H & MeOH & 150 \\
\hline
\end{tabular}

\begin{tabular}{|l|l|l|l|}
\hline Peak \# & Ret. Time & Area & Area \% \\
\hline 1 & $24.35 \mathrm{~min}$ & 14790.9718 & 92.0674 \\
\hline 2 & $28.44 \mathrm{~min}$ & 1274.4019 & 7.9326 \\
\hline
\end{tabular}

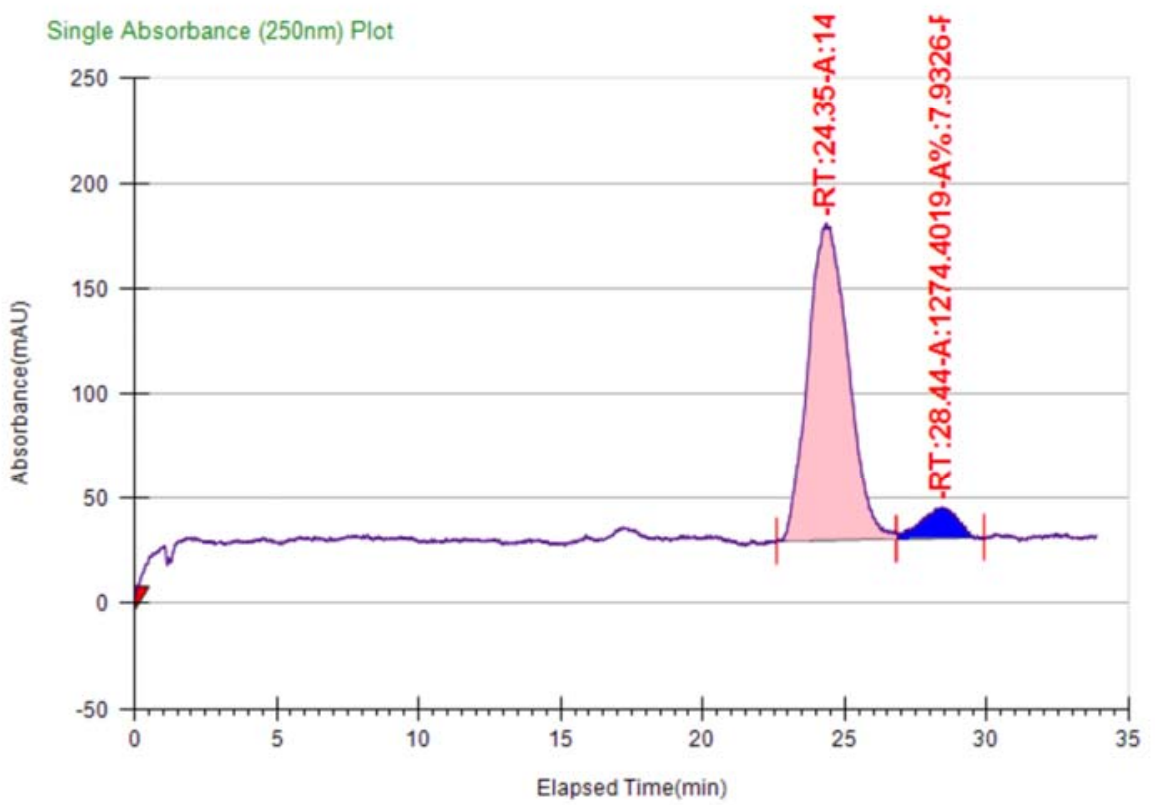




\begin{tabular}{|l|l|l|l|l|}
\hline Co-Solvent \% & Total Flow & Column & Co-Solvent & Back Pressure \\
\hline 3 & 4 & OD-H & MeOH & 150 \\
\hline
\end{tabular}

\begin{tabular}{|l|l|l|l|}
\hline Peak \# & Ret. Time & Area & Area \% \\
\hline 1 & $9.8 \mathrm{~min}$ & 1024.1707 & 50.0813 \\
\hline 2 & $11.12 \mathrm{~min}$ & 1020.8462 & 49.9187 \\
\hline
\end{tabular}

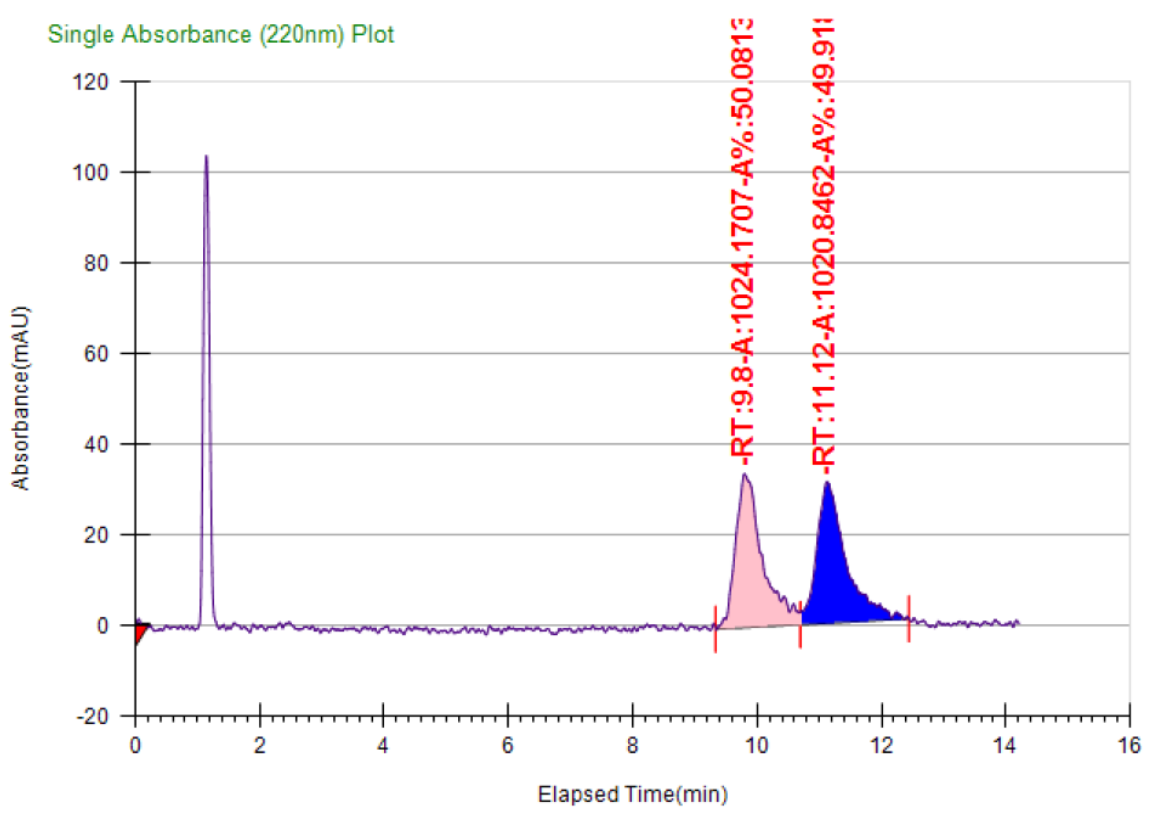

\begin{tabular}{|l|l|l|l|l|}
\hline Co-Solvent \% & Total Flow & Column & Co-Solvent & Back Pressure \\
\hline 3 & 4 & OD-H & MeOH & 150 \\
\hline
\end{tabular}

\begin{tabular}{|l|l|l|l|}
\hline Peak \# & Ret. Time & Area & Area \% \\
\hline 1 & $10.05 \mathrm{~min}$ & 353.5843 & 2.762 \\
\hline 2 & $11.32 \mathrm{~min}$ & 12447.9404 & 97.238 \\
\hline
\end{tabular}

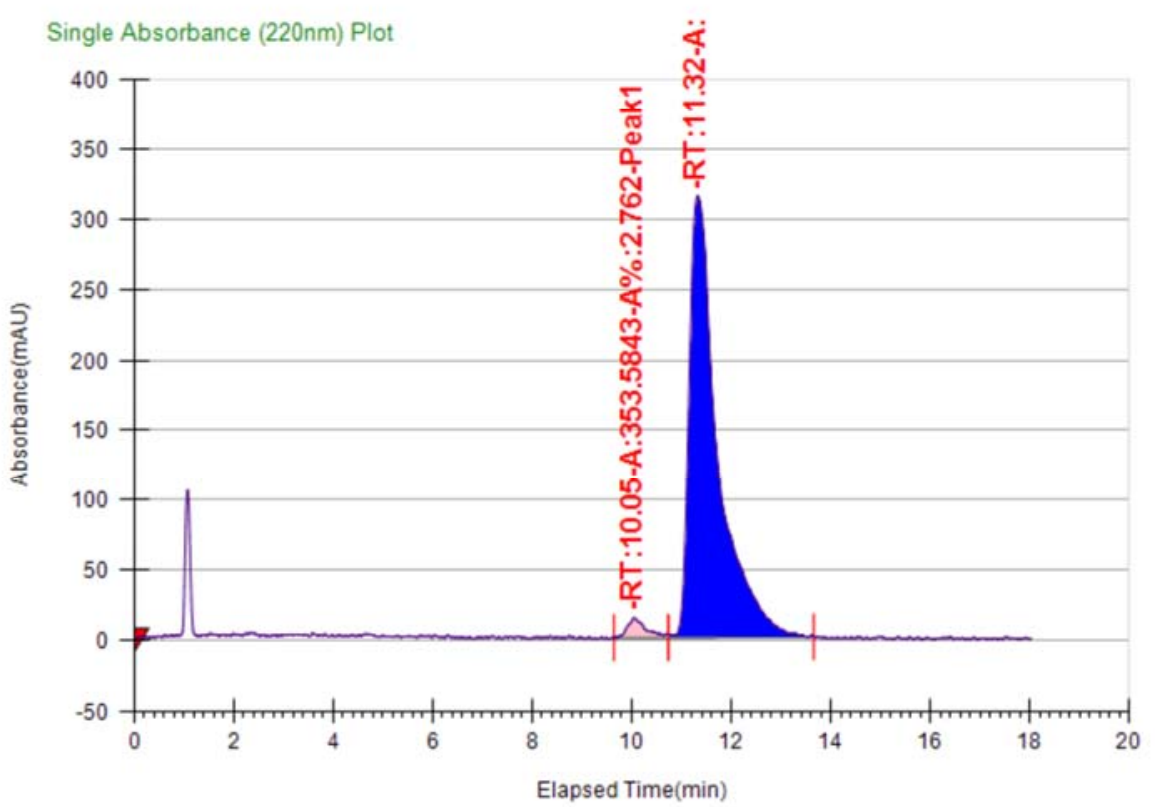




\begin{tabular}{|l|l|l|l|l|}
\hline Co-Solvent \% & Total Flow & Column & Co-Solvent & Back Pressure \\
\hline 3 & 4 & OD-H & MeOH & 150 \\
\hline
\end{tabular}
\begin{tabular}{|l|l|l|l|}
\hline Peak \# & Ret. Time & Area & Area \% \\
\hline 1 & 22.44 min & 9986.6052 & 49.6696 \\
\hline 2 & 26.44 min & 10119.4721 & 50.3304 \\
\hline
\end{tabular}
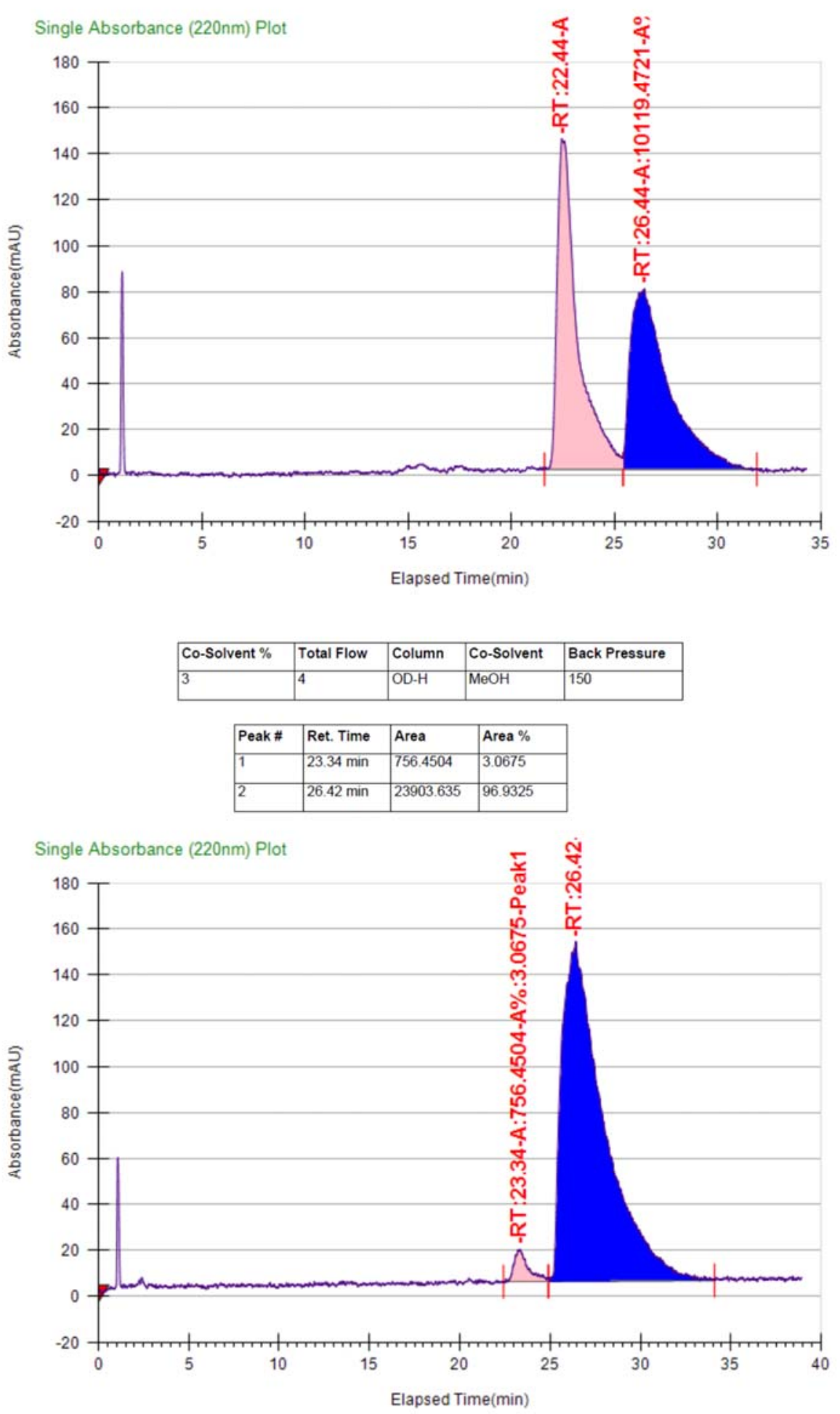


\begin{tabular}{|l|l|l|l|l|}
\hline Co-Solvent \% & Total Flow & Column & Co-Solvent & Back Pressure \\
\hline 10 & 4 & OD-H & MeOH & 150 \\
\hline
\end{tabular}

\begin{tabular}{|l|l|l|l|}
\hline Peak \# & Ret. Time & Area & Area \% \\
\hline 1 & $3.61 \mathrm{~min}$ & 13117.7511 & 49.7137 \\
\hline 2 & $4.54 \mathrm{~min}$ & 13268.8672 & 50.2863 \\
\hline
\end{tabular}

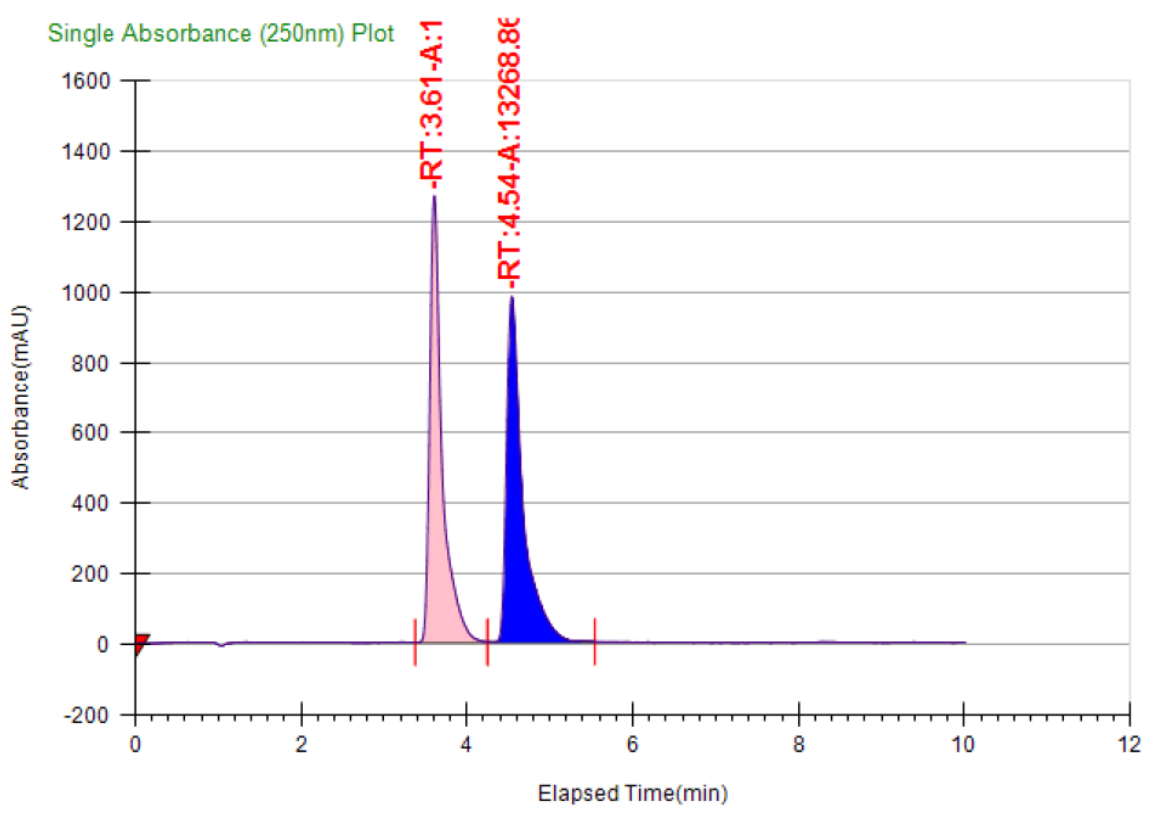

\begin{tabular}{|c|c|c|c|c|}
\hline Co-Solvent \% & Total Flow & Column & Co-Solvent & Back Pressure \\
\hline 10 & 4 & OD-H & $\mathrm{MeOH}$ & 150 \\
\hline Peak \# & Ret. Time & Area & Area \% & \\
\hline 1 & $3.55 \mathrm{~min}$ & 1967.3841 & 8.1829 & \\
\hline 2 & $4.48 \mathrm{~min}$ & 22075.3883 & 91.8171 & \\
\hline
\end{tabular}

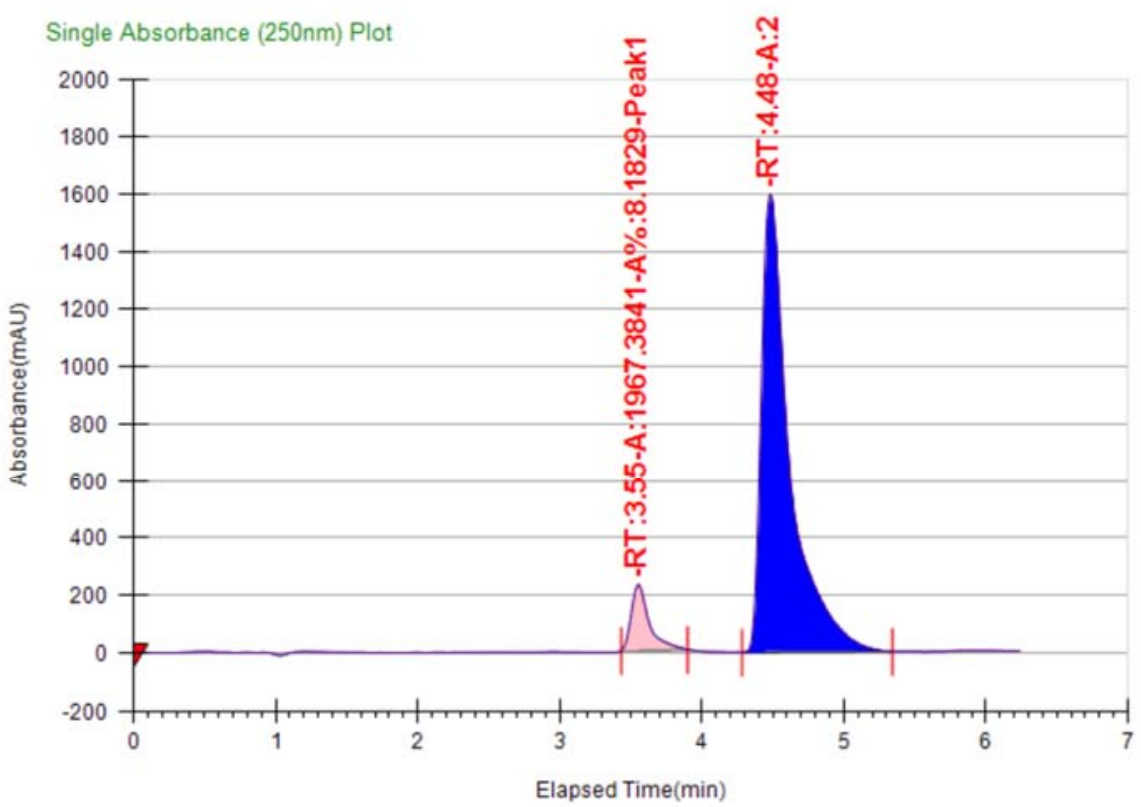




\begin{tabular}{|l|l|l|l|}
\hline Peak \# & Ret. Time & Area & Area \% \\
\hline 1 & $14.97 \mathrm{~min}$ & 7080.5364 & 49.4996 \\
\hline 2 & $17.54 \mathrm{~min}$ & 7223.6862 & 50.5004 \\
\hline
\end{tabular}

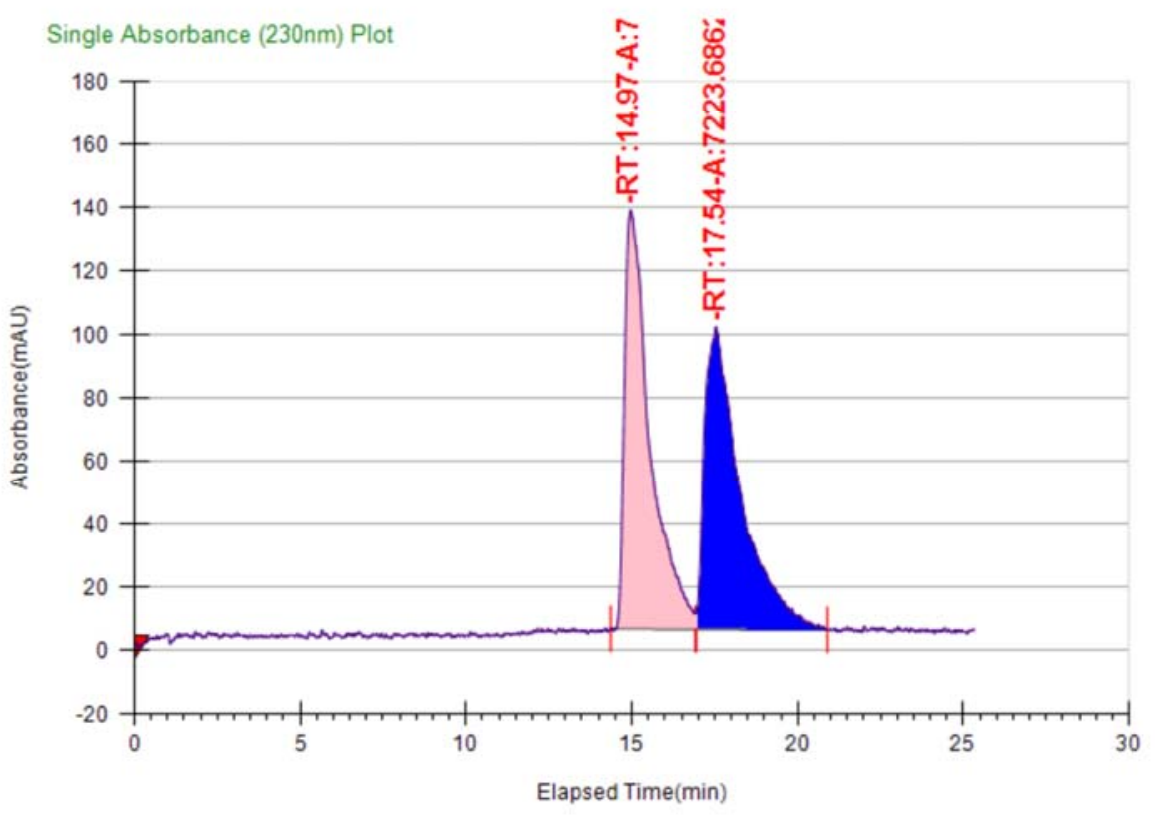

\begin{tabular}{|l|l|l|l|l|}
\hline Co-Solvent \% & Total Flow & Column & Co-Solvent & Back Pressure \\
\hline 2 & 4 & OD-H & MeOH & 150 \\
\hline
\end{tabular}

\begin{tabular}{|l|l|l|l|}
\hline Peak \# & Ret. Time & Area & Area \% \\
\hline 1 & $14.92 \mathrm{~min}$ & 12623.8015 & 95.2661 \\
\hline 2 & $17.98 \mathrm{~min}$ & 627.2884 & 4.7339 \\
\hline
\end{tabular}

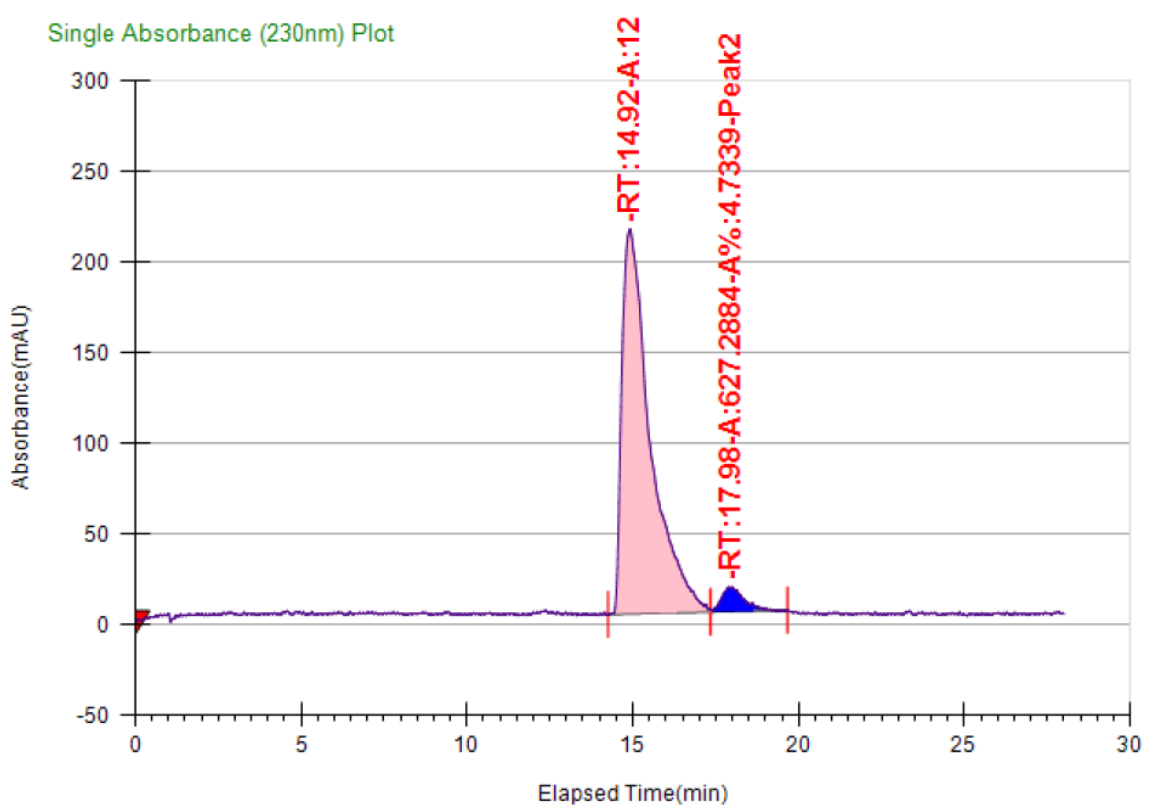




\begin{tabular}{|l|l|l|l|l|}
\hline Co-Solvent \% & Total Flow & Column & Co-Solvent & Back Pressure \\
\hline 1 & 4 & OD-H & $\mathrm{MeOH}$ & 150 \\
\hline
\end{tabular}

\begin{tabular}{|l|l|l|l|}
\hline Peak \# & Ret. Time & Area & Area \% \\
\hline 1 & $8.74 \mathrm{~min}$ & 7297.8228 & 49.6757 \\
\hline 2 & $10.73 \mathrm{~min}$ & 7393.0944 & 50.3243 \\
\hline
\end{tabular}

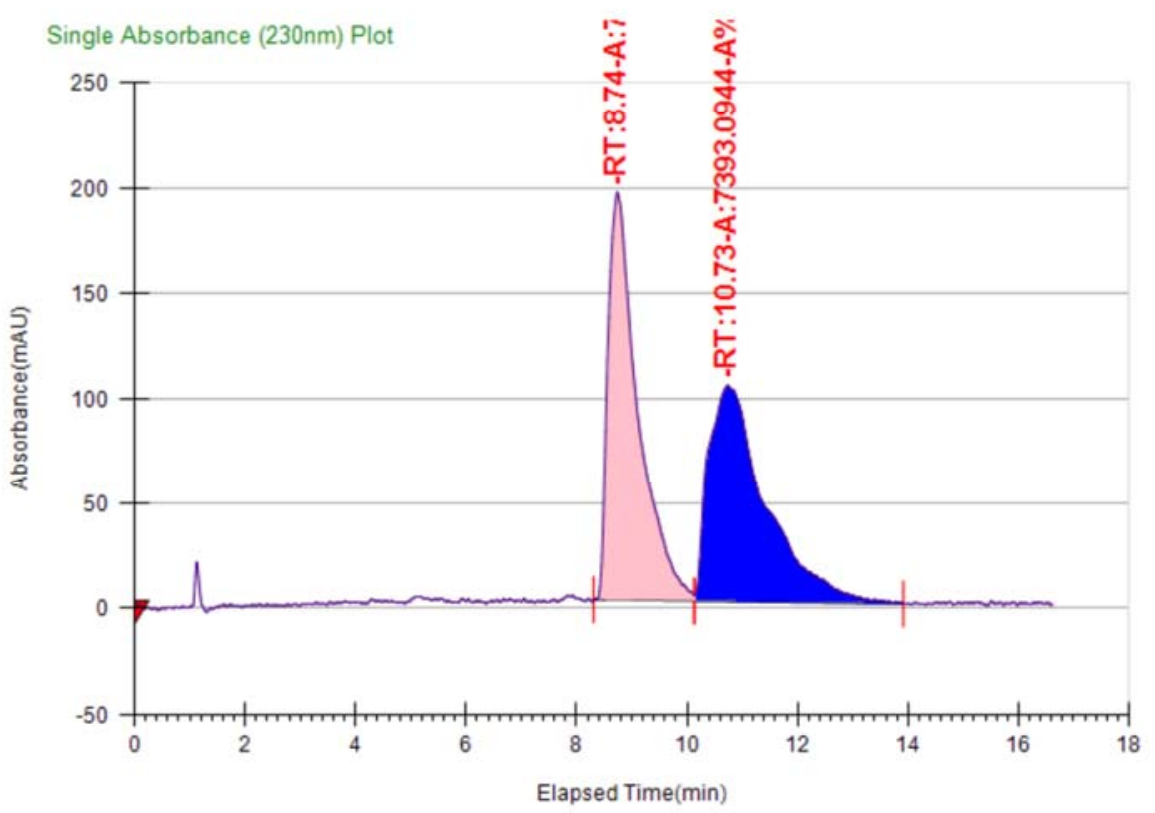

\begin{tabular}{|l|l|l|l|l|}
\hline Co-Solvent \% & Total Flow & Column & Co-Solvent & Back Pressure \\
\hline 1 & 4 & OD-H & MeOH & 150 \\
\hline
\end{tabular}

\begin{tabular}{|l|l|l|l|}
\hline Peak \# & Ret. Time & Area & Area \% \\
\hline 1 & $8.49 \mathrm{~min}$ & 11936.998 & 96.0158 \\
\hline 2 & $11.13 \mathrm{~min}$ & 495.3293 & 3.9842 \\
\hline
\end{tabular}

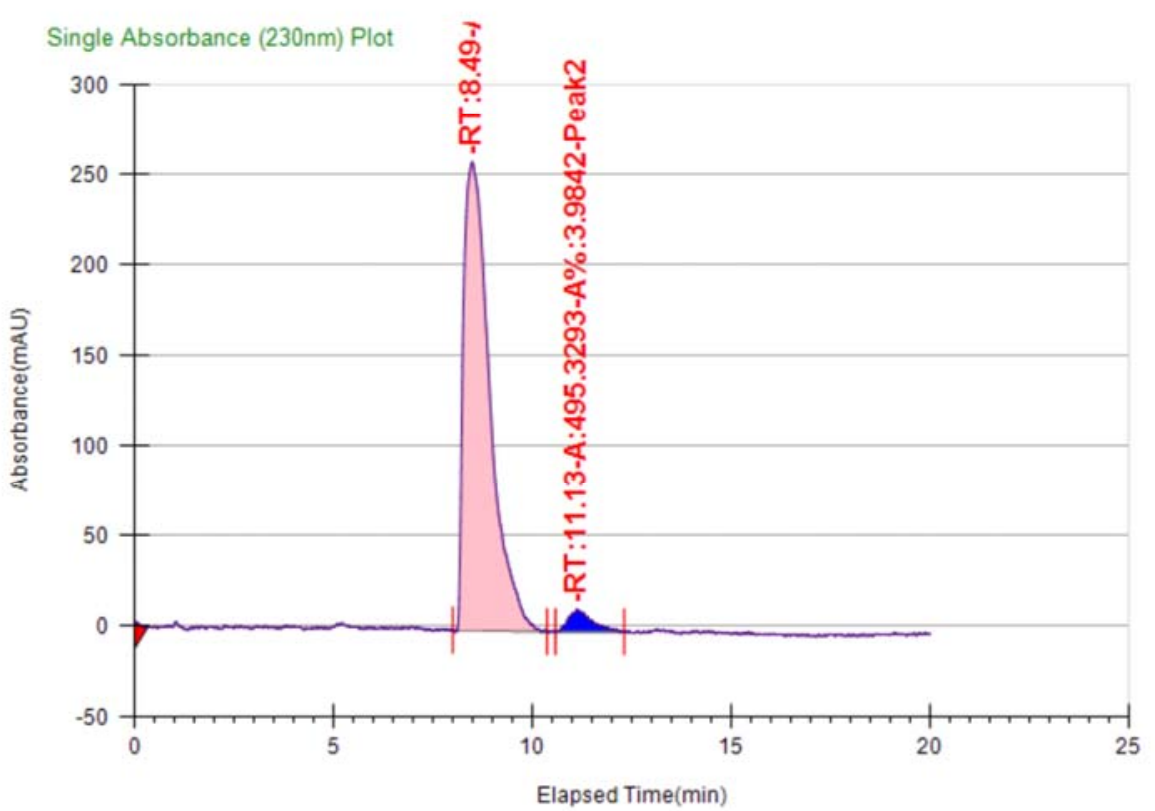




\begin{tabular}{|l|l|l|l|l|}
\hline Co-Solvent \% & Total Flow & Column & Co-Solvent & Back Pressure \\
\hline 4 & 4 & AD-H & MeOH & 150 \\
\hline
\end{tabular}

\begin{tabular}{|l|l|l|l|}
\hline Peak \# & Ret. Time & Area & Area \% \\
\hline 1 & $10.87 \mathrm{~min}$ & 6959.295 & 50.6234 \\
\hline 2 & $13.46 \mathrm{~min}$ & 6787.9046 & 49.3766 \\
\hline
\end{tabular}

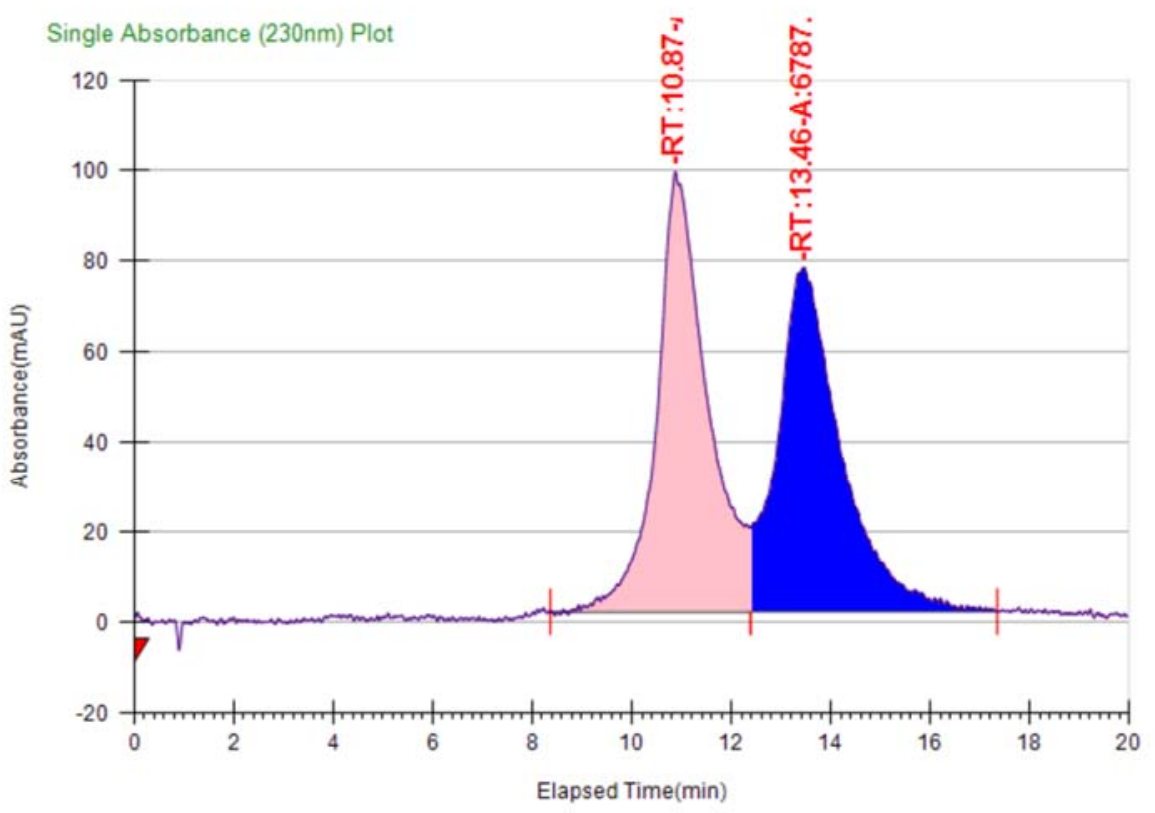

\begin{tabular}{|l|l|l|l|l|}
\hline Co-Solvent \% & Total Flow & Column & Co-Solvent & Back Pressure \\
\hline 4 & 4 & AD-H & MeOH & 150 \\
\hline
\end{tabular}

\begin{tabular}{|l|l|l|l|}
\hline Peak \# & Ret. Time & Area & Area \% \\
\hline 1 & $10.88 \mathrm{~min}$ & 12782.7327 & 93.4135 \\
\hline 2 & $13.81 \mathrm{~min}$ & 901.2929 & 6.5865 \\
\hline
\end{tabular}

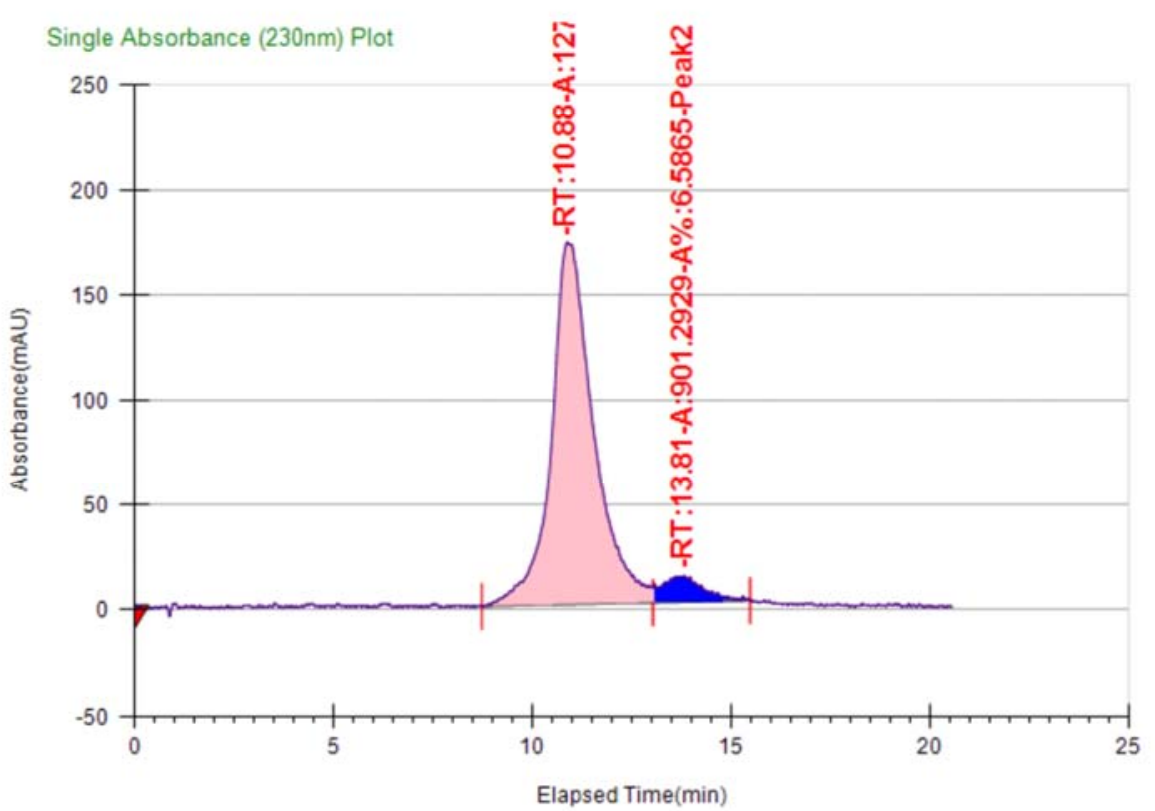




\begin{tabular}{|l|l|l|l|l|}
\hline Co-Solvent \% & Total Flow & Column & Co-Solvent & Back Pressure \\
\hline 3 & 4 & OD-H & MeOH & 150 \\
\hline
\end{tabular}

\begin{tabular}{|l|l|l|l|}
\hline Peak \# & Ret. Time & Area & Area \% \\
\hline 1 & $15.64 \mathrm{~min}$ & 12951.1001 & 48.908 \\
\hline 2 & $17.01 \mathrm{~min}$ & 13529.4378 & 51.092 \\
\hline
\end{tabular}

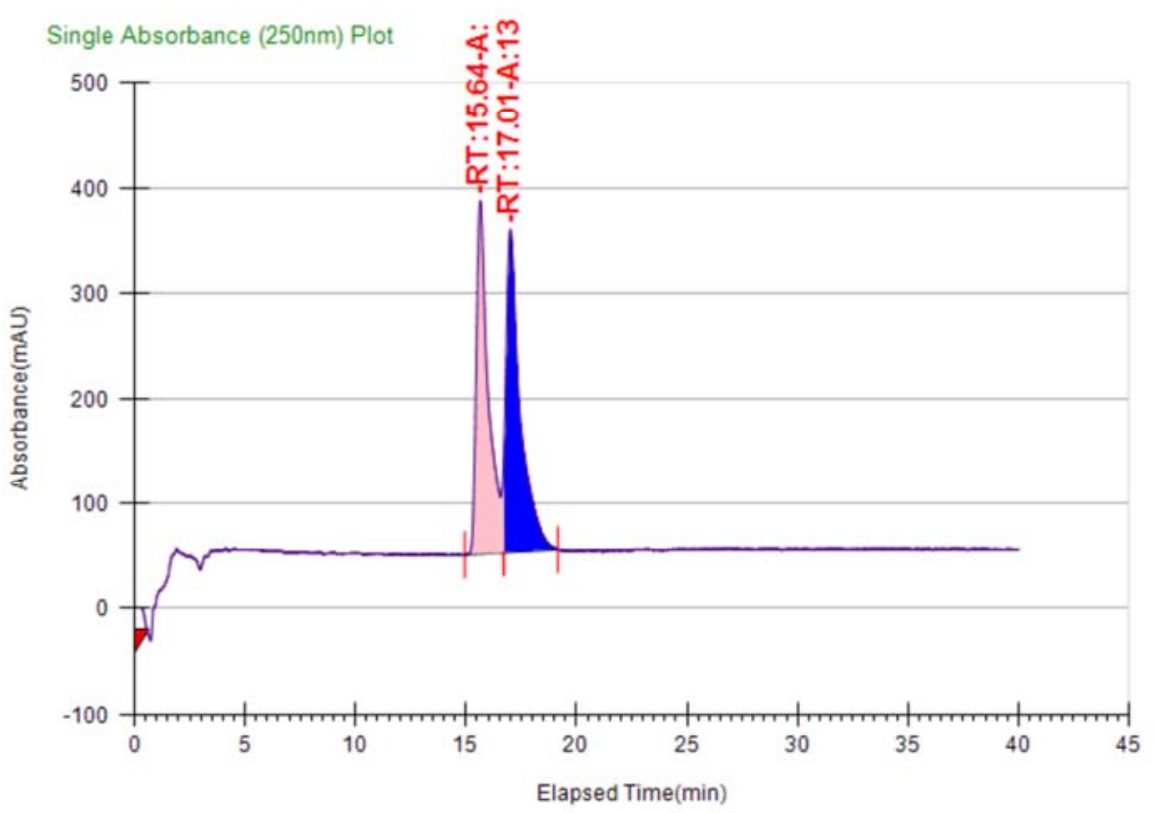

\begin{tabular}{|l|l|l|l|l|}
\hline Co-Solvent \% & Total Flow & Column & Co-Solvent & Back Pressure \\
\hline 3 & 4 & OD-H & MeOH & 150 \\
\hline
\end{tabular}

\begin{tabular}{|l|l|l|l|}
\hline Peak \# & Ret. Time & Area & Area \% \\
\hline 1 & $15.09 \mathrm{~min}$ & 924.5007 & 5.1003 \\
\hline 2 & $16.27 \mathrm{~min}$ & 17202.0641 & 94.8997 \\
\hline
\end{tabular}

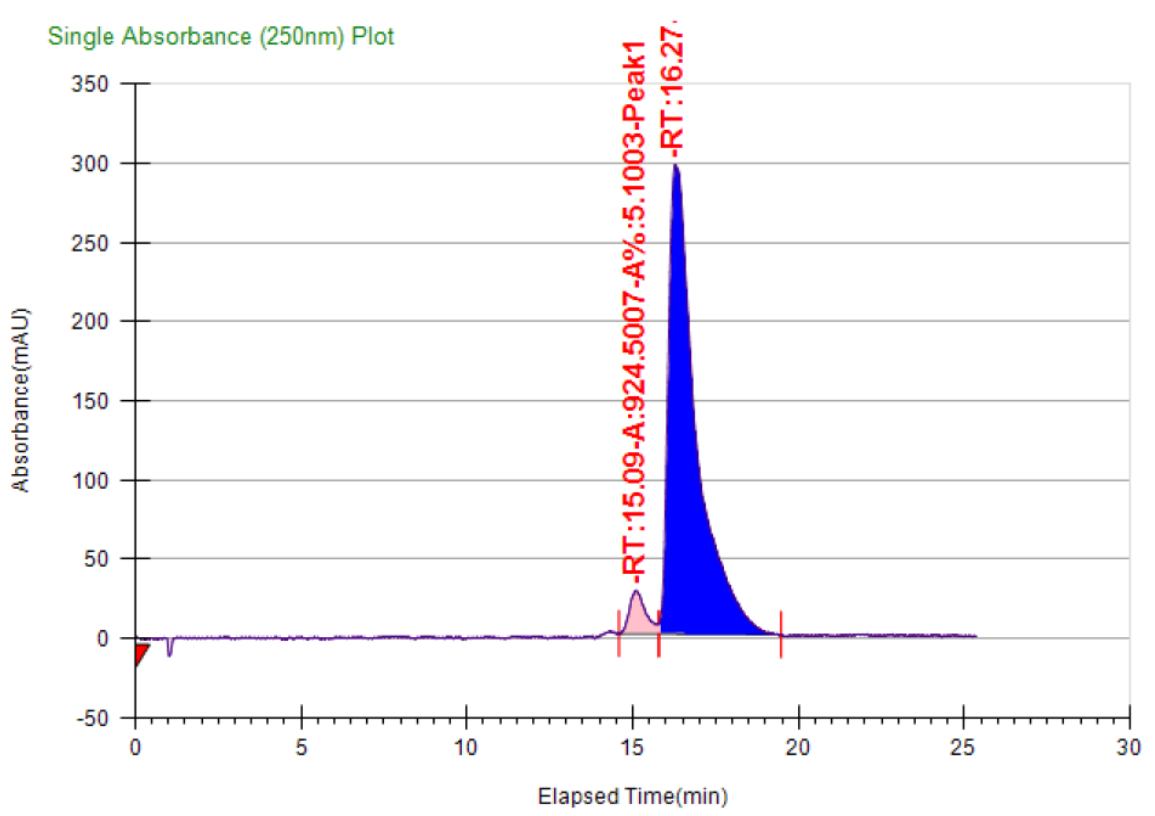




\begin{tabular}{|l|l|l|l|l|}
\hline Co-Solvent \% & Total Flow & Column & Co-Solvent & Back Pressure \\
\hline 3 & 4 & OD-H & MeOH & 150 \\
\hline
\end{tabular}

\begin{tabular}{|l|l|l|l|}
\hline Peak \# & Ret. Time & Area & Area \% \\
\hline 1 & $2.14 \mathrm{~min}$ & 1790.6996 & 49.8772 \\
\hline 2 & $2.87 \mathrm{~min}$ & 1799.5184 & 50.1228 \\
\hline
\end{tabular}

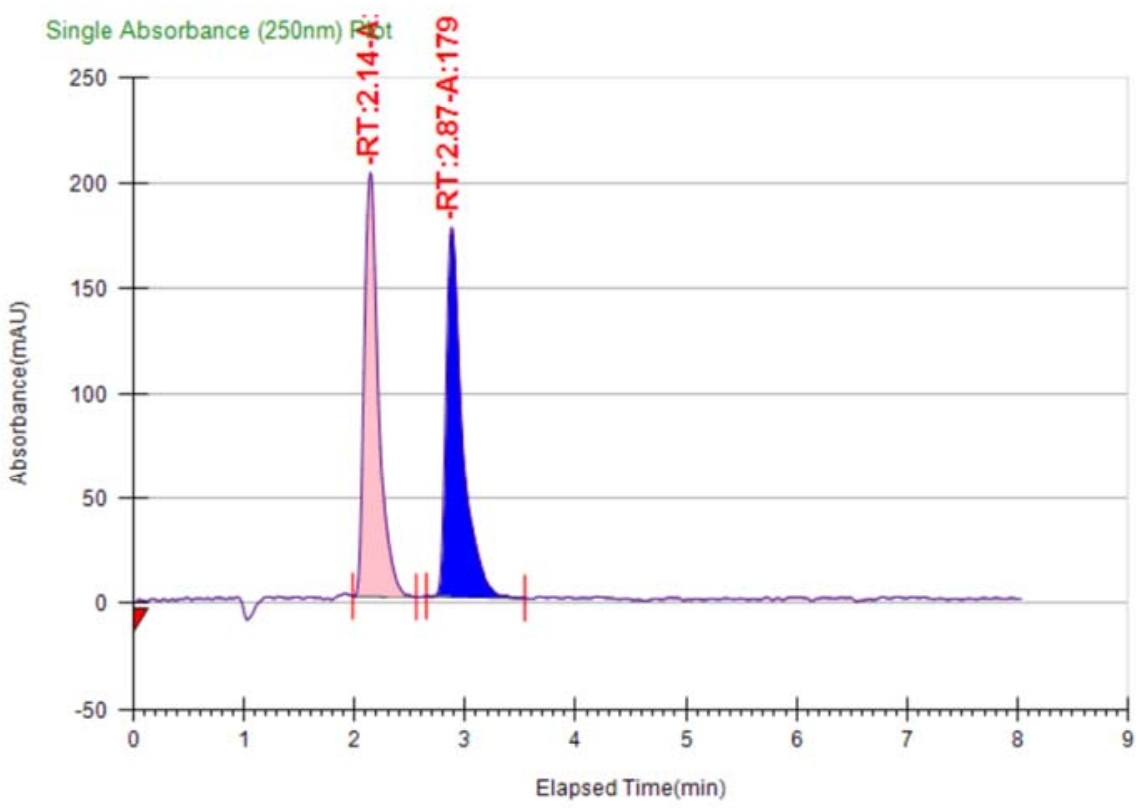

\begin{tabular}{|l|l|l|l|l|}
\hline Co-Solvent \% & Total Flow & Column & Co-Solvent & Back Pressure \\
\hline 3 & 4 & OD-H & MeOH & 150 \\
\hline
\end{tabular}

\begin{tabular}{|l|l|l|l|}
\hline Peak \# & Ret. Time & Area & Area \% \\
\hline 1 & $2.15 \mathrm{~min}$ & 144.1413 & 8.4962 \\
\hline 2 & $2.87 \mathrm{~min}$ & 1552.4022 & 91.5038 \\
\hline
\end{tabular}

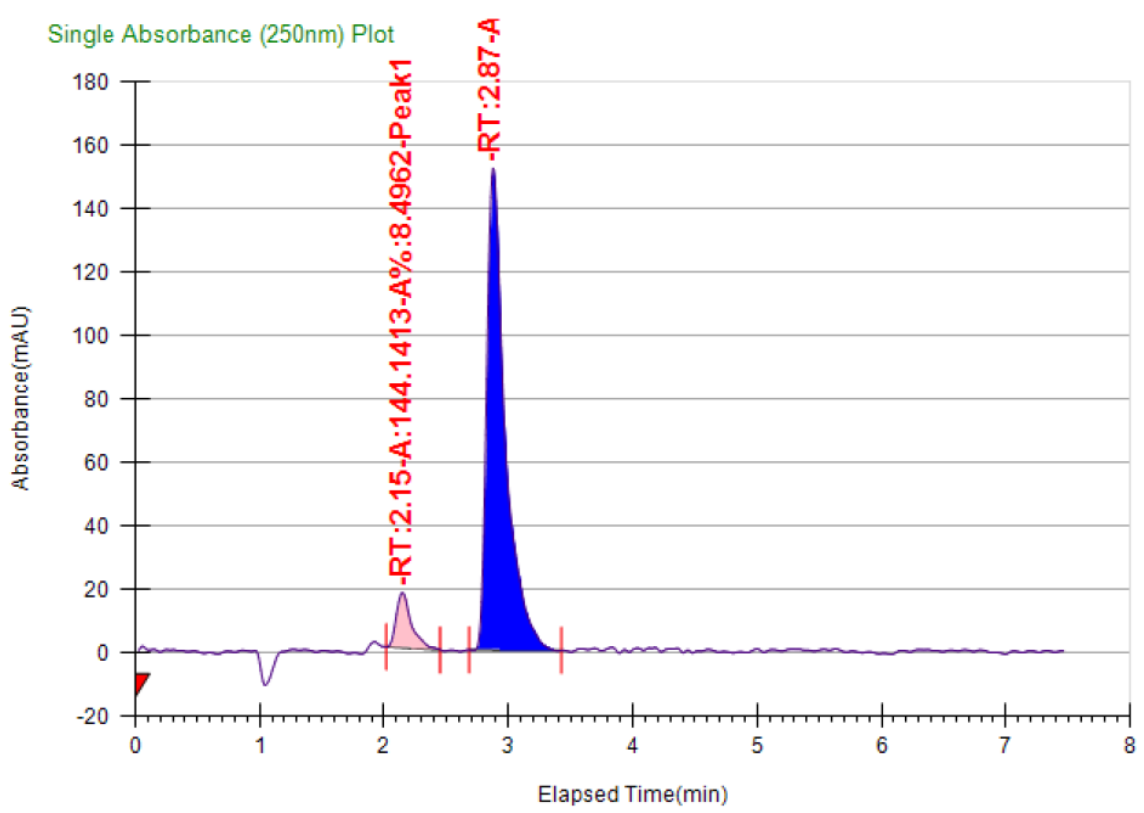




\begin{tabular}{|c|c|c|c|c|}
\hline Co-Solvent \% & Total Flow & Column & Co-Solvent & Back Pressure \\
\hline 2 & 4 & OD-H & $\mathrm{MeOH}$ & 150 \\
\hline Peak \# & Ret. Time & Area & Area $\%$ & \\
\hline 1 & $14.54 \mathrm{~min}$ & 53945.9053 & \begin{tabular}{l|l}
3 & 48.2535
\end{tabular} & \\
\hline 2 & $16.58 \mathrm{~min}$ & 57850.9139 & \begin{tabular}{l|l}
9 & 51.7465
\end{tabular} & \\
\hline
\end{tabular}

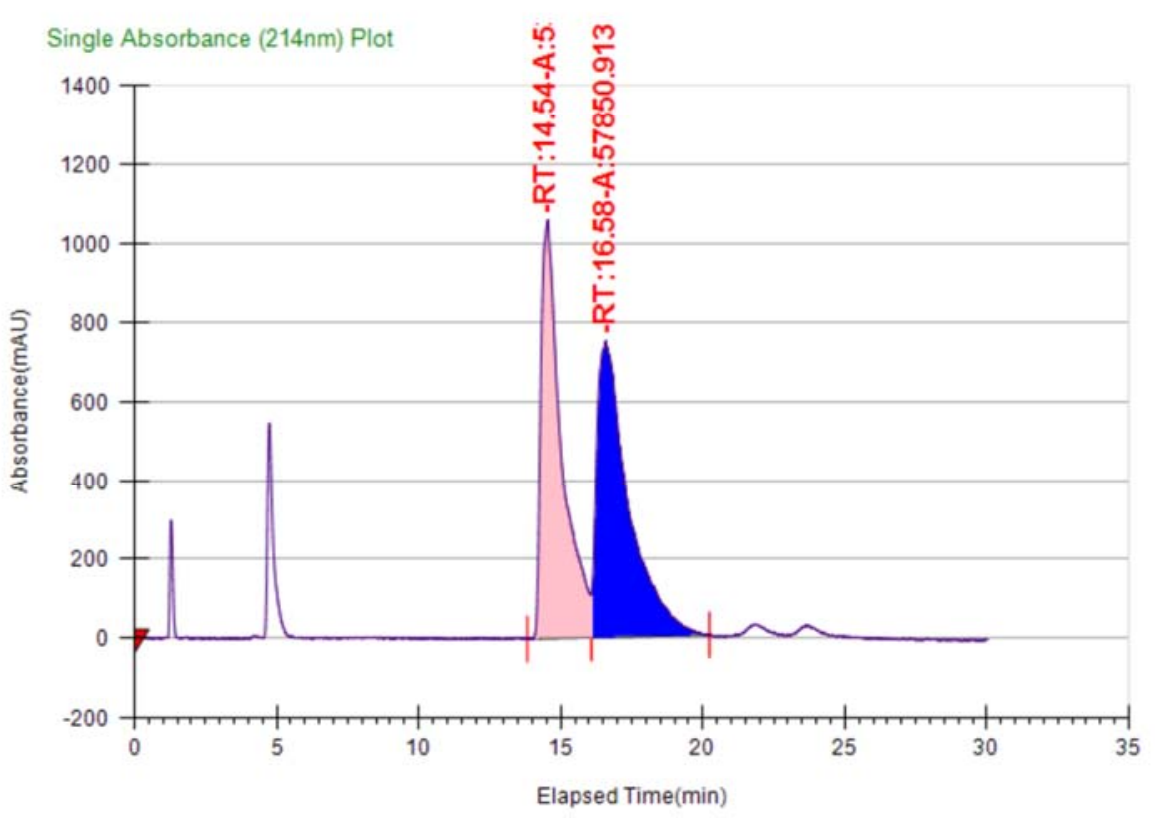

\begin{tabular}{|l|l|l|l|l|}
\hline Co-Solvent \% & Total Flow & Column & Co-Solvent & Back Pressure \\
\hline 2 & 4 & OD-H & MeOH & 150 \\
\hline
\end{tabular}

\begin{tabular}{|l|l|l|l|}
\hline Peak \# & Ret. Time & Area & Area \% \\
\hline 1 & $14.93 \mathrm{~min}$ & 1167.5326 & 9.1401 \\
\hline 2 & $17.24 \mathrm{~min}$ & 11606.1485 & 90.8599 \\
\hline
\end{tabular}

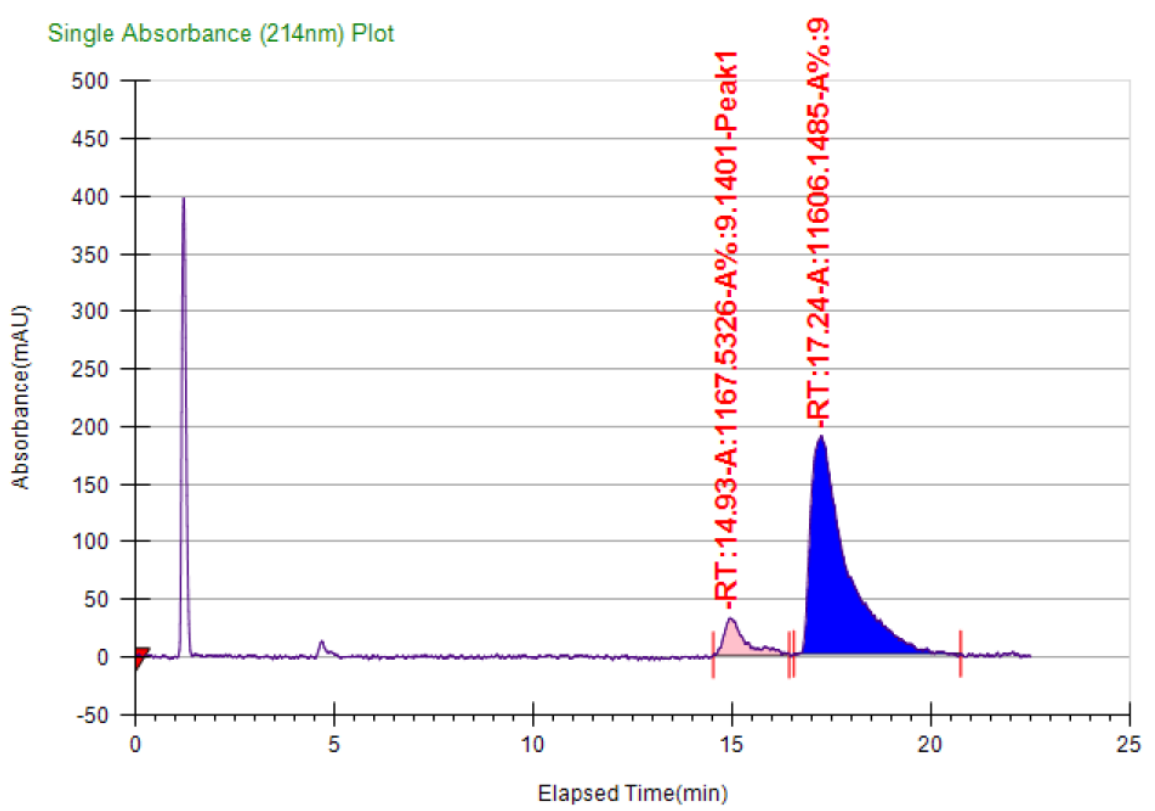




\begin{tabular}{|l|l|l|l|l|}
\hline Co-Solvent \% & Total Flow & Column & Co-Solvent & Back Pressure \\
\hline 3 & 4 & OD-H & MeOH & 150 \\
\hline
\end{tabular}

\begin{tabular}{|l|l|l|l|}
\hline Peak \# & Ret. Time & Area & Area \% \\
\hline 1 & $6.68 \mathrm{~min}$ & 10869.8029 & 49.6253 \\
\hline 2 & $7.75 \mathrm{~min}$ & 11033.9709 & 50.3747 \\
\hline
\end{tabular}

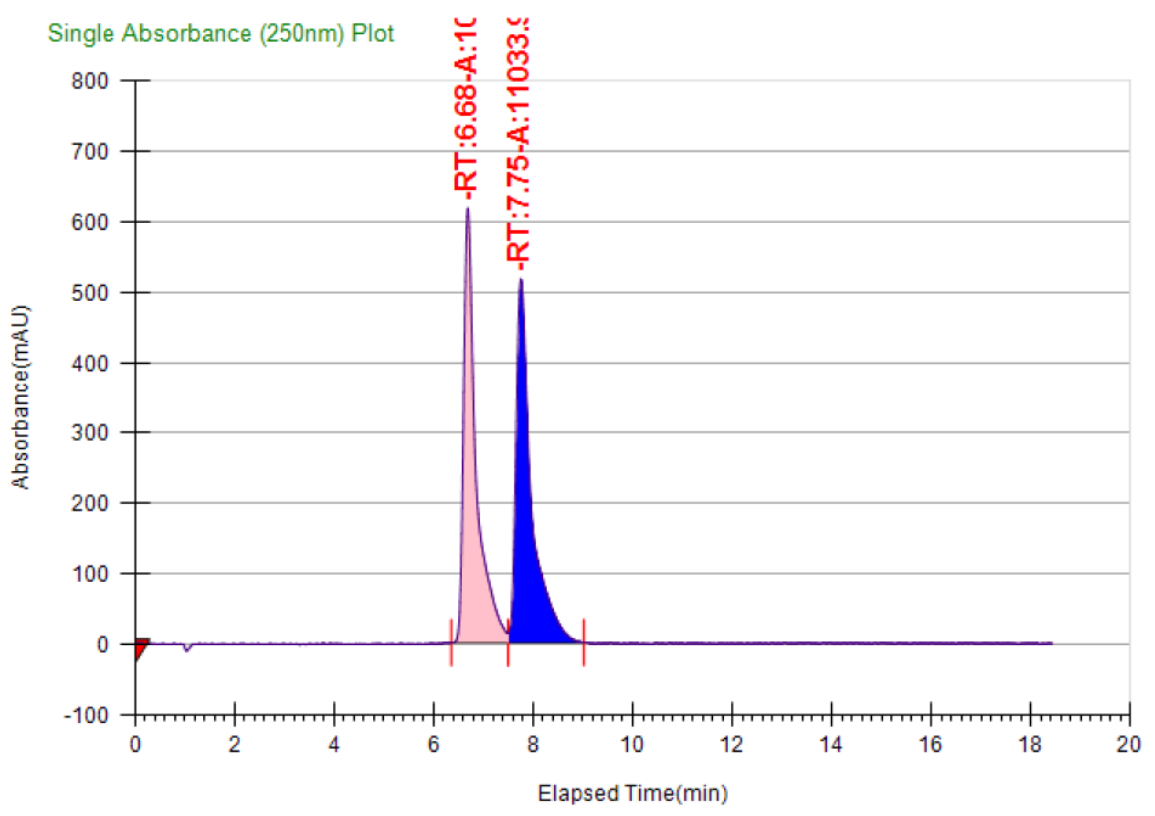

\begin{tabular}{|l|l|l|l|l|}
\hline Co-Solvent \% & Total Flow & Column & Co-Solvent & Back Pressure \\
\hline 3 & 4 & OD-H & MeOH & 150 \\
\hline
\end{tabular}

\begin{tabular}{|l|l|l|l|}
\hline Peak \# & Ret. Time & Area & Area \% \\
\hline 1 & $6.82 \mathrm{~min}$ & 1321.9463 & 8.7227 \\
\hline 2 & $7.87 \mathrm{~min}$ & 13833.3115 & 91.2773 \\
\hline
\end{tabular}

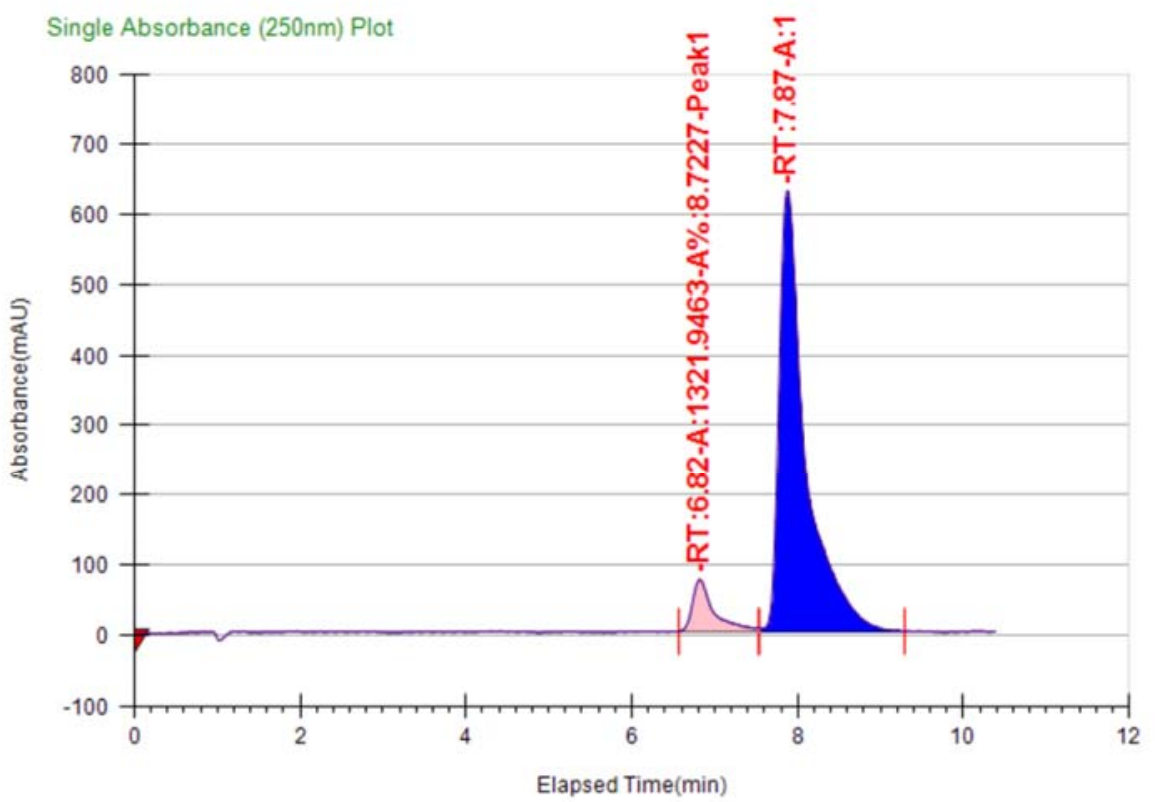




\begin{tabular}{|l|l|l|l|l|}
\hline Co-Solvent \% & Total Flow & Column & Co-Solvent & Back Pressure \\
\hline 5 & 4 & OD-H & MeOH & 150 \\
\hline
\end{tabular}

\begin{tabular}{|l|l|l|l|}
\hline Peak \# & Ret. Time & Area & Area \% \\
\hline 1 & $5.92 \mathrm{~min}$ & 15539.2102 & 49.9007 \\
\hline 2 & $7.25 \mathrm{~min}$ & 15601.0509 & 50.0993 \\
\hline
\end{tabular}

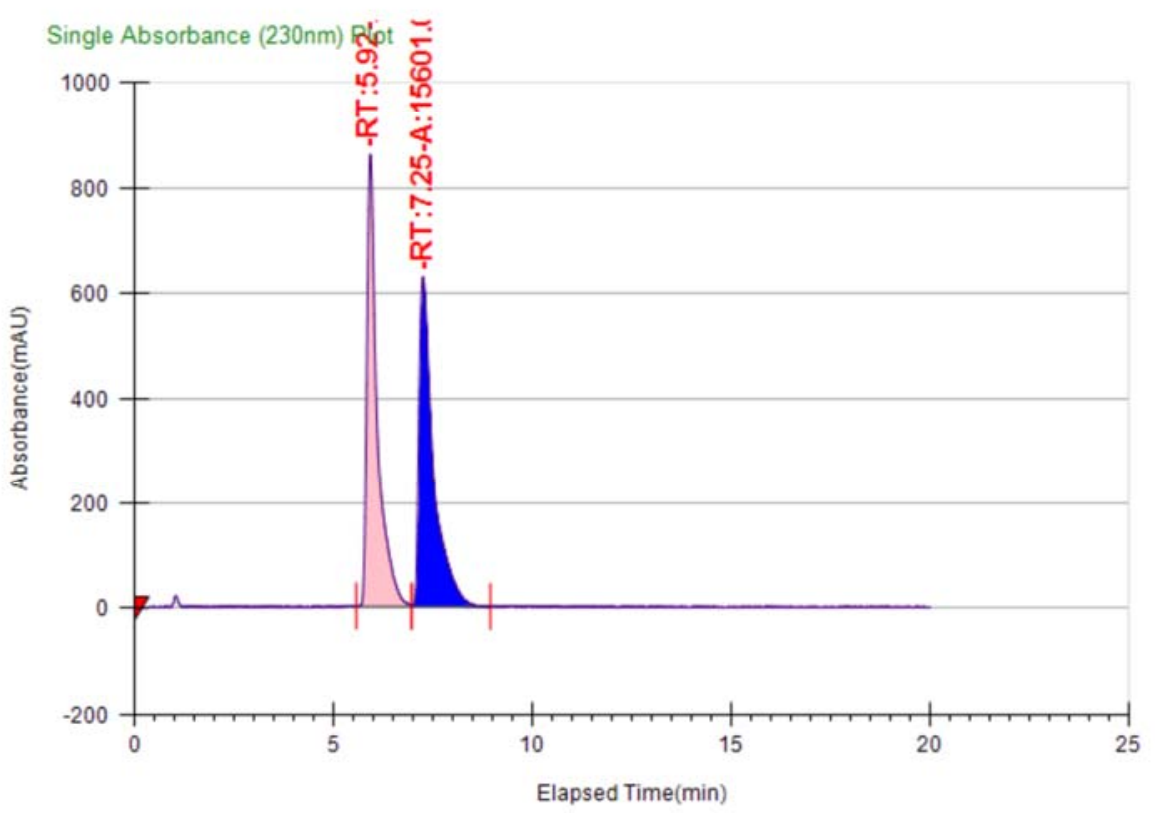

\begin{tabular}{|l|l|l|l|l|}
\hline Co-Solvent \% & Total Flow & Column & Co-Solvent & Back Pressure \\
\hline 5 & 4 & OD-H & MeOH & 150 \\
\hline
\end{tabular}

\begin{tabular}{|l|l|l|l|}
\hline Peak \# & Ret. Time & Area & Area \% \\
\hline 1 & $5.86 \mathrm{~min}$ & 503.5873 & 9.5745 \\
\hline 2 & $7.23 \mathrm{~min}$ & 4756.1023 & 90.4255 \\
\hline
\end{tabular}

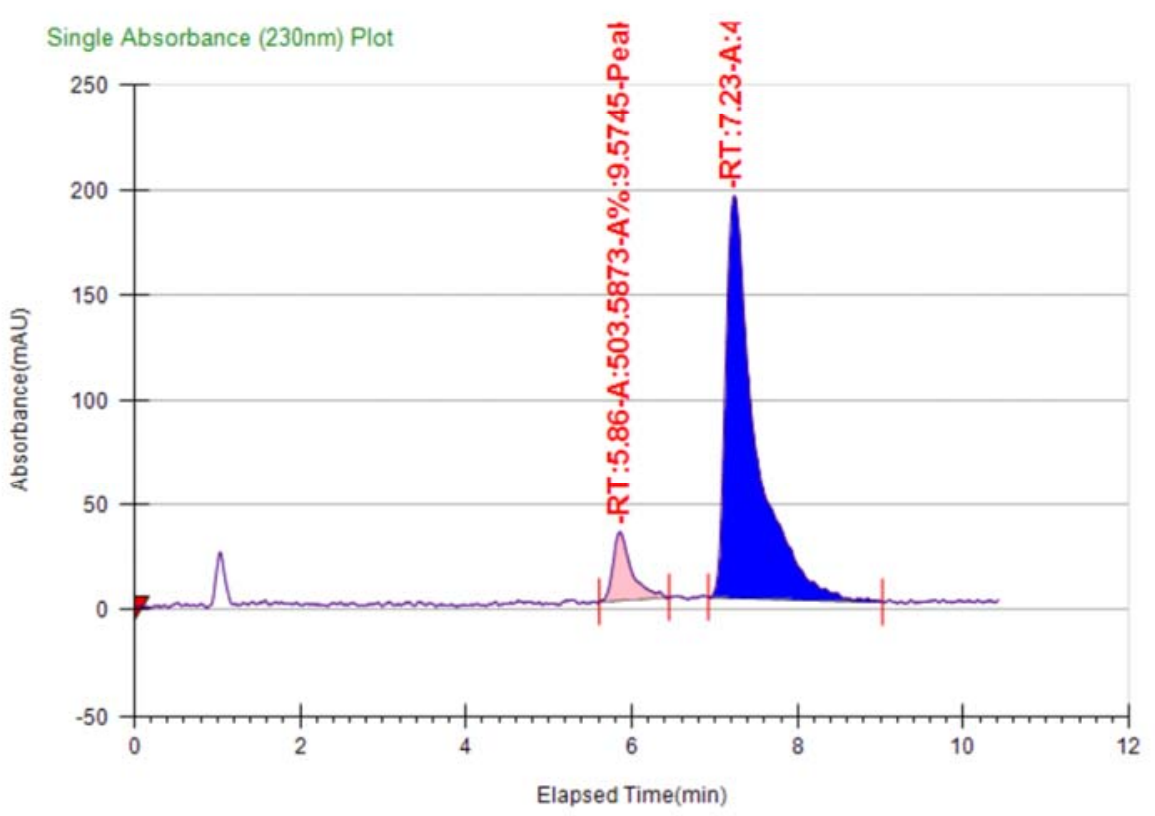




\begin{tabular}{|l|l|l|l|l|}
\hline Co-Solvent \% & Total Flow & Column & Co-Solvent & Back Pressure \\
\hline 2 & 4 & OD-H & MeOH & 150 \\
\hline
\end{tabular}

\begin{tabular}{|l|l|l|l|}
\hline Peak \# & Ret. Time & Area & Area \% \\
\hline 1 & $7.81 \mathrm{~min}$ & 19884.8867 & 49.5722 \\
\hline 2 & $9.1 \mathrm{~min}$ & 20228.1175 & 50.4278 \\
\hline
\end{tabular}

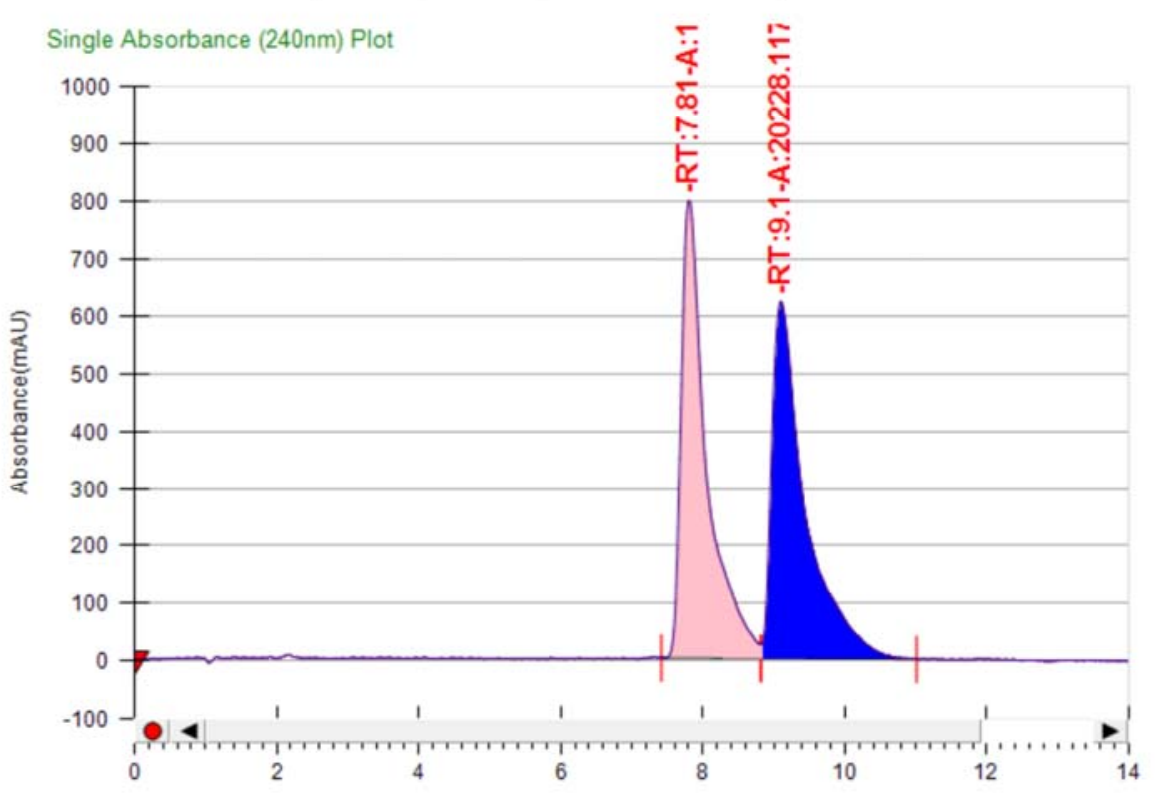

\begin{tabular}{|l|l|l|l|l|}
\hline Co-Solvent \% & Total Flow & Column & Co-Solvent & Back Pressure \\
\hline 2 & 4 & OD-H & MeOH & 150 \\
\hline
\end{tabular}

\begin{tabular}{|l|l|l|l|}
\hline Peak \# & Ret. Time & Area & Area \% \\
\hline 1 & $7.78 \mathrm{~min}$ & 6014.0351 & 17.6909 \\
\hline 2 & $9.01 \mathrm{~min}$ & 27980.9667 & 82.3091 \\
\hline
\end{tabular}

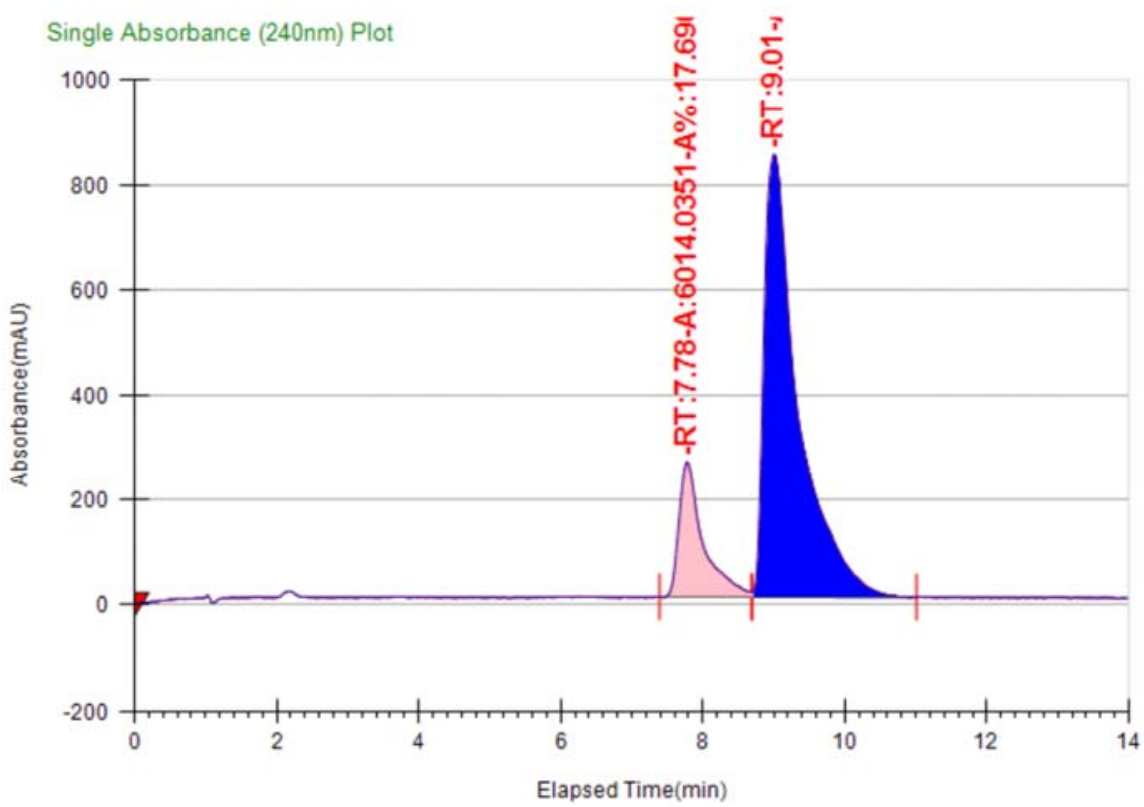




\begin{tabular}{|l|l|l|l|l|}
\hline Co-Solvent \% & Total Flow & Column & Co-Solvent & Back Pressure \\
\hline 5 & 4 & OD-H & MeOH & 150 \\
\hline
\end{tabular}

\begin{tabular}{|l|l|l|l|}
\hline Peak \# & Ret. Time & Area & Area \% \\
\hline 1 & $19.3 \mathrm{~min}$ & 10631.9492 & 49.5954 \\
\hline 2 & $22.59 \mathrm{~min}$ & 10805.4363 & 50.4046 \\
\hline
\end{tabular}
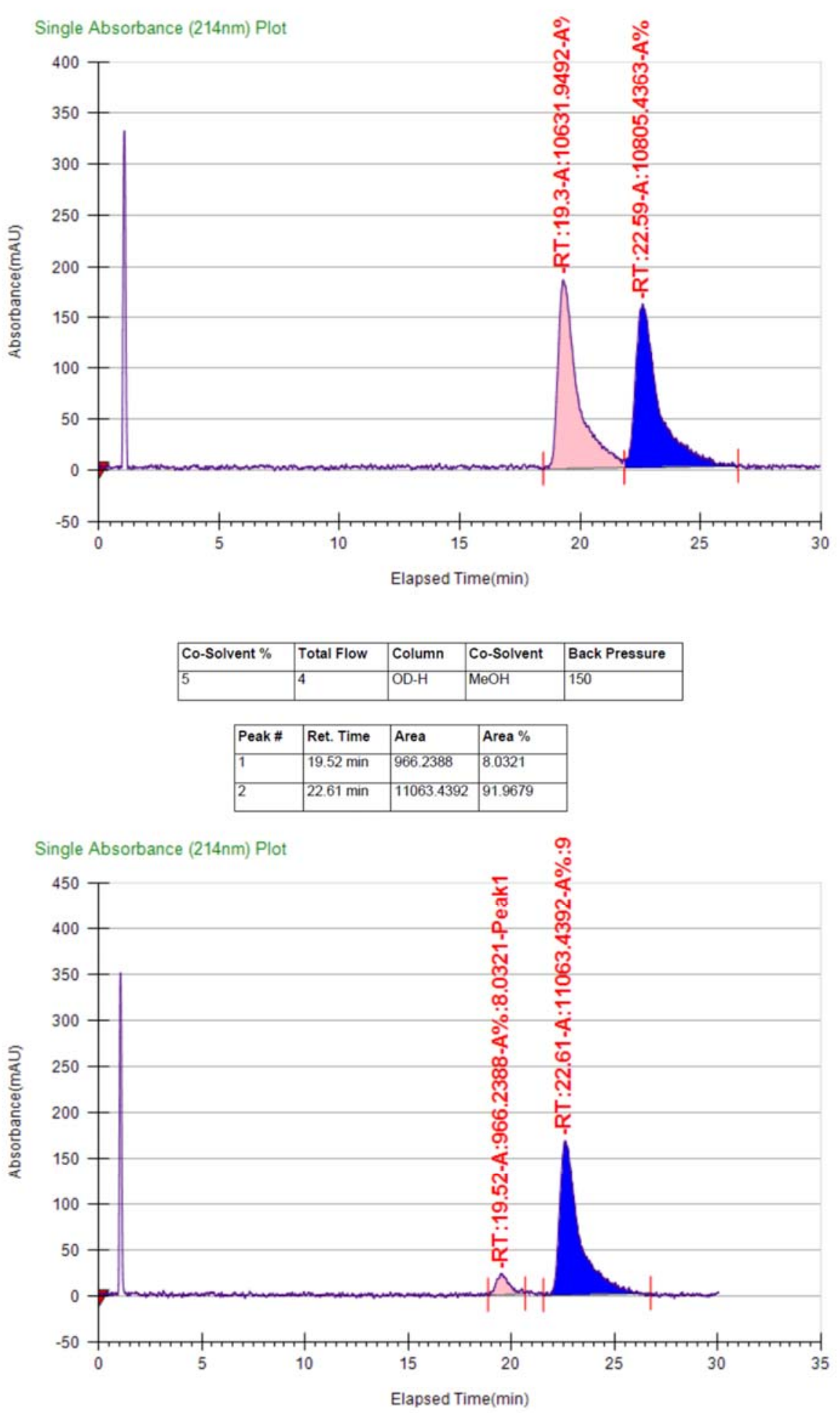


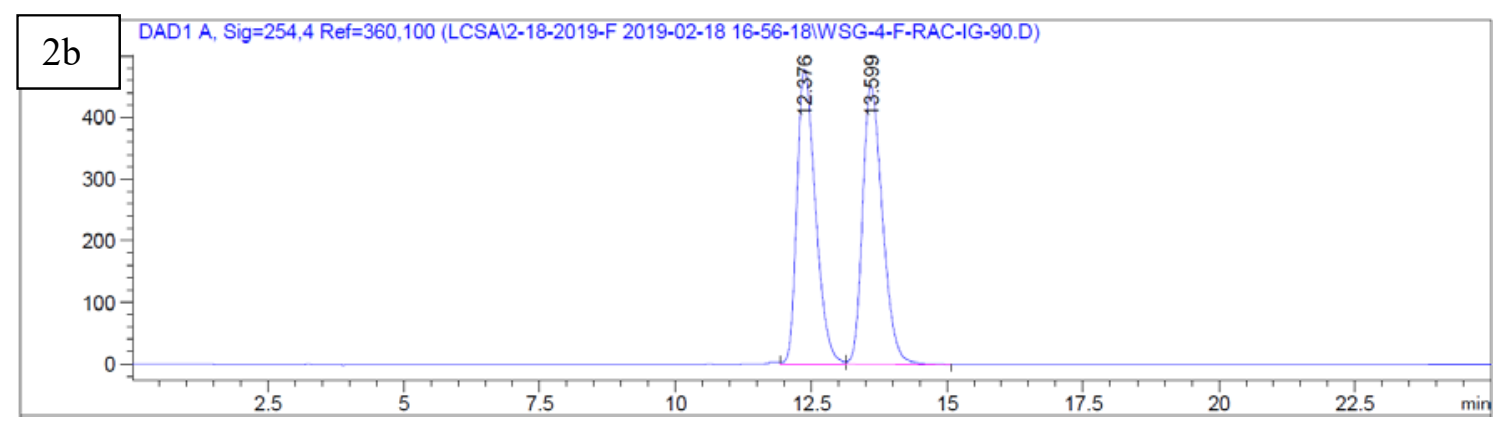

Signal 1: DAD1 A, Sig=254,4 Ref=360,100

\begin{tabular}{|c|c|c|c|c|c|c|}
\hline $\begin{array}{c}\text { Peak } \\
\#\end{array}$ & $\begin{array}{c}\text { RetTime } \\
\text { [min] }\end{array}$ & Type & $\begin{array}{l}\text { Width } \\
\text { [min] }\end{array}$ & $\begin{array}{c}\text { Area } \\
{\left[\mathrm{mAU}{ }^{\star} \mathrm{s}\right]}\end{array}$ & $\begin{array}{l}\text { Height } \\
{[\mathrm{mAU}]}\end{array}$ & $\begin{array}{c}\text { Area } \\
\frac{\%}{\circ}\end{array}$ \\
\hline & ------1 & & & 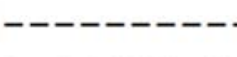 & - & $5---1$ \\
\hline 1 & 12.376 & VV & 0.3652 & $1.13402 \mathrm{e} 4$ & 477.30685 & 49.6616 \\
\hline 2 & 13.599 & VB & 0.3929 & $1.14948 \mathrm{e} 4$ & 451.68295 & 50.3384 \\
\hline
\end{tabular}

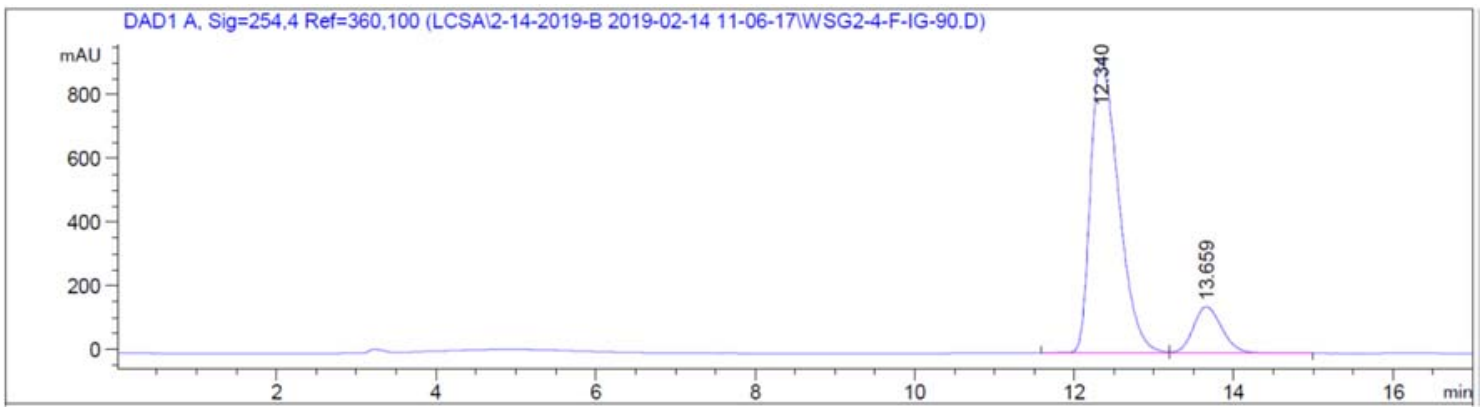

Signal 1: DAD1 A, Sig=254, 4 Ref $=360,100$

\begin{tabular}{|c|c|c|c|c|c|c|}
\hline $\begin{array}{c}\text { Peak } \\
\#\end{array}$ & $\begin{array}{c}\text { RetTime } \\
\text { [min] }\end{array}$ & Type & $\begin{array}{l}\text { Width } \\
\text { [min] }\end{array}$ & $\begin{array}{c}\text { Area } \\
{\left[\mathrm{mAU}{ }^{\star} \mathrm{s}\right]}\end{array}$ & $\begin{array}{l}\text { Height } \\
\text { [mAU] }\end{array}$ & $\begin{array}{c}\text { Area } \\
\quad \%\end{array}$ \\
\hline 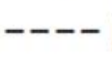 & ------- & 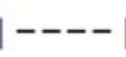 & 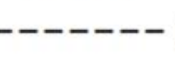 & $------\mid$ & --- & ---1 \\
\hline 1 & 12.340 & BV & 810 & $2.25855 \mathrm{e} 4$ & 924.66925 & 9959 \\
\hline 2 & 13.659 & VB & 0.3909 & 3677.96924 & 146.51579 & 0041 \\
\hline
\end{tabular}




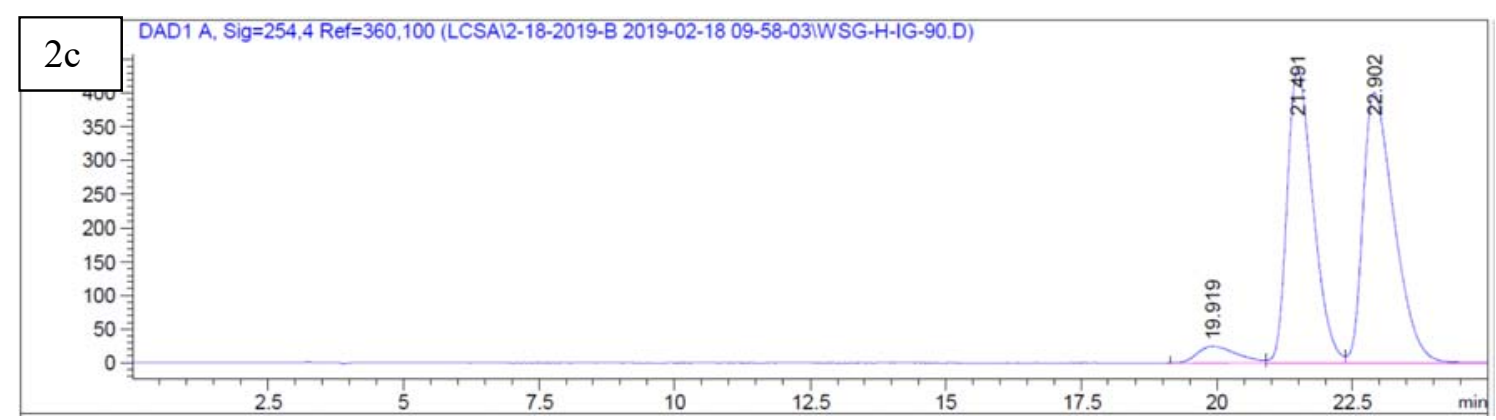

Signal 1: DAD1 A, Sig=254,4 Ref=360,100

\begin{tabular}{|c|c|c|c|c|c|c|}
\hline $\begin{array}{c}\text { Peak } \\
\#\end{array}$ & $\begin{array}{c}\text { RetTime } \\
\text { [min] }\end{array}$ & Type & $\begin{array}{c}\text { Width } \\
\text { [min] }\end{array}$ & $\begin{array}{c}\text { Area } \\
{\left[\mathrm{mAU}{ }^{\star} \mathrm{S}\right]}\end{array}$ & $\begin{array}{c}\text { Height } \\
{[\mathrm{mAU}]}\end{array}$ & $\begin{array}{c}\text { Area } \\
\frac{\circ}{8}\end{array}$ \\
\hline & & & & --------- & $---------\mid$ & \\
\hline 1 & 19.919 & BV & 0.6926 & 1255.76184 & 24.88947 & 3.9478 \\
\hline 2 & 21.491 & VV & 0.5115 & $1.45645 \mathrm{e} 4$ & 436.23581 & 45.7875 \\
\hline 3 & 22.902 & VBA & 0.6047 & $1.59887 \mathrm{e} 4$ & 401.39572 & 50.2647 \\
\hline
\end{tabular}

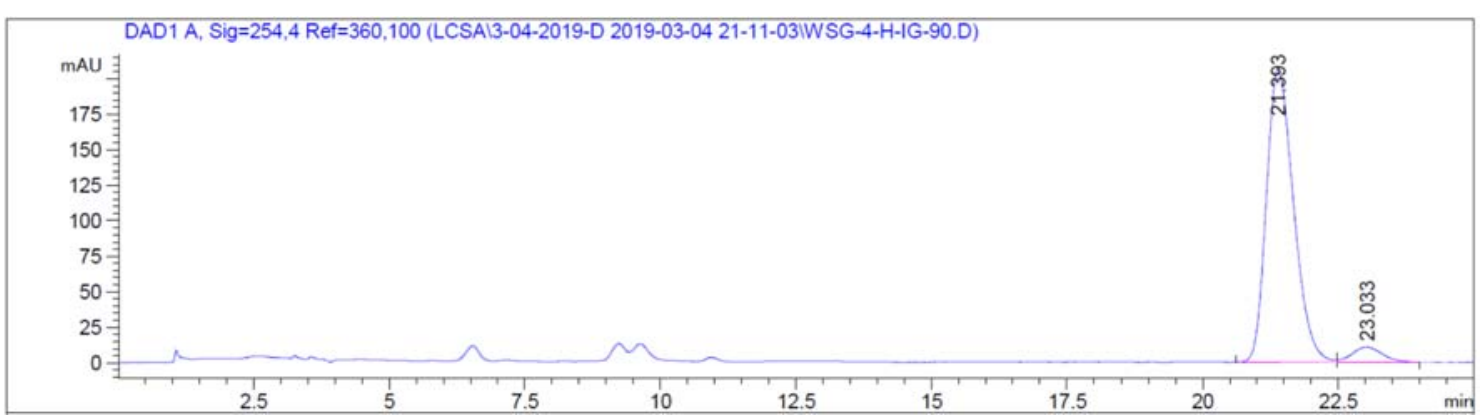

Signal 1: DAD1 A, Sig=254, 4 Ref=360,100

\begin{tabular}{|c|c|c|c|c|c|c|}
\hline $\begin{array}{c}\text { Peak } \\
\#\end{array}$ & $\begin{array}{c}\text { RetTime } \\
\text { [min] }\end{array}$ & Type & $\begin{array}{l}\text { Width } \\
\text { [min] }\end{array}$ & $\begin{array}{c}\text { Area } \\
{\left[\mathrm{mAU}^{\star} \mathrm{s}\right]}\end{array}$ & $\begin{array}{l}\text { Height } \\
{[\mathrm{mAU}]}\end{array}$ & $\begin{array}{c}\text { Area } \\
\frac{\circ}{8}\end{array}$ \\
\hline & & & & ---------- & ---------- & $--------\mid$ \\
\hline 1 & 21.393 & BV & 0.5361 & 7184.48193 & 206.40697 & 94.6993 \\
\hline 2 & 23.033 & VB & 0.4899 & 402.14401 & 10.52244 & 5.3007 \\
\hline
\end{tabular}




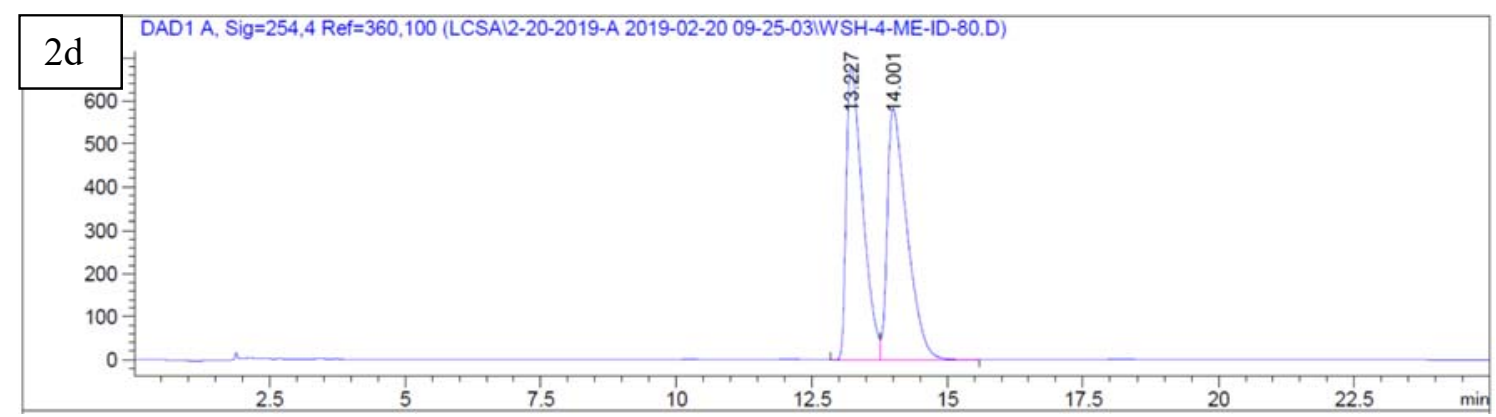

Signal 1: DAD1 A, Sig=254,4 Ref=360,100

\begin{tabular}{|c|c|c|c|c|c|c|}
\hline $\begin{array}{c}\text { Peak } \\
\#\end{array}$ & $\begin{array}{c}\text { RetTime } \\
\text { [min] }\end{array}$ & Type & $\begin{array}{l}\text { Width } \\
\text { [min] }\end{array}$ & $\begin{array}{c}\text { Area } \\
{\left[\mathrm{mAU}{ }^{*} \mathrm{~s}\right]}\end{array}$ & $\begin{array}{l}\text { Height } \\
{[\mathrm{mAU}]}\end{array}$ & $\begin{array}{c}\text { Area } \\
\frac{\circ}{8}\end{array}$ \\
\hline & & & & ---------- & ---------- & $--------\mid$ \\
\hline 1 & 13.227 & $\mathrm{BV}$ & 0.3163 & $1.42588 \mathrm{e} 4$ & 680.90033 & 49.2167 \\
\hline 2 & 14.001 & VB & 0.3731 & $1.47127 \mathrm{e} 4$ & 581.74835 & 50.7833 \\
\hline
\end{tabular}

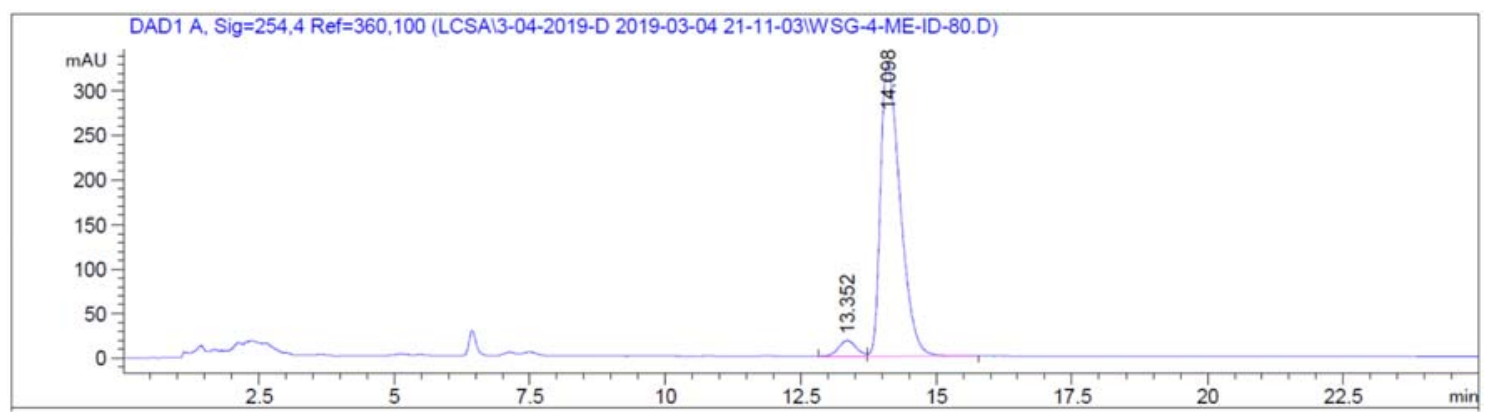

Signal 1: DAD1 A, Sig=254, 4 Ref=360,100

\begin{tabular}{|c|c|c|c|c|c|c|}
\hline $\begin{array}{c}\text { Peak } \\
\#\end{array}$ & $\begin{array}{c}\text { RetTime } \\
\text { [min] }\end{array}$ & Type & $\begin{array}{l}\text { Width } \\
\text { [min] }\end{array}$ & $\begin{array}{c}\text { Area } \\
{\left[\mathrm{mAU}{ }^{*} \mathrm{~s}\right]}\end{array}$ & $\begin{array}{l}\text { Height } \\
\text { [mAU] }\end{array}$ & $\begin{array}{c}\text { Area } \\
\frac{8}{\delta}\end{array}$ \\
\hline & - & & -- & ---- & --- & ---- \\
\hline 1 & 13.352 & BV & 0.3428 & 389.47064 & 17.42265 & 4.4908 \\
\hline 2 & 14.098 & VB & 3885 & 8283.06836 & 328.18408 & 95.5092 \\
\hline
\end{tabular}




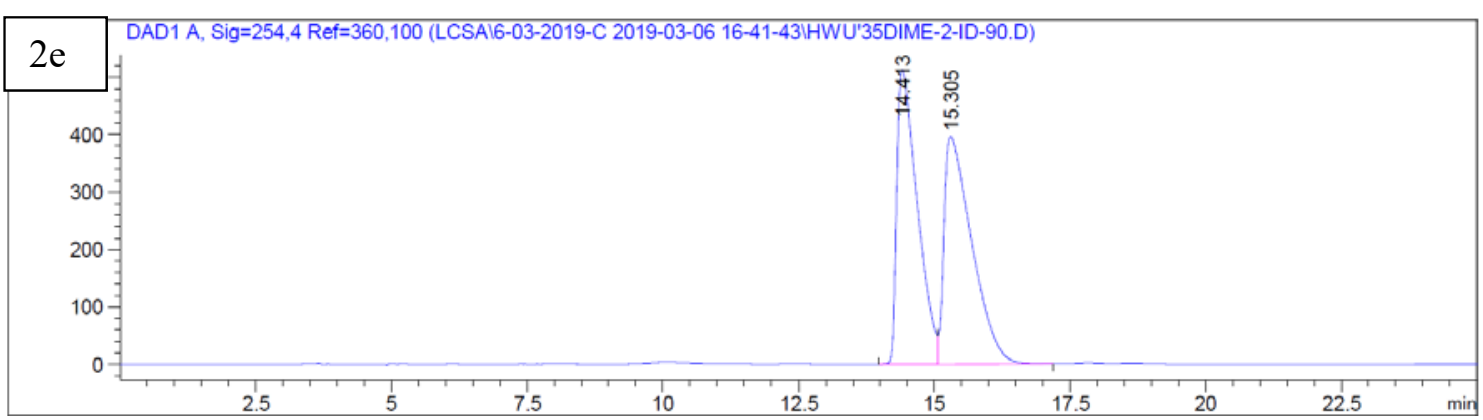

Signal 1: DAD1 A, Sig=254, 4 Ref=360,100

\begin{tabular}{|c|c|c|c|c|c|c|}
\hline $\begin{array}{c}\text { Peak } \\
\#\end{array}$ & $\begin{array}{c}\text { RetTime } \\
\text { [min] }\end{array}$ & Type & $\begin{array}{l}\text { Width } \\
\text { [min] }\end{array}$ & $\begin{array}{c}\text { Area } \\
{\left[\mathrm{mAU}{ }^{\star} \mathrm{s}\right]}\end{array}$ & $\begin{array}{l}\text { Height } \\
\text { [mAU] }\end{array}$ & $\begin{array}{c}\text { Area } \\
\frac{\circ}{\circ}\end{array}$ \\
\hline & & & & $\mid---------$ & ---------- & $-------\mid$ \\
\hline 1 & 14.413 & BV & 0.3967 & $1.34666 \mathrm{e} 4$ & 512.27045 & 48.7829 \\
\hline 2 & 15.305 & VB & 0.5204 & $1.41385 \mathrm{e} 4$ & 396.17529 & 51.2171 \\
\hline
\end{tabular}

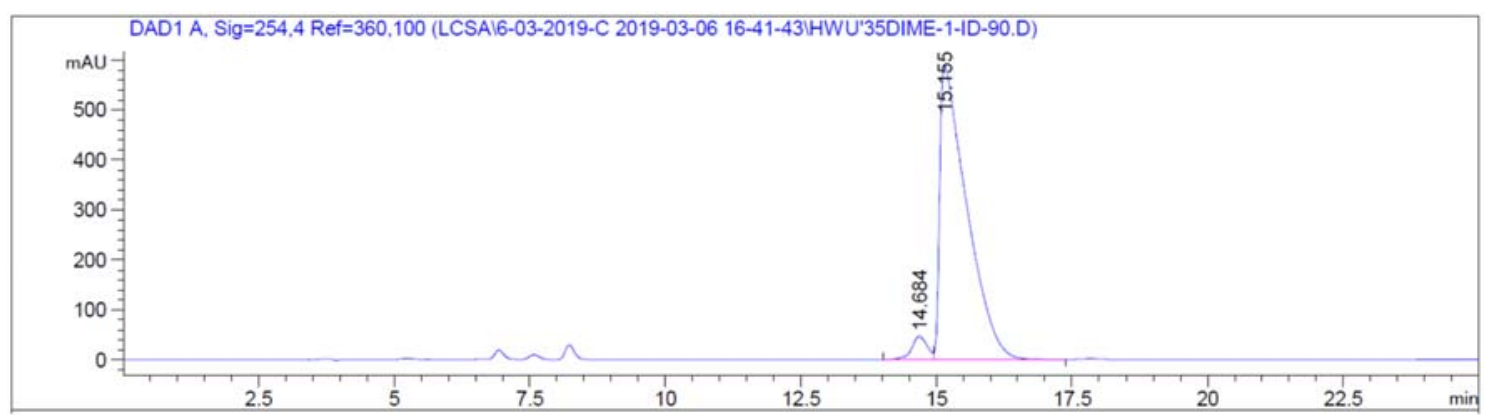

Signal 1: DAD1 A, Sig=254,4 Ref=360,100

\begin{tabular}{|c|c|c|c|c|c|c|}
\hline $\begin{array}{c}\text { Peak } \\
\#\end{array}$ & $\begin{array}{c}\text { RetTime } \\
\text { [min] }\end{array}$ & Type & $\begin{array}{l}\text { Width } \\
\text { [min] }\end{array}$ & $\begin{array}{c}\text { Area } \\
{\left[\mathrm{mAU}{ }^{*} \mathrm{~s}\right]}\end{array}$ & $\begin{array}{l}\text { Height } \\
{[\mathrm{mAU}]}\end{array}$ & $\begin{array}{c}\text { Area } \\
\quad \%\end{array}$ \\
\hline--- & - & & $=-$ & ----- & --- & $-------\mid$ \\
\hline 1 & 14.684 & BV & 0.3094 & 930.72644 & 46.53011 & 4.3655 \\
\hline 2 & 15.155 & VB & 0.4909 & $2.03893 e 4$ & 587.54742 & 95.6345 \\
\hline
\end{tabular}




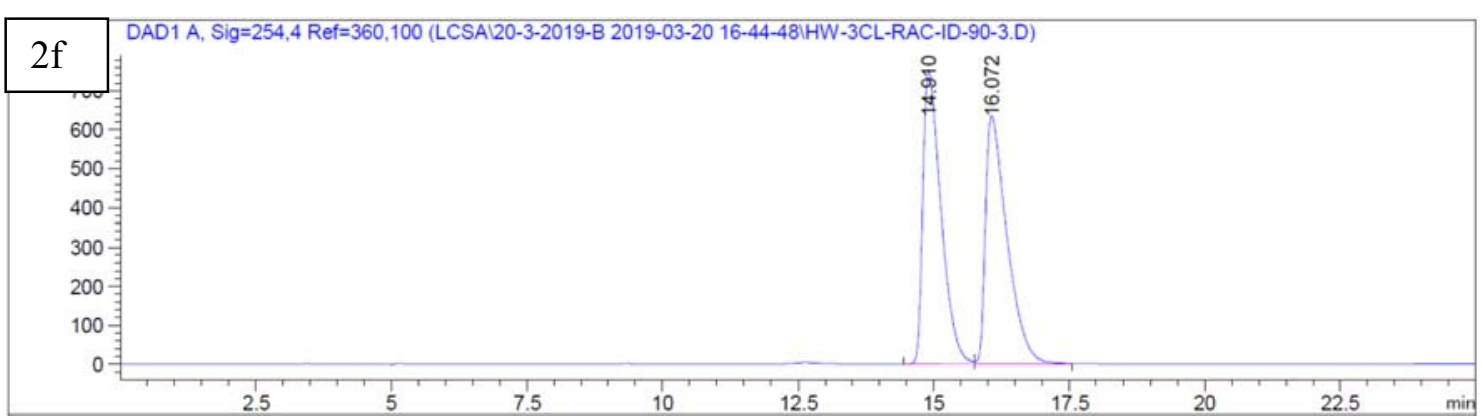

Signal 1: DAD1 A, Sig=254,4 Ref=360,100

\begin{tabular}{|c|c|c|c|c|c|c|}
\hline $\begin{array}{c}\text { Peak } \\
\#\end{array}$ & $\begin{array}{c}\text { RetTime } \\
\text { [min] }\end{array}$ & Type & $\begin{array}{l}\text { Width } \\
\text { [min] }\end{array}$ & $\begin{array}{c}\text { Area } \\
{\left[\mathrm{mAU}{ }^{\star} \mathrm{s}\right]}\end{array}$ & $\begin{array}{l}\text { Height } \\
{[\mathrm{mAU}]}\end{array}$ & $\begin{array}{c}\text { Area } \\
\quad \frac{\circ}{8}\end{array}$ \\
\hline & ----- & & & --------- & -------- & $------\mid$ \\
\hline 1 & 14.910 & BV & 0.3553 & $1.75244 \mathrm{e} 4$ & 753.62244 & 50.0311 \\
\hline 2 & 16.072 & VB & 0.4096 & $1.75026 \mathrm{e} 4$ & 635.01282 & 49.9689 \\
\hline
\end{tabular}

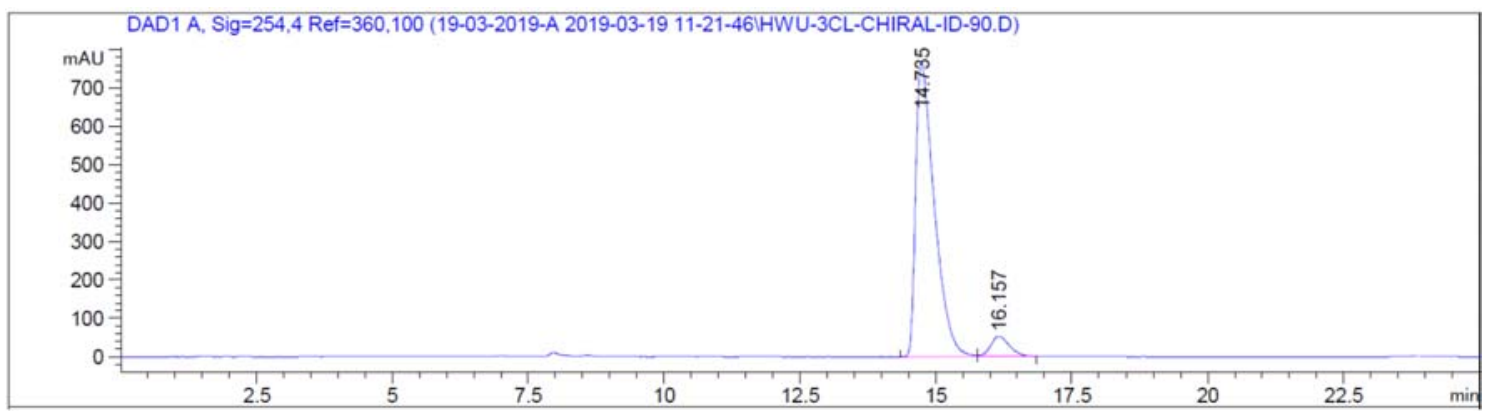

Signal 1: DAD1 A, Sig=254,4 Ref=360,100

\begin{tabular}{|c|c|c|c|c|c|c|}
\hline $\begin{array}{c}\text { Peak } \\
\#\end{array}$ & $\begin{array}{c}\text { RetTime } \\
\text { [min] }\end{array}$ & Type & $\begin{array}{l}\text { Width } \\
\text { [min] }\end{array}$ & $\begin{array}{c}\text { Area } \\
{\left[\mathrm{mAU}{ }^{\star} \mathrm{S}\right]}\end{array}$ & $\begin{array}{c}\text { Height } \\
\text { [mAU] }\end{array}$ & $\begin{array}{c}\text { Area } \\
\frac{\circ}{8}\end{array}$ \\
\hline & & & - & ---- & -1 & \\
\hline 1 & 14.735 & BV & 602 & $1.82907 \mathrm{e} 4$ & 767.04907 & 93.5391 \\
\hline 2 & 16.157 & VB & 0.3643 & 1263.36755 & 52.21482 & 6.4609 \\
\hline
\end{tabular}




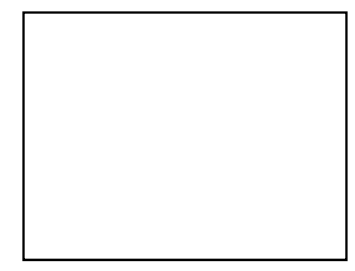

\begin{tabular}{|l|l|l|l|l|}
\hline Co-Solvent \% & Total Flow & Column & Co-Solvent & Back Pressure \\
\hline 5 & 4 & IA & MeOH & 150 \\
\hline
\end{tabular}
\begin{tabular}{|l|l|l|l|}
\hline Peak \# & Ret. Time & Area & Area \% \\
\hline 1 & $23.26 \mathrm{~min}$ & 1633.6367 & 11.6074 \\
\hline 2 & $25.87 \mathrm{~min}$ & 5324.9638 & 37.8351 \\
\hline 3 & $29.97 \mathrm{~min}$ & 1685.8677 & 11.9785 \\
\hline 4 & $31.95 \mathrm{~min}$ & 5429.6536 & 38.579 \\
\hline
\end{tabular}
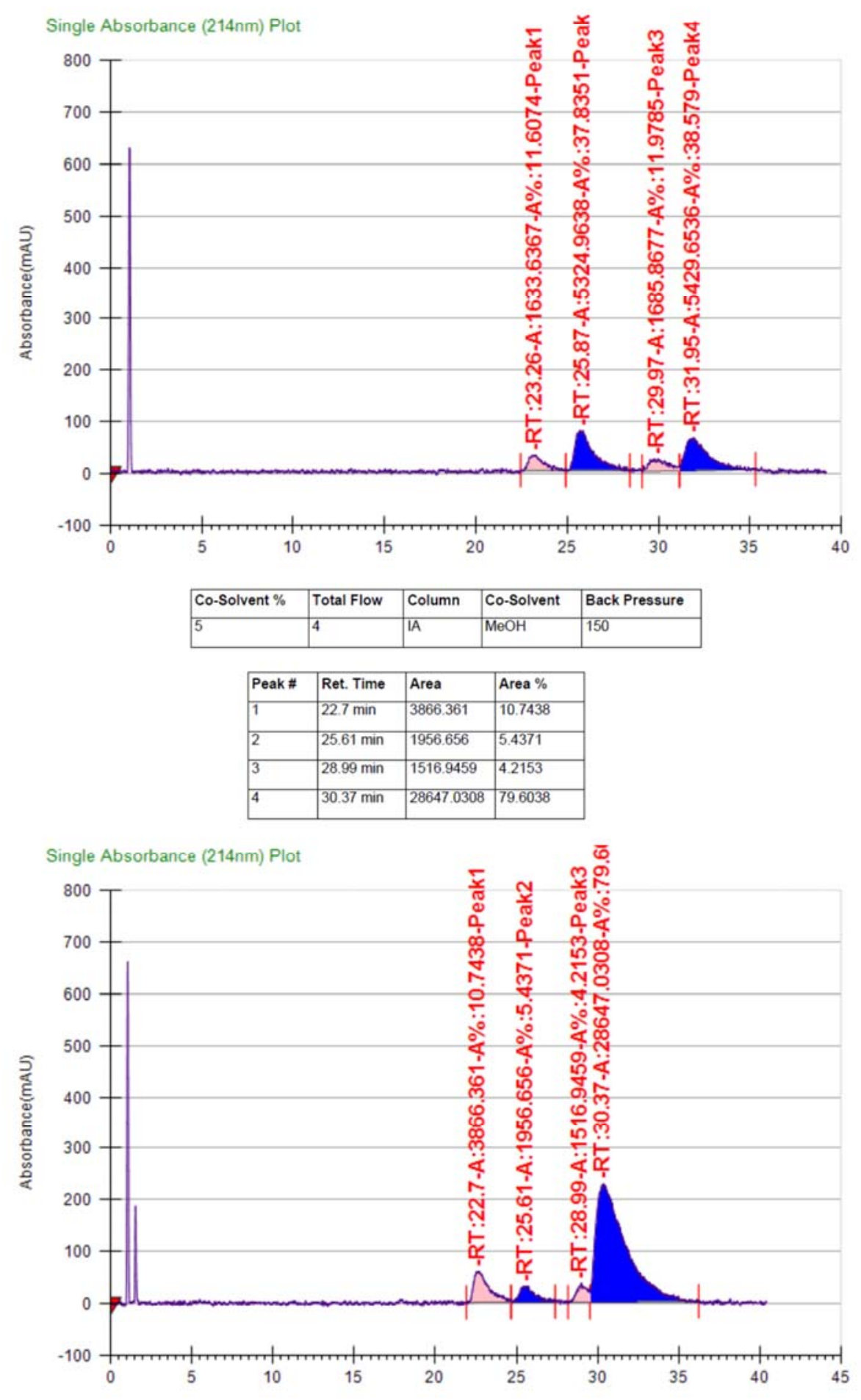


\section{References}

[1] González, M. J.; González, J.; Vicente, R. An Alternative Reaction Outcome in the GoldCatalyzed Rearrangement of 1-Alkynyloxiranes. Eur. J. Org. Chem. 2012, 6140.

[2] Mihovilovic, M. D.; Müller, B.; Kayser, M. M.; Stanetty, P. Microbial Baeyer-Villiger Oxidation of Bicyclo[4.3.0]ketones by Two Recombinant E. coli Strains. A Novel Access to Indole Alkaloids. Synlett 2002, 700.

[3] Mori, A.; Mizusaki, T.; Ikawa, T.; Maegawa, T.; Monguchi, Y.; Sajiki, H. Mechanistic Study of a Pd/C-Catalyzed Reduction of Aryl Sulfonates Using the Mg-MeOH-NH $4 \mathrm{OAc}$ System. Chem. Eur. J. 2007, 13, 1432.

[4] Guérard, K. C.; Sabot, C.; Racicot, L.; Canesi, S. Oxidative Friedel-Crafts Reaction and its Application to the Total Syntheses of Amaryllidaceae Alkaloids. J. Org. Chem. 2009, 74, 2039. [5] Li, L.; Zeng, M.; Herzon, S. B. Broad-Spectrum Catalysts for the Ambient Temperature Anti-Markovnikov Hydration of Alkynes. Angew. Chem. Int. Ed. 2014, 53, 7892.

[6] Das, M. K.; De, S.; Shubhashish; Bisai, A. Concise Total Syntheses of ( \pm )-Mesembrane and $( \pm)$-Crinane. Org. Biomol. Chem. 2015, 13, 3585.

[7] Verma, P.; Chandra, A.; Pandey, G. Diversity-Oriented Approach Toward the Syntheses of Amaryllidaceae Alkaloids via a Common Chiral Synthon. J. Org. Chem. 2018, 83, 9968. 